\title{
CUADERNOS
}

de

\section{INVESTIGACIÓN GEOGRÁFICA}

\section{GEOGRAPHICAL RESEARCH LETTERS}

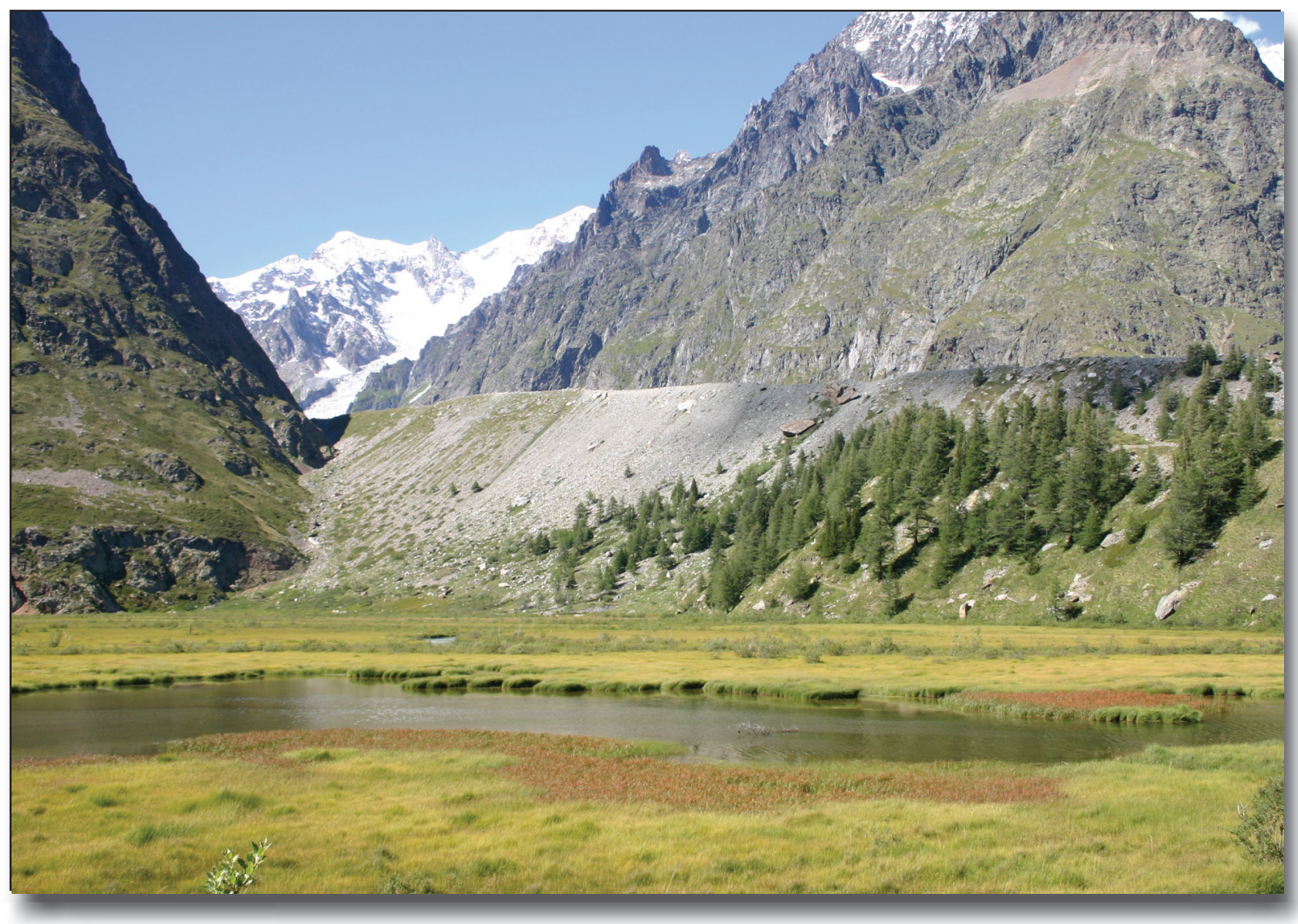

\section{UNIVERSIDAD DE LA RIOJA LOGROÑO (ESPAÑA)}




\section{CUADERNOS DE INVESTIGACIÓN GEOGRÁFICA GEOGRAPHICAL RESEARCH LETTERS}

Editor Principal / Editor-in-Chief

José Arnáez (Universidad de La Rioja)

Editores Adjuntos / Associate Editors

Jorge Lorenzo-Lacruz (Universidad de La Rioja)

Amelia Gómez-Villar (Universidad de León)

Noemí Lana-Renault Monreal (Universidad de La Rioja)

Purificación Ruiz-Flaño (Universidad de La Rioja)

\section{Consejo Editorial / Editorial Board}

S. Beguería (Estación Experimental de Aula Dei, CSIC) Spatial analysis, Climate change, Water resources management, Environmental hydrology

E. Cammeraat (Universiteit van Amsterdam, Holanda) Geomorphology, Soil Science, Soil Conservation

S.R. Fassnacht (Colorado State University, EEUU) Environmental hydrology, Snow hydrology, Hydrological Modeling

H. Holzmann (Universität für Bodenkultur Wien, Austria) Hydrological modeling, Surface hydrology, Soil hydrology, Snow Hydrology, Climate change

P. Hughes (University of Manchester, Reino Unido) Glacial and Periglacial Geomorphology, Climate change

V. Jomelli (Université Paris I, Francia) Climate change, Climatology, Paleoclimatology, Glaciology, Periglacial Geomorphology

J.J. Keizer (Universidade de Aveiro, Portugal) Ecological impact of wildfire, Soil erosion, Wind erosion

M.A. Lenzi (Universitá di Padova, Italia)

Fluvial geomorphology, Natural hazards, Sedimentology

M. López-Vicente (Universidad de Jaén) Sustainability, Water Resources Management, Soils, Hydrological modelling

Y. Onda (University of Tsukuba, Japón)

Water Quality, Hydrology, Soil Science, Freshwater Ecology, Soil erosion, Geomorphology

J.L. Peña (Universidad de Zaragoza)

Geomorphology, Geoarchaeology, Natural hazards, Holocene
D. Palacios (Universidad Complutense de Madrid)

Palaeoclimatology, Glaciology

J. Poesen (Katholieke Universiteit Leuven, Alemania) Geomorphology, Soil erosion, Badland geomorphology, Environmental change

J.B. Ries (Universität Trier, Alemania)

Water resources management, Soil erosion, Land degradation

M.A. Romero Díaz (Universidad de Murcia)

Soil erosion, Badland geomorphology, Reforestation, Piping geomorphology

E. Serrano Cañadas (Universidad de Valladolid)

Geomorphology, Glacial and periglacial environments, Quaternary

M. Vanmaercke (Katholieke Universiteit Leuven, Alemania)

Soil erosion, Sediment Transport, Geomorphology

S. Vicente-Serrano (Instituto Pirenaico de Ecología,

CSIC)

Climatology, Droughts, Climate change, Environmental hydrology, Remote sensing

L. Vázquez Selem (Universidad Nacional Autónoma de

México, México)

Paleoclimatology, Glacial geomorphology, Volcanoes, Natural Hazards

G-L. Wu (Northwest Agriculture \& Forestry University, Chinese Academy of Sciences, China)

Soil and Water Conservation, Soil erosion, Land degradation, Desertification

Cuadernos de Investigación Geográfica = Geographical Research Letters (ISSN: 0211-6820) is a scientific journal that publishes two issues per year. It includes papers on Physical Geography and other related environmental sciences (Hydrology, Ecology, Climatology). Interdisciplinary studies with Human Geography are also welcome. The peer review and publication of articles in Cuadernos de Investigación Geográfica is free of charge, in agreement with the policy of the journal of making accessible the advances in Physical Geography to the scientific community. All papers are subject to full peer review.

Since 2015 Cuadernos de Investigación Geográfica = Geographical Research Letters has been included in the Emerging Sources Citation Index (ESCI) of Clarivate Analytics, a new edition of the Web of Science, within the subject category of Geography (Physical). The journal is also indexed in SCOPUS and SCIMAGO Journal \& Country Rank within the subject categories of Geography, Planning and Development, Environmental Sciences, and Earth and Planetary Sciences.

Fotografía de portada/Cover photo: Morrena lateral del glaciar de Miage (Alpes italianos) / Lateral moraine of Miage glaciar (Italian Alps) (Autor / Author: J. Arnáez) 


\section{CUADERNOS DE INVESTIGACIÓN GEOGRÁFICA GEOGRAPHICAL RESEARCH LETTERS}

ISSN 1697-9540

Tomo 47 (2) 2021

\section{CONTENIDO / CONTENT}

Josep Bonsoms, Ferran Salvador-Franch, Marc Oliva. Snowfall and snow cover evolution in the Eastern Pre-Pyrenees (NE Iberian Peninsula)

Evolución de la cobertura nival y la nivosidad en el Prepirineo oriental (NE de la Península Ibérica)

Ana Paula Forte, Cristian Daniel Villarroel, María Yanina Esper Angillieri. Rock glacier and protalus rampart inventory in Las Salinas river basin, Central Andes of Argentina Inventario de glaciares rocosos y protalus rampart en la cuenca del río Las Salinas, Andes centrales argentinos

Felix I. Contreras, Gian M. Mavo Mastretta, María C. Piccolo, Gerardo M. E. Perillo. Spatio-temporal variability monitoring of the floods in the center-west of the Buenos Aires Province (Argentina) using remote sensing techniques

Seguimiento de la variabilidad espacio-temporal de las inundaciones en el centro-oeste de la Provincia de Buenos Aires (Argentina) mediante técnicas de teledetección. El papel de las dunas de arena

Carolina Lauro, Alberto Vich, Stella M. Moreiras, Luis Bastidas, Sebastián Otta, Emilce Vaccarino. Regionalización del caudal máximo anual en cuencas del sistema hidrográfico del río Colorado, Argentina

Annual maximum flow regionalization of Colorado River System, Argentina

Annick Delapierre, Juan A. Ballesteros-Canovas, Jorge Buzzi Marcos, Vera I. Slaveykova, Markus Stoffel. Trees as sentinels of metallic pollution induced by mining along the Odiel River (Southern Iberian Peninsula)

Árboles como indicadores de la contaminación metálica inducida por la minería a lo largo del río Odiel (sur de la Península Ibérica) 
Michal Fojutowski, Piotr Gierszewski, Dariusz Brykala, Alicja Bonk, Mirosław Blaszkiewicz, Mateusz Kramkowski. Spatio-temporal differences of sediment accumulation rate in the Lake Gościąż (Central Poland) as a response of meteorological conditions and lake basin morphometry

Diferencias espacio temporales de la tasa de acumulación de sedimentos en el lago Gościąż (Polonia central) como respuesta a las condiciones meteorológicas y a la morfometría del lago

Álvaro Tena, Damià Vericat, Ramón J. Batalla. Balance sedimentario del embalse de Ribarroja

Sediment balance of the Ribarroja Reservoir

Aarón Santana Cordero. Human-derived environmental consequences for three sedimentary systems of the Canary Islands (Spain) - a study of changes and impacts: A synthesis

Consecuencias ambientales derivadas del desarrollo humano en tres sistemas sedimentarios de las islas Canarias - estudio de cambios e impactos: una sintesis

María B. Alfonso, Andrea S. Brendel, Alejandro J. Vitale, María C. Piccolo. Impact of heatwave events on latent and sensible surface heat flux and future perspectives in shallow lakes based on climate change models

Impacto de las olas de calor en el flujo de calor superficial latente y sensible y perspectivas de futuro para las lagunas someras basadas en modelos de cambio climático

Teodoro Lasanta, Estela Nadal-Romero, Makki Khorchani, Asunción Romero-Díaz. Una revisión sobre las tierras abandonadas en España: de los paisajes locales a las estrategias globales de gestión

A review of abandoned lands in Spain: from local landscapes to global management strategies

Francisco J. Vázquez Pinillos, Manuel J. Marchena Gómez. Territorial impacts of sealevel rise in marsh environments. The case of the Bay of Cádiz, Spain

Impactos territoriales del ascenso del nivel del mar en entornos de marisma. El caso de la Bahía de Cádiz, España

Parviz Jokar, Masoud Masoudi, Fatemeh Karimi. An MCE-based innovative approach to evaluating ecotourism suitability using GIS

Una aproximación innovadora basada en MCE y SIG para evaluar la idoneidad del ecoturismo 


\title{
SNOWFALL AND SNOW COVER EVOLUTION IN THE EASTERN PRE-PYRENEES (NE IBERIAN PENINSULA)
}

\author{
JOSEP BONSOMS $^{1 *}$, FERRAN SALVADOR FRANCH ${ }^{2}$, MARC OLIVA $^{2}$
}

${ }^{1}$ Centre d'Etudes Spatiales de la Biosphère (CESBIO), CNES/CNRS/IRD/UPS, 31401 Toulouse CEDEX 9, France.

\author{
${ }^{2}$ Department of Geography, Universitat de Barcelona, 08001 Barcelona, Spain.
}

\begin{abstract}
Snow cover has significant impacts on geoecological dynamics as well as on socio-economical systems. An accurate quantification of snow precipitation patterns in mountain regions is needed to better understand the spatio-temporal implications of snow cover. The objective of this work is to characterize the patterns of solid precipitation and snow cover in two high Mediterranean massifs. To this purpose, we analyse instrumental data series of snowfall and snow depth of Port del Comte (2316 m a.s.l.) and Cadí-Nord (2134 m). Both stations are situated in the eastern Pre-Pyrenees and include 14 consecutive snow seasons from November to May, allowing to (i) explore the dependence of the main drivers of snowpack: temperature and snowfall; (ii) find out the most frequent circulation weather types associated with high intensity snowfall events, and finally (iii) investigate the role of the North Atlantic Oceanic (NAO) teleconnection pattern explaining snow cover evolution during the winter season. Data show that snowfall is controlled by similar weather types in both stations that resulted in similar snowfall averages: $205 \mathrm{~cm}$ and $258 \mathrm{~cm}$ at Port del Comte and Cadí-Nord, respectively. Nevertheless, local factors interfere with the amount of snow depth recorded, which is moderately different between stations. Whereas Cadí-Nord records a seasonal mean of $66 \mathrm{~cm}$, Port del Comte records a smaller quantity of $25 \mathrm{~cm}$ with a high interannual and seasonal variability. In fact, snowfall recurrence, snow amount or duration in the ground is considerably variable among years $(\mathrm{CV}>20)$. In these stations, snow cover duration is determined by the precipitation in the form of snow falling during the previous months. Snowfalls in moderate to severe episodes ( $>15 \mathrm{~cm}$ in $24 \mathrm{~h}$ ) are mainly driven by Atlantic flows, mostly from NW. In addition, NAO pattern is negatively correlated with snowfall in November and December months $(\mathrm{R}>-0.50)$, showing a weaker and not statistically significant correlation during the rest of the winter season.
\end{abstract}

\section{Evolución de la cobertura nival y la nivosidad en el Prepirineo oriental (NE de la Península Ibérica)}

RESUMEN. La presencia de cobertura nival en el Prepirineo es crucial, tanto para la dinámica geoecológica presente como para el sistema socioeconómico, ambos dependientes a partes iguales de este elemento natural. Una precisa cuantificación de los patrones de precipitación nivales son necesarios para entender las implicaciones espaciales de la cobertura nival. El objetivo del presente estudio es caracterizar la precipitación en forma de nieve y la cobertura nival de dos enclaves montañosos de elevada altitud situados en la cuenca Mediterránea. Por este motivo, a continuación, se analizan los datos instrumentales de las estaciones nivometeorológicas del Port del Comte (2316 m. a.s.l.) y del Cadí-Nord (2134 m). Ambas estaciones están localizadas en el Prepirineo oriental, y contienen una cobertura temporal de 14 temporadas nivales desde noviembre a mayo. Con este fin, (i) se ha verificado la correlación existente entre la temperatura y la precipitación nival con el grosor de nieve existente a final de temporada. Además, (ii) se han descrito los patrones sinópticos que se repiten con más frecuencia en los episodios nivales de mayor intensidad, y finalmente (iii) se ha analizado la dependencia de la nivosidad con el 
patrón de teleconexión principal de la Península Ibérica en los meses invernales, la Oscilación del Atlántico Norte (NAO). Los resultados demuestran cómo ambas estaciones están influenciadas por los mismos patrones sinópticos de precipitación, mostrando una media de precipitación nival por temporada similar: de $205 \mathrm{~cm}$ y de $258 \mathrm{~cm}$ en el Port del Comte y el Cadí-Nord, respectivamente. No obstante, debido a factores locales, el espesor de nieve registrado por temporada es moderadamente diferente. Mientras que el Cadí-Nord registra una media de $66 \mathrm{~cm}$, el Port del Comte registra una cuantía inferior, de $25 \mathrm{~cm}$ con una remarcable variación interanual y estacional. De hecho, tanto la nivosidad, cobertura nival, periodicidad o duración del manto nivoso tienen una considerable variación entre años $(\mathrm{CV}>20)$. En estos dos enclaves geográficos, la explicación de la duración nival a final de temporada se encuentra en la precipitación sólida de los meses precedentes. Esta precipitación en los episodios de moderados a severos ( $>15 \mathrm{~cm}$ en $24 \mathrm{~h}$ ) es caracterizada por flujos Atlánticos con un patrón sinóptico de componente noroeste. Además, el patrón de teleconexión NAO está negativamente correlacionado con la precipitación nival durante los meses de noviembre y diciembre $(\mathrm{R}>-0.50)$, mientras que la correlación es débil y estadísticamente no

significativa los subseque de meses invernales.

Key words. Pre-Pyrenees, climate variability, snowfall, snow depth.

Palabras clave. Prepirineo, variabilidad climática, cobertura nival, nivosidad.

Received: 18 September 2020

Accepted: 7 January 2021

*Corresponding author: Josep Bonsoms, Centre d'Etudes Spatiales de la Biosphère (CESBIO), CNES/CNRS/IRD/UPS, 31401 Toulouse CEDEX 9, France. E-mail address: josepbonsoms5@gmail.com

\section{Introduction}

Solid precipitation and the consequent accumulation as snowpack is crucial for the stability of the global climate system. Seasonal snow extent, duration or characteristics have large interactions and impacts in the surrounding landscapes. By its own nature, snow albedo delays ground warming (Hall, 2004), provides a protective layer for vegetation when temperatures are severely low, conditions the plant growing season, reduces thermodynamics and affects biogeochemical cycles (e.g., Wipf et al., 2009). Snow is in essence water storage, available for downstream areas. Snow melting determines the temporal distribution of the river flow in mountain areas and supplies nutrients both for the ecological and social systems (García-Ruiz et al., 2011). Indeed, snow presence and abundance favours human activities in winter, for example, guaranteeing the opening of ski resorts, a major business in the Pyrenees area, or in springtime, providing hydroelectricity, hydric resources for agriculture or water for settlements and population (e.g., Lasanta et al., 2005). These dependencies will increase in the future, when droughts will be more frequent and severe, and water availability will be more demanding across the Mediterranean basin (García-Ruiz et al., 2011).

Future climatic scenarios are expected to impact the timing of snow cover in the ground as well as the frequency and intensity of snowfalls in mid-latitude mountain environments, affecting thus ecological and socio-economic issues (IPCC, 2013). Snowpack is mainly conditioned by the combination of temperatures and precipitation. A long-term warming of ca. $1{ }^{\circ} \mathrm{C}$ has been recorded following the Little Ice Age in the Iberian mountains (Oliva et al., 2018), with temperatures expected to further increase in the next decades. In fact, high elevation areas in mid-latitude mountain ranges are considered to record faster warming rates compared with lowlands (Pepin et al., 2015). On the other hand, annual and winter precipitation in the Iberian Peninsula between 1961 and 2011 years has decreased at a rate of $18.7 \mathrm{~mm} /$ decade (Vicente-Serrano et al., 2017). In the Pyrenees, the decrease of 
precipitation has been more accentuated in the southern slopes of the eastern mountain range (LemusCanovas et al., 2019a). Furthermore, several studies pointed to a decrease of snow depth in the mountain range during the second half of the 20th and early 21 st centuries (e.g López-Moreno, 2005, 2020). However, this trend is variable depending on the time frame analysed, showing a decrease of snow days between 1971 and 2000, and a positive trend between 1985 and 2010 (Buisan et al., 2016). With regards to teleconnection and circulation patterns, numerous studies identified the NAO - often defined as the difference of anomalies of the sea level pressure (SLP) between Ponta Delgada (Azores) and Reykjavick (Iceland) (Hurrell et al., 1995), as the main driver of precipitation in Iberian Peninsula in winter as well as snowfall in the western and central Pyrenees (López-Moreno, 2005; Revuelto et al., 2012). Additionally, a predominance of NAO positive phases in winter has been found over the $20^{\text {th }}$ century, leading to a reduction of circulation weather types associated with precipitation (López-Bustins et al., 2008).

Nonetheless, an accurate characterization of the snowfall and snowpack in high altitudes of the eastern Pyrenees and Pre-Pyrenees is still missing. Previous works focused on snow stations of the eastern Pyrenees (Salvador Franch et al., 2014, 2016) are now complemented with a detailed characterization of the recent evolution of snow variables. With this aim, this study examines snow data from two stations from the eastern Pre-Pyrenees: Port del Comte $(2316 \mathrm{~m})$, located in the massif of Port del Comte, and Cadí-Nord station (2134 m), at the northern foot of the Cadí massif. We analyse snow records of 14 snow seasons examining snowfall records, as well as their frequency and intensity, together with snow cover, thickness and duration. With the objective of providing a better characterization of the spatio-temporal patterns of snow cover in these areas, we will give answer to the following specific objectives: i) Characterise the seasonal snowfall and snow cover evolution in the PrePyrenees, ii) explore the relationship between snow cover, precipitation and temperature, iii) investigate the main weather types associated with snowfall events y iv) analyse the role of NAO teleconnection pattern during the snowfall season.

\section{Study sites and regional climate}

The Pre-Pyrenees mountains are located in the NE of Iberian Peninsula (Fig. 1). This mountain system is aligned $\mathrm{W}-\mathrm{E}$ along the southern and northern foothills of the Pyrenees, a major mountain system stretching $450 \mathrm{~km}$ between the Mediterranean Sea and the Atlantic Ocean. This research focuses on two high elevation meteorological stations from two massifs of the SE Pre-Pyrenees: (i) Port del Comte, situated at $42^{\circ} 18^{\prime} 25^{\prime \prime} \mathrm{N}$ and $1^{\circ} 52^{\prime} 40^{\prime \prime} \mathrm{E}$, SW of the highest peak of the massif (Pedró dels Quatre Batlles, $2378 \mathrm{~m}$ ), and (ii) the Cadí-Nord snow station, at latitude of $42^{\circ} 29^{\prime} 26^{\prime \prime} \mathrm{N}$ and a longitude of $1^{\circ} 71^{\prime} 49^{\prime \prime} \mathrm{E}, 20 \mathrm{~km}$ NE Port del Comte. It is situated at an altitude of $2134 \mathrm{~m}$, in the northern slope of the Cadí massif and near the Vulturó summit (2648 m) (Fig. 2).

The climate of the Iberian Peninsula is defined by a dominant westerly circulation from November to May, and prevailing anticyclone conditions during summer (Cortesi et al., 2013). In the Pyrenees, the larger geographical differences generate a wide range of sub regional climate types, mainly caused by the distance to the Atlantic Ocean or Mediterranean Sea, and the altitude and aspect which also condition relief barrier effects (Xercavins, 1985). Throughout December to March, precipitation in mid-altitude areas (around $1500 \mathrm{~m}$ ) is regionalized in three different zones: (i) the western Pyrenees, with an average of precipitation $>600 \mathrm{~mm}$; (ii) central axial of the range, with $<400 \mathrm{~mm}$; and (iii) eastern fringe, with high interannual variations and $<200 \mathrm{~mm}$ recorded (Buisan et al., 2016); herein, both studied snow stations are located. According to precipitation criteria, the study area corresponds to the Mediterranean eastern Pyrenees (SMC, 2008). Annual precipitation totals around $1050 \mathrm{~mm}$ at the summit of the Port del Comte and $1100 \mathrm{~mm}$ at $2100 \mathrm{~m}$ of the Cadí-Nord (SMC, 2008). During the warm season, and due to adiabatic heating forced by orography and high elevations, convective rainfall is frequent. This explains a higher precipitation in summer and spring, followed by lower values in autumn and winter. The mean annual air temperature is $3^{\circ} \mathrm{C}$ at Port del Comte and $4^{\circ} \mathrm{C}$ at Cadí-Nord (SMC, 
2008). Between 2100 and $2300 \mathrm{~m}$, the annual air temperature amplitude exceeds $14^{\circ} \mathrm{C}$, with the coldest and warmest months averaging $-3^{\circ} \mathrm{C}$ (February) and $12^{\circ} \mathrm{C}$ (August), respectively. Moreover, nuanced thermal inversions are observed between the culmination zones and the valley bottoms. Temperature elevation gradients in the eastern Pyrenees are slightly lower $\left(0.52^{\circ} \mathrm{C} / 100 \mathrm{~m}\right)$ than the average due to the maritime influence of the Mediterranean Sea (Xercavins, 1985). The wind shows different patterns in these two stations, particularly during the cold semester of the year (November-May), when WNW winds prevail at Port del Comte station with maximum speeds of $>20 \mathrm{~m} / \mathrm{s}$, whereas at Cadí-Nord station WSW are most frequent, rarely exceeding $10 \mathrm{~m} / \mathrm{s}$ (SMC, 2019).

The mountain landscape in these two regions is controlled by the presence of the prevailing limestone lithology that has favoured the development of a wide variety of karstic landforms, such as dolines, caves and deep gorges. Soils are thin and poorly developed, which constraints a scarce vegetation cover dominated mostly by alpine meadows at the summit level with above the tree line (2200-2300 m) drawn by small pines (Pinus uncinata). Additionally, part of the study area is under regional and Nature 2000 protection figures. The combination of low temperatures with moderate and high precipitation in the winter makes possible the practice of tourism related with snow in several ski resorts established in the surroundings.
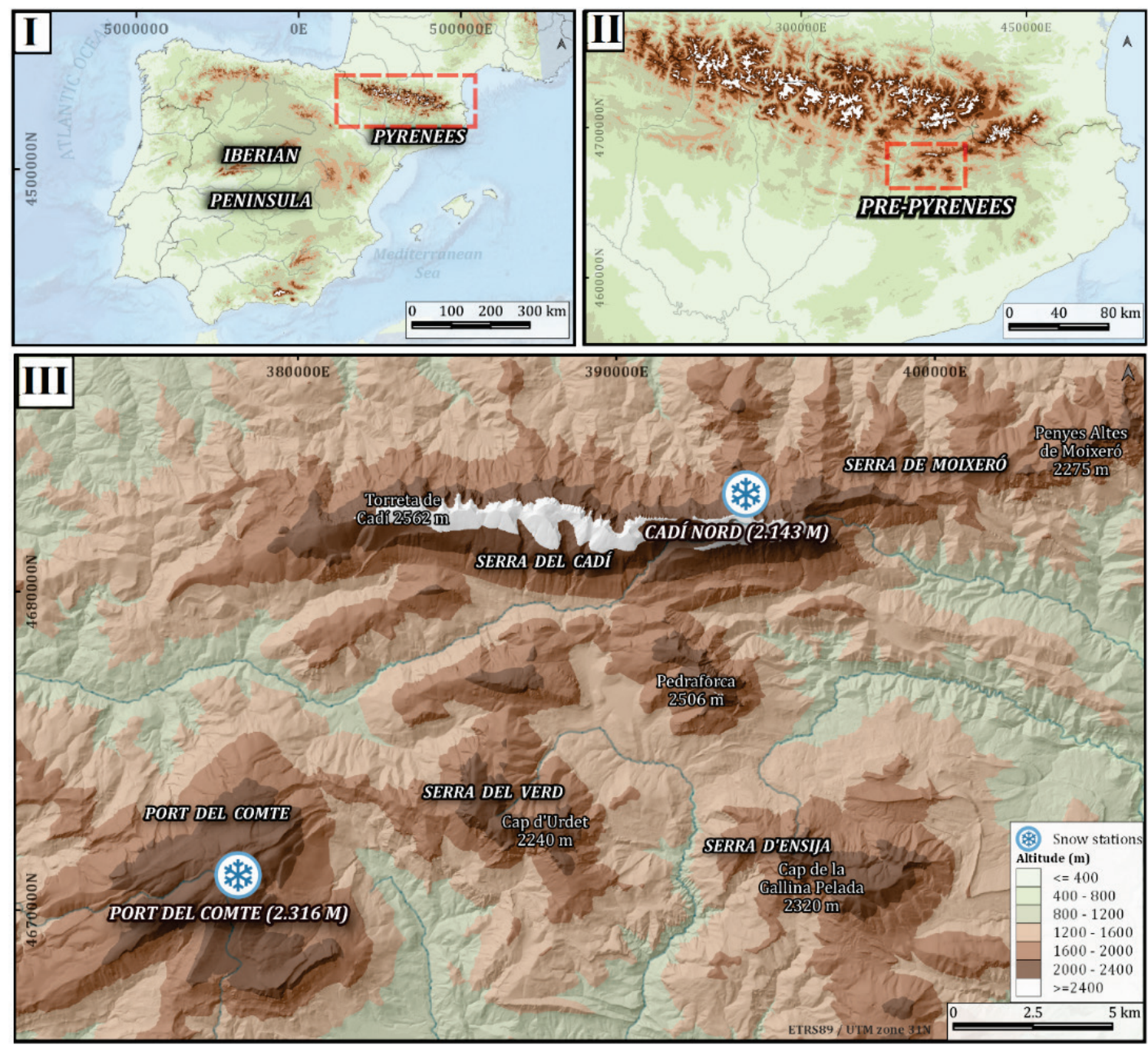

Figure 1. Location of the Pyrenees within the Iberian Peninsula: (I) Eastern Pre-Pyrenees; (II) Distribution of the stations in the study area (III). 

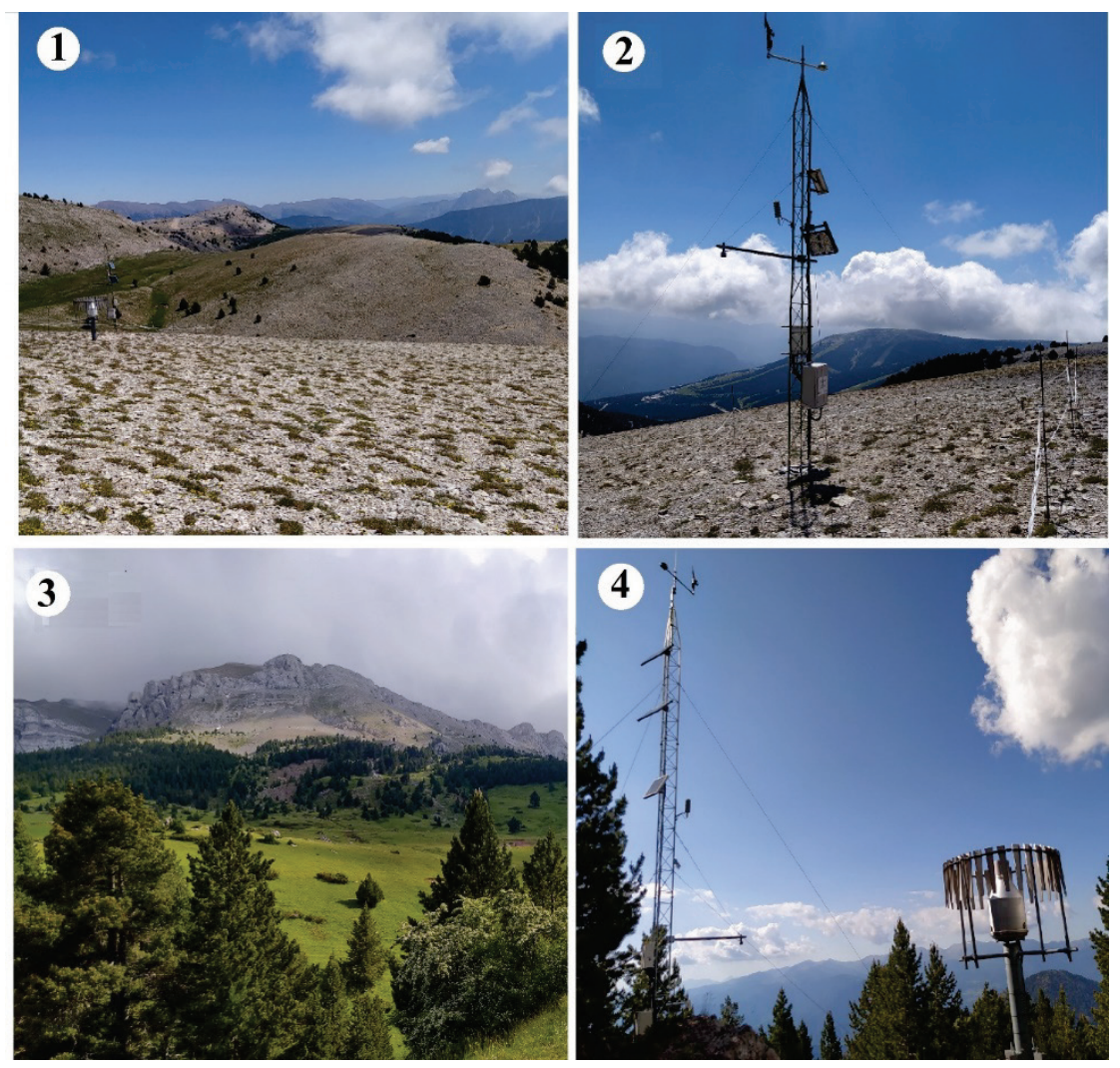

Figure 2. Several pictures of: (1) Panoramic view of Port del Comte massif, near Pedró dels Quatre Batlles peak (2378 m) towards the Cadi massif; (2) meteorological and snow station of Port del Comte; (3) View of Prat d'Aguiló, a meadow close to Cadi-Nord station; (4) meteorological and snow station of Cadi-Nord.

\section{Data and methodology}

\subsection{Instrumental data}

The instrumental meteorological records provided by the Meteorological Service of Catalonia include daily records of temperature, precipitation and snow depth. All analysed data have passed a quality control and homogeneity test. For the purposes of this work, we considered the snowfall season from November to May including two consecutive years of the Gregorian calendar. Port del Comte snow data cover a total of 16 seasons (2002 to 2018) whereas the Cadí-Nord station encompasses data from 15 years (2004 to 2019). In order to compare patterns in both stations, we examine snow seasons from 2004 to 2018.

\subsection{Snowfall characterization}

Daily snowfall was calculated by the difference with the maximum snow depth of the previous day, assuming that the increase corresponds to snowfall. From the whole dataset, missing data occurred in $0.9 \%$ of the total cases at Port del Comte and only $0.2 \%$ at Cadí-Nord. For such cases, we compared both snow stations and if there have been any snowfall events; we assumed the mean of snow depth between the last and the following day. The average of snow depth and duration calculated include only days with $>0.1 \mathrm{~cm}$ of snow depth. Finally, monthly snowfall anomalies (Fig. 3) are the result of the difference between the monthly average and the corresponding monthly value of the season.

To determine whether snowfall or temperature are the main drivers of the variations in the snow depth in late snow season, we followed a previous approach carried out in the western and central Pyrenees (López-Moreno, 2005). A Pearson's correlation was performed between the snowfall and 
temperature recorded in the preceding months - January, February, March and April - and the last month with measurable snow depth, April. We considered April as the melting month due to the general lack of snow in May, particularly at Port del Comte station (Fig. 3).

\subsection{Weather types for snowfall events}

The synoptic classification of the major snowfall days $(>15 \mathrm{~cm}$ in $24 \mathrm{~h})$ was based on the SynoptReg R package (Lemus-Canovas et al., 2019b). This method was successfully implemented at the Pyrenees (e.g., Esteban et al., 2005; Lemus-Canovas et al., 2019a). It uses a principal component analysis applied a to spatial mode matrix, where the days are the observations and the grid points (latitude and longitude) are the variables. Then a clustering method is implemented, and the number of components retained are decided by a Scree test (Cattell, 1966). We selected 12 clusters (CL), which explained $90 \%$ of the variance. Subsequently, the principal components were rotated by means of Varimax rotation (Kaiser, 1958) and the extreme scores method (Esteban et al., 2005) was applied. This method uses the scores $(>2$ and -2$)$ determining a positive and negative phase for every principal component. The extreme scores established the number of groups and centroids for the K-means method. Further details of SynoptReg package are described at Lemus-Canovas et al. (2019b). The Database used was Daily SLP (Sea Level Pressure) and Geopotential Height at $500 \mathrm{hPa}$ obtained from NCEP/NCAR Reanalysis 2 (Kalnay et al., 1996) for $30^{\circ} \mathrm{N}-60^{\circ} \mathrm{N}$ by $30^{\circ} \mathrm{W}-10^{\circ} \mathrm{E}$ with 2.5 resolution.

\subsection{NAO and snowfall}

In order to detect the relationship between NAO and snowfall during the season, a linear regression was undertaken between monthly snowfall records and monthly NAO values. NAO was calculated by the difference of sea-level pressure in Reykjavik (Iceland) and Ponta Delgada (Azores), and is provided by the Climate Research Unit (CRU), University of East Anglia (https://crudata.eua.ac.uk/cru/ data/nao/)

\section{Results}

We present an accurate characterization of the snowfall and snow depth at Port del Comte and Cadí-Nord over 14 consecutive seasons, together with an analysis of the relationship between snowpack during the late season and the main meteorological drivers and the role of the NAO.

\subsection{Snowfall and snow depth}

\section{Port del Comte}

From November to May, the total measured snowfall averages $205 \mathrm{~cm}$ at Port del Comte, which represents $21 \%$ of annual precipitation. Late season snowfall is sometimes beyond the monthly average (for instance 2008-2009 season, when $78 \mathrm{~cm}$ were recorded in April). Occasionally, about once in every two seasons, there is a snowfall episode in early summer. There is a considerable interannual variation $(\mathrm{CV}=41 \%)$ and snowy years (i.e. 2009-2010) alternate with snow-poor seasons (2011-2012). The maximum snowfall season was recorded in 2017-2018, with a total accumulation of $411 \mathrm{~cm}$. On the opposite site, minimum peaks occurred in the 2014-2015 season, with a solid precipitation of only 89 $\mathrm{cm}$ (Table 1 and Fig. 4). On average, the frequency of snowfall days during a season is 51 . The higher recurrence of snowfall days was recorded in the 2008-2009 season (70 days) and a minimum in the 2016-2017 season (30 days). The amount of snow accumulated in $24 \mathrm{~h}$ is on average $26 \mathrm{~cm}$, with a minimum of $11 \mathrm{~cm}$ (2004-2005). The most intense snowfall episode was recorded on 26th of January of 2018, when $41 \mathrm{~cm}$ of fresh snow accumulated in less than 24 hours. 
Table 1. Basic parameters of snowfall and snow depth at Port del Comte and Cadi Nord stations.

\begin{tabular}{|c|c|c|c|c|c|c|c|c|c|c|c|c|c|c|}
\hline & $\begin{array}{r}\text { Sno } \\
(\mathrm{c} \\
\text { P. Comt } \\
\end{array}$ & $\begin{array}{l}\text { wfall } \\
\text { m) } \\
e / C a d i\end{array}$ & $\begin{array}{r}\text { Max } \\
(\mathrm{c}\end{array}$ & $\begin{array}{l}24 \mathrm{~h} \\
\mathrm{~m})\end{array}$ & $\begin{array}{r}\text { Freq } \\
(\mathrm{d} a\end{array}$ & $\begin{array}{l}\text { ncy } \\
\text { ) }\end{array}$ & $\begin{array}{r}\mathrm{PPt} \\
\quad(0\end{array}$ & & $\begin{array}{r}\text { Snow } \\
\text { Thic } \\
\text { (c }\end{array}$ & $\begin{array}{l}\text { depth } \\
\text { kness } \\
\text { m) }\end{array}$ & $\begin{array}{r}\text { Dur: } \\
\text { (da }\end{array}$ & & $\begin{array}{r}\text { Dur } \\
\geq 10 \mathrm{~cm}\end{array}$ & $\begin{array}{l}\text { tion } \\
\text { (days) }\end{array}$ \\
\hline Mean & 205 & 258 & 26.2 & 38 & 51 & 50 & 21.5 & 26.2 & 25.5 & 66.3 & 148 & 175 & 108 & 151 \\
\hline Max. & $\begin{array}{c}411 \\
(17-18)\end{array}$ & $\begin{array}{c}431 \\
(17-18)\end{array}$ & $\begin{array}{c}41.8 \\
(17-18)\end{array}$ & $\begin{array}{c}62 \\
(17-18)\end{array}$ & $\begin{array}{c}70 \\
(08-09)\end{array}$ & $\begin{array}{c}70 \\
(10-11)\end{array}$ & $\begin{array}{c}29.7 \\
(08-09)\end{array}$ & $\begin{array}{c}33.1 \\
(04-05)\end{array}$ & $\begin{array}{c}59.8 \\
(17-18)\end{array}$ & $\begin{array}{c}117 \\
(08-09)\end{array}$ & $\begin{array}{c}191 \\
(08-09)\end{array}$ & $\begin{array}{c}206 \\
(08-09)\end{array}$ & $\begin{array}{c}190 \\
(08-09)\end{array}$ & $\begin{array}{c}205 \\
(08-09)\end{array}$ \\
\hline Min. & $\begin{array}{c}89 \\
(14-15)\end{array}$ & $\begin{array}{c}135.5 \\
(14-15)\end{array}$ & $\begin{array}{c}11 \\
(04-05)\end{array}$ & $\begin{array}{c}12 \\
(04-05)\end{array}$ & $\begin{array}{c}30 \\
(16-17)\end{array}$ & $\begin{array}{c}32 \\
(16-17)\end{array}$ & $\begin{array}{c}8.6 \\
(14-15)\end{array}$ & $\begin{array}{c}13.0 \\
(14-15)\end{array}$ & $\begin{array}{c}5.1 \\
(14-15)\end{array}$ & $\begin{array}{c}24.7 \\
(04-05)\end{array}$ & $\begin{array}{c}83 \\
(11-12)\end{array}$ & $\begin{array}{c}131 \\
(11-12)\end{array}$ & $\begin{array}{c}18 \\
(14-15)\end{array}$ & $\begin{array}{c}54 \\
(11-12)\end{array}$ \\
\hline $\mathrm{CV}$ & 38.6 & 29.5 & 28.6 & 51.6 & 24.7 & 27.7 & 28.6 & 23.3 & 59.4 & 50.7 & 22.8 & 10.8 & 48.6 & 26.0 \\
\hline
\end{tabular}

( ) = Years; PPt year*(\%): Percentage of annual precipitation corresponding to snowfall $(\mathrm{cm})$.
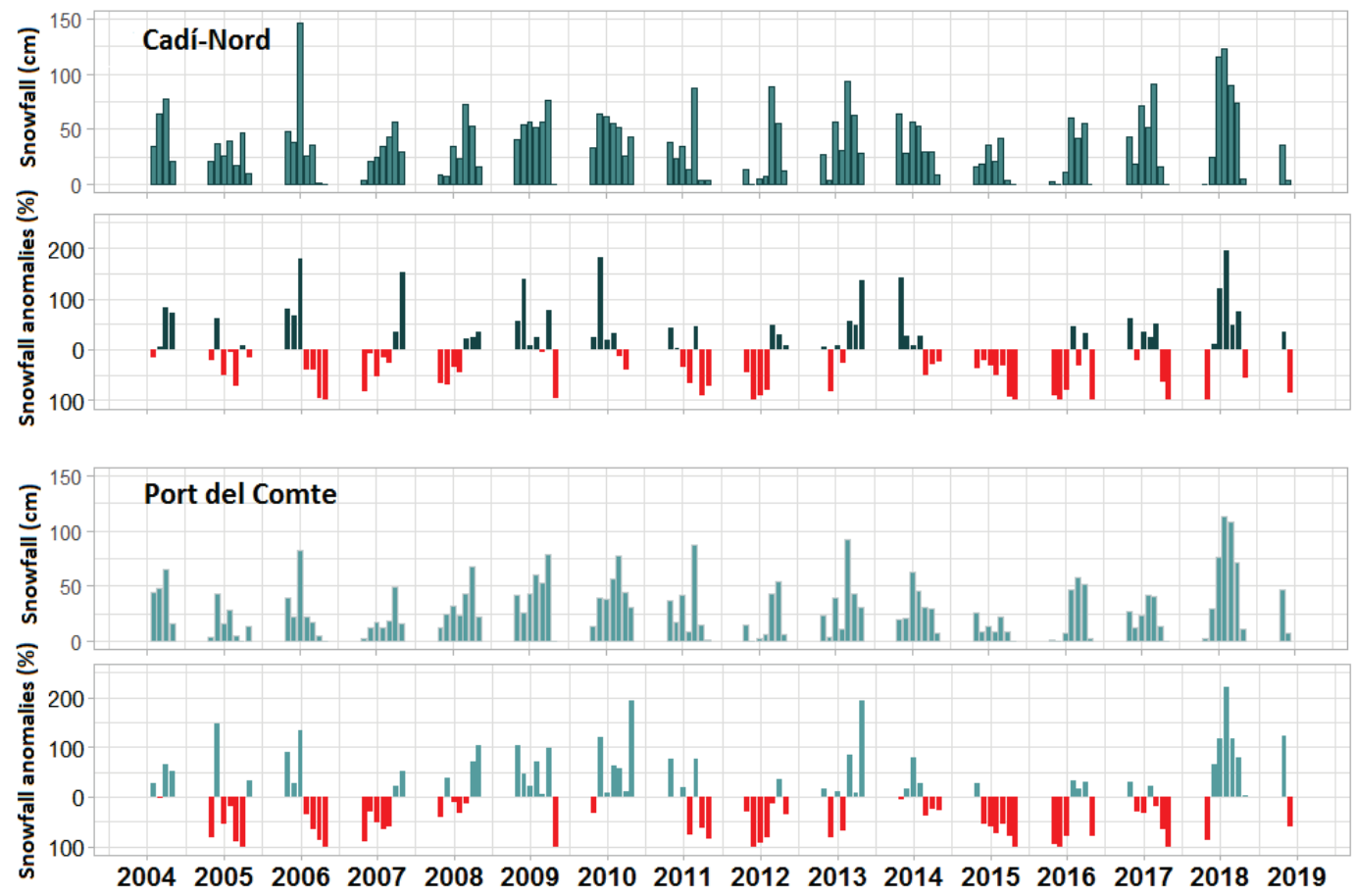

Figure 3. Time series of monthly snowfall and snowfall anomalies at Cadi-Nord and Port del Comte during the snow season.

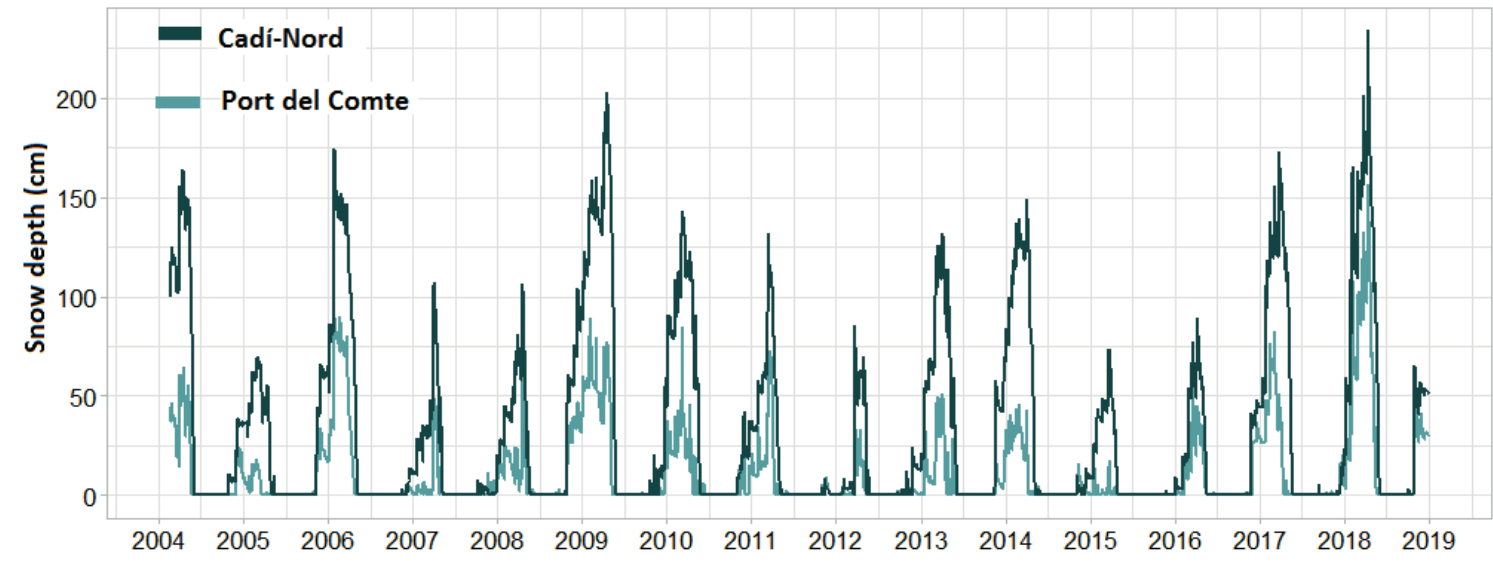

Figure 4. Annual evolution of snow depth (cm) at Cadi-Nord and Port del Comte. 
Snow persists on the ground during 148 days on average, with maximum values in 2003 and 2004 years, when 211 days of snow were recorded. On the other extreme, the minimum snow persistence occurred in the 2011-2012 season, with only 83 days. If we only consider the days when snow cover was higher than $10 \mathrm{~cm}$, the mean duration of snow depth decreases to 108 days. The minimum was recorded in 2014-2015, with a snow thickness exceeding $10 \mathrm{~cm}$ only during 18 days. Snow cover thickness $(>10 \mathrm{~cm})$ shows also a high interannual variability as shown by its high CV $(48 \%)$. When the ground is snow-covered, the average thickness is $25 \mathrm{~cm}$, with peaks in 2017-2018 $(59 \mathrm{~cm})$ and a minimum in 2014-2015 with $5 \mathrm{~cm}$. The maximum snow depth normally occurs in February at Port del Comte (Fig. 5), although the highest monthly values were recorded in April 2018, when $114 \mathrm{~cm}$ were measured.
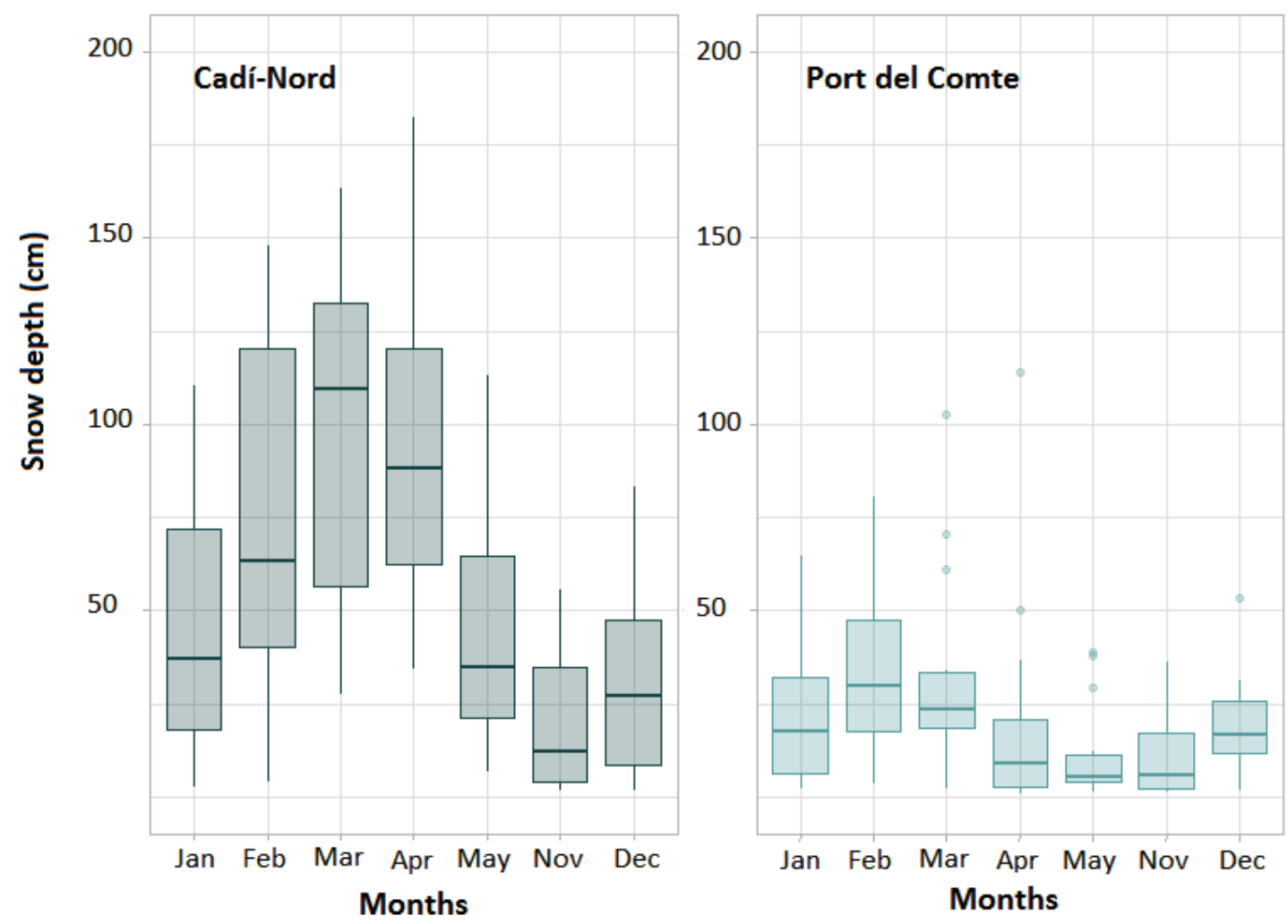

Figure 5. Monthly distribution of snow depth (cm).

\section{Cadi-Nord $(2143 \mathrm{~m})$}

Over these 14 snow seasons, the average snowfall at Cadí-Nord was $258 \mathrm{~cm}$. In comparison with the annual precipitation, snow accounts for $26 \%$ of the total recorded at Cadí-Nord. From November to May, snowfalls represent almost half of the total precipitation (42\%). There are seasons above the mean, such as 2013-2014 $(213 \mathrm{~cm})$, followed by two snow-poor seasons (89 and $166 \mathrm{~cm})$. The 2017-2018 period was extraordinary in terms of snow as it reached a maximum accumulation of $431 \mathrm{~cm}$, three times higher than the minimum snowfall recorded during the 2014-2015 season $(135 \mathrm{~cm})$ (Fig. 4). As well as at Port del Comte, high interannual variability is a common characteristic of snowfall at Cadí-Nord $(\mathrm{CV}=29.5 \%)$. On average, there are 50 days of snowfall at Cadí-Nord, with maximum snow days in 2010-2011 (70 days) and minimum in 2016-2017 (32 days). Intensity record on a snow day is on average $38 \mathrm{~cm}$, with maximum peaks in the 2017-2018 season $(62 \mathrm{~cm})$ and minimum in 2004$2005(12 \mathrm{~cm})$. 
In terms of snow cover, snow is present more than $80 \%$ of the days between November and May at Cadí-Nord station. The maximum duration took place in the 2008-2009 season, with 206 days. Taking into account only the days with $>10 \mathrm{~cm}$ of snow, there were 151 days with snow covering the ground. In both cases, the 2011-2012 season recorded minimum values, with 131 days with snow cover and 54 of these with more than $10 \mathrm{~cm}$ of snow. Considering days with snow depth $>0.1 \mathrm{~cm}$, the snowpack is on average $66 \mathrm{~cm}$. Snow depth $(>10 \mathrm{~cm})$ at Cadí-Nord showed also moderate interannual variations, with a CV around $26 \%$. This explains maximum years when snow thickness exceeds $1 \mathrm{~m}$ in punctual dates (2008-2009 and 2018-2019, among others) and years with values lower than $30 \mathrm{~cm}$ (i.e., 20062007 or 2015-2016). Seasonally, March is the month with the highest mean snow depth, although the highest values were measured in April of 2018 (182 cm) (Fig. 5). Usually, snow cover lasts from November to May but it persisted until June in three years (2008, 2013 and 2018).

\subsection{Climatic parameters affecting snow cover}

\section{Temperature}

Figure 6 shows evidence of the control of winter temperatures on spring snow depth. Results show that grouped months have stronger correlation for both stations, however, at a non-significant level of confidence $(p>0.05)$. The influence of temperature on the snow depth persisting in April is weak at Cadí-Nord $(\mathrm{R}=-0.30)$ as well at Port del Comte $(\mathrm{R}=-0.27)$. The occurrence of snow in April is more influenced by temperatures at Cadí-Nord than at Port del Comte because of its lower elevation. In detail, mean monthly temperatures from January to April have a moderate negative correlation with snow depth $(\mathrm{R}=-0.34$ at Cadí-Nord and $\mathrm{R}=-0.28$ at Port del Comte). Similar negative correlations are found from January to March. In March, temperature effects are weaker at Cadí-Nord $(\mathrm{R}=-0.13)$ but moderate at Port del Comte $(\mathrm{R}=-0.43)$. Finally, April mean temperature is not related to snow depth variations, $\mathrm{R}=-$ 0.18 at Cadí-Nord and $\mathrm{R}=-0.1$ at Port del Comte.
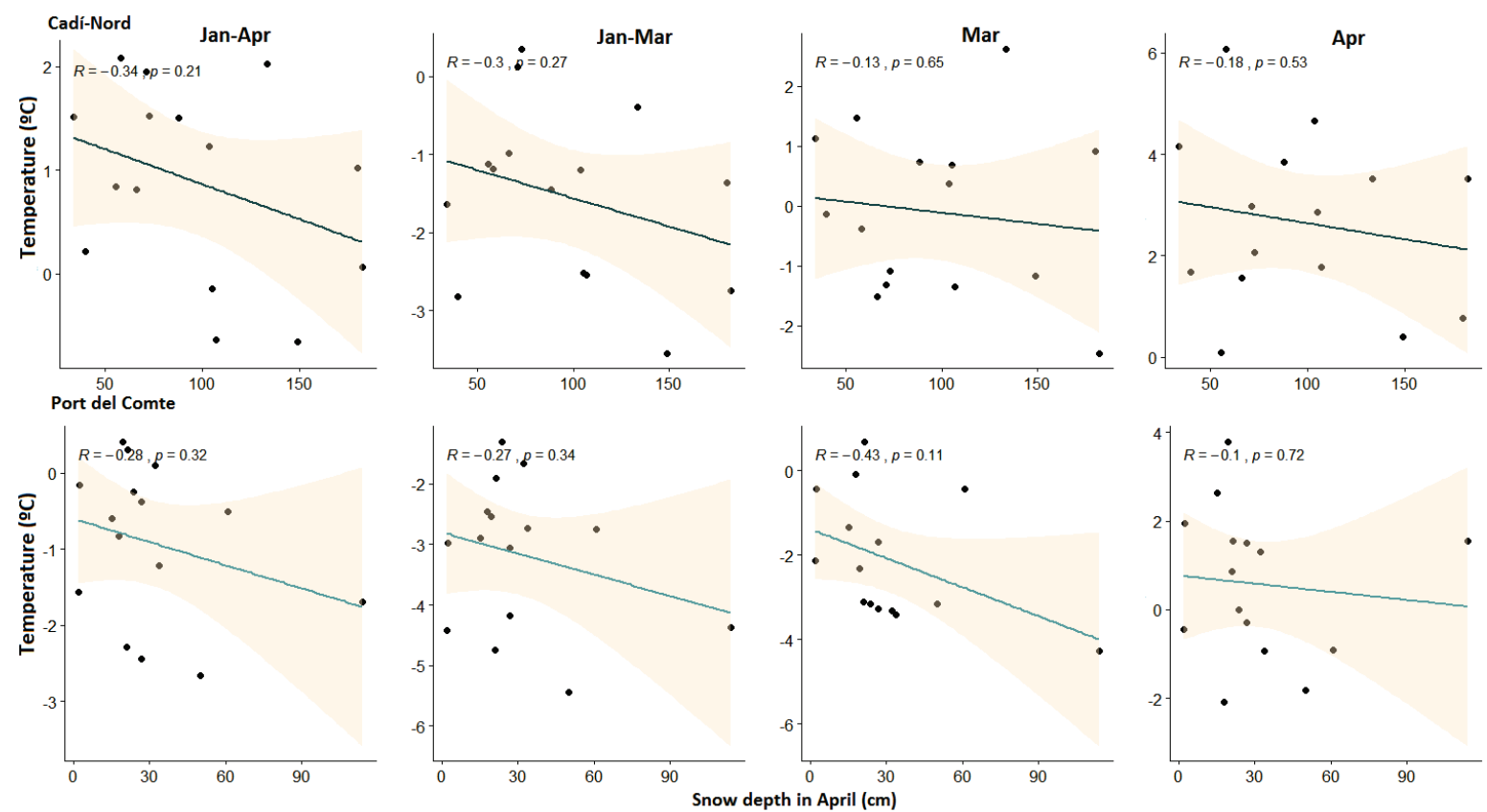

Figure 6. Correlation between snow depth in April $(\mathrm{cm})$ and mean temperatures $\left({ }^{\circ} \mathrm{C}\right)$. 


\section{Snowfall}

The effect of mid to late season snowfall over late season snow depth at Cadí-Nord and Port del Comte is significant (Fig. 7). For both stations, snowfall is the key factor that controls the thickness of snow in April. In fact, a robust positive correlation is found, stronger at Port del Comte than at CadíNord. Snowfall from January to April shows a higher correlation at Port del Comte $(\mathrm{R}=0.84)$ than at Cadí-Nord $(\mathrm{R}=0.79)$ with a significant level of confidence $(p<0.05)$. Correlations are lower if we consider less months (Fig. 7). While at Cadí-Nord the correlation in March is weak $(\mathrm{R}=0.35, p>0.05)$ at Port del Comte is higher $(\mathrm{R}=0.58, p<0.05)$. Lastly, April snowfall is strongly correlated with snow depth at Port del Comte $(\mathrm{R}=0.67, p<0.05)$, more than Cadí-Nord $(\mathrm{R}=0.49)$ but at a non-significant level in the later $(p=0.06)$.
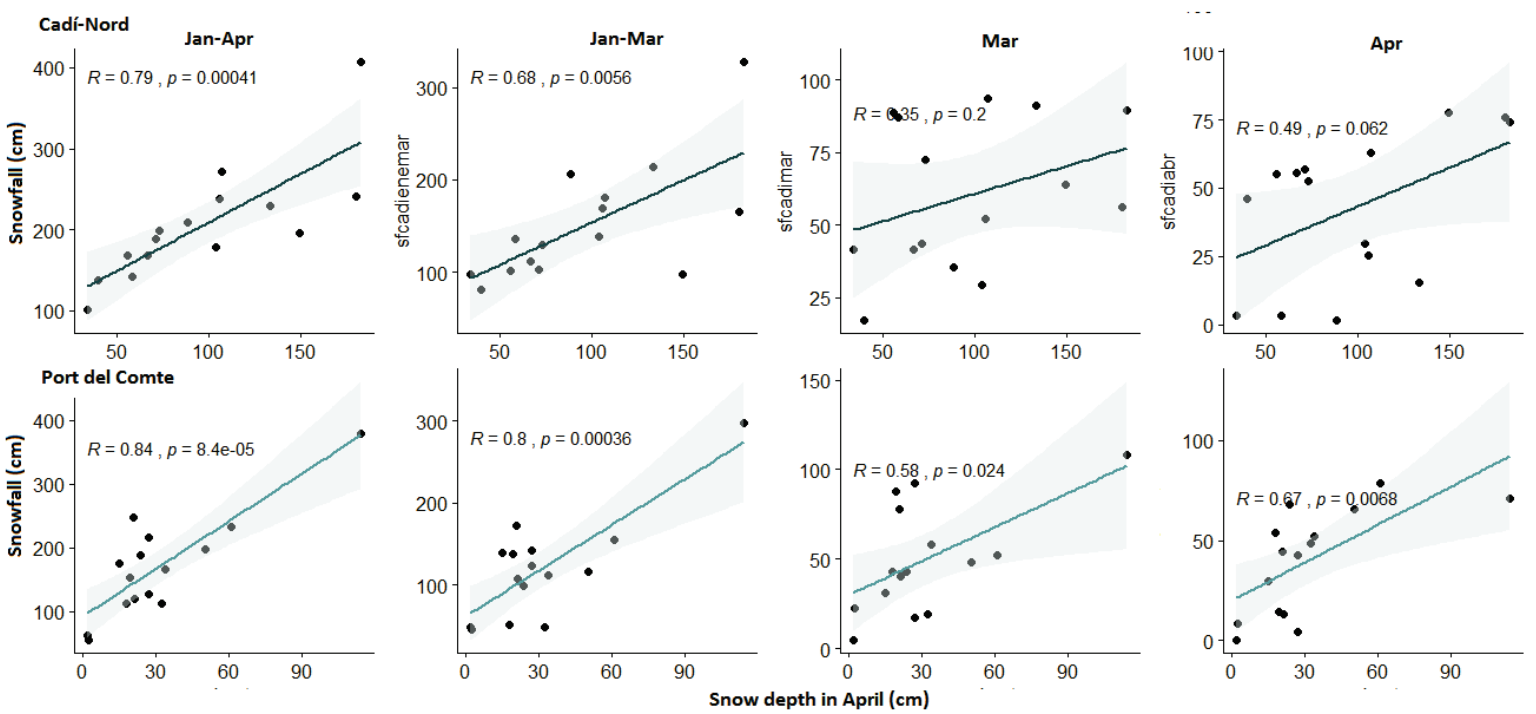

Figure 7. Correlation between snowfall (cm) and snow depth in April (cm).

\subsection{Types of weather associated with major snowfalls}

The synoptic classification of the most frequent weather types revealed $12 \mathrm{CL}$ more usual in the study area grouped in stable or unstable air masses at $500 \mathrm{hPa}$. The first category corresponds with $\mathrm{CL}$ 1, typified as high pressure in Azores and low pressures around Great Britain; CL 2, a zonal situation and high pressure in NW Europe; CL 3, an anticyclone situated over Iberia, a typical situation in January month; CL 4 low pressures with the advection of a polar maritime air mass, CL 5 high pressures over NW Iberian and low pressures circulating in central and North Europe; CL 6 Northern Europe anticyclone; CL 7 high pressures over NW Iberia, blocking northern and mid Mediterranean depressions; CL 8 an extended continental high pressure; CL 9 advection of a depression situated over Gulf of Genoa; CL 10 an anticyclone over western Europe and low pressure around Great Britain; CL 11 high pressures affecting NW Iberian and Bay of Biscay; CL 12 low pressure affecting western Europe from NW origin (Fig. 8). 


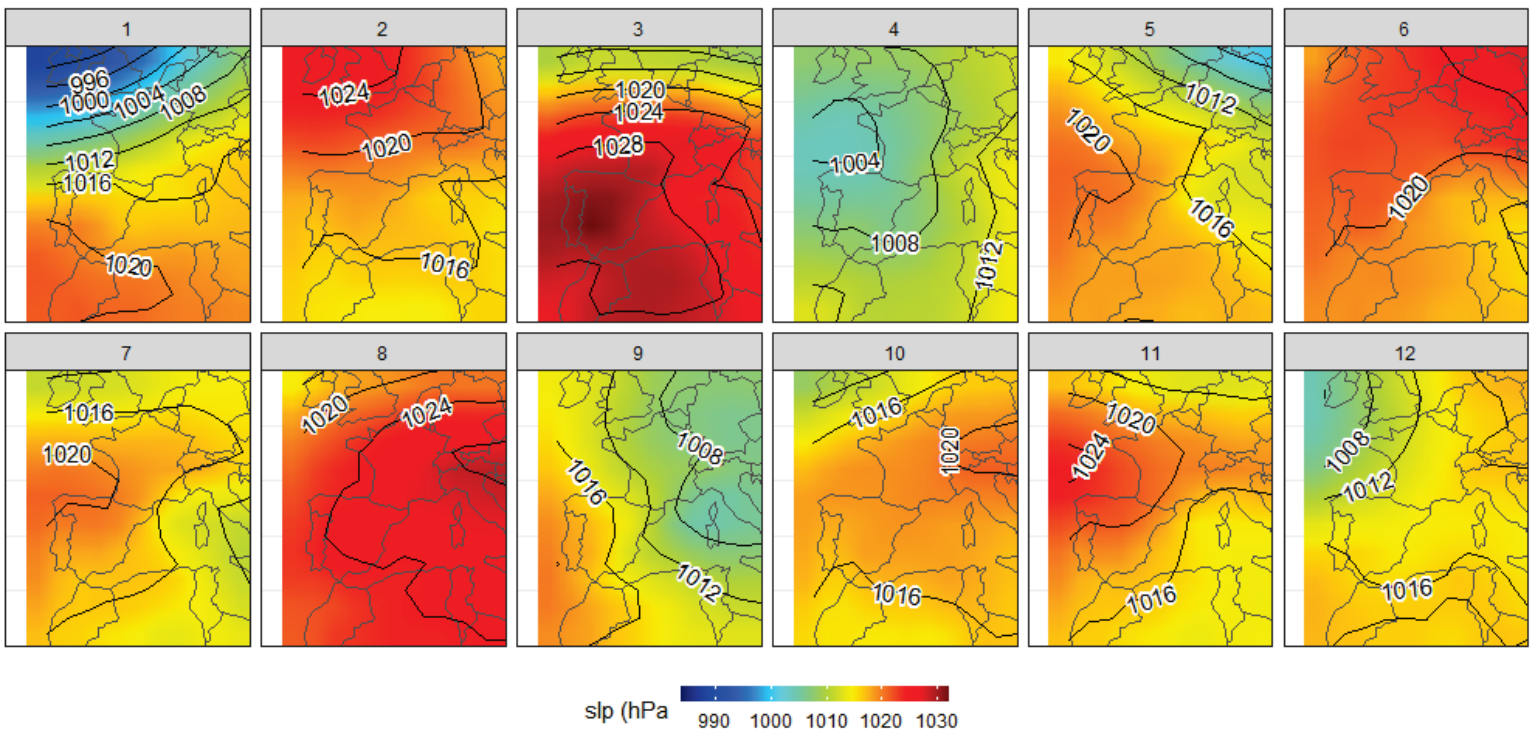

Figure 8. Mean SLP of the main CL in the study area.

Nevertheless, there are $5 \mathrm{CL}$ more recurrent categories explaining heavy snowfalls $(>15 \mathrm{~cm})$ in the eastern Pre-Pyrenees that concentrate more than $80 \%$ of such events. These large snow events are more frequent at Cadí-Nord $(n=74)$ than at Port del Comte $(n=36)$. During the snow season they are concentrated mainly in March, both at Port del Comte (32\% of the total) and at Cadí-Nord (27\%); followed by April (20 and 18\%, respectively) and January (15 and 20\%). The NW pattern (CL 4) is the synoptic configuration explaining the most frequent snowfalls ( $40 \%$ of the total). It is characterized by low pressure systems from a polar maritime origin, associated with wet and cold unstable air masses that generate SW air flow over NE Iberia; it is a frequent pattern in winter and the preceding and subsequent months. The second type of situation that led to more intense snowfalls was associated with the advection of a cold polar continental air mass (CL 8). Mediterranean cyclonic activity, formed by the interaction between cold-dry continental and warm-wet air masses from the Mediterranean Sea, characterize CL 9 and 11. These situations are mainly present at the beginning and final months of the season, and bring notable snow events at Cadí-Nord.

\subsection{The role of $N A O$ teleconnection pattern}

Practically the same correlation indexes between snowfall and NAO are observed at Port del Comte and at Cadí-Nord (Fig. 9). NAO is negatively correlated with snowfall in November at Port del Comte $(\mathrm{R}=-0.51)$ and in December at Cadí-Nord $(\mathrm{R}=-0.61)$, with a significant level of confidence in both cases. NAO also shows a high negative correlation in November at Cadí-Nord $(\mathrm{R}=-0.41)$ and in December at Port del Comte $(\mathrm{R}=-0.49)$, although at a non-significant level $(p>0.05)$. During the next months, the correlation is weak, with the exception of April $(\mathrm{R}=0.3, p=0.2)$. For example, in January and February we found positive correlations with NAO $(\mathrm{R}=0.16, p=0.58$ at Cadí Nord and $\mathrm{R}=0.03$, $p=0.89$ at Port del Comte), while in March is negative at Port del Comte $(\mathrm{R}=-0.23, p=0.28)$ and slightly positive at Cadí-Nord $(\mathrm{R}=0.06, p=0.2)$. In April the correlation is moderately negative, but at a not significant level ( $\mathrm{R}=-0.35, p=0.2$ at Cadí-Nord and $\mathrm{R}=-0.29, p=0.28$ at Port del Comte). Finally, snowfall in May shows no correlation with the $\mathrm{NAO}(\mathrm{R}=-0.075, p=0.79$ at Cadí-Nord and $\mathrm{R}=-0.07, p=0.8$ at Port del Comte). 

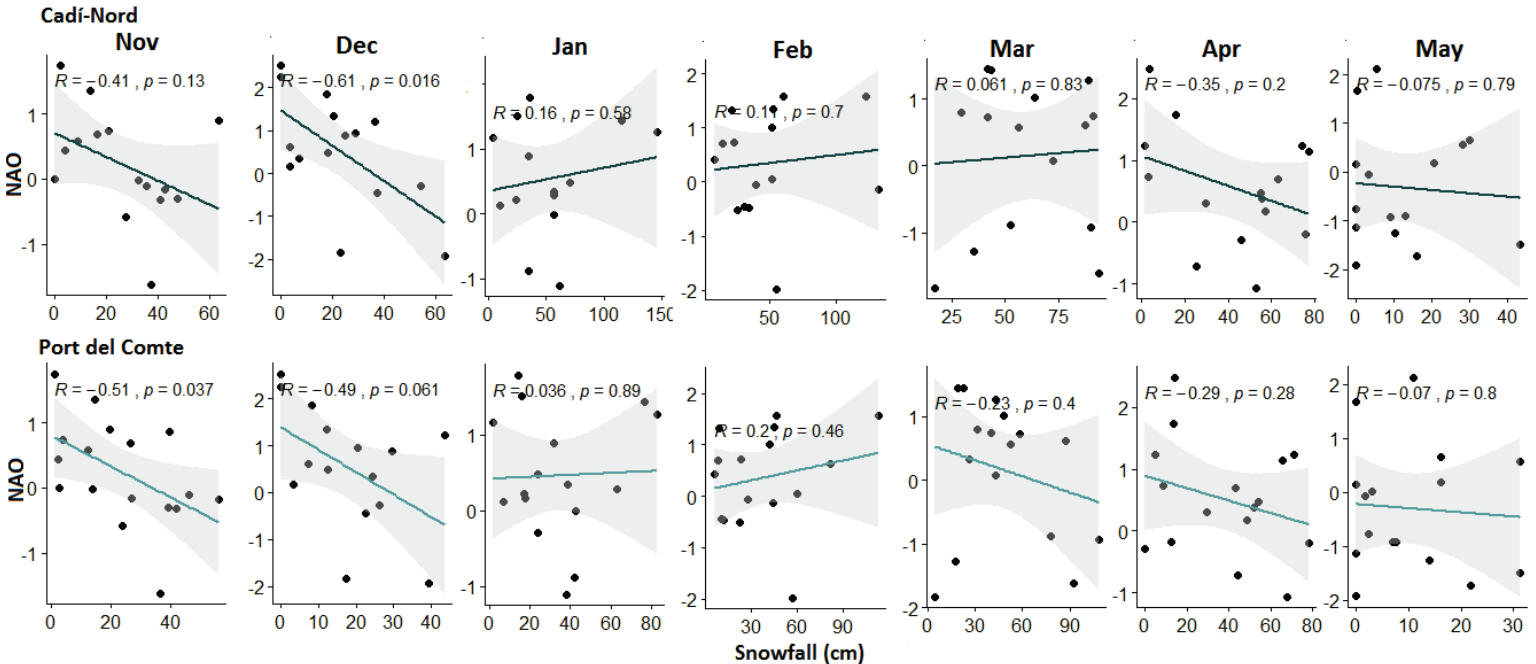

Figure 9. Correlation between mean monthly snowfall (cm) and NAO.

In conclusion, data confirm that the NAO plays a prominent role in determining frequency and amount of solid precipitation during the early snow season (November and December). The negative correlation indicating a negative NAO phase suggests that snowfall during these months is driven by low pressure systems located in the southern position, with a weakened Azores anticyclone. In this case, $\mathrm{W}$ and SW flows are active, with cyclonic genesis in the western sector of the Iberian Peninsula that favours snow precipitation in the eastern Pre-Pyrenees. During the rest of the season, the relationship with NAO and snowfall is inexistent or weaker and non-statistically significant.

\section{Discussion}

The proximity between Port del Comte and Cadí-Nord snow stations explains that the climate regime is mostly ruled by the same regional factors that determine a similar snow cover evolution. Nevertheless, local factors introduce some singularities at each site; Cadí-Nord snow station is located in a place affected by weak WSW winds that do not favour snow drift, whereas Port del Comte snow station is situated in an open area, exposed to WNW air flows that affect effectively the redistribution of the snow cover.

\subsection{Snowfall and snow depth patterns}

A high correlation between snowfall and snow depth is found between the two stations $(\mathrm{R}=0.86)$. Snowfall events from December to April include ca. $85 \%$ of the total events at both sites. Port del Comte, despite being located almost $200 \mathrm{~m}$ higher, records ca. 20\% less snow depth than Cadí-Nord. Xercavins (1985) showed that precipitation in the southern slopes of the eastern Pyrenees is strongly influenced by the W-E alignment of the mountain range, the distance to moisture sources (Atlantic vs Mediterranean) and the massifs distributed in the surroundings. While the northern face of Pyrenees intercepts the Atlantic low pressures - precisely the most frequent in high snow episodes (Fig. 10) -, the eastern sector of Pyrenees intercepts Mediterranean flows (Xercavins, 1985). Probably for this reason, Port del Comte massif is rain shadowed and climatologically isolated by the orographic in the surrounding. Therefore, instead of altitude, the lesser precipitation recorded at Port del Comte can be explained by its geographical setting, showing also slight continental influences. By contrast, the northern face of Cadí massif is slightly more exposed to the Mediterranean flows channelled through the Tet and Segre valleys, as shown by the higher frequency of heavy snowfall episodes associated with CL 9 and 11 (Fig. 10). 


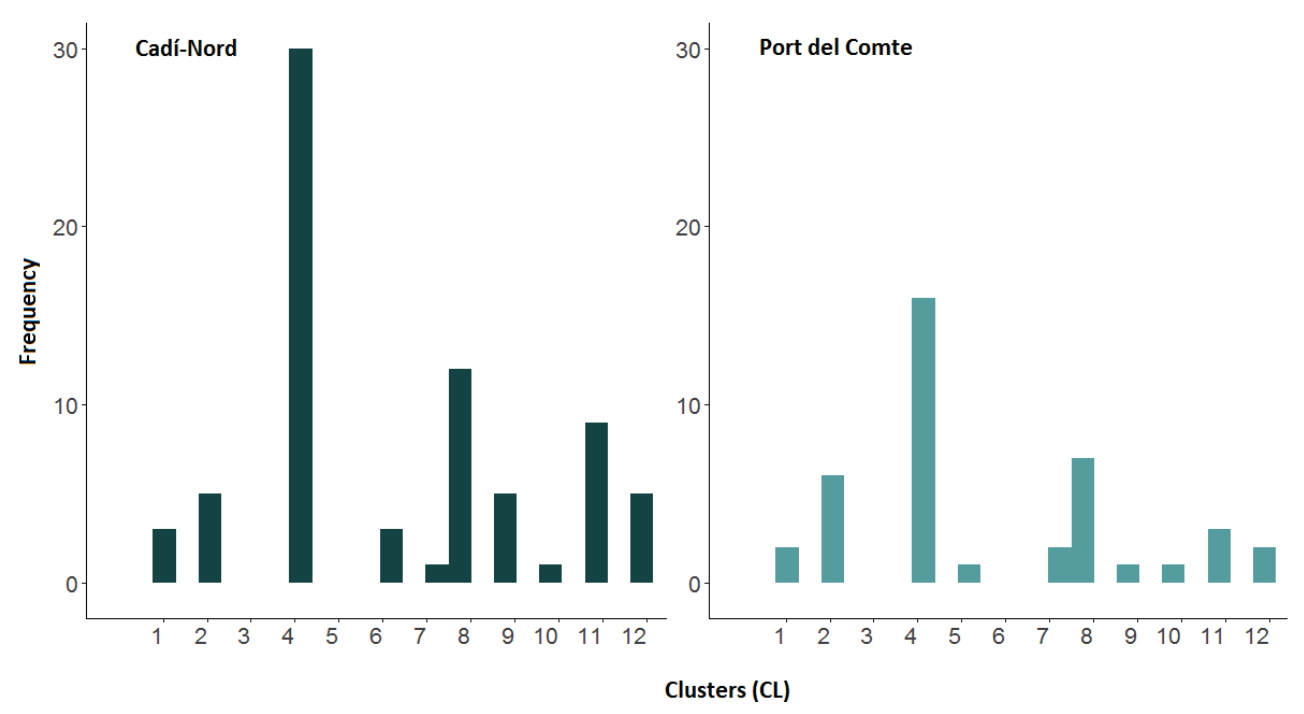

Figure 10. Frequency of days with $>15 \mathrm{~cm}$ of snowfall in both stations grouped by CL.

Our results are in agreement with Vigo et al. (2003), who showed an increase of drier conditions in the Pre-Pyrenees moving from the Mediterranean Sea towards NW, particularly in low elevations. The annual snowfall at Cadí-Nord is practically the same than in La Molina (251 cm; $1703 \mathrm{~m})$; just 20 $\mathrm{km}$ NE (Salvador-Franch et al., 2016). Patterns of precipitation in both regions are driven mostly by wet advections from the Mediterranean Sea uplifted with the first foothills of the eastern Pyrenees (Xercavins, 1985). The number of snowfall days in both stations is constrained by the lower elevation of La Molina station (50 vs 39). In La Molina, snowfall events can be more intense due to shorter distance to Mediterranean Sea, but tends to rain in ca. $20 \%$ of the episodes when at Port del Comte and at Cadí-Nord is snowing, because of the altitude difference.

The eastern Pre-Pyrenees show an alternation of snow-rich years with snow-poor years as detected in both stations. However, Port del Comte tends to have greater interannual variability ( $C V=41 \%$ ) than Cadí-Nord (CV=29\%). Snow-poor seasons (i.e. 2007-2008, 2014-2015) recorded less than half snow precipitation than the snowiest years (i.e. 2008-2009 or 2017-2018). This high interannual variability is linked with the Mediterranean climate of the eastern Pre-Pyrenees that is much reduced in the western fringe of the range where interannual variability is lower (CV $25 \%$; NavarroSerrano et al., 2017). Port del Comte and Cadí-Nord share approximately the same snowfall days, around 50 per season. Nevertheless, Cadí-Nord records higher snowfalls every season $(38 \mathrm{~cm})$ in comparison with Port del Comte $(26 \mathrm{~cm})$. Therefore, snowstorms are usually more intense at Cadí-Nord.

Generally, snow accumulation started in late November, reaching a maximum in February (Port del Comte) or in March (Cadí-Nord). The melting season is faster at Port del Comte, beginning mainly in April until late May in most cases. Along this period, snow cover at Cadí-Nord was thicker and more persistent on average than at Port del Comte. Snow thickness contrasts between both stations, with a thicker snow cover at Cadí-Nord $(66 \mathrm{~cm})$ and a thinner one at Port del Comte $(25 \mathrm{~cm})$. It also persisted for a longer duration at Cadí-Nord, 175 days and 148 days at Port del Comte. Yet, if we consider only days with notable snow cover, the difference is still greater for the Cadí-Nord. Even so, compared with nearby snow stations, Cadí-Nord station records higher snow depths than in the eastern Pyrenees, such as Núria snow station $(1970 \mathrm{~m})$, with $50 \mathrm{~cm}$ of snow depth per season, or La Molina (1703 m) with 21 $\mathrm{cm}$ (Salvador-Franch et al., 2014, 2016). Nonetheless, these differences are apparently related to the higher altitude of Cadí-Nord, the northern slope aspect and the low exposition to the prevailing winds. Snow accumulation in the eastern Pre-Pyrenees is fairly low compared with the snow amount measured in the western Pyrenees, which exceeds $600 \mathrm{~cm}$ in mountain areas above $2000 \mathrm{~m}$ (Navarro-Serrano et 
al., 2017). Nonetheless, snow covers the ground at ca. $40 \%$ of the year at Port del Comte and $47 \%$ at Cadí-Nord. These values are similar to other Iberian mountains: Alonso-González et al. (2020) demonstrated that the landscape in the Pyrenees was snow-covered above $2000 \mathrm{~m} \mathrm{ca} .40 \%$ of the days between 2000 and 2014, decreasing to 37\% in the Cantabrian Mountains and 27\% in the Central Iberian Range.

\subsection{Climatic factors affecting snow cover and weather types associated with snow accumulation}

The correlation between snow cover and temperature decreases gaining altitude, and vice versa (López-Moreno et al., 2005). For this reason, at the altitude where both stations are placed, the snow depth at the end of the snow season showed a high correlation with snowfall, and a weaker influence of temperatures. The accumulation of snowfall between January to April is the main driver of April snowpack followed by snowfall occurred in the same month. In comparison, Cadí-Nord showed a weaker relationship with snowfall in aggregated and isolated months, and a stronger correlation with temperature than at Port del Comte. In the western Pyrenees above $2200 \mathrm{~m}$ the correlation between January to April snowfall and the snow depth in April to May is very high ( $\mathrm{R}=0.72$; López-Moreno, $2005)$, similarly to Cadí-Nord $(\mathrm{R}=0.79)$ and Port del Comte $(\mathrm{R}=0.84)$. This difference between CadíNord and Port del Comte could be explained by the higher altitude of the last one.

The broad regional climatic influences existing across the Pyrenees from $\mathrm{W}$ to $\mathrm{E}$, and $\mathrm{N}$ to $\mathrm{S}$, explain the range of synoptic patterns that determine snowfall events in the Pyrenees. In the case of the eastern Pre-Pyrenees, medium to high $(>15 \mathrm{~cm})$ snow storms are usually recorded by a few circulation weather types, namely NW and N flows. Results from the eastern Pre-Pyrenees are in accordance with those observed in other areas of the Pyrenees. In high elevation areas of western Pyrenees $(>1800 \mathrm{~m})$, heavy snowfall episodes were linked also with NW, N and W air masses (Navarro-Serrano et al., 2017). Similar results were found in the central-eastern Pyrenees (Andorra), where NW and N advections are the situations that record more snow episodes, followed by NE advections (Esteban et al., 2005). Navarro-Serrano et al. (2017) pointed out the increasing influence of $\mathrm{NE}$ and $\mathrm{E}$ advections towards the $\mathrm{E}$ in the Pyrenees. Nonetheless, in the eastern Pre-Pyrenees the snowfall recorded by Mediterranean patterns (CL 9 and 11) are less frequent in medium to high snowfall episodes. Indeed, this frequency of this weather configuration driven by a low-pressure system centred around the Gulf of Genova has decreased between 1960 and 2010 (Lemus-Canovas et al., 2019a).

As regards to weather types associated with precipitation events over the Iberian Peninsula, W weather types, consisting of a high pressure system in the $\mathrm{W}$ of Canary Island and a low in the $\mathrm{W}$ of Ireland records the highest amounts of precipitation from September to March (Cortesi et al., 2013). In the lowlands of the NE Iberian Peninsula, between November and January the highest precipitation episodes correspond also with W weather types (Martin-Vide et al., 2008). These periods during the early snow season supply more solid precipitation in the eastern Pre-Pyrenees, statistically significant in November (Port del Comte) and December (Cadí-Nord), $\mathrm{R}=-0.51$ and $\mathrm{R}=-0.60$, respectively. These findings are in accordance with previous studies that identified December as the month with strong and significant negative correlation with precipitation and NAO in NE Iberia (Esteban et al., 2001). In the spring season, as expected by the dynamics of the general atmospheric circulation, the relation between snowfall and NAO is weak or inexistent. During winter, NAO takes an NW to SE alignment above the North Hemisphere, and lower than normal SLP around Azores leads the entrance of northern depressions to Iberia. In boreal spring, has less capacity of explanation, due to the NW to SE alignment and the fewer than winter extension and SLP amplitude (Hurrell et al., 1995). The lack of NAO influence in snowfall in the rest of the months at eastern Pre-Pyrenees is in accordance with previous studies on rainfall in the eastern fringes of the Iberian Peninsula. As well as negative NAO phases are strongly correlated with precipitation in the western Iberia, in the eastern parts of the Iberia there is a weak link between autumn and winter precipitation and the NAO pattern probably due to orographic factors, such as high altitudes 
or steep slopes (Martin-Vide et al., 2006). Indeed, an opposite influence between NAO and snow has been detected depending on the aspect and surrounding topography (Alonso-González et al., 2020). In addition, NAO negative phases are more significantly correlated with precipitation in SW-exposed valleys in the NE Iberia (Esteban et al., 2001).

The impact of the NAO on winter precipitation shows marked spatial differences over Iberian mountain ranges. Robust negative correlations of winter precipitation (and snowfall) and NAO were found in Sierra Nevada $(\mathrm{R}=-0.7)$, Cantabrian Mountains and the Central Iberian Range $(\mathrm{R}=-0.6)$ (LópezMoreno et al., 2011). In other mountain systems such as the Alps, positive NAO values are correlated with years with a thin snowpack; a negative correlation $(\mathrm{R}=-0.3)$ between NAO and snow depth was detected between 1931 and 1999 (Scherrer et al., 2006). Lastly, the temporal evolution in the last half of the 20th century has shown a trend to increase positive phases of NAO, high pressures in central Europe and anticyclone patterns over IP in winter, translated in a reduction of the WT associated with precipitation (López-Bustins et al., 2008).

\section{Conclusions}

There are still significant gaps on spatio-temporal patterns of snowfall in high mountain areas, such as the eastern Pyrenees, where snow cover plays a critical role for socio-economic activities. Here, the distribution of tens of ski resorts in elevations slightly below $2000 \mathrm{~m}$, together with the high interannual variability of the snow cover characteristic of this range, shows evidence of their critical future in the warming scenario projected by international reports. Changes in the spatio-temporal regime of precipitation, including snow, may also affect water availability in this Mediterranean area where droughts are recurrent and where major cities depend on water supply from the neighbouring mountains. A better characterization of snowfall and snow cover evolution is therefore needed to assess future changes of snow regime in the Pyrenees.

This work characterizes recent patterns of snowfall and snow depth evolution in two high altitude snow stations of the eastern Pre-Pyrenees (Port del Comte and Cadí-Nord) since the early 2000s. Snow cover has shown to be highly variable, with values ranging from 5 to $60 \mathrm{~cm}$ at Port del Comte, and from 24 to $117 \mathrm{~cm}$ at Cadí-Nord. The presence of snow on the ground at the end of the season is highly controlled by previous months snowfall. Snowfalls are mostly associated with NW weather configurations driven by negative NAO phases during November and December.

Snow cover in both areas is therefore close to limiting climatic conditions for snow ski resorts. A warming scenario or an increase of snowfall variability could impact the feasibility of these activities, or they should be adapted to the new climatic setting.

\section{Acknowledgements}

The work complements the research topics examined by the research groups "Paisatge $\mathrm{i}$ paleoambients a la muntanya mediterrània" (SGR2014-0373) and "Antarctic, Artic, Alpine Environments-ANTALP" (2017-SGR-1102), both funded by the Government of Catalonia through the Agència de Gestió d'Ajuts Universitaris i de Recerca. Marc Oliva is grateful for the support of the Ramón y Cajal research program (RYC-2015-17597). The authors acknowledge the Servei Meteorològic de Catalunya for the data provided for this study.

\section{References}

Alonso-González, E., López-Moreno, J.I., Navarro-Serrano, F., Sanmiguel-Vallelado, A., Revuelto, J., Domínguez-Castro, F., Ceballos, A. 2020. Snow climatology for the mountains in the Iberian Peninsula 
using satellite imagery and simulations with dynamically downscaled reanalysis data. International Journal of Climatology 40 (1), 477-491. https://doi.org/10.1002/joc.62235

Alonso-González, E., López-Moreno, J.I., Navarro-Serrano, F.M., Revuelto, J. 2020. Impact of North Atlantic Oscillation on the Snowpack in Iberian Peninsula Mountains. Water, 12, 105. https://doi.org/10.3390/w12010105

Buisan, S., López-Moreno, J.I., Saz, M.A., Kochendorfer, J. 2016. Impact of weather type variability on winter precipitation, temperature and annual snowpack in the Spanish Pyrenees. Climate Research 69, 79-92. https://doi.org/10.3354/cr01391

Cattell, R.B. 1966. The Scree Plot Test for the Number of Factors. Multivariate Behavioral Research. 1, 140-161.

Cortesi, N., Trigo, R., Gonzalez-Hidalgo J.C., Ramos, A. 2013. Modelling monthly precipitation with circulation weather types for a dense network of stations over Iberia. Hydrology and Earth System Sciences 17, 665678. https://doi.org/10.5194/hess-17-665-2013

Esteban, P., Soler, X., Prohom, M., Planchón, O. 2001. La distribución de la precipitación a través del índice NAO. El efecto del relieve a escala local: el Pirineo Oriental. In III Congreso de la AEC: El agua y el clima, Marratxí, Mallorca, pp. 594.

Esteban, P., Jones, P., Martin-Vide, J., Mases, M. 2005. Atmospheric Circulation patterns related to heavy snowfall days in Andorra Pyrenees. International Journal of Climatology 25, 319-329. https://doi.org/10.1002/joc. 1103

García-Ruiz, J.M., López-Moreno, J.I., Vicente-Serrano, S., Lasanta, T., Beguería, S. 2011. Mediterranean Water Resources in a Global Change Scenario. Earth-Science Reviews 105, 121-139. https://doi.org/10.1016/j.earscirev.2011.01.006

Hall, A. 2004. The Role of Surface Albedo Feedback in Climate. Journal of Climate 17, 1550-1568. https://doi.org/10.1175/1520-0442(2004)017<1550:TROSAF>2.0.CO;2

Hurrell, J.W. 1995. Decadal trends in the North Atlantic Oscillation: regional temperatures and precipitation. Science 269, 676-679. https://doi.org/10.1126/science.269.5224.676

IPCC, 2013. Climate Change: The Physical Science Basis. In: T.F. Stocker, D. Qin, G.K. Plattner, M. Tignor, S.K. Allen, J. Boschung, A. Nauels, Y. Xia, V. Bex, P.M. Midgley (Eds.). Contribution of Working Group I to the Fifth Assessment Report of the Intergovernmental Panel on Climate Change, Cambridge University Press, Cambridge, United Kingdom and New York, NY, 1535.

Kalnay, E., Kanamitsu, M., Kistler, R., Collins, W., Deaven, D., Gandin, L., Iredell, M., Saha, S., White, G., Woollen, J., Zhu, Y., Chelliah, M., Ebisuzaki, W., Higgins, W., Janowiak, J., Mo, K.C., Ropelewski, C., Wang, J., Leetmaa, A., Reynolds, R., Jenne, R., Jospeh, D. 1996. The NCEP/NCAR 40-Year Reanalysis Project. Bulletin American Meteorological Society 77, 437-472. https://doi.org/10.1175/15200477(1996)077<0437:TNYRP >2.0.CO;2

Kaiser, H.E. 1958. The varimax criterion for analytic rotation in factor analysis. Psikometrica 23, 187-200. https://doi.org/10.1007/BF02289233

Lasanta, T., Vicente-Serrano, S., Cuadrat, J. 2005. Mountain Mediterranean landscape evolution caused by the abandonment of traditional primary activities: A study of the Spanish Central Pyrenees. Applied Geography 25, 47-65. https://doi.org/10.1016/j.apgeog.2004.11.001

Lemus-Canovas, M., Lopez-Bustins, J.A., Trapero, L., Martin-Vide, J. 2019a. Combining circulation weather types and daily precipitation modelling to derive climatic precipitation regions in the Pyrenees. Atmospheric Research 220, 181-193. https://doi.org/10.1016/j.atmosres.2019.01.018

Lemus-Canovas, M., Lopez-Bustins, J.A., Martin-Vide J., Royé, D. 2019b. synoptReg: An R package for computing a synoptic climate classification and a spatial regionalization of environmental data. Environmental Modelling \& Software 118, 114-119. https://doi.org/10.1016/j.envsoft.2019.04.006

Lopez-Bustins, J.A, Martin-Vide, J., Sanchez-Lorenzo, A. 2008. Iberia winter rainfall trends based upon changes in teleconnection and circulation patterns. Global and Planetary Change 63, 171-176. https://doi.org/10.1016/j.gloplacha.2007.09.002

López-Moreno, J.I. 2005. Recent variations of snowpack depth in the central Spanish Pyrenees. Arctic Antarctic Alpine Research 37(2), 253-260. 
López-Moreno, J.I., Soubeyroux, J.M., Gascoin, S., Alonso-González, E., Durán-Gómez, N., Lafaysse, M., Vernay, M., Carmagnola, C., Morin, S. 2020. Long-term trends (1958-2017) in snow cover duration and depth in the Pyrenees. International Journal of Climatology, 1-15. https://doi.org/10.1002/joc.6571

López-Moreno, J.I., Vicente-Serrano S.M., Morán-Tejeda E., Lorenzo J., Kenawy, A., Beniston, M. 2011. NAO effects on combined temperature and precipitation winter modes in the Mediterranean mountains: Observed relationships and projections for the 21st century. Global and Planetary Change 77, 62-76.

Navarro-Serrano, F., López-Moreno, J.I. 2017. Spatio-Temporal analysis of snowfall events in the Spanish Pyrenees and their relationship to Atmospheric Circulation. Cuadernos de Investigación Geográfica (Geographical Research Letters) 43(1), 233-254. https://doi.org/10.18172/cig.3042

Martin-Vide, J., Lopez-Bustins, J.A. 2006. The Western Mediterranean Oscillation and rainfall in Iberian Peninsula. International Journal of climatology 26, 1455-1475. https://doi.org/10.1002/joc.1388

Martin-Vide, J., Sanchez-Lorenzo, A., Lopez-Bustins, J.A., Cordobilla, M.J., Garcia-Manuel, A., Raso, J. 2008. Torrential rainfall in northeast of the Iberian Peninsula: Synoptic patterns and WeMO influence. Advances in Science and Research (2), 99-105. https://doi.org/10.5194/asr-2-99-2008

Oliva, M., Ruiz-Fernández, J., Barriendos, M., Benito, G., Cuadrat, J.M., García-Ruiz, J.M., Giralt, S., GómezOrtiz, A., Hernández, A., López-Costas, O., López-Moreno, J.I., López-Sáez, J.A., Martínez- Cortizas, A., Moreno, A., Prohom, M., Saz, M.A., Serrano, E., Tejedor, E., Trigo, R., Valero-Garcés, B.L., VicenteSerrano, S. 2018. The Little Ice Age in Iberian mountains. Earth-Science Review 177, 175-188. https://doi.org/10.1016/j.earscirev.2017.11.010

Pepin, N., Bradley, R., Diaz, H. 2015. Elevation-dependent warming in mountain regions of the world. Nature Climate Change 5, 424-430. https://doi.org/10.1038/nclimate2563

Revuelto, J., López-Moreno, J.I., Morán, E., Fassnacht, S., Vicente, S.M., 2012. Variabilidad interanual del manto de nieve en el Pirineo: tendencias observadas y su relación con índices de teleconexión durante el periodo 1985-2011. En: C. Rodríguez, A. Ceballos, N. González, E. Morán, S. Pacheco, A. Hernández (Eds.). Asociación Española de Climatología, pp. 613- 621, Madrid. http://hdl.handle.net/20.500.11765/8325

Salvador Franch, F., Salvà, G., Vilar, F., García, C. 2014. Nivometría y perfiles de innivación en Núria (1970 m, Pirineo Oriental): 1985-2013. En: IX Congreso de la AEC, pp. 729-738, Almería. http://hdl.handle.net/20.500.11765/8229

Salvador-Franch, F., Salvà, G., Vilar, F., García, C. 2016. Contribución al análisis nivométrico del Pirineo Oriental: La Molina, periodo 1956-1996. En: X Congreso Internacional AEC: Clima, sociedad, riesgos y ordenación del territorio, pp. 365-375, Alicante. http://hdl.handle.net/10045/58002

Scherrer, S.C., Appenzeller, C. 2006. Swiss Alpine snow pack variability: major patterns and links to local climate and large-scale flow. Climatic Research 32, 187-199. https://doi.org/10.3354/cr032187

SMC. 2008. Atlas climàtic de Catalunya: periode 1961-1990. Termopluviometria. ICC. Barcelona.

SMC. 2019. Roses dels vents climàtiques. https:/www.meteo.cat/wpweb/climatologia/serveis-i-dadesclimatiques/roses-dels-vents-climatiques/ (last access: 10/05/2020).

Vicente-Serrano, S.M., Rodríguez-Camino, E., Domínguez-Castro, F., El Kenawy, A., Azorín-Molina, C. 2017. An updated review on recent trends in observational surface atmospheric variables and their extremes over Spain. Cuadernos de Investigación Geográfica (Geographical Research Letters) 43 (1), 209-232. https://doi.org/10.18172/cig.3134

Vigo, J., Soriano, I., Carreras, J., Aymerich, P., Carrillo, E., Font, X., Masalles, R.M., Ninot, J.M. 2003. Flora del Parc Natural del Cadi-Moixeró i de les serres vë̈nes. Monografies del Museu de Ciències Naturals, 1. Barcelona.

Wipf, S., Stoeckli, V., Bebi, P. 2009. Winter climate change in alpine tundra: plant responses to changes in snow depth and snowmelt timing. Climatic Change 94, 105-121. https://doi.org/10.1007/s10584-009-9546-x

Xercavins-Comas, A. 1985. Els climes del Pirineu Oriental: des de les terres gironines fins a la Catalunya Nord i Andorra. Documents d'Anàlisi Geogràfica 7, 81-102. 



\title{
ROCK GLACIER AND PROTALUS RAMPART INVENTORY IN LAS SALINAS RIVER BASIN, CENTRAL ANDES OF ARGENTINA
}

\author{
ANA PAULA FORTE ${ }^{1,2^{*}}$, CRISTIAN DANIEL VILLARROEL ${ }^{1,2,}$ \\ MARÍA YANINA ESPER ANGILLIERI ${ }^{2}$
}

\author{
${ }^{1}$ Facultad de Ciencias Exactas y Naturales (FCEN), \\ Universidad Nacional de Cuyo (UNCuyo), Argentina. \\ ${ }^{2}$ Centro de Investigaciones de la Geosfera y la Biósfera (CIGEOBIO), Centro de Ciencia y Técnica de \\ San Juan (CCT - San Juan), Universidad Nacional de San Juan - CONICET, \\ Av. Ignacio de la Roza 590 (O), Complejo Universitario «Islas Malvinas», \\ Rivadavia, San Juan, CPA: J5402DCS, Argentina.
}

\begin{abstract}
This paper presents a detailed rock glacier and protalus rampart inventory from Las Salinas river basin, an arid subtropical mountain hydrological system (between $31^{\circ} 02^{\prime}$ and $31^{\circ} 22^{\prime} \mathrm{S}$ latitude) located in the northern sector of the Central Andes of Argentina, where permafrost and cryogenic processes prevail. The inventory is based on a geomorphological characterization by means of optical remote sensing and field description data. The study region covers $630 \mathrm{~km}^{2}$, with $3.25 \%$ of the area containing $405 \pm 8.2$ rock glaciers and protalus ramparts in total, of which $231 \pm 2.5$ are considered protalus rampart and $49 \pm 2$ are considered active, $61 \pm 1$ inactive and $64 \pm 3$ fossil rock glaciers. Frequency ratio and logistic regression were used as statistical methods to determine the relationship between the distribution of these periglacial landforms and different geological, morphometric and climatic variables as elevation, potential incoming solar radiation, slope, aspect and lithology. Results show that elevation, lithology, and aspect are the most influencing factors for the occurrence of active rock glaciers. According to rock glaciers and protalus ramparts distribution, the permafrost occurrence probability is above 3690 $\mathrm{m}$ a.s.l. (current and high periglacial environment). However, some inactive rock glaciers and protalus rampart were found below this elevation, thus between 3300 and $3690 \mathrm{~m}$ a.s.l. the landscapes are dominated by an unstable periglacial environment.
\end{abstract}

\section{Inventario de glaciares rocosos y protalus rampart en la cuenca del río Las Salinas, Andes centrales argentinos.}

RESUMEN. Este artículo presenta un inventario detallado de glaciares rocosos y protalus rampart en la cuenca del río Las Salinas, un sistema hidrológico de montaña subtropical (entre $31^{\circ} 02^{\prime}$ y $31^{\circ} 22^{\prime} \mathrm{S}$ de latitud), localizado en el sector septentrional de los Andes centrales de Argentina, donde predominan el permafrost y los procesos criogénicos. El inventario se basa en una caracterización geomorfológica mediante teledetección óptica y datos de descripción de campo. La región cubre $630 \mathrm{~km}^{2}$. Un 3,25\% del área contiene un total de 405 $\pm 8,2$ glaciares rocosos y protalus rampart, de los que $231 \pm 2,5$ pueden considerarse protalus rampart, $49 \pm 2$ pueden catalogarse como activos, $61 \pm 1$ inactivos y $64 \pm 3$ glaciares rocosos fósiles. Ratio de frecuencias y regresión logística se utilizaron como métodos estadísticos para determinar la relación entre la distribución de estas formas periglaciares y diferentes variables geológicas, morfométricas y climáticas como la altitud, radiación solar entrante potencial, pendiente, exposición y litología. Los resultados muestran que la altitud, la litología y la exposición son los factores más influyentes en la aparición de glaciares rocosos activos. Según la distribución de los glaciares rocosos y protalus rampart, el permafrost se sitúa por encima de los $3690 \mathrm{~m}$ s.n.m. (medioambiente periglacial actual). Sin embargo, por debajo de esta elevación se encontraron algunos glaciares rocosos y protalus rampart inactivos, por lo que entre los 3300 y 3690 m s.n.m., los paisajes están dominados por un ambiente periglacial inestable. 
Key words: Rock glacier, mountain permafrost, protalus rampart, Central Andes of Argentina, environmental variables.

Palabras clave: Glaciar rocoso, permafrost de montaña, protalus rampart, Andes centrales argentinos, variables ambientales.

Received: 6 November 2020

Accepted: 7 February 2021

\begin{abstract}
*Corresponding author: Ana P. Forte, Centro de Investigaciones de la Geosfera y la Biósfera (CIGEOBIO), Centro de Ciencia y Técnica de San Juan (CCT - San Juan), Universidad Nacional de San Juan - CONICET. Av. Ignacio de la Roza 590 (O), Complejo Universitario «Islas Malvinas», Rivadavia, San Juan, CPA: J5402DCS, Argentina. Email address: anapau.forte@gmail.com
\end{abstract}

\title{
1. Introduction
}

Cryospheric science shows a special interest in regions of arid mountain ranges, such as the Central Andes of Argentina, where permafrost and periglacial processes are prevalent. A reason for this is that periglacial environments are one of the main hydrological resource suppliers (Ohlanders et al., 2013). More specifically, intact rock glaciers and protalus ramparts are considered solid water reserves (Scapozza et al., 2011; Jones et al., 2018; Villarroel et al., 2020) and indicators of mountain permafrost (Barsch D., 1996; Haeberli et al., 2010).

Rock glaciers are periglacial landforms comprised of debris and interstitial ice, ice lenses or even massive ice bodies. They have a root zone (upper part) with a moderate incline and a frontal slope (lower part) with a very steep hillside (Barsch, 1996; Haeberli et al., 2010). Being an expression of creeping mountain permafrost, they are constituted by a permafrost core and a surface layer (active layer) that is in contact with the atmosphere. Some rock glaciers have an intermediate layer (transition layer), which at times acts as part of the permafrost and at other times as a part of the active layer. Whereas, protalus ramparts are periglacial landforms that are typically smaller than rock glaciers and exhibit a greater width/length ratio. They have a pronounced frontal slope and superficially do not show ridges and furrows superficially. They have been mentioned as the result of ice rich ground creep (Colucci et al., 2016) and some authors deem them as embryonic rock glaciers since they are also considered an expression of mountain permafrost (Barsch, 1996; Scapozza et al., 2011; Hedding, 2011; Scotti et al., 2013). Other authors associate the occurrence of protalus ramparts with the existence of old patches of perennial snow (Whalley and Azizi, 2003). In this work the definition provided by Barsch (1996) has been adopted.

Intact rock glaciers are classified as active and inactive based on their dynamics (Haeberli et al., 2010). Active rock glaciers show movement rates in the order of tens of centimeters per year, while inactive rock glaciers show almost no signs of surface movements (Roer and Nyenhuis, 2007; Kääb et al., 2007). The state of activity of the intact rock glaciers is closely related to their capacity as solidwater reserves (Azócar and Brenning, 2010; Jones et al., 2018, 2019) and to the degree of permafrost stability, given the surrounding environmental conditions (Kääb et al., 2007). Thus, their activity could be considered as a very accurate indicator of current climatic conditions and the state of the permafrost and the periglacial environment (stable or in degradation). However, climatic conditions in the past have also influenced the current distribution of rock glaciers (Haeberli et al., 2010, Jones et al, 2018).

The classification of rock glaciers as active, inactive and fossil is of great environmental significance; therefore, it is important to consider the highest number of criteria to reduce possible errors (Barsch, 1996; Haeberli et al., 2010; Ikeda and Matsuoka, 2006; Harrison et al., 2008; Esper Angillieri, 
2010, 2017; Jones et al., 2019). Active rock glaciers preserve and increase their ice content; whereas inactive rock glaciers, due to their unbalance with the environment, keep losing ice and, thus, are considered water sources. Moreover, rock glaciers that have lost all their ice content and indicate unfavorable periglacial environmental conditions, are called fossil, relict or pseudo-relict rock glaciers (Scotti et al., 2013; Colucci et al., 2019). Nevertheless, some periglacial processes could still exist around this kind of rock glaciers.

The Central Andes of Argentina mountain range is characterized by a wide periglacial environment development and for having the highest density of rock glaciers in the world (Trombotto et al., 2012). However, a detailed inventory along with descriptions of the landforms of the region is still scarce. This paper presents a rock glacier and protalus rampart inventory carried out in the northern sector of the Central Andes of Argentina, more specifically, in Las Salinas river basin, which encompasses the Frío, La Embarrada, El Bagre, and De Las Salinas or De Las Lagunas rivers. In the past decade, a number of rock glacier inventory surveys (Esper Angillieri, 2017; Trombotto et al., 2012; Villarroel and Forte, 2020; Tapia Baldis, 2018) and two regional studies (ING, 2016; Villarroel et al., 2018) have been conducted in nearby areas.

The relationship between environmental variables (climatic, lithological, topographic and morphometric) and the spatial distribution of rock glaciers is essential for conducting regional studies on periglacial environment distribution. Especially in the case of high mountain areas, where poor accessibility reduces the chances of obtaining climatic data, while it steeply increases the costs of systematic field studies. In this paper, we employ frequency ratio and logistic regression analyses as statistical methods. Various researches of this kind have been performed in other regions e.g.: Brenning (2005), Brenning and Trombotto (2006), Ikeda and Matsuoka (2006), Johnson et al. (2007) and Esper Angillieri (2010, 2017).

The results presented in this study will therefore prove to be a significant contribution to knowledge available on the number, features and distribution of rock glaciers and protalus ramparts in a scarcely studied sector of the Central Andes of Argentina. Furthermore, they could be useful for both regional and larger-scale assessments, mainly for permafrost distribution and geological risk studies.

\section{Regional setting}

The study region is Las Salinas river basin, which is situated on a northern sector of the Central Andes, in the Blanco river headwaters, covering about $13 \%$ of the Blanco river basin $\left(4861 \mathrm{~km}^{2}\right)$. Las Salinas basin includes the De Las Lagunas or De Las Salinas, El Bagre, La Embarrada and Frío river basins (Fig. 1). This hydrological system feeds north-western tributaries of the San Juan river basin, one of the two most important rivers of the region, with a total mean annual discharge of $56 \mathrm{~m}^{3} / \mathrm{s}$ (Subsecretaría de Recursos Hídricos, 2002). The Las Salinas river basin spreads over an area of 630 $\mathrm{km}^{2}$, with a total perimeter of $155 \mathrm{~km}$ and elevations between 2940 and $4750 \mathrm{~m}$ a.s.l. Its closing point is located at the geographical coordinates: $31^{\circ} 21.30^{\prime} \mathrm{S}$ and $70^{\circ} 23.35^{\prime} \mathrm{O}$, where the Salinas river flows into the Blanco river. 

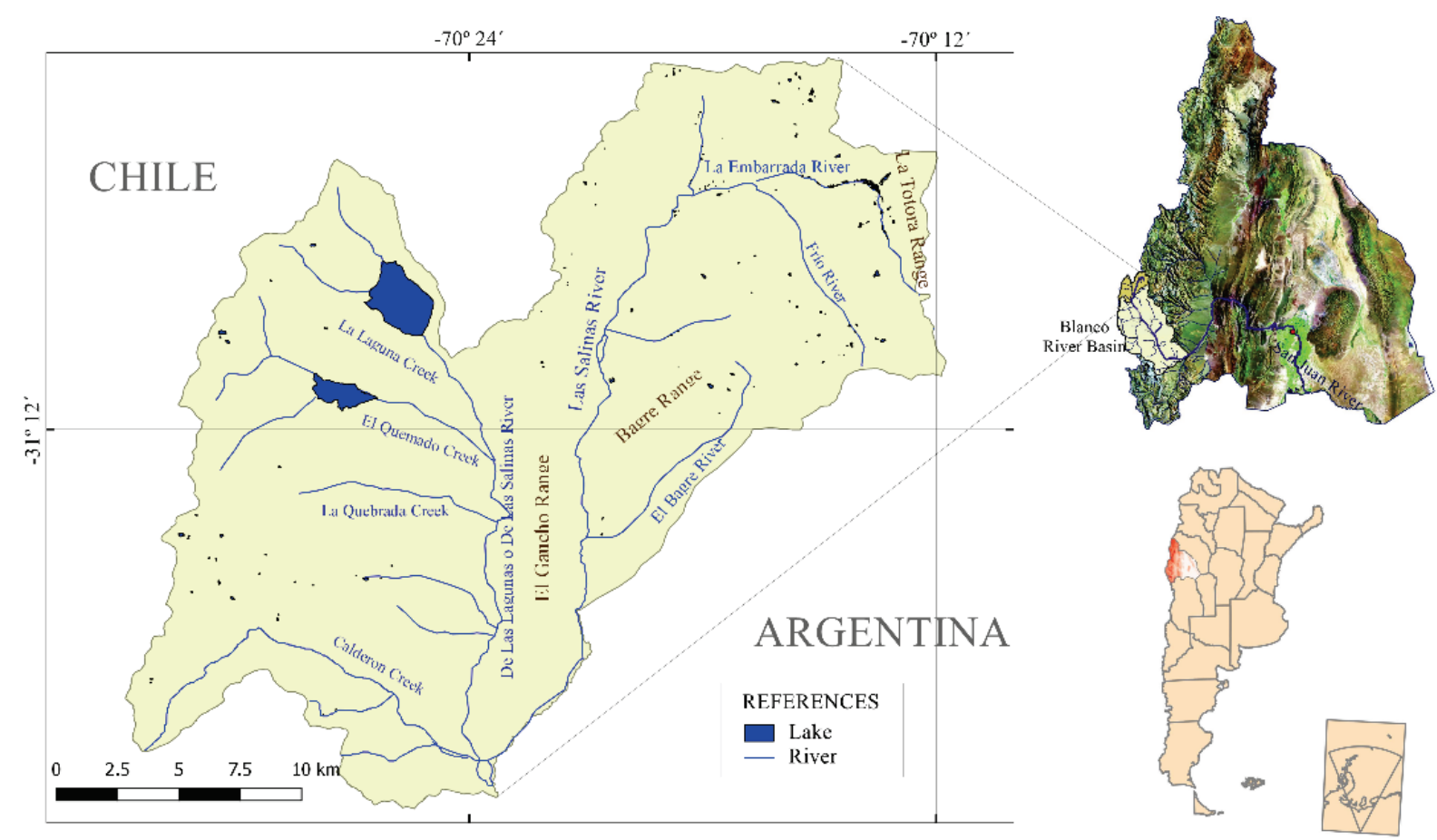

Figure 1. Location of Las Salinas river basin, part of Blanco river basin in the province of San Juan, Argentina

\subsection{Climatic and geomorphological setting}

The semi-arid climate of the study region, which lies on the rain shadow on the leeward side of the Andean Range, is influenced by the South American Arid Diagonal (Bruniard, 2014) and the interaction between the high-pressure systems of the Atlantic and Pacific Oceans (Rivera et al., 2013). Warm moist air collides and travels the Andes orography, which acts as a barrier for the moisture transfer from the Pacific (Strecker et al., 2007). Therefore, upon precipitations on the Chilean side, a dry and warm Föhn effect wind, starts blowing on the leeward side of the Andes mountain ranges. Annual moisture input in the region is highly variable, which correlates with the El Niño Southern Oscillation (or ENSO) phenomena (Corripio et al., 2007) and the wet atmospheric south-east masses coming from Atlantic winds. In the study area, the mean annual air temperature (MAAT) is $2.4^{\circ} \mathrm{C}$ at $3375 \mathrm{~m}$ a.s.l., based on measurements between April 2011 and February 2014 from three weather stations placed in the study area - two at El Altar and one at Los Azules mining projects (Schreiber 2015).

Other nearby meteorological stations indicate: a MAAT of $+0.59^{\circ} \mathrm{C}(3600 \mathrm{~m}$ a.s.l. during the period 1981-2003) at the Pachón meteorological station of Hydraulic Department of the government of San Juan (Villarroel, 2019); a MAAT of $+0.55^{\circ} \mathrm{C}(3740 \mathrm{~m}$ a.s.l. for the period 2009-2015) at the Pachón mining project meteorological station (Villarroel, 2019). While the General Water Directorate (DGA, Chile) in the upper portion of the Choapa valley indicates a MAAT of $+0.5^{\circ} \mathrm{C}$ at $3700 \mathrm{~m}$ a.s.l. (Monnier and Kinnard, 2013). It is worth noting that in Forte (2020) a regional isotherm of $0^{\circ} \mathrm{C}$ was calculated at $3800 \mathrm{~m}$ a.s.l. for the Central Andes.

In addition, the meteorological stations of the region show a mean annual precipitation value of $350 \mathrm{~mm}$ for an elevation of $4019 \mathrm{~m}$ a.s.l. (Portezuelo de la Guardia Station for the period 2009-2014; Tapia Baldis, 2018) and $297.6 \mathrm{~mm}$ for an elevation of $3600 \mathrm{~m}$ a.s.l. during the period 1981-2003, at the Pachón meteorological station of Hydraulic Department of the government of San Juan (Villarroel, 2019).

The relief in the Central Andes is mainly shaped by tectonic system activity (Strecker et al., 2007) and by Pleistocene glaciations (Forte and Villarroel, 2019; Palacios et al., 2020), thus, there are landforms caused by glacial erosion, e.g.: glacial striae, U-shaped and asymmetric valleys, smooth 
polished bedrocks, erratic blocks, cirques, rams, truncated spurs, edges, tarns, horns, hanging or suspended valleys, among others. Accumulation landforms conforming glacial deposits, such as moraines, erratic blocks or other sedimentary deposits (Till) are also frequently found. Currently, the glacial landscape is being modified and weathered by gravitational, fluvial, alluvial and periglacial processes. As regards periglacial landforms, there is a striking number of active, inactive and fossil rock glaciers, as well as other periglacial landforms such as protalus ramparts, solifluction hillsides, stones rings and belts, stratified slope waste deposits, convex-concave debris-mantled slopes and polygonal grounds. Furthermore, the local permafrost models show a high probability of permafrost occurrence in the study area (Esper Angillieri, 2017; Tapia Baldis, 2018). In this study area, lakes and lacustrine deposits are associated to moraines and mass wasting deposits. There is a noticeable change in the hydrological system below 3200 m a.s.1., where high Andean wetlands which grow on the foothills and lagoon systems (locally known as Vegas) has been identified.

\subsection{Geological setting}

The structural, topographic, stratigraphic and geological aspects of the region are highly dependent on the geometry of the Wadatti Benioff zone, where the interaction between the South American and Nazca tectonic plates takes place.

In the study area, Eo-Paleozoic rock deposits consist of strongly deformed Intrusive Rocks (DPig). Overlying the Paleozoic rocks, the Mesozoic deposits are found, which are mainly represented by the Choiyoi Group (Rolleri and Criado, 1970). This group is constituted by Permian-Triassic volcanic and pyroclastic rocks (PTrv) of alkaline composition, i.e. basalt, andesite, dacite and rhyolite rocks. The upper section of the group is characterized by aciditic association of intrusive and volcanic rocks (PTrg), consisting of gabbros, granodiorites and tonalities rocks. There is also a Jurassic and Lower Cretaceous marine sedimentary sequence ( $\mathrm{Jk}$-ism). The Cenozoic deposits are represented by Paleogene rocks (EOp and $\mathrm{OMp}$ ) in close relation with volcanic and plutonic events. In addition, there is a wide development of Neogene sedimentary and volcanic sequences (Ms and Mv) corresponding to the Lower Miocene. Finally, Quaternary deposits overlie the surface of the valleys (P1Qs), which consists mainly of glacial accumulation, proof of Pleistocene glacial advance and retreat sequences.

\section{Materials and methods}

In order to analyze the influence of environmental variables on the periglacial system, we applied a threefold approach: (1) Detailed mapping of rock glacier and protalus ramparts, (2) setup of a rock glacier inventory database, and (3) statistical analysis of rock glacier distribution.

The rock glacier and protalus ramparts inventory has been framed within the corresponding hydrological system. The network and basin drainage were mapped using a digital elevation model with a 12.5 x 12.5 m spatial resolution provided by ALOS Palsar (AP_07965_FBD_F6550). Rock glacier identification, classification, mapping and inventory were carried out based on geomorphological criteria (Roer and Nyenhuis, 2007) using field information and optical satellite imageries Terra/ASTER; CBERS2B/HRC; Sentinel 2; SPOT5 and SPOT 7. The landforms were manually (on-screen) digitized as vectors, using projection UTM zone 19 south and WGS84 datum. Mapping was performed on scenes from Autumn 2016, due to the diversity and good quality of the images available. Around $78 \%$ of the rock glacier and protalus rampart identifiers on optical images were checked on site.

For each rock glacier and protalus rampart mapped, the abrupt slope change in the root zone was considered as the upper limit. While the lower limit was delimitated by the marked slope change of the frontal and lateral talus.

The criterion used to distinguish protalus ramparts was their geomorphological characteristics: size (usually small), shape (similar width and length), steep frontal talus, smooth surfaces (no furrows and ridges structures); and their spatial location (frequently on steep slopes). 
The geomorphological criterion for identification and classification of rock glaciers included aspects as the degree of furrow and ridges development, slope angle of the frontal talus, presence/absence of vegetation, presence of springs, streams or bodies of liquid water on its surface or edges, weathering of exposed block faces and thermokarst occurrences. Rock glaciers were classified as fossil or relict and intact, the latter were subclassified as active and inactive.

- Active rock glaciers are characterized by a steep front talus $\left(>35^{\circ}\right)$, generally with exposure of fine debris and overlying loose boulders on the top and an accumulation of large boulders at the bottom (Ikeda and Matsuoka, 2006). Also, they show a pronounced development of furrow and ridges, evidence of on-going movement and are formed on favorable environmental conditions for the occurrence of geocryological processes (Haeberli et al., 2010).

- Inactive rock glaciers still contain ice but actually do not move and keep their shape. Unlike active rock glaciers, they do not show notable development of ridges and furrows on their surface. They are characterized by depressed surfaces, low angle talus slopes, and possibly by the presence of vegetation and collapsed structures. Ikeda and Matsuoka (2006) have considered talus slopes as criteria to classify a rock glacier: if the angle is less than $35^{\circ}$, it is inactive - in the case it is greater, it is active. As mentioned before, inactive rock glaciers still conserve ice, but their permafrost conditions are in a degradation state, so it is possible to find liquid water (springs, small streams or lagoons) and vegetation, as well as, collapse structures such as depressions or thermokarst.

- Fossil rock glaciers tend to have gentler slopes and a major collapsed surface due to ice melting. They have lost all their ice content and indicate current unfavorable environmental conditions for rock glacier or mountain permafrost development. The presence of vegetation on their surface is common, and their frontal talus has a lower angle and is also usually underdeveloped.

A detailed field control was conducted on the Frío, La Embarrada, Los Patos Norte and Bagres river basins and near the Salinas drainage. Geomorphological field studies allowed a clear characterization of the periglacial landforms, especially to detect vegetation, collapse structures, weathering blocks, springs and other geomorphological structures and processes. Field controls were conducted by late summer and early autumn (March and April), because in that period the area was clear of snow, which facilitated fieldwork.

Some of the geomorphological criteria used, in exceptional cases, may present ambiguities, for example active rock glaciers with depressions product of collapse or fossil rock glaciers without such depression, can be sometimes found. However, though this method of classifying rock glaciers may present errors, it has been shown to be a fairly accurate approach. In order to assess the rock glacier and protalus rampart inventory, an uncertainty study was performed. The processes of identification, classification and mapping of periglacial landforms were carried out by two different individuals. Firstly, the uncertainty for each type of landform was described. The results were manually compared and the criteria were discussed in detail. Then, the total amount of rock glaciers and protalus ramparts mapped (n: total amount) was analyzed.

Database for each landform mapped included area, elevation, lithology, slope, aspect and potential incoming solar radiation values.

Frequency ratio and logistic regression methods were performed in order to analyze the relationship between active rock glacier occurrence and environmental variables (lithology, elevation, aspect, slope and potential incoming solar radiation). These variables were classified into categories, which were defined taking into account the rock glacier distribution over each category, and representative values were chosen for each one. 
Nine types of lithological units were identified in the area based on the Geological and Mining Service of Argentina Republic (SEGEMAR, 2008) (Table 1). Elevation, slope, aspect and potential incoming solar radiation were obtained using a digital elevation model with $12.5 \times 12.5 \mathrm{~m}$ spatial resolution provided by ALOS Palsar (Dataset: ASF DAAC 2015, ALOS 235140 PALSAR_Radiometric_Terrain_Corrected_Hi_res; Includes Material (C JAXA/METI 2007. Accessed through ASF DAAC, https://www.asf.alaska.edu 16 May 2016 DOI: 237 10.5067/JBYK3J6HFSVF). Physical parameters were calculated using the open-source Quantum GIS, GV GIS, SAGA GIS and Kosmo GIS softwares. In order to categorize these variables (elevation, slope, aspect and solar radiation), the mean, minimum and maximum rock glacier values were taken into account.

Table 1. Lithological Units.

\begin{tabular}{|l|l|l|}
\hline Symbol & \multicolumn{1}{|c|}{ Age } & \multicolumn{1}{c|}{ Description } \\
\hline PlQs & Pliocene - Quaternary & Sedimentary Deposits \\
\hline Mv & Middle to Upper Miocene & Volcanic Rocks \\
\hline Ms & Middle to Upper Miocene & Sedimentary Clastic Rocks \\
\hline OMp & Oligocene - Lower Miocene & Intrusive Porphyry and Subvolcanic Dome Rocks \\
\hline EOp & Eocene - Oligocene & Intrusive Porphyry Complex Rocks \\
\hline Jk ism & Jurassic - Lower Cretaceous & Sedimentary Clastic Rocks \\
\hline PTrg & Permian - Triassic & Intrusive Granitic Acid Rocks \\
\hline PTrv & Permian - Triassic & Volcanic, Pyroclastic and Sedimentary Alkaline Rocks \\
\hline DPig & Devonian - Lower Permian & Intrusive Basic Rocks \\
\hline
\end{tabular}

For analyzing the altitudinal distribution of the rock glaciers, the study area was divided into four main altitudinal ranges based on their elevation values (Table 2). The mean aspect units were calculated using the WGMS methodology (Paul et al., 2010). The aspect cartography was performed using the Travis et al (1975) algorithm over the ALOS PALSAR DEM (Table 3). Also, sky view factor, aspect and slope (derivatives from ALOS PALSAR DEM) were used to obtain the annual potential solar radiation cartography. In addition, the latitudinal geographic position of the study area (position of incidence of sun rays) was taken into account. In order to consider the seasonal differences that characterize subtropical areas, the measurements were calculated every 7 days in periods of 1.5 hours for a whole year by using SAGA GIS software. The measuring unit for output radiation raster was watthours per square meter $\left(\mathrm{W} \cdot \mathrm{hr} / \mathrm{m}^{2}\right)$. The chosen solar constant was $1,367 \mathrm{~W} / \mathrm{m}^{2}$, a value assigned by the World Radiation Reference Centre (WRRC) proposed by the WMO (World Meteorological Organization).

Table 2. Elevation units.

\begin{tabular}{|c|c|c|c|c|}
\hline \multirow{2}{*}{\multicolumn{2}{|c|}{ Elevation Units }} & \multicolumn{2}{|c|}{ Elevation (m.a.s.l.) } & \multirow{2}{*}{$\operatorname{Area}\left(\mathrm{Km}^{2}\right)$} \\
\hline & & Min. & Max. & \\
\hline \multicolumn{2}{|c|}{ High Periglacial Environment } & 4225 & 4750 & 17.23 \\
\hline \multirow{3}{*}{$\begin{array}{l}\text { Current } \\
\text { Periglacial } \\
\text { Environment }\end{array}$} & Upper & 4047 & 4225 & 36.26 \\
\hline & Middle & 3868 & 4047 & 70.12 \\
\hline & Lower & 3690 & 3868 & 104.3 \\
\hline \multicolumn{2}{|c|}{ Unstable Periglacial Environment } & 3300 & 3690 & 283.58 \\
\hline \multicolumn{2}{|c|}{ Seasonal Frost } & 2940 & 3300 & 118.45 \\
\hline
\end{tabular}


Table 3. Aspect units.

\begin{tabular}{|c|c|c|}
\hline Aspect units & \multicolumn{2}{|c|}{ Degree } \\
\hline $\mathrm{N}$ & 337.5 & 22.5 \\
\hline $\mathrm{NE}$ & 22.5 & 67.5 \\
\hline $\mathrm{E}$ & 67.5 & 112.5 \\
\hline $\mathrm{SE}$ & 112.5 & 157.5 \\
\hline $\mathrm{S}$ & 157.5 & 202.5 \\
\hline $\mathrm{SW}$ & 202.5 & 247.5 \\
\hline $\mathrm{W}$ & 247.5 & 292.5 \\
\hline $\mathrm{NW}$ & 292.5 & 337.5 \\
\hline
\end{tabular}

Frequency ratio analyzes the relationship between the occurrences of a landform (in this case rock glaciers) and the different categories of the environmental variables studied (Bonham-Carter, 1994; Esper Angillieri, 2010). This method takes the number of pixels with rock glacier occurrence over a determinate category for each variable as input data, and then considers the percentage of the total number of pixels with respect to the complete study area (Eq. 1).

$$
F_{r}=\left(N_{i} / N\right) / S_{i} / S
$$

Therefore, the frequency ratio $\left(F_{r}\right)$ can be calculated through the following Eq. (1). Where $S$ is the total number of pixels; $N$ is the number of pixels with rock glacier occurrence; $S_{i}$ is the number of pixels, being $i$ the factor or variable; and $\mathrm{N}_{\mathrm{i}}$ is the number of pixels in which the rock glaciers occurred for the $i$-attribute or factor. If $F_{r}$ is greater than 1 , it means a higher correlation. A value smaller than 1 means a lower correlation.

Logistic regression estimates the probability of rock glacier existence based on independent variables or predictors (Hosmer and Lemeshow, 1989; Atkinson et al., 1998). It is a multivariate regression model where the dependent variable is categorical, this paper covers the case of a binary dependent variable: absence and presence of rock glaciers (values 0 and 1). The predictor variables used were lithology, elevation, slope, aspect and potential incoming solar radiation. The lithological and aspect units were treated as categorical variables, while slope, elevation, and solar radiation were considered as continuous variables. A bilateral and Pearson correlation coefficient was applied to verify the existence of correlation between variables and to evaluate their independence.

For logistic regression analysis, the number of points representing areas with rock glacier occurrence or non-occurrence should be the same (Ayalew and Yamagishi, 2005). In the study area, 1000 points represent the active rock glacier presence. Therefore, 1000 points without rock glacier occurrence were randomly selected for logistic regression; while $\mathrm{N}$ is the total number of analyzed points $=2000$. This data is used to incorporate predictor variables, thus allowing to estimate the probability of rock glacier occurrence from the presence of some category of each variable.

Also, a multicollinearity checking was performed in order to assess the correlation between independent variables (Hosmer and Lemeshow, 1989). Multicollinearity is a statistical situation in which two or more predictor variables are highly correlated, meaning that one can be linearly predicted from the others with a non-trivial degree of accuracy. Tolerance (TOL) and the variance inflation factor (VIF) are two important indexes that are widely used for multicollinearity checking. According to Menard (1995), a TOL value of less than 0.2 is one indicator for multicollinearity, and serious multicollinearity occurs between independent variables when the TOL values are smaller than 0.1 . The VIF is calculated by $1 /$ tolerance. If the VIF value exceeds 10 , it is often regarded as indicating multicollinearity. 


\section{Results}

The study area, which covers $630 \mathrm{~km}^{2}$, is characterized by a wide development of periglacial environment. The total number of rock glaciers and protalus ramparts inventoried is 405 with an accuracy of \pm 8.2 (Fig. 2, Table 4).

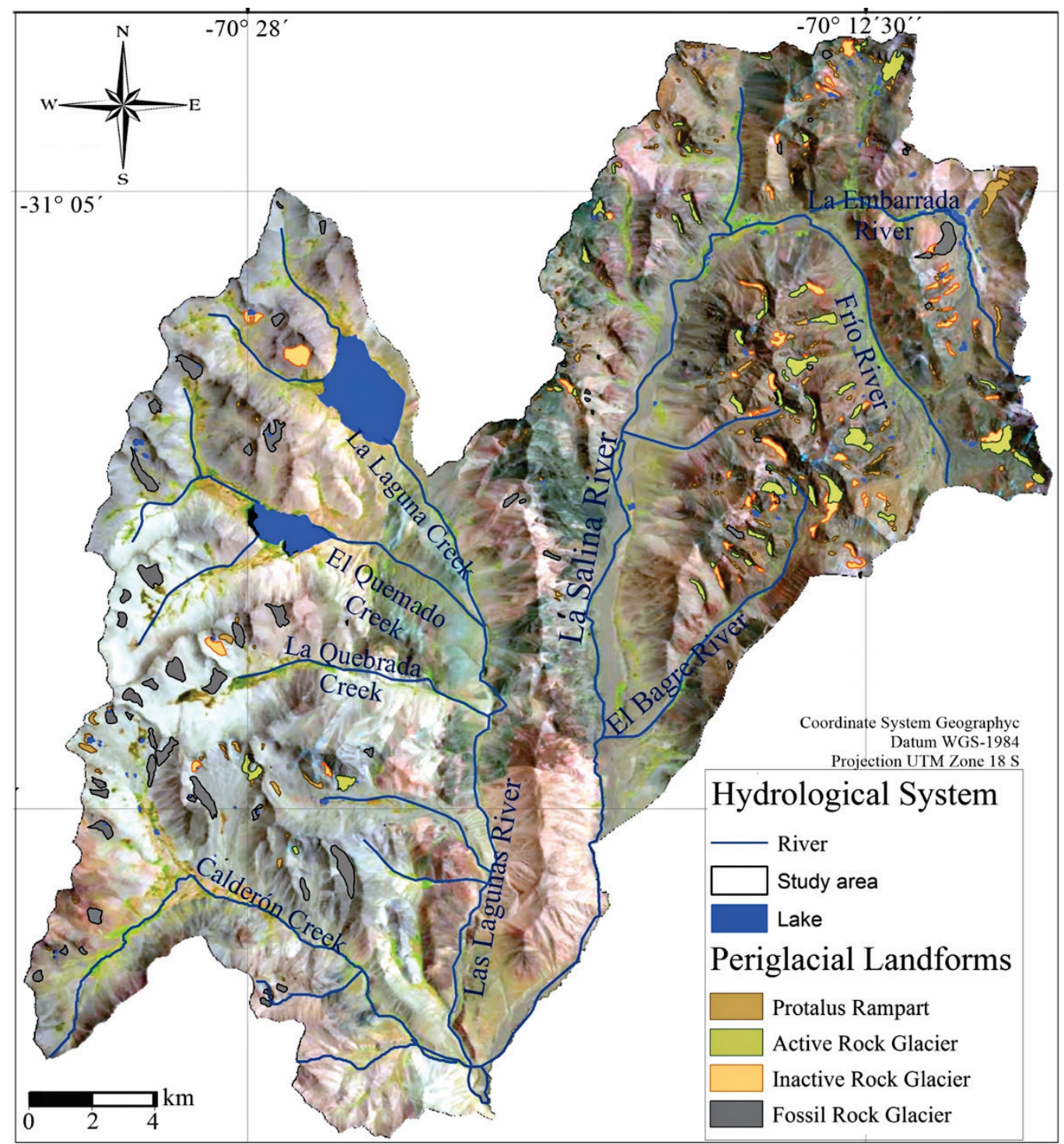

Figure 2. Rock glacier and protalus rampart inventory.

Table 4. Inventory uncertainty assessment.

\begin{tabular}{|l|c|c|c|c|c|c|}
\hline \multicolumn{1}{|c|}{ Type of landforms } & $\mathbf{n}_{\mathbf{a}}{ }^{*}$ & $\mathbf{n}_{\mathbf{b}}{ }^{* * *}$ & $\mathbf{n}_{\mathbf{m}}{ }^{* * *}$ & variance & Stand. dev. & According number \\
\hline Active rock glacier & 53 & 49 & 51 & 4 & 2 & $49+/-2$ \\
\hline Inactive rock glacier & 62 & 60 & 61 & 1 & 1 & $61+/-1$ \\
\hline Fossil rock glacier & 66 & 60 & 63 & 9 & 3 & $64+/-3$ \\
\hline Protalus rampart & 234 & 229 & 231.5 & 6.25 & 2,5 & $231+/-2,5$ \\
\hline Total of landforms & 415 & 398 & 406.5 & 67.24 & 8.2 & $405+/-8.2$ \\
\hline
\end{tabular}

${ }^{*} \mathrm{Na}$ : Amount of landforms identified by Person A.

${ }^{* *} \mathrm{Nb}$ : Amount of landforms identified by Person B.

${ }^{* * *} \mathrm{Nm}$ : average of both values. 
A number of 231 protalus ramparts and 174 rock glaciers have been identified -49 considered active rock glaciers. Field studies were performed over $80 \%$ of the total inventory (Fig. 3).

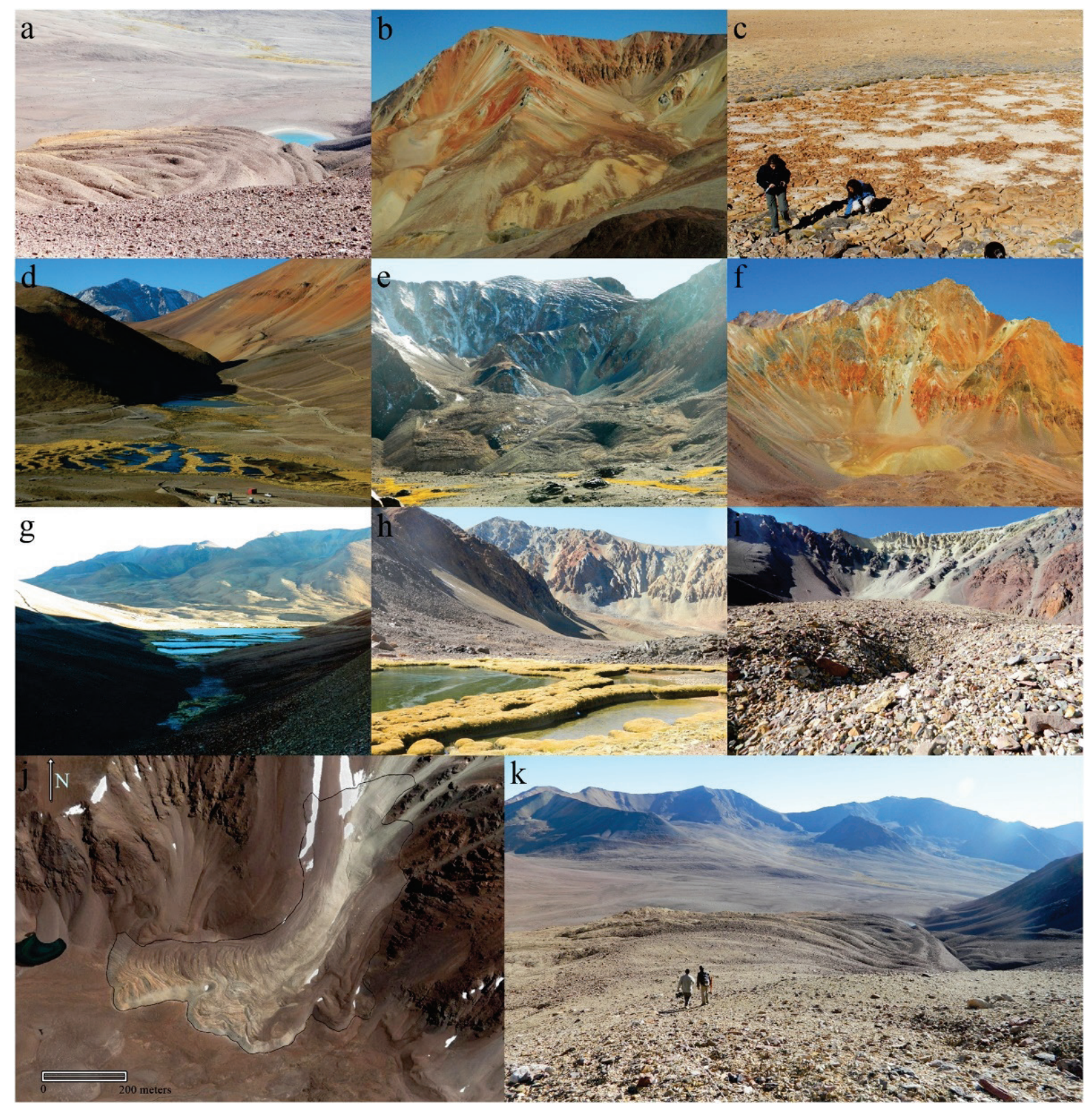

Figure 3. Periglacial landforms identified in the field. a) Furrows and ridges on an active rock glacier (geographic coordinates: $31^{\circ} 9.75^{\prime} \mathrm{S}$ and $70^{\circ} 12.8^{\prime} \mathrm{W}$ ). b) Inactive rock glacier (geographic coordinates: $31^{\circ} 12^{\prime} \mathrm{S}$ and $70^{\circ} 14.5^{\prime} \mathrm{W}$ ). c) Patterned ground (geographic coordinates: $31^{\circ} 3^{\prime} 50^{\prime \prime S}$ and $70^{\circ} 14^{\prime} 10^{\prime \prime} \mathrm{W}$ ). d) Vegas or Andean wetlands associated to lagoon systems (geographic coordinates: $31^{\circ} 6^{\prime} S$ and $70^{\circ} 13^{\prime} 40^{\prime \prime} \mathrm{W}$ ). e) Los Patos Norte active rock glacier (geographic coordinates: $31^{\circ} 3.1^{\prime} \mathrm{S}$ and $70^{\circ} 15^{\prime} \mathrm{W}$ ). $\mathrm{f}$ ) Active rock glacier (geographic coordinates: $31^{\circ} 10^{\prime}$ and $70^{\circ} 12.1^{\prime} \mathrm{W}$ ). g) Vegas or Andean wetlands associated to water springs and lagoon systems (geographic coordinates: $31^{\circ} 5^{\prime} 25^{\prime \prime}$ S and 70 $73^{\circ} 11^{\prime \prime} \mathrm{W}$ ). h) Vegas or Andean wetlands associated to water springs and lagoon systems (geographic coordinates: $31^{\circ} 31^{\circ} 6^{\prime} 40^{\prime \prime S}$ and $70^{\circ} 13^{\prime} 7^{\prime \prime} \mathrm{W}$ ). i) Thermokarst on Amarillo active rock glacier surface (geographic coordinates: $31^{\circ} 9^{\prime} 45^{\prime \prime} \mathrm{S}$ and $70^{\circ} 12^{\prime} 57^{\prime \prime} \mathrm{W}$ ). j) Amarillo active rock glacier mapping (geographic coordinates: $31^{\circ} 9.75^{\prime} \mathrm{S}$ and $70^{\circ} 12.8^{\prime} \mathrm{W}$ ). $\mathrm{k}$ ) Furrows and ridges on Amarillo active rock glacier surface. two persons as scale. 
The altitudinal distribution of the periglacial landforms identified varies between $2940 \mathrm{~m}$ a.s.l. and $4750 \mathrm{~m}$ a.s.1. The total elevation range was fragmented into 4 altitudinal units, based on minimum and maximum elevation of intact rock glaciers (Table 2 and Fig. 4 and 5) and, in turn, the third unit was subdivided into three classes.

The first unit (between 2940 and $3300 \mathrm{~m}$ a.s.1.) was considered a seasonal frost unit because no periglacial landforms were identified under it, only fossil landforms (Fig. 5c). So, it is probable that periglacial phenomena are mainly limited to seasonal freezing and thawing. The second unit comprises the area between 3300 and $3690 \mathrm{~m}$ a.s.l. (lower inactive and active rock glacier elevation); which is considered an unstable periglacial environment unit, since there are only inactive rock glaciers (Fig. 5b). The third unit includes areas comprehended between the lower and upper active rock glacier elevations (Fig. 5a). As mentioned above, this unit was subdivided into three intervals corresponding to the lower, middle and upper current periglacial environments (3690-3868, 3868-4047 and 4047-4225 m a.s.1.), considering the area below $3300 \mathrm{~m}$ a.s.l. the lower intact rock glacier altitude. The fourth and highest unit includes areas with higher elevations than $4225 \mathrm{~m}$ a.s.l., generally mountain peaks, where landforms lie on the highest slopes (Fig. 4 and 5).

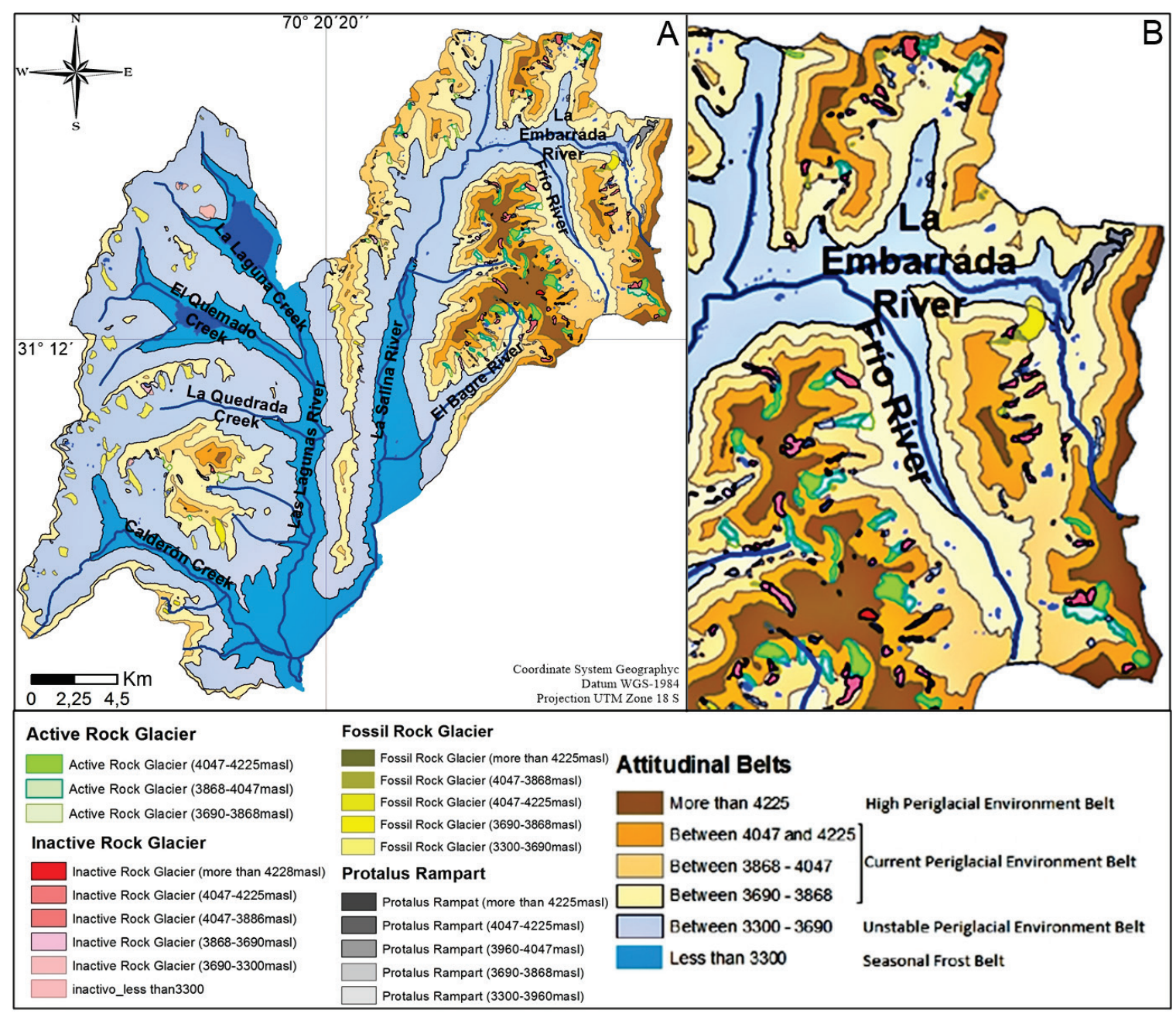

Figure 4. Rock glacier and protalus rampart distribution over the six altitudinal units. A) Regional map. B) Detailed view of the area with the highest density of periglacial landforms. 
Forte et al.

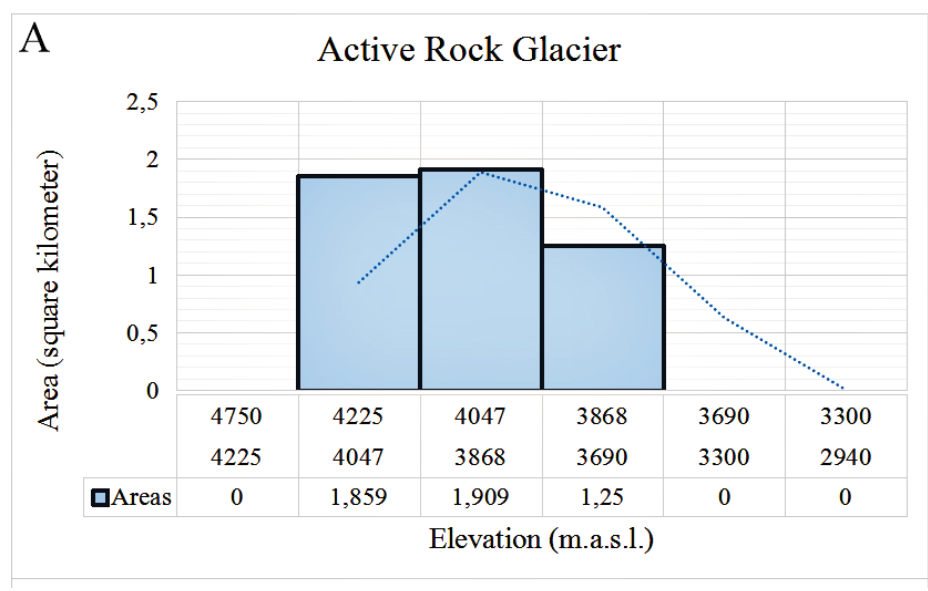

$\mathrm{B}$

Inactive Rock Glacier

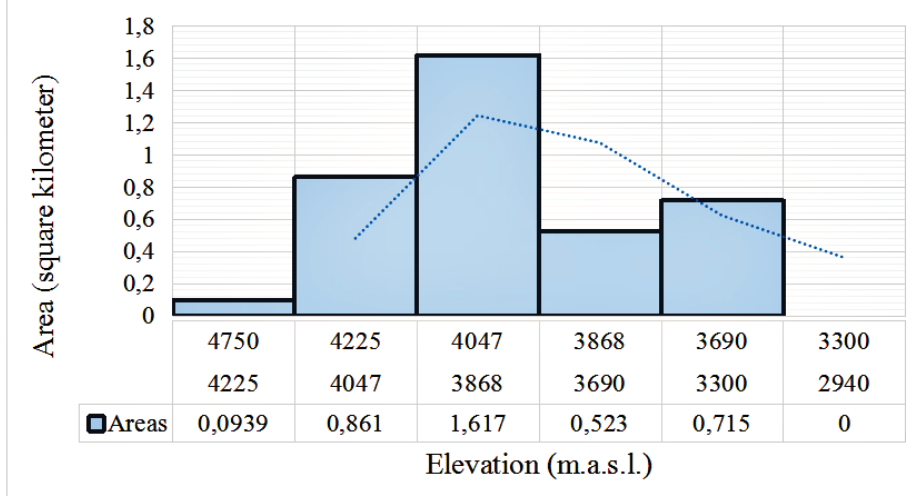

C Fossil Rock Glacier

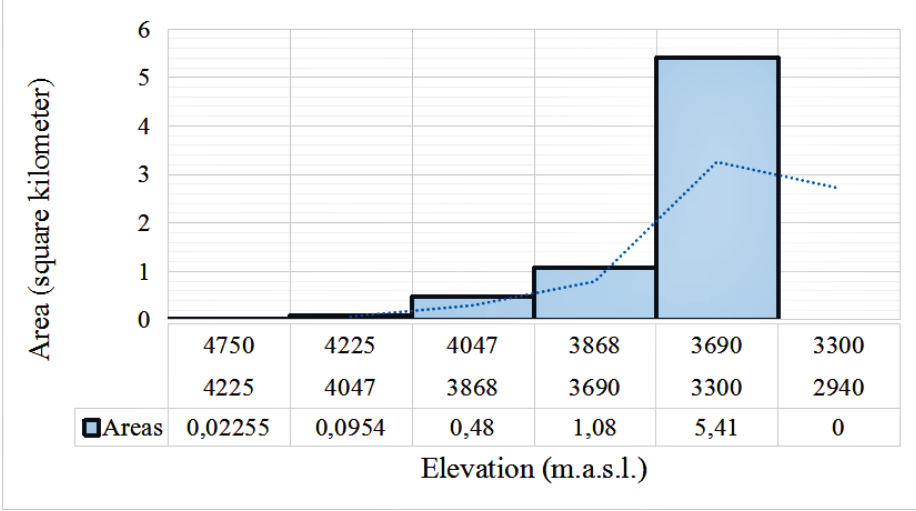

$\mathrm{D}$

Protalus Rampart

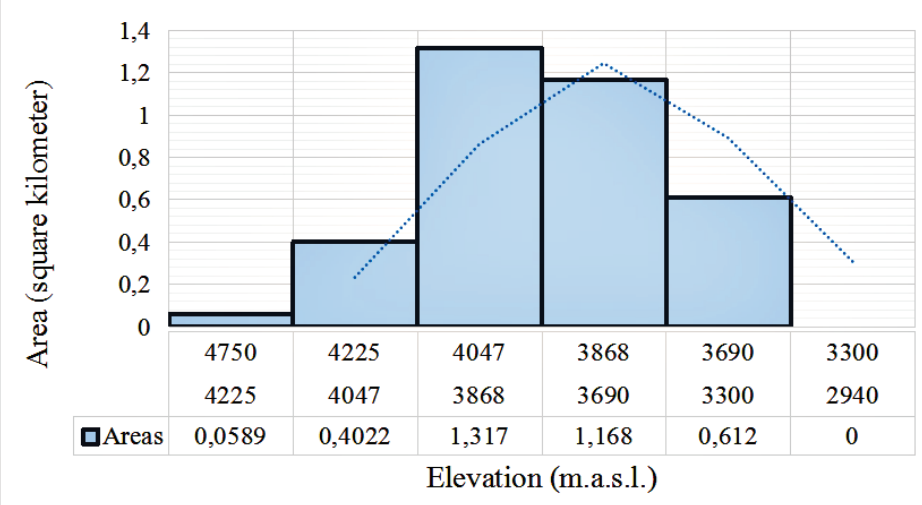

Figure 5. Hypsometry of the studied periglacial landforms. A) Active rock glaciers. B) Inactive rock glaciers. C) Fossil rock glaciers. D) Protalus rampart. 
The spatial distribution of rock glaciers and protalus ramparts over the nine lithological units shows that rock glaciers are usually made up of volcanic, pyroclastic and sedimentary alkaline rocks (PTrv) and, to a lesser extent, by intrusive granitic rocks (PTrg); both lithologies are Permian-Triassic rocks with a significant degree of Cenozoic hydrothermal alteration (Fig. 6 and 7). Also, active rock glaciers trend to appear over areas with Middle to Upper Miocene volcanic rock outcrops (Mv). While inactive rock glaciers are developed more frequently on intrusive porphyric and subvolcanic rocks from the Oligocene-Lower Miocene (OMp) and Eocene-Oligocene (EOp) periods.

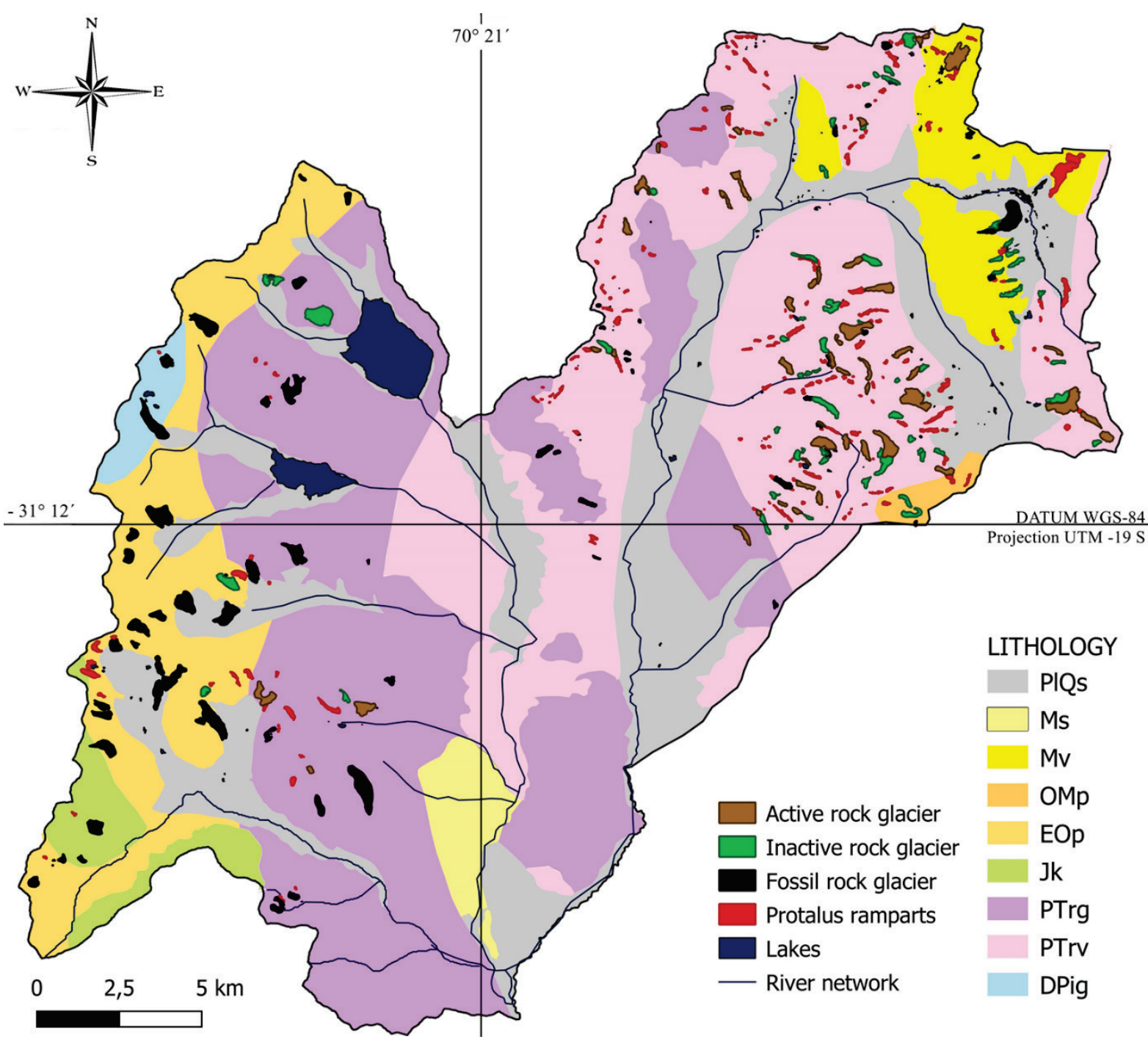

Figure 6. Rock glacier and protalus rampart distribution over the nine lithological units based on SEGEMAR geological map (2008). PlQs: Pliocene-Quaternary sedimentary deposits. Mv: Middle to upper Miocene volcanic rocks. Ms: middle to upper Miocene sedimentary clastic rocks. OMp: Oligocene-lower Miocene intrusive porphyry and subvolcanic rocks. EOp: Eocene-Oligocene intrusive porphyry complex rocks. Jk ism: Jurassic-lower Cretaceous sedimentary clastic rocks. PTrg. Permian-Triassic intrusive granitic acid rocks. PTrv: Permian-Triassic volcanic, pyroclastic and sedimentary alkaline rocks. DPig: Devonian-lower Permian intrusive basic rocks 
Forte et al.
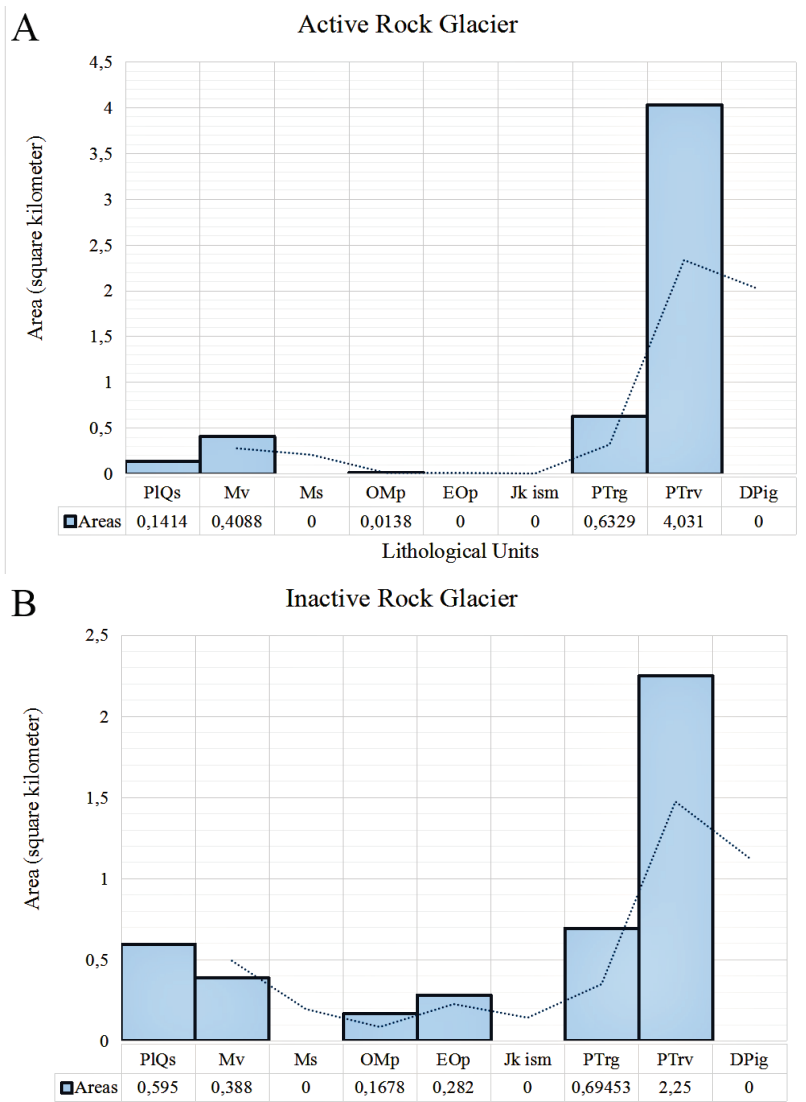

\begin{tabular}{l|l|l|l|l|r|r|r|r|r|} 
口Areas & 0,595 & 0,388 & 0 & 0,1678 & 0,282 & 0 & 0,69453 & 2,25 & 0
\end{tabular}
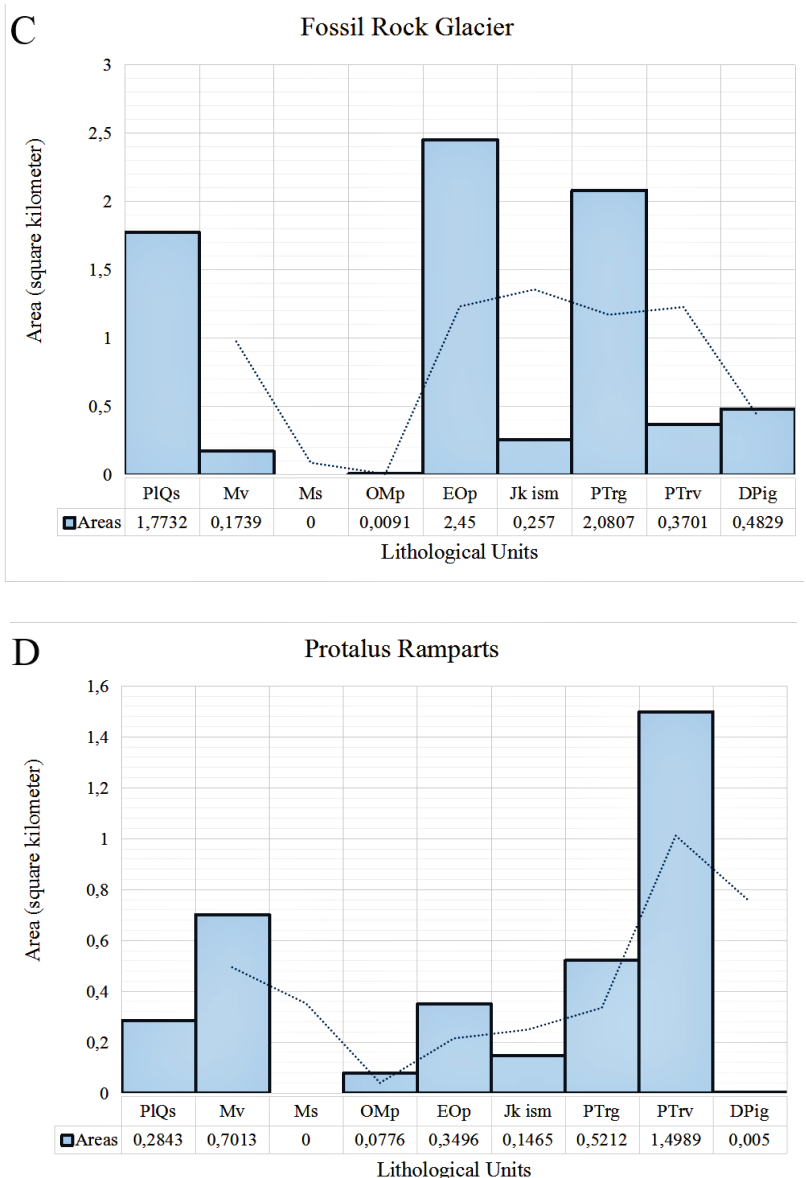

Figure 7. Area occupied. over lithological units. by: A) Active rock glaciers. B) Inactive rock glaciers. C) Fossil rock glaciers. D) Protalus rampart. 
Slope has been classified in 4 units with intervals: $0-11 \% ; 11-20 \% ; 20-35 \%$ and $35-75 \%$. The mean slope of active rock glaciers is between $11.2 \%$ and $28.5 \%$ with an average of $18.9 \%$. The mean slope of inactive rock glaciers has minimum values of $8.5 \%$, maximum values of $26 \%$ and averages of $18.5 \%$. Lastly, the mean slope of fossil rock glaciers has minimum values of $3.9 \%$, maximum values of $31.3 \%$ and averages of $12.7 \%$; and the mean slope of protalus ramparts has minimum values of $4.5 \%$, maximum values of $48.8 \%$ and averages of $4.5 \%$. This shows that a very high slope (greater than $35 \%$ ) is not related to rock glacier occurrence (Fig. 8 and 9).

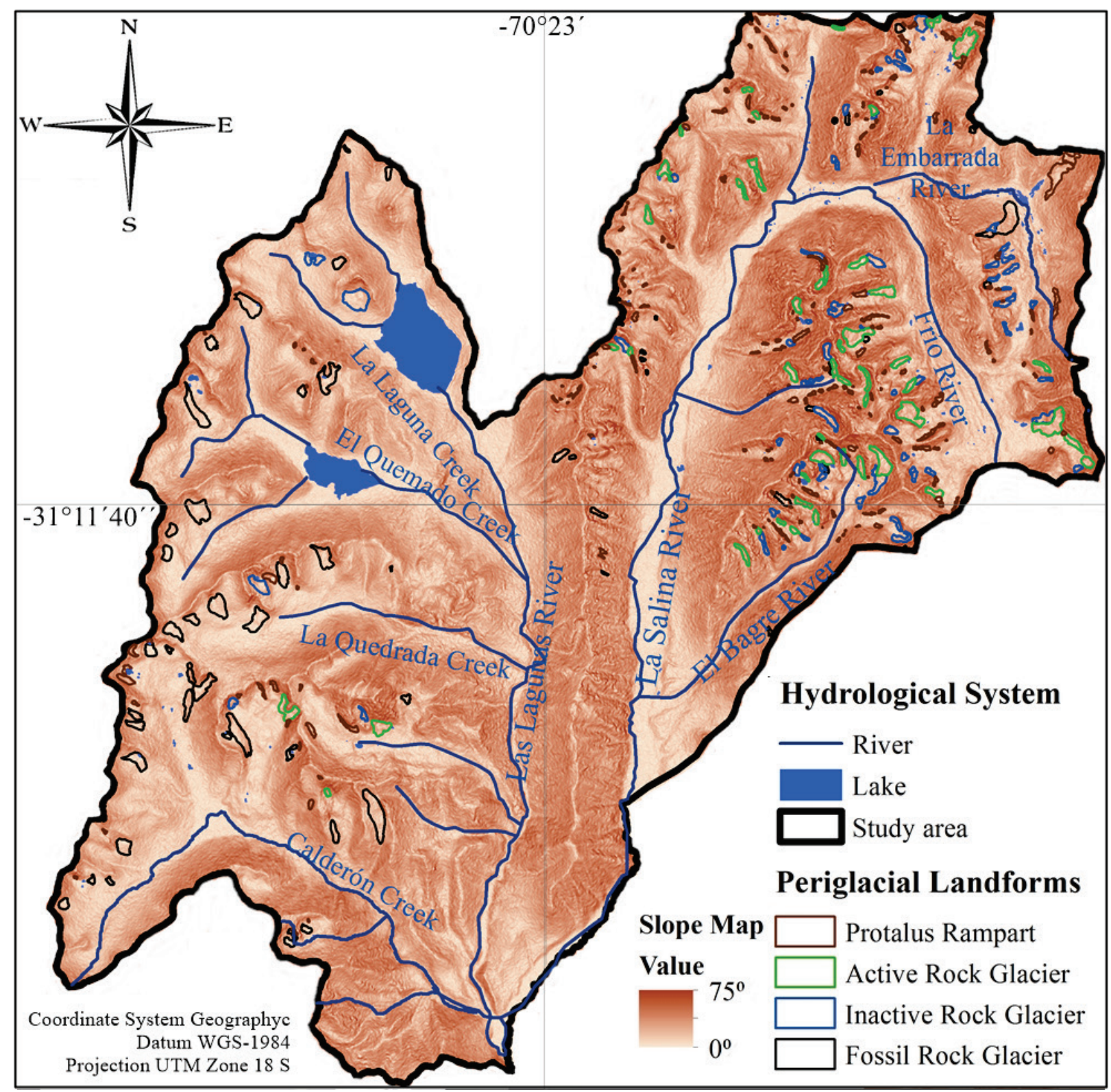

Figure 8. Rock glacier and protalus rampart distribution over the four slope units. 
Forte et al.
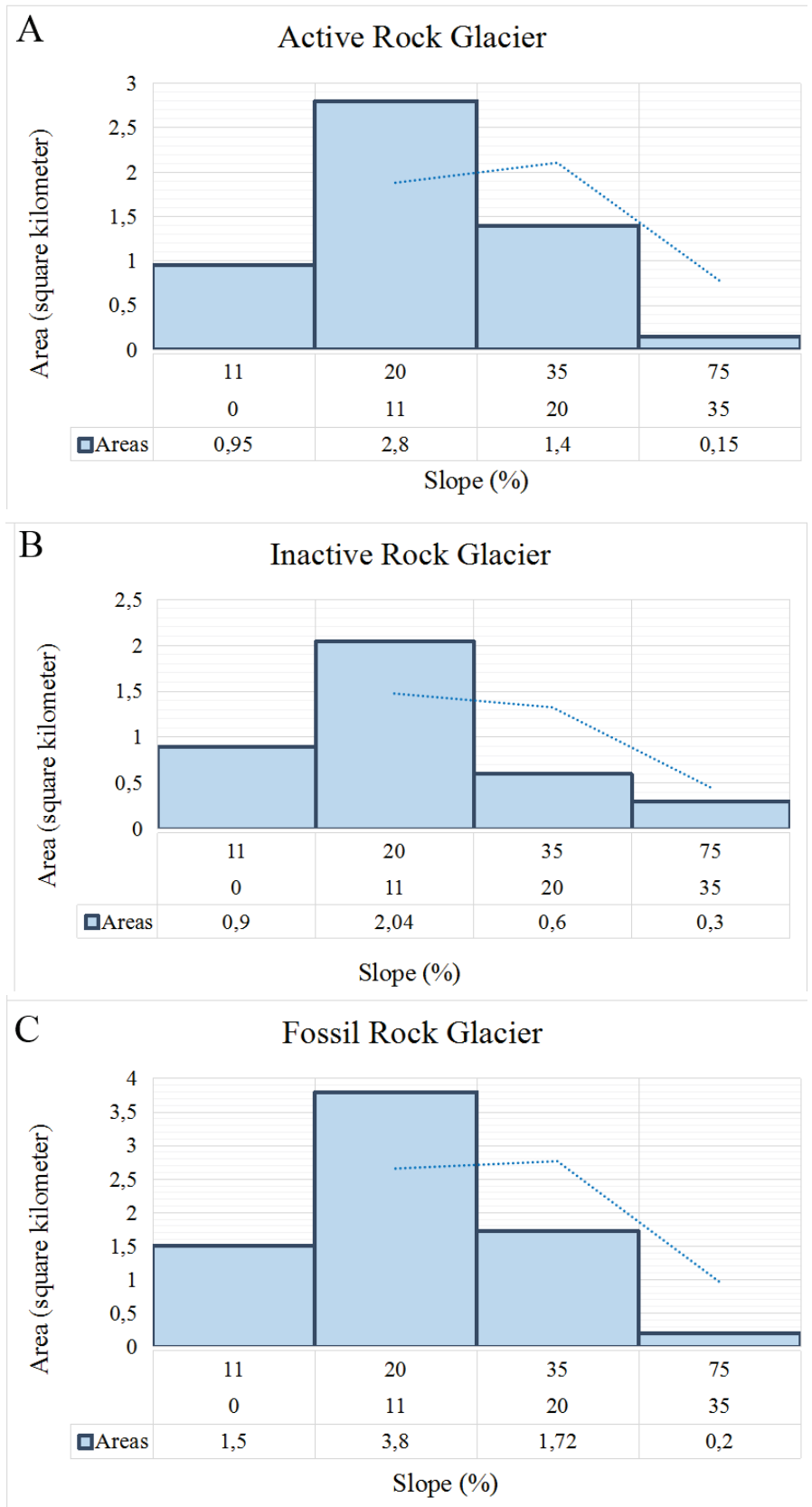

D Protalus Rampart

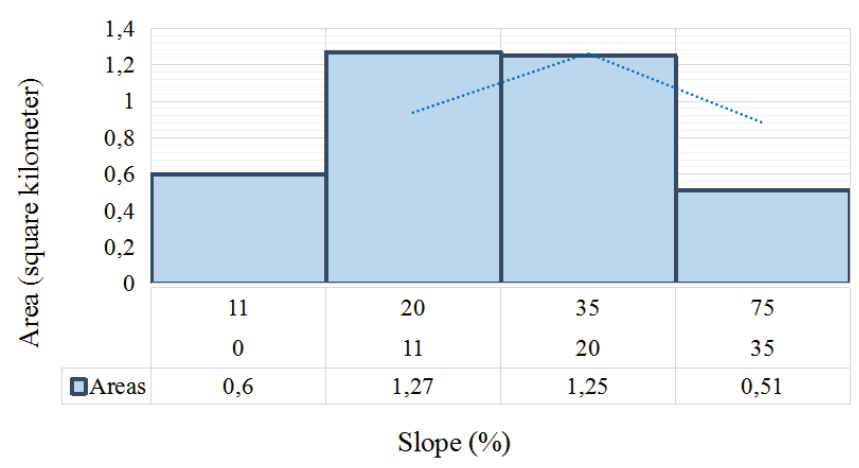

Figure 9. Area occupied by periglacial landforms over slope units. A) Active rock glaciers. B) Inactive rock glaciers. C) Fossil rock glaciers. D) Protalus rampart. 
The aspect analysis shows that active rock glaciers are generally south and south-west oriented. Some are oriented to the east due to the Bagres range layout which accompanies the rock glacier development in said direction. North-faces contain almost no active rock glaciers. In addition, inactive rock glaciers are predominant on the south-face of mountains and, to a lesser extent, on the east and west faces, as shown mainly on the Bagres and La Totora ranges (Fig. 10). While the aspect of fossil rock glaciers has a clear trend toward the southeast, protalus ramparts show a highly diverse characterization. This type of feature is practically present in all directions, though occurrences are most common on west-facing slopes and are less frequent on north-faces (Fig. 10 and 11).

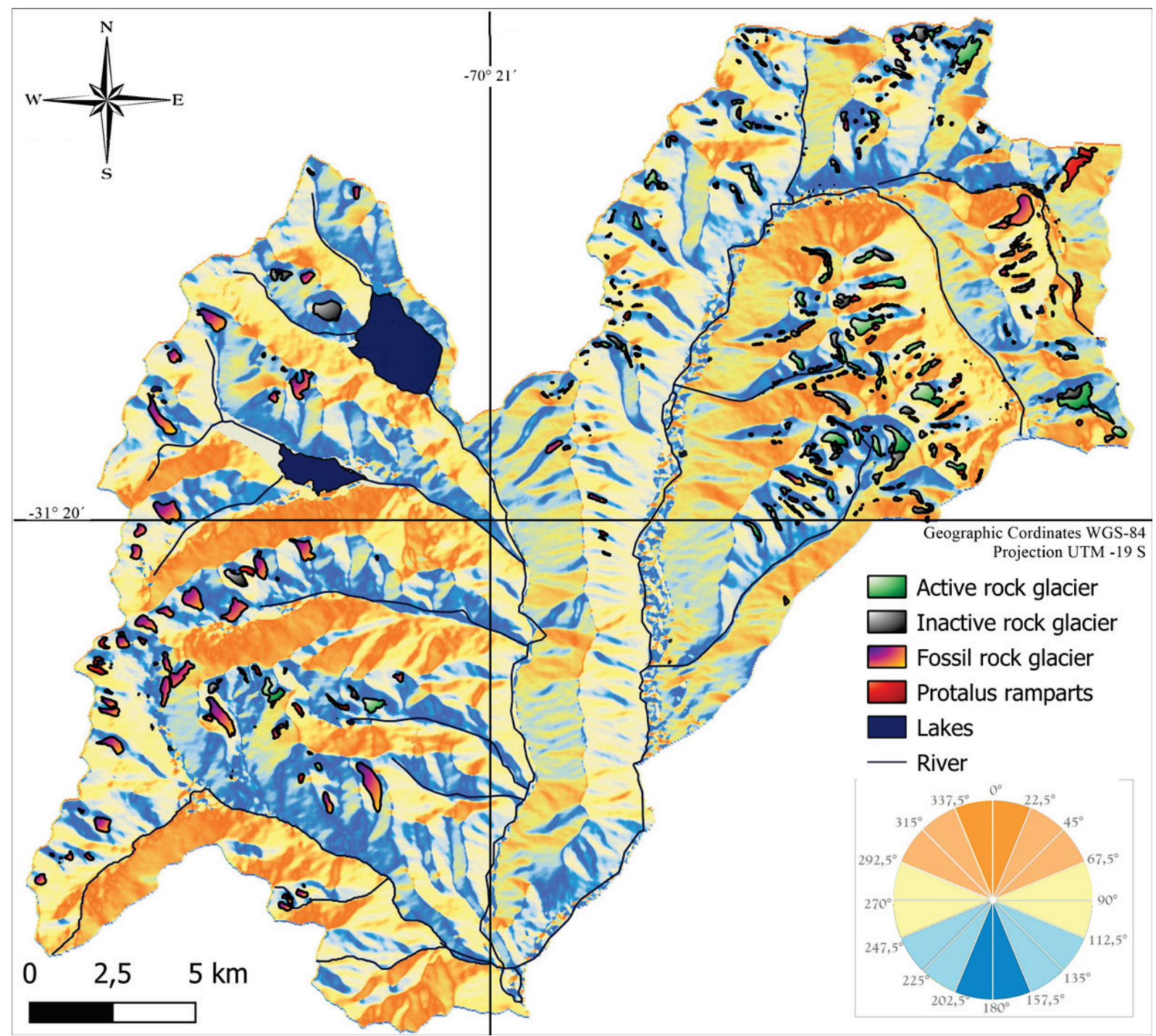

Figure 10. Rock glacier and protalus rampart distribution over the eight aspect units. Red and orange represent the warm north faces; yellow represents the west and east faces; while blue and light green show the cold south faces. 
A

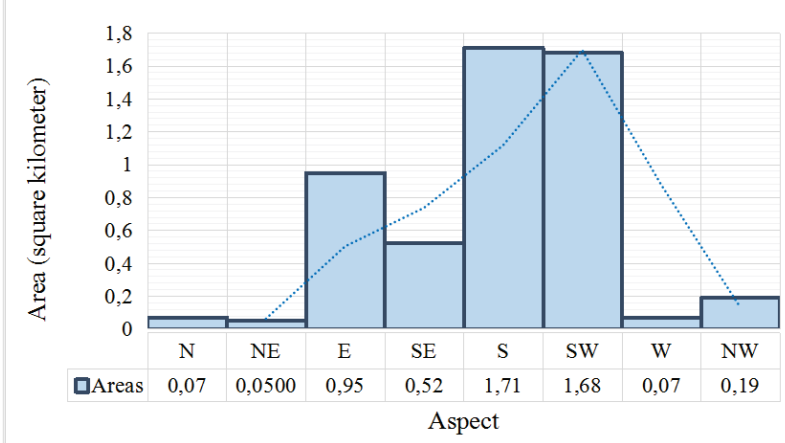

C

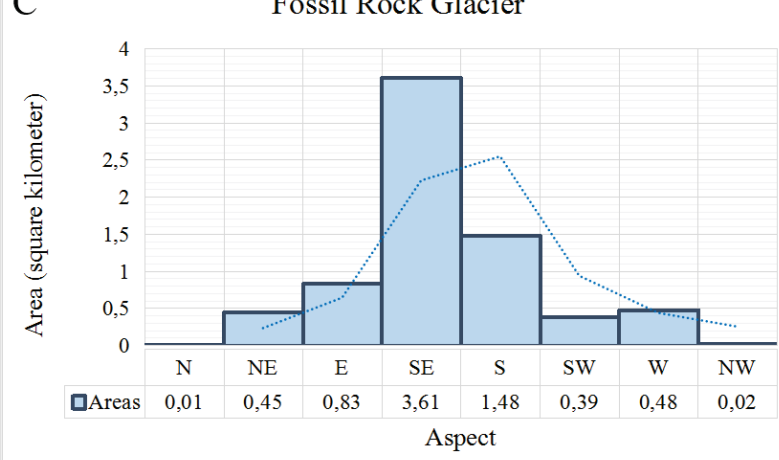

B

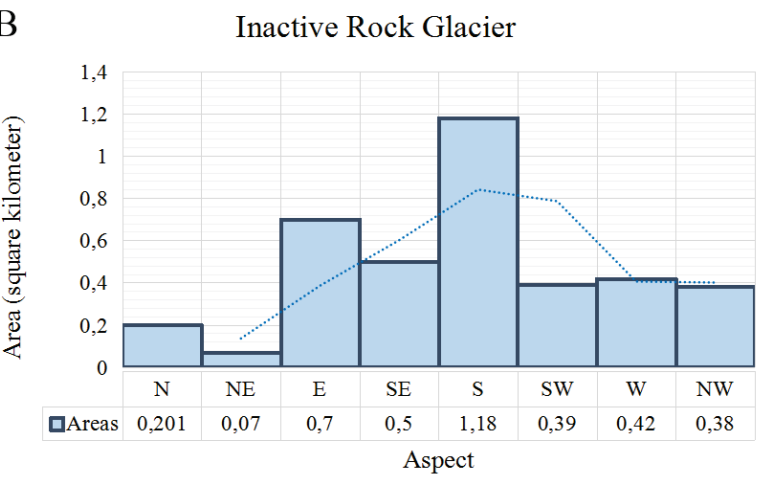

D

Protalus Rampart

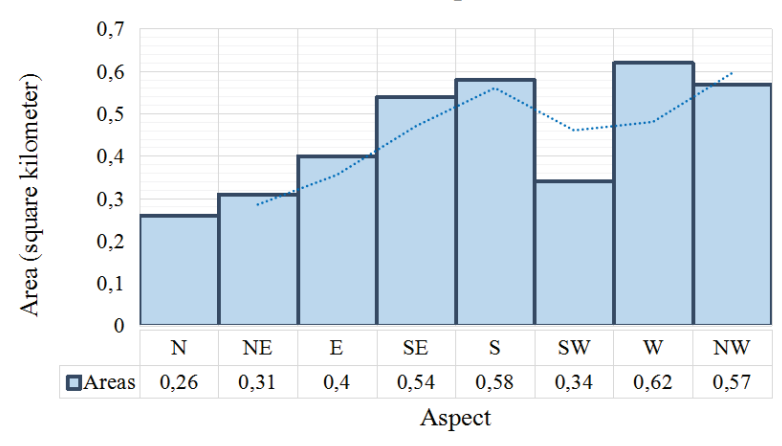

E ACTIVE ROCK GLACIERS

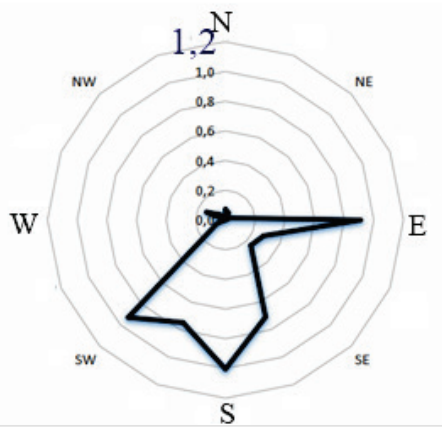

$\mathrm{G}^{\text {PSEUDO-RELICT ROCK GLACIERS }}$

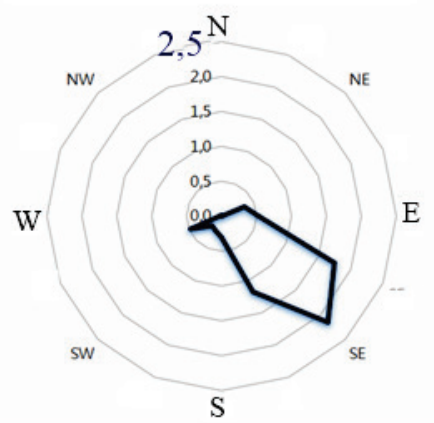

\section{F INACTIVE ROCK GLACIERS}

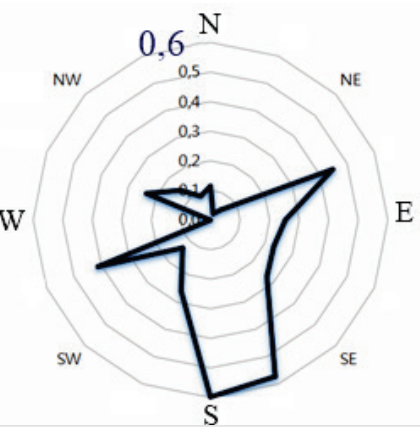

\section{PROTALUS RAMPART}

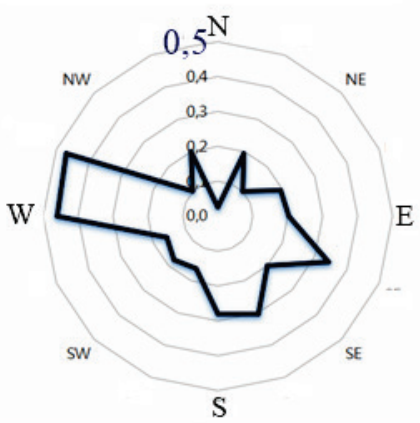

Figure 11. Periglacial landforms over the eight aspect units. A) Area occupied by active rock glaciers over each aspect unit. B) Area occupied by inactive rock glaciers over each aspect unit. C) Area occupied by fossil rock glaciers over each aspect unit. D) Area occupied by protalus ramparts over each aspect unit. E) Amount of active rock glaciers with mean aspect over the same unit. F) Amount of inactive rock glaciers with mean aspect over the same unit. G) Amount of fossil rock glaciers with mean aspect over the same unit. H) Amount of protalus ramparts with mean aspect over the same unit. 
Potential direct annual solar radiation shows a minimum of $660 \mathrm{Wh} / \mathrm{m}^{2}$ and a maximum of 2600 $\mathrm{KWh} / \mathrm{m}^{2}$ for the study area (Fig. 12). This variable was divided into four units to analyze the rock glacier and protalus rampart distribution (Fig. 13). Values for active rock glaciers vary between 857 and 2322 $\mathrm{KWh} / \mathrm{m}^{2}$ and the average values for rock glaciers and protalus ramparts are $1800-1900 \mathrm{KWh} / \mathrm{m}^{2}$. The potential incoming solar radiation in the valleys is irregular and it usually shows low values in southfacing slopes, lower lying terrain and shade-casted areas; which allows rock glaciers to develop at lower elevations. However, the potential incoming solar radiation values for intact rock glaciers are lower than those for fossil rock glaciers and protalus ramparts (Fig. 13). On active rock glaciers, the radiation readings show a bit less solar exposure. Over south-facing slopes, there is a minor heliophany which decreases the solar radiation and allows rock glaciers to develop or to be preserved.

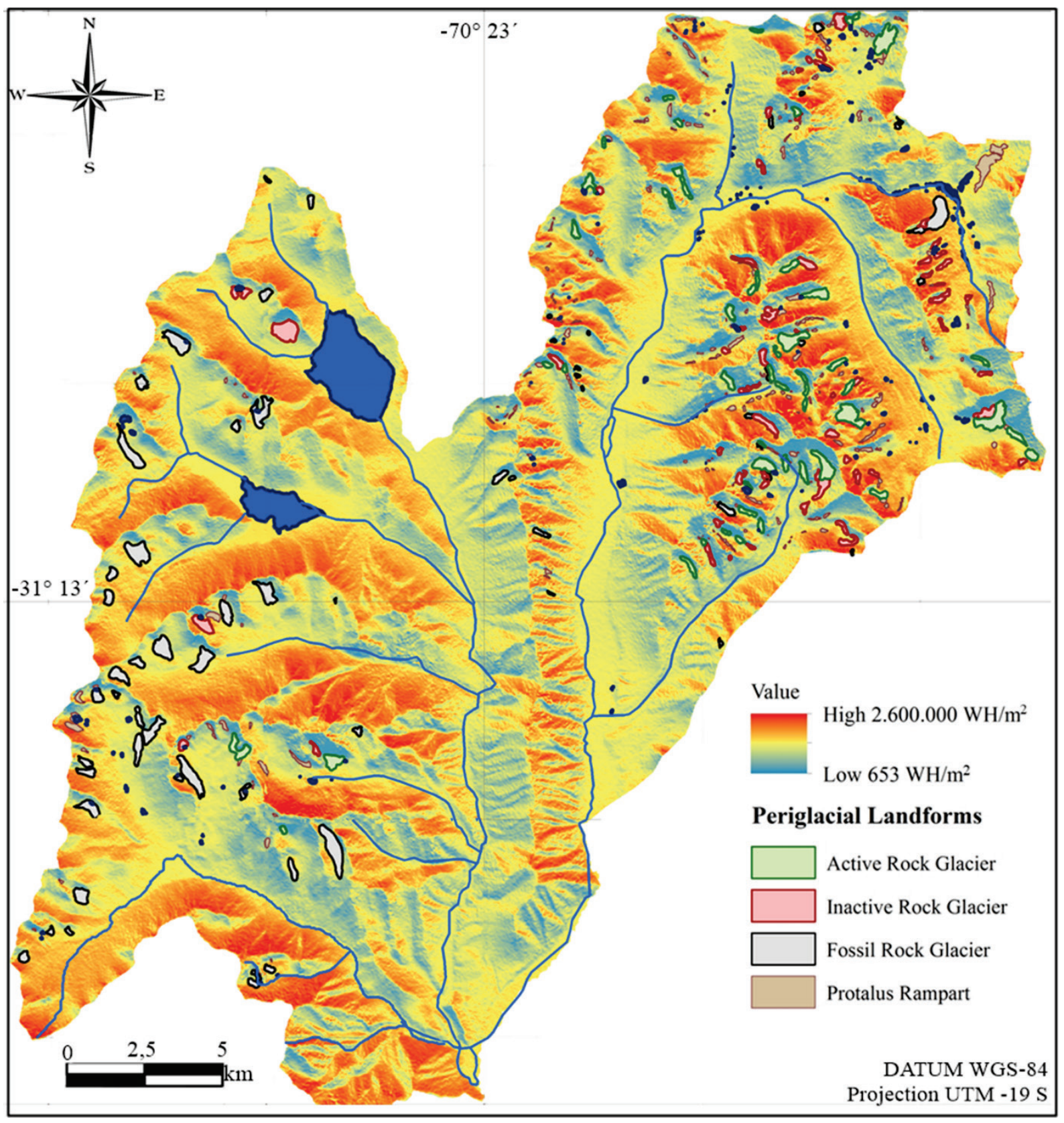

Figure 12. Rock glacier and protalus rampart over the four solar radiation units. 

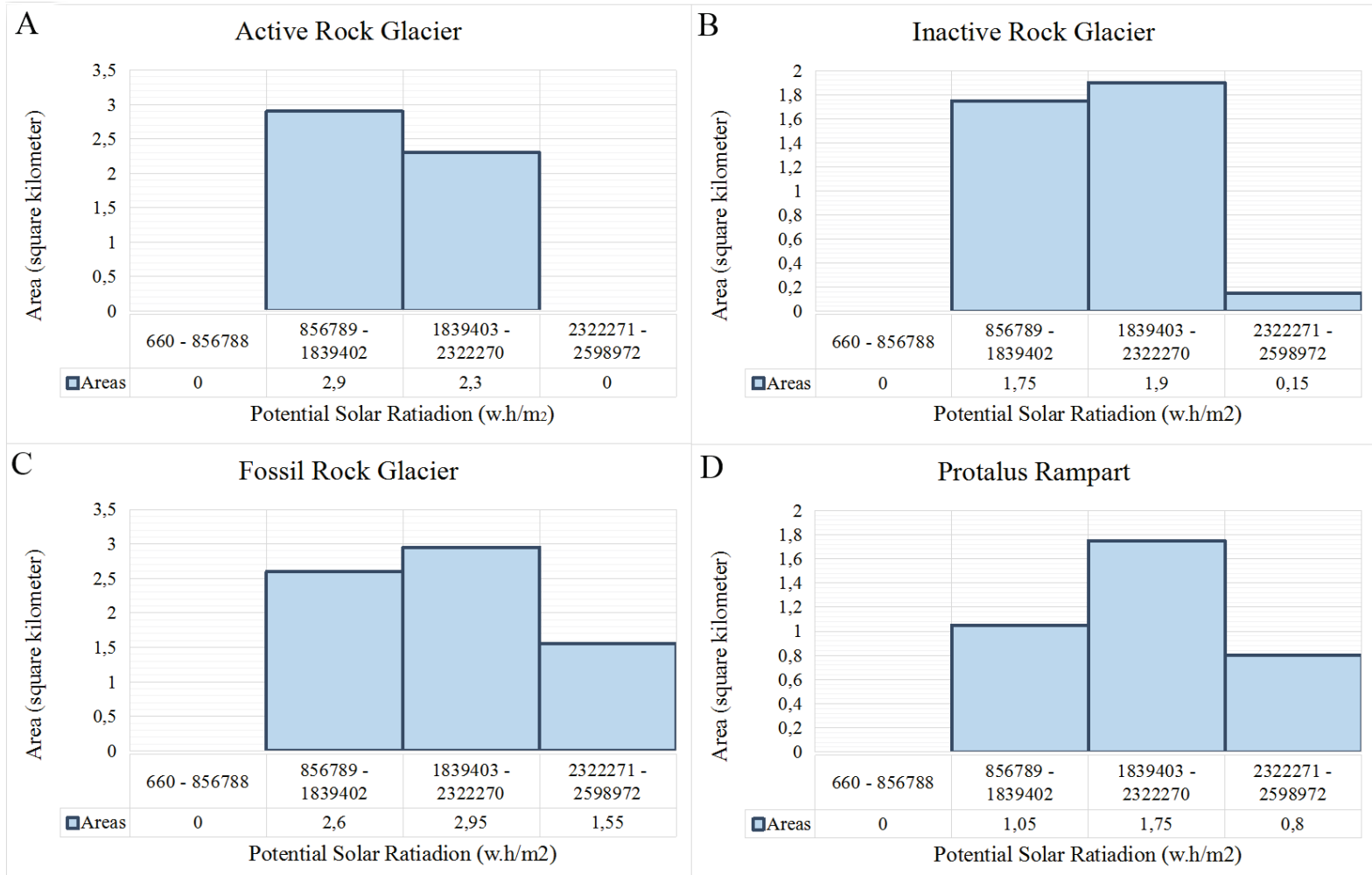

Figure 13. Area occupied over solar radiation units by: A) Active rock glaciers. B) Inactive rock glaciers. C) Fossil rock glaciers. D) Protalus rampart.

The frequency ratio (Fr) analysis over active rock glaciers was calculated for each environmental variable. Elevation is shown as the most important parameter, evidencing the highest frequency ratio value of 6.2 for elevations above $4048 \mathrm{~m}$ a.s.1.

The influence of lithology is the second most important parameter with $\mathrm{Fr}=2.5$ for PermianTriassic volcanic, pyroclastic and sedimentary rocks (PTrv), which are mainly compounded by basalt, andesite, dacite and rhyolits rocks.

Aspect is the third most relevant parameter for active rock glacier occurrence, with a maximum value of frequency ratio on the south-faces $(\mathrm{Fr}=2.66)$.

The remaining parameters -potential incoming solar radiation and slope- show a lower influence. However, slopes between 11 and $20 \%$ and values of radiation between 856,789 to 2,322,270 $\mathrm{Wh} / \mathrm{m}^{2}$ display frequency ratio values greater than 1 (Table 5).

The logistic regression coefficients (RL) for the distribution of active rock glaciers are also shown in Table 5 . The system designed is valid, its shows that an $89.1 \%$ of the predicted pixels are being used correctly $(90.9 \%$ of the active rock glacier pixels and $87.3 \%$ of the areas with non-rock glacier pixels). Collinearity correlation between predictor variables express independently in the regression model, so can predict the value of the dependent variable (Table 6). 
Table 5. Ratio frequency and logistic regression analyses.

\begin{tabular}{|c|c|c|c|c|c|c|c|}
\hline Factor & Class & $\begin{array}{c}\text { Pixels } \\
\text { showing } \\
\text { active rock } \\
\text { glaciers }^{\mathrm{a}} \\
\end{array}$ & $\begin{array}{l}\text { \% of pixels } \\
\text { showing } \\
\text { rock } \\
\text { glaciers }^{\mathrm{b}} \\
\end{array}$ & $\begin{array}{l}\text { Pixels in } \\
\text { domain }^{c}\end{array}$ & $\begin{array}{l}\text { Pixels } \\
\%^{\mathrm{d}}\end{array}$ & $\begin{array}{c}\text { Frequenc } \\
\text { y ratio }\end{array}$ & $\begin{array}{c}\text { Logistic } \\
\underset{\text { RR }}{\text { Regression }}\end{array}$ \\
\hline \multirow{6}{*}{$\begin{array}{l}\text { Elevation } \\
\text { (m.a.s.l.) }\end{array}$} & $2950-3299$ & 0 & 0.000 & 723601 & 18.104 & 0.000 & -19.966 \\
\hline & $3300-3689$ & 0 & 0.000 & 1252217 & 31.330 & 0.000 & -0.748 \\
\hline & $3690-3867$ & 9057 & 27.188 & 1178607 & 29.488 & 0.922 & 1.441 \\
\hline & $3868-4047$ & 12186 & 36.580 & 427470 & 10.695 & 3.420 & 1.993 \\
\hline & $4048-4225$ & 12070 & 36.232 & 232191 & 5.809 & 6.237 & 2.328 \\
\hline & $4226-4763$ & 0 & 0.000 & 182801 & 4.574 & 0.000 & 1 \\
\hline \multirow{8}{*}{$\begin{array}{c}\text { Aspect } \\
\text { (degree) }\end{array}$} & $338-22$ & 703 & 2.110 & 504867 & 12.632 & 0.167 & 17.989 \\
\hline & $23-67$ & 1250 & 3.752 & 497005 & 12.435 & 0.302 & 18.084 \\
\hline & $68-112$ & 3819 & 11.464 & 603825 & 15.107 & 0.759 & 18.545 \\
\hline & $113-157$ & 6286 & 18.870 & 544826 & 13.631 & 1.384 & 20.54 \\
\hline & $158-202$ & 9124 & 27.389 & 411352 & 10.292 & 2.661 & 21.507 \\
\hline & $203-247$ & 8039 & 24.132 & 494739 & 12.378 & 1.950 & 21.117 \\
\hline & $248-293$ & 2953 & 8.864 & 545267 & 13.642 & 0.650 & 19.284 \\
\hline & 294-337 & 1139 & 3.419 & 395006 & 9.883 & 0.346 & 18.225 \\
\hline \multirow{4}{*}{$\begin{array}{c}\text { Slope } \\
\text { (percentage) }\end{array}$} & $0-10$ & 6109 & 18.338 & 1072973 & 26.845 & 0.683 & -0.423 \\
\hline & 11-19 & 17930 & 53.823 & 1219425 & 30.509 & 1.764 & 1.33 \\
\hline & $20-35$ & 8525 & 25.591 & 1495870 & 37.426 & 0.684 & 1.585 \\
\hline & $35-75$ & 749 & 2.248 & 208619 & 5.220 & 0.431 & 1 \\
\hline \multirow{9}{*}{ Lithology } & $\begin{array}{l}\text { Pliocene-Quaternary } \\
\text { Sedimentary deposits }\end{array}$ & 900 & 2.702 & 855951 & 21.415 & 0.126 & -1.534 \\
\hline & $\begin{array}{c}\text { Middle to Upper } \\
\text { Miocene-Volcanic } \\
\text { Rocks }\end{array}$ & 2570 & 7.715 & 192497 & 4.816 & 1.602 & -0.995 \\
\hline & $\begin{array}{c}\text { Middle to Upper } \\
\text { Miocene- Sedimentary } \\
\text { rocks } \\
\end{array}$ & 0 & 0.000 & 73069 & 1.828 & 0.000 & -19.516 \\
\hline & $\begin{array}{c}\text { Oligocene to Lower } \\
\text { Miocene-Intrusive } \\
\text { Rocks }\end{array}$ & 84 & 0.252 & 22802 & 0.570 & 0.442 & -1.385 \\
\hline & $\begin{array}{l}\text { Eocene to Oligocene- } \\
\text { Intrusive Rocks }\end{array}$ & 0 & 0.000 & 374766 & 9.376 & 0.000 & -20.213 \\
\hline & $\begin{array}{l}\text { Jurassic to Lower } \\
\text { Cretaceous- } \\
\text { Sedimentary rocks } \\
\end{array}$ & 0 & 0.000 & 90136 & 2.255 & 0.000 & -19.28 \\
\hline & $\begin{array}{l}\text { Permian to Triassic- } \\
\text { Intrusive rocks }\end{array}$ & 4050 & 12.157 & 1227578 & 30.713 & 0.396 & -1.001 \\
\hline & $\begin{array}{l}\text { Permian to Triassic- } \\
\text { Vulcano-sedimentary } \\
\text { rocks }\end{array}$ & 24909 & 74.773 & 1117440 & 27.958 & 2.674 & 1 \\
\hline & $\begin{array}{c}\text { Devonian to Lower } \\
\text { Permia Intrusive rocks }\end{array}$ & 0 & 0.000 & 42648 & 1.067 & 0.000 & -19.845 \\
\hline \multirow{4}{*}{$\begin{array}{l}\text { Potential } \\
\text { Solar } \\
\text { Radiation } \\
\left(\mathrm{WH} / \mathrm{m}^{2}\right)\end{array}$} & $660-856788$ & 0 & 0.000 & 743104 & 18.592 & 0.000 & -0.21 \\
\hline & $856789-1839402$ & 18568 & 55.738 & 1236587 & 30.939 & 1.802 & 21.003 \\
\hline & $1839403-2322270$ & 14745 & 44.262 & 991562 & 24.808 & 1.784 & 21.589 \\
\hline & $2322271-2598972$ & 0 & 0.000 & 1025634 & 25.661 & 0.000 & 1 \\
\hline
\end{tabular}

$\mathrm{a}=$ Total number of pixels showing active rock glacier occurrence $=33313$

$\mathrm{b}=(\mathrm{a} / 33313) * 100$

$\mathrm{c}=$ Total number of pixels in domain $=3.996 .887$

$\mathrm{d}=(\mathrm{c} / 3996887) * 100$

$\mathrm{e}=\mathrm{b} / \mathrm{d}$ 
Table 6. Collinearity statistics.

\begin{tabular}{|l|c|c|}
\hline \multirow{2}{*}{ Model } & \multicolumn{2}{|c|}{ Collinearity Statistics } \\
\cline { 2 - 3 } & Tolerance & FIV: variance inflation factor \\
\hline Lithology & 0.858 & 1.166 \\
\hline Slope & 0.951 & 1.052 \\
\hline Aspect & 0.997 & 1.003 \\
\hline Elevation & 0.807 & 1.239 \\
\hline Potential Solar Radiation & 0.924 & 1.083 \\
\hline
\end{tabular}

The test proved that the equation and model could be admissible, because the values of Cox and Snell $\mathrm{R}^{2}(0.584)$ and Nagelkerke $\mathrm{R}^{2}(0.779)$ are greater than 0.2 (Clark and Hosking, 1986). The goodness of fit of our statistical model is based on Hosmer and Lemeshow Test (Table 7) and Pearson's chi-squared test (Table 8). These reveal that there is no multicollinearity among any of the variables and that they are weakly correlated with each other. In addition, Pearson correlations (Table 8) once again confirm that the variables used in the present study are only weakly correlated with each other, thus, they are considered independent variables.

Table 7. Hosmer and Lemeshow Test (H-L Test).

\begin{tabular}{|c|c|c|c|}
\hline Step & Chi squared & Gl. & Sig \\
\hline 1 & 7.678 & 8 & 0.466 \\
\hline
\end{tabular}

Table 8. Bilateral and Pearson correlation.

\begin{tabular}{|l|c|c|c|c|c|c|}
\hline & & Lithology & Slope & Aspect & Elevation & $\begin{array}{c}\text { Potential Solar } \\
\text { Radiation }\end{array}$ \\
\hline \multirow{2}{*}{ Lithology } & Pearson Correlation & 1 & 0.11 & 0.41 & 0.368 & 0.158 \\
\cline { 2 - 7 } & SIG (Bilateral) & & 0 & 0.064 & 0 & 0 \\
\hline \multirow{3}{*}{ Slope } & Pearson Correlation & 0.11 & 1 & 0.028 & 0.199 & 0.135 \\
\cline { 2 - 7 } & SIG (Bilateral) & 0 & & 0.205 & 0 & 0 \\
\hline \multirow{3}{*}{ Espect } & Pearson Correlation & 0.041 & 0.028 & 1 & 0.021 & -0.01 \\
\cline { 2 - 7 } & SIG (Bilateral) & 0 & 0.205 & & 0.338 & 0.654 \\
\hline \multirow{2}{*}{$\begin{array}{l}\text { Potential Solar } \\
\text { Radiation }\end{array}$} & Pearson Correlation & 0.368 & 0.199 & 0.021 & 1 & 0.253 \\
\cline { 2 - 7 } & SIG (Bilateral) & 0 & 0 & 0.338 & & 0 \\
\cline { 2 - 7 } & SIG (Bilateral) & 0 & 0 & 0.654 & 0 & 1 \\
\hline
\end{tabular}

Taking everything into account, active rock glacier occurrence is mainly influenced by elevation and, to a lesser extent, by lithology and aspect. As their elevation range had been divided into three zones: lower, middle and upper current periglacial environment belt; it was possible to analyze the altitudinal distribution in detail. The highest belt (4048 to $4225 \mathrm{~m}$ a.s.1.) presents the most suitable conditions for active rock glacier occurrence: $\mathrm{Fr}=6.237$ and $\mathrm{LR}=2.328$. The analysis of lithology influence over active rock glaciers demonstrates that they are mainly developed over Permo-Triassic volcanic complex rocks (PTrv). $\mathrm{Fr}=2.674$ and $\mathrm{LL}=1$; while aspect analyses show a major occurrence on south-facing slopes $(\mathrm{Fr}=2.661$ and $\mathrm{LR}=21.507)$. 


\section{Discussion}

The Central Andes of Argentina is the mountain range with the highest density of rock glaciers in the world (Trombotto et al., 2012). The results obtained in this paper show that rock glaciers and protalus ramparts are indeed abundant in the northern sector of the Central Andes of Argentina, with 405 rock glaciers and protalus ramparts occupying $3.14 \%$ of the total area $\left(630 \mathrm{~km}^{2}\right)$. Other rock glaciers inventories in the region show similar results as regards the spatial density of these landforms (Esper Angillieri, 2017; Trombotto et al., 2012; ING, 2016; Villarroel and Forte, 2020; Villarroel et al., 2018, 2020; Tapia Baldis, 2018).

Rock glaciers are generally easily recognized in the field or on satellite imagery. In their upper sector, they are usually delimited by a noticeable change in slope between the rockwall and the landform, while in their lower sector, the boundary is denoted by a frontal talus with a pronounced slope. On the surface, the presence of furrow and ridges or collapsed structures is frequent, in the form of thermokarst associated with ice melting. However, the delimitation of the landforms in the rooting zone or in the area of connection with other lobes presents some difficulties (Roer and Neynhuis, 2007). In order to avoid ambiguity and obtain a more accurate inventory, the mapping has been performed by two individuals and controlled with field information. Previous research, including that by Scotti et al. (2013) and Brardinoni et al. (2019), has also applied the methodology of using two or more mappers in the same sector to record rock glacier inventories. In this study, the uncertainty is relatively less, possibly because the landforms are clearly shaped and thus easily identified, even in the case of inactive rock glaciers.

One of the most significant aspects in arid regions is that intact rock glaciers and protalus ramparts are considered solid-water reserves (Scapozza et al., 2011; Jones et al., 2018; Villarroel et al., 2020) and indicators of mountain permafrost (Barsch, 1996; Haeberli et al., 2010). Therefore, the classification as active and inactive takes special relevance for the climatic and environmental information available because it is closely related to the capacity of these landforms to be solid-water reserves and/or water resources (Jones et al., 2018, 2019; Ohlanders et al., 2013; Villarroel et al., 2020). Despite the possible ambiguity, in several studies the state of activity has been assessed by taking into account morphological characteristics (Barsch 1996; Haeberli et al., 2010; Ikeda and Matsuoka 2006; Roer and Neynhuis, 2007; Esper Angillieri, 2010, 2017; Jones et al., 2019). The observed active and inactive rock glaciers are usually located in continuity, active overlapping inactive ones. A similar situation was found between inactive and fossil rock glaciers, where inactive shapes developed over fossil ones.

The minimum elevation of active rock glaciers is considered as altitudinal limit from which the permafrost develops (Barsch, 1996). Thus, the Current Periglacial Environment (where mountain permafrost exists) in the study area is above $3690 \mathrm{~m}$ a.s.l. Other studies in the Central Andes show similar elevations, for example: at a $34^{\circ} \mathrm{S}$ latitude, the lower elevation is $3750 \mathrm{~m}$ a.s.l. (Brenning and Trombotto, 2006); at a $30^{\circ} 50^{\prime}$ to $33^{\circ} 50^{\prime}$ latitude, it is $3700 \mathrm{~m}$ a.s.l. (Villarroel et al., 2018); at $31^{\circ} 50^{\prime}$ ', it is $3593 \mathrm{~m}$ a.s.1. (Villarroel and Forte, 2020) and at $32^{\circ} 20^{\prime}$, it is $3280 \mathrm{~m}$ a.s.l. (Forte, 2020). Regional data have shown that there is an important effect on latitude due to the increase in elevation of active rock glaciers from south to north (IGN, 2016; Villarroel et al., 2018). In addition, it has been shown that the cryosphere also changes latitudinally given the increasing aridity towards the north that enters the South American arid diagonal in the north of the country; and that the glacial environment is noticeably reduced, while the periglacial environment and the mountain permafrost develop more frequently (Garcia et al., 2017).

As regards the classification of intact rock glaciers, Barsch (1996) mentions two types of inactive rock glaciers: climatic or dynamic. In the first case, inactivity is due to the ice melting, while in the second case, the inactivity is due to the considerable distance between the surface of the rock glacier and the debris source. In the study region, rock glaciers have a small size and also a short length, hence it was assumed that activity could be mainly controlled by climatic variables. 
Protalus ramparts are considered an expression of current mountain permafrost creep (Barsch, 1996; Scapozza et al., 2011; Hedding, 2011; Scotti et al., 2013). This paper shows that these are the most frequent landforms (231). Thus, there is evidence of widespread current permafrost conditions in the study area.

The relationship between environmental variables (climatic, lithology, topographic and morphometric) and the spatial distribution of rock glaciers is essential for conducting regional studies on periglacial environment distribution. Especially in the case of high mountain areas, where poor accessibility reduces the chances of obtaining climatic data, while it steeply increases the costs of systematic field studies. In this paper, we present this spatial analysis using frequency ratio and logistic regression as statistical methods.

The results show that the occurrence of active rock glaciers is mainly influenced by their altitudinal distribution and, in the study area, all of them lie above $3600 \mathrm{~m}$ a.s.l. Thus, this altitude is assigned as the minimum elevation for the development of the current periglacial environment. Under this elevation, inactive rock glaciers and protalus ramparts can be found, thus it has been deemed an unstable periglacial environment area, where their existence is possible due to the terrain and environment capacities for permafrost conservation. The belt between 4048 and $4225 \mathrm{~m}$ a.s.1. presents the most suitable conditions for active rock glacier occurrence: $\mathrm{Fr}=6.237$ and $\mathrm{LR}=2.328$. These results are consistent with other similar researches performed in other regions, e.g.: Brenning (2005), Brenning and Trombotto (2006), Ikeda and Matsuoka (2006), Johnson et al. (2007) and Esper Angillieri, (2010, 2017).

Statistical analysis also shows that lithologic characteristics are a key variable for the occurrence of rock glaciers. Active rock glaciers are mainly found over Permo-Triassic volcanic complex rocks (PTrv), $\mathrm{Fr}=2.674$ and $492 \mathrm{LR}=1$. This lithology possibly affects the debris production (and their size), as well as the capacity of infiltration and water and ice storage of rock glaciers. Furthermore, it could influence their behavior since there are lithologies that favor rock glacier development, but in doing so, could also make these landforms prone to higher degradation. More specifically, due to their chemical composition, high porosity (availability for water infiltration) and/or subjection to a high degree of weathering, these rocks tend to have a greater capacity for the development of debris and, therefore, for rock glacier development and degradation (French and Williams, 2007; Johnson et al., 2007). For example, rocks from the PTrv unit (Permo-Triassic sedimentary, pyroclastic, subvolcanic and volcanic or plutonic rocks with hydrothermal alteration) evidence greater weathering and erosion processes, mainly cryoclastism. Hence, inactive rock glaciers are as common as active rock glaciers in such lithologies. On the other hand, there are other lithologies over which rock glaciers develop less frequently but seem to withstand environmental changes in a state of preservation for a quite longer period of time. Over these lithological units, there is usually a smaller number of inactive rock glaciers than active ones. The results of this work indicate that intrusive and granitic rocks, despite not being the most favorable rocks for periglacial landform development, seem to be a suitable lithology for active rock glacier conservation. While areas with lithologies as EOp, Mv, JK ism and DPig units, despite their favorable elevation, slope, aspect and solar radiation conditions for rock glacier occurrence, there are relatively rare, occupying only $0.4 \mathrm{~km}^{2}$ in total. Instead, active rock glaciers commonly locate in areas dominated by pyroclastic, subvolcanic and intrusive rocks of the Permo-Triassic volcanic complex (PTrv), covering $4.03 \mathrm{~km}^{2}$. Therefore, the study suggests some intriguing topics related to lithologies and their capacity for rock glacier development and/or conservation.

The larger number of rock glaciers is found in the Frío river basin $(n=15)$. Whereas the basin with the major development of active rock glaciers is found on the Bagre river watersheds $(n=12)$. Over the latter, the most favorable lithology and highest peaks are found.

Although intact rock glaciers and protalus ramparts are indicators of periglacial environment and mountain permafrost occurrence, different authors have indicated that the lower altitudinal limit of active rock glaciers represents the lower limit of discontinuous mountain permafrost (Brenning, 2005). 
In the study area, this limit is assigned at $3690 \mathrm{~m}$ a.s.l. and, in this paper, the resulting area is called 'current periglacial environment'. Above said elevation, the $90 \%$ of inactive rock glaciers and $61 \%$ of fossil rock glaciers are located. These results are consistent with the elevation of $3725 \mathrm{~m}$ a.s.l. as the lower limit of permafrost identified from geophysical surveys in three rock glaciers over the Frío river basin (Forte et al., 2013; Villarroel et al., 2020). In addition, two altitudinal units are differentiated below $3690 \mathrm{~m}$ a.s.1.: one between 3300 to $3690 \mathrm{~m}$ a.s.1., where there are protalus ramparts and inactive rock glaciers, an another below $3300 \mathrm{~m}$ a.s.l., where there is a large number of fossil rock glaciers and other periglacial landforms, indicating paleo-environmental conditions in favor of cryogenic processes. At $3375 \mathrm{~m}$ a.s.l., the altitudinal belt is considered an unstable periglacial environment, because the mean annual air temperature (MAAT) is $2.4{ }^{\circ} \mathrm{C}$ (Schreiber, 2015). However, the existence of inactive rock glaciers and protalus ramparts in this region demonstrates that elevation is not the only variable controlling the occurrence of periglacial landforms.

Another noteworthy topic is the influence of the subtropical position of the region over the distribution of periglacial landforms. The study area is located about $1000 \mathrm{~km}$ south of the Capricorn Tropic. This is reflected in their distribution with regards to the orientation of the slopes (aspect), along with a remarkable development and conservation on south-facing slopes $(\mathrm{Fr}=2.661$ and $\mathrm{LR}=21.507)$. On the other hand, slope and solar radiation are not considered significant variables for rock glacier occurrence. Solar radiation is high on the entire studied area, due to the semi-arid climate and the large number of sunny days. For active rock glacier occurrence, the most favorable solar radiation obtained from the frequency relation is the range between 857 and $1840 \mathrm{Kw} \cdot \mathrm{h} / \mathrm{m}^{2}(\mathrm{Fr}=1.802)$; while logistic regression shows the range between 1840 to $2322 \mathrm{Kw} \cdot \mathrm{h} / \mathrm{m}^{2}$ as the best condition. So, as also recorded by other studies (Esper Angillieri, 2010), solar radiation does not indicate a clear pattern or a significant influence over the occurrence of rock glaciers.

During recent years, activities related to mining projects have modified the landscape and the original environmental characteristics of the study area. If these projects become mines, they could affect the behavior of the natural hydrological and cryospheric systems significantly. Thus, the findings presented in this work constitute a contribution by documenting the original and natural number, features and distribution of rock glacier bodies currently present in the region.

\section{Conclusion}

An important periglacial environment development is evidenced in the study area. The environmental conditions are optimal for the development and conservation of cryospheric landforms with potential ice content in solid state, shape of intact rock glaciers or protalus ramparts.

The statistical methods used in this paper, show how five environmental variables influence the occurrence of active rock glaciers in a sector of the Central Andes of Argentina, obtaining valuable results for the assessment of the environmental situation of the region. The methodology applied reveals that the spatial distribution of active rock glaciers is influenced mainly by elevation, followed by lithology and aspect, and, to a lesser extent, by slope and solar radiation. The analysis of their spatial distribution in relation to aspect shows their larger development and conservation on south-facing slopes. The subtropical location of the study area seems to have a greater influence on their development than slope orientation.

Considering that elevation is the variable with the greatest influence in the occurrence of active rock glaciers, and that this aspect is also directly linked to air temperature, it is important to monitor the stability and behavioral changes of these landforms. Especially, taking into account the current context of increasing environmental temperatures and the potential of rock glaciers as water resources and reserves in many arid mountainous regions. 
Forte et al.

\section{References}

Atkinson, P., Jiskoot, H., Massari, R., Murray, T. 1998. Generalized linear modelling in geomorphology. Earth Surface Processes and Landforms 23 (13), 1185-1195. https://doi.org/10.1002/(SICI)10969837(199812)23:13<1185::AID-ESP928>3.0.CO;2-W

Ayalew, L., Yamagishi, H. 2005. The application of GIS-based logistic regression for landslide susceptibility mapping in the Kakuda-Yahiko Mountains, Central Japan. Geomorphology 65 (1-2), 15-31. https://doi.org/10.1016/j.geomorph.2004.06.010

Azócar, G.F., Brenning, A. 2010. Hydrological and Geomorphological Significance of Rock Glaciers in the Dry Andes, Chile $\left(27^{\circ}-33^{\circ} \mathrm{S}\right)$. Permafrost and Periglacial Processes 21, 42-53. https://doi.org/10.1002/ppp.669

Barsch, D., 1996. Rockglaciers. Indicators for the Present and Former Geoecology in High Mountain Environments Springer, Berlin.

Bonham-Carter, G.F. 1994. Geographic information systems for geoscientists-modeling with GIS. Computer Methods in the Geoscientists 13, 398.

Brardinoni, F., Scotti, R., Sailer, R., Mair, V. 2019. Evaluating sources of uncertainty and variability in rock glacier inventories. Earth Surface Processes and Landforms 44 (12), 2450-2466. https://doi.org/10.1002/esp.4674

Brenning, A. 2005. Climatic and geomorphological controls of rock glaciers in the Andes of Central Chile: Combining statistical modelling and field mapping. Ph.D. thesis. Humboldt Universität. Berlin, Alemania, $137 \mathrm{pp}$.

Brenning, A., Trombotto, D. 2006. Logistic regression modelling of rock glacier and glacier distribution: Topographic and climatic controls in the semi-arid Andes, Geomorphology 81, 141-154. https://doi.org/10.1016/j.geomorph.2006.04.003

Bruniard, E.D., 2014. La diagonal árida argentina: un límite climático real. Revista Geográfica 95, 5-20.

Clarck, W.A.V., Hosking, P.L. 1986. Statistical methods for geographers. John Wiley and Sons, 518 p., New York.

Colucci, R.R., Boccali, C., Žebre, M., Guglielmin, M. 2016. Rock glaciers, protalus ramparts and pronival ramparts in the south-eastern Alps. Geomorphology 269, 112-121. https://doi.org/10.1016/j.geomorph.2016.06.039

Colucci, R.R., Forte, E., Zebre, M., Maset, E., Zanetti, C., Guglielmin, M. 2019. Is that a relict rock glacier? Geomorphology 330, 177-189. https://doi.org/10.1016/j.geomorph.2019.02.002

Corripio, J., Purves, R., Rivera, A. 2007. Modeling climate-change impacts on mountain glaciers and water resources in the Central Dry Andes, Darkening Peaks: Glacier Retreat, Science and Society, University of California Press, pp. 126-136, USA.

Esper Angillieri, M.Y., 2010. Application of frequency ratio and logistic regression to active rock glacier occurrence in the Andes of San Juan, Argentina. Geomorphology 114, 396-405. https://doi.org/10.1016/j.geomorph.2009.08.003

Esper Angillieri, M.Y. 2017. Permafrost distribution map of San Juan Dry Andes (Argentina) based on rock glacier sites. Journal of South American Earth Sciences $73 \quad$ (C), 42-49. https://doi.org/10.1016/j.jsames.2016.12.002

Forte, A.P. 2020. Evolución reciente de glaciares en las nacientes del arroyo Laguna Blanca y su importancia hidrológica. Calingasta, San Juan, Argentina. Tesis Doctoral. Facultad de Ciencias Exactas, Físicas y Naturales. Universidad Nacional de San Juan.

Forte, A.P., Güell, A.E., Villarroel, C.D. 2013. Tomografía sísmica en glaciares de escombros en las nacientes del arroyo Los Patos Norte. Calingasta, San Juan, Argentina. Boll. Geof. Teor. Appl. 25, 263-265.

Forte, A.P., Villarroel, C.D., 2019. Reconstrucción geomorfológica del último máximo glaciar en la cuenca del río San Juan, Argentina. Cuaternario y Geomorfología 33, 62-82.

French, H. M., Williams, P. 2007. The periglacial environment (Vol. 458). Chichester: John Wiley and Sons. 
García Piña A., Ulloa C., Amigo G., Milana J.P., Medina C., 2017. An inventory of cryospheric landforms in the arid diagonal of South America (high Central Andes, Atacama region, Chile). Quaternary International 1-16. https://doi.org/10.1016/j.quaint.2017.04.033

Haeberli W., Noetzli J., Arenson L., Delaloye R., Gärtner-Roer I., Gruber S., Isaksen K., Kneisel C., Krautblatter M., Phillips M., 2010. Mountain permafrost: development and challenges of a young research field. Journal of Glaciology 56 (200), 1043-1058. https://doi.org/10.3189/002214311796406121

Harrisson, S., Glasser, N., Winchester, V., Haresign, E., Warren, C.H., Duller, G., Bailey, R., Ivy-Ochs, S., Jansson, K., Kubik, P., 2008. Glaciar León, Chilean Patagonia: late-Holocene chronology and geomorphology. The Holocene 18 (4), 643-652. https://doi.org/10.1177/0959683607086771

Hedding, D.W., 2011. Pronival rampart and protalus rampart: A review of terminology. Journal of Glaciology 57 (206), 1179-1180. https://doi.org/10.3189/002214311798843241

Hosmer, D.W., Jovanovic, B., Lemeshow, S. 1989. Best subsets logistic regression. Biometrics 45 (4), 1265-1270. https://doi.org/10.2307/2531779

Ikeda, A., Matsuoka, N. 2006. Pebbly versus bouldery rock glaciers: Morphology, structure and processes. Geomorphology 73, 279-296. https://doi.org/10.1016/j.geomorph.2005.07.015

ING. 2016. Informe de la subcuenca del río Blanco, Cuenca del río San Juan. Inventario Nacional de Glaciares. Ministerio de Ambiente y Desarrollo Sustentable. IANIGLA- CCT Mendoza - CONICET.

Johnson, B., Thackray, G., Van Kirk, R. 2007. The effect of topography, latitude and lithology on rock glacier distribution in Lemhi Range, central Idaho, USA, Geomorphology 91, 38-50. https://doi.org/10.1016/j.geomorph.2007.01.023

Jones, D.B., Harrison, S., Anderson, K., Selley, H.L., Wood, J.L., Betts, R.A. 2018. The distribution and hydrological significance of rock glaciers in the Nepalese Himalaya. Glob. Planet. Chang. 160 (C), 123142. https://doi.org/10.1016/j.gloplacha.2017.11.005

Jones, D.B., Harrison, S., Anderson, K., Walley, B. 2019. Rock glaciers and mountain hydrology: A review. EarthScience Reviews 193, 66-90. https://doi.org/10.1016/j.earscirev.2019.04.001

Kääb, A., Frauenfelder, R., Roer, I., 2007. On the response of rockglacier creep to surface temperature increase. Global and Planetary Change 56, 172-187. https://doi.org/10.1016/j.gloplacha.2006.07.005

Menard, S. 2000. Coefficients of determination for multiple logistic regression analysis. The American Statistician 54(1), 17-24.

Monnier, S., Kinnard, C. 2013. Internal structure and composition of a rock glacier in the Andes (upper Choapa valley, Chile) using borehole information and ground-penetrating radar. Annals of Glaciology 54 (64), 61-72. https://doi.org/10.3189/2013AoG64A107

Ohlanders, N., Rodríguez, M., McPhee, J. 2013. Stable water isotope variation in a Central Andean watershed dominated by glacier and snowmelt. Hydrology and Earth System Sciences 17 (3), 1035-1050. https://doi.org/10.5194/hess-17-1035-2013

Palacios, D., Stokes, C. R., Phillips, F. M., Clague, J.J., Alcalá-Reygosa, J., Andrés, N., Angel, I., Blard, P.H., Briner, P.J., Hall, B.L., Dahms, D., Heins, A.S., Jomelli, V., Mark, B.G., Martine, M.A., Moreno, P., Riedel, J., Sagredo, E., Stansell, N.D., Vázquez-Selem, L., Vuille, M., Ward, D.J. 2020. The deglaciation of the Americas during the Last Glacial Termination. Earth-Science Reviews 203, 103113. https://doi.org/10.1016/j.earscirev.2020.103113

Paul, F., Barry, J.G., Cogley, H., Frey, H., Haeberli, W., Ohmura, A., Ommanney, C.S.L., Raup, B., Rivera, A., Zemp, M., 2010. Guidelines for the compilation of glacier inventory data from digital sources. WGMS, GLIMS, Globglacier, University of Zürich.

Rivera, J.A., Penalba, O.C., Betolli, M.L., 2013. Inter-annual and inter-decadal variability of dry days in Argentina. International Journal Climatology 33, 834-842. https://doi.org/10.1002/joc.3472

Roer, I., Nyenhuis, M. 2007. Rock glacier activity studies on a regional scale: comparison of geomorphological mapping and photogrammetric monitoring. Earth Surface Processes Landforms 32, 1747-1758. https://doi.org/10.1002/esp.1496

Rolleri, E.O., Criado R.P., 1970. Geología de la provincia de Mendoza. $4^{a}$ Jornadas Geológicas Argentinas, Actas 2, 1-60. 
Scapozza, C., Lambiel C., Baron L., Marescot L., Reynard E., 2011. Internal structure and permafrost distribution in two alpine periglacial talus slopes, Valais, Swiss Alps. Geomorphology 132 (3-4), 208221.https://doi.org/10.1016/j.geomorph.2011.05.010

Schreiber, E., 2015. Modeling the distribution of Mountain Permafrost in Central Andes, San Juan, Argentina. M. $\mathrm{S}$. Thesis, University of Delaware, United States.

Scotti, R., Brardinoni, F., Alberti, S., Frattini, P., Crosta, G.B. 2013. A regional inventory of rock glaciers and protalus ramparts in the central Italian Alps. Geomorphology 186, 136-149. https://doi.org/10.1016/j.geomorph.2012.12.028

SEGEMAR, 2008. SIG SEGEMAR, 2016. http://sig.segemar.gov.ar/, last access: 7 September 2016.

Strecker, M.R., Alonso, R.N., Bookhagen, B., Carrapa, B., Hilley, G.E., Sobel, E. R., Trauth, M.H., 2007. Tectonics and climate of the southern central Andes. Annual Review of Earth and Planetary Sciences 35, 747-787.

Tapia Baldis, C. 2018. Distribución y características del ambiente periglaciar en el extremo oeste del departamento Calingasta, provincial de San Juan, Argentina. Tesis Doctoral. Facultad de Ciencias Exactas, Físicas y Naturales. Universidad Nacional de San Juan.

Travis, M.R., Elsener, G.H., Iverson, W.D., Johnson, C.G. 1975. View it computation of seen areas, slope, and aspect for land-use planning. US Department of Agriculture Forest Service General Technical Report PSW, Pacific Southwest Forest and Range Experimental Station, Berkeley, California, U.S.A, 11, 70 p.

Trombotto, D., Lenzano, M.G., Castro, M. 2012. Inventory and monitoring of cryoforms and cryogenic processes in the Central Andes of Mendoza, Argentina: birth and extinction of a periglacial lake. $10^{\circ}$ International Conference on Permafrost, Proceedings 1, 419-424, Salekhard, Russia.

Villarroel, C.D. 2019. Características hidrológicas del ambiente periglacial de montaña y estructura interna de glaciares de escombros en los Andes centrales de San Juán. Tesis Doctoral. Facultad de Ciencias Exactas, Físicas y Naturales. Universidad Nacional de San Juan.

Villarroel, C.D., Tamburini Beliveau, G., Forte, A.P., Monserrat, O., Morvillo, M. 2018. DInSAR for a Regional Inventory of Active Rock Glaciers in the Dry Andes Mountains of Argentina and Chile with Sentinel-1 Data. Remote Sensing 10, 1-21. https://doi.org/10.3390/rs10101588

Villarroel, C.D., Forte, A. P., 2020. Spatial distribution of active and inactive rock glaciers and protalus ramparts in a sector of the Central Andes of Argentina. Cuadernos de Investigación Geográfica (Geographical Research Letters) 46 (1), 141-158. https://doi.org/10.18172/cig.4272

Villarroel, C.D., Forte, A.P., Ortiz, D.A., Beliveau, G.T., Güell, A. 2020. Active layer and permafrost thickness in rock glaciers derived from geophysical methods in the semiarid Andes of Argentina. Geomorphology, 107249. https://doi.org/10.1016/j.geomorph.2020.107249

Whalley W.B., Azizi F., 2003. Rock glaciers and protalus landforms: Analogous forms and ice sources on Earth and Mars. Journal of Geophysical Research: Planets 108-E4. https://doi.org/10.1029/2002JE001864 


\title{
SPATIO-TEMPORAL VARIABILITY MONITORING OF THE FLOODS IN THE CENTER-WEST OF THE BUENOS AIRES PROVINCE (ARGENTINA) USING REMOTE SENSING TECHNIQUES. THE ROLE OF SAND DUNES
}

\author{
FELIX I. CONTRERAS ${ }^{1,2 *}$, GIAN M. MAVO MANSTRETTA ${ }^{3}$, \\ MARIA C. PICCOLO ${ }^{3,4}$, GERARDO M.E. PERILLO ${ }^{3,5}$ \\ ${ }^{1}$ Centro de Ecología Aplicada del Litoral (CONICET - UNNE), \\ Ruta $5 \mathrm{~km} \mathrm{2,5} \mathrm{s/n,} 3400$ Corrientes, Argentina. \\ ${ }^{2}$ Departamento de Agrimensura, Universidad Nacional del Nordeste, \\ Avda. Libertad 5460, 3400 Corrientes, Argentina. \\ ${ }^{3}$ Instituto Argentino de Oceanografía (CONICET-UNS), \\ Camino a la Carrindanga km 7, 8000 Bahía Blanca, Argentina. \\ ${ }^{4}$ Departamento de Geografía y Turismo, Universidad Nacional del Sur, \\ 12 de octubre y San Juan, 8000 Bahía Blanca, Argentina. \\ ${ }^{5}$ Departamento de Geología, Universidad Nacional del Sur, \\ Avda. Alem 1253, 8000 Bahía Blanca, Argentina.
}

\begin{abstract}
Floods are recurring events in Buenos Aires Province, mainly caused by high-intensity precipitation, and in some cases, they persist for long periods. The objective of this study is to analyze how the topography of the central-western portion of Buenos Aires Province determines the occurrence and the extent of floods. The presence of shallow lakes characterizes the area which is controlled by the topography. LANDSAT 5 and 8 images corresponding to periods of drought and flooding in the area were compared with topographic profiles and digital elevation models generated from SRTM $3 \mathrm{arc} / \mathrm{sec}$ data. The results showed that in those areas where there are aligned dunes, and after significant storm drain engineering works, during the wet periods, the lakes, their overflows, and the subsequent runoff to the east of Buenos Aires Province flow freely, limiting the flood areas. Due to this topography, the lakes became a more permanent feature of the landscape, even during extreme drought events. In contrast, in those areas where parabolic dunes predominate, the landscape is more affected by droughts and floods. During drought events, we observed a small number of shallow lakes, and during strong storms with high-intensity rainfall, the number of lakes increases and large flooded areas generate damage to agricultural fields and neighboring cities, either due to natural causes or improvised and illegal storm drains. Early warning of flood risks and a systematic territorial ordaining would be the key to the management of the area.
\end{abstract}

\section{Seguimiento de la variabilidad espacio-temporal de las inundaciones en el centro-oeste de la Provincia de Buenos Aires (Argentina) mediante técnicas de teledetección. El papel de las dunas de arena}

RESUMEN. Las inundaciones son eventos recurrentes en la provincia de Buenos Aires causados principalmente por precipitaciones de gran intensidad. En algunos casos, persisten durante prolongados periodos de tiempo. El objetivo de este trabajo es analizar cómo la topografía del centro-oeste de la provincia de Buenos Aires ha determinado la ocurrencia y extensión de las inundaciones. El área se caracterizada por la presencia de lagunas 
que son controladas por la topografía. Imágenes de satélite LANDSAT 5 y 8 correspondientes a períodos de sequía e inundación fueron comparadas con perfiles topográficos y modelos de elevación digital generados a partir de datos SRTM de $3 \mathrm{arc} / \mathrm{seg}$. Los resultados demostraron que, en aquellas zonas donde existen dunas alineadas, con importantes obras de ingeniería (desagües pluviales), las lagunas, sus desbordamientos y la posterior escorrentía se realiza de manera encauzada hacia el este de la provincia de Buenos Aires, limitando las áreas de inundación. Debido a esta disposición topográfica, los lagos tuvieron un mayor período de permanencia, incluso en eventos extremos de sequía. Por otro lado, en aquellos lugares donde predominan las dunas parabólicas, el paisaje se ve más afectado por las sequías e inundaciones. Durante los eventos de sequía se observa un menor número lagos poco profundos, y durante tormentas fuertes, con lluvias de alta intensidad, el número de lagos aumenta y las áreas inundadas se extienden, generando daños a los campos agrícolas y a las ciudades vecinas, ya sea por causas naturales o por drenajes improvisados e ilegales. La alerta temprana de los riesgos de inundación y una ordenación territorial serían la clave para la gestión de la zona.

Key words: Dunes, topography, floods, shallow lakes, runoff.

Palabras clave: Dunas, topografía, inundaciones, lagunas, escorrentía.

Received: 7 January 2020

Accepted: 25 May 2020

* Corresponding author: Félix Ignacio Contreras, Centro de Ecología Aplicada (CONICET-Universidad Nacional del Nordeste), Ruta 5 km 2.5 s/n, 3400 Corrientes, Argentina. E-mail: ignaciocontreras@conicet.gov.ar

\section{Introduction}

The manifestation of droughts and floods in the distribution and extension of water bodies in a certain region depends on a series of factors. In one hand, several studies had shown that the precipitation, either by its scarcity or by its excesses, constitutes one of the main variable that determine the manifestation of floods and droughts (Quirós, 2002; Kwak and Kondoh, 2008; Ferrelli and Aliaga, 2016). On the other hand, the geology and its related processes, the terrain slope, the vegetation, the topography and the soil types (Scarpati et al., 2008; Frot and Wesemael, 2009) are key factors in the landscape evolution (Kasprak et al., 2019). Although such factors should be considered when studying floods and droughts (Tripaldi et al., 2018); they are seldom discussed. Even more, for aeolian environment, there is a lack of information about the role of the topography and the aeolian morphologies associated, mainly dunes, in the development of the surface drainage and water accumulations process.

The modern dune systems (Perillo, 2001) constitute one of the most prominent morphologies created by sedimentary deposits driven by wind (Blumberg, 2006). These systems have complex arrangements of geomorphic elements, including the dunes and interdune areas. These areas occur on a wide range of scales, are characterized by a variety of morphologies (Al-Masrahy and Mountney, 2013), and contain important paleoenvironmental and paleoclimatic information (Ivester and Leigh, 2003, Tripaldi and Zarate, 2016). The dune system of Buenos Aires Province (Argentina) in the study area have mainly longitudinal and parabolic dunes. The longitudinal dunes are arranged in parallel ridges, with a well-defined wavelength when they are large (Courrech du Pont, 2015). Meanwhile, parabolic dunes typically have a U- or V-shaped lobe with two trailing arms pointing upwind encompassing a deflation basin (Yan and Baas, 2017). They are known to be genetically associated with an adequate sand supply (Yan and Baas, 2017), moderate to high vegetation cover (Tsoar, 2001; Ivester and Leigh, 2003; Pye and Tsoar, 2009), unidirectional wind (Ivester and Leigh, 2003) and are mostly found in humid and cold areas (Tsoar, 2001). As in other areas worldwide, these dunes are mostly inactive and 
stabilized by vegetation (Ivester and Leigh, 2003), but also deeply disturbed by cattle grazing and agriculture (Zarate and Tripaldi, 2012; Contreras et al, 2018; Tripaldi et al., 2018).

There are shallow lakes between the dunes that intervene in the hydrological characteristics. Water coming from both rainfall and groundwater in low runoff areas and from what is stored in the dunes recharges these shallow lakes (Yang et al., 2003). In the case of the Pampean region, water from rainfall is not always able to infiltrate because the water table is very close to the surface and only minor amounts of floodwaters percolate through the dune field; so, this water would be transported to form shallow lakes (Forte Lay et al., 2008). Topography, climatic regime, drainage, soils and anthropogenic activities explain the structure and functioning of these lakes (Ferrelli and Aliaga, 2016).

There may be considerable portions of known aeolian systems that remain under-sampled, or systems that remain undocumented (Barrineau et al., 2019); but thanks to the studies of dunes using remote sensing (Blumberg, 2006; White et al., 2015; Telfer et al., 2015; Saadouda et al., 2018; Contreras et al, 2018; Tripaldi et al., 2018), significant advances in the knowledge of their evolution and morphological dynamics have been made. Morphometric analysis is cost-effective (Möller et al., 2008; Mashimbye et al., 2014) and is an established technique for determining the geomorphology of aeolian environments, providing relevant information about processes and controls of dune formation (White et al., 2015). Digital elevation models (DEM), as those derived from Shuttle Radar Topographic Mission (SRTM) data have also been used to determine the heights of dunes and delineate possible flood areas depending on the geomorphological characteristics of these sites (Blumberg, 2006; Bubenzer and Bolten, 2008; Ho et al., 2010; Tripaldi et al., 2018). The recent increase in the availability of highresolution remote sensing data offers important information for the study of aeolian environments (Telfer et al., 2015).

During recent decades, several hydrological events in the Pampean region have been studied due to their effects on agricultural production and soil cover, and their impact on the population and its access to drinking water (Sierra et al., 1994; Scarpati et al., 2008). During the 1949-50, 1962-63, and 1988-89 harvests seasons, severe droughts reduced national production of grains by more than $30 \%$, while during the 1985-1987 and 2001-2002 periods, successive floods in the area made agricultural tasks difficult if not impossible in some sectors.

The west-center of Buenos Aires Province is characterized by cycles of floods and droughts (Sosnovsky and Quirós, 2006; Labraga et al., 2011). In general terms, landform characteristics give information on flow characteristics, such as direction, depth, and behaviour (Carrivick and Rushmer, 2006). In this case, the flat topography and low slopes cause these events to be enhanced due to the low water retention capacity in dry years and limited expulsion capacity of local water excesses in wet periods. In this last scenario, water can be preserved both in the sediments, as it happens in the landscape (McLaren et al, 2009) for longer periods. The lakes located in the west-center of Buenos Aires Province were referred by Frengüelli (1956) as basins originated by aeolian excavation. However, in this region, most of the lakes and creeks are relict basins, whose waters occupy the remains of ancient river channels barely discernible today in the field (Dangavs, 2005). In the last fifty years, the succession of severe meteorological droughts and significant flood periods (Labraga et al., 2011) have not only affected economic activities but also the dynamics of the water bodies and, with it, the biodiversity and land cover of the Pampean region (Quirós et al., 2002; Taboada et al., 2009; Ferrelli, 2012).

Floods not only alter landscapes over different space-time scales but also provide sudden and abrupt inputs of water and sediment (Carrivick and Rushmer, 2006). Floods are also related to changes in social, economic and land use conditions (Kwak and Kondoh, 2008). The use of efficient climate forecasts and knowledge about the repetition of extreme events are the basis for planning the management of water resources (Labraga et al., 2011), control policies and early warning of risks. These studies can also provide valuable information as to how effective management actions have been in mitigating effects of anthropogenic land use (Sankey et al., 2018). In short, the objective of this study is to demonstrate how the topographic conditions associated with a dune system located in the Buenos 
Aires Province determine the characteristics of the extension, permanence, and recurrence of floods in the region.

\section{Study area}

Southern South America, particularly the central-western region of Argentina, shows an extensive Quaternary aeolian cover (Zarate and Tripaldi, 2012; Contreras et al., 2018; Mehl et al., 2018), known as the Mar de Arena Pampeano (Pampean Sand Sea) (Iriondo, 1990; Tripaldi et al., 2018), where stabilized dune fields are common and potentially reflect drought variability in the Late Quaternary (Tripaldi and Forman, 2007). These dune systems comprise a large variety of dune morphologies and occur in a variety of environmental settings under different climatic conditions, from humid-subhumid to semiarid-arid (Tripaldi and Zarate, 2016). An important part of these dunes was locally reformed in the past 100 years, which apparently coincides with intensified agrarian use and drying in the late $19^{\text {th }}$ and early $20^{\text {th }}$ century (Tripaldi and Forman, 2007). The shallow lakes in the Pampean region record water run-off, as well as are affected by the wind action, whose predominant direction is from the north and have a wide range of intensities. These dynamics were associated with pronounced droughts followed by floods, likely because of a marked seasonality in the annual precipitation regime and a regionally unstable environment (Stutz et al., 2014; Mehl et al., 2018). The study area covers 21,040 $\mathrm{km}^{2}$ in the central-western region of Buenos Aires Province (Fig. 1), described by Dangavs (2005) as: "sandy Pampa, which lacks drainage, with abundant troughs in interdune depressions in an N-NE direction and relicts of old drainage ditches". The fact that the region is crossed by dunes, that hinder the runoff, is one of the factors that determine the development of surface drainage (Taboada et al., 2009). This region has smooth slopes (Quirós et al., 2002), is made up of a large plain with a smooth regional gradient from west to east, the average of slopes is $25 \mathrm{~cm}$ per km (Taboada et al., 2009). The regional hydrography is characterized by a diagonal system of stagnant waters with a remarkable alignment of its shallow lakes and waterlogged areas. This system contains shallow lakes as reservoirs associated with the longitudinal dunes, partly active due to current removals and, in part, strongly devastated in fixed or semifixed sandy areas, that may continue approximately along the $61^{\circ} 30^{\prime} \mathrm{W}$ meridian, following the sinuous course of old drainage lines. Throughout this region, numerous basins, mostly small, are often only flooded during prolonged rainy periods, arranged in wavy lines distributed like a string of beads (Frengüelli, 1956).

At the S and SW there is a parabolic dune field of approximately $20,200 \mathrm{~m}^{2}$ with a SW-NE orientation in which agricultural and livestock activities are developed. The width of the dunes ranges from 2 to $5 \mathrm{~km}$, whose heights range between 2 and $6 \mathrm{~m}$. Like the longitudinal dunes there are shallow lakes, with an average length of $200 \mathrm{~m}$ in normal periods, dry completely or occupy the entire interdune sector, reaching a maximum length of $6 \mathrm{~km}$ (Contreras et al., 2018). The water bodies in this region were characterized by Quirós and Drago (1999) as a shallow, whose depth average range between 0.7 and $7.3 \mathrm{~m}$. Consequently, these strong morphometric changes represent potential flood risks, considering that it is an area of great agricultural exploitation and that, in many cases, these buckets are over the boundaries of the peri-urban areas of the different localities of the region. Finally, this field dune field is divided into Eastern (wet) and Western (dry) sectors separated by the $650 \mathrm{~mm}$ isohyet.

The region is characterized by a temperate climate, with significant climate variability, where the occurrence of long periods of floods and droughts affect the availability of water resources. Most of the region receives a range of precipitation between 650 and 1,100 mm/year (Aliaga et al., 2017) and presents a NE-SW precipitation gradient (Tripaldi et al., 2018). This regime of precipitation can also amplify the role of vegetation as an active agent in the sandy landscape evolution (Durán et al., 2008). Regionalization of the Pampean climate in the Buenos Aires Province carried out by Aliaga et al. (2016), according to its rainfall regime and temperature distribution, indicates that the study area is located in the humid subregion. Within this subregion, annual precipitation is higher than $650 \mathrm{~mm}$, and moving water is often the dominant natural process shaping the land surface (Charlton, 2007; Liu and Coulthard, 
2015). The annual precipitation shows a marked seasonality of high rainfall during the autumn (MarchApril) and spring months (October-November), whereas winter is the dry season. This average difference is inherited from the inter-annual variability in rainfall across the region (Barrineau et al., 2019). This zone is affected by extreme precipitation and drought events with variable duration and intensity. In the last 50 years in Argentina, a succession of severe droughts and significant flood periods occurred, all of them affecting both the economic activities and the number of shallow lakes that characterized the area. These events included the plain areas, where the dynamics of water resources are directly related to the regularity, intensity, and frequency of rainfall. On the other hand, during dry events a decrease in the cultivated areas and an increase in aeolian erosion processes were observed (Aliaga et al., 2016).

The shallow lakes in this region are not located in an environmentally protected area, therefore its resources are anthropically exploited, although in many cases in low use. Furthermore, from the trophic standpoint, they were characterized as very variable, from eutrophic to hypertrophic and has been found high biomass of their biotic communities, with a variable abundance of piscivore, planktivore (with a predominance of Odontesthes bonariensis) and filtering fishes (Quirós et al., 2002). The fauna present in these shallow lakes is in turn affected by both the annual climatic seasons and drought and flood periods. In droughts have been documented generalized fish mortalities, while in periods with positive hydric regimes have been found algal blooms and high abundance of fish (Quirós et al., 2002).
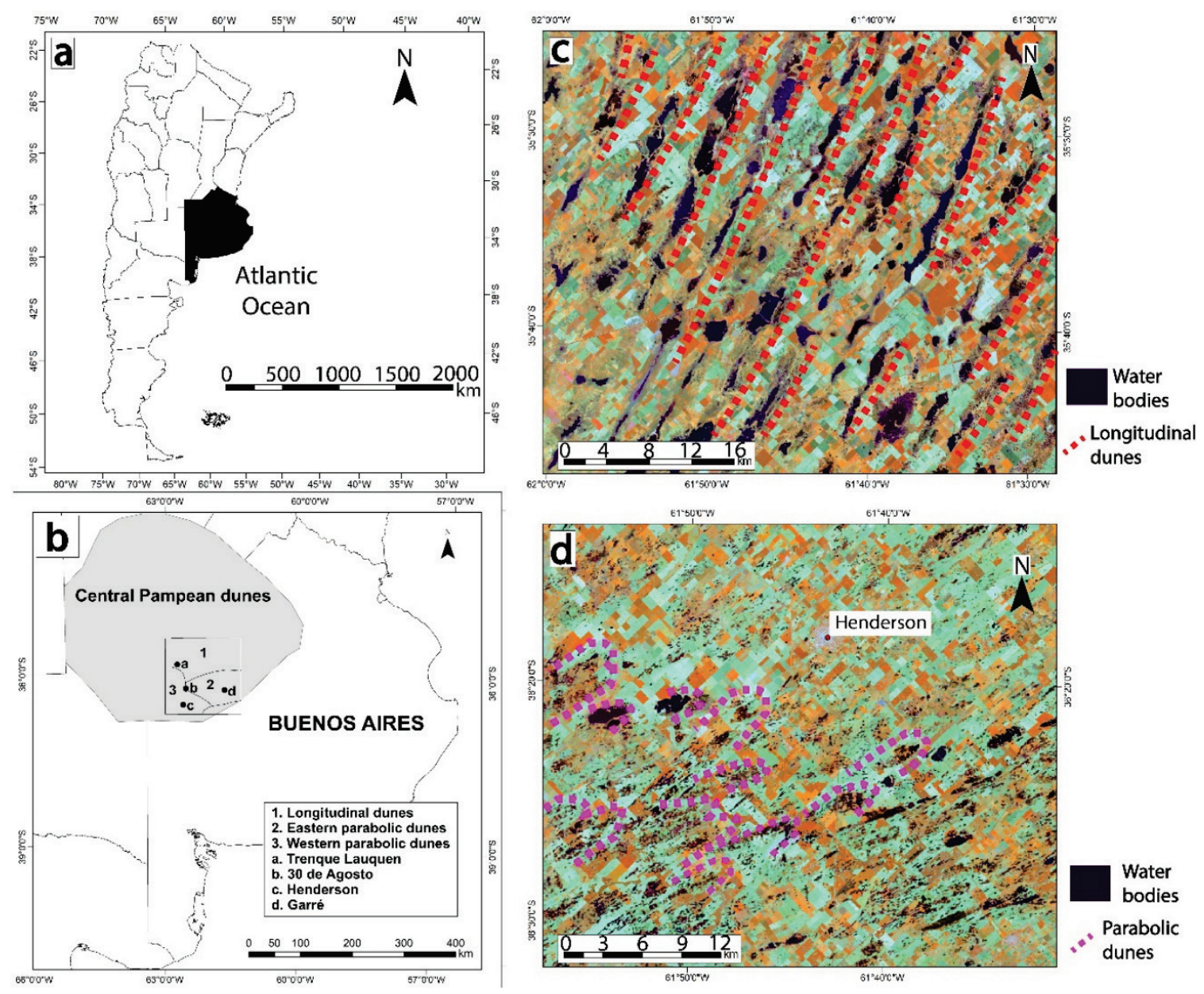

Figure 1. Study area and comparison between water bodies and dunes a) Argentina and Buenos Aires Province. b) Delimitation of dune fields. c) Longitudinal dunes. d) Parabolic dunes. Modified from LANDSAT 5 TM Path/Row 227/85 image (corresponding to 12/16/2008). 


\section{Materials and Methods}

To analyze wet and dry conditions, we used the Standardized Precipitation Evapotranspiration Index (SPEI), which takes into account both precipitation and potential evapotranspiration in its formulation to expresses the water supply-demand relationships in order to understand for climate change influences (Vicente-Serrano et al., 2012).

The SPEI is a drought index that incorporates the effect of evapotranspiration on drought analysis to identify changes in water demand. The SPEI was computed at 1 (month) and 12 (annual) timescales using a 34 years-long monthly. The monthly values (SPEI-1) allow knowing the distribution and the percentage of occurrence of extreme events during the year, while the annual values (SPEI-12) allow intra-annual comparisons during the study period. The datasets have a spatial resolution of $0.5^{\circ}$ by $0.5^{\circ}$ and were extracted for grid point in Lat. -35.75 and Long: -62.25 . For drought analysis, the grading is similar to the SPI with drought categories as: extreme (SPEI $>-2.0)$, severe $(-1.5<$ SPEI $<$ - 1.99), moderate $(-1.0<$ SPEI $<-1.49)$ and near normal $(1.0<$ SPEI $<-1.0)$.

The spatial analysis of 31 satellite images over the period (1986-2017) and its relationship with the SPEI 12-month made it possible to make a comparison of the study area during extreme events of flood and drought. To determine the role that dunes play in the distribution and morphometric characteristics of the shallow lakes that characterize the area during extreme events, two historical cases of flood and drought were selected. The LANDSAT 5 TM Path/Row 227/85 image (corresponding to 12/16/2008) was used for the extreme drought event, while the image LANDSAT 8 OLI Path/Row 227/85 was employed for the extreme flooding event corresponding to 13/03/2017.

The distribution and number of shallow lakes in both years were determined by a combination of bands 4-5-3 for the LANDSAT 5 TM image, while 5-6-4 for the LANDSAT 8 OLI image, both generated in ArcGIS 10.1 (Odriozola and Contreras, 2016). The use of this methodology was chosen based on the least amount of errors that are generated in the unsupervised classification using the IsoClusters tool of ArcGIS 10.1. The results were represented cartographically.

Morphometric variables of the shallow lakes were estimated through the comparison of both years. The studied variables were surface area and lake density. The lake density is defined as the area occupied by lakes in a region, referred to as the total area and expressed as a percentage. The SPEI-12 was used to determine their relationship to lake density.

The topographic description was made based on the 90m SRTM image corresponding to the study area. Once the digital elevation model was generated, topographic profiles were generated using Path Profile spatial analysis tool of the Global Mapper 19.1 software. Flooding and flood models were generated using the Water level tool of the Global Mapper 19.1. The determination of the topographic characteristics corresponding to the dune area and its possible responses during extreme flood and drought events was carried out using $1 \mathrm{~m}$ of water level increase with the water level increase tool.

\section{Results and Discussion}

The intra-annual analysis of SPEI 1 has shown that in the study area there is a predominance of extreme floods over extreme droughts. On the other hand, the highest humidity values are distributed from spring to autumn, but mainly between late summer and early autumn (Fig. 2). This situation is due to the predominance of winds from the $\mathrm{N}$ and $\mathrm{NE}$ coming from the South Atlantic anticyclone, which contributes humidity to the region. On the other hand, extreme drought events are found in the winter, at which time the SW winds from the South Pacific Ocean predominate, characterized by being cold and dry. 


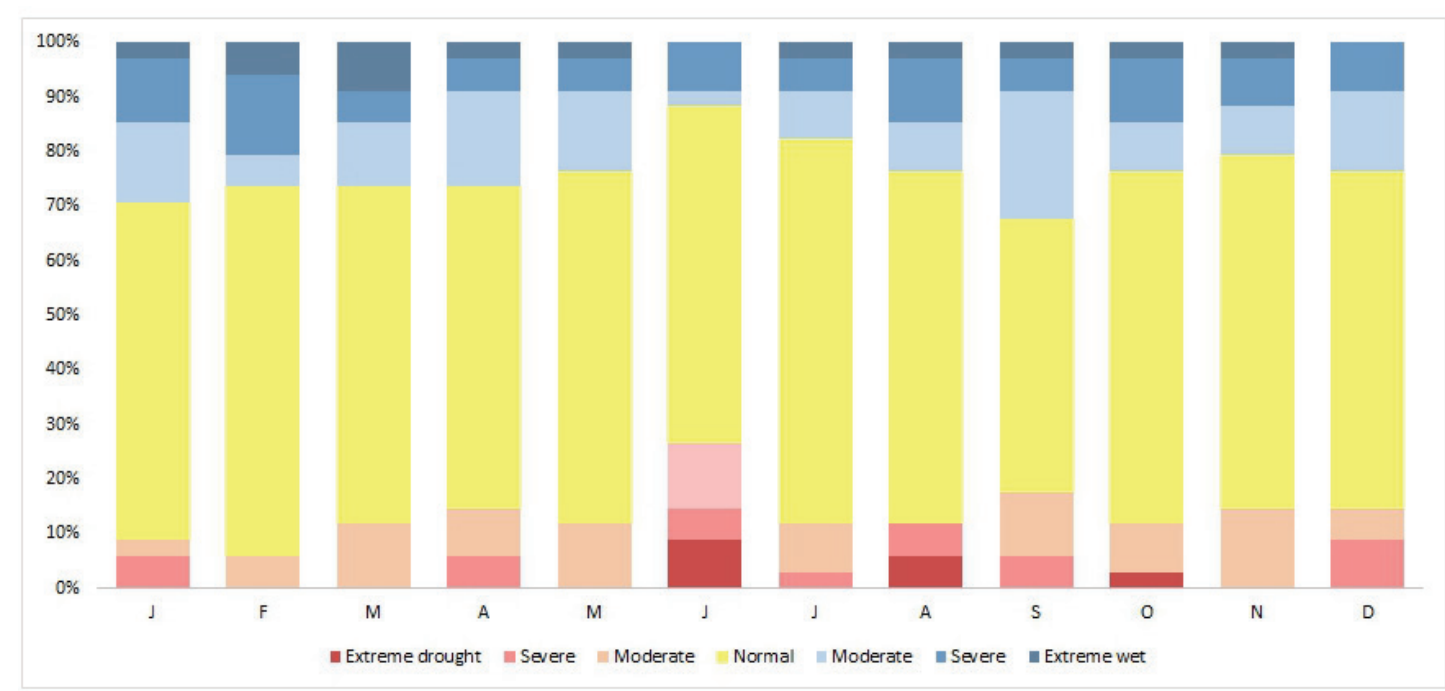

Figure 2. Annual distribution and percentage of occurrence of extreme flood and drought events. Period 1984-2017.

The comparison of SPEI 1 between 2008 and 2017 shows significant differences in both extreme events (Fig. 3). Although the year 2017 was characterized by extreme floods, this event was fueled by the manifestation of the El Niño phenomenon during 2015 and 2016, according to information from the National Oceanic and Atmospheric Administration (NOAA, 2020). In this sense, the water-saturated soils, added to the abundant rainfall during 2017, generated the overflow of the shallow lakes, which have a limited capacity for water retention, mainly those located to the S and SW of the study area.

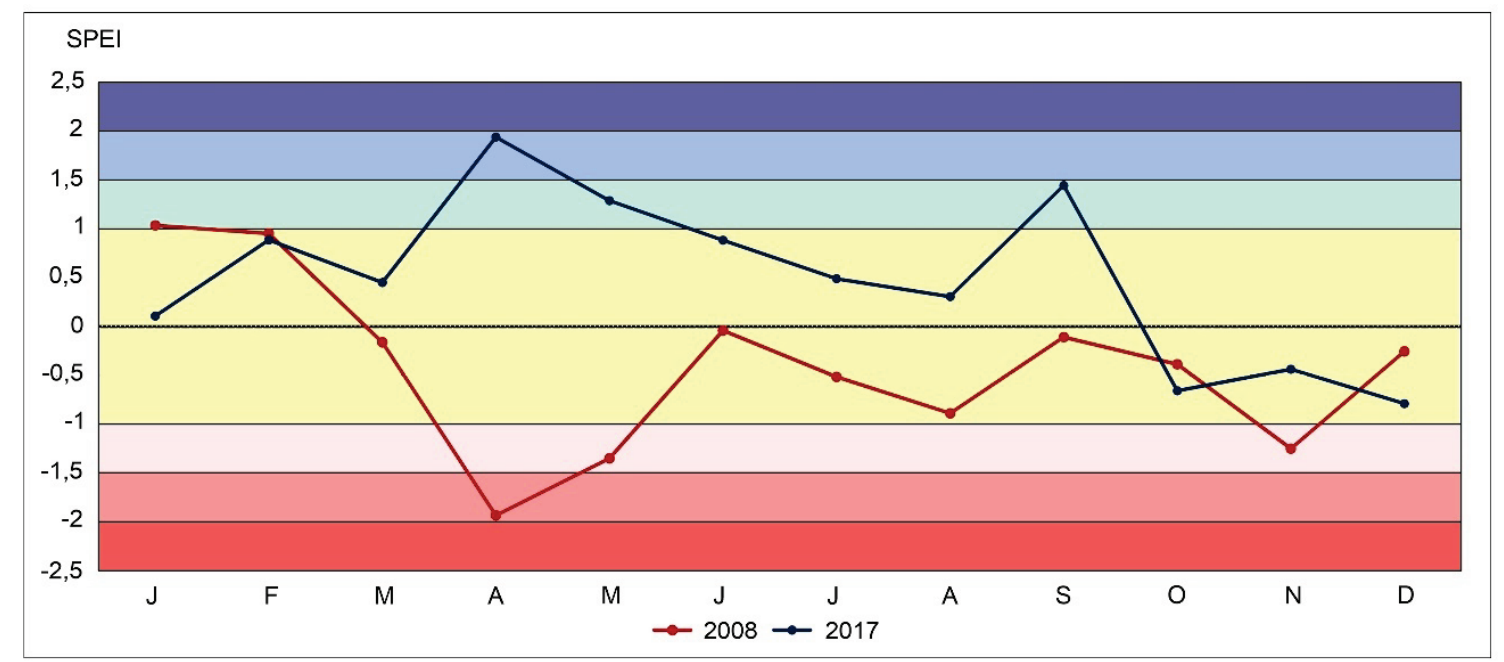

Figure 3. Comparison between SPEI 1 values for the years 2008 and 2017

On the other hand, in the first half of 2008, mainly during April, the values of SPEI 1 were inverse to those of 2017. This is not a minor fact since an extreme drought event was manifested at the same time than an increased annual rainfall and during the month with the greatest recurrence of the expected occurrence of extreme flood events. Subsequently, during June, in both years there was a slight trend towards extreme drought events; however, they remain within normal values until September, when a rebound was observed. In this case, in 2017 reaches severe flooding events again, allowing water to enter in the region. 
In summary, these results evidence that, in the study area, the precipitations registered during the first quarter of the year, determine the hydrological levels of the water bodies, being significant the influence of the winds from the NE to the continental interior, mainly for the western portion of the study area.

In the region of parabolic dunes (Fig. 4), the magnitude of the floods did not change in relation to the low percentage of lake density in extreme flood events. However, the response of the lake density between the longitudinal dunes and the eastern parabolic dunes, except for the difference in values, was similar because both dune systems are in a more humid sector of the study area.

The western parabolic dunes, being in a more arid sector, only exceeded $10 \%$ in lake density in the most extreme years of flood events with a difference of 30 years. The limited recurrence of extreme flood events could explain the lack of investments in storm drainage works in the region, which is why, in the floods of 2017, the channeling was done privately and without impact studies, which generated numerous legal conflicts, as will be explained later.

Comparing the distribution of floods to the north of the study area between 2008 (driest year of the period) and 2017 (last year of extreme flooding), the shallow lakes were aligned following the orientation of the longitudinal dunes in both meteorological conditions of flood and drought. Towards the south, in the period of drought, surface water was basically absent in the area (Fig. 4). In the wet period (2017), large flooded areas were observed (Fig. 5), which allowed the establishment of a boundary between the elongated shallow lakes within the longitudinal dunes to the north and significant extensions of small shallow lakes interdunes to the south (Fig. 5).

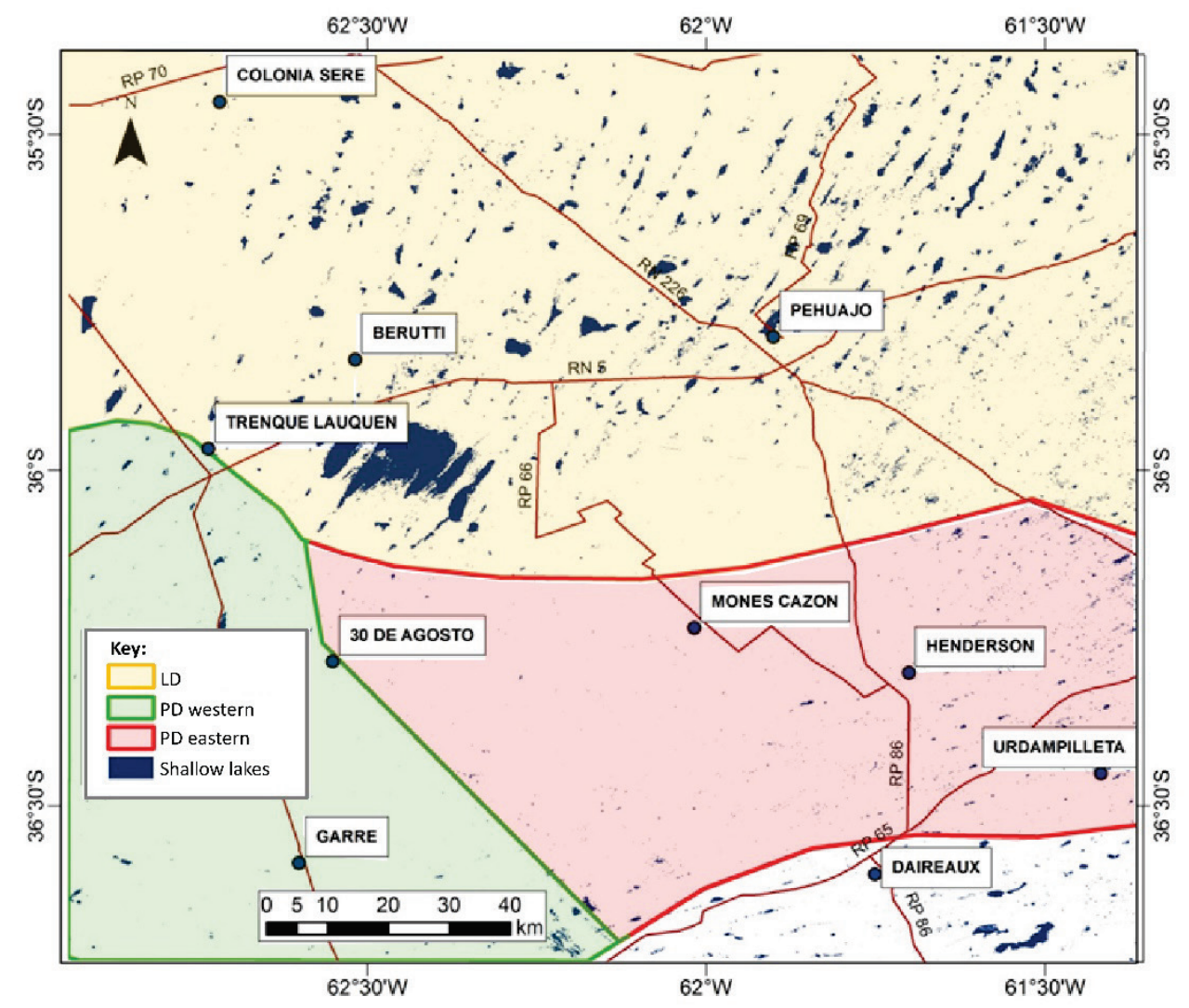

Figure 4. Distribution of shallow lakes and ponds in the central west of Buenos Aires Province during an extreme drought event, December 2008. Delimitation of the area of longitudinal (LD) and parabolic (PD) dunes in the study area. 


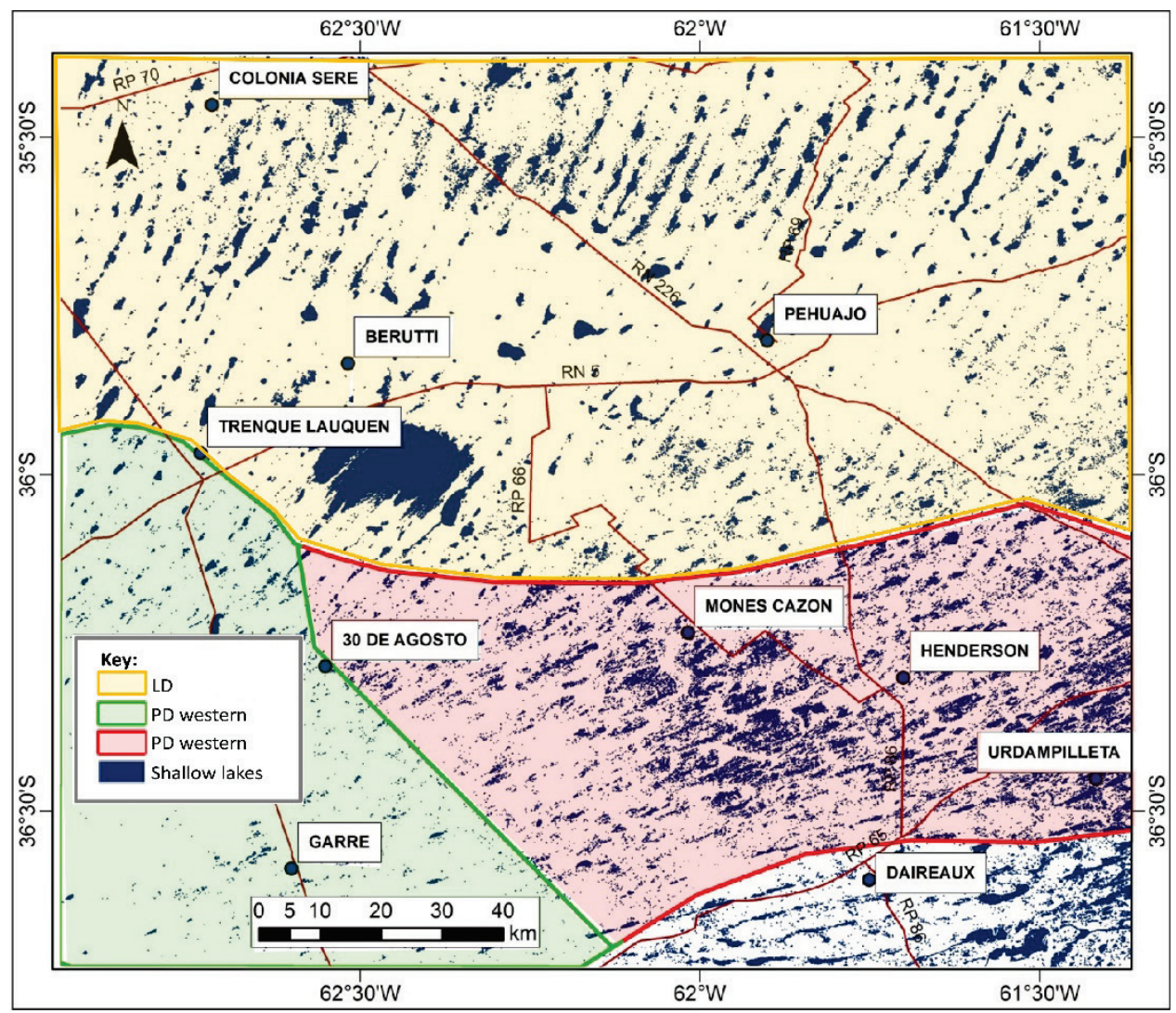

Figure 5. Distribution of shallow lakes and ponds in the central west of Buenos Aires during an extreme flood event, March 2017. Delimitation of the area of longitudinal (LD) and parabolic (PD) dunes in the study area.

Lake density (Table 1) was employed to compare the total study area into sub-regions (Fig. 4): the sector of longitudinal dunes and the sector of parabolic dunes, which in turn is subdivided into eastern areas (more significant presence of shallow lakes) and western areas (drier). Therefore, the water fluctuation of the landscape could be estimated from the values of lacustrine density in both events and, on this basis, to establish temporal and spatial comparisons.

Table 1. Comparison between the occurrence of shallow lakes and ponds during extreme events of drought and flood in the center-west of Buenos Aires (years 2008 and 2017, respectively)

\begin{tabular}{|c|r|r|r|r|r|}
\cline { 2 - 6 } \multicolumn{1}{c|}{} & Area & \multicolumn{2}{c|}{2008} & \multicolumn{2}{c|}{2017} \\
\cline { 2 - 6 } \multicolumn{1}{c|}{} & $\mathrm{km}^{2}$ & $\mathrm{~km}^{2}$ & \multicolumn{1}{c|}{$\mathrm{km}^{2}$} & $\%$ \\
\hline Study area & 21,040 & 6,928 & 33 & 14,389 & 68 \\
PD Total & 9,017 & 732 & 8 & 4,592 & 51 \\
PD Eastern & 5,358 & 584 & 11 & 4,106 & 77 \\
PD Western & 3,659 & 147 & 4 & 486 & 13 \\
LD & 12,023 & 6,196 & 52 & 9,797 & 81 \\
\hline
\end{tabular}

The longitudinal dunes sector has more stable conditions regarding the temporality and extension of its lakes. In contrast, the shallow lakes associated with parabolic dunes, which are particularly sensitive to changes in environmental controls (e.g., temperature, precipitation, and wind regime) and human disturbances (Yan and Baas, 2017), present a rapid response and, therefore, would act as sentinels in scenarios of change, both current and future. 
The longitudinal dunes allow channeling and temporary connections between the depresions seen in the landscape with the reappearance of ponds in extreme flood events, which accommodate excess water and can achieve considerable size (Stanistreet and Stollhofen, 2002). There are no overflow processes, or they are reduced and, therefore, the flooded or waterlogged surfaces are smaller (Fig. 5). On the contrary, the interdune area of the parabolic dunes is extensive (reaching values of up to $6 \mathrm{~km}^{2}$ ) and flat, which enhances the processes of overflow and waterlogging of the shallow lakes, and consequently, large areas of flooded ground are generated during heavy precipitations.

Aeolian dune-field patterns reflect the complex external environment within which the pattern evolves (Ewing and Kocurek, 2010; Yan and Baas, 2017) and are sensitive to climate change (Xu et al, 2015) over a wide range scales (Ewing et al., 2015). In this case, regions with different type of dunes (parabolic and longitudinal) show essential differences in terms of the number of lakes or the area covered by water or floods in the interdune areas. These lake fluctuations can be associated with subdecadal variability (Forman and Pierson, 2003). The differences found are related to the levels of moisture availability (Bullard and Livingstone, 2002) and the amount of rainfall in the wet period. The study area is located in the transition zone between dry and humid climates of the Pampean region (Aliaga et al., 2016), separated by the $650 \mathrm{~mm}$ isohyet. During extreme flood events, this limit shifts to the SW, incorporating the western parabolic dune sector into the wet region. On the contrary, in extreme drought events, the limit displaces towards the NE, which results in the shallow lakes of the eastern parabolic dunes sector drying out completely; during such periods the longitudinal dunes maintain a higher number of permanent shallow lakes, being within the humid region.

The highest percentage of lake density occurred in the longitudinal dunes, coinciding with a very strong flood event in 1987 (Fig. 6). Subsequently, the flood peaks reached $20 \%$, a number that reversed in 2004, with heavy investments in projects of storm drainage engineering towards the Salado River basin, in direction to the NE. According to the Ministry of Economy of the Argentine Republic, more than 300 US million dollars were invested in rural areas to prevent future floods (UCOFIN, 2012). After that date, a decade associated with aridity began, only increasing the values of lake density to 15 $\%$ during the first months of 2017 , where abundant rainfall occurred.

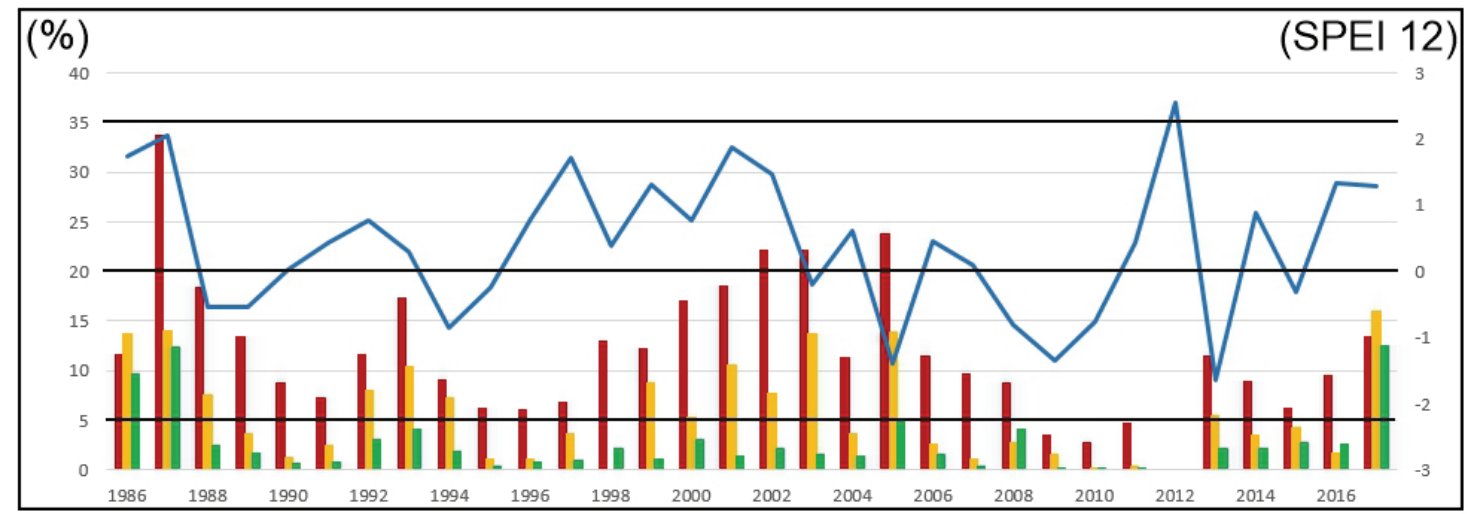

Figure 6. Comparison of lake density between the three regions and the SPEI 12. In brown, the longitudinal dunes field, in yellow, the field of eastern parabolic dunes, and in green, western parabolic dunes field.

In 2014, Argentina returned to a wet period with abundant rainfall during 2015 and 2016 associated with "El Niño" phenomenon that lasted until mid-2017 when droughts returned (Figure SPEI 12). During the wet period in the study area, we observed significant differences in the landscape, mainly in the density and area of the shallow lakes. In such a small area, we distinguished how the rainfall and the landforms (the dunes type) condition the distribution, density, and expansion of the water bodies. 
The quantitative analysis of both satellite images in the wet period allowed us to calculate the total area corresponding to lakes and ponds as well as the flooded areas (Fig. 7). On 15/01/2014, there were $8,626.92 \mathrm{~km}^{2}$ of water in the region, where $60 \%$ corresponded to shallow lakes and ponds and the remaining $40 \%$, to flooded areas. On 10/30/2014, the water inundated region covered $21,573.89 \mathrm{~km}^{2}$, where $59 \%$ belonged to shallow lakes and ponds, and the rest to flooded areas.

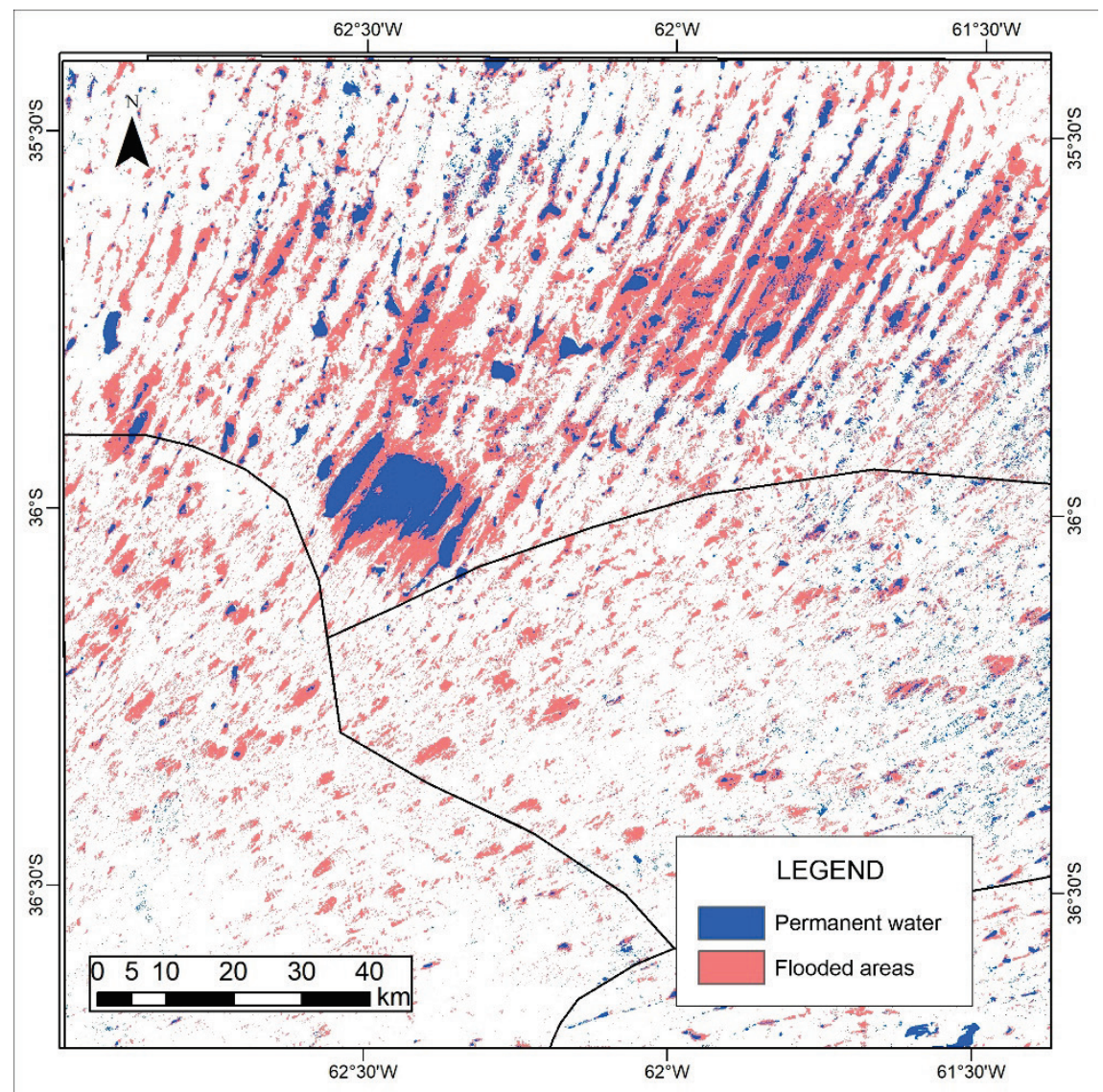

Figure 7. Comparison between water bodies and flooded areas in the study area between 2008-2017.

The results of the comparisons between the two dates and between the geomorphological regions reveal even more significant differences. On $01 / 15 / 2014$, of the $8,626.92 \mathrm{~km}^{2}$ with shallow lakes and flooded areas, only $303.45 \mathrm{~km}^{2}$ belonged to the parabolic dunes' region, and of that surface, $72 \%$ corresponded to flooded environments. The remaining $28 \%$ were water bodies, i.e., before the wet period started, $91 \%$ of the water in the study area was in the humid wetland region. Ten months later, the area with shallow lakes and flood increased to $23,506.65 \mathrm{~km}^{2}$, where $92 \%$ corresponded to the sector of the longitudinal dunes and the remaining $8 \%$ to the parabolic dunes. Although the proportions between the areas of shallow lakes and the waterlogged sectors are maintained for longitudinal dunes (60\% shallow lakes and $40 \%$ waterlogged sectors), the most significant differences are those of the parabolic dunes, where the area goes from $28 \%$ in January to $49 \%$ of water bodies in October and the remaining $51 \%$ to flooding areas. In summary, whereas in the humid, aligned dunes region, the landscape responded to flood events with an increased water-covered ground, the patterns that dominate the landscape remained the same. In the region of parabolic dunes with a drier climate, the changes are more significant, generating substantial landscape changes associated with the water-covered ground. Undoubtedly, it is a complex situation, since environments that vary so much in short periods expose the population to issues most associated with the risk of flooding (Contreras and Paira, 2016).

In the longitudinal dunes, the greater availability of water, in addition to the channeling of the interdune areas, allows the development of agricultural activities in the higher interfluvial areas (Fig. 
1c), leaving the waterlogged areas for livestock exploitation. On the other hand, parabolic dunes have important implications for land use as vegetated dune fields often carry significant socio-economic activity (Yan and Baas, 2017). Therefore, the landscape with parabolic dunes and a drier climate allows for agricultural activities to be carried out on both the dunes and the interdune areas. The use of these sectors with higher risk is not only linked to larger farms, but it is also due to the presence of fine sedimentary material in the soil composition that provides more nutrients than the poor soils on sandy dunes.

Changes in these types of systems have many local environmental and economic impacts (Xu et al., 2015). In this region, as a result of these socio-environmental interactions, the most significant flood damages in agriculture will be found in the parabolic dunes region. Although the levels of the overflow of the shallow lakes or the increase in the water level are inferior to that of the longitudinal dunes, the floods are sufficiently significant to harm an annual harvest (Fig. 1d).

Postdepositional modification of dune morphology is primarily a result of erosion by rain and wind, bioturbation and human disturbance (Ivester and Leigh, 2003). Under certain conditions, parabolic dunes may be transformed into other dune morphologies (Yan and Baas, 2017), including longitudinal dunes (Meurisse et al., 2005). In this study area, abundant and frequent precipitations erode the crest of the parabolic dunes, leaving the disposition of their arms in the landscape and generating the longitudinal dunes characteristic of the region (Contreras et al., 2018). In the parabolic dunes sector, extreme drought events are more frequent and, therefore, the water bodies dry up completely. On the surface of the dry interdune areas, a large quantity of loose fine sediments rebuilds the parabolic dunes when transported by the wind (Contreras et al., 2018). The parabolic dunes remain active due to either anthropogenic pressure or prolonged drought (Yizhaq et al., 2007).

Sand dunes can deflect and confine flows, dam and divert water courses, and thus, determine the position of many contemporary depressions and channels (Maroulis et al., 2007; Liu and Coulthard, 2015). In accordance to these effects, the DEM and topographic profiles of both types of dunes (Figs. 8 and 9) showed that the lakes in the longitudinal dune area (Fig. 8) have channel-like shapes and, in general, the interdune area are permanently inundated. In contrast, in the parabolic dunes area, the lakes are only filled during floods (Fig. 9).

The sedimentary records in dune systems and their related geomorphic forms respond to climatic and environmental changes (McLaren et al., 2009; Thomas and Bailey, 2019). Dangavs (2005) correlated the degree of eutrophication of shallow lakes in relation to age. Shallow lakes occupying older (Pleistocene) depressions are deeper; therefore, they tend to have higher water transparency and presence of macrophytes. On the other hand, in depressions formed during the Holocene, lakes are shallower and either turbid or directly dry during drought periods.

During events of extreme drought in the region, there are significant socioeconomic consequences, such as the reduction in the area of crops and pastures as the area occupied by bare ground increases and so does evaporation (Ferrelli, 2012). In addition, the effects of this phenomenon are observed in a reduction of the depth of the shallow lakes (Quirós et al., 2002). In contrast, an increase in rainfall generates an extension of the cultivated area, an increase in drinking water for the inhabitants and an increase in lake surface area (Sosnovsky and Quirós, 2006; Ferrelli, 2012) as well. If this increase exceeds the load capacity of these shallow lakes, flooding and waterlogging processes begin.

In summary, the topographic profiles help to explain why the two dune landscapes do not originate the same responses during the same extreme event, despite being very close to each other. The region of parabolic dunes is in a latent state of vulnerability to the occurrence of floods. The topographic differences are observed in both the windward and the leeward sides of the dunes, where we perceived that the water level on one side was two or three meters higher than the other (Fig. 9b). The crest of the dune would act as a barrier or natural dam, preventing the passage of water to the next interdune area, considering that there is a general slope with direction W-E. 


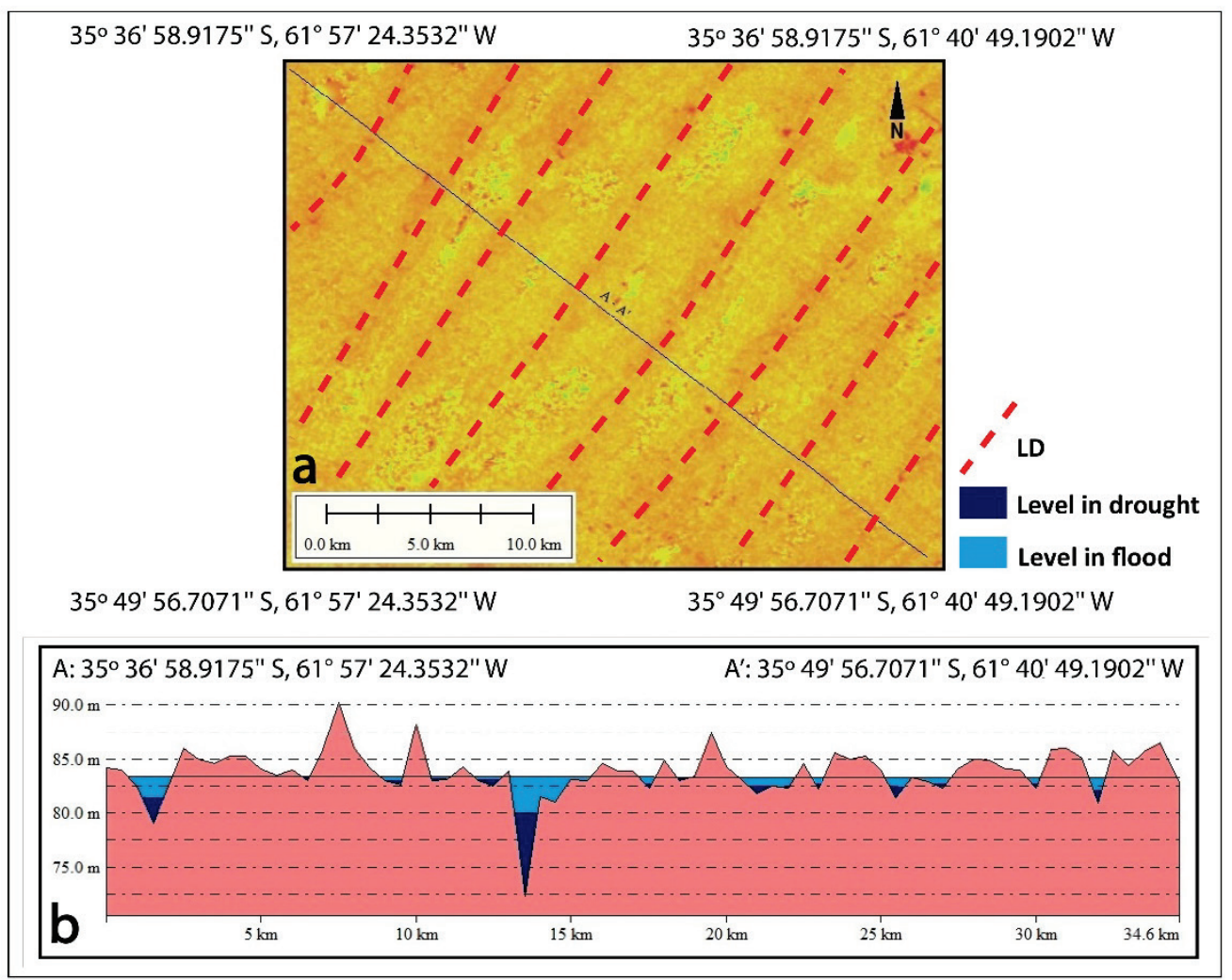

Figure 8. a) Location of cross section $A-A^{\prime}$ in the DEM. b) Topographical profile of the area of longitudinal dunes and distribution of shallow lakes with their respective water levels during extreme events of drought and flood.

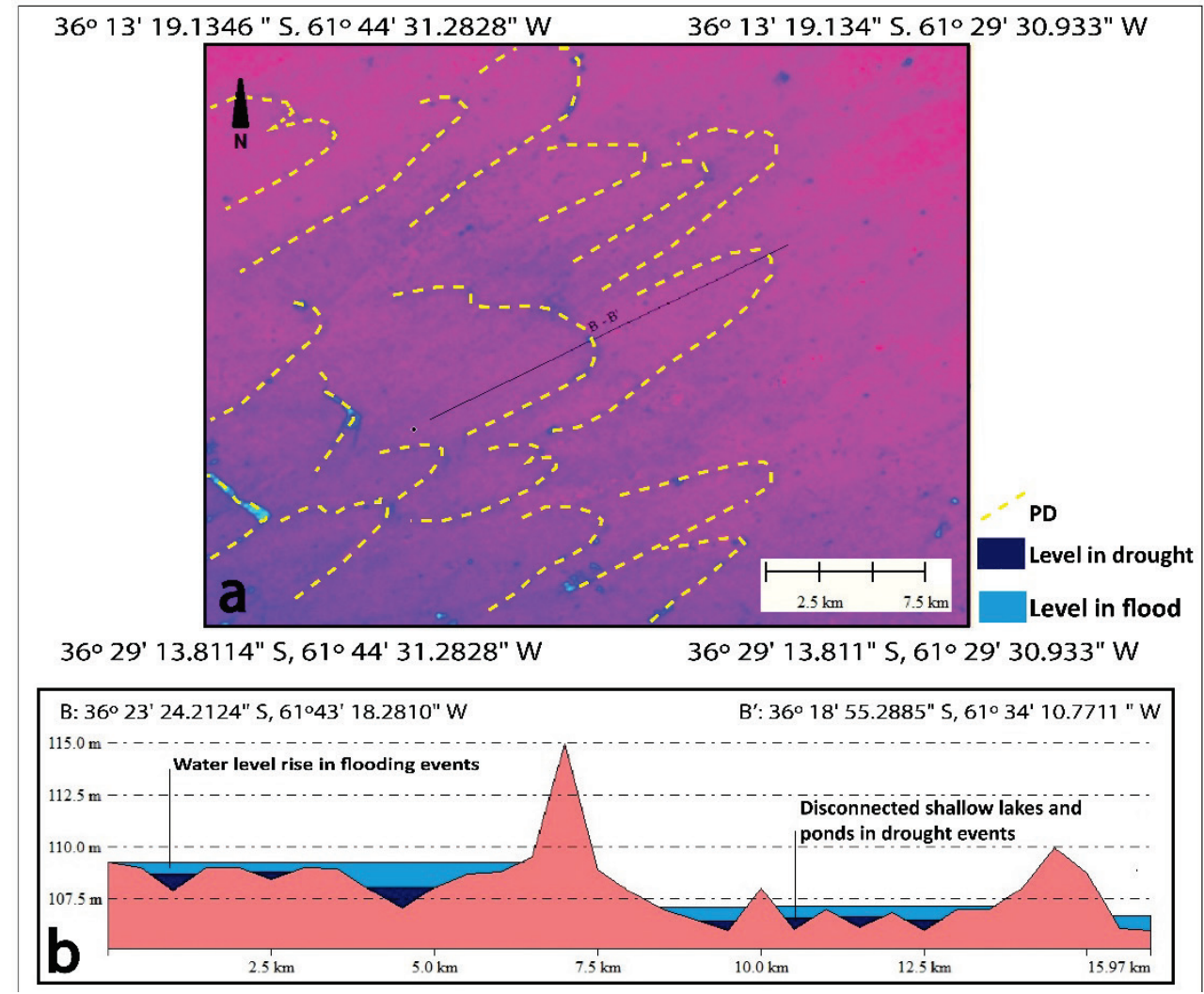

Figure 9. a) Location of the cross-section $B-B^{\prime}$ in the digital elevation model. b) Topographical profile of the parabolic dunes and distribution of shallow lakes with their respective water levels during extreme drought and flood events. 
In this framework, in mid-2017, different localities were affected by flooding, in both urban and rural areas. Stakeholders proceeded to reduce the inundated zones by clandestine ditches and channels (InfoCielo, September 20,2017). Thus, they opened up a way through the dunes, in the same way as in other areas worldwide -especially in those that have been fixed due to agricultural activities and recent engineering projects (Zeng et al., 2018). This allowed the circulation of water from one interdune area to another, generating an extraordinary increase in the water levels registered, even surpassing historical values.

It is not the first time that districts in the region have come into conflict because of floods, and in this occasion, the municipality of Trenque Lauquen filed a criminal complaint against the municipality of Daireaux (Fig. 1) and, at the same time, made a formal complaint about clandestine channels to the Water Authority of Buenos Aires Province. In the meantime, the municipality of Trenque Lauquen stopped the drainage by closing the passage of water (InfoCielo, September 20, 2017). These social conflicts caused by emergency actions carried out without contemplating any possible environmental impacts are persistent in locations at risk and could even affect those communities who were not under any risk.

The severity and complexity of the water crisis are, for Somlyody and Varis (2006), due to sectoral response management and responses to crises and problems without a predictive attitude and systematic approach, among other things. The flood events that occurred in the west of Buenos Aires Province during 2017 was a clear example of a lack of territorial planning and management that included the geographical variables, both human and natural. The management of the early warning occurrence of, for example, floods should not be focused strictly on climatic variables because, as it has been shown, differences in the topographic conditions of a place can result in reducing or enhancing the risk.

\section{Conclusions}

Buenos Aires Province has witnessed extreme flooding and drought events through historical times; however, in recent years, due to climate variability, the events characterized by heavy rainfall are increasingly recurrent. In this study, we show how topography increases the risk of flooding, shortage of drinking water, stakeholder conflict, etc. The same precipitation event does not affect limiting areas in the same way, due to the presence of geomorphological differences.

The development of aligned longitudinal dunes allows the channeling of the shallow lakes overflow in flood events while contributing to the water permanence during droughts. However, the parabolic dunes respond inversely, in extreme drought events the interdune areas are arid, whereas in flood events the overflows of the shallow lakes occupy large areas of the landscape that contains them. Furthermore, these dunes constitute a natural barrier to the general slope W-E that, when crossed by engineering works (in many cases clandestine), flood events take on historical dimensions.

Using the map with the distribution and variability of precipitations in the Pampean region and the distribution of dunes as a reference, it was possible to verify that both, the longitudinal and the eastern parabolic dunes are within the humid region, whereas the western parabolic dunes are in the dry one. The $650 \mathrm{~mm}$ isohyet displacements determine the presence of shallow water bodies, such as semipermanent puddles.

Predictions about flooding or drought risk are crucial for the management of a region affected by climate variability and with certain topographic features. The need for territorial management that provides adequate guidelines on how to mitigate natural disasters are needed. A systematic view of flood vulnerability is the key to minimizing the adverse effects these events bring with them.

This paper determines how three aspects linked to flood risk converge in a relatively small area but present significant differences: 1.- Climatic characteristics: longitudinal dunes in the NE have larger and recurring precipitations, and their water elasticity varied considerably between the years of extreme 
moisture and drought in comparison with the SW area, which is more arid and where flood events occurred after 30 years; 2.- Types and distribution of geoforms: aligned dunes generate more channeled floods, simulating river runoff, while parabolic dunes create large flood areas similar to shallow lakes or ponds; and 3.- Anthropic intervention: strong investments against the reduction of flooded areas in the aligned dunes has reduced more than half the lake density during extreme flood events. In contrast, the reduced or lack of works in the region of parabolic dunes led not only to floods similar to those that occurred 30 years ago, but in turn led to illegal engineering works, further aggravating the situation generated by the natural disaster.

\section{Acknowledgements}

This work was carried out with the aid of a grant from the Inter-American Institute for Global Change Research (IAI) CRN3038 which is supported by the US National Science Foundation (Grant GEO-1128040). This paper is partially financed by the projects 19Q002 of the SGCyT - UNNE, PICT2018-00636-DT and the Universidad Nacional del Sur.

\section{References}

Aliaga, V.S., Ferrelli, F., Alberdi Algañaraz, E.D., Piccolo, M.C., 2016. Distribución y variabilidad de la precipitación en la región Pampeana, Argentina. Cuadernos de Investigación Geográfica 42(1), 261-280. http://doi.org/10.18172/cig.2867

Aliaga, V., Ferrelli, F., Piccolo, M.C., 2017. Regionalization of climate over the Argentine Pampas. International Journal of Climatology 37(1), 1237-1247. http://dx.doi.org/10.1002/joc.5079

Al-Masrahy, M.A., Mountney, N.P., 2013. Remote sensing of spatial variability in aeolian dune and interdune morphology in the Rub' Al-Khali, Saudi Arabia. Aeolian Research 11, 155-170. https://doi.org/10.1016/j.aeolia.2013.06.004

Barrineau, P., Dobreva, I., Bishop, M.P., Houser, C., Forman, S., 2019. Deconstructing aeolian landscapes. Catena 174, 452-468. https://doi.org/10.1016/j.catena. 2018.11.038

Blumberg, D.G. 2006. Analysis of large aeolian (wind-blown) bedforms using the Shuttle Radar Topography Mission (SRTM) digital elevation data. Remote sensing of environment 100(2), 179-189. https://doi.org/10.1016/j.rse.2005.10.011

Bubenzer, O., Bolten, A., 2008. The use of new elevation data (SRTM/ASTER) for the detection and morphometric quantification of Pleistocene megadunes (draa) in the eastern Sahara and the southern Namib. Geomorphology 102(2), 221-231. https://doi.org/10.1016/j.geomorph.2008.05.003

Bullard, J.E., Livingstone, I. 2002. Interactions between aeolian and fluvial systems in dryland environments. Area 34(1), 8-16. https://doi.org/10.1111/1475-4762.00052

Carrivick, J.L., Rushmer, E.L., 2006. Understanding high-magnitude outburst floods. Geology Today 22(2), 6065. https://doi.org/10.1111/j.1365-2451.2006.00554.x

Charlton, R., 2007. Fundamentals of fluvial geomorphology. Routledge. London-New York, pp. 1-224.

Contreras, F.I., Mavo Manstretta, G.M., Perillo, G., Piccolo, M.C., 2018. Caracterización de médanos parabólicos de la región pampeana oriental, centro oeste de la provincia de Buenos Aires (Argentina). Latin American Journal of Sedimentology and Basin Analysis 25(1), 1-15.

Contreras, F.I., Paira, A.R., 2016. Aplicación del "índice de cambio" a las variaciones morfométricas de las lagunas de lomadas arenosas. El caso de Bella Vista (Corrientes, Argentina). Revista de Geografía 21, 31-38.

Courrech du Pont, S. 2015. Dune morphodynamics. Comptes Rendus Physique 16, 118- 138. https://doi.org/10.1016/j.crhy.2015.02.002

Dangavs, N. 2005. Ambientes acuáticos de la provincia de Buenos Aires. Actas del XVI Congreso Geológico Argentino. Geología y Recursos Minerales de la provincia de Buenos Aires, 2019-2035. 
Durán, O., Silva, M.V.N., Bezerra, L.J.C., Herrmann, H.J., Maia, L.P., 2008. Measurements and numerical simulations of the degree of activity and vegetation cover on parabolic dunes in north-eastern Brazil. Geomorphology 102(3-4), 460-471. https://doi.org/10.1016/j.geomorph.2008.05.011

Ewing, R.C., Kocurek, G., 2010. Aeolian dune-field pattern boundary conditions. Geomorphology 114(3), 175187. https://doi.org/10.1016/j.geomorph.2009.06.015

Ewing, R.C., McDonald, G.D., Hayes, A.G., 2015. Multi-spatial analysis of aeolian dune-field patterns. Geomorphology 240, 44-53. https://doi.org/10.1016/j.geomorph. 2014.11.023

Ferrelli, F., 2012. La sequía 2008-2009 en el sudoeste de la provincia de Buenos Aires (Argentina). Ecosistemas, $21,235-238$.

Ferrelli, F., Aliaga, V.S., 2016. Variabilidad de las precipitaciones y sus efectos sobre la respuesta espaciotemporal de cuerpos de agua en la Región Pampeana, Argentina. Huellas 20, 239-246.

Forman, S.L., Pierson, J., 2003. Formation of linear and parabolic dunes on the eastern Snake River Plain, Idaho in the nineteenth century. Geomorphology 56(1-2), 189-200. https://doi.org/10.1016/S0169$555 X(03) 00078-3$

Forte Lay, J., Scarpati O., Capriolo A., 2008. Precipitation variability and soil water content in Pampean Flatlands (Argentina). Geofisica Internacional 47(4), 341-354.

Frengüelli, J., 1956. Rasgos generales de la hidrografía de la provincia de Buenos Aires. Ministerio de Obras Públicas de la provincia de Buenos Aires. 17 p.

Frot, E., Wesemael, B. 2009. Predicting runoff from semi-arid hillslopes as source areas for water harvesting in the Sierra de Gador, southeast Spain. Catena 79, 83-92. https://doi.org/10.1016/j.catena.2009.06.004

Ho, L.T.K., Umitsu M., Yamaguchi Y., 2010. Flood hazard mapping by satellite images and SRTM DEM in the Vugia-Thu Bon alluvial plain, Central Vietnam. International Archives of the Photogrammetry, Remote Sensing and Spatial Information Science, Volume XXXVIII, Part 8, Kyoto, Japan.

Infocielo, 2017. Municipios en guerra por el agua: canales clandestinos y denuncias penales por inundaciones. Infocielo. http://www.infocielo.com/nota/83930/

Iriondo, M.H., 1990. The map of the South American Plains. Its Present State. Quaternary of South America and Antarctic Peninsula 6, 297-306.

Ivester, A.H., Leigh, D.S., 2003. Riverine dunes on the coastal plain of Georgia, USA. Geomorphology 51(4), 289-311. https://doi.org/10.1016/S0169-555X(02)00240-4

Kasprak, A., Bransky, N.D., Sankey, J.B., Caster, J., Sankey, T.T., 2019. The effects of topographic surveying technique and data resolution on the detection and interpretation of geomorphic change. Geomorphology 333, 1-15. https://doi.org/10.1016/j.geomorph.2019.02.020.

Kwak, Y., Kondoh, A., 2008. A study on the extraction of multi-factor influencing floods from RS image and GIS data; a case study in Nackdong basin, S. Korea. The International Archives of the Photogrammetry, Remote Sensing and Spatial Information Sciences 37 (Part B8), 421-425.

Labraga, J.C., Brandizi, L.D., López, M.A., 2011. Avances en el pronóstico climático de las anomalías de lluvia en la región pampeana. Meteorológica 36 (2), 59-71.

Liu, B., Coulthard, T.J., 2015. Mapping the interactions between rivers and sand dunes: implications for fluvial $\begin{array}{lllll}\text { and aeolian } & \text { geomorphology. } & \text { Geomorphology }\end{array}$ https://doi.org/10.1016/j.geomorph.2014.12.011

Maroulis, J.C., Nanson, G.C., Price, D.M., Pietsch, T., 2007. Aeolian-fluvial interaction and climate change: source-bordering dune development over the past $100 \mathrm{ka}$ on Cooper Creek, central Australia. Quaternary Science Reviews 26(3-4), 386-404. https://doi.org/10.1016/j.quascirev.2006.08.010

Mashimbye, Z.E., de Clercq, W.P., Van Niekerk, A., 2014. An evaluation of digital elevation models (DEMs) for delineating land components. Geoderma 213, 312-319. https://doi.org/10.1016/j.geoderma.2013.08.023 
McLaren, S. J., Al-Juaidi, F., Bateman, M.D., Millington, A. C., 2009. First evidence for episodic flooding events in the arid interior of central Saudi Arabia over the last $60 \mathrm{ka}$. Journal of Quaternary Science 24(2), 198207. https://doi.org/10.1002/jqs.1199

Mehl, A., Tripaldi, A., Zárate, M., 2018. Late Quaternary aeolian and fluvial-aeolian deposits from southwestern Pampas of Argentina, southern South America. Palaeogeography, Palaeoclimatology, Palaeoecology 511, 280-297. https://doi.org/10.1016/j.palaeo.2018.08.014

Meurisse, M., Van Vliet-Lanoë, B., Talon, B., Recourt, P., 2005. Holocene dune and peat complexes along the shore of northern France. Comptes Rendus Géoscience 337, 675-684.

Möller, M., Volk, M., Friedrich, K., Lymburner, L., 2008. Placing soil-genesis and transport processes into a landscape context: A multiscale terrain-analysis approach. Journal of Plant Nutrition and Soil Science 171(3), 419-430. https://doi.org/10.1002/jpln.200625039

NOAA, 2020. Historical El Nino/ La Nina episodes (1950-present). Available at: https://origin.cpc.ncep.noaa.gov/products/analysis_monitoring/ensostuff/ONI_v5.php (last access: 18/05/2020).

Odriozola, M.P., Contreras, F.I., 2016. Distribución de las lagunas de la Lomada Norte basada en una clasificación no supervisada de imágenes satelitales LANDSAT 5 TM. En: F.I. Contreras y M.P. Odriozola, M.P. (Comp). Libro de la Junta de Geografia de la Provincia de Corrientes, 23-32.

Perillo, G.M.E., 2001. Hacia una nomenclatura y clasificación de formas de fondo transversales formadas por flujos de agua. Revista de la Asociación Argentina de Sedimentología 8(2), 15-34.

Pye, K., Tsoar, H., 2009. Eolian sand and sand dunes. Springer, pp. 1-475.

Quirós, R., Drago, E. 1999. The environmental state of Argentinean lakes: an overview. Lakes and Reservoirs 4(12), 55-64. https://doi.org/10.1046/j.1440-1770.1999.00076.x.

Quirós, R., Rennella, A., Boveri, M., Rosso, J., Sosnovsky, A., 2002. Factores que afectan la estructura y el funcionamiento de las lagunas pampeanas. Ecología Austral 12, 175-185.

Saadouda, D., Hassanib, M., Peinadoc, F.J.M., Guettouchea, M. S., 2018. Application of fuzzy logic approach for wind erosion hazard mapping in Laghouat region (Algeria) using remote sensing and GIS. Aeolian Research 32, 24-34. https://doi.org/ 10.1016/j.aeolia.2018.01.002.

Sankey, J.B., Caster, J., Kasprak, A., East, A. E., 2018. The response of source-bordering aeolian dunefields to sediment-supply changes 2: Controlled floods of the Colorado River in Grand Canyon, Arizona, USA. Aeolian Research 32, 154-169. https://doi.org/10.1016/j.aeolia.2018.02.004

Scarpati, O.E., Forte Lay, J.A., Capriolo, A.D., 2008. La inundación del año 2001 en la provincia de Buenos Aires, Argentina. Mundo Agrario 9(17).

Sierra, M.E., Fernández Long, M.E., Bustos, C., 1994. Cronología de inundaciones y sequías en el noreste de la provincia de Buenos Aires 1911-89. Revista de la Facultad de Agronomía 14(3), 241-249.

Somlyody, L., Varis, O., 2006. Freshwater under pressure. International Review for Environmental Strategies. 6(2), 181-204.

Sosnovsky, A., Quirós, R., 2006. El estado trófico de pequeñas lagunas pampeanas, su relación con la hidrología y el uso de la tierra. Ecología Austral 16, 115-124.

Stanistreet, I.G., Stollhofen, H., 2002. Hoanib River flood deposits of Namib Desert interdunes asanalogues for thin permeability barrier mudstone layers in aeolianite reservoirs. Sedimentology 49(4), 719-736. https://doi.org/10.1046/j.1365-3091.2002.00458.x

Stutz, S., Tonello, M.S., González Sagrario, M.A., Navarro, D., Fontana, S.L., 2014. Historia ambiental de los lagos someros de la llanura Pampeana (Argentina) desde el Holoceno medio: Inferencias paleoclimáticas. Latin American Journal of Sedimentology and Basin Analysis 21, 119-138.

Taboada, M.A., Damiano, F., Lavado, R.S., 2009. Inundaciones en la Región Pampeana. Consecuencias sobre los suelos. Alteraciones de la fertilidad de los suelos: el halomorfismo, la acidez, el hidromorfismo y las inundaciones. EFA (Editorial Facultad de Agronomía), Universidad de Buenos Aires, Buenos Aires, pp $103-127$. 
Telfer, M.W., Fyfe, R.M., Lewin S., 2015. Automated mapping of linear dunefield morphometric parameters from remotely-sensed data. Aeolian Research 19, 215-224. https://doi.org/10.1016/j.aeolia.2015.03.001.

Thomas, D.S., Bailey, R. M., 2019. Analysis of late Quaternary dune field development in Asia using the accumulation intensity model. Aeolian Research 39, 33-46. https://doi.org/10.1016/j.aeolia.2019.04.005

Tripaldi, A., Forman, S.L., 2007. Geomorphology and chronology of Late Quaternary dune fields of western Argentina. Palaeogeography, Palaeoclimatology, Palaeoecology 251(2), 300-320. https://doi.org/10.1016/j.palaeo.2007.04.007

Tripaldi, A., Mehl, A., Zárate, M.A., 2018. Parabolic megadunes in a subtropical Quaternary inland dune field, southwestern Pampas, Argentina. Geomorphology, 321, 103-116. https://doi.org/10.1016/j.geomorph.2018.08.021

Tripaldi, A., Zárate, M.A., 2016. A review of Late Quaternary inland dune systems of South America east of the Andes. Quaternary International, 410, 96-110. https://doi.org/10.1016/j.quaint.2014.06.069

Tsoar, H., 2001. Types of aeolian sand dunes and their formation. In N. J. Balmforth and A. Provenzale (Ed.), Geomorphological fluid mechanics. Springer, Berlin, Heidelberg, pp. 403-429. https://doi.org/10.1007/3-540-45670-8_17

UCOFIN, 2012. Fondo fiduciario de infraestructura hídrica. Ministerio de Hacienda de la República Argentina.

Vicente-Serrano, S.M., Zouber, A., Lasanta, T., Pueyo, Y., 2012. Dryness is accelerating degradation of vulnerable shrublands in semiarid Mediterranean environments. Ecological Monographs 82, 407-428. https://doi.org/10.1890/11-2164.1

White, K., Bullard, J., Livingstone I., Moran L., 2015. A morphometric comparison of the Namib and southwest Kalahari dunefields using ASTER GDEM data. Aeolian Research, 19 (Part A), 87-95. https://doi.org/10.1016/j.aeolia.2015.09.006

Xu, Z., Lu, H., Yi, S., Vandenberghe, J., Mason, J. A., Zhou, Y., Wang, X., 2015. Climate-driven changes to dune activity during the Last Glacial Maximum and deglaciation in the Mu Us dune field, north-central China. Earth and Planetary Science Letters 427, 149-159. https://doi.org/10.1016/j.epsl.2015.07.002

Yan, N., Baas, A.C., 2017. Environmental controls, morphodynamic processes, and ecogeomorphic interactions of barchan to parabolic dune Parabolic dunes and their transformations under environmental and climatic changes: Towards a conceptual framework for understanding and prediction. Global and Planetary Change 124, 123-148. https://doi.org/10.1016/j.geomorph.2016.10.033

Yang, X., Liu T., Xiao, H., 2003. Evolution of megadunes and lakes in the Badain Jaran Desert, Inner Mongolia, China during the last 31,000 years. Quaternary International 104, 99-112. https://doi.org/10.1016/S10406182(02)00138-6

Yizhaq, H., Ashkenazy, Y., Tsoar, H., 2007. Why do active and stabilized dunes coexist under the same climatic conditions? Physical Review Letters, 98(18), 188001. https://doi.org/10.1103/PhysRevLett.98.188001

Zeng, L., Yi, S., Lu, H., Chen, Y., Lei, F., Xu, Z., Wang, X., Zhang, W., 2018. Response of dune mobility and pedogenesis to fluctuations in monsoon precipitation and human activity in the Hulunbuir dune field, northeastern China, since the last deglaciation. Global and Planetary Change, 168, 1-14. https://doi.org/10.1016/j.gloplacha.2018.06.001 


\title{
REGIONALIZACIÓN DEL CAUDAL MÁXIMO ANUAL EN CUENCAS DEL SISTEMA HIDROGRÁFICO DEL RÍO COLORADO, ARGENTINA
}

\author{
CAROLINA LAURO ${ }^{1 *}$, ALBERTO VICH ${ }^{1,2}$, STELLA M. MOREIRAS $^{1,3}$, \\ LUIS BASTIDAS ${ }^{1}$, SEBASTIÁN OTTA ${ }^{1}$, EMILCE VACCARINO ${ }^{1}$
}

${ }^{1}$ Instituto Argentino de Nivología, Glaciología y Ciencias Ambientales, CCT-Mendoza, CONICET, Argentina.

${ }^{2}$ Instituto de Estudios del Ambiente y los Recursos Naturales (IDEARN), Facultad de Filosofía y Letras, Universidad Nacional de Cuyo, Argentina.

${ }^{3}$ Cátedra de Edafología, Facultad de Ciencias Agrarias, Universidad Nacional de Cuyo, Argentina.

\begin{abstract}
RESUMEN. La predicción del caudal máximo anual resulta de utilidad para la gestión de eventos extremos relacionados con crecidas. Para realizar estimaciones con bajo grado de incertidumbre es necesario contar con una gran cantidad de información hidrométrica. Las cuencas que integran el sistema hidrográfico del río Colorado presentan una topografía que dificulta el mantenimiento de las estaciones hidrométricas, razón por la cual, se carece de continuidad en los registros y se presentan en varios casos sectores sin aforar. La regionalización consiste en la transferencia de información de los sitios aforados a los no aforados para la predicción de caudales. El objeto del trabajo fue encontrar la función que relaciona el caudal máximo anual con las características climáticas y morfométricas de las cuencas. Para ello, se emplearon modelos de regresión simple. A partir de dicha relación y la curva de frecuencia regional es posible predecir caudales máximos anuales para diferentes períodos de retorno en cuencas sin aforo pertenecientes al sistema hidrográfico del río Colorado, Argentina. De los doce modelos analizados, los mejores resultados se presentan cuando la variable predictora es el área y perímetro de la cuenca. Los errores de los modelos de regionalización para diversos sitios del sistema resultaron entre el $6 \%$ y el $67 \%$. Estos modelos constituyen una herramienta para la gestión de crecidas en el centro oeste argentino.
\end{abstract}

\section{Annual maximum flow regionalization of Colorado River System, Argentina}

\begin{abstract}
The prediction of the maximum annual flow is necessary for flood management. Large amounts of hydrological information are required to make meaningful estimates. The Colorado River System basins have a topography that makes it difficult to maintain hydrometric stations, so there is a lack of continuity in records and in several cases there are ungauged basins. Regionalization methods consist of transferring information from gauged to ungauged sites in order to make predictions. The objective is to find regional regression models that relate the climate and morphometric characteristics of the basins with the maximum annual flow. For this purpose simple linear regression models were used. From this relationship and the regional frequency curve it will be possible to predict the maximum annual flows for different return periods in ungauged basins of the Colorado River System, Argentina. Regionalization models show that the best estimates occur when the predictor variable is the area and perimeter of the basin. Errors in the regionalization models of various sites in the system resulted between $6 \%$ and $67 \%$. The models found are a tool for flood management in central-western Argentina.
\end{abstract}


Palabras clave: Regionalización, crecida, modelos de regresión, período de retorno.

Keywords: Regionalization, flood, regression models, return period.

Recibido: 26 Diciembre 2019

Aceptado: 16 Septiembre 2020

*Correspondencia: Carolina Lauro, Instituto Argentino de Nivología, Glaciología y Ciencias Ambientales, CCTMendoza, CONICET, Argentina. E-mail: clauro@mendoza-conicet.gob.ar

\section{Introducción}

La predicción de caudales es de utilidad para la planificación de la gestión de los recursos hídricos, por ejemplo, el diseño de la infraestructura hídrica, restauración de ríos, determinación de caudales ambientales y gestión de riesgos, entre otros. Particularmente, la predicción de caudales máximos resulta de gran importancia para la prevención y mitigación de los impactos de desastres naturales asociados a las crecidas e inundaciones. Además, la correcta estimación de la magnitud y frecuencia de los eventos hidrológicos es un factor limitante en la viabilidad económica de obras hidráulicas y gestión de los recursos hídricos.

La manera directa de estudiar el comportamiento hidrológico de una cuenca es a través del análisis de series de datos temporales de caudales disponibles para el sitio de interés. Cuando la información hidrométrica no está disponible o la longitud temporal de los registros no es la apropiada para realizar extrapolaciones a largo plazo, se debe recurrir a métodos indirectos para estudiar las características hidrológicas de la cuenca. Estos métodos consisten en transferir información de sitios donde existe información hidrométrica hacia sitios donde no existen tales registros. Se basan en el principio de compensar la ausencia de datos temporales a través de la abundancia espacial (Hosking y Wallis, 1997).

En la región de Los Andes Centrales, la escasez de sitios de observación de variables hidrológicas y la discontinuidad en las mediciones sistemáticas incrementan la dificultad en la obtención de información hidrológica de base. Ante esta problemática, resulta necesario buscar formas de transferencia de información de un sitio a otro en una cuenca o región (Vich et al., 2014; Lauro et al., 2018).

En los últimos años se ha avanzado en la exploración y aplicación de nuevas herramientas matemáticas, estadísticas y computacionales para la evaluación de los recursos hídricos utilizando toda la información disponible en una región (Poveda et al., 2002; Blöschl et al., 2013). Por un lado, se puede mencionar el análisis regional de frecuencias, cuya principal ventaja es que permite paliar el déficit temporal de información con la abundancia espacial, aumentando la longitud de los registros disponibles mediante la transferencia de información de los diferentes sitios pertenecientes a una región homogénea (Hosking y Wallis, 1997; Teklu y Knut, 2017). Por otro lado, la regionalización hidrológica, definida como un conjunto de herramientas que a partir del análisis de datos hidrométricos, de variables climáticas y características geológicas, permite estimar la magnitud de las variables hidrológicas en sitios con información insuficiente o sin datos (Franchini y Suppo, 1996; Tucci, 1997; Viglione et al., 2007; Song et al., 2016; Kuzmin et al., 2019). La principal ventaja de los estudios regionales es la de extrapolar o interpolar los parámetros para lugares sin datos, optimizando al máximo la información disponible en la región (Mesa Sánchez et al., 2003).

Éstas son prácticas poco usadas en la región de Los Andes Centrales. Puede mencionarse los trabajos realizados por Vich et al. (2014) y el de Lauro et al. (2018) en la región de Cuyo. Los autores 
realizaron la estimación de cuantiles y de parámetros de las funciones de distribución de frecuencias para diversas variables hidrológicas empleando momentos regionales adimensionales, con el método de momentos-L. Además, encontraron que el análisis de frecuencia regional presenta mejor comportamiento y menor incertidumbre en las estimaciones, principalmente para eventos de baja frecuencia, en comparación con el análisis de frecuencia local (Lauro et al., 2018).

El objetivo de este trabajo es determinar la función que relaciona las características morfométricas y climáticas de las cuencas con el caudal máximo anual. Su finalidad es obtener el factor de escala de la curva de crecimiento regional adimensional, para predecir el caudal máximo para diferentes períodos de retorno en cuencas sin aforo pertenecientes al sistema hidrográfico del río Colorado.

\section{2. Área de estudio}

El sistema hidrográfico del río Colorado en la región centro-oeste de Argentina (Fig.1a) abarca una extensión de más de $360.000 \mathrm{Km}^{2}$. Las corrientes fluyen desde la cordillera de Los Andes hacia el este hasta el colector del río Desaguadero; éste drena en el río Colorado, el cual está formado por la confluencia de los ríos Grande y Barrancas.

El régimen hidrológico es nivo-glaciar, con ocurrencia de caudales máximos en el período primavera-verano, entre los meses de noviembre a febrero (Lauro et al., 2019). La variabilidad interanual y decenal del caudal máximo anual de la región se asocia con el índice climático Niño 3.4 y el índice de la Oscilación Decenal del Pacífico (Lauro et al., 2019).

El análisis de regionalización se realizó considerando 12 cuencas del sistema hidrográfico del río Colorado (Fig.1b). Éstas constituyen una región homogénea, es decir, que los caudales diarios máximos de todos los sitios se ajustan a la función de distribución General de Valores Extremos (GEV), diferenciándose sólo por un factor de escala particular para cada sitio (Lauro et al., 2018). Se considera como factor de escala el valor medio de la serie completa de caudales diarios máximos anuales para cada sitio. Todos los sitios que integran la región homogénea acumulan un total de 567 años de registros. Las características estadísticas de las series para cada sitio se presentan en la Tabla 1. Las series de caudal máximo anual se construyeron a partir de los caudales máximo diario por año, promedio para la longitud registro de la serie. Los datos se obtuvieron de la Base de Datos Hidrológica Integrada de la Secretaría de Infraestructura y Política Hídrica de Argentina.

La temperatura es un parámetro importante para caracterizar el régimen fluvial de las corrientes con nacientes en alta montaña. Su incremento influye en la fusión nivo-glacial. Las temperaturas son menores conforme aumenta la altitud de la cordillera y la latitud. Las temperaturas máximas anuales correspondientes al período de verano se registran en la estación Buta Ranquil en la cuenca del río Colorado. Las temperaturas de invernales son menores en la cuenca del río Tupungato (Tabla 2). Las condiciones climáticas y la topografía del lugar dificultan la instalación y mantenimiento de las estaciones meteorológicas; por ello, los registros instrumentales en la cordillera son escasos.

En la región de Los Andes Centrales, el ciclo de precipitación al este de la cordillera está influido por masas de aire continental que contribuyen a los totales de precipitación de verano (Compagnucci et al., 2002; Masiokas et al., 2006). El patrón de precipitación, con un máximo en invierno en la zona más alta de Los Andes, es una consecuencia de la interacción entre los flujos de humedad provenientes de los centros de acción de latitudes medias del Pacífico Sur, la topografía de Los Andes, la ocurrencia de bajas segregadas durante la estación fría (Garreaud y Fuenzalida, 2007) y el paso de frentes fríos (Seluchi et al., 2006). La precipitación media entre los $32^{\circ} \mathrm{S}-36^{\circ} \mathrm{S}$ y $69^{\circ} \mathrm{O}-68^{\circ} \mathrm{O}$ alcanza los $200-600 \mathrm{~mm}$ anuales (Viale et al., 2019). En la cuenca del río Mendoza, la cobertura nival muestra variaciones estacionales con máximos en invierno que alcanzan el 58\% de cobertura promedio anual y variaciones interanuales que marcan períodos secos donde la cobertura alcanza el 12\%, contra los períodos con 
mayor incidencia de nevadas que cubren el 32\% anual promedio (Cara et al., 2016). La mayor precipitación media anual se registra en la cuenca del río Tunuyán en la estación Valle de Uco, mientras que los registros más bajos pertenecen a la estación Punta de Vacas en la cuenca del río Mendoza (Tabla 2).

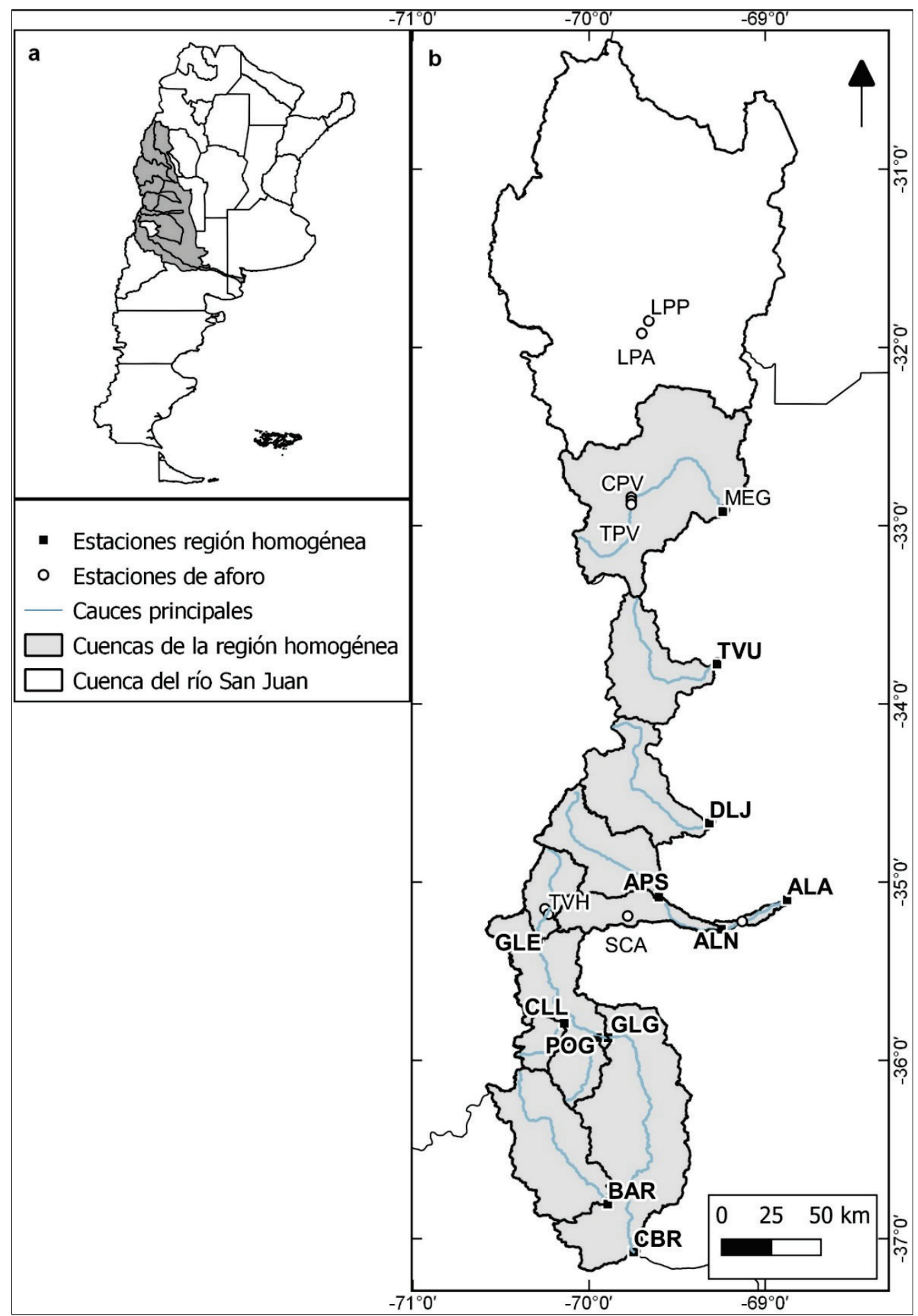

Figura 1. a) Localización regional de las cuencas que integran el sistema hidrográfico del río Colorado. b) Localización de las estaciones de aforo del sistema hidrográfico del río Colorado; sitios que integran la región homogénea y sitios empleados para la predicción. Ver siglas en Tabla 1. 
Tabla 1. Características estadísticas del caudal máximo anual para cada cuenca.

\begin{tabular}{|c|c|c|c|c|c|c|c|c|c|}
\hline Cuenca & Río & Estación & Sigla & Registro & $\begin{array}{c}\text { Caudal } \\
\text { máximo } \\
\text { anual } \\
\left(\mathbf{m}^{3} / \mathbf{s}\right) \\
\end{array}$ & $\begin{array}{c}\text { Máx } \\
\left(\mathbf{m}^{3} / \mathbf{s}\right)\end{array}$ & $\begin{array}{c}\text { Mín } \\
\left(\mathrm{m}^{3} / \mathbf{s}\right)\end{array}$ & $\begin{array}{c}\text { Desvío } \\
\text { estandar } \\
\left(\mathrm{m}^{3} / \mathbf{s}\right)\end{array}$ & $\begin{array}{c}\text { Coef. } \\
\text { Variación } \\
(\%)\end{array}$ \\
\hline Mendoza & Mendoza & Guido & MEG & 1956-11 & 149,6 & 401,5 & 51,8 & 73,3 & 49,0 \\
\hline Tunuyán & Tunuyán & Valle de Uco & TVU & 1944-11 & 92,8 & 211,0 & 41,1 & 39,6 & 42,7 \\
\hline Diamante & Diamante & La Jaula & DLJ & 1971-11 & 102,7 & 227,0 & 29,0 & 47,3 & 46,1 \\
\hline \multirow{3}{*}{ Atuel } & Atuel & El Sosneado & APS & 1972-11 & 121,3 & 306,5 & 54,3 & 50,5 & 41,7 \\
\hline & Atuel & Loma Negra & ALN & $1982-11$ & 95,8 & 210,5 & 35,8 & 39,3 & 41,0 \\
\hline & Atuel & $\begin{array}{c}\text { La } \\
\text { Angostura }\end{array}$ & ALA & 1931-11 & 87,8 & 207,5 & 37,0 & 31,5 & 35,8 \\
\hline \multirow{4}{*}{ Grande } & Chico & Las Loicas & CLL & 1991-11 & 56,5 & 128,4 & 8,8 & 26,2 & 2,2 \\
\hline & PotiMalal & $\begin{array}{c}\text { Puesto } \\
\text { Gendarmería }\end{array}$ & POG & 1971-11 & 31,2 & 80,0 & 3,6 & 19,7 & 1,6 \\
\hline & Grande & $\begin{array}{c}\mathrm{La} \\
\text { Estrechura }\end{array}$ & GLE & $1977-11$ & 127,8 & 320,0 & 29,3 & 63,2 & 49,4 \\
\hline & Grande & La Gotera & GLG & 1971-11 & 391,5 & 840,0 & 70,5 & 142,8 & 2,7 \\
\hline Barrancas & Barrancas & Barrancas & BAR & $1960-11$ & 117,0 & 344,4 & 23,3 & 56,3 & 2,1 \\
\hline Colorado & Colorado & Buta Ranquil & CBR & 1939-11 & 486,7 & $1.404,1$ & 96,1 & 233,1 & 2,1 \\
\hline
\end{tabular}

Tabla 2. Datos de precipitación y temperatura en distintas cuencas del sistema hidrográfico del río Colorado. Los datos se extrajeron de la Base de Datos Hidrológica Integrada de la Secretaría de Infraestructura y Política Hidrica de Argentina.

\begin{tabular}{|c|c|c|c|c|c|c|c|c|c|c|c|}
\hline \multirow{2}{*}{ Cuenca } & \multirow{2}{*}{ Estación } & \multirow{2}{*}{ Sigla } & \multirow{2}{*}{$\begin{array}{c}\text { Elevación } \\
\text { (msnm) }\end{array}$} & \multicolumn{4}{|c|}{ Temperatura $\left({ }^{\circ} \mathrm{C}\right)$} & \multicolumn{4}{|c|}{ Precipitación (mm) } \\
\hline & & & & Serie & Anual & Verano & Invierno & Serie & Anual & Verano & Invierno \\
\hline \multirow{2}{*}{ Mendoza } & Guido & MEG & 1550 & $1994-11$ & 15,0 & 20,9 & 9,0 & $1958-11$ & 197,9 & 79,1 & 38,1 \\
\hline & Punta de Vacas & TPV & 2450 & 1994-11 & 10,9 & 16,5 & 4,9 & 1993-11 & 182,4 & 14,9 & 70,0 \\
\hline Tunuyán & Valle de Uco & TVU & 1200 & 1998-11 & 14,1 & 20,4 & 7,9 & 1955-11 & 419,6 & 133,1 & 89,5 \\
\hline Diamante & La Jaula & DLJ & 1500 & 1994-11 & 13,1 & 19,5 & 6,8 & $1975-11$ & 225,7 & 71,9 & 59,9 \\
\hline Colorado & Buta Ranquil & CBR & 850 & 1994-11 & 16,2 & 23,5 & 9,2 & 1960-11 & 198,0 & 35,1 & 55,1 \\
\hline
\end{tabular}

\section{Materiales y métodos}

\subsection{Obtención de parámetros morfométricos}

Los parámetros morfométricos (Tabla 3) se obtuvieron a partir del modelo digital de elevación (MDE) elaborado por el Instituto Geográfico Nacional (IGN, 2014). Este tiene una resolución de 45x45 $\mathrm{m}$, georreferenciado en coordenadas geográficas y Datum WGS84. Los sectores limítrofes de la cordillera se complementaron con el modelo Shuttle Radar Topography Mission (SRTM) de la NASA. El tamaño de pixel es de 39x39 m, con coordenadas planas UTM (faja 19S). Los procedimientos se realizaron con el software QGIS y planillas de cálculo.

La red hídrica considerada en este trabajo es la correspondiente a las cartas topográficas 1:250.000 del Instituto Geográfico Nacional. 
Tabla 3. Parámetros morfométricos que se utilizaron en el estudio.

\begin{tabular}{|c|c|c|c|c|}
\hline & Parámetro & Unidad & Ecuación/Definición & Referencia \\
\hline \multirow{4}{*}{ 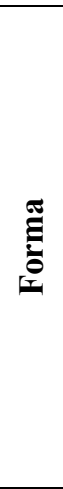 } & Área(A) & $\mathrm{km}^{2}$ & $\begin{array}{c}\text { Área resultante de la proyección de la cuenca en un } \\
\text { plano. }\end{array}$ & \\
\hline & Perímetro(P) & $\mathrm{km}$ & $\begin{array}{l}\text { Longitud del contorno de la proyección de la cuenca en } \\
\text { un plano. }\end{array}$ & \\
\hline & $\begin{array}{c}\text { Relación de } \\
\text { circularidad (C) }\end{array}$ & --- & $\begin{array}{c}C=\frac{4 \pi A_{u}}{P_{u}^{2}} \text { Relaciona el área de la cuenca con la } \\
\text { superficie de un círculo que posee igual perímetro que } \\
\text { la unidad de estudio. }\end{array}$ & Miller, 1953 \\
\hline & $\begin{array}{l}\text { Relación de } \\
\text { elongación (RE) }\end{array}$ & --- & $\begin{array}{c}E=\frac{\sqrt{\frac{4 A_{u}}{\pi}}}{L} \text { Es la relación entre el diámetro de un círculo } \\
\text { con igual área que la de la cuenca y la longitud máxima } \\
\text { de la misma. }\end{array}$ & Schumm, 1956 \\
\hline \multirow{8}{*}{ 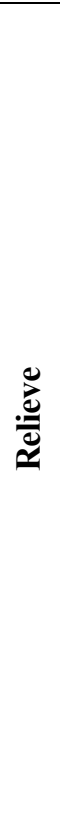 } & Cota máxima(Hmax) & msnm & $\begin{array}{l}\text { Altura máxima de la cuenca. Valor máximo del pixel } \\
\text { correspondiente a la cuenca. }\end{array}$ & \\
\hline & Cota mínima(Hmin) & $\mathrm{msnm}$ & $\begin{array}{l}\text { Altura mínima de la cuenca. Valor mínimo del pixel } \\
\text { correspondiente al MDE de la cuenca. }\end{array}$ & \\
\hline & $\begin{array}{l}\text { Diferencia cota } \\
\text { máxima y mínima } \\
\text { (DH) }\end{array}$ & msnm & $\begin{array}{l}\text { Diferencia entre altura máxima y mínima. Diferencia } \\
\text { entre los valores de pixel máximo y mínimo del MDE } \\
\text { de cada cuenca. }\end{array}$ & \\
\hline & Cota media (Hm) & msnm & Valor medio de las alturas en la cuenca. & \\
\hline & $\begin{array}{l}\text { Pendiente media de la } \\
\text { cuenca (S) }\end{array}$ & $\%$ & Pendiente promedio de todas las laderas. & \\
\hline & $\begin{array}{c}\text { Longitud del cauce } \\
\text { principal (LCP) }\end{array}$ & $\mathrm{km}$ & $\begin{array}{l}\text { Longitud del cauce que conecta el punto de aforo con el } \\
\text { punto más alejado de la cuenca. }\end{array}$ & \\
\hline & $\begin{array}{l}\text { Longitud total de } \\
\text { cauces (LCT) }\end{array}$ & $\mathrm{km}$ & Longitud de todos los cauces presentes en la cuenca. & Linsley et al., 1977 \\
\hline & $\begin{array}{c}\text { Tiempo de } \\
\text { Concentración }(\mathrm{Tc})\end{array}$ & hs & $\begin{array}{l}\text { Tiempo en el cual la escorrentía superficial del punto } \\
\text { más alejado de la cuenca alcanza el punto de desagüe. } \\
\text { Donde J es la pendiente del cauce principal. } \\
\qquad T C=0,3\left(\frac{L C P}{J^{0,25}}\right)^{0,76}\end{array}$ & Témez, 1987 \\
\hline 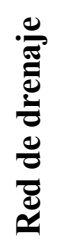 & $\begin{array}{l}\text { Densidad de drenaje } \\
\text { (DD) }\end{array}$ & $\mathrm{km} / \mathrm{km}^{2}$ & $\begin{array}{l}D_{d}=L_{t} / A_{u} \text { Relación entre la longitud de todos los } \\
\text { cauces de cualquier orden (Lt) y el área de la cuenca. }\end{array}$ & Horton 1932 \\
\hline
\end{tabular}

\subsection{Obtención de variables climáticas: precipitación y temperatura}

Debido a baja cantidad de registros instrumentales en la región, se emplearon datos de precipitación y temperatura pertenecientes a la base de datos del Climate Research Unit (CRU) TS4.03 (Harris et al., 2014), expuestos como datos grillados mensuales con resolución espacial de $0,5^{\circ} \times 0,5^{\circ}$. Esta base de datos representa de forma adecuada las variaciones climáticas en la región de Los Andes Centrales (Rusticucci et al., 2014).

Para cada una de las cuencas se obtuvo la precipitación y temperatura media anual para el período 1950-2011. El procedimiento se basó en la obtención de promedios ponderados entre las variables climáticas consideradas, de acuerdo a la superficie de cada cuenca, es decir, la sumatoria de los productos entre los valores de las celdas involucradas, por las superficies relativas de estas (en función del área de la cuenca en cuestión). 
Para evaluar el grado de asociación entre el dato grillado y los datos instrumentales, se calculó el coeficiente de correlación para el período común entre ambas bases de datos.

\subsection{Relación entre parámetros morfométricos y variables climáticas}

En primer lugar, se realizó un análisis de correlación para encontrar los parámetros que poseen mayor relación con la variable dependiente con la intención de reducir la cantidad de variables predictoras a analizar.

La regionalización de caudales se realizó a través de un análisis de regresión simple, donde se considera el caudal máximo promedio como variable respuesta (dependiente) y los parámetros morfométricos y las variables climáticas como variables predictoras (independientes). La estimación de los parámetros del modelo se realizó por medio de mínimos cuadrados ordinarios. Se emplearon tres tipos de modelos: lineal, potencial y exponencial. Mediante las transformaciones correspondientes los modelos potenciales y exponenciales fueron linealizados.

La eficacia de los modelos se evaluó por medio del coeficiente de determinación $\left(\mathrm{R}^{2}\right)$ y el error cuadrático medio (RMSE) cuantificado en las mismas unidades que la variable respuesta $\left(\mathrm{m}^{3} / \mathrm{s}\right)$. Además, se calculó el porcentaje de error aproximado del modelo, dividiendo el valor de RMSE por el valor promedio de la variable respuesta.

\subsection{Aplicación de los modelos}

Los modelos regionales de regresión se aplicaron para calcular el caudal máximo anual de diversas cuencas cuyo caudal máximo anual es conocido con la finalidad de evaluar la capacidad predictiva de los mismos. Los sitios empleados pertenecen a cuencas sistema hidrográfico del río Colorado (Fig. 1b).

A partir de los valores medios obtenidos con los modelos de regresión y la curva de frecuencia regional (Lauro et al., 2018) se calcularon los caudales máximo anuales para diferentes períodos de retorno.

\section{Resultados}

\subsection{Características morfométricas y climáticas de la región homogénea}

En la Tabla 4 se muestran los valores medios, máximos, mínimos y desviación estándar de los parámetros morfométricos y climáticos estimados para las 12 cuencas de la región homogénea.

El caudal anual máximo promedio para la región integrada por 12 cuencas es de $155,1 \mathrm{~m}^{3} / \mathrm{s}$. Los valores mínimos y máximos corresponden a las cuencas de los ríos Potimalal y Colorado, respectivamente.

El promedio del área de las cuencas que integran la región es de $4.033,1 \mathrm{~km}^{2}$, siendo las cuencas del río Chico y del río Colorado las de menor y mayor extensión respectivamente. Los bajos valores del índice de circularidad y el índice de elongación indican que las cuencas se alejan de la forma de círculo, presentando formas ovalo-oblonga a rectangular-oblonga.

En la región de estudio, la elevación del terreno (Fig. 2b) disminuye de norte a sur, alcanzando la altitud máxima en la cuenca del río Mendoza y la mínima en la del río Colorado. Las pendientes medias de las cuencas son características de un relieve irregular y muy fuertemente accidentado (Heras, 1972), con un rango entre $25 \%$ y $48 \%$, siendo representativos de las cuencas del río Colorado y Tunuyán respectivamente. En la Figura 2a se presenta el mapa de pendientes de donde se extrajeron los valores promedio para cada cuenca. 
Tabla 4. Características descriptivas de los parámetros morfométricos y climáticos para la región homogénea.

\begin{tabular}{|c|c|c|c|c|c|}
\hline Variable & n & Mínimo & Máximo & Media & Desv. típica \\
\hline $\mathrm{A}\left(\mathrm{km}^{2}\right)$ & 12 & 595,8 & $14.875,2$ & 4033,1 & 3906,8 \\
\hline $\mathrm{P}(\mathrm{km})$ & 12 & 141,4 & 1038,8 & 447,4 & 251,9 \\
\hline $\mathrm{RC}$ & 12 & 0,1 & 0,4 & 0,3 & 0,1 \\
\hline $\mathrm{RE}$ & 12 & 0,5 & 1,0 & 0,7 & 0,1 \\
\hline $\mathrm{H} \mathrm{Max}(\mathrm{m})$ & 12 & 3807,0 & 6903,0 & 5034,8 & 853,2 \\
\hline $\mathrm{H} \mathrm{Min}(\mathrm{m})$ & 12 & 815,0 & 1994,0 & 1390,0 & 303,4 \\
\hline $\mathrm{Dh}(\mathrm{m})$ & 12 & 2233,0 & 5493,0 & 3644,8 & 941,6 \\
\hline $\mathrm{H} \mathrm{m}(\mathrm{m})$ & 12 & 2169,0 & 3650,0 & 2793,8 & 467,0 \\
\hline $\mathrm{S} \%$ & 12 & 25,0 & 48,0 & 34,0 & 7,0 \\
\hline $\mathrm{LCP}(\mathrm{km})$ & 12 & 42,2 & 365,3 & 143,4 & 87,0 \\
\hline $\mathrm{LCT}(\mathrm{km})$ & 12 & 205,1 & 3542,8 & 1111,0 & 988,0 \\
\hline $\mathrm{Tc}(\mathrm{hs})$ & 12 & 10,4 & 66,5 & 28,9 & 15,38 \\
\hline $\mathrm{DD}\left(\mathrm{km} / \mathrm{km}^{2}\right)$ & 12 & 0,2 & 1,4 & 0,4 & 0,3 \\
\hline $\mathrm{PPM}(\mathrm{mm})$ & 12 & 353,9 & 539,7 & 458,8 & 61,5 \\
\hline $\mathrm{TM}\left({ }^{\circ} \mathrm{C}\right)$ & 12 & 1,8 & 8,3 & 5,6 & 2,2 \\
\hline $\mathrm{QMAX}\left(\mathrm{m}^{3} / \mathrm{s}\right)$ & 12 & 31,2 & 486,7 & 155,1 & 137,8 \\
\hline
\end{tabular}

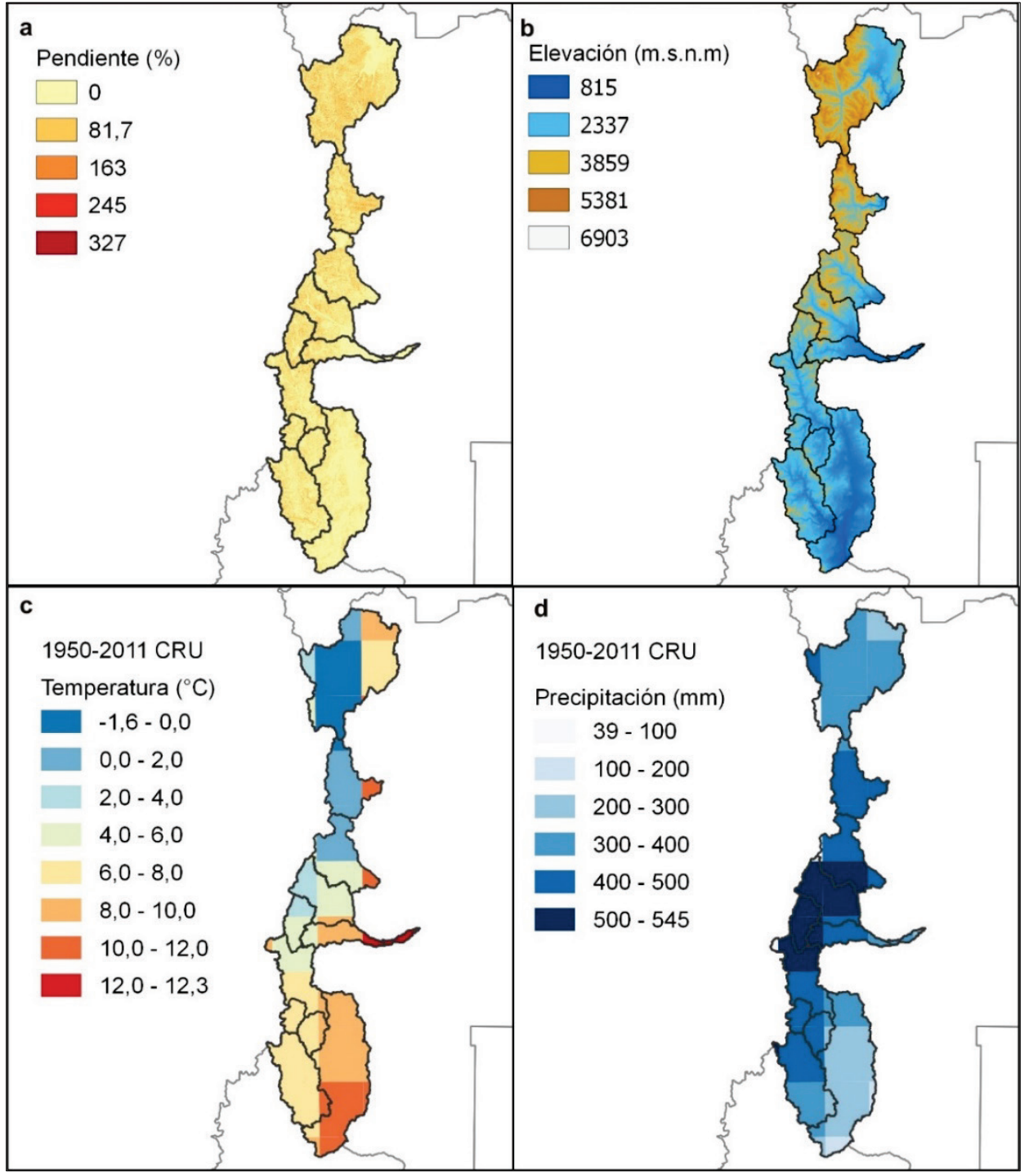

Figura 2. a) Mapas de pendientes (\%), b) elevación (msnm), c) temperatura media anual $\left({ }^{\circ} \mathrm{C}\right), d$ ) precipitación media anual $(\mathrm{mm})$ para la región homogénea. 
En promedio, la longitud del cauce principal y la longitud de todos los cauces es de 143,4 km y $1111 \mathrm{~km}$ respectivamente; en ambos casos, los valores mínimos corresponden a la cuenca del río Chico y los máximos a la cuenca del río Colorado. Las densidades de drenajes son bajas, lo cual es característico de cuencas de zona de montaña con alta permeabilidad.

El tiempo de concentración se encuentra entre 10,04 horas y 66,5 horas correspondientes a las cuencas del río Chico y río Colorado respectivamente.

En líneas generales, la temperatura aumenta hacia el sur y hacia el este de las cuencas conforme disminuye la elevación (Fig. 2c). El promedio de la temperatura media anual de las 12 cuencas es de $5,6^{\circ} \mathrm{C}$, con un rango de $1,8^{\circ} \mathrm{C}$ a $8,3^{\circ} \mathrm{C}$ correspondientes a las cuencas del río Tunuyán y Colorado respectivamente. Dichos valores son inferiores a los procedentes de registros instrumentales (ver Tabla 2) debido a que las estaciones meteorológicas se encuentran en la parte más baja de la cuenca. Los coeficientes de correlación entre la base de datos grillada y los datos instrumentales de temperatura resultaron significativos, con coeficientes entre 0,5 y 0,62 .

Los valores máximos de precipitación se encuentran en la zona central de la región analizada, en las cuencas del río Atuel y río Grande, dejando a los valores mínimos en los extremos norte y sur correspondientes a las cuencas del Mendoza y Colorado (Fig.2d). Los coeficientes de correlación entre los datos instrumentales y grillados de precipitación resultaron significativos, con magnitudes entre 0,42 y 0,75 .

\subsection{Análisis de correlación}

El caudal máximo anual se correlaciona positiva y significativamente con el área, el perímetro, la longitud del cauce principal, la longitud de todos los cauces y el tiempo de concentración (Tabla 5). A su vez, estas variables tienen correlaciones significativas entre ellas, por lo que incorporarlas todas juntas en un modelo de regresión múltiple no generaría aportaciones sustanciales al modelo, pudiendo ocasionar efecto de co-linealidad. La multicolinealidad trae como consecuencia errores en la interpretación de la contribución individual de cada parámetro (Costa, 2017). Para la elección del modelo, se sugiere considerar un número veraz de parámetros de regresión, a fin de garantizar la fiabilidad y solidez del modelo estadístico, considerando el principio de parsimonia (Noto y La Loggia, 2009; Cassahlo, 2018). 


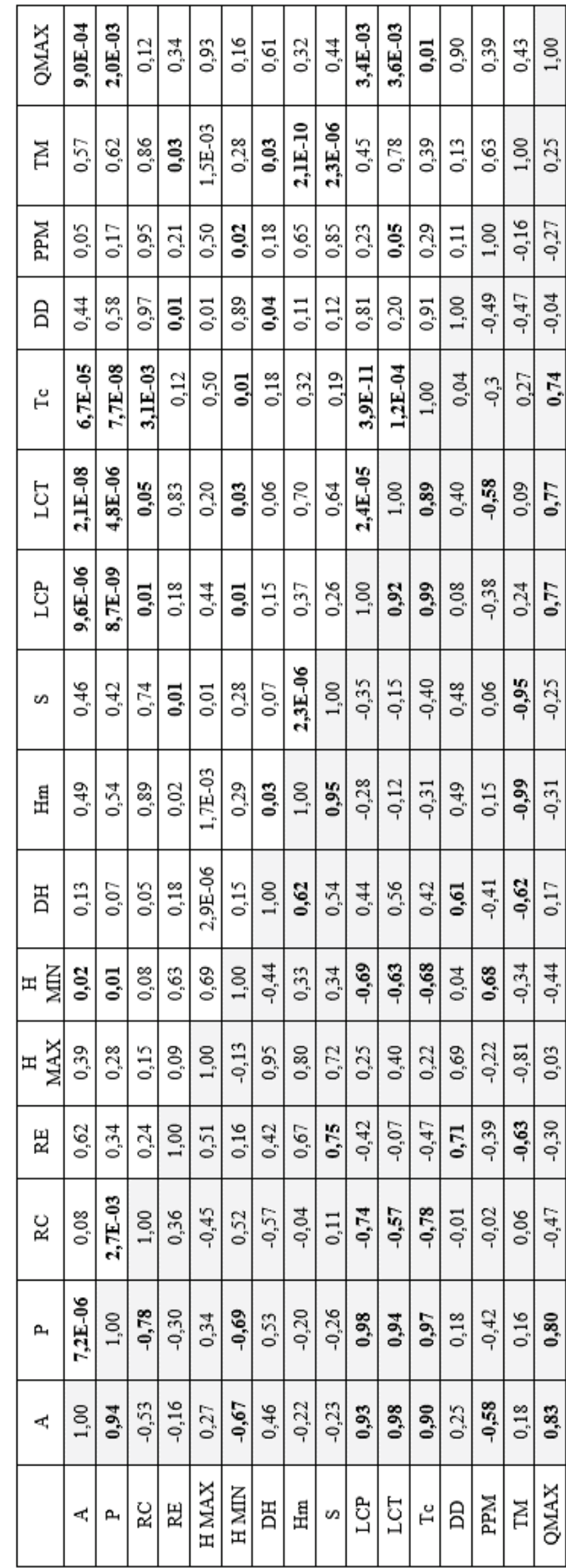

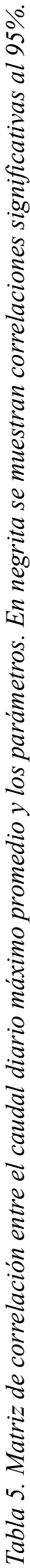




\subsection{Análisis de regresión}

Dada la poca cantidad de sitios que integran la región homogénea $(\mathrm{n}=12)$, no es conveniente utilizar más de un parámetro por modelo para evitar efectos de colinealidad y sobreparametrización, como se mencionó anteriormente. Por ello, se realizaron regresiones lineales con las cinco variables predictoras que tenían mayor correlación con la variable respuesta. Las variables predictoras son: área, perímetro, longitud del cauce principal, longitud total de todos los cauces que integran la cuenca y el tiempo de concentración.

Tras comparar los modelos, el modelo lineal presentó el coeficiente de determinación mayor y el menor RSME en todos los casos, excepto para el modelo exponencial con la variable perímetro, donde el RSME es levemente inferior (Tabla 6).

Tabla 1. Resumen de las características estadísticas de los modelos obtenidos para cada uno de los parámetros (A, área; P, perímetro; LCP, longitud cauce principal; LCT, longitud total de cauces).

\begin{tabular}{|c|c|c|c|}
\hline & Modelo Lineal & Modelo Potencial & Modelo exponencial \\
\hline $\mathbf{A}$ & 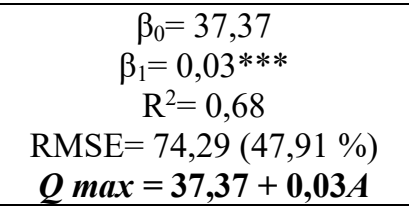 & $\begin{array}{c}\beta_{0}=-0,17 \\
\beta_{1}=0,65^{* *} \\
\mathrm{R}^{2}=0,62 \\
\mathrm{RMSE}=83,93(54,13 \%) \\
\boldsymbol{Q} \boldsymbol{m a x}=\mathbf{0 , 6 7} \boldsymbol{A}^{\mathbf{0 , 6 5}}\end{array}$ & $\begin{array}{c}\beta_{0}=4,18 * * * \\
\beta_{1}=1,5 \mathrm{E}-04 * * \\
\mathrm{R}^{2}=0,59 \\
\mathrm{RMSE}=79,85(51,5 \%) \\
\boldsymbol{Q} \boldsymbol{\operatorname { m a x }}=\mathbf{6 5 , 6} \boldsymbol{e}^{\boldsymbol{A 1} \mathbf{1}, \mathbf{E - 0 4}}\end{array}$ \\
\hline $\mathbf{P}$ & $\begin{array}{c}\beta_{0}=-39,68 \\
\beta_{1}=0,44 * * * \\
\mathrm{R}^{2}=0,63 \\
\mathrm{RMSE}=79,98(51,58 \%) \\
\boldsymbol{Q} \boldsymbol{m a x}=\mathbf{- 3 9 , 6 8}+\mathbf{0 , 4 4 P}\end{array}$ & $\begin{array}{c}\beta_{0}=-0,33 \\
\beta_{1}=0,93 * * \\
\mathrm{R}^{2}=0,62 \\
\mathrm{RMSE}=90,62(58,45 \%) \\
\boldsymbol{Q} \boldsymbol{\operatorname { m a x }}=\mathbf{0 , 4 7 P} \mathbf{0 , 9 3}\end{array}$ & $\begin{array}{c}\beta_{0}=3,74 * * * \\
\beta_{1}=2,3 \mathrm{E}-03 * * \\
\mathrm{R}^{2}=0,62 \\
\mathrm{RMSE}=73,78(47,58 \%) \\
\boldsymbol{Q} \boldsymbol{\operatorname { m a x }}=\mathbf{4 2} \boldsymbol{e}^{\boldsymbol{P 2 . 3 E - 0 3}}\end{array}$ \\
\hline LCP & $\begin{array}{c}\beta_{0}=-19,97 \\
\beta_{1}=1,22 * * * \\
\mathrm{R}^{2}=0,59 \\
\mathrm{RMSE}=84,12(54,25 \%) \\
\boldsymbol{Q} \boldsymbol{m a x}=\mathbf{- 1 9 , 9 7 + 1 , 2 2 L \boldsymbol { C P }}\end{array}$ & $\begin{array}{c}\beta_{0}=0,13 \\
\beta_{1}=0,93 * * \\
\mathrm{R}^{2}=0,56 \\
\mathrm{RMSE}=91,49(59 \%) \\
\boldsymbol{Q} \max =\mathbf{1 , 3 5 L C P} \mathbf{C , 9 3}\end{array}$ & $\begin{array}{c}\beta_{0}=3,86 * * * \\
\beta_{1}=0,006^{* *} \\
\mathrm{R}^{2}=0,55 \\
\mathrm{RMSE}=84,63(54,58 \%) \\
\boldsymbol{Q} \max =\mathbf{4 7 , 4} \boldsymbol{e}^{\boldsymbol{L C P \boldsymbol { 0 } , 0 1}}\end{array}$ \\
\hline LCT & $\begin{array}{c}\beta_{0}=36,24 \\
\beta_{1}=0,11 * * * \\
\mathrm{R}^{2}=0,59 \\
\mathrm{RMSE}=84,83(54,71 \%) \\
\boldsymbol{Q} \boldsymbol{m a x}=\mathbf{3 6 , 2 4}+\mathbf{0 , 1 1} \boldsymbol{L C T}\end{array}$ & $\begin{array}{c}\beta_{0}=0,28 \\
\beta_{1}=0,62 * * \\
\mathrm{R}^{2}=0,57 \\
\mathrm{RMSE}=93,84(60,52 \%) \\
\boldsymbol{Q} \text { max }=\mathbf{1 , 9 0 L} \boldsymbol{C} \boldsymbol{T}^{\mathbf{0 , 6 2}}\end{array}$ & 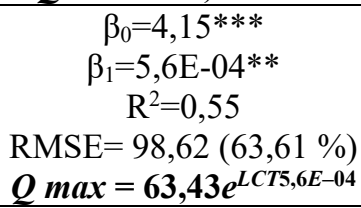 \\
\hline Tc & $\begin{array}{c}\beta_{0}=-37,75 \\
\beta_{1}=6,6^{* *} \\
\mathrm{R}^{2}=0,55 \\
\mathrm{RMSE}=88,25(56.91 \%) \\
\boldsymbol{Q} \boldsymbol{m a x}=\mathbf{- 3 7 , 7 5}+\mathbf{6 , 6 T c}\end{array}$ & 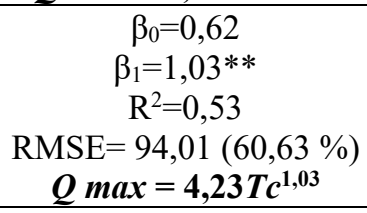 & $\begin{array}{c}\beta_{0}=3,76^{* * *} \\
\beta_{1}=0,035^{* *} \\
\mathrm{R}^{2}=0,52 \\
\mathrm{RMSE}=98,52(63,54 \%) \\
\boldsymbol{Q} \max =\mathbf{4 3 , 2 1} \boldsymbol{e}^{\boldsymbol{T c 0 , 0 3}}\end{array}$ \\
\hline
\end{tabular}

$0 \leq$ Valor-p $\leq 0,001 * * * ; 0,001<$ Valor-p $\leq 0,01 * * ; 0,01<$ Valor-p $\leq 0,05^{*}$

De los doce modelos obtenidos, el que explica mejor el comportamiento del caudal medio máximo es el modelo lineal, con el área de la cuenca como variable predictora. El modelo es significativo (p-valor 0,0009), el cual explica un 68\% de la variabilidad, con un error residual estándar de 74,29 $\mathrm{m}^{3} / \mathrm{s}$, lo que en términos porcentuales representa el $47,91 \%$.

Alternativamente, el modelo exponencial con el perímetro como variable predictora también presenta similares resultados. El modelo es significativo (p-valor 0,0024 ) pues explica el $62 \%$ de la variabilidad. El error residual estándar es $73,78 \mathrm{~m}^{3} / \mathrm{s}$, lo que en términos porcentuales respecto a la media es del $47,58 \%$.

Por lo general, el área de la cuenca es la variable que presenta mayor relación con el caudal en diversos trabajos (Noto y La Loggia, 2009; Malekinezhad et al., 2011; Lujano et al., 2016, 2017). 
Comúnmente se emplean diversas variables ambientales (como precipitación, temperatura o evaporación); sin embargo, en el presente estudio no se encontró una correlación significativa con las variables climáticas.

\subsection{Aplicación de los modelos}

Con los modelos regionales encontrados se realizaron predicciones para los diversos sitios del sistema hidrográfico del río Colorado que reunían las condiciones límites de los modelos; es decir, que tuvieran un área entre 595,5-14.875,2 $\mathrm{km}^{2}$, un perímetro entre $141,4-1038,8 \mathrm{~km}$ y cuyos caudales fueran conocidos para poder evaluar los modelos. Los detalles de los sitios y resultados de los modelos se detallan a continuación (Tabla 7).

Tabla 7. Aplicación de los modelos regionalizados en diversos ríos.

\begin{tabular}{|c|c|c|c|c|c|c|c|c|c|}
\hline Río & Estación & Sigla & $\begin{array}{c}\text { Qmax } \\
\left(\mathrm{m}^{3} / \mathbf{s}\right)\end{array}$ & $\begin{array}{c}\text { Área } \\
\left(\mathrm{km}^{2}\right)\end{array}$ & $\begin{array}{c}\mathbf{Q} \mathbf{m a x} \\
\mathbf{M} . \\
\mathbf{L i n e a l} \\
\left(\mathbf{m}^{\mathbf{3} / \mathbf{s}}\right)\end{array}$ & $\begin{array}{c}\text { Error } \\
(\%)\end{array}$ & $\begin{array}{c}\text { Perímetro } \\
(\mathrm{km})\end{array}$ & $\begin{array}{c}\widehat{\mathbf{Q} m a x} \mathbf{M} . \\
\mathbf{e x p o n e n c i a l} \\
\left(\mathbf{m}^{\mathbf{3} / \mathbf{s})}\right.\end{array}$ & $\begin{array}{c}\text { Error } \\
(\%)\end{array}$ \\
\hline Los Patos & La Plateada & LPP & 166,6 & 8539,6 & 293,6 & 43,3 & 845,6 & 293,7 & 43,3 \\
\hline Los Patos & Álvarez Condarco & LPA & 89,6 & 3581,1 & 114,8 & 38,1 & 475,8 & 125,5 & 28,6 \\
\hline Cuevas & Punta de vacas & CPV & 21,0 & 667,2 & 57,7 & 63,6 & 151,9 & 59,6 & 64,7 \\
\hline Tupungato & Punta de Vacas & TPV & 85,4 & 1783,5 & 90,9 & 6,0 & 257,7 & 76,0 & 12,4 \\
\hline Salado & Cañada Ancha & SCA & 37,4 & 668,5 & 57,4 & 34,8 & 157,8 & 60,8 & 38,0 \\
\hline Tordillo & Valle Hermoso & TVH & 37,4 & 628,5 & 56,2 & 33,5 & 151,9 & 59,6 & 37,3 \\
\hline
\end{tabular}

En los casos del modelo lineal se sobreestimaron los valores de caudal máximo promedio. La mejor estimación fue para el río Tupungato (TPV), con un error del $6 \%$. El modelo exponencial también sobrestima los valores de caudal, salvo para el sitio TPV, donde se subestima, y en este caso, el modelo también tuvo el menor error de estimación (12\%), aunque no menor que el estimado por el modelo lineal. Para el caso del río Los Patos (LPA), el modelo exponencial presentó menor error que el modelo lineal. Para el mismo río, en la estación La Plateada ambos modelos tuvieron similares resultados. En la Figura 3 se muestran los modelos y las predicciones realizadas.

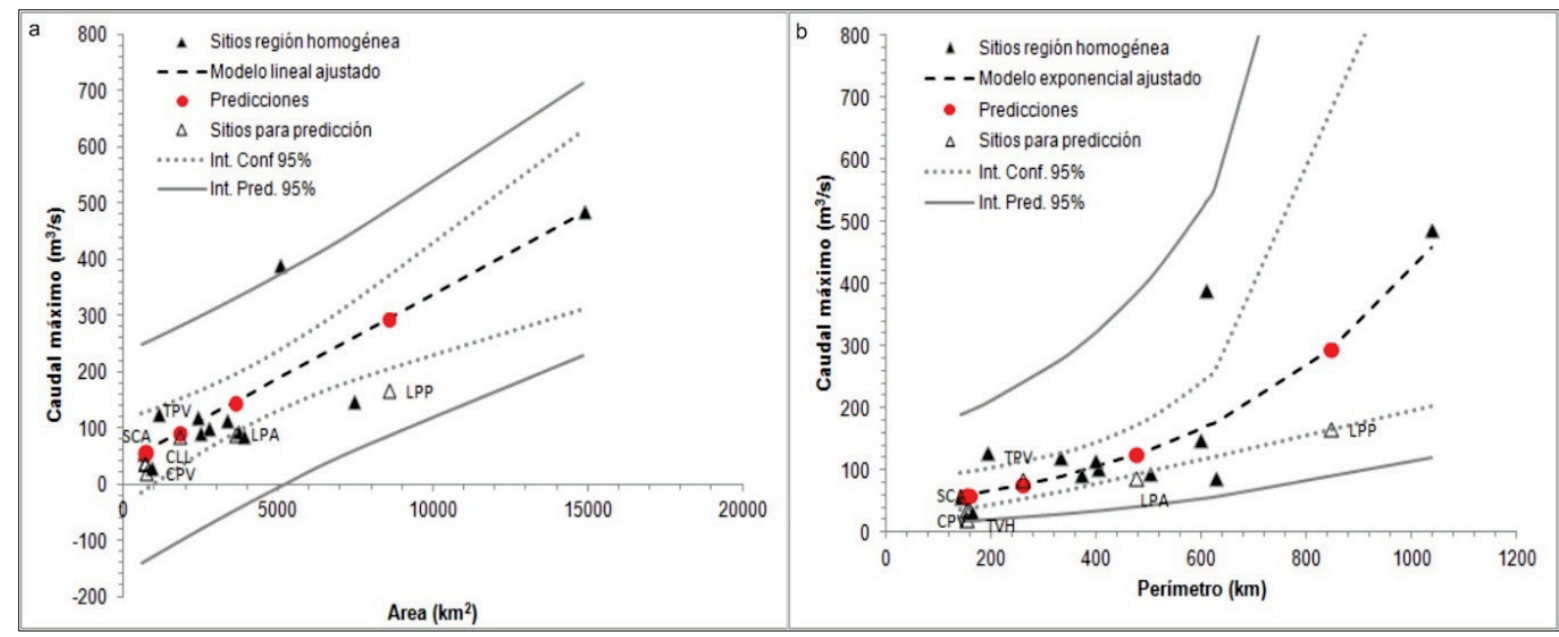

Figura 3. Modelo de regresión lineal (a) y exponencial (b) ajustado a los sitios pertenecientes a la región homogénea. Se muestran los caudales medio máximo anual para seis sitios del sistema del río Colorado y su correspondiente predicción utilizando los modelos de regionalización. 


\subsubsection{Estimación del caudal para diversos períodos de retorno}

Se estimó el caudal máximo para diferentes períodos de retorno (TR), que van desde $\mathrm{TR}=2$ años hasta TR=10.000 años. Para ello se empleó la curva de crecimiento regional adimensional, derivada del análisis de frecuencia regional (Lauro et al., 2018) y los caudales máximos medios obtenidos de las predicciones realizadas con los modelos de regresión. En la Figura 4 se muestran las estimaciones.

Se observa una sobrestimación del caudal cuya menor diferencia se obtuvo para el sitio TPV, con el valor medio del caudal estimado a partir del modelo lineal. Para el sitio LPP se obtuvo la mayor diferencia.

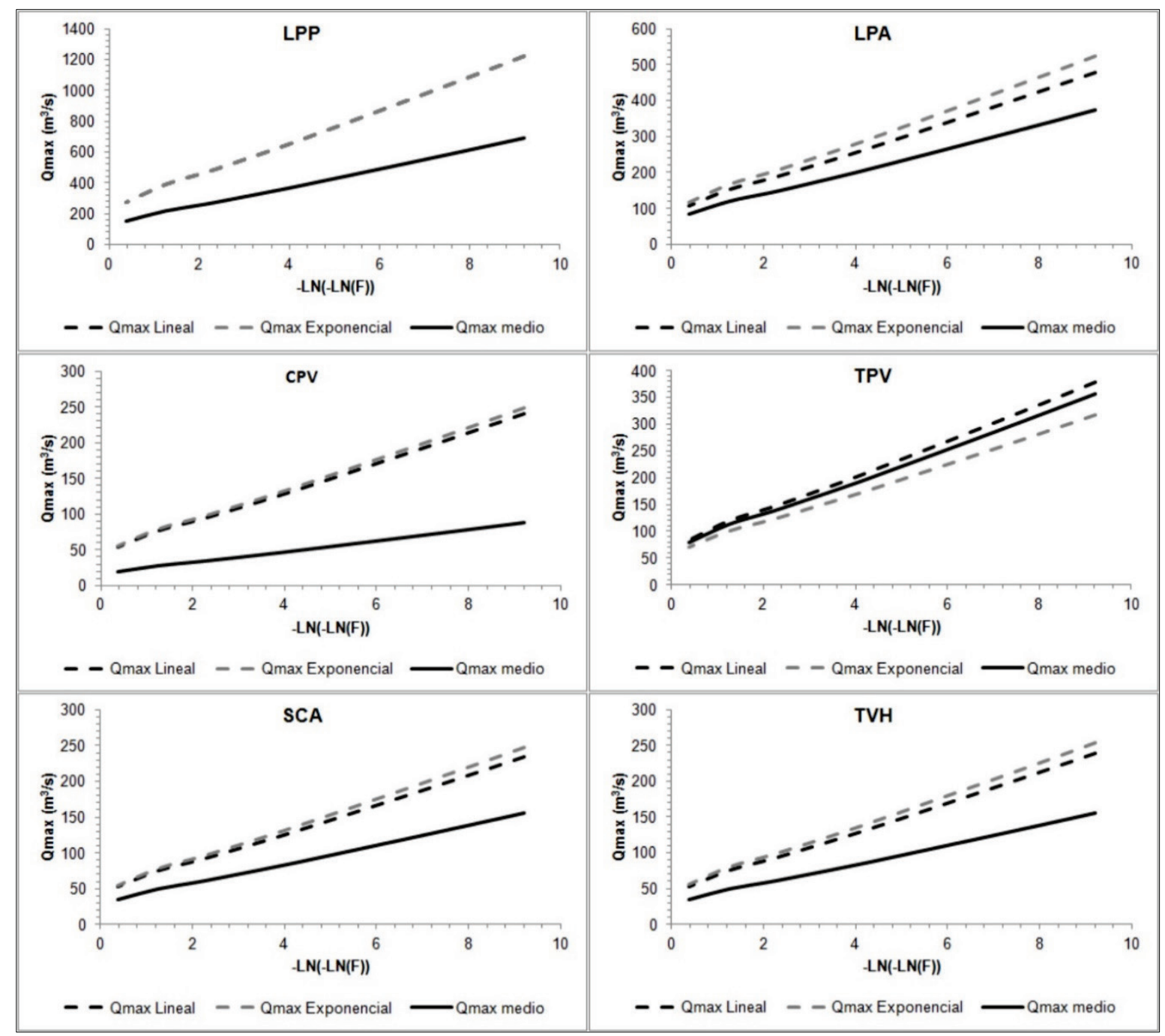

Figura 4. Estimación de caudales máximos para diferentes períodos de retorno.

\section{Conclusiones}

El presente estudio consistió en encontrar modelos regionales que estimasen los caudales máximos anuales en función de las características morfométricas y climáticas de las cuencas que integran el sistema hidrográfico del río Colorado. Estas cuencas poseen la particularidad de presentar sus nacimientos en regiones montañosas de difícil acceso, lo que dificulta el mantenimiento de las estaciones de medición y conduce a la falta de información hidrométrica. 
El bajo número de estaciones hidrométricas que integran la región homogénea condicionó los modelos de regresión a emplear, pudiendo utilizar solo una variable explicativa por regresión, para evitar la sobreparemetrización. Los modelos encontrados permiten obtener el valor de caudal máximo anual en función del área o perímetro de la cuenca. Los modelos son válidos para rangos del área y perímetro para los cuales fueron establecidos y para cuencas en la que se verifique la homogeneidad regional.

Los caudales máximos anuales regionalizados constituyen el factor de escala de la curva regional de frecuencias que fueron empleados para la estimación de caudales para distintos períodos de retorno. En líneas generales se observó una sobreestimación de los caudales, siendo esta mayor para períodos de retornos más grandes.

En futuros estudios deberían considerarse otras variables ambientales que expliquen el comportamiento de los caudales en la región. También deberá contarse con mediciones de campo que permitan verificar la predictibilidad de los modelos.

\section{Referencias}

Blöschl, G., Sivapalan, M., Wagener, T., Viglione, A., Savenije, H. 2013. Runoff prediction in ungauged basins. Synthesis across processes, places and scales. Cambridge University Press. 490 p.

Cara, L., Masiokas, M., Viale, M., Villalba, R. 2016. Análisis de la cobertura nival de la cuenca superior del río Mendoza a partir de imágenes MODIS. Meteorológica 41(1), 21-36.

Cassalho, F., Beskow, S., de Mello, C.R., de Moura, MM. 2018. Regional flood frequency analysis using Lmoments for geographically defined regions: An assessment in Brazil. Journal of Flood Risk Management 12 (2). https://doi.org/10.1111/jfr3.12453

Compagnucci, R.H., Agosta, E.A., Vargas, W.M. 2002. Climatic change and quasi-oscillations in central-west Argentina summer precipitation: main features and coherent behavior with southern African region. Climate Dynamics 18, 421-435. https://doi.org/10.1007/s003820100183

Costa, V. 2017. Correlation and regression. In: M. Naghettini (Ed.), Fundamentals of statistical hydrology (pp. 391-440). Switzerland: Springer. https://doi.org/10.1007/978-3-319-43561-9

Franchini, M., Suppo, M. 1996. Regional analysis of flow duration curves for a limestone region. Water Resources Management 10(3), 199-218. https://doi.org/10.1007/BF00424203

Garreaud, R., Fuenzalida, H. 2007. The Influence of Andes on cutoff lows: A modeling study. Monthly Weather Review 135, 1596-1613. https://doi.org/10.1175/MWR3350.1

Harris, I., Jones, P.D., Osborna, T.J., Lister, D.H. 2014. Updated high-resolution grids of monthly climatic observations-the CRU TS3.10 Dataset. International Journal of Climatology 34 (3), 623-642. https://doi.org/10.1002/joc.3711

Heras, R. 1972. Manual de hidrología. Tomo IV: hidrología de las crecidas. Servicio de Edición del Centro de Estudios Hidrográficos y Dirección General de Obras Hidráulicas.

Horton R.E. 1932. Drainage-Basin characteristics. EOS, Transactions American Geophysical Union. 13 (1), $350-$ 361. https://doi.org/10.1029/TR013i001p00350

Hosking, J., Wallis, J. 1997. Regional Frequency Analysis. An approach based on L-Moments. Cambridge University Press. U K. 240 p.

IGN. 2014. Modelo Digital de elevaciones de la República Argentina MDE-Ar (2014). http://www.ign.gob.ar/NuestrasActividades/Geodesia/ModeloDigitalElevaciones/Mapa

Kuzmin, V., Pivovarova, I., Shemanaev, K., Sokolova, D., Batyrov, A., Tran, N., Dang D. 2019. Method of Prediction of the Stream Flows in Poorly Gauged and Ungauged Basins. Journal of Ecological Engineering 20(1), 180-187. https://doi.org/10.12911/22998993/94915. 
Lauro, C., Vich, A., Moreiras, S.M. 2018. Regional flood frequency analysis in the Central Western River Basins $\left(28^{\circ}-37^{\circ} \mathrm{S}\right)$ of Argentina. River Research and Applications 34 (7), 721-733. https://doi.org/10.1002/rra.3319

Lauro, C., Vich, A., Moreiras, S.M. 2019. Streamflow variability and its relationship with climate indexes in western river basins of Argentina. Hydrological Science Journal 57 (1). http://doi.org/10.1080/02626667.2019.1594820

Linsley, R.K., Kholer, M.A., Paulus, J.L.H. 1977. Hidrología para ingenieros. McGraw-Hill Latinoamericana. Bogotá. 398 p.

Lujano, A., Quispe, J., Lujano, E., Tapia, B. 2017. Regionalización de caudales mensuales en la región hidrográfica del Tititaca, Perú. Revista Investigaciones Altoandinas 19 (2), 219-230. http://doi.org/10.18271/ria.2017.281

Lujano, A., Lujano, E., Quispe, J.P. 2016. Regionalización de caudales anuales en cuencas del altiplano peruano. Revista de Investigaciones Altoandinas 18(2), 189-194. http://doi.org/10.18271/ria.2016.199

Malekinezhad, H., Nachtnebel, H.P., Klik, A. 2011. Comparing the index-flood and multiple-regression methods using L-moments. Physics and Chemistry of the Earth 36, 54-60. https://doi.org/10.1016/j.pce.2010.07.013

Masiokas, M., Villalba, R., Luckman, B., Le Quesne, C., Aravena, J.C. 2006. Snowpack variations in the Central Andes of Argentina and Chile, 1951-2005: Large-scale atmospheric influences and implications for water resources in the region. Journal of Climate 19, 6334-6352. https://doi.org/10.1175/JCLI3969.1

Mesa, Ó.J., Vélez, J.I., Giraldo, J.D., Quevedo, D.I. 2003. Regionalización de características medias de la cuenca con aplicación en la estimación de caudales máximos. Meteorológica Colombiana 7, 141-147.

Miller, V.C. 1953. A quantitative geomorphic study of drainage basin characteristics inthe Clinch Mountain area, Virginia and Tennessee. Office of Naval Research, GeographyBranch, Project NR 389-042, Technical Report, 3, Columbia University.

Noto, L.V., La Loggia, G. 2009. Use of L-moments approach for regional flood frequency analysis in Sicily, Italy. Water Resources Management 23, 2207-2229. https://doi.org/10.1007/s11269-008-9378-x.

Poveda, G., Vélez J., Mesa O., Ceballos L., Zuluaga M. y Hoyos, C. 2002. Estimación de caudales mínimos para Colombia mediante regionalización y aplicación de la curva de recesión de caudales. Meteorológica Colombiana 6, 73-80.

Rusticucci, M., Zazulie, N., Raga G.B. 2014. Regional winter climate of the southern central Andes: Assessing the performance of ERA-Interim for climate studies. Journal of Geophysical Research Atmospheres 119, 8568-8582. https://doi.org/10.1002/2013JD021167

Schumm, S.A. 1956. Evolution of drainage systems and slopes in badlands at Perth Amboy, New Jersey. GSA Bulletin, 67 (5), 597-646. https://doi.org/10.1130/0016-7606(1956)67[597:EODSAS]2.0.CO;2

Seluchi, M.E., Garreaud, R.D., Norte, F.A., Saulo, A.C. 2006. Influence of the subtropical Andes on baroclinic disturbances: A cold front case study. Monthly Weather Review 134, 3317-3335. https://doi.org/10.1175/MWR3247.1

Song, J., Xia, J., Zhang, L., Wang, Z., Wan, H., She, D. 2016. Streamflow prediction in ungauged basins by regressive regionalization: a case study in Huai River Basin, China. Hydrology Research 47 (5). https://doi.org/10.2166/nh.2015.155

Teklu, T.H., Knut, A. 2017. Regional flood frequency analysis and prediction in ungauged basins including estimation of major uncertainties formid-Norway. Journal of Hydrology: Regional Studies 9, 104-126.

Témez, J.R. 1987. Cálculo hidrometeorológico de caudales máximos en pequeñas cuencas naturales. Dirección General de Carreteras, MOPU. 124 pp.

Tucci, C.E.M. 1997. Regionalização de vazões. $2^{\circ}$ ed. En: Hidrologia: Ciência e Aplicação. Tucci, C (Org). ABRH, Porto Alegre, p.573-620.

Viale, M., Bianchi, E., Cara, L., Ruiz, L., Villalba, R., Pitte, P., Masiokas, M., Rivera, J., Zalazar., L. 2019. Contrasting climates al both sides of the Andes in Argentina and Chile. Frontiers in Environmental Science 7 (69). http://doi.org/10.3389/fenvs.2019.00069. 
Vich, A.I.J., Norte, F.A., Lauro, C. 2014. Análisis regional de frecuencias de caudales de ríos pertenecientes a cuencas con nacientes en la Cordillera de los Andes. Meteorológica 39 (1), 3-26.

Vich, A.I.J. Norte, F. 2012. Análisis de frecuencias regional en cuencas con nacientes en la cordillera de Los Andes de Argentina. XI Congreso Argentino de Meteorología. CongreMet XI. Mendoza junio de 2012.

Vich, A.I.J. 1999. Aguas continentales. Formas y procesos. Manual de Aplicaciones prácticas. Mendoza. ZETA Editores.

Viglione, A., Claps P., Laio, F. 2007. Mean annual runoff estimation in North-Western Italy, In: G. Water resources assessment and management under water scarcity scenarios. La Loggia (Ed.). CDSU Publ. Milano. 


\title{
TREES AS SENTINELS OF METALLIC POLLUTION INDUCED BY MINING ALONG THE ODIEL RIVER (SOUTHERN IBERIAN PENINSULA)
}

\author{
ANNICK DELAPIERRE ${ }^{1,2,4}$, JUAN A. BALLESTEROS-CÁNOVAS ${ }^{1,2^{*} \text {, }}$ \\ JORGE BUZZI MARCOS ${ }^{3}$, VERA I. SLAVEYKOVA ${ }^{2,4}$, MARKUS STOFFEL ${ }^{1,2,4}$
}

\author{
${ }^{1}$ Department of Earth Sciences, University of Geneva, Switzerland. \\ ${ }^{2}$ Institute for Environmental Sciences, University of Geneva, Switzerland. \\ ${ }^{3}$ Department of Prospective Geoscientific, Spanish Geological Service, Spain.
}

${ }^{4}$ Department F.A. Forel for Environmental and Aquatic Sciences, University of Geneva, Switzerland.

\begin{abstract}
Mining activity is often responsible for the drainage of acid or metal-enriched waters to fluvial systems. The release of metals is especially disturbing due to the toxicity and persistence of these products and their accumulation in the biosphere. Hence, a systematic detection and delimitation of highly polluted floodplains and linkages between pollution and high-flow stages would likely assist the improvement of land management and ease the design of mitigation or rehabilitation measures. Here we test how trees growing in different geomorphic positions along a fluvial system uptake metal during floods and how these uptakes can be documented "a posteriori". To this end, we apply dendrogeochemical analyses to twenty Pinus pinaster Ait. trees growing on the banks of Odiel River (south-western Spain) as well as to five reference trees growing outside the river channel. In the field, trees were sampled with a large-diameter $(1 \mathrm{~cm})$ increment borer. In the lab, tree-ring series were dendrochronologically cross dated and separated into 5-yr blocks, so that wood blocks contained the dates of major floods. Then, Inductively Coupled Plasma Mass Spectrometry (ICPM) was employed to evaluate toxic metal concentrations in trees. Results point to clear correlations between the accumulation of toxic metals and the geomorphic position of trees within the fluvial network. We show that morphological units along a river exert control on toxic metal concentrations in trees, with uptake being much higher in trees located on meander cut banks than in trees growing on point-bar structures. Besides, we detect chemical signatures in trees located farthest away from the main river channel after the largest floods, but not in the aftermath of smaller events. We conclude that tree position is the single-most important determinant for metallic pollution in an environment controlled by fluvial processes, but also find that more studies are still needed to determine linkages with individual floods and interactions of metal uptake in roots via the water table in the river corridor.
\end{abstract}

\section{Árboles como indicadores de la contaminación metálica inducida por la minería a lo largo del río Odiel (sur de la Península Ibérica)}

RESUMEN. La actividad minera es responsable de vertidos de aguas enriquecidas con ácidos y metales. La liberación de metales es especialmente preocupante debido a su toxicidad y persistencia en los ecosistemas. La detección y delimitación sistemática de zonas contaminadas en la llanura de inundación puede contribuir a una mejor gestión de dichas zonas en relación con el ciclo hidrológico. En este trabajo se analiza si los árboles, que crecen en diferentes posiciones geomorfológicas, registran la absorción de metales durante eventos de inundaciones. Para ello, se aplicaron análisis dendroquímicos a veinticinco ejemplares de la especie Pinus pinaster Ait. que crecen en las orillas del río Odiel (suroeste de España). También se analizaron cinco árboles de referencia 
que crecen alejados del cauce del río. Los árboles fueron muestreados con una barrena de Pressler (diámetro $1 \mathrm{~cm}$ ) y las muestras fueron dendrocronológicamente fechadas, aislando bloques de 5 años que coincidían con eventos de inundaciones. Las concentraciones de metales tóxicos en muestras de árboles se midieron en dichos bloques mediante espectrometría de masas de plasma (ICPM). Los resultados revelan una clara correlación entre la acumulación de metales tóxicos y la ubicación geomorfológica de los árboles. La absorción de elementos metálicos fue mucho mayor en los árboles ubicados en el banco de orilla que en los árboles que crecen en las barras de sedimentos. Por otro lado, en los árboles más alejados del canal principal del río solo detectamos señales químicas después de las mayores inundaciones, pero no después de eventos más pequeños. Concluimos que la posición del árbol condiciona la señal dendroquímica asociada a procesos fluviales, aunque todavía se requieren más estudios para discernir los vínculos con eventos de inundaciones.

Key words: Tree ring, flood, fluvial geomorphology, dendrochemistry, heavy metal, pollution, Odiel River.

Palabras clave: Anillos de crecimiento, inundaciones, geomorfología fluvial, dendroquímica, elementos metálicos, contaminación, río Odiel.

Received: 15 June 2020

Accepted: 20 October 2020

* Corresponding author: J. A. Ballesteros-Canovas, Department of Earth Sciences, University of Geneva, Switzerland. E-mail: juan.ballesteros@unige.ch

\section{Introduction}

The incorporation of metallic pollutants in the hydrological cycle poses major environmental problems, with very direct impacts on living organism and human population worldwide (Foster et al., 1996; Ali et al., 2019). Anthropogenic activities, such as mining, have been hold responsible for the drainage of acid and metal-enriched waters, thereby contaminating river ecosystem downstream of their source as a result of the weathering of exposed minerals or tailing dam failures (Robles-Arenas et al., 2006; Rico et al., 2008). The release of metallic pollutants is particularly disturbing as they are typically highly toxic, persist, and as they have the capacity to accumulate in organisms and thus will ultimately end up in the food chain (Tchounwou et al., 2012). Even if multiple mining activities can be traced back easily into Roman times in the Old World (Hong et al., 1996; Nriagu et al., 1996; Ballesteros-Canovas et al., 2017), the recent industrialization has prompted a decentralized demand of metals for different purposes, with an unprecedented increase in legal and illegal mining activities worldwide (Banchirigah, 2008; Obeng et al., 2019).

In fluvial ecosystems, tracing metallic pollutants is essential for a proper management of sediments containing heavy metals and to limit harmful effects farther downstream in the river system (Hürkamp et al., 2009). One can reasonably anticipate that over the decades to come, mining activities will likely increase due to the ever-increasing demand of metals and rare earth elements. In addition, climate change has been demonstrated to enhance the hydrological cycle, which in turn could lead to more floods and again increase the spread of pollutants in river systems (Foulds et al., 2014). To adequately cope with these environmental issues of rising concern, it is essential to adopt adaption strategies at various levels: At the European level, the European Commission has issued two directives (2000/60/CE and 2006/11/CE) defining the obligation to evaluate and monitor the chemical status of water bodies in all member states. National governments have therefore put protocols in place through which water or sediment samples can be collected and analyzed ad hoc (Islam et al., 2015) with spectrophotometry, chromatography, or mass spectrometry (Fifield and Haines, 2000). These methods 
allow replicability and consistency of analyses over time, but they remain limited to the time period covered by sampling.

Long-term chemical records could instead contribute to a better identification of long-term trends in chemical concentrations and their linkages with environmental changes (Corella et al., 2017; Bing et al., 2016), but data does not readily exist so far. In this longer-term context, tree-ring series could become an interesting alternative as they can extend the time window covered by analysis farther back in time and thereby offer valuable insight into the temporal evolution of chemical compounds over decades to centuries (Cutter and Guyette, 1993). In addition to their longevity, the seasonal growth and dormancy of trees would also allow analysis with intra-annual resolution. Tree-ring series can also help in the creation of chronologies of environmental processes (Schweingruber, 2007), and thus assist determination of changes in chemical composition of soils and trees in the past (Smith and Shortle, 1996; Kabata and Pendias, 2001). Dendrochemistry deals with the analysis of trace elements in tree-ring series by assuming that a tree-ring represents the environmental chemistry of the year in which it was formed (Wright et al., 2014). As such, the discipline has lately evolved into a sub-branch of environmental forensic research (Balouet et al., 2009; Lageard et al., 2008; Stoffel et al., 2020) and now contributes to the understanding of physiological processes controlling uptake, transport and sequestration of chemical elements in the secondary xylem (Cutter and Guyette, 1993).

The potential of dendrochemistry in tracing historic pollution has gained in interest, primarily also in catchments where human-induced mining has led to contamination of rivers. Pioneering studies in the field go back to the 1970s when Sheppard and Funk (1975) found that Pinus ponderosa trees growing next to Spokane River (Idaho) can be employed to monitor changes in metal ion concentrations. More recently, Yanosky et al. (1995) analyzed chloride concentrations in Taxodium distichum growing on the Cape Fear River freshwater estuary to date the onset of saltwater intrusions induced by ongoing sea level rise. Saint-Laurent et al. (2010) analyzed tree-ring records from Fraxinus sp. growing along the Massawippi and Saint-François rivers (Quebec) contamination of trees with heavy metals released by industry. Likewise, tree rings have been used to record groundwater contamination with chlorinated solvents (Yanosky and Vroblesky, 1992; Balouet et al., 2009), but also to identify metal concentrations in water downstream of mines (Witte et al., 2004). The ability of tree rings to record pollution depends on the nature (or type) of pollutant and the exposure of a tree to the source of pollution (Cutter and Guyette, 1993, Rodríguez-Martin et al., 2018). Besides, some trace elements have been demonstrated to behave like microelements in such a way that they can translocate within the tree and across ring boundaries, therefore preventing dendrochemical analyses at annual resolution (Hagemeyer, 1993). Despite these possible limitations, the analysis of the chemical signatures in tree rings has the potential to improve our understanding of past pollution trends in fluvial ecosystems, even more so as trees are often ubiquitously present in fluvial corridors and have discernible tree rings, which also enables them to record fluvial processes and floods (Ballesteros-Canovas et al., 2015).

Here we test whether the accumulation of metallic pollutants in trees growing along a fluvial channel of the Odiel River is driven by their connectivity to the channel. We do so by analyzing trees growing at different positions with respect to the channel and that are affected by different magnitudes of floods. The study is based on the following premises: (i) temporal variations in metal concentrations in tree rings are in line with the historical development of mining activities upstream of the site where trees have been sampled; (ii) metals released into the river through acid drainage are absorbed by pine trees growing in the flood plain and contained in the rings of trees growing at locations where an interaction with the waters of Odiel River occurs; (iii) floods in a river impacted by mining drainage can be defined according to the concentrations of metals found in the tree rings (St. George et al., 2006). We assume that the application of dendrochemical analysis in trees affected by floods in the highly anthropogenic Odiel catchment will contribute to a better understanding of linkages between future floods and floodplain pollution. 


\section{Study site}

\subsection{Geomorphic description}

Odiel River is located in the Iberian Pyrite Belt (IPB), in the Province of Huelva, southwestern Spain (Fig. 1). The watershed has an area is $2333 \mathrm{~km}^{2}$ and the river flows on permeable bedrock over a distance of $140 \mathrm{~km}$ (Buzzi Marcos, 2012), i.e. between the Sierra de Aracena and the estuary of the Ría de Huelva (a UNESCO Biosphere Reserve, listed since 1983). The Odiel Basin contains three geological domains: in the uppermost part of the watershed, plutonic and metamorphic rocks (i.e. sericite schists, granites, gneiss, quartzites, marbles, and tuff) of the Upper Precambrian to Devonian can be found. In its middle part, bedrock is formed by volcanic and sedimentary rocks of the Iberian Pyrite Belt, locally known as the "PQ Group" (phyllite and quartzite group), the "Volcanic-Sedimentary Complex" (i.e. shales, greywackes, acid to basic volcanic and volcanoclastic rocks), and the "Culm Group" composed of flysch-like sequences of shales and greywackes belonging to the Upper Devonian to Middle Carboniferous. The lowest and southernmost portion of the basin is composed of siliciclastic materials of Miocene age (i.e. sands, silts, and clays) (Silva et al., 1990).

The volcanic-sedimentary complex holds enormous amounts of massive sulphides, forming one of the biggest polymetallic sulphide mining districts in the world (Leistel et al., 1998). These sulphides have been exploited for centuries, and more than a hundred mining sites can be found along Odiel River, corresponding to about 200 million $\mathrm{m}^{3}$ of sulphide-rich mine waste (Pérez López et al., 2011). Even if mining activities have ceased almost completely today, surficial weathering of pyrite-rich minerals still generates a continuous flow of acid mine drainage (AMD) into Odiel River. This drainage is responsible for low pH values and an increase of dissolved metal concentrations (Fig. 1B) (Borrego, 1992; Braungardt et al., 1998; Buzzi Marcos, 2012).

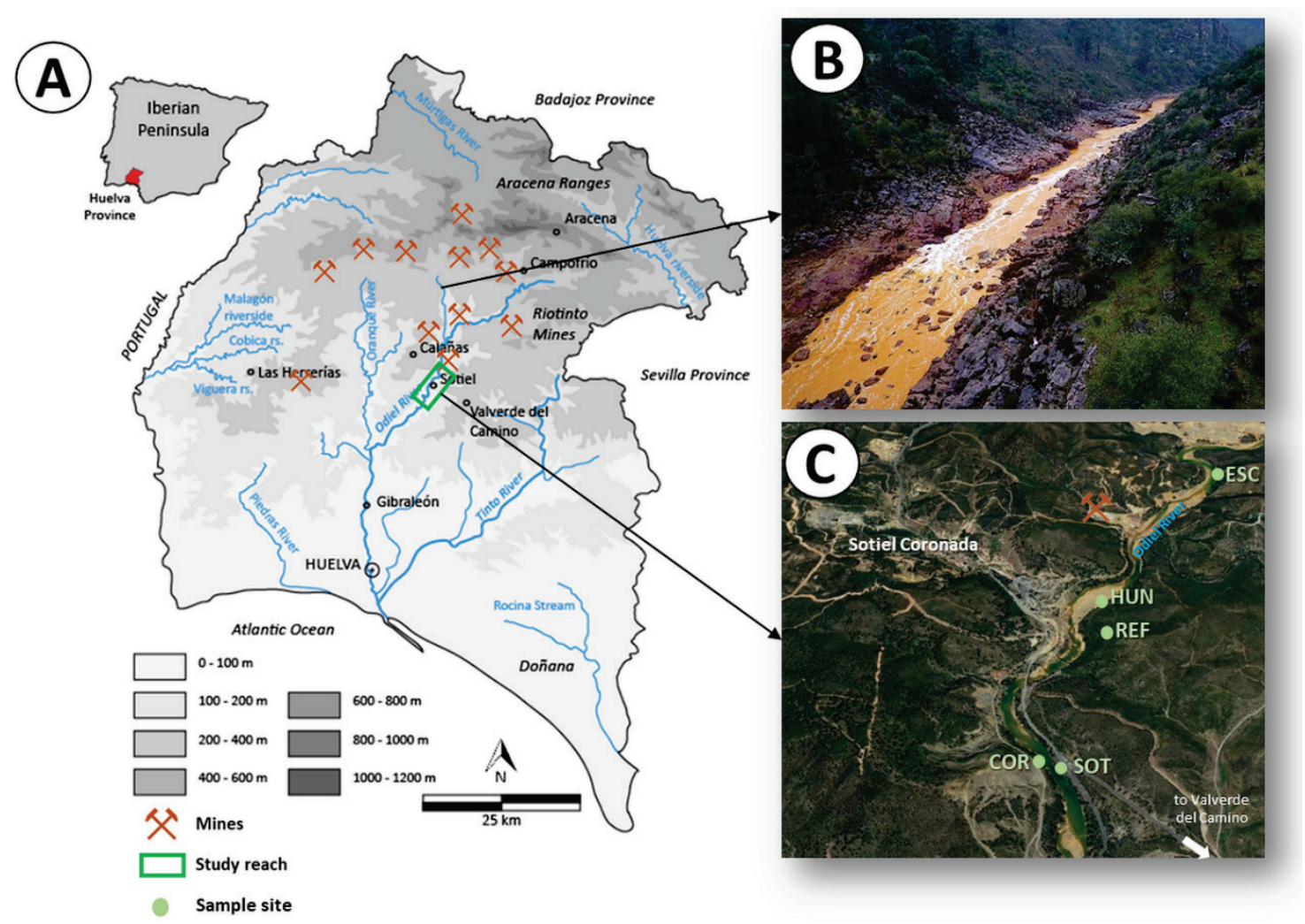

Figure 1. A) Location of the river reach analyzed along Odiel River. B) Metal-enriched waters in the Odiel River at Puente de los Cinco Ojos bridge. C) Localization of the five sampling sites along Odiel River (source: GoogleEarth). 
The Odiel River flows in a channel with a changing floodplain lithology and is characterized by low sinuosity but considerable lateral mobility, thereby favoring the development of sandbars along its course, as typical for bedload transport stream. The channel often diverts into different paths, forming braided side channels that are separated from each other by sandy and gravelly bars. The flow path is mostly linear, with smaller changes occurring where the Odiel River crosses rocks of differing competence. Flow is also controlled by walls from historic watermills, causing water diversion while favoring the retention of sediment. In its lower part, the Odiel River forms an estuary in which saltwater fluxes are controlled by tidal processes.

Climate in the study area is Mediterranean. Annual average precipitation is $617 \mathrm{~mm}$, whereas mean annual temperature is $18.5^{\circ} \mathrm{C}$, oscillating from below $0^{\circ} \mathrm{C}$ in winter to more than $40^{\circ} \mathrm{C}$ in summer. Vegetation is typical of Mediterranean sclerophyllous forests with several oak (Quercus suber, Q. ilex, Q. faginea) and conifer (Pinus pinaster and P. pinea) species accompanied by Mediterranean shrubs. In the Odiel catchments, allochthonous species are present as well, in particular eucalypts (Eucalyptus globulus and E. rostrata) (Cánovas, 2009). The riparian zone is characterized by acidophilic algae typical for mining environments (Euglena mutabilis and Klebsormidium sp., Grande et al., 2016).

River flow has been measured close to the estuary (1980-2011), at Gibraleón (lat: 37.3 N, long: $\left.-6.97^{\circ} \mathrm{W}\right)$, but the time series is highly fragmented with important data gaps. Highest discharge was typically observed in December and January, corresponding to the passage of Atlantic storms, whereas low runoff wass commonly found during dry summers. The rocky substrate of the Odiel catchment makes the river very reactive to any changes in precipitation: In January 1996 and December 2009, floods were triggered after intense rainfall over the study region, as reported by Morales et al. (2005) and Galván (2012); these events were also recorded partly by the gauge records. In the context of this study, we selected these two floods for the evaluation of their effects on metal concentrations in tree rings.

\subsection{Mining history in the Odiel catchment}

Archaeological evidence allows tracking back incipient mining activity for more than 4500 years (i.e. to the Copper Age; Grande et al., 2016). Since the Bronze Age and until the $6^{\text {th }}$ century BCE, Tartessians, Phoenicians, and Carthaginians exploited the mines in the Sierra de Aracena to extract lead, silver, and copper (Galván, 2012). Since the arrival of the Romans in the $3^{\text {rd }}$ century BCE, mining of copper, silver, and iron gained momentum as new technologies evolved, leaving several million tons of slag after the melting of minerals at the mining sites (Galván and Olías, 2015; Grande et al., 2016). Activity gradually slowed down thereafter to disappear completely during the $5^{\text {th }}$ century CE. During the Middle Ages, iron mining resumed in the area, especially during the Visigoths $\left(5^{\text {th }}\right.$ to $8^{\text {th }}$ centuries) and Arabic reigns ( $8^{\text {th }}$ to $15^{\text {th }}$ centuries). At the end of the $18^{\text {th }}$ century, mine exploitation gained again in importance as a result of relevant technological advances; it was fueled further by Industrial Revolution in the $19^{\text {th }}$ century (Grande et al., 2016). The demand for raw materials during this time was in fact important: copper was used for electrical industry, whereas sulphur was relevant to extract sulfuric acid used in chemical industry. Ever since, the revival of large-scale mining, supported by the appearance of the railway industry, triggered the export of raw materials from the IPB to most European countries (López et al., 2008; Grande et al., 2016). During the second half of the $20^{\text {th }}$ century, exploitation benefited from the reconstruction of infrastructure after the end of World War II. However, with the gradual but steady decline of copper prices on the market, environmental regulations of the European Union and the depletion of raw materials in various mines, activity slowed down again substantially and gradually disappeared from the area in the 1990s (Galván, 2012).

The mines remained abandoned for almost twenty years, but recently, international companies have explored possibilities to resume exploitation of certain historical mines in the IPB, and particularly in the Odiel Basin (Galván, 2012). These re-openings take place in a normative context that is quite 
different from that in place during the $20^{\text {th }}$ century, and companies are now subject to legal obligations and the realization of Environmental Impact Assessments, as prescribed by the National Environmental Assessment Act (Law 21/2013), in application of the European Directive 2014/52/EU.

\section{Material and methods}

\subsection{Sampling and tree-ring dating}

Tree-ring sampling along Odiel River was realized in early 2018 in the locality of Sotiel (Huelva, Spain). To test the influence of tree position on metal uptake, twenty trees were sampled at different positions within the river reach, i.e. at sites characterized by a straight channel and at sites characterized by meanders. Figure 2 provides insights into the sampling strategy and shows the sites that have been sampled: Trees from SOR $\left(37.58^{\circ},-6.84^{\circ}\right)$ and $\operatorname{COR}\left(37.59^{\circ},-6.84^{\circ}\right)$ grow in straight channel reaches, whereas those selected at $\operatorname{HUN}\left(37.59^{\circ},-6.84^{\circ}\right)$ and $\operatorname{ESC}\left(37.60^{\circ},-6.83^{\circ}\right)$ are located on point bars and cut banks, respectively, in a reach characterized by meanders.
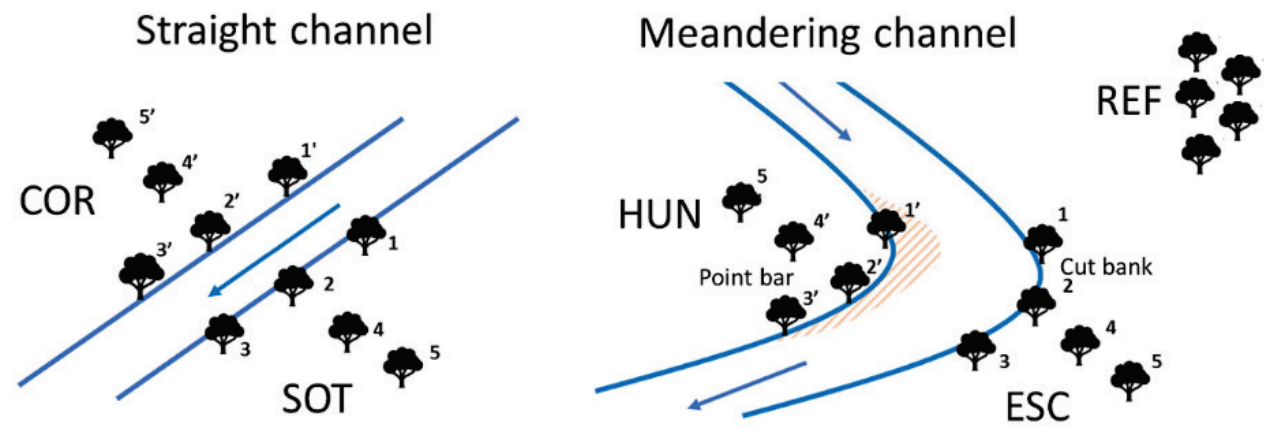

Figure 2. Trees sampled at different distances from the main course of the Odiel river at the four sites investigated: SOT, COR, HUN, and ESC. At the reference site (REF), tree selection was random.

In addition, we sampled five trees growing in the same area, but without any connection with the river, so as to use them as reference trees representing undisturbed growth (REF, $37.59^{\circ},-6.84^{\circ}$ ). Sampling was performed with increment borers of differing size. We used a 5-mm increment borer (i.e. inner diameter) to extract cores from the reference trees and to perform standard tree-ring dating. We also used a 10-mm increment borer to get increment cores for chemical analysis. To avoid contamination during sampling, the increment borers were cleaned with a metal-free oil before alcohol $\left(\right.$ at $\left.70^{\circ}\right)$ was used for further cleaning. Once the samples extracted, cores were stored plastic tubes filled with milliQ water and frozen at $-20^{\circ} \mathrm{C}$ until they were analyzed in the lab. In the field, additional information was recorded for each tree analyzed, such as tree diameter and its exact location (GPS).

In the lab, all samples were mounted on wooden supports, sanded with increasingly finer grit and then polished following standard dendrochronological procedures (Ballesteros-Canovas et al., 2015). Then, we carried out a visual reconnaissance and performed skeleton plots to evaluate the representativity of samples. Afterwards, tree-ring widths were measured with a Lintab ${ }^{\mathrm{TM}}$ measurement device connected to TSAP-Win software. We employed the cross-dating index (CDI) - a combination of the Gleichläufigkeit index (GLK) and Student's t-value test - to evaluate reliability of the cross dating between annual tree-ring chronologies (Rinn, 1996). 


\subsection{Sample preparation and analysis by inductively coupled plasma mass spectrometry (ICP-} $M S)$

The cores sampled with the 10-mm increment borer were prepared for chemical analysis and to quantify the evolution of metal uptake over time. To maximize the material available from each sample used for ICP-MS, we cut tree rings into blocks of 5 years: 1978-82, 1983-87, 1988-92, 1993-97, 19982002, 2003-07, 2008-12, and 2013-17. In addition, we assessed the impacts of floods by preparing 4year blocks prior and after the main floods in 1996 and 2009. Analysis of metal concentrations was adapted from Watmough and Hutchinson (2003): Samples were weighed after drying at $60^{\circ} \mathrm{C}$ for $72 \mathrm{~h}$, before they were calcined at $400^{\circ} \mathrm{C}$ for $6 \mathrm{~h}$. The resulting ashes were then weighed again and dissolved in $1 \mathrm{ml}$ of nitric acid supra-pure $\left(65 \% \mathrm{HNO}_{3}\right)$ for $24 \mathrm{~h}$. Ashes were then dissolved in $9 \mathrm{ml}$ of ultrapure water. Metal concentrations were analyzed by inductively coupled plasma mass spectrometry (ICP-MS, model 7700x, Agilent technologies, Morges, Switzerland) and multi-element calibration with certified standards (Certipur ICP multi-element standard VI Reference Material, Merck KGaA). Accuracy of the measurements was checked by BCR ${ }^{\circledR}-482$ Certified Reference Material (Lichen).

\subsection{Statistical analysis}

Results from the ICP-MS analyses were analyzed statistically using visual and descriptive approaches, including the analysis of linear trends and matrix correlation at $95 \%$ least significant difference (LSD), with the aim to identify linkages between metal uptake as a function of the vertical/horizontal distance of trees from the main channel of Odiel River. We also employed principal component analysis (PCA) to distinguish patterns associated with the analyzed sites. Finally, to determine whether metal uptake was statistically significant after the main floods events, we carried out non-parametric Wilcoxon tests (Sprent and Smeeton, 2001) at 95\% LSD.

\section{Results and discussion}

\subsection{Temporal evolution in the uptake of metallic pollutant in trees}

Average age of analyzed trees was $49 \pm 11$ years. The reference chronology and the trees sampled along the Odiel River cross-dated successfully with an average Gleichläufigkeit (GLK) of 65 and a cross-dating index (CDI) of 20. The PCA points to the presence of two main groups of elements present in samples that account for $58 \%$ of the variance (Fig. 3A): the first group (37.8\%) is formed by $\mathrm{Ag}, \mathrm{Cd}$, $\mathrm{Co}, \mathrm{Mn}$, and $\mathrm{Zn}$ whereas the second group (20.2\%) contains $\mathrm{Al}, \mathrm{Cu}, \mathrm{Cr}, \mathrm{Fe}, \mathrm{Mo}$, Ni and $\mathrm{V}$. In the first group, $\mathrm{Mn}, \mathrm{Cd}$ and Co are highly correlated and contribute significantly to the PCA, whereas the contribution of $\mathrm{Zn}$ and $\mathrm{Ag}$ is more limited. In the second group, Mo and Fe are overlapping, confirming a strong correlation between these two elements. $\mathrm{Cr}$, Ni and V show similar distributions, whereas the contribution of $\mathrm{Cu}$ is significantly lower if compared to other elements in the group. Interestingly, $\mathrm{Pb}$ does not contribute to any factor and appears isolated from the other elements, suggesting that its presence in trees is related to causes other than river contamination. The first group of elements is characterized by a centripetal pattern, with higher concentrations towards older rings, whereas the second group shows a centrifugal pattern, with higher concentrations towards the youngest rings (Fig. S.1). Fig. 3B shows the temporal evolution of average metal accumulation at each of the sites, including the reference site. Overall, $\mathrm{Al}, \mathrm{Ni}$, and $\mathrm{Cu}$ show the smallest changes over time at all sites and generally remain below the $25^{\text {th }}$ percentile (i.e. changes $<28 \%$ ). By contrast, $\mathrm{Zn}, \mathrm{Ag}$ or $\mathrm{Cd}$ show much larger changes over time, exceeding the $75^{\text {th }}$ percentile on average $(>42 \%)$.

Several environmental and physiological factors can explain the distribution patterns of metallic pollutants we observe in the analyzed trees. It is possible that the concentration of elements at the site is controlled by translocation processes between the sapwood and the heartwood (Meerts 2002; Scharnweber et al., 2016). Although analysis was constrained mostly to sapwood in our study, we 
observed an increase in the concentration of metals toward the limit between the sapwood and the heartwood (Fig. S.2), particularly for $\mathrm{Cr}, \mathrm{Mn}, \mathrm{Fe}, \mathrm{Ni}, \mathrm{Mo}, \mathrm{Ag}$, and $\mathrm{Pb}$, and to a lesser degree for $\mathrm{Al}, \mathrm{V}$, $\mathrm{Co}, \mathrm{Cu}, \mathrm{Zn}$, and $\mathrm{Cd}$ as well. Similar trends in metal concentration between the sap- and heartwood were described previously (Watmough and Hutchinson, 2002; Hagemeyer and Schäfer, 1995; Prohaska et al., 1998) and interpreted as a detoxification process induced by the storing of phytotoxic elements in dead heartwood cells (Donnelly et al., 1990). Likewise, correlations between elements could also be explained by physiological factors as $\mathrm{Fe}, \mathrm{Zn}$ and Mo as they are involved in different physiological processes (Nobel, 1999), In particular, Mo will depend on Fe for its assimilation, as well as on $\mathrm{Cr}$ and V (Hänsch and Mendel, 2009). Interestingly, Fe is generally considered as an element with low mobility in xylem (Prohaska et al., 1998), which would imply that the tendencies observed in trees would indeed depend on environmental factors, probably linked to the acidification of soils (Kabata and Pendias, 2001). Moreover, we find that $\mathrm{Zn}$ shows an irregular pattern, in agreement with observations made in previous studies focusing on Pinus strobus (Doucet, 2011), Acer pseudoplatanus (Watmough and Hutchinson, 1996) or Quercus robur (Hagemeyer, 1995). The fact that $\mathrm{Zn}$ is bioavailable to plants in its soluble form suggests that these variations could also be linked to the acid drainage of Odiel River. This assumption can be supported with results from the reference trees (REF) for which we could not find comparable variations in $\mathrm{Zn}$ or Co concentrations.

Results thus clearly evidence that mining activities upstream of the study sites are the main source of metallic pollution recorded in the analyzed trees. Therefore, and even if the most intense period of mine exploitation ceased in the 1990s, activity has resumed recently with a production of 4.6 million tons of $\mathrm{Co}$ and $\mathrm{Zn}$ annually. At the study site, metallic sulphide weathering occurs rapidly when these minerals are exposed to air, thereby releasing sulphates into running water bodies and lowering their $\mathrm{pH}$ values (Nordstrom and Alpers, 1999). As a consequence, the pyrite weathering products precipitate on the banks of the Odiel River in the form of complex sulphate crusts and efflorescence, and they change their composition during the meteorological year, following a well-known mineralogical evolution controlled by climatic and geomorphic factors (Buzzi Marcos, 2012). The mineral uptake that we identified in trees is in line with results from the chemical signature of sediments analyzed with spectroscopy (using hyperspectral sensors), and both processes are causally related to mine waste deposits. Analysis of hyperspectral time series can assist in the detection of changes in precipitated crusts and efflorescence along the flow path of Odiel River, thereby evidencing that the mine at Sotiel is indeed a notable source of acid mine drainage to the Odiel River (Buzzi Marcos, 2012; Buzzi et al., 2014; Riaza et al., 2017). 


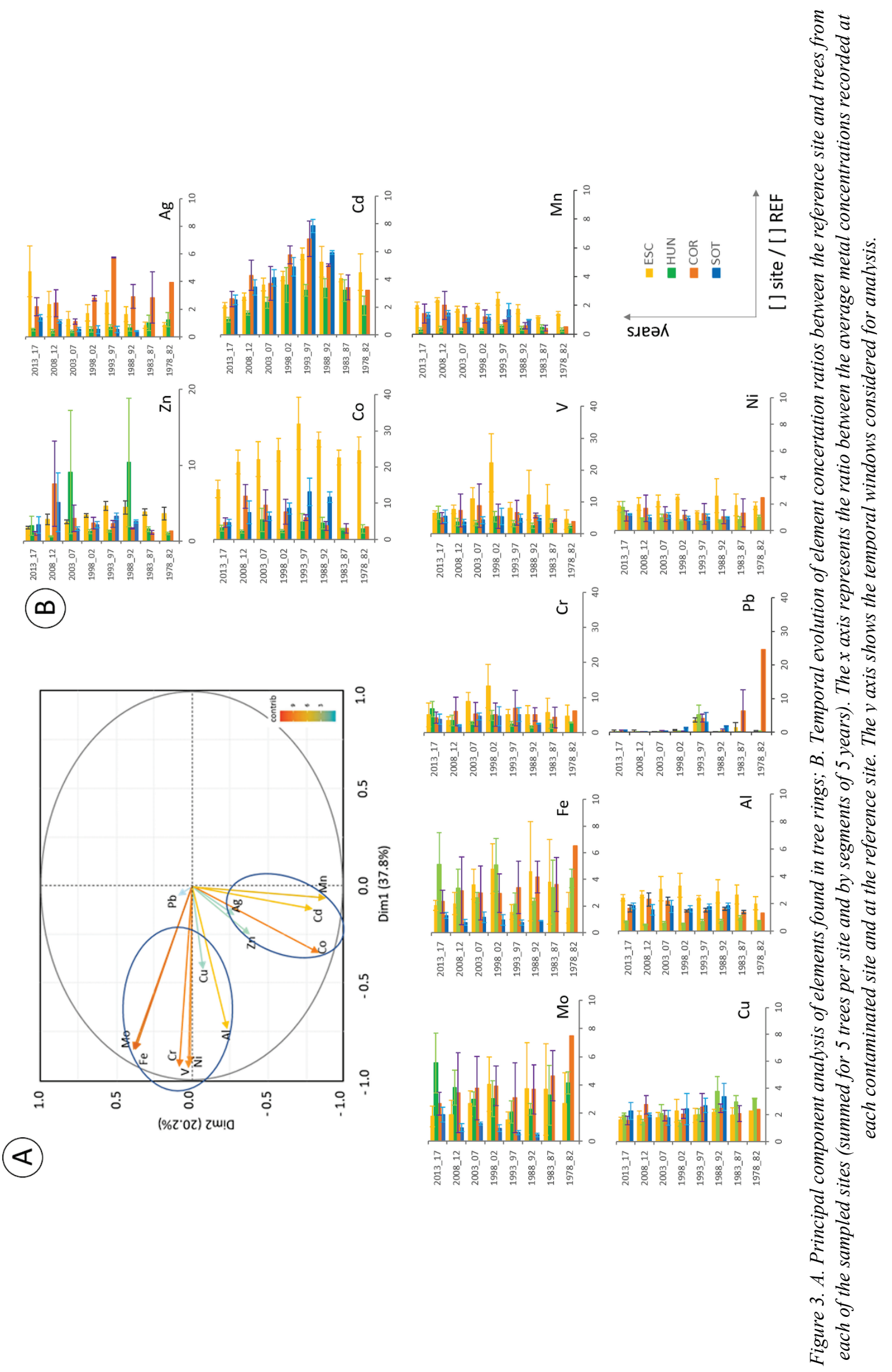




\subsection{Effect of the geomorphic position of trees on metal accumulation in trees}

Figure 4A shows significantly negative $(p<0.05)$ correlations between metal concentrations and the position of sampled trees, such as their distance to and height above the river channel: the greater the vertical and horizontal distances from the river, the lower the metal concentrations measured in the tree-ring series. The graph also shows that most elements are significantly positively correlated among each other, especially in the case of $\mathrm{Fe}, \mathrm{Cr}, \mathrm{V}, \mathrm{Ni}$, and $\mathrm{Mo}(\mathrm{r}>0.5)$. Mn is the exception to the rule and shows a negative correlation with $\mathrm{Fe}$ and $\mathrm{Mo}-$ as is $\mathrm{Pb}$ because it did not correlate with any other variable.
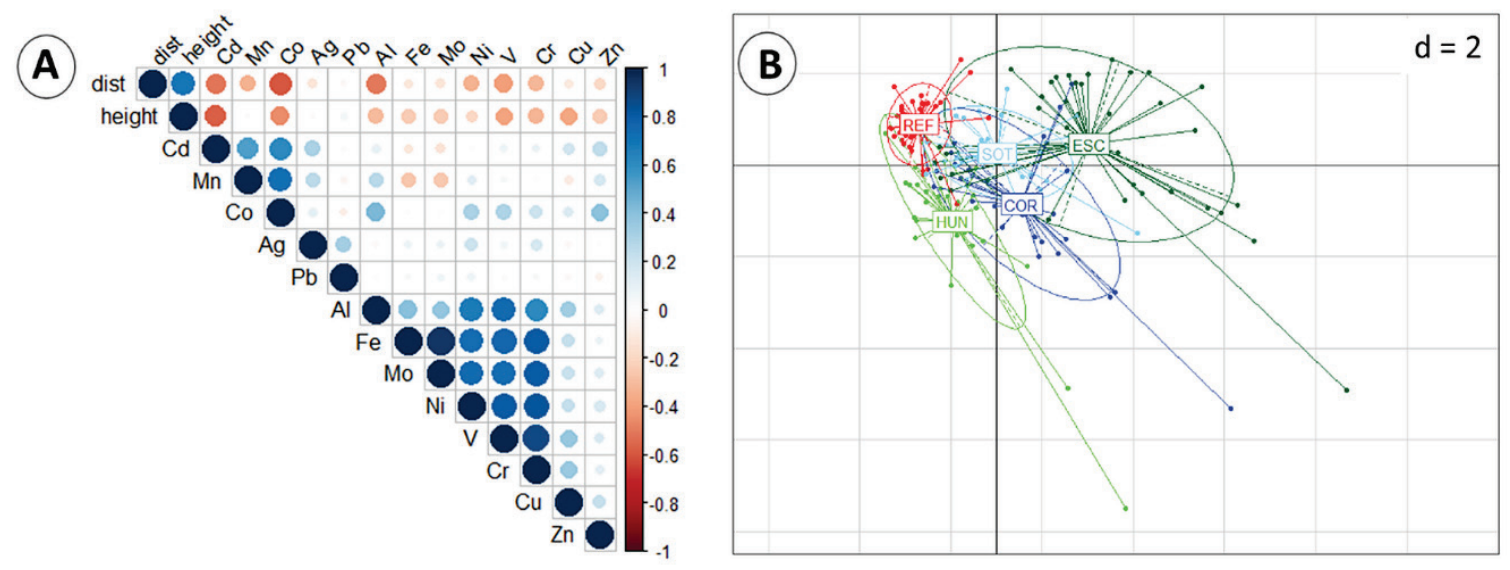

Figure 4. A) Correlations between metal concentrations in trees and the horizontal (dist) and vertical (height) distance of sampling sites from the channel. B) Principal Component Analysis of the element concentration measured in grouped trees at each sampling site.

Figure 4B illustrates results of the PCA with elements measured in trees classified by sampling site. By applying a Monte Carlo permutation test to this ordination, we show that $23.7 \%$ of the variability described by the PCA can be explained by fluvio-geomorphic differences between the sites ( $p$-value = 0.001). Overall, the highest metallic concentrations were found in trees growing on the cut banks of the meandering river reach (ESC). The distribution of concentrations was, by contrast, similar for trees growing in straight reaches (SOT and COR), whereas trees growing on the point bar of the meandering reach (HUN) showed lower concentrations. The reference site (REF), located on a nearby hillslope and thus without any connection to the channel network, showed the smallest metal concentrations.

The negative correlations between the horizontal and vertical distances of trees from the channel and metal concentration uptake support the initial assumption that the connectivity of trees to the fluvial system will control metal concentration uptake, resulting not only in lower, but also in more stable levels of metal concentrations in trees growing at REF. Results thus evidence a gradient of exposure of trees to metals transported by the river, and confirm findings of Madejón et al. (2004) stating that metal concentrations in Populus sp. growing in contaminated riparian zones of the Guadiamar River (southern Spain) were higher than in trees growing in unaffected neighboring areas. Moreover, our results are in line with observations of St. Laurent et al. (2009) by showing that the distance between analyzed trees and the channel plays a major role in contaminant assimilation.

In this study, we also provide insights into the effect of fluvio-geomorphic positions on metallic concentrations in trees: Whereas trees growing in straight channel reaches (i.e. sites COR and SOT) showed comparable metallic concentrations in their xylem, a clear difference was observed between the cut bank and point bar in the meandering reach, with higher metallic concentration found in those trees growing on the external side of the meander. This finding was unexpected insofar as sediments located on the inner side of a meander were generally thought to serve as reservoirs for metallic elements (e.g., 
Ciszewski, 2004; Ciszewski, 1998). On the other hand, the acidity of water (or at least its permanent proximity) may have played a key role favoring the release of metals from sediments to the water column, thereby increasing their solubility and mobility, which could then lead to enhanced bioavailability. Indeed, elements in acid rivers were shown to be present in a bioavailable ion form and could thus have been assimilated more easily by plants (Buzzi et al., 2014; Riaza et al., 2017). In addition, even if deposited sediments may contain significant quantities of metals (Morillo et al., 2002), the latter could form complexes with humic compounds or the clays contained in river sediments. If so, it would be possible that metal ions will be retained in a form that is not bioavailable to plants (Nobel, 1999).

\subsection{Effect of floods on metal accumulation in trees}

To analyze the effect of floods on metal uptake, blocks of five consecutive rings were prepared around the two major floods of 1996 and 2009. Sample COR4 was discarded due to fragmentation and the lack of sufficient wood material for analysis. Table 1 compares average metal concentrations observed in trees based on the Wilcoxon-test. We observe enhanced metal accumulation in trees located farther away from the channel (i.e. trees 4 and 5), especially after the flood of $2009(p<0.05)$. At the same time, data does not point to significant changes in the accumulation of metallic concentrations after the 1996 flood. Figure 5 therefore shows a dissimilar trend of element concentrations before and after floods with concentration of $\mathrm{Cu}, \mathrm{Zn}$ and $\mathrm{Pb}$ being lower (higher) after the 1996 (2009) floods. Although not significant, larger relative changes in metal concentration were observed for $\mathrm{Cr}, \mathrm{V}$, Mo and $\mathrm{Fe}\left(>75^{\text {th }}\right.$ percentile) after the 1996 flood, whereas lower concentration were observed for $\mathrm{Cu}, \mathrm{Zn}$, $\mathrm{Ag}, \mathrm{Pb}\left(<25^{\text {th }}\right.$ percentile $)$. By contrast, after the 2009 flood, larger relative changes in metal concentrations were measured for $\mathrm{Cu}, \mathrm{Zn}, \mathrm{Cr}, \mathrm{Pb}\left(>75^{\text {th }}\right.$ percentile), whereas lower concentrations were measured for $\mathrm{Ag}, \mathrm{Mn}$, and $\mathrm{Cd}\left(<25^{\text {th }}\right.$ percentile). These results imply a dissimilar response of trees to floods in terms of metal concentrations.

Table 1. Mean metallic concentrations before and after the 1996 and 2009 floods given as p-values obtained with Wilcoxon comparison tests (measured in 4-year blocks prior to and after the floods).

\begin{tabular}{llll}
\hline & $\mathbf{1 9 9 6}$ & Trees & p-value \\
\hline REF & All & 0.05759 \\
SOT, COR, HUN, ESC & $1-3$ & 0.4706 \\
SOT, COR, HUN, ESC & & $4-5$ & 0.2319 \\
\hline & $\mathbf{2 0 0 9}$ & Trees & p-value \\
\hline REF & All & 0.5206 \\
SOT, COR, HUN, ESC & $1-3$ & 0.7978 \\
SOT, COR, HUN, ESC & $4-5$ & 0.000668 \\
\hline
\end{tabular}




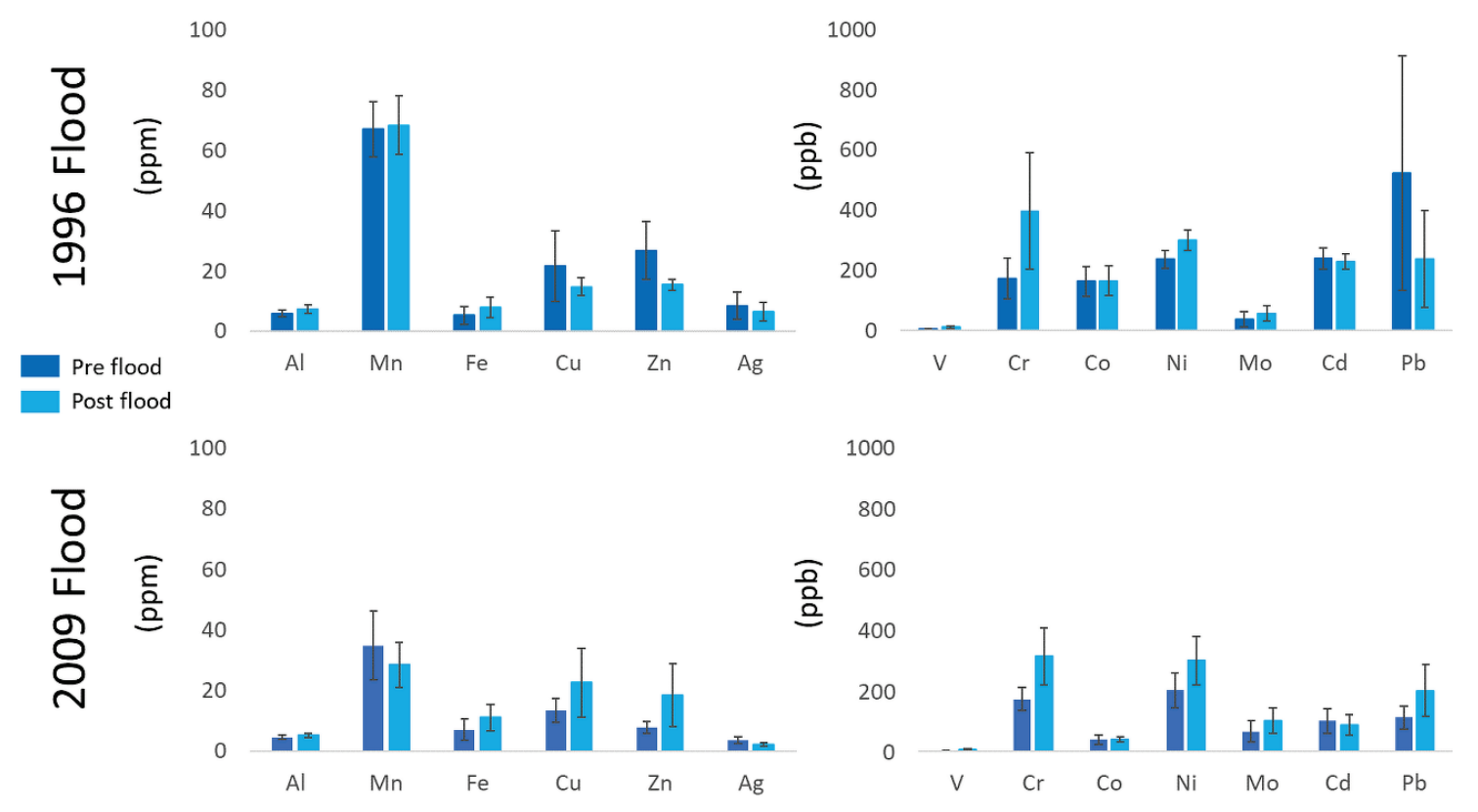

Figure 5. Response of element concentrations in trees after the major floods in the Odiel River in 1996 and 2009.

The assessment of rings in blocks of five before and after the events was realized with the idea to minimize translocation of elements through the xylem (Cheng et al., 2007), but also because a certain lag in the chemical response of trees to contamination has been described in the literature (Hagemeyer et al., 1992; Hagemeyer, 1995; Hagemeyer and Lohrie, 1995; Kabata and Pendias, 2001; Balouet et al., 2009). In the past, several studies analyzed chemical signatures based on aggregate measurements in consecutive tree rings so as to minimize the effects of translocation processes (Hagemeyer et al., 1992), as the latter are commonly considered to prevent interpretation at annual timescales (Guyette and Cutter, 1994).

The influence of floods on tree-ring records has been postulated by St. George et al. (2006) in his analysis of element concentrations in Quercus macrocarpa Michx. affected by persistent floods in the Red River (Canada). The authors did not find a clear and consistent chemical signature in the growthring records of trees that could be related to floods, but observed lower concentration of $\mathrm{Mg}, \mathrm{Mn}$ and $\mathrm{Sr}$ in trees after the largest $20^{\text {th }}$ century flood. More recently, St-Laurent et al. (2009) found anomalous concentrations of $\mathrm{As}, \mathrm{Cd}, \mathrm{Cu}, \mathrm{Ni}$, and $\mathrm{Zn}$ in riparian trees, and linked increased concentrations to floods along the Massawippi River (Canada), suggesting that floods were an important agent in transporting and depositing contaminants along the floodplains. In our study, we documented significant differences in element concentrations in those trees located far away from the channel after the 2009 flood but did not find comparable changes after the 1996 flood. Like St. George et al. (2006), we measured lower Mn concentrations in trees after the 2009 flood. Similar to St. Laurent et al. (2009), we found higher $\mathrm{Cu}, \mathrm{Ni}$, and $\mathrm{Zn}$ concentrations after the 2009 flood, but also realized that $\mathrm{Cd}$ showed invariant behavior. Interestingly, the response of $\mathrm{Zn}$ was not quite clear and even showed a completely opposite reaction after the 1996 flood. We explain these differences in response to the 1996 and 2009 floods by differences in flood magnitude, as discharge during the 1996 flood $\left(111.84 \mathrm{~m}^{3} / \mathrm{s}\right)$ corresponds to only c. $60 \%$ of that recorded during the 2009 flood $\left(186.10 \mathrm{~m}^{3} / \mathrm{s}\right)$. We argue that changes in metal concentrations after the 2009 flood can be explained by (i) the much larger connectivity between the main channel and those trees that are normally located far away from the river course and (ii) the mobilization of sediments transported by the flood to portions of the river corridor that are only activated by major events (St. Laurent et al., 2009). This assumption is supported further by the fact that trees at the REF site did not 
exhibit any changes after the two floods. In our case, the low Mn concentration found in trees after the two floods cannot be explained as a secondary process in wood formation during anoxic conditions as found as a result of the permanent flooding of riparian vegetation over several weeks (St. George et al., 2006), because, unlike Red River (Canada), the Odiel River is characterized by a quick hydrological response (Aguirre et al., 2003) and short-lived, almost flashy, flood episodes.

Nonetheless, we cannot rule out the possibility that floods could also have an opposite effect on metal concentrations in tree rings. Floods could in fact mobilize and transport metallic elements and thereby affect trees located far away from the main channel, but also dilute metallic elements contained in soil by the simple supply of excessive precipitation and surface runoff as often observed in Mediterranean climates during floods. This mechanism could be particularly relevant for floods in autumn when the flush can mobilize efflorescences of soluble salts that have been deposited on the banks of Odiel River during the low water stages of the summer months. Besides, long-lasting rainfall events could saturate soils in the catchment and modify its $\mathrm{pH}$ (Norton, 1977; Riaza et al., 2012); these persistent rainfall events are indeed a typical cause of floods in the Atlantic basin (Benito et al., 1996; Ballesteros-Cánovas et al., 2018). On the other hand, changes in soil $\mathrm{pH}$ may affect the bioavailability of metallic pollutants and their uptake by trees (Augustin et al., 2005). This latter effect could be even more relevant in the current context of soil acidification in the study area, as illustrated by the Mn/Al ratio found in the xylem of the trees analyzed (Fig. S.3).

\section{Conclusions}

Anthropogenic activities result in an incorporation of metallic pollutants in the hydrological cycle, posing major environmental problems. In this study, we analyzed twenty-five $P$. pinaster trees growing along Odiel River, a Mediterranean stream that is highly affected by mining activities. Results of the dendrochemical analysis of trees growing in the river corridor were compared with reference trees growing at a site that has not been affected by floods. We hypothesized that $P$. pinaster trees show a chemical signature that is controlled by the magnitude of floods and the fluvio-geomorphic position of trees within the river corridor. We conclude that the fluvial position of trees and their horizontal and vertical distance from the river channel determine metal accumulations found in trees. We observe that trees located farther away from the fluvial channel show a significant response to the 2009 flood, but trees lack a clear signal after the 1996 flood, presumably as a result of the much smaller magnitude of the 1996 flood. Despite certain limitations, we also conclude that trees are valuable sentinels for toxic metals and herewith call for follow-up research to test such linkages, with the aim to determine metal uptake by trees in a way that it could soon support land management strategies in fluvial ecosystem affected by mining in various contexts worldwide.

\section{Acknowledgements}

This study has been partly funded by the Foundation Ernst et Lucie Schmidheiny. Warm thanks are extended to Isabelle Worms for performing the ICPMS measurements, to Asuncion Riaza for initial discussion as well as to the anonymous external reviewers.

\section{References}

Aguirre, J.A.M., Sánchez, J.C.R., Arce, P.J. S. 2003. Avenidas torrenciales en el Arroyo del Partido y su incidencia en la Marisma del Parque Nacional de Doñana. Organismo Autónomo Parques Nacionales, Madrid.

Ali, H., Khan, E. Ilahi, I. 2019. Environmental Chemistry and Ecotoxicology of Hazardous Heavy Metals: Environmental Persistence, Toxicity, and Bioaccumulation. Journal of Chemical, 6730305. https://doi.org/10.1155/2019/6730305 
Alpers, C.N., Nordstrom, D.K., Spitzley, J. 2003. Extreme acid mine drainage from a pyritic massive sulfide deposit: The Iron Mountain endmember. In: J.L. Jambor, D.W. Blowes, A.I.M. Ritchie (Eds.). Environmental Aspects of Mine-Wastes. Mineralogical Association of Canada, pp. 407-430.

Augustin, S., Stephanowitz, H., Wolff, B., Schröder, J., Hoffmann, E. 2005. Manganese in Tree Rings of Norway Spruce as an Indicator for Soil Chemical Changes in the Past. European Journal of Forest Research 124 (4), 313-318. https://doi.org/10.1007/s10342-005-0084-4

Ballesteros-Cánovas, J.A., Stoffel, M., St George, S., Hirschboeck, K. 2015. A review of flood records from tree rings. Progress in Physical Geography 39 (6) 794-816. https://doi.org/10.1177/0309133315608758

Ballesteros-Cánovas, J.A., Stoffel, M., Benito, G., Rohrer, M., Barriopedro, D., García-Herrera, R., Beniston, M., Brönnimann, S. 2018. On the extraordinary winter flood episode over the North Atlantic Basin in 1936. Annals of the New York Academy of Sciences 1436, 206-216. https://doi.org/10.1111/nyas.13911

Ballesteros-Cánovas, J.A., Stoffel, M., Martín-Duque, J.F., Corona, C., Lucía, A., Bodoque, J.M., Montgomery, D.R. 2017. Gully evolution and geomorphic adjustments of badlands to reforestation. Scientific Reports 7,45027 .

Balouet, J.C., Smith, K.T., Vroblesky, D., Oudijk, G. 2009. Use of dendrochronology and dendrochemistry in environmental forensics: does it meet the Daubert criteria? Environmental Forensics 10 (4), 268-276. https://doi.org/10.1080/ 15275920903347545

Banchirigah, S.M. 2008. Challenges with eradicating illegal mining in Ghana: A perspective from the grassroots. Resources Policy 33(1), 29-38. https://doi.org/10.1016/ j.resourpol.2007.11.001

Benito, G., Machado, M.J., Pérez-González, A. 1996. Climate change and flood sensitivity in Spain. Geological Society, London, Special Publications, 115(1), 85-98. https://doi.org/10.1144/GSL.SP.1996.115.01.08

Bing, H., Wu, Y., Zhou, J., Li, R., Wang, J. 2016. Historical trends of anthropogenic metals in Eastern Tibetan Plateau as reconstructed from alpine lake sediments over the last century. Chemosphere 148, 211-219. https://doi.org/10.1016/j.chemosphere.2016.01.042

Borrego, J. 1992. Sedimentología del estuario del Río Odiel, Huelva, S.O. España. PhD thesis, Univ. of Sevilla, Sevilla, Spain.

Braungardt, C.B., Achterberg, E.P., Mimmo, M. 1998. Behaviour of disolved trace metals in the Rio Tinto/Rio Odiel Esturine System. In: J.A. Morales, J. Borrego, J. (Eds). European land-ocean interaction studies. Second annual scientific conference. Abstract 51.

Buzzi Marcos, J. 2012. Imaging Spectroscopy to Evaluate the Contamination from Sulphide Mine Waste in the Iberian Pyrite Belt Using Hyperspectral Sensors (Huelva, Spain). Ph.D. Thesis, Universidad de León, León, Spain.

Buzzi, J., Riaza, A., García-Meléndez, E., Weide, S., Bachmann. M. 2014. Mapping Changes in a Recovering Mine Site with Hyperspectral Airborne Hymap Imagery (Sotiel, SW Spain). Minerals 4, 313-329. https://doi.org/10.3390/min4020313

Cánovas, C.R. 2009. La calidad del agua de los ríos Tinto y Odiel: evolución temporal y factores condicionantes de la movilidad de los metales. Universidad de Huelva. Huelva.

Cheng, Z., Buckley, B.M., Katz, B., Wright, W., Bailey, R., Smith, K.T., van Geen, A. 2007. Arsenic in tree rings at a highly contaminated site. Science of the Total Environment 376 (1-3), 324-334. https://doi.org/10.1016/j.scitotenv.2007.01.074

Ciszewski, D. 1998. Channel processes as a factor controlling accumulation of heavy metals in river bottom sediments: consequences for pollution monitoring (Upper Silesia, Poland). Environmental Geology 36 (1), 45-54. https://doi.org/10.1007/s002540050319

Ciszewski. D. 2004. Pollution of Mala Panew river sediments by heavy metals: Part I. Effect of changes in river bed morphology. Polish Journal of Environmental Studies 13 (6), 589-595.

Consejería de Agricultura, Pesca y Medio Ambiente (Junta de Andalucía), 2014. Anejo 5 Implantación del régimen de caudales ecológicos. Demarcación Hidrográfica del Tinto, Odiel y Piedras. Memoria del Ciclo de Plan Hidrológico de la Planificación Hidrológica 2015/2021. 
Corella, J.P., Valero-Garcés, B.L., Wang, F., Martínez-Cortizas, A., Cuevas, C.A., Saiz-Lopez, A. 2017. 700 years reconstruction of mercury and lead atmospheric deposition in the Pyrenees (NE Spain). Atmospheric Environment 155, 97-107. https://doi.org/10.1016/j.atmosenv.2017.02.018

Cutter, B.E., Guyette. R.P. 1993. Anatomical, Chemical, and Ecological Factors Affecting Tree Species Choice in Dendrochemistry Studies. Journal of Environment Quality 22 (3), 611-619. https://doi.org/10.2134/jeq1993.00472425002200030028x

Donnelly, J.R., Shane, J.B., Schaberg P.G. 1990. Lead mobility within the xylem of red spruce seedlings: implications for the development of pollution histories. Journal of Environmental Quality 19, 268-271. https://doi.org/10.2134/jeq1990.00472425001900020012x

Doucet, A. 2011. Perspective spatio-temporelle et impacts des contaminants atmosphériques d'origine diffuse sur les forêts périurbaines du sud-est du Canada : une approche dendrogéochimique. Université du Québec, Quebec, Canada.

Fifield, F.W., Haines, P.J. (Eds.). 2000. Environmental analytical chemistry. Wiley.

Foster, I.D.L., Charlesworth, S.M. 1996. Heavy metals in the hydrological cycle: trends and explanation. Hydrological Processes $10 \quad$ (2), 227-261. https://doi.org/10.1002/(SICI)10991085(199602)10:2<227::AID-HYP357>3.0.CO;2-X

Foulds, S.A., Brewer, P.A., Macklin, M.G., Haresign, W., Betson, R.E., Rassner, S.M.E., 2014. Flood-related contamination in catchments affected by historical metal mining: an unexpected and emerging hazard of climate change. Science of the Total Environment 476, 165-180. https://doi.org/10.1016/j.scitotenv.2013.12.079

Galván González, L. 2012. Modelización hidrológica del rio Odiel: aplicación al estudio de la contaminación por drenaje ácido de minas. Ph.D. Thesis, Universidad de Huelva, Huelva, Spain.

Galván, L., Olías, M., 2015. Estudio de la contaminación por drenaje ácido de minas en la cuenca del Río Odiel. Boletín de la Sociedad Española de Mineralogía 20, 51-52.

George, S.S., Outridge, P.M., Nielsen, E. 2006. High-resolution dendrochemical analysis of flood-affected oaks using laser ablation ICP-mass spectrometry. IAWA Journal 27(1), 19-31. https://doi.org/10.1163/22941932-90000134

Grande, J.A., Pérez Ostalé, E., de la Torre Sánchez, M.L., Fernandes Valente, T.M., Borrego Flores, J., Pérez Macias, J.A., Santisteban Fernández, M., Garrido Morillo, R., Romero Macías, E., Salmerón García, I. 2016. Drenaje ácido de mina en la Faja Pirítica Ibérica: Técnicas de estudio e inventario de explotaciones. Servicio de Publicaciones Universidad de Huelva, Huelva, Spain.

Gross, J.H. 2006. Mass spectrometry: a textbook. Springer, 1-518 pp., Heilderberg,

Guyette, R.P., Cutter. B.E. 1994. Barium and manganese trends un tree-rings as monitors of sulfur deposition. Water, Air, and Soil Pollution 73, 213-223. https://doi.org/10.1007/BF00477987

Hagemeyer, J. 1995. Radial distributions of Cd in stems of oak trees (Quercus robur L.) re-analyzed after 10 years. Trees-Structure and Function 9 (4): 200-203. https://doi.org/10.1007/BF00195273

Hänsch, R., Mendel, R. R. 2009. Physiological Functions of Mineral Micronutrients (Cu, Zn, Mn, Fe, Ni, Mo, B, Cl). Current Opinion in Plant Biology 12 (3): 259-266. https://doi.org/10.1016/j.pbi.2009.05.006

Hong, S., Candelone, J.P., Patterson, C.C., Boutron, C.F. 1996. History of ancient copper smelting pollution during Roman and medieval times recorded in Greenland ice. Science 272(5259), 246-249. https://doi.org/10.1126/science.272.5259.246

Hürkamp, K., Raab, T., Völkel, J. 2009. Lead pollution of floodplain soils in a historic mining area-age, distribution and binding forms. Water, air, and soil pollution 201(1-4), 331-345. https://doi.org/10.1007/s11270-008-9948-9

Islam, M.S., Ahmed, M.K., Raknuzzaman, M., Habibullah-Al-Mamun, M., Islam, M.K. 2015. Heavy metal pollution in surface water and sediment: a preliminary assessment of an urban river in a developing country. Ecological Indicators 48, 282-291. https://doi.org/10.1016/j.ecolind.2014.08.016 
Kabata-Pendias, A., Pendias, H. 2001. Trace elements in soils and plants. 3rd ed. FI CRC Press, 1-413 pp., Boca Raton, US.

Lageard, J.G.A., Howell, J.A., Rothwell, J.J., Drew, I.B. 2008. The utility of Pinus sylvestris L. in dendrochemical investigations: Pollution impact of lead mining and smelting in Darley Dale, Derbyshire, UK. Environmental Pollution 153 (2), 284-294. https://doi.org/10.1016/j.envpol.2007.08.031

Leistel, J.M., Marcoux, E., Thiéblemont, D., Quesada, C., Sánchez, A., Almodóvar, G.R., Sáez, R.J.M.D. 1997. The volcanic-hosted massive sulphide deposits of the Iberian Pyrite Belt Review and preface to the Thematic Issue. Mineralium Deposita 33 (1-2), 2-30.

Lewis, T.E. (Ed.). 1995. Dendrochronology and dendrochemistry in regional ecosystem health assessments: the forest health monitoring experience. In: Tree Rings as Indicators of Ecosystem Health, 25 June 1993, Penn State University, University Park, Pa. CRC Press, Boca Raton, Fla. pp. 1-16.

López, E., Sánchez, J., Diez, M., Santofimia, E., Reyes J., 2008. Cortas mineras inundadas de la Faja Piritica: inventario e hidroquímica. Instituto Geológico y Minero de España. Madrid.

Madejon, P., Marañón, T., Murillo, J. M., Robinson, B. 2004. White poplar (Populus alba) as a biomonitor of trace elements in contaminated riparian forests. Environmental Pollution 132 (1), 145-155. https://doi.org/10.1016/j.envpol.2004.03.015

Meerts, P. 2002. Mineral nutrient concentrations in sapwood and heartwood: a literature review. Annals of Forest Science 59 (7), 713-722. https://doi.org/10.1051/forest:2002059

Morales, J.A., Pons, J.M., Cantano, M. 2005. Introducción al análisis de los riesgos de inundación en las riberas de las áreas estuarinas: El caso de las poblaciones adyacentes a la Ría de Huelva (SO España). Geogaceta 37, 243-246.

Morillo, J., Usero, J., Gracia, I. 2002. Partitioning of metals in sediments from the Odiel River (Spain). Environment International 28 (4), 263-271. https://doi.org/10.1016/S0160-4120(02)00033-8

Nobel, P.S. 1999. Physicochemical and environmental plant physiology. Academic Press, San Diego, California, USA.

Nordstrom, D.K., Alpers, C.N. 1999. Geochemistry of acid mine waters. In: The environmental geochemistry of mineral deposits, 6A: 133-160. Rev Econ Geol. Plumlee GS, Logsdon MJ.

Norton, S.A. 1977. Changes in chemical processes in soils caused by acid precipitation. Water, Air, and Soil Pollution, 7(3), 389-400. https://doi.org/10.1007/BF00284133

Nriagu, J.O. 1996. A history of global metal pollution. Science 272 (5259), 223-223. https://doi.org/10.1126/science.272.5259.223

Obeng, E.A., Oduro, K.A., Obiri, B.D., Abukari, H., Guuroh, R.T., Djagbletey, G.D., Appiah, M., 2019. Impact of illegal mining activities on forest ecosystem services: local communities' attitudes and willingness to participate in restoration activities in Ghana. Heliyon 5 (10), e02617. https://doi.org/10.1016/j.heliyon.2019.e02617

Pérez-López, R., Nieto, J.M., López-Cascajosa, M.J., Díaz-Blanco, M.J., Sarmiento, A.M., Oliveira, V., SánchezRodas, D. 2011. Evaluation of heavy metals and arsenic speciation discharged by the industrial activity on the Tinto-Odiel estuary, SW Spain. Marine Pollution Bulletin 62, 405-411. https://doi.org/10.1016/j.marpolbul.2010.12.013

Riaza, A., Buzzi, J., García-Meléndez, E., Carrère, V., Sarmiento A., Müller, A. 2012. River acid mine drainage: sediment and water mapping through hyperspectral Hymap data. International Journal of Remote Sensing 33 (19), 6163-6185. https://doi.org/10.1080/01431161.2012.675454

Riaza, A., Buzzi, J., García-Meléndez, E., Del Moral, B., Carrère, V., Richter, R. 2017. Monitoring salt crusts on an AMD contaminated coastal wetland using hyperspectral Hyperion data (Estuary of the River Odiel, SW Spain). International Journal of Remote Sensing 38 (12), 3735-3762. https://doi.org/10.1080/01431161.2017.1302621

Rico, M., Benito, G., Diez-Herrero, A. 2008. Floods from tailings dam failures. Journal of Hazardous Materials 154 (1-3), 79-87. https://doi.org/10.1016/j.jhazmat.2007.09.110. 
Rinn F. 1996. Tsap V. 3.6 Reference Manual: Computer Program for Tree-Ring Analysis and Presentation, 263 pp., Heidelberg, Germany,

Robles-Arenas, V.M., Rodríguez, R., García, C., Manteca, J.I., Candela, L. 2006. Sulphide-mining impacts in the physical environment: Sierra de Cartagena-La Unión (SE Spain) case study. Environmental Geology 51(1), 47-64. https://doi.org/0.1007/s00254-006-0303-4

Rodríguez-Martín, J.A.R., Gutiérrez, C., Torrijos, M., Nanos, N. 2018. Wood and bark of Pinus halepensis as archives of heavy metal pollution in the Mediterranean Region. Environmental Pollution 239, 438-447. https://doi.org/10.1016/ j.envpol.2018.04.036

Prohaska, T., Stadlbauer, C., Wimmer, R. Stingeder, G., Latkoczy, Ch., Hoffmann, E., Stephanowitz, H. 1998. Investigation of element variability in tree rings of young Norway spruce by laser-ablation-ICPMS. Science of the Total Environment 219 (1): 29-39. https://doi.org/10.1016/S0048-9697(98)00224-1

Saint-Laurent, D., St-Laurent, J., Duplessis, P., Lavoie, L. 2010. Isotopic record of lead contamination in alluvial soils and tree rings on recent floodplains (Southern Québec, Canada). Water, Air, \& Soil Pollution 20, 9(1-4), 451-466. https://doi.org/10.1007/s11270-009-0213-7

Scharnweber, T., Hevia, A., Buras, A., van der Maaten, E., Wilmking, M. 2016. Common trends in elements? Within-and between-tree variations of wood-chemistry measured by X-ray fluorescence-A dendrochemical study. Science of the Total Environment 566, 1245-1253. https://doi.org/10.1016/j.scitotenv.2016.05.182

Schweingruber, F.H., 2007. Wood structure and environment. Springer, Heidelberg, Germany.

Sheppard, J.C., Funk. W.H. 1975. Trees as environmental sensors monitoring long-term heavy metal contamination of Spokane River, Idaho. Environmental Science \& Technology 9 (7), 638-642.

Silva, J.B., Oliveira, J.T., Ribeiro, A. 1990. Structural outline. In: R.D. Dallmeyer, E.M. García (Eds.). PreMesozoic Geology of Iberia. IGCP-Project 233 (Terranes in the Circum-Atlantic Palezoic Orogens), Springer, pp. 348-362, Berlin, Heidelberg. https://doi.org/10.1007/978-3-642-83980-1_24

Smith, K.T., Shortle, W.C. 1996. Tree biology and dendrochemistry. In: J.S. Dean, D.M. Meko, T.W. Swetnam (Eds.). Tree rings, Environment and Humanity. Proceedings of an International Conference. Tucson, AZ: Radiocarbon: 629-635.

Sprent, P., Smeeton, N.C. 2001. Applied Nonparametric Statistical Methods. 3rd Edition, Chapman \& Hall/CRC, 463 pp., Boca Raton, US.

St. George, S., Outridge, P.M., Nielsen, E. 2006. High-resolution dendrochemical analysis of flood-affected oaks using laser ablation ICP-mass spectrometry. IAWA journal 27(1), 19-31.

St. Laurent, J., Saint-Laurent, D., Duplessis, P., Hähni, M., Begin, C. 2009. Application of dendrochronological and dendrochemical methods for dating contamination events of the Saint-François and Massawippi riverbanks (Québec, Canada). Soil, Sediment and Contamination 18 (5), 642-668. https://doi.org/10.1080/15320380903113626

Stoffel, M., Slaveykova, V.I., Corona, C., Ballesteros-Cánovas, J.A. 2020. When scientists become detectives: investigating systematic tree poisoning in a protected cove. Heliyon 6(2), e03386. https://doi.org/10.1016/j.heliyon.2020.e03386

Tchounwou, P.B., Yedjou, C.G., Patlolla, A.K., Sutton, D.J. 2012. Heavy metal toxicity and the environment. In: A. Luch (Ed.). Molecular, Clinical and Environmental Toxicology. Springer, pp. 133-164, Basel.

Watmough, S.A., Hutchinson, T.C. 1996. Analysis of Tree Rings Using Inductively Coupled Plasma Mass Spectrometry to Record Fluctuations in a Metal Pollution Episode. Environmental Pollution 93 (1), 93 102. https://doi.org/10.1016/0269-7491(95)00107-7

Watmough, S.A., Hutchinson, T.C. 2002. Historical changes in lead concentrations in tree-rings of sycamore, oak and Scots pine in north-west England. Science of the Total Environment 293 (1): 85-96. https://doi.org/10.1016/S0048-9697(01)01149-4 
Watmough, S.A., Hutchinson, T.C. 2003. A comparison of temporal patterns in trace metal concentration in tree rings of four common European tree species adjacent to a $\mathrm{Cu}-\mathrm{Cd}$ refinery. Water, Air, \& Soil Pollution 146 (1): 225-241. https://doi.org/10.1023 /A:1023952417583

Witte, K.M., Wanty, R.B., Ridley, W.I. 2004. Engelmann Spruce (Picea engelmannii) as a biological monitor of changes in soil metal loading related to past mining activity. Applied Geochemistry 19 (9), 1367-1376. https://doi.org/10.1016/j.apgeochem.2004.01.022

Wright, G., Woodward, C., Peri, L., Weisberg, P.J., Gustin, M.S. 2014. Application of tree rings [dendrochemistry] for detecting historical trends in air $\mathrm{Hg}$ concentrations across multiple scales. Biogeochemistry 120 (1-3), 149-162. https://doi.org/10.1007/s10533-014-9987-9

Yanosky, T.M., Hupp, C.R., Hackney, C.T. 1995. Chloride concentrations in growth rings of Taxodium distichum in a saltwater-intruded estuary. Ecological Applications 5 (3), 785-792. https://doi.org/10.2307/1941986

Yanosky, T.M., Vroblesky, D.A. 1992. Relation of nickel concentrations in tree rings to groundwater contamination. Water Resources Research 28 (8), 2077-2083. https://doi.org/10.1029/92WR00731 


\section{Supplementary material}

Al

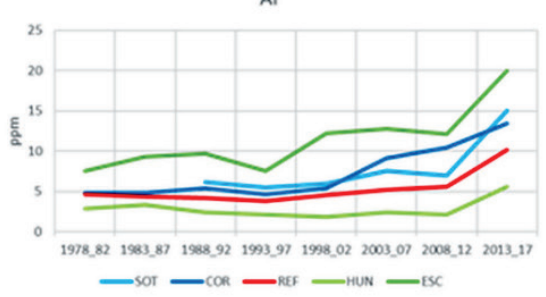

$\mathrm{Mn}$

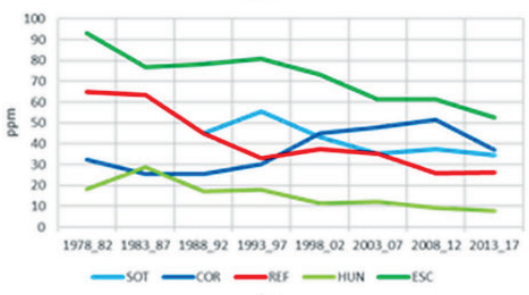

$\mathrm{Ni}$

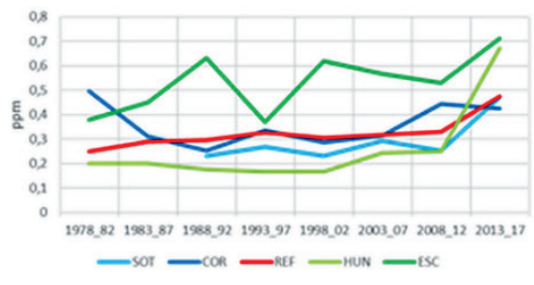

Mo

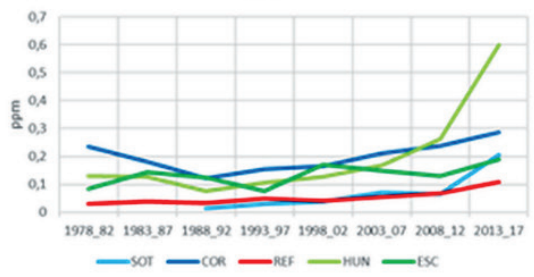

$\mathrm{Pb}$

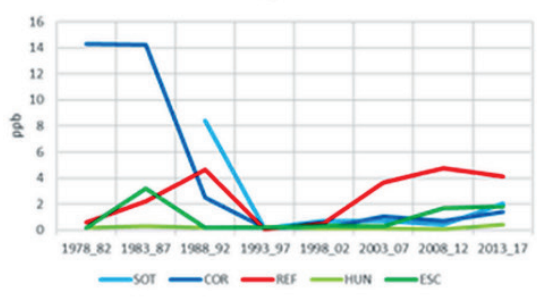

v

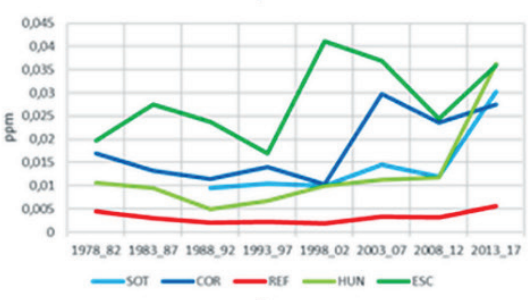

$\mathrm{Fe}$
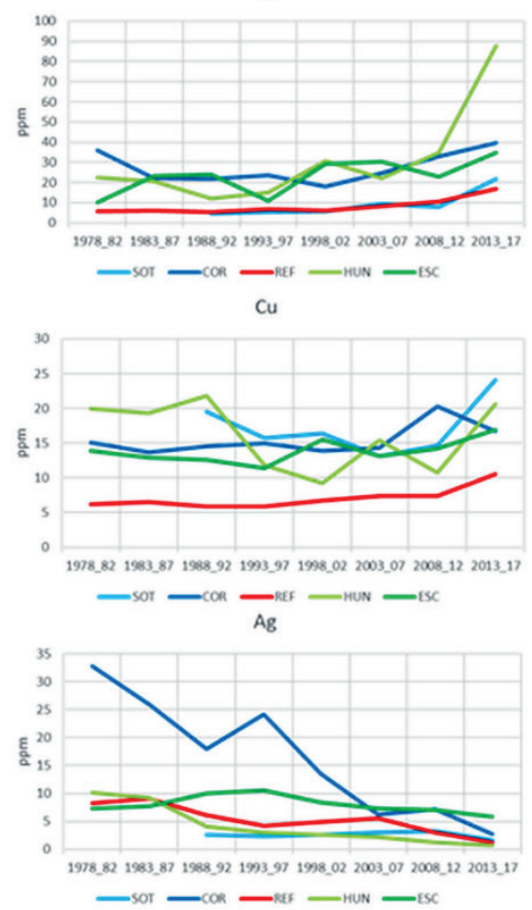

$\mathrm{Cr}$

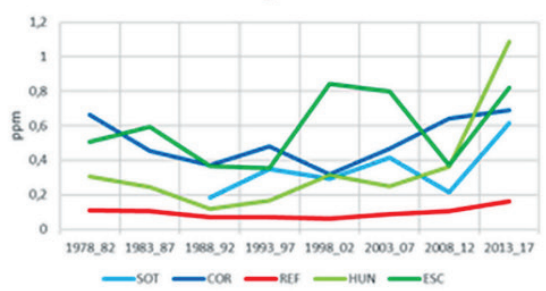

co

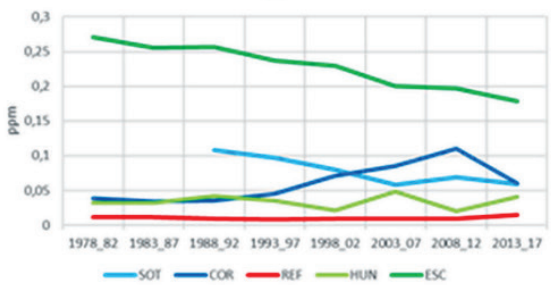

$\mathrm{Zn}$

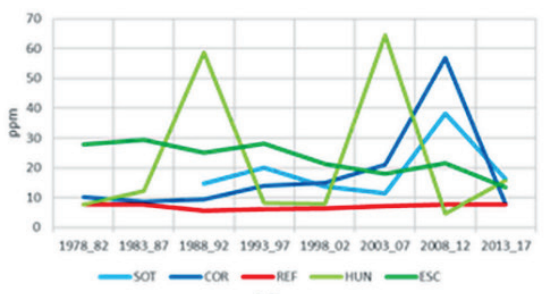

$\mathrm{Cd}$

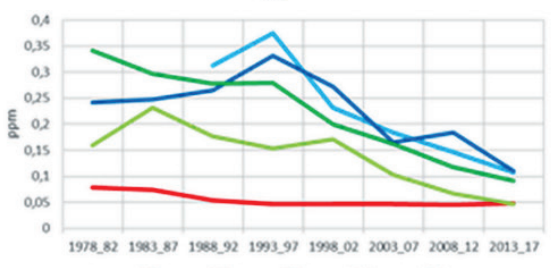

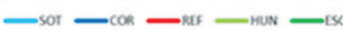

Figure S1. Temporal evolution of the average concentration of each element in the five sampling sites. 
Delapierre et al.
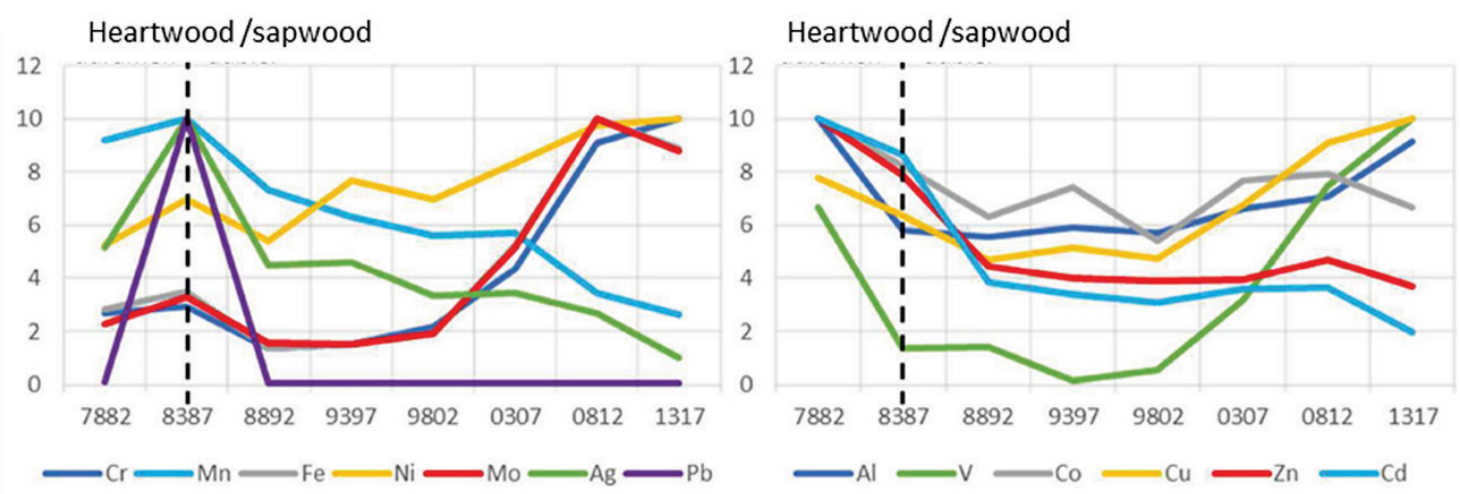

Figure S2. Example of changes in element concentration in the interface between hearwood and sapwood (Tree \# R3). In the left panel, peak in element concetrations can be seen in the border between sapwood/hearwood; while in the right pannel element concentrations tend to increase towards the pith from the sapwood / heartwood limit.

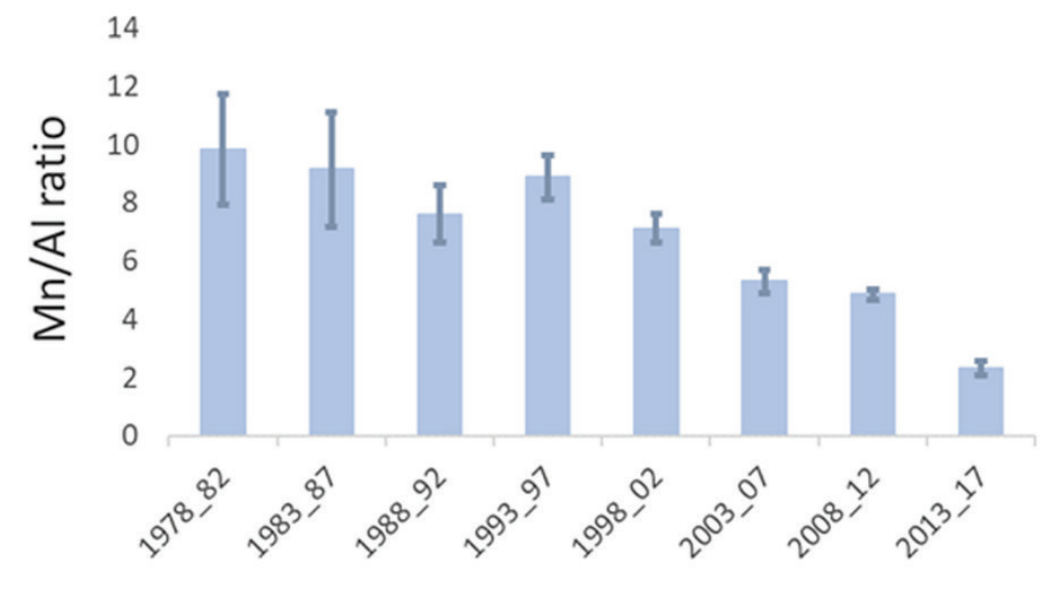

Figure S3. Temporal evolution of the Mn / Al ratio (averages of all sites) 


\title{
SPATIO-TEMPORAL DIFFERENCES OF SEDIMENT ACCUMULATION RATE IN THE LAKE GOŚCIĄŻ (CENTRAL POLAND) AS A RESPONSE OF METEOROLOGICAL CONDITIONS AND LAKE BASIN MORPHOMETRY
}

\author{
MICHAŁ FOJUTOWSKI*, PIOTR GIERSZEWSKI, DARIUSZ BRYKALA, \\ ALICJA BONK, MIROSŁAW BLASZKIEWICZ, MATEUSZ KRAMKOWSKI
}

Department of Environmental Resources and Geohazards, Institute of Geography and Spatial Organization, Polish Academy of Sciences, Poland.

\begin{abstract}
Weather conditions and lake basin morphometry are of key importance in the study of sediment accumulation rate in lakes. This study aims to determine how these factors affect spatial and seasonal variations in sedimentation rate in the epilimnion and hypolimnion of Lake Gościąż. To determine sedimentation rates, six sedimentation traps were set up at different locations and depths in the lake. Weather data were obtained from a meteorological station near the lake. Furthermore, temperature in the lake water column was measured continuously, and during field work oxygenation and transparency were also measured. Seasonal changes in sediment composition were analyzed on smear slides under microscope. The study showed that sedimentation rate increased as bottom steepness increased, and that there was more sediment in the hypolimnion than the epilimnion, especially in spring and autumn. There was a clear seasonal variation in early-spring and autumn peaks in sedimentation. The obtained results were significantly dependent on bottom relief, wind and air temperature through these factors' influence on water temperature. The results show that the sediment accumulation rate in Lake Gościąż depends on the hydrodynamic conditions, which are determined by wind speed, wind direction, water temperature, and the shape and steepness of the lake basin. The relief features of the lake bottom and its orientation relative to the prevailing wind are significant factors in the spatial differentiation in sediment accumulation rate and composition of sedimenting material. It has been shown that the lake's shallow-water zone (littoral and sublittoral) is an important source of the material accumulated in the profundal zone. The patterns and mechanisms of the course of contemporary sedimentation in Lake Gościąż, as determined based on the conducted investigations, can be applied in the study of other lakes and in assessing the representativeness of sampling sites for laminated bottom sediments to be used in palaeo-environmental studies.
\end{abstract}

Diferencias espacio temporales de la tasa de acumulación de sedimentos en el lago Gościąż (Polonia central) como respuesta a las condiciones meteorológicas y a la morfometría del lago

RESUMEN. Las condiciones meteorológicas y la morfometría de la cuenca son clave en el estudio de las tasas de sedimentación en lagos. Este trabajo tiene como objetivo analizar cómo estos factores afectan a las variaciones espaciales y estacionales de la tasa de sedimentación en el epilimnion y el hipolimnion del lago Gościąż. Para determinar las tasas de sedimentación, se instalaron seis trampas de sedimentos en diferentes localizaciones dentro del lago y a diferentes profundidades. Los datos meteorológicos se obtuvieron de una estación próxima al lago. Además, se midió de manera constante la temperatura de la columna de agua y, durante campañas de campo, su grado de oxigenación y transparencia. Se analizaron cambios estacionales en la composición del sedimento mediante frotis para microscopio. El estudio indica que la tasa de sedimentación incrementó conforme la pendiente 
del fondo del lago aumentaba, y que había más sedimento en el hipolimnion que en el epilimnion, especialmente en verano y en otoño. Se observó una clara variación estacional en los picos de sedimentación al principio de la primavera y en otoño. Los resultados dependieron significativamente del relieve del fondo del lago, del viento y de la temperatura del aire, que a su vez influenciaron la temperatura del agua. Los resultados muestran que la acumulación de sedimento en el Lago Gościąż depende de las condiciones hidrodinámicas, las cuales están determinadas por la velocidad y dirección del viento, la temperatura del agua y la pendiente del fondo del lago. Las características del relieve del fondo del lago y su orientación relativa a los vientos predominantes son factores significativos que explican la diferenciación espacial de la tasa de sedimentación y la composición del material sedimentado. Las zonas someras del lago (litorales y sublitorales) son una fuente importante de sedimento del material acumulado en la zona profunda. Los patrones y mecanismos de la sedimentación contemporánea del lago Gościąż, definidos en este trabajo pueden ser aplicados al estudio de otros lagos, así como en la evaluación de la representatividad de sitios de muestreo para sedimentos laminados en estudios paleoambientales.

Key words: Lake morphometry, dimictic lake, sediment resuspension, sediment accumulation rate, hydrometeorological monitoring.

Palabras clave: Morfometría lacustre, lago dimíctico, resuspensión de sedimentos, tasa de acumulación de sedimento, seguimiento hidrometeorológico.

Received: 10 June 2020

Accepted: 30 October 2020

* Corresponding author: Michał Fojutowski, Department of Environmental Resources and Geohazards, Institute of Geography and Spatial Organization, Polish Academy of Sciences, Kopernika 19, PL87100 Toruń, Poland. E-mail address: mfojutowski@twarda.pan.pl

\section{Introduction}

Lake sediments are excellent archives of changes taking place in the natural environment, because lakes react very quickly to changes in their surroundings (Vos et al., 1997). Of particular usefulness in this regard are annually laminated sediments, i.e. varves, which show regional and local changes at high-resolution (Segerstrom et al., 1984; Goslar, 1993; Leeman and Niessen, 1994; Schaller et al., 1997; Moore et al., 2001; Schettler et al., 2006; Ojala et al., 2008; Kaal et al., 2015; Zolitschka et al., 2015; Bonk et al., 2016; Ott et al., 2017) but are relatively rare (Tylmann, 2011; Ojala et al., 2012; Zolitschka et al., 2015). Anaerobic conditions at the lake bottom are required for the preservation of varves, as confirmed by the fact that they coincide spatially with bottom areas covered by the hypolimnion in dimictic lakes or the monimolimnion in meromictic lakes (Petterson et al., 1993; Tylmann et al., 2012; Salminen et al., 2019). Therefore, the potential drivers of annual lamination discontinuity or its absence are syn- and metasedimentary disturbances to the sediment (O'Sullivan, 1983) caused by dwelling organisms, strong winds leading to intense water column mixing or a deeplying thermocline (Horppila and Niemistö, 2008).

In order to fully exploit the information on environmental changes preserved in laminated sediments, and thus to create a reliable and accurate chronology, it is necessary to understand the mechanisms affecting the formation of these sediments (Lotter and Birks, 1997; Bonk et al., 2015). For this, monitoring of contemporary lake sediments, which reflect current seasonal changes, is an excellent tool. Observing changes requires sediment traps, as well as monitoring of the meteorological and hydrological conditions in the catchment (Leeman and Niessen, 1994; Ojala et al., 2014). Sediment traps are a fairly common limnological research method but in the study of annually laminated lake sediments, 
their popularity is relatively recent, and is still growing (Flower, 1990; Mieszczankin, 1997; Bluszcz et al., 2008; Bonk et al., 2015; Kienel et al., 2017; Maier et al., 2018; Johansson et al., 2019). The results of this type of research are difficult to integrate with morphometric and meteorological conditions, and hence it is relatively rarely done. The basic research involves capturing suspended matter (also known as "seston" [Ruttner, 1963]) in the lake's water; this is a component of the lake's primary production and a supply of allochthonous material (Wetzel et al., 1972; Gasith, 1976). Primary production can affect the amount of sediment directly by biological particles, and indirectly through calcium carbonate precipitating in biogeochemical processes (Groleau et al., 2000). The biological production volume in the lake is affected most strongly by nutrient supply (Pace and Lovett, 2013). Meteorological impacts are usually taken into consideration less frequently in studies of lake productivity, although they are becoming more important as climate conditions change dynamically and human impact increases. Factors related to meteorological conditions may also affect the redistribution of plankton within the lake and sediment resuspension, especially in very shallow parts (Dearing, 1997). The spatial diversity of sedimentation is also influenced by lake basin morphology, i.e. the steepness of the lake basin slopes and the number and arrangement of the basins composing the water body (Punning et al., 2004).

Lake Gościąż is widely known for its annually laminated lake sediments, which occur throughout almost the entire sediment profile of the deepest part of the lake (Ralska-Jasiewiczowa et al., 1998). Only recently, a detailed investigation of varve microfacies combined with multiproxy dating techniques have allowed a new chronology to be developed for the Lake Gościąż sediment record, establishing the onset of lacustrine sedimentation in the late Allerød at 12,844 +213/-311 varve yrs BP (Bonk et al., 2021; Müller et al., in press). Studies of contemporary sedimentation using sediment traps were first used in Lake Gościąż in 1988. Further studies were performed in 1991 (Kentzer and Żytkowicz, 1993) and in 1993-95 (Mieszczankin, 1997). They were conducted to analyse sediment composition (content of $\mathrm{N}, \mathrm{P}, \mathrm{CaCO}_{3}$, organic matter and plant pollen), differentiation of sedimentation rate in the water column, and seasonal variability of sedimentation rate (Kentzer and Żytkowicz, 1993; Mieszczankin and Noryśkiewicz, 2000). Research conducted in the 1990s showed that the sedimentation rate in Lake Gościąż is as much as several times higher than in other lakes with annually laminated sediments, and these differences result from its higher trophy and large contribution of resuspended matter (Mieszczankin, 1997). These studies were conducted using material collected from sediment traps located along a north-south axis. Our research analysed the course of sedimentation in an east-west transect, along the lake's longer axis and the prevailing wind direction. Thus, only the results of the analysis of the sedimentation rate at the site in the deepest part of the lake will be comparable.

The main objective of the presented research is to recognise the determinants of the course of contemporary sedimentation and the qualitative composition of sedimenting material. This study therefore aims to show the impact of meteorological factors and basin topography on the spatial differentiation of the sediment accumulation rate in the lake's epilimnion and hypolimnion. This issue will be presented against the seasonal variability in deposited matter. To achieve this goal, complementary meteorological and hydrological monitoring, which has not been conducted in the Lake Gościąż catchment area before, was carried out.

\section{Material and methods}

\subsection{Study area}

Lake Gościąż is located in the Gostynin Lakeland (Central Poland) at an elevation of $64.3 \mathrm{~m}$ a.s.l. (Fig. 1). The lake is located in a widening in the valley of the lower (lowland) course of the Vistula River (the longest river in Poland). This area was initially formed under the influence of glacial erosion and, later, fluvioglacial and fluvial erosion. The coexistence of accumulation and erosion processes led to the deposition of a series of sandy-gravelly sediments with an average thickness of 40$45 \mathrm{~m}$ (Skompski, 1969). The area's relief was shaped during the Weichselian glaciation and the 
Holocene. Its essential features are subglacial channels and fluvioglacial terraces (Wiśniewski, 1976). Sandy dunes created by aeolian activity in the Late Glacial are also abundant in this area (Urbaniak, 1967; Rychel et al., 2018; Kruczkowska et al., 2020). Lake Gościąż lies at the bottom part of a subglacial channel that developed during the recession of the Last Glacial ice sheet around 18,000 years ago. The lake formation started during the transition from Bølling to Allerød as a result of dead ice melting (Ralska-Jasiewiczowa et al., 1998). The properties of the relief and geological structure allow the Vistula's valley and bed to maintain hydrogeological connections to their adjacent areas. In such conditions, subglacial channels whose course runs vertically relative to the direction of groundwater flow are fed by groundwaters of local and regional circulation system (Gierszewski, 2000). In combination with other environmental features (including the poorly-varied subsoil lithology, high afforestation, and low human impact on the environment), this ensures that Lake Gościąż receives a steady supply of groundwater of relatively stable chemical composition, which in turn also results in a steady amount of sedimentation and stable sediment characteristics.

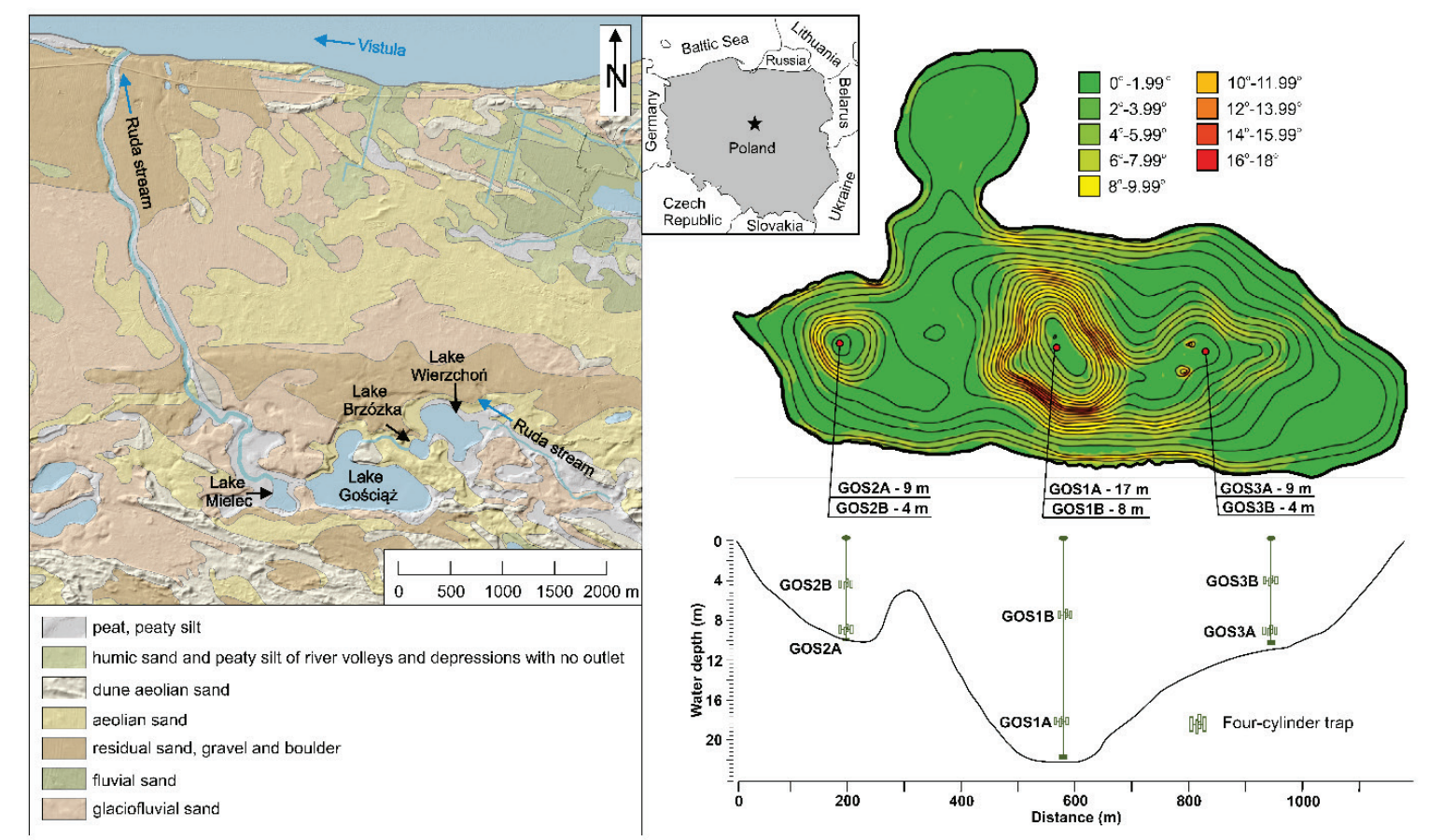

Figure 1. Location of the study area and the bathymetry and slope steepness map of Lake Gościaż with marked locations of sediment traps (geological background of the Lake Gościąz catchment area on the basis of Rychel et al., 2014).

The climate in this area is classified as cold temperate with warm summers (Peel et al., 2007). According to data from $1967-2015$, the annual average temperature is $8.2^{\circ} \mathrm{C}$, while the average sum of precipitation is $533 \mathrm{~mm}$ (Bartczak et al., 2019). The growing period lasts 210-215 days (Demidowicz et al., 1998). Westerly winds prevail in this area (Woś, 1999).

The lake catchment area is covered with pine forests. There are communities of Querco roborisPinetum wetter habitats, and Peucedano-Pinetum in drier locations (Kępczyński and Noryśkiewicz, 1998). There is a community of Carici elongatae-Alnetum sensu lato immediately around the lake, and a Tilio-Carpinetum community slightly further away. On submerged shores there are also brushwood communities represented by Salicetum pentandro-cinereaes. Aquatic vegetation inhabits the shallowest parts of the lake, especially in the shallow bay to the north. This vegetation is represented mainly by communities of Lemno-Spirodeletum polyrrhizae, Hydrocharitetum morsus-ranae, Elodeetum Canadensis and Potamogetonetum pectinati, and less commonly by Potamogetonetum filiformis, and 
also, at the south-western shore of the lake, by Potamogetonetum perfoliati. The slightly silty littoral parts of Gościąż Lake are overgrown with reedswamps. The largest areas are occupied by Phragmitetum communis and Typhetum angustifoliae. Smaller areas are occupied by Glycerietum maximae, Eleocharitetum palustris and Acoretum calami (Kępczyński and Noryśkiewicz, 1993).

Lake Gościąż is small $\left(0.417 \mathrm{~km}^{2}\right)$ and relatively deep (maximum depth $\left.22.1 \mathrm{~m}\right)$ (Table 1). It consists of two basins: a shallow northern one (Tobyłka Bay) and a main one with two clearly distinguished sub-basins. The lake is deepest in the middle (Fig. 1). Along with three other lakes Mielec, Brzózka and Wierzchoń - Lake Gościąż forms a lake system called "Na Jazach", which is connected by the stream Ruda. In the middle course of the stream, below Lake Gościąż, there is a weir that has existed in its present form since 1989 and is a remnant of a mill built in the 16th century. The groundwater watershed of the catchment area is $56 \mathrm{~km}^{2}$, of which $39 \mathrm{~km}^{2}$ is endorheic (Glazik, 1978; Gierszewski, 2000). The lakes in the studied catchment area are fed primarily (ca. 90\%) by groundwater, mainly from the south (Gierszewski, 2000). The groundwater supplying the Lake Gościąż is of Ca$\mathrm{HCO}_{3}-\mathrm{SO}_{4}$ type, with low mineralisation (averaging $260 \mathrm{mg} \cdot \mathrm{dm}^{-3}$ ) (Gierszewski,, 2000; 2001). The biogeochemical changes in the lake have a slight effect - a decrease of $20 \mathrm{mg} \cdot \mathrm{dm}^{-3}$ in the total mineralisation of the water, mainly seen in lower concentrations of $\mathrm{Ca}$ and $\mathrm{SO}_{4}$ ions. As a result, the ionic type of the water also changes to $\mathrm{Ca}-\mathrm{Mg}-\mathrm{HCO}_{3}$. The supply of nutrients in the groundwater exceeds safe thresholds for the harmonious functioning of the lake. In the years 1991-93 this averaged about $3 \mathrm{~g}$ $\mathrm{P} \mathrm{m}^{-2}$ and about $40 \mathrm{~g} \mathrm{~N} \mathrm{~m}^{-2}$. The N:P ratio of 14:1 indicates that in terms of fertility, Lake Gościąż is eutrophic (Giziński et al., 1998).

Table 1. Morphometric parameters of the Lake Gościąż.

\begin{tabular}{|c|c|}
\hline Location & $52^{\circ} 34 ' 58^{\prime \prime} \mathrm{N} 19^{\circ} 20^{\prime} 23^{\prime} ' \mathrm{E}$ \\
\hline Water level (m a.s.1.) & 64.3 \\
\hline Surface $\left(\mathrm{km}^{2}\right)$ & 0.417 \\
\hline Maximum length (m) & 1180 \\
\hline Maximum width (m) & 723 \\
\hline Medium width (m) & 357 \\
\hline Maximum depth (m) & 22.1 \\
\hline Average depth (m) & 5 \\
\hline Elongation indicator (m) & 1.59 \\
\hline Shoreline length (m) & 3452 \\
\hline Development of the coastline & 1.51 \\
\hline Capacity $\left(\mathrm{m}^{3}\right)$ & 2073000 \\
\hline
\end{tabular}

The lake freezing period averages about 75 days in this region (Choiński et al., 2015). In terms of mixing, Lake Gościąż represents a bradimictic type (Churski and Marszelewski, 1998). The lake has a well-developed summer stratification, with a distinct thermocline with a strong gradient of $3.5^{\circ} \mathrm{C} \mathrm{m}^{-1}$. At the same time, the level of oxygenation diminishes steadily down though the water column (Gierszewski, 2000). The deoxygenated waters of the hypolimnion have a higher concentration of $\mathrm{Ca}^{2+}$ $(25 \%), \mathrm{HCO}_{3}{ }^{2-}, \mathrm{NH}_{4}{ }^{+}$and $\mathrm{PO}_{4}{ }^{3-}$ ions. However, the concentration of $\mathrm{SO}_{4}(20 \%)$ and the $\mathrm{pH}$ of the water are significantly lower. The chemical properties of the water in Lake Gościąż (in addition to the assimilation of biogenic substances by aquatic organisms) is transformed by changes related to the functioning of the carbonate system and redox reactions (Gierszewski, 2000). 
Due to its morphological and hydrobiological features, Lake Gościąż has two zones with different water dynamics: a shallow-water zone (to a depth of 5-6 m), which covers about $50 \%$ of the lake's bottom surface, and a deep-water zone. The bottom sediments in the polymictic shallow-water zone are subject to frequent redeposition and displacement towards the middle of the lake. The highest accumulation of sediments occurs in the deep-water zone, which is static and deoxygenated during the summer stratification (Giziński et al., 1998). The studies conducted in the 1990s showed the mean sedimentation rate to be $5.9 \mathrm{~g} \mathrm{~m}^{-2} \mathrm{~d}^{-1}$ in the epilimnion and $13.1 \mathrm{~g} \mathrm{~m}^{-2} \mathrm{~d}^{-1}$ in the hypolimnion (Mieszczankin, 1997). The maxima occurred during overturn, and the minima in summer stratification periods. The low summer sedimentation rate is conditioned by the thermocline, which is a natural barrier to the sedimentation of matter. The greater sedimentation in traps in the hypolimnion, especially during overturns, is associated with resedimentation processes, and during summer stratifications should be connected with the "funnel effect". The most important component of sediment was $\mathrm{CaCO}_{3}$ (Giziński et al., 1998).

\subsection{Sampling and analyses}

Sediment samples were collected from August 2017 till May 2019 at four- to seven-week intervals except for periods of ice cover on the lake. The research was carried out using sediment traps at three different points in the lake (GOS 1, GOS 2 and GOS 3) (Fig. 1). At each, two sediment traps were installed: one in the lower epilimnion (traps GOS 1B, 2B and 3B) and the other in hypolimnion above the bottom (traps GOS 1A, 2A and 3A). Each trap consisted of four cylinders of $8 \mathrm{~cm}$ in diameter and $50 \mathrm{~cm}$ high. In the deepest part of the lake (GOS 1) thermal monitoring was also conducted using a HOBO Water Temperature Pro v2 Data Logger. The water temperature was measured at 30-minute resolution at depths of $0,1,2,3,4,5,6,7,8,9,10,12,14,18$ and $20 \mathrm{~m}$. During each sediment traps receiving, a Secchi disc was also used to measure water transparency, and in the period from July 2017 to December 2018, a Hanna HI 9829 multiparametric meter was also used to measure the dissolved oxygen content at the lake's deepest point (GOS 1), from the surface down to the bottom. In 2017, bathymetric measurements of the lake were taken with a Lowrance HDS-7 echosounder.

The course of weather conditions was recorded using a HOBO RX300 Station - CELL-3G automatic weather station at $2 \mathrm{~km}$ from the lake. The station records the following parameters hourly: air temperature, precipitation, and direction, speed and gusts of wind. Lake water levels were recorded every 0.5 h using a HOBO U20-001-04 water level recorder.

To determine dry weight of sediment, the material from sediment traps was centrifuged and dried for $24 \mathrm{~h}$ at $105^{\circ} \mathrm{C}$, and then weighted to an accuracy of $0.001 \mathrm{~g}$. Mean sediment-accumulation rate was expressed in grams of dry matter per square metre per day $\left(\mathrm{g} \mathrm{m}^{-2} \mathrm{~d}^{-1}\right)$.

To determine the quantitative composition, sediment samples were analysed for the content of organic matter, calcium carbonate, and biogenic and terrigenous silica. Organic matter content was determined by roasting of the sample for 4 hours at $550^{\circ} \mathrm{C}$ (Heiri et al., 2001), and calcium carbonate by the Scheibler method. Biogenic silica concentration was calculated as the difference in weight between total silica content and the share of terrigenous silica. Total silica was determined after dissolution of acid-soluble fractions and organic matter in $\mathrm{H}_{2} \mathrm{SO}_{4}$, and the terrigenous silica was the residue after removal of opaline (biogenic) silica from the total silica with $0.5 \mathrm{n} \mathrm{NaOH}$ in a water bath at $100^{\circ} \mathrm{C}$ for 2 hours (Bechtel et al., 2007). The difference between $100 \%$ and the total sum of organic matter, $\mathrm{CaCO}_{3}$, terrigenous and biogenic silica represents "mineral residue" (MR). The "mineral residue" includes hot-acid-extractable compounds excluding carbonates (e.g. sulphides, some clay minerals, etc.) (Woszczyk et al., 2009). 
Prior to smear slide preparation, 66 fresh sediment trap samples were transferred to a microscope slide and dried on a heating plate. Then, a cover slip was placed on the dried sediment using glycerine. Ready smear slides were analysed under a light microscope at $20 \times$ to $200 \times$ magnification to track the seasonal changes within the sediment components. This analysis is only a semi-quantitative analytical tool, but it provides insights into the sediment components and allows to recognize a relative change in lithology (Schnurrenberger et al., 2003). The components of material retrieved from sediment traps can be grouped in three categories: organic, diatoms and carbonates.

The twenty-month-long measurement period of water temperature made it possible to construct depth profiles of water temperature (Surfer Software) for the deepest part of the lake (GOS 1). The data from bathymetric measurements were next converted into numerical .csv files, which were processed by Arc GIS version 10.2.2 software (ESRI 2014) to generate a bathymetric map and a map of bottom slope steepness. The bathymetric data were used to calculate the morphometric parameters of the lake basin.

\section{Results}

\subsection{Sediment accumulation rate}

Mean sediment accumulation rate in each of the three monitored locations was higher in the traps in the hypolimnion (deeper trap). In the epilimnion traps, the accumulation varied from $0.94 \mathrm{~g} \mathrm{~m}^{-2} \mathrm{~d}^{-1}$ to $5.95 \mathrm{~g} \mathrm{~m}^{-2} \mathrm{~d}^{-1}$ (Fig. 2). The lowest sediment accumulation rate for traps GOS 1B and GOS 3B were measured in winter 2018-19 and reached only $1.24 \mathrm{~g} \mathrm{~m}^{-2} \mathrm{~d}^{-1}$ and $1.18 \mathrm{~g} \mathrm{~m}^{-2} \mathrm{~d}^{-1}$, respectively. In trap GOS 2B, the accumulation rate was lowest between 27 July and 25 August 2017, and amounted to $0.94 \mathrm{~g} \mathrm{~m}^{-2} \mathrm{~d}^{-1}$. The largest amount of sediment was accumulated in trap GOS 1B between 13 July and 13 August 2018 (4.24 $\left.\mathrm{g} \mathrm{m}^{-2} \mathrm{~d}^{-1}\right)$, in GOS 2B between 26 August and 9 October $2017\left(5.95 \mathrm{~g} \mathrm{~m}^{-2} \mathrm{~d}^{-1}\right)$, and in GOS 3B between 14 March and 11 April $2019\left(4.54 \mathrm{~g} \mathrm{~m}^{-2} \mathrm{~d}^{-1}\right)$. In the traps located in the hypolimnion, the sediment accumulation rate varied from $1.93 \mathrm{~g} \mathrm{~m}^{-2} \mathrm{~d}^{-1}$ to $10.21 \mathrm{~g} \mathrm{~m}^{-2} \mathrm{~d}^{-1}$. The lowest accumulation rate in GOS $1 \mathrm{~A}$ was measured between 25 May and 12 July $2018\left(1.93 \mathrm{~g} \mathrm{~m}^{-2} \mathrm{~d}^{-1}\right)$, whereas in traps GOS 2A and GOS 3A, it was between 5 December 2018 and 13 March $2019\left(2.17 \mathrm{~g} \mathrm{~m}^{-2} \mathrm{~d}^{-1}\right.$ and $1.46 \mathrm{~g} \mathrm{~m}^{-2} \mathrm{~d}^{-1}$, respectively). In GOS 1A, the sediment accumulation rate was highest between 10 October and 10 November 2017, and reached $10.21 \mathrm{~g} \mathrm{~m}^{-2} \mathrm{~d}^{-1}$. In GOS 2A and GOS 3A it was the highest from 14 March till 11 April 2019, amounting up to $8.28 \mathrm{~g} \mathrm{~m}^{-2} \mathrm{~d}^{-1}$ and $6.47 \mathrm{~g} \mathrm{~m}^{-2} \mathrm{~d}^{-1}$, respectively. There is no data on the course of sedimentation in the GOS 1A and GOS 2B traps from October 2018 to April 2019 and in the GOS 3A trap from May 2018 to October 2018 because the traps disappeared. It should also be noted that the results obtained for April 2018 (containing sedimentation material from the winter season) are a value averaged over the five-month period. Therefore, these samples contain material from the end of the autumn overturn, the winter and early spring. Mean sediment accumulation rate in the deepest location (GOS 1) was 71\% higher in the hypolimnion than in the epilimnion. In the western location (GOS 2), the difference reached $57 \%$ in favour of the deeper trap, while in the eastern part of the lake (GOS 3) it was only $29 \%$. In the deepest lake sub-basin (near GOS 1), slope steepness on the south-western side is up to $18^{\circ}$ (Fig. 1). In the western part (near GOS 2), it is up to $14^{\circ}$, while in the eastern part (near GOS 3), where sedimentation values are the lowest, slope steepness does not exceed $4^{\circ}$. In the hypolimnion, the trap in the western part (GOS 2A) accumulated on average $41 \%$ more sediment than the one in the eastern part (GOS 3A), while in the deepest part of the lake (GOS 1A) the western trap averaged 38\% more than the eastern trap (GOS $3 \mathrm{~A})$. In the traps in the epilimnion, the average sediment accumulation rate was $16 \%$ higher in the western part of the lake (GOS 2B) than in the eastern part (GOS 3B), and in the deepest part (GOS 1B) it was 5\% higher than in the east (GOS 3B). 
Fojutowski et al.

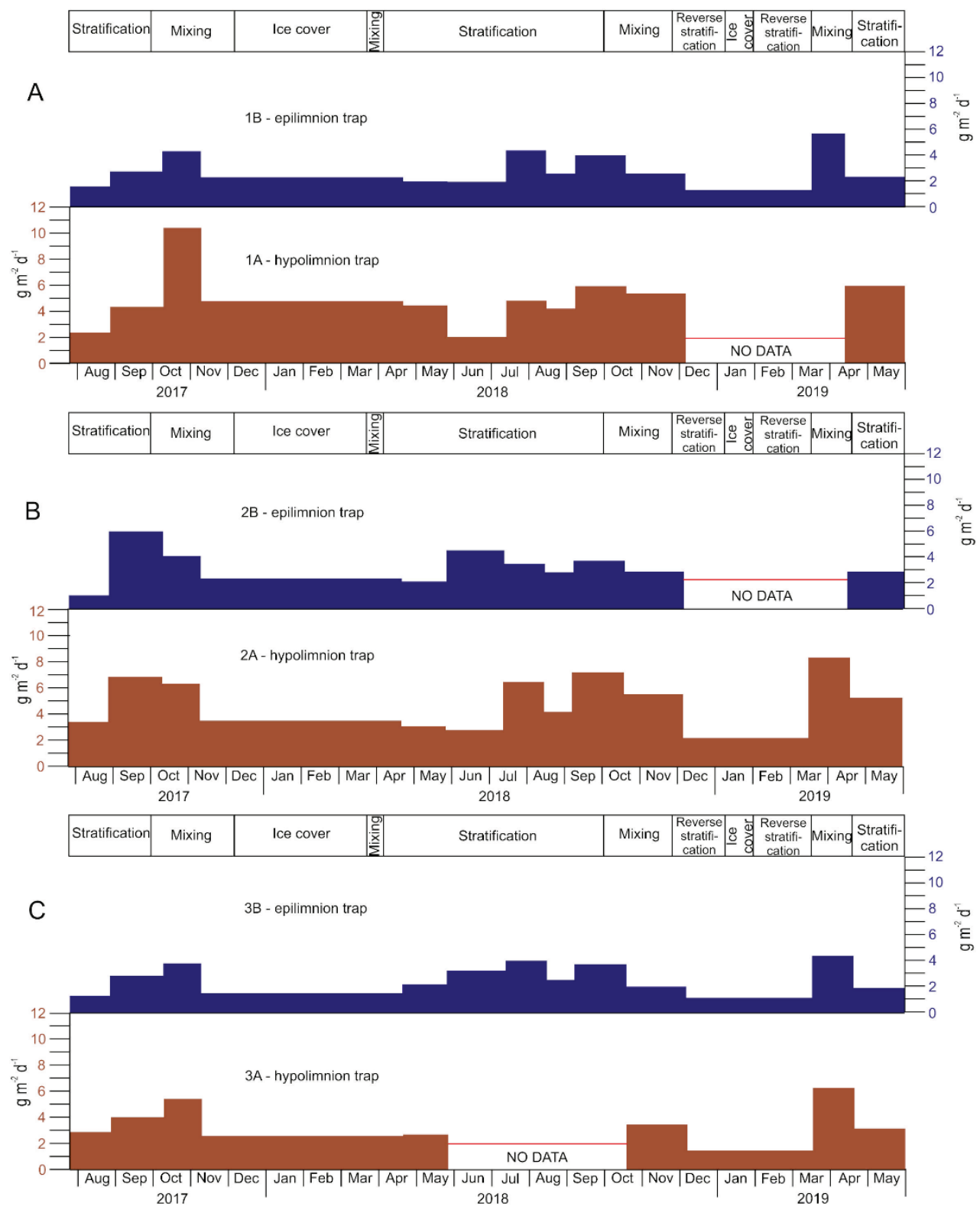

Figure 2. Sediment accumulation rate in Lake Gościqż: A - sediment traps GOS1A and $1 B ; B$ - sediment traps

GOS2A and 2B; C-sediments traps GOS3A and 3B. No data $1 A$ (December 2018 - April 2019), $2 A$

(December 2018 - April 2019), 3B (May 2018 - October 2018); D - air temperature (blue line), precipitation (orange line) and time of the sediment trap retrieving (black bars); $E$-variability in thermal conditions at the deepest site in Lake Gościaż. 

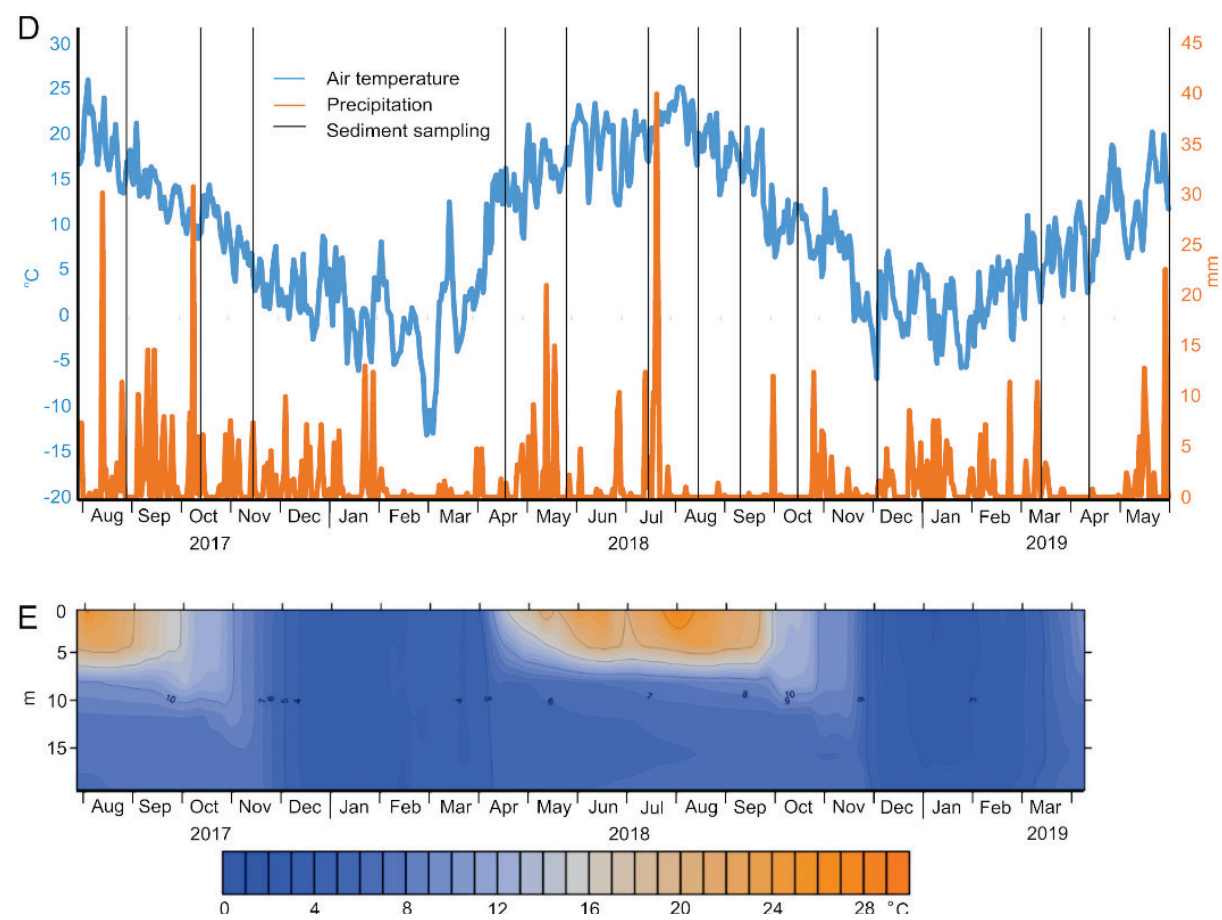

Figure 2. Sediment accumulation rate in Lake Gościąz: $A$ - sediment traps GOS1A and $1 B ; B$ - sediment traps GOS2A and 2B; C-sediments traps GOS3A and 3B. No data 1A (December 2018 - April 2019), $2 A$ (December 2018 - April 2019), 3B (May 2018 - October 2018); D - air temperature (blue line), precipitation (orange line) and time of the sediment trap retrieving (black bars); E-variability in thermal conditions at the deepest site in Lake Gościąz. (cont.)

\subsection{Meteorological conditions}

The highest air temperature in the study period was $35.3^{\circ} \mathrm{C}$, recorded on 9 August 2018, whereas the lowest value was $-19.6^{\circ} \mathrm{C}$, recorded on 27 February 2018. Mean daily temperatures (Fig. 2D) varied from $26.0^{\circ} \mathrm{C}$ (1 August 2017) to $-13.1^{\circ} \mathrm{C}(26$ February 2018). The highest monthly precipitation of 103.4 $\mathrm{mm}$ was observed in July 2018, compared to only $1 \mathrm{~mm}$ in April 2019. Daily precipitation was the highest on 18 July 2018 (40 mm).

The prevailing winds were southerly (26.9\%), south-westerly $(21.9 \%)$, northerly $(18.2 \%)$, and north-westerly $(15.6 \%)$. In autumn and winter, we detected markedly higher contributions of southerly winds $\left(157.5^{\circ}-202.5^{\circ}\right)$, with up to $51.6 \%$ in October 2017 , and south-westerly winds $\left(202.5^{\circ}-247.5^{\circ}\right)$, with up to $36.9 \%$ in December 2018. In spring and summer of each year, winds were mostly northerly $\left(337.5^{\circ}-22.5^{\circ}\right)$, north-westerly $\left(292.5^{\circ}-337.5^{\circ}\right)$, and westerly $\left(247.5^{\circ}-292.5^{\circ}\right)$ (Fig. 3). Generally, during the studied seasons, winds along the NE-SW axis dominated from September till April. Particularly conspicuous was the dominance of winds from the directions $180^{\circ}-270^{\circ}$, which in October-November 2017 reached as much as $85 \%$ of the total. In the summer half-year (April-October), the dominant wind directions were $270^{\circ}-360^{\circ}$, as their percentage contributions were then around $70 \%$, with up to $78 \%$ in May-July 2018. The average wind speeds in individual measurement series ranged from $0.58 \mathrm{~m} \mathrm{~s}^{-1}$ (AugSept 2018) to $0.85 \mathrm{~m} \mathrm{~s}^{-1}$ (Mar-Apr 2019) (Table 2). However, in terms of their impact on the lake-water hydrodynamics, momentary wind speeds - gust speeds - are important. Longer periods of wind with higher gust speeds were found in October 2017, March, June, July and October 2018, and March 2019. The maximum wind speed values measured ranged from 6 to $13.1 \mathrm{~m} \mathrm{~s}^{-1}$. 
Fojutowski et al.

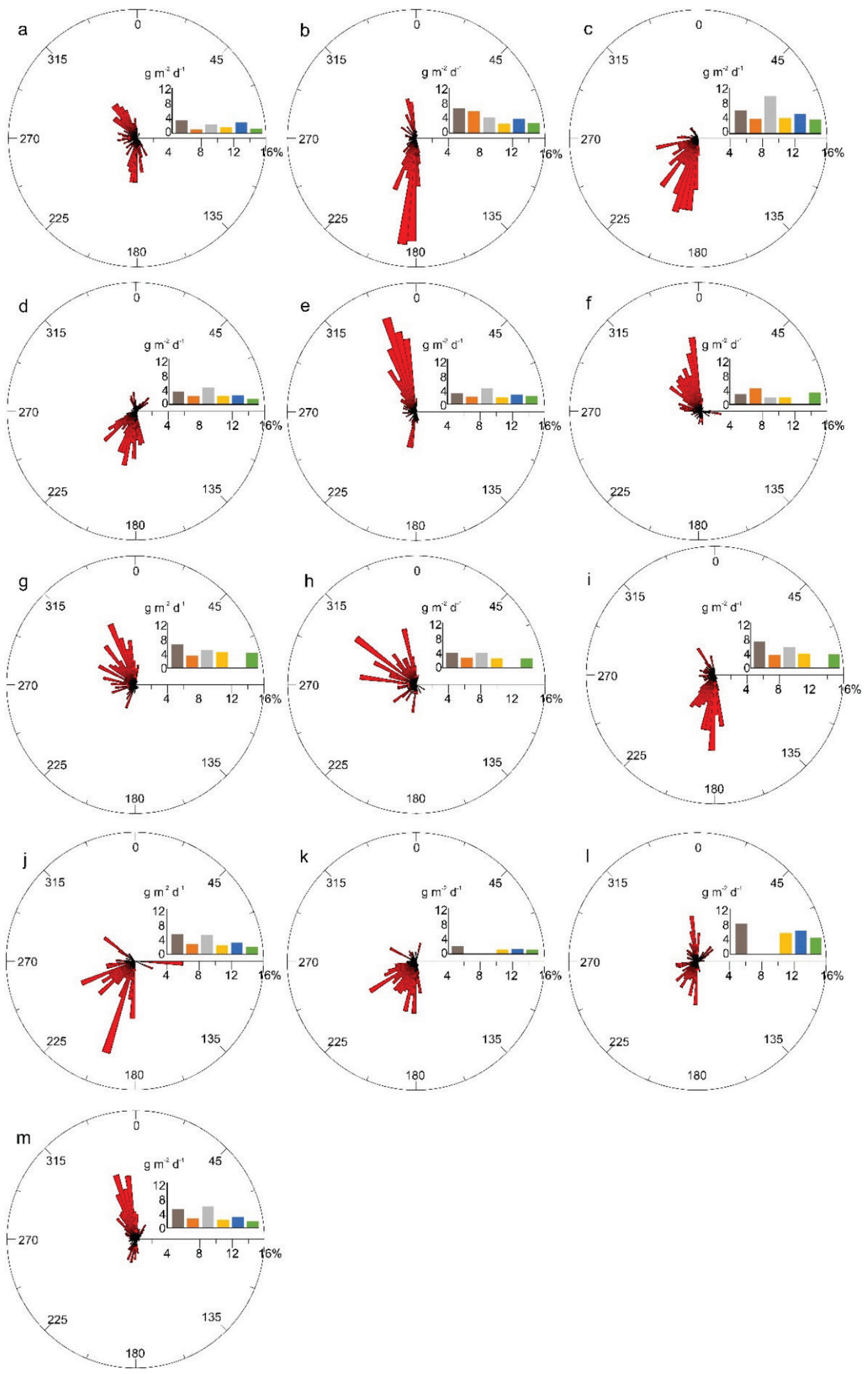

Figure 3. Wind direction: $a$ - 27 July 2017- 25 Aug 2017; b- 25 Aug 2017 - 9 Oct 2017; c - 9 Oct 2017-10 Nov 2017; d-10 Nov 2017-19 Apr 2018; e-19 Apr 2018-25 May 2018; f- 25 May 2018-12 Jul 2018; g- 12 Jul 2018-13 Aug 2018; $h$ - 13 Aug 2018-7 Sep 2018; i- 7 Sep 2018-15 Oct 2018; j- 15 Oct 2018-4 Dec 2018; $k-4$ Dec 2018-13 Mar 2019; l-13 Mar 2019-11 Apr 2019; m - 11 Apr 2019-30 May 2019; Sediment traps: brownGOS 1A, orange - GOS 1B, gray - GOS $2 A$, yellow - GOS $2 B$, blue - GOS 3 A, green - GOS $3 B$. 


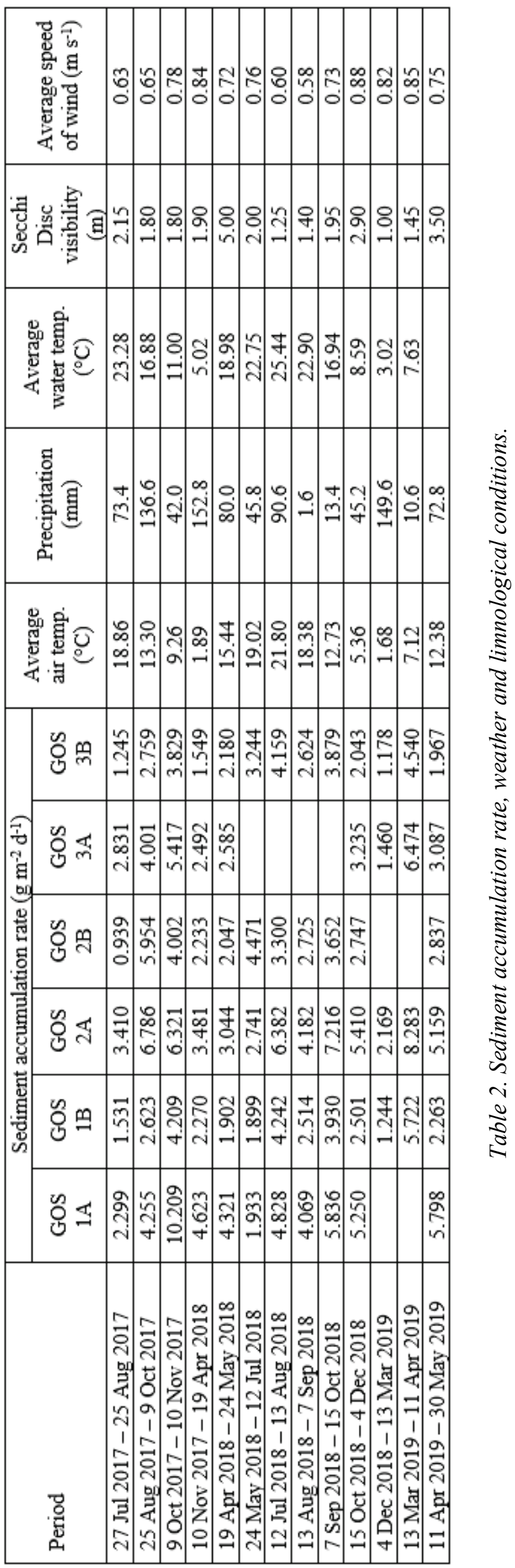




\subsection{Limnological conditions}

The amplitude of water level fluctuations in Lake Gościąż in the period from July 2017 to May 2019 was $40 \mathrm{~cm}$. In the first (incomplete) monitoring season (from July 2017), a thermal stratification was developed until the end of September 2017 (Fig. 2E). At the beginning of October 2017, the autumn mixing began. The water column mixed fully in the second half of November 2017. At the beginning of December 2017 an ice cover appeared on the lake and lasted until mid-March 2018. During this time, reverse stratification was observed. After the ice cover melted, the spring mixing took place. The first days of May 2018 showed the beginning of stratification, which progress was briefly interrupted by a cooling mid-month when the air temperature during the day did not exceed $20^{\circ} \mathrm{C}$. In the second half of June 2018, the water temperature in the epilimnion fell again, caused by the onset of a sharp chill. Within an hour, the air temperature dropped dramatically by $15^{\circ} \mathrm{C}$, and did not exceed $16^{\circ} \mathrm{C}$ for next several days. In the first decade of July 2018 , the temperature of the surface layer rose again and remained stable until the second half of September 2018. During the thermal stratification of the lake, the hypolimnion was completely anoxic, as recorded in measurements of oxygen concentration in the water (Fig. 4A). In May 2018, a total oxygen deficit was found from a depth of $10 \mathrm{~m}$ downwards, and from $8 \mathrm{~m}$ in June 2018. At the beginning of August 2018, oxygen conditions in the hypolimnion improved slightly. In October 2018, the autumn mixing began, and was completed in the beginning of December 2018. Ice cover appeared on the lake in the second half of January 2019 and lasted for only about a month. Reverse stratification then developed. After the lake had thawed, there was a spring mixing. The thermal stratification phase of the lake began in the first half of April 2019 already. The water measurements revealed substantial variations in temperature (up to $3.1^{\circ} \mathrm{C}$ ) in a short time (30 min) during the stratification period at the depth thermocline ( $8 \mathrm{~m})$ (Fig. 4B).

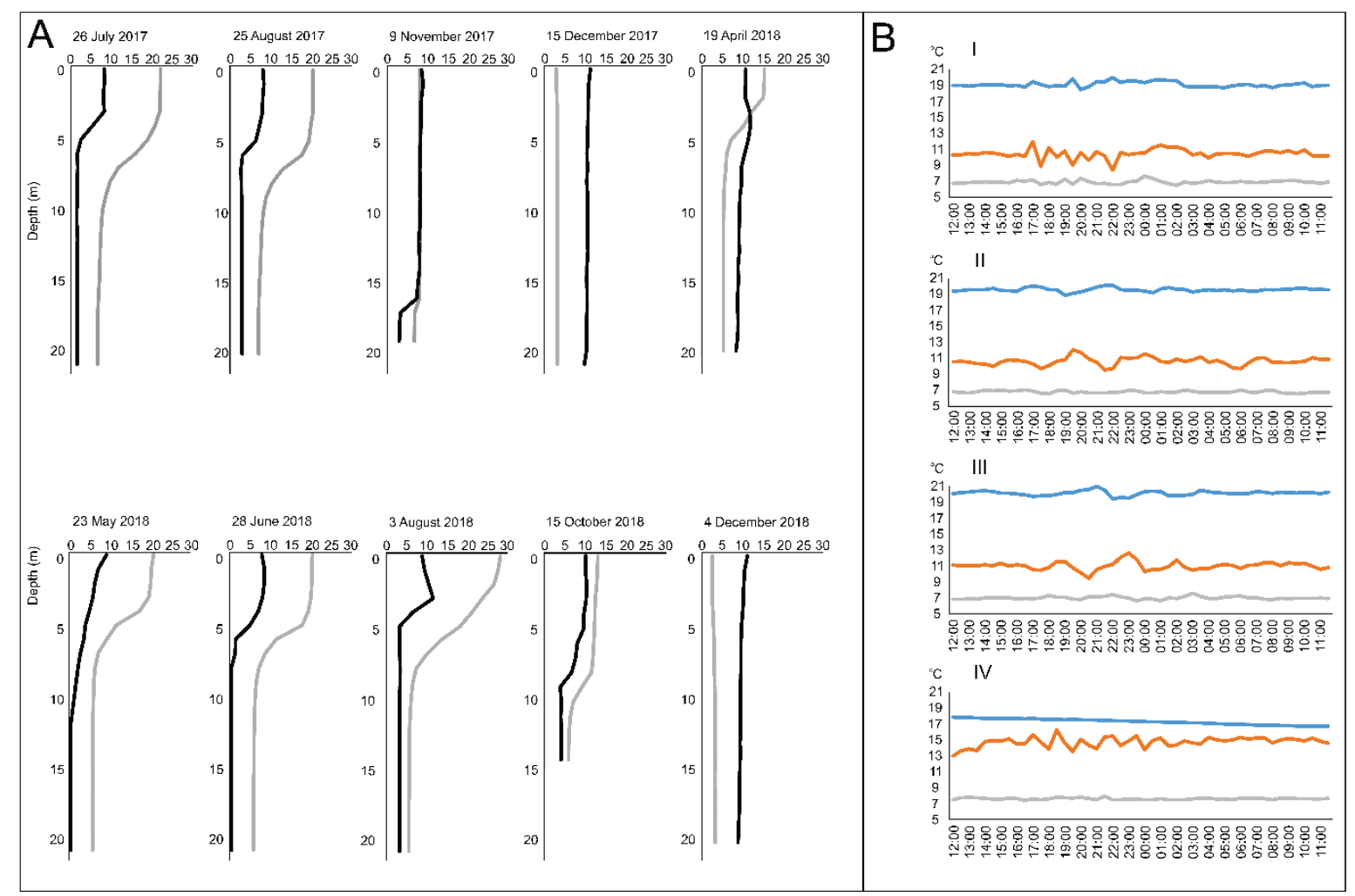

Figure 4. A: The water temperature in ${ }^{\circ} \mathrm{C}$ (gray line) and dissolved oxygen in $\mathrm{mg} \mathrm{dm}^{-3}$ (black line) in Lake Gościąz; B: selected examples of water temperature at $6 \mathrm{~m}$ (blue line), $8 \mathrm{~m}$ (orange line), $10 \mathrm{~m}$ (gray line) depth. I - 18.07.2018 (12:00) - 19.07.2018 (12:00); II - 25.07 .2018 (12:00) - 26.07.2018 (12:00); III $3.08 .2018(12: 00)$ - 4.08.2018 (12:00); IV - 24.09.2018 (12:00) - 25.09.2018 (12:00). 
Measurements of Secchi disc visibility determined that water transparency was lowest (about $1 \mathrm{~m}$ ) in early spring (March 2018 and April 2019). A noticeable increase in Secchi disc visibility was recorded in May (from $3.5 \mathrm{~m}$ to $5.0 \mathrm{~m}$ ). In summer and early autumn, the water transparency again decreased to $1.0-1.5 \mathrm{~m}$. After this period, Secchi disc visibility increased to $3 \mathrm{~m}$.

\subsection{Sedimentation cycle}

The qualitative analysis of 66 smear slides showed a clear seasonal variation in sample composition. In general, sample content in a given season was similar at all locations and both depths. The annual cycle started in spring, immediately after ice cover disappearance (Fig. 5). The spring sedimentation was dominated by phytoplankton appearance (diatoms) and followed by calcite (ca. 6-8 $\mu \mathrm{m})$ autochthonous precipitation. We also observed coarse $(>6-8 \mu \mathrm{m})$ but rare calcite agglomerates, which in contrary to fresh, single grains, could have been transported to the lake bottom through resuspension from the littoral zone. During summer, the amount of diatoms was very low in comparison to the number of zooplankton remains, which increased markedly. Fresh calcite grains were abundant. During the autumn, sediment was enriched with fresh calcite and its agglomerates and diatoms. However, the amount of diatoms was much lower than during the spring, which leads to a conclusion that the calcite was the main component of the retrieved sediment. Deposition of mineral matter (rare grains of quartz), scarce diatoms, fungi and remains of plant tissues prevailed in the winter. During the whole cycle, a green alga (Phacotus) with a calcite lorica was observed.

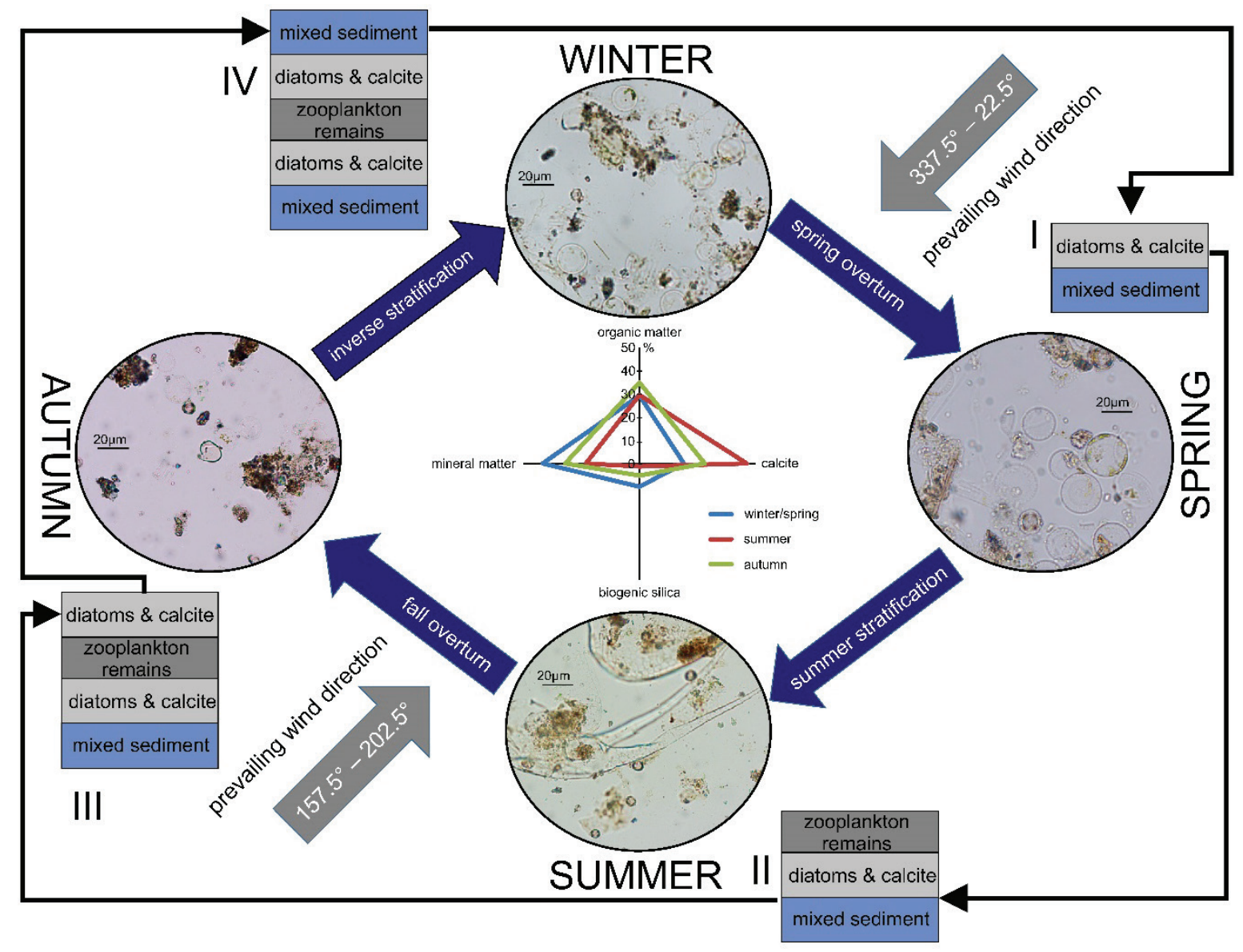

Figure 5. The seasonal changes within sedimentation cycle within Lake Gościąż. 
The smear slide analysis results are generally consistent with the results of the quantitative geochemical studies on the 57 sediment samples (Fig. 6). They showed that the tested sediments comprise $18.7-53.3 \%$ of organic matter (averaging 30.9\%) and $9.7-64.8 \%$ of calcium carbonate (averaging 28.7\%). The highest contribution of organic material was found in samples representing the summer and autumn months. Calcium carbonate sedimented mainly in summer (July, August). The lowest content of calcium carbonate was measured in samples taken in spring (April, May). Biogenic silica accounted for $0.4-27.3 \%$ (averaging $6.2 \%$ ) and terrigenous silica for $0.8-41 \%$ (averaging $14.5 \%$ ). Both forms of silica were most strongly represented in spring and autumn samples. The seasonal variability of the sediment composition was, however, very similar across all locations for particular measurement series. Against this background, however, the GOS 3A site stands out, as its sediments had a significantly higher content of terrigenous material and a lower content of calcium carbonate. The GOS 2 site was also particular, as, unlike the other two, it had a clearly greater vertical differentiation in biogenic silica content.
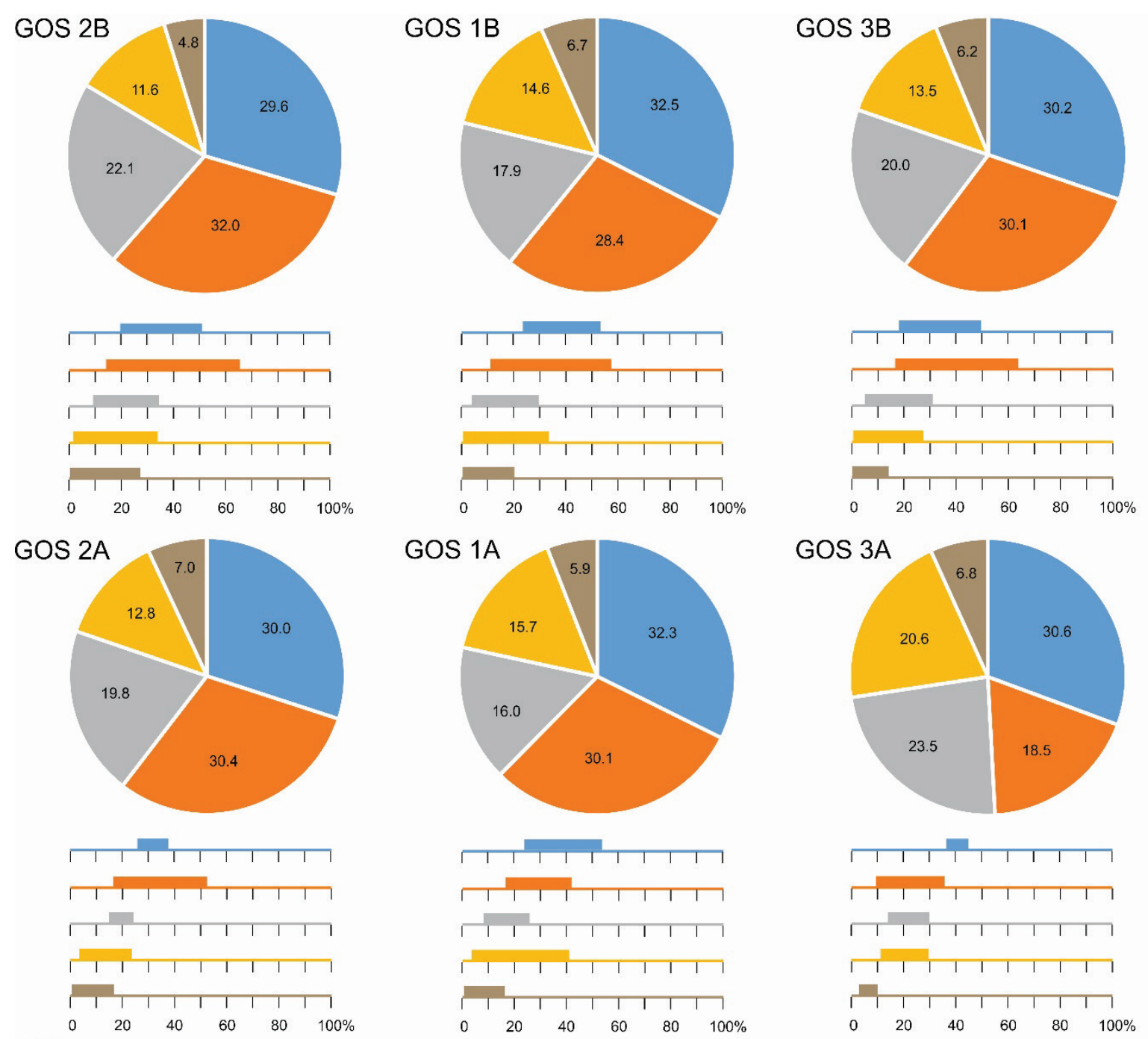

W
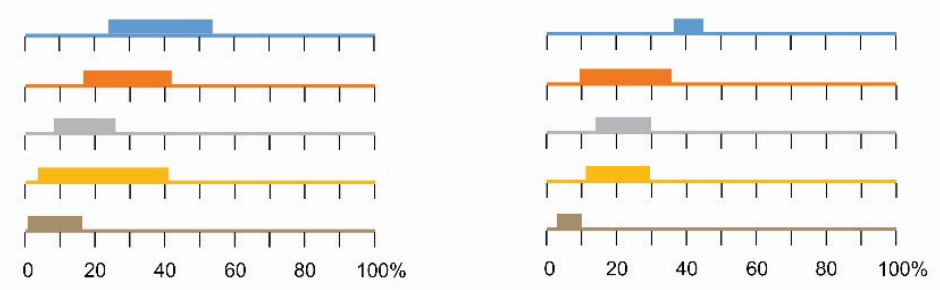

Figure 6. Properties of the sediment collected in the sedimentation traps in Lake Gościaż: average values and ranges (in percent). Organic matter (blue), calcium carbonate (orange), mineral residue (gray), terrigenous silica (yellow) and biogenic silica (brown). Epilimnion traps (GOS 2B, GOS 1B, GOS 3B), hypolimnion traps (GOS 2A, GOS 1A, GOS 3A). 


\section{Discussion}

\subsection{Spatial variation in sedimentation}

Previous studies on sediment accumulation rates have shown cases of sediment both decreasing with increasing depth (Bloesch and Uehlinger, 1986; Botwe et al., 2017) and the reverse (Kentzer and Żytkowicz, 1993; Ott et al., 2018). In almost all cases, our study results on contemporary sediment accumulation rates in Lake Gościąż showed the amount of material deposited in traps increasing with depth. The only case of sediment accumulation rate in a trap installed in the epilimnion (in the GOS 2B trap) being greater than that in the hypolimnion (GOS 2A) occurred in July 2018, and may have been caused by an increased concentration of phytoplankton in the western part of the lake. This should be associated with the effect of the surface water layer being pushed or displaced by a strong easterly wind (gusts of up to $7.5 \mathrm{~m} \mathrm{~s}^{-1}$ for 3 days) at the end of June 2018. During the lake mixing (early spring and autumn) differences in the amount of sediment between the traps in the hypolimnion (A traps) and those in the epilimnion (B traps) were largest. More than once, especially in autumn, the amount of sediment in the A traps was more than double that in the B traps. During the summer stratification, when a wellestablished thermocline is a natural barrier to falling sediment (Lastein, 1976; Mieszczankin, 2004), the differences between the traps in the epilimnion and those in the hypolimnion were smaller. Similar relationships were noted during the research conducted on Lake Gościąż in 1991 (Kentzer and Żytkowicz, 1993) and in 1993-95 (Mieszczankin, 2004). Then too, summer quantities of sediment were similar at various depths, while in spring, and especially in late autumn, the rate of accumulation in the hypolimnion was several times greater than that in the epilimnion. The higher rate of sedimentation during the lake's overturn should be associated with an increased proportion of material from resuspension (Mieszczankin, 2004).

Assuming that the volume of primary production is similar in various places in such a small lake, the spatial variation in the amount of sedimentation is determined by other factors. In the case of lakes fed by river discharge, the influence of the density currents on the course and the distribution of sedimentation is greater (Lewis et al., 2002; Cohen, 2003; Schiefer and Gilbert, 2008). The significance of this factor may be dismissed in the case of Lake Gościąż. The small inflow (the average annual discharge is about $13 \mathrm{dm}^{3} \mathrm{~s}^{-1}$ ), which disappears in summer and autumn, plays practically no role in supplying matter. In small, shallow lakes, this variability is mainly determined by the topography (Terasmaa, 2005) steepness of the lake basin (Blais and Kalff, 1995) and the distance of the measuring point from the shore (Bennett and Buck, 2016; Graham et al., 2016). Higher sediment accumulation rates are found in a lake's deepest places, and in positions closer to the littoral zone. This is also true of Lake Gościąż. The lake basin morphology favours this. It consists of two steep-sloped basins (Fig. 1) separated by a clear threshold. This kind of the topography means that the sediment accumulation rate at the GOS 1 and GOS 2 stations is markedly higher than at GOS 3. This can be attributed to a "funnel effect", which involves the stream of falling particles increasing in concentration, translated into a higher sediment accumulation rate in the steepest zones of the lake basin. Material from the shallower parts of the lake being supplied can also not be discounted. Particularly favourable conditions in terms of sedimentation rate occurred at the GOS 2 site. This is due to the specific features of the location. The most important are the good sheltered of the local pool and the proximity of the littoral zone. The distal location of the site is also important, as it favours the supply of material from shallower parts of the lake via the action of internal waves along the bottom. This last factor has a particularly large impact on the course of sedimentation at the GOS 3 site. The supply of material from the littoral zone to the sediment trap, which is in the hypolimnion, is evidenced by the high share of mineral material in the sediment composition (Fig. 6).

During the 1991 study, the sediment accumulation rate at the deepest point of the lake averaged $8.65 \mathrm{~g} \mathrm{~m}^{-2} \mathrm{~d}^{-1}$, while this value once reached $28.56 \mathrm{~g} \mathrm{~m}^{-2} \mathrm{~d}^{-1}$ (in October 1991). Sediment accumulation rate values in other months ranged from $1.84 \mathrm{~g} \mathrm{~m}^{-2} \mathrm{~d}^{-1}$ to $13.61 \mathrm{~g} \mathrm{~m}^{-2} \mathrm{~d}^{-1}$ (Kentzer and Żytkowicz, 1993) and were comparable with the 2017-19 results. This is indirect evidence that environmental conditions 
in the catchment are stable. Over the past 30 years there have been no changes in land use. Since 2001, Lake Gościąż's drainage basin has been protected as a reserve and human impact has been negligible. The form and structure of forest use has not changed in that time.

\subsection{Seasonal variation in sedimentation cycle and its potential for varve formation and preservation}

When examining the sediment accumulation rate in lakes, the local climate should be taken into consideration, since it may affect the seasonal variability of sedimentation. The amount of sediment can have either one or two peaks in a year and vary in duration. One commonly observed case is the clear increase in the amount of sediment in early spring. Sometimes this peak is persisted through the summer (Ott et al., 2018; Johansson et al., 2019). It can also happen that the peak occurs only in the summer (Bloesch and Uehlinger, 1986). Usually, however, two peaks - in early spring and autumn - can be found (Bonk et al., 2015; Ott et al., 2018). This last case applies to Lake Gościąż. The spring and autumn increase in sedimentation rate primarily result from increased primary production in the lake. This is favoured by the increase in air temperature in spring, the supply of nutrients from the lake bottom during periods of water mixing, and from the lake catchment in spring. According to our observations, a significant proportion of the material gathered in the sedimentation traps (especially traps in the hypolimnion) is redeposited from the lake basin.

The thermal conditions of Lake Gościąż are typical for dimictic water bodies. The temperature measurements showed clear thermal stratification from April 2018 to October 2018, and overturn in spring and autumn. Stratification and mixing periods significantly affect the amount of dissolved oxygen in the water column. Oxygen content is particularly important when considering the preservation of laminated sediments. For conditions to be conducive to preserving lamination, bottom waters should be devoid of, or relatively low in oxygen for at least part of the year (Anderson and Dean, 1988). The extent to which oxygen-free (anoxic) conditions are created is influenced by both how much organic matter is produced and the intensity of circulation in the water column (Kienel et al., 2013). The relatively short period of anoxia in Lake Gościąż (from May 2018 to July 2018) may have adversely affected the preservation of sediment lamination. Compared to studies from the 1990s, when anoxia lasted from May to October, anaerobic conditions now last considerably shorter. However, detailed investigation by Bonk et al. (2021) showed that the annual lamination can be recognised in the sediment structure up to coring time (2015 CE).

The increase in the dissolved oxygen concentration below the thermocline observed in July 2018 and August 2018 is probably attributable to temporary circulation introduced by internal waves. Internal waves drive baroclinic shear instabilities that enhance the vertical turbulent diffusivity and reduce the rate of dissolved oxygen depletion in the hypolimnion (Bouffard et al., 2014). Deep motions characterised by larger amplitudes at lower frequencies are favoured by the excitation of internal waves of the second vertical mode in strongly thermally stratified lakes (Valerio et al., 2019). Internal waves are a common response to wind forcing in not only large but also small stratified lakes (Boegman, 2009). Internal waves at the density discontinuity border along the course of the thermocline are a record of short-term water temperature variations in the metalimnion (Thorpe and Jiang, 1998; Pannard et al., 2011; Bonhomme et al., 2011; Filatov et al., 2012). Measurements taken in the middle of the lake (GOS 1) showed just such a temperature variability at a depth of $8 \mathrm{~m}$ (Fig. 4B). This can be considered indirect evidence of the occurrence of internal waves in Lake Gościąż.

In addition to the general effect that air temperature has on a lake's thermal and oxygen regimes, the short-term effects that meteorological parameters have on the hydrodynamic state of a lake should also be taken into account. In this regard, it is crucial to determine the effect of wind on lake mixing, the formation of internal waves, and the water circulation caused by their appearance. Increased water dynamics, not only in the epilimnion layer but also in the deeper layers of the lake, cause previously 
deposited sediment to be resuspended and increase the amount of material recorded in sedimentation traps. In Lake Gościąż, during the early spring and autumn, when the sediment accumulation rate increased, the prevailing winds were southerly and south-westerly. In the summer, when there was less sediment in the traps, northerlies prevailed. During prolonged strong winds, sedimentation increased, and in the GOS 2A trap in particular. When wind conditions were stable, the GOS 1A trap accumulated the most sediment, i.e. the one in the deepest studied location. The major impact of storm phenomena, when the strongest gusts were recorded, is visible in the course of sedimentation in the lake's western sub-basin (GOS 2A). The steep slope in the western part of the lake and the closeness of the sediment traps to the shore increased the supply of redeposition material. Internal waves formed under the influence of strong gusts of wind triggered the resuspension of material accumulated in the littoral zone and on the slope of the lake basin. The course of sedimentation was similar in the eastern part of the lake (GOS 3A), where the supply of material redeposited from the littoral zone is confirmed by the high content of terrigenous matter in the analysed samples.

The two-year investigation of lake productivity and weather conditions revealed clear, seasonal changes in sediment composition. Weather conditions have a direct influence on the water column, and thereby on variations in sediment composition. The sedimentation cycle starts when the ice cover begins to melt. Because the water density is uniform throughout the water column in that period, low wind speed is required to start the spring overturn (Löffler, 2004). Northerly winds $\left(337.5^{\circ}-22.5^{\circ}\right)$ were the most frequent in spring in both study years and could have caused the intensive water column mixing (Fig. 2). Smear slides analysis suggests that green algae developed rapidly in spring (Fig. 5). The increased abundance of phytoplankton and rising temperatures caused, in turn, authigenic calcite production (Groleau et al., 2000), which is visible in the increased $\mathrm{CaCO}_{3}$ content (Fig. 6). As the heating proceeded, a thermocline developed and caused the development of the summer stratification of waters.

After the summer stratification, the epilimnion cooled down and, as a result, the temperature was the same throughout the water column. In autumn, southerly $\left(157.5^{\circ}-202.5^{\circ}\right)$ and south-westerly $\left(202.5^{\circ}-247.5^{\circ}\right)$ winds dominated, which caused the mixing of the water column to the bottom. The mixing could be the reason for the higher deposition and may be related to the remobilisation of nutrients, so the nutrients accumulated in the hypolimnion may have reached the surface layer of water and caused the phytoplankton bloom.

Reworked calcite forms agglomerates, in contrast to the single calcite grains that are newly precipitated in biochemical processes. The resuspended calcite grains were first observed in spring next to fresh grains. However, they were most abundant during autumn. The appearance of phytoplankton along with both types of calcite suggests a second calcite precipitation event and supports the idea of previously deposited material having been remobilised. It is not possible, however, to distinguish whether the resuspended calcite represents littoral sediments or comes from deeper slopes of the lake.

The seasonality in the course of sedimentation is partly reflected in water transparency measurements. Under conditions of thermal stratification, when the sediment accumulation rate was lowest, water transparency was highest. By contrast, water transparency was lowest during lake mixes, and during the spring peak in primary production, when the sediment accumulation rate was higher.

In dealing with the various factors that might affect the seasonal distribution of sedimentation, it was also necessary to consider how the lake water level fluctuated throughout the year (Lopez et al., 2016). During the study period, however, this was minor, with the fluctuation amplitude not exceeding $40 \mathrm{~cm}$. The weir on the stream Ruda, downstream of Lake Gościąż, moderates water level fluctuations in the lake. In connection with the above, this factor has a negligible role in the course of contemporary sedimentation in this area. 


\section{Conclusions}

The results of this study show a spatio-temporal variation in sediment accumulation rate in the relatively small Lake Gościąż, Poland. The mean sediment accumulation rate in each of the three monitored locations was higher in the traps in the hypolimnion than in the traps in the epilimnion, as well as in locations with greater maximum depth. These results depend not so much on the spatial variation of the biological productivity of the lake, but mostly on the topography of the lake bottom (funnel effect). The seasonal variations were influenced by air temperature, and the speed and direction of the wind. These factors favoured the development of internal waves, which in turned caused an oxygen increase in the deeper parts of the lake. The confirmed relationship between sediment accumulation rate and both wind speed and direction indicates that relocated material is supplied to sediment traps, especially those located deeper. Redeposition of the material was also evidenced by the results of our analysis of smear slides and sediment composition. They showed that sediment composition is affected by season and the occurrence of strong north-westerly and south-westerly winds. The patterns and mechanisms of the course of contemporary sedimentation in Lake Gościąz, as determined based on the conducted investigations, can be applied in the study of other lakes and in assessing the representativeness of sampling sites for laminated bottom sediments to be used in palaeoenvironmental studies.

\section{Acknowledgments}

This study is a contribution to the scientific project financed by the National Science Centre, Poland - No. UMO-2015/19/B/ST10/03039 "New high-resolution and integrated analyses of environmental response to climate change over the last 15,000 years from the Lake Gościąż sediment record within the frame of a WE European transect". We would like to sincerely thank Sebastian Tyszkowski for making the bathymetric map of Lake Gościąż.

\section{References}

Anderson, R.Y., Dean, W.E. 1988. Lacustrine varve formation through time. Palaeogeography, Palaeoclimatology, Palaeoecology 62 (1-4), 215-235. https://doi.org/10.1016/0031-0182(88)90055-7

Bartczak, A., Słowińska, S., Tyszkowski, S., Kramkowski, M., Kaczmarek, H., Kordowski, J., Słowiński, M. 2019. Ecohydrological changes and resilience of a shallow lake ecosystem under intense human pressure and recent climate change. Water 11 (1), 32. https://doi.org/10.3390/w11010032

Bechtel, A., Woszczyk, M., Reischenbacher, D., Sachsenhofer, R.F., Gratzer, R., Püttmann, W., Spychalski, W. 2007. Biomarkers and geochemical indicators of Holocene environmental changes in coastal Lake Sarbsko (Poland). Organic Geochemistry 38 (7), 1112-1131. https://doi.org/10.1016/j.orggeochem.2007.02.009

Bennett, K.D., Buck, C.E. 2016. Interpretation of lake sediment accumulation rates. The Holocene 26 (7), 10921102. https://doi.org/10.1177/0959683616632880

Blais, J.M., Kalff, J. 1995. The influence of lake morphometry on sediment focusing. Limnology and Oceanography 40 (3), 582-588. https://doi.org/10.4319/lo.1995.40.3.0582

Bloesch, J., Uehlinger, U. 1986. Horizontal sedimentation differences in a eutrophic Swiss lake. Limnology and Oceanography 31 (5), 1094-1109. https://doi.org/10.4319/1o.1986.31.5.1094

Bluszcz, P., Kirilova, E., Lotter, A.F., Ohlendorf, C., Zolitschka, B. 2008. Global radiation and onset of stratification as forcing factors of seasonal carbonate and organic matter flux dynamics in a hypertrophic hardwater lake (Sacrower See, Northeastern Germany). Aquatic Geochemistry 14 (1): 73-98. https://doi.org/10.1007/s10498-008-9026-3

Boegman, L. 2009. Currents in stratified water bodies 2: Internal waves. In: G.E. Likens, (Ed.) Encyclopedia of Inland Waters. Volume 1. Elsevier, Oxford, pp. 539-558. 
Bonhomme, C., Poulin, M., Vinçon-Leite, B., Saad, M., Groleau, A., Jézéquel, D., Tassin, B. 2011. Maintaining meromixis in Lake Pavin (Auvergne, France): The key role of a sublacustrine spring. Comptes Rendus Geoscience 343 (11-12), 749-759. https://doi.org/10.1016/j.crte.2011.09.006

Bonk, A., Tylmann, W., Amann, B.J.F., Enters, D., Grosjean, M. 2015. Modern limnology and varve-formation processes in Lake Żabińskie, northeastern Poland: comprehensive process studies as a key to understand the sediment record. Journal of Limnology 74 (2), 358-370. https://doi.org/10.4081/jlimnol.2014.1117

Bonk, A., Kinder, M., Enters, D., Grosjean, M., Meyer-Jacob, C., Tylmann, W. 2016. Sedimentological and geochemical responses of Lake Żabińskie (north-eastern Poland) to erosion changes during the last millennium. Journal of Paleolimnology 56 (2-3), 239-252. https://doi.org/10.1007/s10933-016-9910-6

Bonk, A., Müller, D., Ramisch, A., Kramkowski, M., Noryśkiewicz, A.M., Sekudewicz, I., Gąsiorowski, M., Luberda-Durnaś, K., Słowiński, M., Schwab, M., Tjallingii, R., Brauer, A., Błaszkiewicz, M. 2021. Varve microfacies and chronology from a new sediment record of Lake Gościąż (Poland). Quaternary Science Review 251, 106715. https://doi.org/10.1016/j.quascirev.2020.106715

Botwe, B.O., Abril, J.M., Schirone, A., Barsanti, M., Delbono, I., Delfanti, R., Nyarko, E., Lens, P.N.L. 2017. Settling fluxes and sediment accumulation rates by the combined use of sediment traps and sediment cores in Tema Harbour (Ghana). Science of the Total Environment 609, 1114-1125. https://doi.org/10.1016/j.scitotenv.2017.07.139

Bouffard, D., Boegman, L., Ackerman, J.D., Valipour, R., Rao, Y.R. 2014. Near-inertial wave driven dissolved oxygen transfer through the thermocline of a large lake. Journal of Great Lakes Research 40 (2), 300307. https://doi.org/10.1016/j.jglr.2014.03.014

Choiński, A., Ptak, M., Skowron, R., Strzelczak, A. 2015. Changes in ice phenology on polish lakes from 1961 to 2010 related to location and morphometry. Limnologica 53, 42-49. https://doi.org/10.1016/j.limno.2015.05.005

Churski, Z., Marszelewski, W. 1998. Hydrology and sedimentation conditions in Lake Gościąż. In: M. RalskaJasiewiczowa (Ed.), Lake Gościąz, Central Poland: A Monographic Study, Part 1. W. Szafer Institute of Botany, Polish Academy of Sciences, Kraków, pp. 39-45.

Cohen, A.S. 2003. Paleolimnology: the history and evolution of lake systems. Oxford University Press, Oxford, $528 \mathrm{pp}$.

Dearing, J.A. 1997. Sedimentary indicators of lake-level changes in the humid temperate zone: a critical review. Journal of Paleolimnology 18 (1), 1-14. https://doi.org/10.1023/A:1007916210820

Demidowicz, G., Deputat, T., Górski, A., Zaliwski, E., Wróblewska, E. 1998. Numerical map of length of vegetation period. IUNG, Puławy. http://www.zazi.iung.pulawy.pl/Images/Maps/dl_oweg.jpg Accessed 12 Jul 2019.

Filatov, N., Terzevik, A., Zdorovennov, R., Vlasenko, V., Stashchuk, N., Hutter, K. 2012. Field Studies of NonLinear Internal Waves in Lakes on the Globe. In: K. Hutter (Ed.), Nonlinear internal waves in lakes. Springer, Berlin, Heidelberg, pp. 23-103. https://doi.org/10.1007/978-3-642-23438-5_2

Flower, R.J. 1990. Seasonal changes in sedimenting material collected by high aspect ratio sediment traps operated in a holomictic eutrophic lake. In: J.P. Smith, P.G. Appleby, R.W. Battarbee, J.A. Dearing, R. Flower, E.Y. Haworth, F. Oldfield, P.E. O’Sullivan (Eds.), Environmental History and Palaeolimnology. Springer, Dordrecht, pp. 311-316. https://doi.org/10.1007/978-94-011-3592-4_40

Gasith, A. 1976. Seston dynamics and tripton sedimentation in the pelagic zone of a shallow eutrophic lake. Hydrobiologia 51 (3), 225-231. https://doi.org/10.1007/BF00005748

Gierszewski, P. 2000. Charakterystyka środowiska hydrochemicznego wód powierzchniowych zachodniej części Kotliny Płockiej. Prace Geograficzne, 176, Instytut Geografii i Przestrzennego Zagospodarowania PAN, Warszawa, $136 \mathrm{pp}$.

Gierszewski, P. 2001. Variability of the concentration of chemical substances in the Ruda river-lake system (Płock Basin). Limnological Review 1, 83-93.

Giziński, A., Kentzer, A., Mieszczankin, T., Żbikowski, J., Żytkowicz, R. 1998. Hydrobiological characteristics and modern sedimentation of Lake Gościąż. In: M. Ralska-Jasiewiczowa (Ed.), Lake Gościąż, Central 
Poland: A Monographic Study, Part 1. W. Szafer Institute of Botany, Polish Academy of Sciences, Kraków, pp. 49-60.

Glazik, R. 1978. Wpływ zbiornika wodnego na Wiśle we Włocławku na zmiany stosunków wodnych w dolinie. Dokumentacja Geograficzna, 2-3, Instytut Geografii i Przestrzennego Zagospodarowania PAN, Warszawa, 119 pp.

Goslar, T., Kuc, T., Ralska-Jasiewiczowa, M., Różański, K., Arnold, M., Bard, E., van Geel, B., Pazdur, M.F., Szeroczyńska, K., Wicik, B., Więckowski, K., Walanus, A. 1993. High - resolution lacustrine record of the Late Glacial/Holocene transition in central Europe. Quaternary Science Review 12 (5), 287-294. https://doi.org/10.1016/0277-3791(93)90037-M

Graham, N.D., Bouffard, D., Loizeau, J.L. 2016. The influence of bottom boundary layer hydrodynamics on sediment focusing in a contaminated bay. Environmental Science and Pollution Research 23 (24), 2541225426. https://doi.org/10.1007/s11356-016-7715-9

Groleau, A., Sarazin, G., Vinçon-Leite, B., Tassin, B., Quiblier-Llobreas, C. 2000. Tracing calcite precipitation with specific conductance in a hard water alpine lake (Lake Bourget). Water Research 34 (17), 41514160. https://doi.org/10.1016/S0043-1354(00)00191-3

Heiri, O., Lotter, A.F., Lemcke, G. 2001. Loss on ignition as a method for estimating organic and carbonate content in sediments: reproducibility and comparability of results. Journal of Paleolimnology 25, 101-110. https://doi.org/10.1023/A:1008119611481

Horppila, J., Niemistö, J. 2008. Horizontal and vertical variations in sedimentation and resuspension rates in a stratifying lake - effects of internal seiches. Sedimentology 55 (5), 1135-1144. https://doi.org/10.1111/j.1365-3091.2007.00939.x

Johansson, M., Saarni, S., Sorvari, J. 2019. Ultra-high-resolution monitoring of the catchment response to changing weather conditions using online sediment trapping. Quaternary 2 (2), 18. https://doi.org/10.3390/quat2020018

Kaal, J., Cortizas, A.M., Rydberg, J., Bigler, C. 2015. Seasonal changes in molecular composition of organic matter in lake sediment trap material from Nylandssjön, Sweden. Organic Geochemistry 83-84, 253-262. https://doi.org/10.1016/j.orggeochem.2015.04.005

Kentzer, A., Żytkowicz, R. 1993. Warunki formowania współczesnych osadów dennych w jeziorze Gościąż. Polish Botanical Studies. Guidebook Series 8, 39-48.

Kępczyński, K., Noryśkiewicz, A. 1993. Współczesna szata roślinna kompleksu jezior 'Na Jazach'. Polish Botanical Studies. Guidebook Series 8, 29-38.

Kępczyński, K., Noryśkiewicz, A. 1998. Vegetation of the Gostynińskie Lake District. In: M. RalskaJasiewiczowa (Ed.), Lake Gościąż, Central Poland: A Monographic Study, Part 1. W. Szafer Institute of Botany, Polish Academy of Sciences, Kraków, pp. 29-33.

Kienel, U., Dulski, P., Ott, F., Lorenz, S., Brauer, A. 2013. Recently induced anoxia leading to the preservation of seasonal laminae in two NE-German lakes. Journal of Paleolimnology 50 (4), 535-544. https://doi.org/10.1007/s10933-013-9745-3

Kienel, U., Kirillin, G., Brademann, B., Plessen, B., Lampe, R., Brauer, A. 2017. Effects of spring warming and mixing duration on diatom deposition in deep Tiefer See, NE Germany. Journal of Paleolimnology 57 (1), 37-49. https://doi.org/10.1007/s10933-016-9925-z

Kruczkowska, B., Błaszkiewicz, M., Jonczak, J., Uzarowicz, Ł., Moska, P., Brauer, A., Bonk, A., Słowiński, M. 2020. The Late Glacial pedogenesis interrupted by aeolian activity in Central Poland - Records from the Lake Gościąż catchment. Catena 185, 104286. https://doi.org/10.1016/j.catena.2019.104286

Lastein, E. 1976. Recent sedimentation and resuspension of organic matter in eutrophic Lake Esrom, Denmark. Oikos 27 (1), 44-49. https://www.jstor.org/stable/3543430

Leemann, A., Niessen, F. 1994. Varve formation and the climatic record in an Alpine proglacial lake: calibrating annually-laminated sediments against hydrological and meteorological data. The Holocene 4 (1), 1-8. https://doi.org/10.1177/095968369400400101 
Lewis, T., Gilbert, R., Lamoureux, S.F. 2002. Spatial and temporal changes in sedimentary processes at proglacial Bear Lake, Devon Island, Nunavut, Canada. Arctic, Antarctic and Alpine Research 34 (2), 119-129.

Lopez, P., Lopez-Tarazon, J.A., Casas-Ruiz, J.P., Pompeo, M., Ordonez, J., Munoz, I. 2016. Sediment size distribution and composition in a reservoir affected by severe water level fluctuations. Science of the Total Environment 540, 158-167. https://doi.org/10.1016/j.scitotenv.2015.06.033

Lotter, A.F., Birks, H.J.B. 1997. The separation of the influence of nutrients and climate on the varve time-series of Baldeggersee, Switzerland. Aquatic Sciences 59 (4), 362-375. https://doi.org/10.1007/BF02522364

Löffler, H, 2004. The origin of lake basins. In: P.E. O'Sullivan, C.S. Reynolds, (Eds.), The lakes handbook, Volume 1: Limnology and Limnetic Ecology. Wiley, New York, pp. 8-60.

Maier, D.B., Gälman, V., Renberg, I., Bigler, C. 2018. Using a decadal diatom sediment trap record to unravel seasonal processes important for the formation of the sedimentary diatom signal. Journal of Paleolimnology 60 (2), 133-152. https://doi.org/10.1007/s10933-018-0020-5

Mieszczankin, T. 1997. A spacio-temporal pattern of pollen sedimentation in a dimictic lake with laminated sediments. Water, Air, and Soil Pollution 99, 587-592. https://doi.org/10.1007/BF02406897

Mieszczankin, T., Noryśkiewicz, B. 2000. Processes that can disturb the chronostratigraphy of laminated sediments and pollen deposition. Journal of Paleolimnology 23 (2), 129-140. https://doi.org/10.1023/A:1008074701468

Mieszczankin, T. 2004. Procesy sedymentacji tryptonu w jeziorze Gościąż - warunki formowania współczesnych osadów dennych oraz rola tryptonu w transformacji i kumulacji fosforu. In: B. Głogowska, A. Kentzer (Eds.), Hydrobiologia toruńska: monografia dorobku prac doktorskich Zakładu Hydrobiologii w latach 1978-2003. Toruń, pp. 117-127.

Moore, J.J., Hughen, K.A., Miller, G.H., Overpeck, J.T. 2001. Little Ice Age recorded in summer temperature reconstruction from varved sediments of Donard Lake, Baffin Island, Canada. Journal of Paleolimnology 25 (4), 503-517. https://doi.org/10.1023/A:1011181301514

Müller, D., Tjallingii, R., Płóciennik, M., Luoto, T.P., Kotrys, B., Plessen, B., Ramisch, A., Schwab, M.J., Błaszkiewicz, M., Słowiński, M., Brauer, A. [in press]. New insights into lake responses to rapid climate change: The Younger Dryas in Lake Gościąż, Central Poland. Boreas

O'Sullivan, P.E. 1983. Annually laminated lake sediments and the study of Quaternary environmental changes a review. Quaternary Science Review 1 (4), 245-313. https://doi.org/10.1016/0277-3791(83)90008-2

Ojala, A.E.K., Heinsalu, A., Kauppila, T., Alenius, T., Saarnisto, M. 2008. Characterizing changes in the sedimentary record in southern central Finland around 8000 cal. yr BP. Journal of Quaternary Science 23 (8), 765-775. https://doi.org/10.1002/jqs.1157

Ojala, A.E.K., Francus, P., Zolitschka, B., Besonen, M., Lamoureux, S.F. 2012. Characteristics of sedimentary varve chronologies - a review. Quaternary Science Review 43, 45-60. https://doi.org/10.1016/j.quascirev.2012.04.006

Ojala, A.E.K., Bigler, C., Weckström, J. 2014. Understanding varved formation processes from sediment trapping and limnological monitoring. Past Global Changes Magazine $22 \quad$ (1), 8-9. https://doi.org/10.22498/pages.22.1.8

Ott, F., Dräger, N., Brykała, D., Kienel, U., Gierszewski, P., Plessen, B., Schwab, M.J., Brademann, B., Pinkerneil, S., Słowiński, M., Błaszkiewicz, M., Brauer, A. 2017. Deciphering varve formation in Lake Czechowskie (N Poland) and Lake Tiefer See (NE Germany) through comprehensive lake monitoring. In: M.J. Schwab, M. Błaszkiewicz, T. Raab, M. Wilmking, A. Brauer (Eds.) ICLEA Final Symposium 2017. Climate Change, Human Impact and Landscape Evolution in the Southern Baltic Lowlands. Abstract Volume \& Excursion Guide. Scientific Technical Report STR17/03. Potsdam, pp. 132-134.

Ott, F., Kramkowski, M., Wulf, S., Plessen, B., Serb, J., Tjallingii, R., Schwab, M., Słowiński, M., Brykała, D., Tyszkowski, S., Putyrskaya, V., Appelt, O., Błaszkiewicz, M., Brauer, A. 2018. Site-specific sediment responses to climate change during the last 140 years in three varved lakes in Northern Poland. The Holocene 28 (3), 464-477. https://doi.org/10.1177/0959683617729448 
Pace, M.L., Lovett, G.M. 2013. Primary production: the foundation of ecosystems. In: K. Weathers, D. Strayer, G. Likens (Eds.), Fundamentals of ecosystem science. Academic Press, pp. 27-51.

Pannard, A., Beisner, B.E., Bird, D.F., Braun, J., Planas, D., Bormans, M. 2011. Recurrent internal waves in a small lake: Potential ecological consequences for metalimnetic phytoplankton populations. Limnology and Oceanography 1 (1), 91-109. https://doi.org/10.1215/21573698-1303296

Peel, M.C., Finalyson, B.L., Mcmahon, T.A. 2007. Updated world map of the Köppen-Geiger climate classification. Hydrology and Earth System Science 11 (5), 1633-1644. https://doi.org/10.5194/hess-111633-2007

Petterson, G., Renberg, I., Geladi, P., Lindberg, A., Lindgren, A. 1993. Spatial uniformity of sediment accumulation in varved lake sediments in northern Sweden. Journal of Paleolimnology 9 (3), 195-208. https://doi.org/10.1007/BF00677213

Punning, J.M., Alliksaar, T., Terasmaa, J., Jevrejeva, S. 2004. Recent patterns of sediment accumulation in a small closed eutrophic lake revealed by the sediment records. Hydrobiologia 529 (1-3), 71-81. https://doi.org/10.1007/s10750-004-4948-2

Ralska-Jasiewiczowa, M., Goslar, T., Madeyska, T., Starkel, L. (Eds.). 1998. Lake Gościąż, Central Poland: A Monographic Study, Part 1. W. Szafer Institute of Botany, Polish Academy of Sciences, Kraków, 340 pp.

Ruttner, F, 1963. Fundamentals of Limnology. University of Toronto, Toronto, 295 pp.

Rychel, J., Błaszkiewicz, M., Brykała, D., Gierszewski, P., Lisicki, S., Roman, M., Tyszkowski, S. 2014, Mapa geologiczno-turystyczna Gostynińsko-Włocławskiego Parku Krajobrazowego. Państwowy Instytut Geologiczny - PIB, Instytut Geografii i Przestrzennego Zagospodarowania PAN, Uniwersytet Łódzki, Warszawa.

Rychel, J., Woronko, B., Błaszkiewicz, M., Karasiewicz, T. 2018. Aeolian processes records within last glacial limit areas based on the Płock Basin case (Central Poland). Bulletin of the Geological Society of Finland 90 (2), 223-237. https://doi.org/10.17741/bgsf/90.2.007

Salminen, S., Saarni, S., Tammelin, M., Fukmoto, Y., Saarinen, T. 2019. Varve distribution reveals spatiotemporal hypolimnetic hypoxia oscillations during the past 200 years in Lake Lehmilampi, Eastern Finland. Quaternary 2 (2), 20. https://doi.org/10.3390/quat2020020

Schaller, T., Moor, H.C., Wehrli, B. 1997. Sedimentary profiles of Fe, Mn, V, Cr, As and Mo as indicators of benthic redox conditions in Baldeggersee. Aquatic Sciences 59 (4), 345-361. https://doi.org/10.1007/BF02522363

Schettler, G., Liu, Q., Mingram, J., Stebich, M., Dulski, P. 2006. East - Asian monsoon variability between 15,000 and 2000 cal. yr BP recorded in varved sediments of Lake Sihailongwan (northeastern China, Long Gang volcanic field). The Holocene 16 (8), 1043-1057. https://doi.org/10.1177/0959683606069388

Schiefer, E., Gilbert, R. 2008. Proglacial sediment trapping in recently formed Silt Lake, upper Lillooet Valley, Coast Mountains, British Columbia. Earth Surface Processes and Landforms 33 (10), 1542-1556. https://doi.org/10.1002/esp.1625

Schnurrenberger, D., Russell, J., Kelts, K. 2003. Classification of lacustrine sediments based on sedimentary components. Journal of Paleolimnology 29 (2), 141-154.

Segerström, U., Renberg, I., Wallin, J.E. 1984. Annual sediment accumulation and land use history; investigations of varved lake sediments: With 7 figures in the text. Internationale Vereinigung für theoretische und angewandte Limnologie: Verhandlungen 22 (3), 1396-1403. https://doi.org/10.1080/03680770.1983.11897507

Skompski, S. 1969. Stratygrafia osadów czwartorzędowych wschodniej części Kotliny Płockiej. Biuletyn Instytutu Geologicznego 220, 175-258.

Terasmaa, J. 2005. Bottom topography and sediment lithology in two small lakes in Estonia. Proceedings of the Estonian Academy of Sciences. Biology. Ecology 54 (3), 171-189.

Thorpe, S.A., Jiang, R. 1998. Estimating internal waves and diapycnal mixing from conventional mooring data in a lake. Limnology and Oceanography 43 (5), 936-945. https://doi.org/10.4319/lo.1998.43.5.0936 
Tylmann, W. 2011. Jeziorne osady rocznie laminowane w północnej Polsce: aktualny stan rozpoznania, postępy metodyczne i perspektywy badawcze. Studia Limnologica et Telmatologica 5 (1), 23-41.

Tylmann, W., Szpakowska, K., Ohlendorf, C., Woszczyk, M., Zolitschka, B. 2012. Conditions for deposition of annually laminated sediments in small meromictic lakes: a case study of Lake Suminko (northern Poland). Journal of Paleolimnology 47 (1), 55-70. https://doi.org/10.1007/s10933-011-9548-3

Urbaniak, U. 1966. Skład mineralny piasków wydmowych w Kotlinie Płockiej. Przegląd Geograficzny 38 (3), 435-453.

Valerio, G., Pilotti, M,. Lau, M.P, Hupfer, M. 2019. Oxycline oscillations induced by internal waves in deep Lake Iseo. Hydrology and Earth System Sciences 23 (3), 1763-1777. https://doi.org/10.5194/hess-23-17632019

Vos, H., Sanchez, A., Zolitschka, B., Brauer, A., Negedank, J.F.W. 1997. Solar activity variations recorded in varved sediments from the crater Lake of Holzmaar - a maar lake in the Westeifel volcanic field, Germany. Surveys in Geophysics 18 (2-3), 163-182. https://doi.org/10.1023/A:1006531825130

Wetzel, R.G., Rich, P.H., Miller, M.C., Allen, H.L. 1972. Metabolism of dissolved and particulate detrital carbon in a temperate hard water lake. Michigan State University, Hickory Corners, 109 pp. https://doi.org/10.2172/4614952

Wiśniewski, E. 1976. Rozwój geomorfologiczny doliny Wisty pomiędzy Kotlina Płocka a Kotlina Toruńską. Prace Geograficzne, 119, Instytut Geografii i Przestrzennego Zagospodarowania PAN, Warszawa, 124 pp.

Woszczyk, M., Cieślinski, R., Spychalski, W. 2009. Geochemistry of surface sediments of a costal Lake Sarbsko (Northern Poland). Studia Quaternaria, 26, 41-53.

Woś, A. 1999. Klimat Polski. Wydawnictwo Naukowe PWN, Warszawa, 301 pp.

Zolitschka, B., Francus, P., Ojala, A.E.K., Schimmelmann, A. 2015. Varves in lake sediments - a review. Quaternary Science Review 117, 1-41. https://doi.org/10.1016/j.quascirev.2015.03.019 



\title{
BALANCE SEDIMENTARIO DEL EMBALSE DE RIBARROJA
}

\author{
ÁLVARO TENA ${ }^{1 *}$, DAMIÀ VERICAT ${ }^{1,2}$, RAMÓN J. BATALLA ${ }^{1,3,4}$ \\ ${ }^{1}$ Fluvial Dynamics Research Group (RIUS), Universidad de Lleida (UdL), Lleida, España. \\ ${ }^{2}$ Centre de Ciencia i Tecnologia Forestal de Catalunya (CTFC), Solsona, España. \\ ${ }^{3}$ Institut Català de Recerca de l'Aigua (ICRA), Girona, España. \\ ${ }^{4}$ Facultad de Ciencias Forestales y Recursos Naturales, Universidad Austral de Chile, Valdivia, Chile.
}

RESUMEN: El objetivo de este estudio ha sido construir un balance de sedimentos del embalse de Ribarroja, situado en el tramo inferior del río Ebro. Para ello, se han analizado datos continuos de caudal y transporte de sedimentos en suspensión tomados en los principales ríos que desembocan en el embalse, así como en la salida de la presa, para el período 2008-2011. La carga de sedimentos estimada aguas arriba del embalse de Mequinenza fue de $0.66 \times 10^{6} \mathrm{ta}^{-1}$, mientras que la salida estimada fue de $0.04 \times 10^{6} \mathrm{t} \mathrm{a}^{-1}$. El aporte de sedimentos proveniente de los grandes afluentes pirenaicos, aunque altamente regulados, Segre y Cinca fue de $0,16 \times 10^{6} \mathrm{t} \mathrm{a}^{-1}$, mientras que los ríos intermitentes que fluyen naturalmente desde la Cordillera Ibérica (Matarraña y Algars) suministraron una carga media de $760 \mathrm{t} \mathrm{a}^{-1}$. La carga de sedimentos en suspensión atrapada en los embalses de Mequinenza y Ribarroja para el período de estudio se estimó en $0,78 \times 10^{6} \mathrm{t} \mathrm{a}^{-1}$, lo que implica una reducción de ca. $95 \%$ de la carga de sedimentos del río. Los resultados ilustran el papel de los embalses en la interrupción de la transferencia de sedimentos en el tramo bajo del Ebro, en un contexto de déficit sedimentario estructural, tras los cambios generalizados de uso del suelo (reforestación) que se produjeron en la cuenca a partir de los años cuarenta del siglo pasado.

\section{Sediment balance of the Ribarroja Reservoir}

\begin{abstract}
The aim of this study was to build up a sediment budget of the Ribarroja Reservoir, which is located in the lower reach of the River Ebro. For this purpose, we analysed continuous discharge and suspended sediment transport data at several river sections entering the reservoir and at the dam outlet, for the period 2008-2011. The sediment load estimated upstream of the Mequinenza reservoir was $0.66 \times 10^{6} \mathrm{t} \mathrm{a}^{-1}$, whereas the estimated output was $0.04 \times 10^{6} \mathrm{t} \mathrm{a}^{-1}$. The sediment input coming from the large but highly regulated Pyrenean tributaries (Segre and Cinca) was $0.16 \times 10^{6} \mathrm{t} \mathrm{a}^{-1}$, whereas intermittent rivers naturally flowing from the Iberian Range (Matarraña and Algars) supplied an average load of $760 \mathrm{t} \mathrm{a}^{-1}$. The suspended sediment load trapped in the Mequinenza and Ribarroja reservoirs for the study period was estimated at $0.78 \times 10^{6} \mathrm{ta}^{-1}$, which implies a reduction of ca. $95 \%$ of the sediment load of the river. The results illustrate the role of reservoirs in interrupting the sediment transfer in the lower reach of the Ebro, within a context of structural sediment deficit, following widespread land use changes (afforestation) that took place in the catchment after the 1940s.
\end{abstract}

Palabras clave: Balance sedimentario, embalses, río Ebro, sedimento en suspensión.

Key words: Sediment balance, reservoirs, Ebro River, suspended sediment.

Recibido: 29 septiembre 2020

Aceptado: 7 enero 2021 
*Correspondencia: Álvaro Tena. Fluvial Dynamics Research Group (RIUS), Universidad de Lleida (UdL), 25198, Lleida, España. E-mail address: alvaro.tena @udl.cat

\section{Introducción}

El impacto antrópico en sistemas fluviales se ha convertido en un tema central para gestores y científicos en las últimas décadas, favoreciendo el incremento de estudios para minimizar sus efectos o restaurar impactos. Entre los impactos destacan los que las grandes presas ejercen sobre la dinámica hidro-sedimentaria en ríos de todo el mundo. En este contexto, la retención de sedimentos en los embalses, y el corte en la transferencia de sedimentos hacia aguas abajo, se ha identificado como uno de los impactos más notables en el ecosistema fluvial, convirtiéndose en uno de los principales responsables de la tendencia decreciente de la transferencia de sedimentos entre las masas continentales y los océanos (e.g. Milliman y Syvitski, 1992; Meybeck y Ragu, 1997; Walling y Fang, 2003; Walling 2006, Milliman y Farnsworth, 2011). Vorosmarty et al. (2003) indicaron que, a nivel global, más de la mitad $(53 \%)$ de la carga sedimentaria transportada por los ríos queda actualmente retenida en los embalses i.e. $73 \mathrm{~km}^{3} \mathrm{a}^{-1}$ (Syvitsky y Kettner, 2011). En el caso del Mississippi se estima que casi 20 millones de toneladas de sedimentos se sedimentan y quedan retenidas anualmente en sus embalses, o del río Yangtze, en el que tan solo una presa, la de las Tres Gargantas, atrapa 34 millones de toneladas de sedimentos por año, lo que equivale al $31 \%$ de la carga sedimentaria transportada por el río (Hu et al., 2009). Milliman y Farnsworth (2011) describen diversos ejemplos (Colorado, Indus, Río Grande, Nilo) sobre el efecto de los grandes embalses en la carga sedimentaria de los ríos. En el caso del río Colorado, la presa Glen Canyon provocó una disminución de aproximadamente el 99,5\% en la cantidad de sedimento fino que circulaba por el Gran Cañón (Topping et al., 2000).

La región mediterránea presenta tasas de producción de sedimento elevadas a escala global (Dedkov y Mozzherin, 1992), alcanzando en algunos casos $>3000 \mathrm{t} \mathrm{km}^{-2} \mathrm{a}^{-1}$ por ej., el río Ombrone, Italia (Milliman y Farnsworth, 2011). Los usos del agua y la variabilidad hidro-climática de la región han sido el motivo histórico de la construcción de embalses para la regulación fluvial, principalmente a lo largo del siglo XX. Los usos de los embalses son múltiples, aunque el almacenaje de agua para riego ha sido el principal, sobre todo en España. Poulos y Collins (2002) ya observaron una reducción en la aportación sedimentaria a la cuenca del Mediterráneo de casi un 50\% desde mediados del siglo XX, asociado, entre otras razones (por ejemplo, cambios en los usos del suelo), al atrapamiento de una parte importante de la carga sólida de los grandes ríos, como el Po, el Ródano, el Ebro y el Nilo por parte de las grandes presas. Milliman y Farnsworth (2011) aumentan la reducción hasta el 75\%.

En este estudio se destaca que el río Ebro ha experimentado una importante reducción en su carga sedimentaria i.e. 95\%, especialmente en su tramo bajo, tras el cierre del complejo de presas Mequinenza-Ribarroja-Flix a final de los años 1960 (para más detalles, ver Batalla et al. 2004 sobre los efectos hidrológicos de las presas, y Batalla y Vericat (2011) sobre los cambios en el flujo de sedimentos en el conjunto de la cuenca sedimentaria debido, entre varias causas, a la retención en embalses). Existen numerosos estudios recientes sobre el transporte de sedimentos y los procesos geomorfológicos en el bajo Ebro en relación con el complejo de presas (e.g. Vericat y Batalla, 2005; Vericat y Batalla, 2006; Vericat et al., 2006; Rovira e Ibañez, 2007; Tena et al., 2011; Tena et al., 2013; Tena y Batalla, 2013; Rovira et al., 2015). A diferencia de los trabajos ya existentes, esta investigación se centra en el análisis de las entradas y salidas de sedimento fino del embalse de Ribarroja, incluyendo la cuantificación de las cargas sedimentarias del río Ebro, así como de los principales afluentes al embalse. Este balance ha sido elaborado principalmente a partir de series en continuo de sedimento en suspensión obtenidas entre 2008 y 2011 a la salida de cada una de las principales subcuencas que desembocan en el embalse de Ribarroja, y aguas abajo de la presa. El estudio se complementa con datos obtenidos anteriormente aguas arriba del embalse de Mequinenza. El objetivo es construir un balance sedimentario del embalse que permita evaluar sus efectos sobre la transferencia de sedimentos desde la cuenca hacia aguas abajo. Los 
resultados tienen interés para determinar la situación sedimentaria reciente del embalse y para re-evaluar las acciones prácticas de restauración ambiental que se pueden llevar a cabo en el tramo bajo del río Ebro.

\section{2. Área de estudio}

El Ebro es uno de los grandes ríos que desembocan en el Mar Mediterráneo (Fig. 1A), sólo superado en longitud por el Nilo. La cuenca del Ebro es, además, la más grande de la península Ibérica, con una superficie de $85.534 \mathrm{~km}^{2}$. El río fluye con dirección NW-SE a lo largo de $910 \mathrm{~km}$, desde la Cordillera Cantábrica hasta el delta del Ebro a través del cual desemboca en el Mar Mediterráneo. La altitud de la cuenca varía desde $>3400$ m s.n.m. en el Pirineo central hasta el nivel del mar en el delta (Fig. 1B). La precipitación media anual es de $600 \mathrm{~mm}$, aunque la variabilidad espacial y temporal es muy alta, variando desde $>2000 \mathrm{~mm} \mathrm{año}^{-1}$ en el Pirineo central hasta $<300 \mathrm{~mm}$ año ${ }^{-1}$ en el centro de la Depresión. El régimen hidrológico del Ebro es pluvio-nival, con máximos en invierno y primavera, mientras que los mínimos se dan en verano (Tena y Batalla, 2013). El caudal medio anual en Tortosa, cerca de la desembocadura, es de $431 \mathrm{~m}^{3} \mathrm{~s}^{-1}$, que corresponde a una aportación hídrica anual de $13.812 \mathrm{hm}^{3}\left(\sigma=5474 \mathrm{hm}^{3} \mathrm{a}^{-1}\right.$, donde $\sigma$ es la desviación estándar de la serie 1912-2018). El caudal medio mínimo anual se ha reducido desde algo más de $600 \mathrm{~m}^{3} \mathrm{~s}^{-1}$, en el período 1912-1930, hasta algo menos de $300 \mathrm{~m}^{3} \mathrm{~s}^{-1}$ durante las dos primeras décadas del siglo XXI. La aportación hídrica varía sustancialmente, desde una aportación máxima anual de $30.821 \mathrm{hm}^{3}$ (1914-1915) hasta un mínimo de $4284 \mathrm{hm}^{3}$ (1989-1990). El caudal máximo instantáneo se estimó en ca. $12.000 \mathrm{~m}^{3} \mathrm{~s}^{-1}$ en Tortosa durante la riada de 1907 (Novoa, 1984). Las crecidas de mayor magnitud se han registrado en 1787, 1907 y 1866 con caudales de 12.900, 12.000 y $7750 \mathrm{~m}^{3} \mathrm{~s}^{-1}$, respectivamente (Balasch et al., 2019). Hoy en día, 133 embalses regulan alrededor de $2 / 3$ de la aportación media anual de la cuenca (i.e. $8000 \mathrm{hm}^{3}$ ). Los 25 embalses más grandes de la cuenca $\left(>50 \mathrm{hm}^{3}\right)$ representan el $90 \%$ de la capacidad total de almacenamiento de la cuenca. El complejo de presas más grande de la cuenca se encuentra en el tramo bajo del río Ebro, y está constituido por las presas de Mequinenza (construida en 1966, $1534 \mathrm{hm}^{3}$ ), Ribarroja $\left(1969,207 \mathrm{hm}^{3}\right)$ y Flix $\left(1948,11 \mathrm{hm}^{3}\right)$, cerrando en este punto el $97 \%$ del área de la cuenca (Fig. 1B).

Este estudio se centra en el embalse de Ribarroja. El Ebro aguas arriba del embalse de Mequinenza drena una cuenca de aproximadamente $51.210 \mathrm{~km}^{2}$, siendo el del Ebro el mayor embalse $\left(500 \mathrm{hm}^{3}\right)$ aguas arriba de este punto (Índice de Regulación IR =0,77; ver Batalla et al., 2004). La aportación media anual al embalse de Mequinenza es de $7174 \mathrm{hm}^{3}$ (periodo 1973-2016). Los principales afluentes al embalse de Ribarroja por el margen izquierdo (Pirineos) son el Segre y el Cinca, mientras que los del margen derecho (Sistema Ibérico) son el Matarraña y el Algars.

Los ríos Segre y Cinca son los afluentes más importantes del Ebro, drenando cuencas de $12.751 \mathrm{~km}^{2}$ y $9608 \mathrm{~km}^{2}$, respectivamente. La aportación hídrica de estos dos ríos al Ebro es la más importante de la cuenca (5122 $\mathrm{hm}^{3}$ año de promedio, series 1949-2016), y dado su régimen nivo-pluvial (alta acumulación de nieve en el Pirineo central durante los meses invernales) presenta un máximo en primavera y caudales relativamente constantes en verano. Ambas cuencas están fuertemente reguladas por grandes presas, con una capacidad total de agua embalsada de $2120 \mathrm{hm}^{3}$ en el caso del Segre y $993 \mathrm{hm}^{3}$ en el caso del Cinca ( $\mathrm{IR}=1,49$ y 0,59 respectivamente). Los afluentes mediterráneos, Matarraña y Algars, se caracterizan por un régimen hidrológico fuertemente influenciado por la precipitación, registrando máximos en primavera y otoño, y el mínimo en verano. El área de la cuenca del Matarraña es de $1717 \mathrm{~km}^{2}$, de los cuales $405 \mathrm{~km}^{2}$ corresponden a su principal afluente, el Algars. Dentro de la cuenca del Matarraña, únicamente el río Pena, un pequeño afluente en la cabecera, está regulado por la presa del mismo nombre con una capacidad de $17,8 \mathrm{hm}^{3}(\mathrm{IR}=1,13)$. La aportación media anual de la cuenca del Matarraña es de $35 \mathrm{hm}^{3}$ (periodo 1974-2016). La cuenca del río Algars no está regulada por embalses, por lo tanto, el IR es 0 . Su aportación media anual es de $21.2 \mathrm{hm}^{3}$ (periodo 1974-2016). 


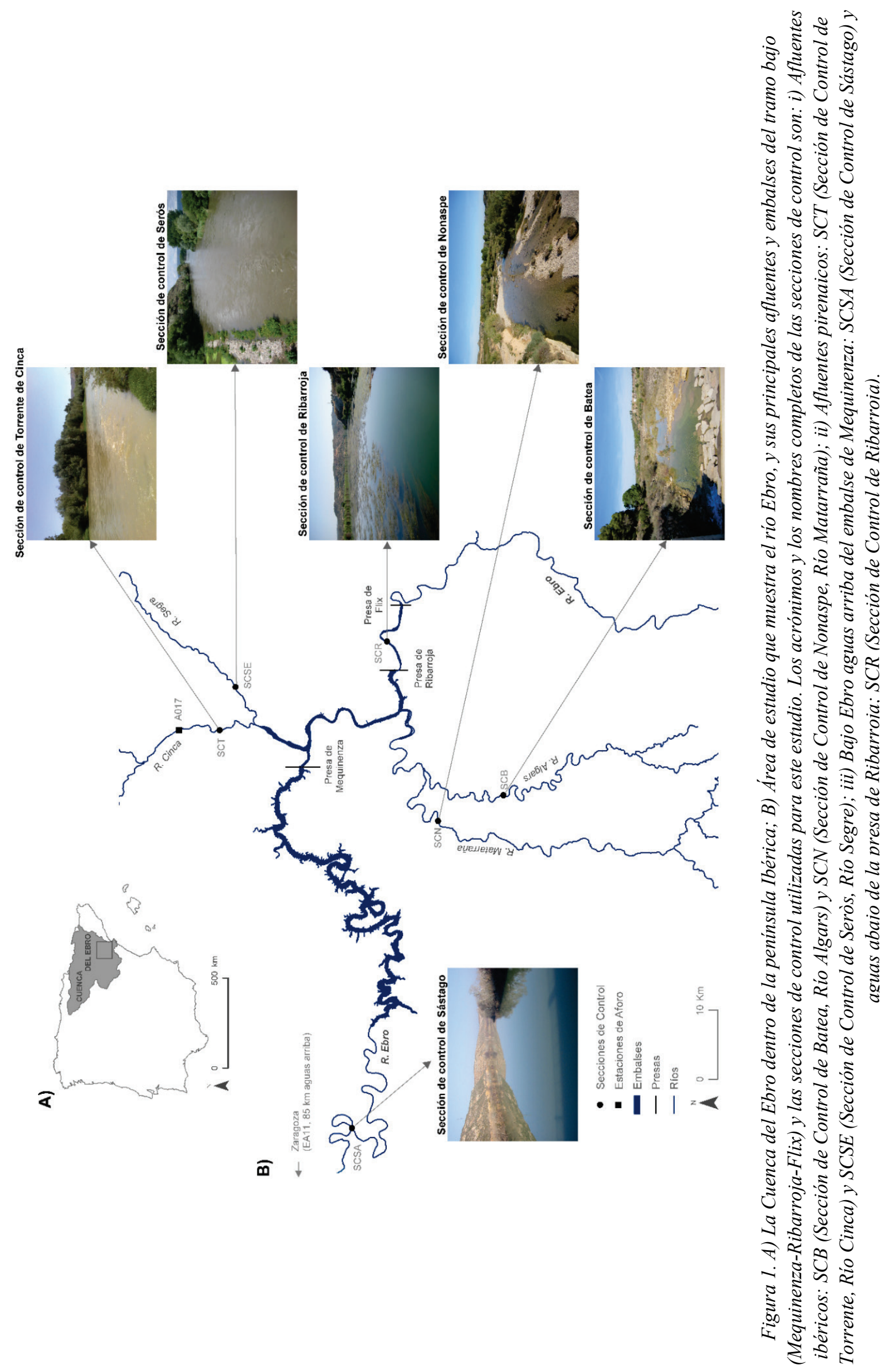




\section{Materiales y métodos}

Los datos utilizados en este estudio consisten en series continuas de caudal y turbidez recogidas entre 2008 y 2011 en diferentes secciones de control, a la entrada y salida del embalse de Ribarroja y en los principales afluentes que desembocan en este embalse. Para el particular caso de la Sección de Control de Sástago (SCSA, entrada al embalse de Mequinenza), se ha utilizado una relación estadística entre caudal y sedimento en suspensión obtenido a partir de muestras discretas durante el periodo 20032004. El análisis de toda la información de manera conjunta permite construir un balance de sedimentos del embalse de Ribarroja (para la localización de todas las secciones de control ver Fig. 1B). A continuación, se presentan los detalles metodológicos específicos.

\subsection{Caudal}

Las series de caudal en continuo (en adelante $Q$ ) se han obtenido de tres maneras diferentes: i) a partir de estaciones de aforo propiedad de Confederación Hidrográfica del Ebro (CHE); ii) a partir de secciones de control sedimentométrico propias (Grupo RIUS-UdL) por medio de sensores de nivel de agua; y iii) a partir del tránsito del caudal desde estaciones de aforo cercanas. Los detalles de las tres fuentes de datos se dan a continuación:

i) Las estaciones de medición de CHE se etiquetan como sección de control de Batea (SCB), sección de control de Nonaspe (SCN) y sección de control de Serós (SCSE) (ver la Figura 1B para localización de las secciones de control). En estas estaciones, se mide el nivel del agua (h) con una frecuencia quinceminutal por medio de un limnígrafo OTT®, y posteriormente se transforma en $Q$ mediante una curva de gasto $h / Q$ específica.

ii) La sección de control propia donde se registra el nivel de agua en continuo es la sección de control de Torrente de Cinca (SCT, ver Fig. 1B). En esta sección, el nivel de agua se midió mediante un TruTrack ${ }^{\circledR}$ WT-HR (sensor capacitivo de nivel de agua con registrador de datos integrado). El error promedio del sensor, estimado a partir de la comparación de mediciones directas en una escala y las lecturas del sensor durante las visitas de campo semanales era de un 2\% (Tena y Batalla, 2013). El nivel de agua se registró a intervalos de 15 minutos. En esta sección, $Q$ se calculó utilizando el método de nivel / área mediante el modelo 1D WinXSPRO ${ }^{\circledR}$. Este software calcula el caudal mediante la ecuación de Manning, a partir de una sección topográfica y la distribución granulométrica representativa del tramo a estudiar, necesaria para estimar el coeficiente de rugosidad $(n)$. Además, para corroborar los valores obtenidos mediante este método, se estableció una relación entre el nivel de agua en SCT y el caudal, obtenida en la estación de aforo más cercana (Fraga EA017; ver Fig. 1B). Una vez conocidos los parámetros hidráulicos (por ejemplo, tiempo de tránsito, atenuación/laminación de caudal), los hidrogramas de las estaciones oficiales se transitaron a SCT, siguiendo el método de Muskingum para toda la serie de datos (Shaw, 1983). La desviación entre los caudales transitados y los caudales modelados fuer inferior al 5\%.

iii) En el caso de la sección de control de Sástago (SCSA) y la sección de control de Ribarroja (SCR, ver Fig. 1B), donde no había sensores de nivel, se ha seguido un procedimiento similar al descrito en ii). En ambos casos, los caudales se han transitado directamente desde las estaciones de medición más cercanas, también mediante el método de Muskingum. En SCR, los caudales se transitaron desde la presa de Ribarroja (datos facilitados por la CHE), ubicada $3 \mathrm{~km}$ aguas arriba. En el caso de SCSA, el caudal se transitó desde la estación de aforo oficial EA11 de Zaragoza (ubicada a $85 \mathrm{~km}$ aguas arriba), teniendo en cuenta que las aportaciones entre estas dos secciones no son significativas (Vericat y Batalla, 2006). 


\subsection{Sedimento en suspensión}

La concentración de sedimentos en suspensión (CSS) se obtuvo a partir de los registros continuos de turbidez. Los datos de turbidez se obtuvieron de tres fuentes diferentes: i) de las estaciones oficiales de calidad del agua de la Agencia Catalana del Agua (ACA), ii) de las secciones de control equipadas con sondas de turbidez propias (Grupo RIUS-UdL; ver la Fig. 1B para más detalles), y iii) a partir de relaciones estadísticas entre el caudal $(Q)$ y el transporte de sedimentos en suspensión (CSS) ( $\tan$ solo en SCSA). Específicamente:

i) Las estaciones de calidad del agua operadas por el ACA. Estos datos (en SCSE) se registran cada 15 minutos por medio de una sonda de turbidez Campbell® OBS-3 + (0-4000 NTU).

ii) En las secciones de control propias ( $\mathrm{SCR}, \mathrm{SCB}, \mathrm{SCN}$ y $\mathrm{SCT}$ ), se registra la turbidez en continuo. En estas secciones, la turbidez se midió mediante turbidímetros ópticos McVann ${ }^{\circledR}$ Analite. Sin embargo, dependiendo de las concentraciones de sedimentos en suspensión estimadas para cada río, se utilizaron modelos diferentes. Por ejemplo, en aquellas secciones aguas abajo de las presas (SCR), donde se esperaba que las concentraciones fueran bajas, se utilizó el NEP9510, una sonda de turbidez de bajo rango (0-1000 NTU). Por otro lado, en los afluentes (SCB, SCN y SCT), donde se esperaba una mayor concentración de sedimentos, se instalaron sondas de turbidez de mayor rango, NEP9530 (0-3000 NTU). El almacenamiento de los datos de turbidez se realizaba en data-loggers Campbell® (CR-510, CR-800 y CR1000) con una frecuencia quinceminutal, obtenidos a partir de los promedios de las lecturas que el instrumento realizaba cada 5 segundos. Con el propósito de evitar problemas de variabilidad y agilizar los cálculos, se utilizaron los promedios horarios.

iii) Aguas arriba de la presa de Mequinenza no se disponía de un turbidímetro. Así, para estimar la carga anual de sedimentos en suspensión en SCSA y poder cerrar el balance sedimentario en Ribarroja se aplicaron las relaciones entre $Q$ y $C S S$ obtenidas previamente por Vericat y Batalla (2006), utilizando el hidrograma transitado previamente. Se utilizó una relación estadísticamente significativa $Q$ - CSS obtenida por Vericat y Batalla (2006) para el año hidrológico 2003-04 para estimar las concentraciones de sedimentos suspensión, y posteriormente las cargas sólidas totales. El año 2003-04 fue similar desde el punto de vista hidrológico al período de estudio 2008-2011, por lo que se considera un modelo representativo del período de estudio. En este caso se trataba de tener un valor de referencia, puesto que como se ha visto en trabajos realizados previamente en este tramo (Roura, 2004; Vericat y Batalla, 2006), el embalse de Mequinenza puede retener hasta el $95 \%$ de los sedimentos en suspensión transportados anualmente desde aguas arriba.

Como parte del programa de muestreo de sedimentos en suspensión implementado desde 2002 en el tramo bajo del río Ebro y sus principales afluentes, se recogieron muestras manuales de 1 litro de manera intensiva durante eventos de crecida y de forma rutinaria (semanal o quincenal) durante períodos de caudales bajos (para más detalles, consultar Vericat y Batalla, 2005, 2006; Tena et al. 2011, 2012; Tena y Batalla, 2013). En total, se utilizaron 737 muestras de sedimentos en suspensión para calibrar los datos de turbidez y complementar los cálculos de cargas sedimentarias. Las muestras se filtraron al vacío por medio de filtros de microfibra de vidrio (Filter-Lab ${ }^{\circledR}$, tamaño de poro de $1,2 \mu \mathrm{m}$ ), se secaron y se pesaron en el laboratorio para determinar la concentración de sedimento en suspensión (CSS en $\mathrm{mg}^{-1}$ ). El contenido de materia orgánica se determinó siguiendo los métodos resumidos por Tena et al. (2011). Una vez que se determinó el contenido en materia orgánica, se restó del peso del filtro. Posteriormente, se estableció una correlación entre NTU y CSS para cada sonda (sección de control). En la mayoría de los casos, la relación siguió una regresión lineal (CSS $=a \times N T U+b$, donde $a$ varía de 0,6 a 0,9 y $b$ de -6 a 3,6, con un coeficiente de determinación $\left(\mathrm{r}^{2}\right)$ entre 0,89 y 0,98$)$. Solo en SCSE la relación entre NTU y CSS siguió una relación del tipo CSS $=0,0093 \times N T U^{2}+1,1303 \times N T U+3,8$ 
$\left(\mathrm{r}^{2}=0,96\right)$. Finalmente, la carga de sedimento en suspensión se calculó multiplicando la CSS (en mg $\mathrm{l}^{-}$ ${ }^{1}$, determinada a partir de la turbidez) y el $Q$ continuo $\left(\mathrm{en}^{3} \mathrm{~s}^{-1}\right)$.

\section{Resultados}

\subsection{Hidrología}

Antes de entrar en el embalse de Mequinenza en la sección de control de Sástago (SCSA), el río Ebro ha recibido ya el aporte de muchos de sus afluentes, aunque todavía no el de los más importantes desde el punto de vista hidro-sedimentario. En SCSA, el aporte medio anual durante los 3 años de estudio fue de $5927 \mathrm{hm}^{3}\left(\sigma=1660 \mathrm{hm}^{3}\right)$ un valor inferior a $\operatorname{los} 7310 \mathrm{hm}^{3}\left(\sigma=2690 \mathrm{hm}^{3}\right)$ registrados históricamente (1912-2016) y, por lo tanto, el período de estudio puede considerarse como seco. El caudal medio $\left(Q_{\mathrm{med}}\right)$ en SCSA para el período de estudio fue de $188 \mathrm{~m}^{3} \mathrm{~s}^{-1}\left(\sigma=201 \mathrm{~m}^{3} \mathrm{~s}^{-1}\right)$, oscilando entre $19 \mathrm{~m}^{3} \mathrm{~s}^{-1}$ y $1545 \mathrm{~m}^{3} \mathrm{~s}^{-1}$, éste último alcanzado durante una crecida en febrero de 2009 (equivalente a un período de retorno de 1,2 años, Tabla 1). El coeficiente de variación interanual (es decir, $\mathrm{CV}=Q_{\sigma} /$ $Q_{\mathrm{med}}$ ) fue del $107 \%$, un valor que confirma la alta variabilidad temporal.

El río Segre, tras recoger las aguas de los grandes afluentes del Pirineo, se convierte en el afluente más importante del Ebro. Pocos kilómetros antes de su entrada en el embalse de Ribarroja, confluye con el río Cinca, su principal afluente. La aportación media anual en la sección de control de Seròs (SCSE, río Segre) durante los 3 años del período de estudio fue de $1427 \mathrm{hm}^{3}\left(\sigma=430 \mathrm{hm}^{3}\right)$, un valor ligeramente inferior a los $1676 \mathrm{hm}^{3}\left(\sigma=189 \mathrm{hm}^{3}\right)$ registrados en la sección de control de Torrente (SCT) en el río Cinca. En ambos casos, la escorrentía está muy por debajo de los $5122 \mathrm{hm}^{3} \mathrm{a}^{-1}(\sigma=1985$ $\mathrm{hm}^{3}$ ) registrados para el sistema Segre-Cinca desde 1950 hasta la actualidad, confirmando que se trató de un periodo seco. Además, la proporción de agua producida en las dos cuencas (45 y 55\% en SCSE y $\mathrm{SCT}$, respectivamente) difiere de la relación histórica, que generalmente es inversa, 55\% en SCSE y $45 \%$ en SCT. El cambio en la proporción de escorrentía relativa entre los dos ríos puede estar relacionado con el incremento reciente de la regulación del caudal en el Segre (Batalla et al., 2004). El $Q_{\text {med }}$ en SCSE fue de $45 \mathrm{~m}^{3} \mathrm{~s}^{-1}\left(\sigma=36 \mathrm{~m}^{3} \mathrm{~s}^{-1}\right)$, con un caudal mínimo de $13 \mathrm{~m}^{3} \mathrm{~s}^{-1} \mathrm{y}$ un caudal máximo de $498 \mathrm{~m}^{3} \mathrm{~s}^{-1}$ (valor que representa un período de retorno de 2 años) alcanzado durante una crecida en la primavera de 2010. El $Q_{\text {med }}$ en SCT fue de $53 \mathrm{~m}^{3} \mathrm{~s}^{-1}\left(\sigma=36 \mathrm{~m}^{3} \mathrm{~s}^{-1}\right)$, y osciló entre $15 \mathrm{~m}^{3} \mathrm{~s}^{-1}$ y 593 $\mathrm{m}^{3} \mathrm{~s}^{-1}$, crecida con un período de retorno de 1,2 años (ver Tabla 1 para obtener información hidrológica completa). El coeficiente de variación interanual fue del 79\% en SCSE y del 68\% en SCT.

Los afluentes que fluyen al embalse de Ribarroja desde el Sistema Ibérico muestran unas características más secas que los pirenaicos. El río Algars se une al Matarraña $22 \mathrm{~km}$ antes de su desembocadura en el embalse de Ribarroja. Las cabeceras de estos dos ríos se encuentran en el mismo macizo montañoso, por lo que sus características hidroclimáticas son muy similares. Al igual que en el caso de los afluentes pirenaicos, los datos reflejan el carácter seco del período de estudio (2008-2011). La aportación hídrica durante el período de estudio fue de $16 \mathrm{hm}^{3} \mathrm{a}^{-1}\left(\sigma=9,6 \mathrm{hm}^{3}\right)$ en la sección de control de Nonaspe (SCN, Matarraña) y $12 \mathrm{hm}^{3} \mathrm{a}^{-1}\left(\sigma=4,6 \mathrm{hm}^{3}\right)$ en la sección de control de Batea (SCB, Algars), estando ambos valores muy por debajo de los $38 \mathrm{hm}^{3} \mathrm{a}^{-1}\left(\sigma=39 \mathrm{hm}^{3}\right)$ y $21 \mathrm{hm}^{3} \mathrm{a}^{-1}\left(\sigma=17 \mathrm{hm}^{3}\right)$ registrados históricamente (desde 1974 hasta la actualidad) (ver Tabla 1). El $Q_{\text {med }}$ en SCN fue de $0,5 \mathrm{~m}^{3} \mathrm{~s}^{-1}\left(\sigma=1,9 \mathrm{~m}^{3} \mathrm{~s}^{-1}\right)$, mientras que en SCB fue $\sigma 0,4 \mathrm{~m}^{3} \mathrm{~s}^{-1}\left(\sigma=1,3 \mathrm{~m}^{3} \mathrm{~s}^{-1}\right)$. El caudal fue especialmente bajo durante los meses de verano, pudiendo encontrarse el cauce seco en ambas secciones durante periodos más o menos largos (7 y 23\% del tiempo en SCN y SCB, respectivamente). El caudal máximo en SCN fue de $50 \mathrm{~m}^{3} \mathrm{~s}^{-1}$ y se alcanzó en octubre de 2011 (con un período de retorno de 1,6 años), mientras que en SCB fue de $39,5 \mathrm{~m}^{3} \mathrm{~s}^{-1} \mathrm{y}$ ocurrió en marzo de 2011 (período de retorno de 1,7 años). La variabilidad interanual fue notable en comparación con los afluentes pirenaicos, alcanzando un $\mathrm{CV}$ de $383 \%$ en $\mathrm{SCN}$ y $357 \%$ en SCB. En estos ríos mediterráneos, las crecidas son las principales responsables de la aportación hídrica de la cuenca. 
Tabla 1 Caudales para el periodo de estudio (2008-2011) en las diferentes secciones de estudio del Ebro y sus principales afluentes (ver Fig. IB para la ubicación y abreviación de las secciones)

\begin{tabular}{|c|c|c|c|c|c|c|c|}
\hline Área & Sección & Año & $\begin{array}{c}Q_{\text {med }} \\
\left(\mathbf{m}^{3} \mathbf{s}^{-1}\right)\end{array}$ & $\underset{\left(\mathbf{m}^{3} \mathbf{s}^{-1}\right)}{Q_{\max }}$ & $\begin{array}{c}Q_{\min } \\
\left(\mathbf{m}^{3} \mathbf{s}^{-1}\right)\end{array}$ & $\begin{array}{c}\sigma^{1} \\
\left(m^{3} s^{-1}\right)\end{array}$ & $\begin{array}{c}\text { Aportación } \\
\left(\mathrm{hm}^{3}\right)\end{array}$ \\
\hline \multirow{8}{*}{ 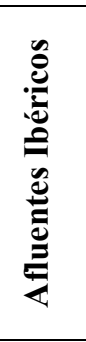 } & \multirow{4}{*}{ 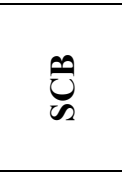 } & 2008-09 & 0,5 & 23,1 & 0,0 & 1,0 & 15,4 \\
\hline & & $2009-10$ & 0,2 & 5,0 & 0,0 & 0,4 & 6,5 \\
\hline & & $2010-11$ & 0,4 & 39,5 & 0,0 & 2,0 & 13,1 \\
\hline & & $2008-11$ & 0,4 & 39,5 & 0,0 & 1,3 & $11,7^{*}$ \\
\hline & \multirow{4}{*}{$Z_{\infty}^{Z}$} & 2008-09 & 0,8 & 32,8 & 0,2 & 1,9 & 26,0 \\
\hline & & $2009-10$ & 0,2 & 3,9 & 0,0 & 0,4 & 7,0 \\
\hline & & $2010-11$ & 0,5 & 49,9 & 0,0 & 2,7 & 14,7 \\
\hline & & 2008-11 & 0,5 & 49,9 & 0,0 & 1,9 & $15,9^{*}$ \\
\hline \multirow{8}{*}{ 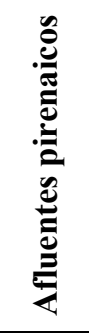 } & \multirow{4}{*}{ t) } & 2008-09 & 54,0 & 230,6 & 18,3 & 35,2 & 1702,0 \\
\hline & & $2009-10$ & 58,7 & 593,2 & 15,0 & 48,2 & 1851,3 \\
\hline & & 2010-11 & 46,8 & 127,4 & 17,6 & 17,3 & 1475,9 \\
\hline & & $2008-11$ & 53,2 & 593,2 & 15,0 & 36,2 & $1676,4^{*}$ \\
\hline & \multirow{4}{*}{ 点 } & 2008-09 & 58,0 & 245,3 & 19,0 & 30,5 & 1829,3 \\
\hline & & $2009-10$ & 46,8 & 498,2 & 12,8 & 48,5 & 1477,1 \\
\hline & & $2010-11$ & 30,9 & 114,0 & 13,8 & 13,7 & 973,4 \\
\hline & & $2008-11$ & 45,2 & 498,2 & 12,8 & 35,8 & $1426,6^{*}$ \\
\hline \multirow{8}{*}{ 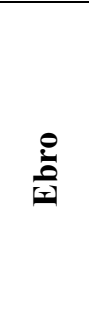 } & \multirow{4}{*}{ 依 } & 2008-09 & 234,2 & 1544,7 & 36,9 & 252,1 & 7384,8 \\
\hline & & $2009-10$ & 199,0 & 1476,4 & 36,5 & 190,8 & 6275,1 \\
\hline & & 2010-11 & 130,7 & 932,4 & 19,1 & 126,6 & 4121,1 \\
\hline & & $2008-11$ & 187,9 & 1544,7 & 19,1 & 201,3 & $5927,0^{*}$ \\
\hline & \multirow{4}{*}{ 萬 } & $2008-09$ & 344,3 & 1259,5 & $1,8^{* *}$ & 251,3 & 10859,4 \\
\hline & & 2009-10 & 314,1 & 2028,3 & $0,8^{* *}$ & 198,7 & 9904,9 \\
\hline & & $2010-11$ & 214,9 & 1123,1 & $1,5^{* *}$ & 119,5 & 6775,7 \\
\hline & & $2008-11$ & 291,1 & 2028,3 & $0,8^{* *}$ & 205,0 & $9180,0^{*}$ \\
\hline
\end{tabular}

\footnotetext{
${ }^{1}$ Desviación estándar.

${ }^{*}$ Valor medio.

** Valor mínimo cuando la presa de Ribarroja estaba cerrada.
}

En la sección de control de Ribarroja (SCR; ver la Fig. 1B para la ubicación exacta), el impacto que ejerce el complejo de presas de Mequinenza-Ribarroja (CPMR en adelante) en la hidrología es notable. La aportación media anual en este punto durante el período de estudio fue de $9180 \mathrm{hm}^{3}$. El caudal medio en esta sección fue de $291 \mathrm{~m}^{3} \mathrm{~s}^{-1}\left(\sigma=205 \mathrm{~m}^{3} \mathrm{~s}^{-1}\right)$, mostrando una variabilidad interanual más baja $(73 \%)$ que las mencionadas estaciones aguas arriba. El caudal máximo en este punto se registró en enero de 2010 durante una crecida natural en la que el caudal máximo liberado por la presa de Ribarroja alcanzó $2028 \mathrm{~m}^{3} \mathrm{~s}^{-1}$, un caudal equivalente a un periodo de retorno de 4 años. Se entiende por crecida natural la que se genera en la cuenca como consecuencia de un episodio lluvioso y que los embalses transitan, en contraposición a las crecidas artificiales que con fines ambientales se generan desde las presas (Batalla y Vericat, 2009).

La dinámica temporal de las aportaciones hídricas en las diferentes secciones de control se ha estudiado mediante un análisis de frecuencias. Los resultados se presentan en diagramas de cajas y bigotes o bloxplots (Fig. 2A). Este método permite conocer el porcentaje de tiempo necesario para transportar distintas fracciones de la aportación anual. Se han elegido un total de 7 percentiles característicos de la aportación anual: 5, 16, 25, 50, 75, 84 y 95\%.

La Figura 2A muestra el tiempo necesario para alcanzar diferentes percentiles característicos de la aportación hídrica media anual. El 50\% de la aportación hídrica se efectúa con un porcentaje de tiempo distinto en los afluentes ibéricos respecto a los pirenaicos y el propio Ebro. En SCB y SCN se requiere del 4 y del 3\% del tiempo para transportar el 50\% de la aportación, mientras que en SCT y SCSE se requiere alrededor del $25 \%$ del tiempo, valores superiores a los requeridos por SCSA y SCR (aproximadamente el 15 y $20 \%$ del tiempo, respectivamente). Estas diferencias muestran el marcado 
carácter mediterráneo de los afluentes ibéricos, en los que la aportación estaría controlada principalmente por las crecidas, frente a los caudales más regulares de los afluentes pirenaicos y del propio Ebro.

Si tomamos como referencia los percentiles más elevados, se puede observar cómo hay parte del tiempo en el que la aportación de los afluentes ibéricos es muy baja o incluso nula. Estos ríos tan solo requieren del $40 \%$ del tiempo o menos para transportar el $95 \%$ de la aportación. En el caso de los afluentes pirenaicos y del propio Ebro, en el que los caudales son más constantes, el tiempo necesario para transportar el $95 \%$ de la aportación en todos los casos es superior al $85 \%$ del tiempo.

\subsection{Transporte de sedimentos}

\subsubsection{Transporte de sedimentos en suspensión}

Las concentraciones de sedimentos en suspensión en la sección de control de SCSA, aguas arriba del embalse Mequinenza, son elevadas comparadas con la estación aguas abajo de la presa de Ribarroja (SCR). Los valores registrados en Sástago son la consecuencia de una menor regulación de este tramo del río y, sobre todo, de la aportación de destacados afluentes como el Gállego y el Jalón. La concentración media de sedimentos en suspensión $\left(C S S_{\mathrm{med}}\right)$ para el período de estudio fue de $56 \mathrm{mg} \mathrm{l}^{-1}$ $\left(\sigma=55,4 \mathrm{mg} \mathrm{l}^{-1}\right)$, mientras que la máxima $\left(C S S_{\max }\right)$ fue alrededor de $510 \mathrm{mg} \mathrm{l}^{-1}$ (ver Tabla 2 para el resumen completo de las CSS registradas)

Tabla 2 Datos de sedimento en suspensión para el periodo de estudio (2008-2011) en las diferentes secciones de estudio del Ebro y sus principales afluentes (ver Fig. 1B para la ubicación y abreviación de las secciones).

\begin{tabular}{|c|c|c|c|c|c|c|c|c|}
\hline Área & Sección & Año & $\begin{array}{l}\mathrm{CSS}_{\text {med }} \\
\left(\mathrm{mg} \mathrm{l}^{-1}\right)\end{array}$ & $\begin{array}{l}\mathrm{CSS}_{\max } \\
\left(\mathrm{mg} \mathrm{l}^{-1}\right)\end{array}$ & $\begin{array}{l}\mathrm{CSS}_{\min } \\
\left(\mathrm{mg} \mathrm{l}^{-1}\right)\end{array}$ & $\begin{array}{c}\sigma^{1} \\
\left(\mathrm{mg} \mathrm{l}^{-1}\right)\end{array}$ & $\begin{array}{c}\text { Carga Total } \\
\left(10^{6} t\right)\end{array}$ & $\begin{array}{l}\text { Producción } \\
\left(\mathrm{t} \mathrm{km}^{2} \mathrm{yr}^{-1}\right)\end{array}$ \\
\hline \multirow{8}{*}{ 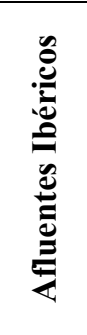 } & \multirow{4}{*}{ 己ै } & 2008-09 & 9,7 & 632,9 & 0 & 14,4 & 0,00024 & 0,7 \\
\hline & & $2009-10$ & 6,9 & 261,7 & 0,1 & 7,4 & 0,00007 & 0,2 \\
\hline & & $2010-11$ & 10 & 510 & 0,4 & 13,1 & 0,00053 & 1,6 \\
\hline & & $2008-11$ & 8,9 & 632,9 & 0 & 12,1 & $0,00083\left(0,00028^{*}\right)$ & 0,9 \\
\hline & \multirow{4}{*}{ Z } & 2008-09 & 4,5 & 309,3 & 0,4 & 13 & 0,00064 & 0,6 \\
\hline & & $2009-10$ & 3,2 & 46 & 0,4 & 2,5 & 0,00003 & 0 \\
\hline & & $2010-11$ & 3,9 & 280,7 & 0,7 & 10,1 & 0,00075 & 0,7 \\
\hline & & $2008-11$ & 3,8 & 309,3 & 0,4 & 9,6 & $0,00143\left(0,00048^{*}\right)$ & 0,5 \\
\hline \multirow{8}{*}{ 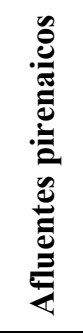 } & \multirow{4}{*}{ 记 } & 2008-09 & 80,4 & 2033 & 7,5 & 167,1 & 0,15 & 16 \\
\hline & & $2009-10$ & 50,7 & 1701,5 & 8,8 & 81,1 & 0,14 & 15 \\
\hline & & $2010-11$ & 35,4 & 1434,5 & 6,7 & 71 & 0,07 & 6,9 \\
\hline & & $2008-11$ & 55,5 & 2033 & 6,7 & 116,3 & $0,365\left(0,12^{*}\right)$ & 12,7 \\
\hline & \multirow{4}{*}{ 己 } & $2008-09$ & 26,6 & 486,5 & 6,1 & 27,9 & 0,06 & 4,5 \\
\hline & & $2009-10$ & 25 & 1551,7 & 7,2 & 38 & 0,05 & 3,7 \\
\hline & & $2010-11$ & 16,9 & 222 & 6,1 & 9,7 & 0,02 & 1,4 \\
\hline & & $2008-11$ & 22,8 & 1551,7 & 6,1 & 28,1 & $0,121\left(0,040^{*}\right)$ & 3,2 \\
\hline \multirow{8}{*}{$\stackrel{\stackrel{0}{0}}{\text { 国 }}$} & \multirow{4}{*}{$\underset{\mathscr{\infty}}{\mathbb{\infty}}$} & 2008-09 & 69,5 & 510 & 16,5 & 73,3 & 1,07 & 20,9 \\
\hline & & 2009-10 & 55,8 & 484,8 & 16,3 & 48,8 & 0,62 & 12,1 \\
\hline & & $2010-11$ & 42,5 & 283,5 & 9 & 33 & 0,29 & 5,6 \\
\hline & & $2008-11$ & 55,9 & 510 & 9 & 55,4 & $1,978\left(0,66^{*}\right)$ & 12,9 \\
\hline & \multirow{4}{*}{$\underset{\mathscr{U}}{\tilde{U}}$} & 2008-09 & 5,5 & 72,4 & 0 & 4,2 & 0,06 & 0,7 \\
\hline & & $2009-10$ & 5,4 & 214,2 & 1,4 & 5,7 & 0,05 & 0,6 \\
\hline & & $2010-11$ & 3,6 & 206,2 & 0,2 & 4,6 & 0,02 & 0,3 \\
\hline & & $2008-11$ & 4,9 & 214,2 & 0 & 4,9 & $0,131\left(0,043^{*}\right)$ & 0,5 \\
\hline
\end{tabular}

${ }^{1}$ Desviación estándar.

*Valor medio. 
En el caso de los afluentes pirenaicos, la $C S S_{\text {med }}$ observada fue generalmente mayor que la de los afluentes ibéricos. La CSS med para el período de estudio en el río Segre (SCSE) fue de $23 \mathrm{mg}^{-1}$ ( $\sigma=$ $\left.28 \mathrm{mg} \mathrm{l}^{-1}\right)$, un valor inferior a los $56 \mathrm{mg} \mathrm{l}^{-1}\left(\sigma=116 \mathrm{mg} \mathrm{l}^{-1}\right)$ registrados en el Cinca (SCT). La CSS $S_{\max }$ también fue mayor en SCT que en SCSE (2033 mg $\mathrm{l}^{-1}$ y $1552 \mathrm{mg} \mathrm{l}^{-1}$, respectivamente). Por su parte, los ríos del Sistema Ibérico mostraron una $C_{S S} S_{\mathrm{med}}$ de $9 \mathrm{mg} \mathrm{l}^{-1}$ en el Algars (SCB, $\sigma=12 \mathrm{mg}^{1-1}$ ), y $4 \mathrm{mg} \mathrm{l}^{-1}$ en el Matarraña ( $\mathrm{SCN}, \sigma=10 \mathrm{mg} \mathrm{l}^{-1}$ ). La CSS $S_{\max }$ también fue mayor en SCB, con un pico de sedimento de $633 \mathrm{mg} \mathrm{l}^{-1}$, dos veces superior al pico registrado en SCN (Tabla 2). La CSS $S_{\max }$ se alcanzó en ambos ríos durante el año 2008-09, pero bajo diferentes condiciones de caudal. Mientras que en el Matarraña coincidió con el caudal máximo $\left(32 \mathrm{~m}^{3} \mathrm{~s}^{-1}\right)$, en el caso del Algars ocurrió durante una crecida de baja magnitud $\left(0,75 \mathrm{~m}^{3} \mathrm{~s}^{-1}\right)$, lo que muestra un claro ejemplo de no linealidad en las relaciones Q-CSS en cuencas donde el suministro y la disponibilidad de sedimentos es variable.

El efecto del complejo de embalses de Mequinenza y Ribarroja se hace patente en el transporte de sedimento en suspensión. La sección de control de Ribarroja, localizada aguas abajo del embalse de Ribarroja, registró CSS de un orden de magnitud inferior a los valores estimados aguas arriba de Mequinenza. Como se indica en la Tabla 2, la $C_{S S}$ med en SCR no superó los $5 \mathrm{mg} \mathrm{l}^{-1}$, mostrando una variabilidad muy baja entre los tres años de estudio $\left(\sigma=2 \mathrm{mg} \mathrm{l}^{-1}\right)$. La CSS $\max$ tuvo lugar durante una crecida de mantenimiento realizada en mayo de 2010, llegando a alcanzar los $214 \mathrm{mg} \mathrm{l}^{-1}$ (Batalla y Vericat, 2009).

\subsubsection{Análisis de frecuencias}

Al igual que en el caso de la aportación hídrica, las cargas sedimentarias y su dinámica temporal se han estudiado en las diferentes secciones de control mediante un análisis de frecuencias (Fig. 2B).

Se distinguen diferentes patrones según el régimen hidrológico del río y el grado de regulación de cada sección. La carga de sedimentos en los afluentes pirenaicos se muestra relativamente constante en el tiempo. La Figura 2B indica que el $84 \%$ del sedimento fue transportado en aproximadamente el $65 \%$ del tiempo en el río Cinca (correspondiente a un $Q>35 \mathrm{~m}^{3} \mathrm{~s}^{-1}$ ); mientras que en el Segre se transportó la misma proporción en aproximadamente $2 / 3$ del tiempo (para $Q>29 \mathrm{~m}^{3} \mathrm{~s}^{-1}$ ). Las crecidas y los caudales altos (tomados como el doble del caudal medio) fueron responsables del 43 y el $52 \%$ de la carga de sedimentos transportada durante el período de estudio en el Segre y el Cinca, respectivamente.

En el caso de los afluentes del Sistema Ibérico (Fig. 2B), el 84\% del sedimento se transportó en el $9 \%$ del tiempo $\left(Q>0,6 \mathrm{~m}^{3} \mathrm{~s}^{-1}\right)$ en el río Algars, y en menos del $2 \%$ en el caso del Matarraña $(Q>$ $\left.3,8 \mathrm{~m}^{3} \mathrm{~s}^{-1}\right)$. En estos dos ríos, la carga está más dominada por las crecidas, que pueden transportar hasta el $95 \%$ de la carga anual. Por ejemplo, si se analizan las frecuencias para cada año de manera independiente, se observa que en 2009 y 2011 las crecidas transportaron el 95\% de la carga en los ríos del Sistema Ibérico. En general, los diagramas están comprimidos en rangos inferiores cuanto más secos son los ambientes (como es el caso de las cuencas mediterráneas del Sistema Ibérico); como se ha indicado, en estas cuencas, las crecidas son responsables de transportar una porción más grande de sedimentos que en otras cuencas con regímenes hidroclimáticos más húmedos.

De las dos secciones localizadas en el curso principal del Ebro, la de SCSA es la que presenta una mayor variabilidad temporal en la carga. Los diagramas de cajas (Fig. 2B) muestran que el 84\% del sedimento en suspensión fue transportado en el $25 \%$ del tiempo $\left(Q>174 \mathrm{~m}^{3} \mathrm{~s}^{-1}\right)$, mostrando una variabilidad intermedia entre las cuencas ibéricas y pirenaicas. Las crecidas y los caudales altos fueron responsables del $74 \%$ de la carga total de sedimentos en suspensión. Aguas abajo, la frecuencia de la carga sedimentaria se ve alterada por las presas de Mequinenza y Ribarroja. La variabilidad natural de los afluentes desaparece y la carga de sedimentos es más constante en el tiempo. La duración requerida para transportar el $86 \%$ de la carga en suspensión en la sección de SCR fue del $42 \%$ del tiempo $(Q>$ $187 \mathrm{~m}^{3} \mathrm{~s}^{-1}$ ). Este patrón está en el mismo rango de variabilidad que el observado en el sistema SegreCinca, sugiriendo por una parte que estos dos afluentes aún ejercen cierta influencia en la carga 
sedimentaria aguas abajo de Ribarroja, a pesar del alto grado de regulación de los dos sistemas (SegreCinca y Ebro). Las crecidas y los caudales altos son responsables del $38 \%$ de la carga total de sedimentos en suspensión transportada en SCR, un valor notablemente más bajo que el obtenido en SCSA y en los afluentes ibéricos.
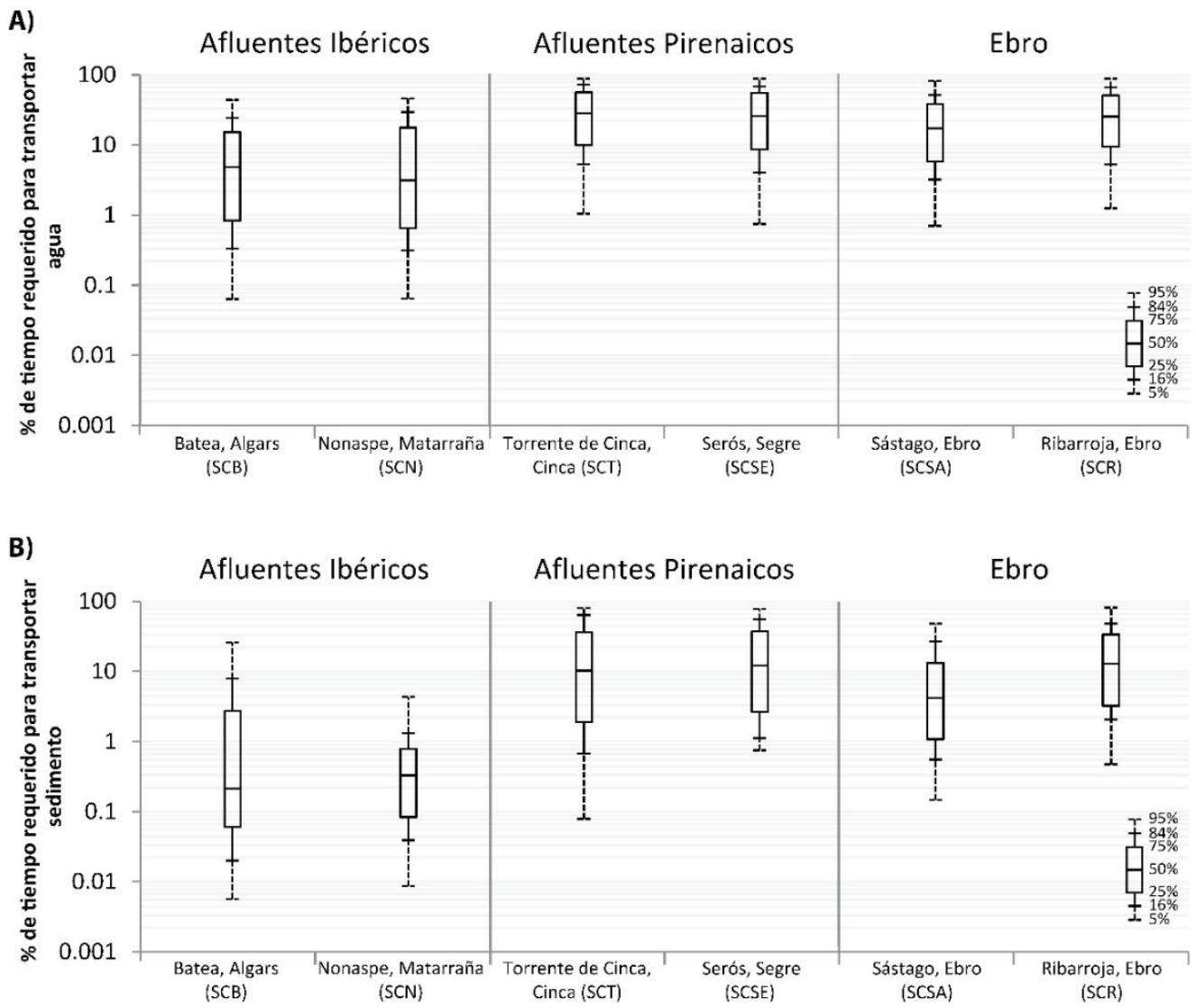

Figura 2. Boxplot (diagrama de caja y bigotes) que muestra el porcentaje de tiempo requerido para alcanzar diferentes percentiles de la aportación hídrica $(5,16,25,50,5,84$ y 95\%) y de la carga sedimentaria en las diferentes secciones de control durante el periodo de estudio (2008-2011). A) Representa la aportación hídrica en las diferentes secciones B) Representa la carga sedimentaria en las diferentes secciones. En la figura las diferentes cajas están separadas por su localización geográfica: Afluentes ibéricos: SCB (Sección de Control de Batea, Río Algars) y SCN (Sección de Control de Nonaspe, Río Matarraña); Afluentes pirenaicos: SCT (Sección de Control de Torrente, Río Cinca) y SCSE (Sección de Control de Seròs, Río Segre); Bajo Ebro aguas arriba del embalse de Mequinenza: SCSA (Sección de Control de Sástago) y aguas abajo de la presa de Ribarroja; SCR (Sección de Control de Ribarroja). Ver Figura 1B para la localización de las secciones.

\subsection{Balance sedimentario del embalse de Ribarroja}

La construcción del balance sedimentario en el embalse de Ribarroja tiene en cuenta la carga sólida estimada aguas arriba del embalse de Mequinenza, las principales entradas de sedimentos en el embalse de Ribarroja y la carga observada aguas abajo del mismo. El objetivo principal es la cuantificación de los flujos sedimentarios en el embalse (entradas y salidas), y el análisis de la influencia de: $i$ ) el diferente régimen hidrológico de las cuencas fluyentes $\mathrm{y}$, ii) la influencia del complejo de presas de Mequinenza y Ribarroja (CPMR) en el tránsito de sedimentos. La Figura 3 resume el balance sedimentario del embalse de Ribarroja y representa los principales flujos de sedimentos para el período de estudio. 


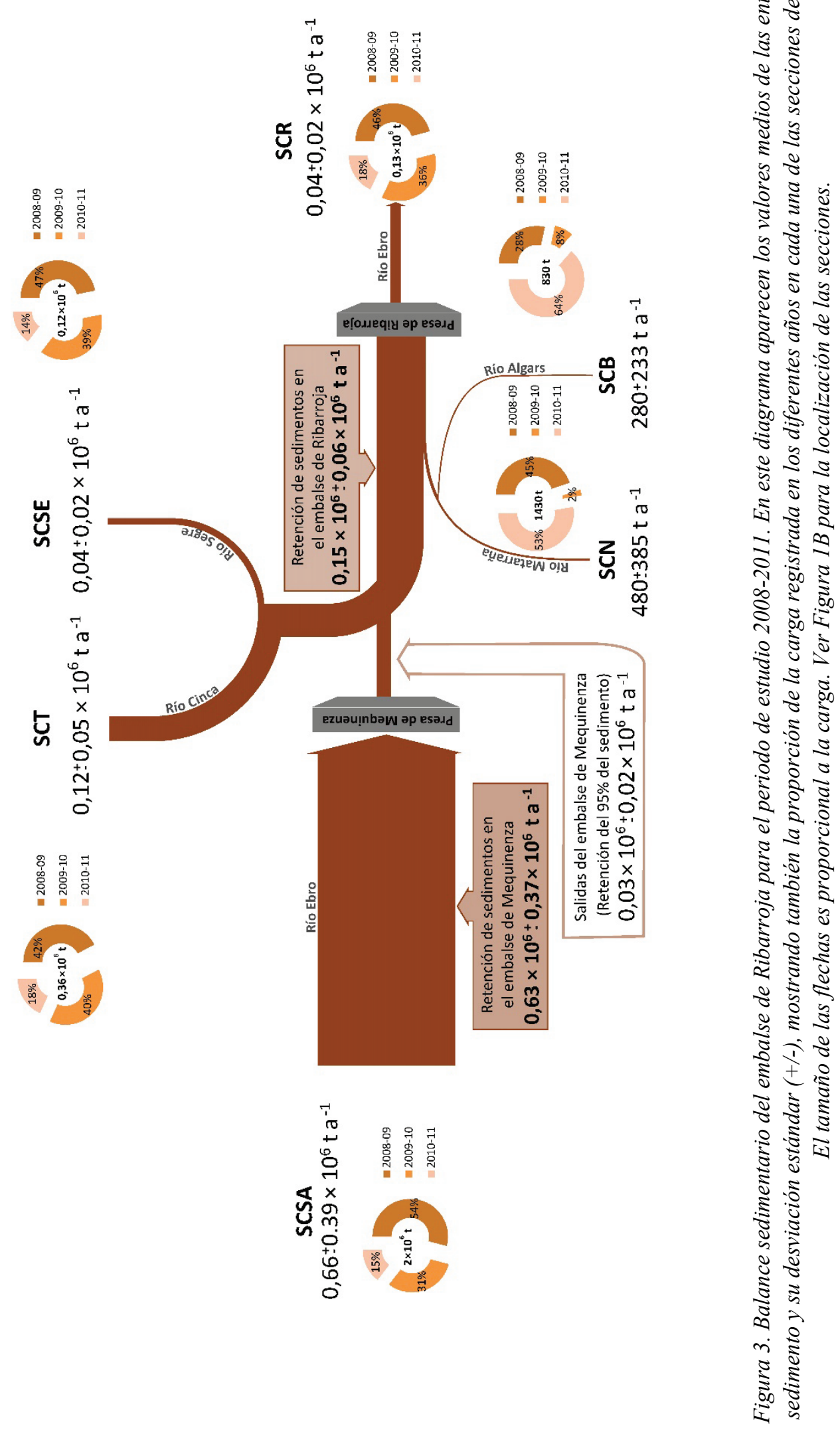




\subsubsection{Entrada de sedimentos al embalse de Mequinenza}

La aportación hídrica y los caudales máximos observados durante el período de estudio fueron similares a los años previamente estudiados por Vericat y Batalla (2006). Por lo tanto, las curvas de duración de caudales para el período 2008-2011, junto con la relación estadísticamente significativa entre $Q$ y $C S S$ obtenida en 2003-2004, proporcionan una base fiable para la estimación de la carga sedimentaria que ha transitado por SCSA durante el período de estudio. A modo de comparación, Vericat y Batalla (2006) calcularon una carga sedimentaria de $1 \times 10^{6} \mathrm{t} \mathrm{a}^{-1}$ para una aportación hídrica de $7263 \mathrm{hm}^{3}$; mientras que en el caso de la carga sedimentaria en 2008-2009 fue de 1,1 $\times 10^{6} \mathrm{t} \mathrm{a}^{-1}$ para una aportación hídrica de $7384 \mathrm{hm}^{3}$. Roura (2004) determinó una carga sedimentaria de $0,5 \times 10^{6}$ t para años con un promedio de aportación hídrica cercano a $5700 \mathrm{hm}^{3}$, un valor que coincide con las estimaciones de este estudio para los años más secos i.e. 2009-10, con una aportación hídrica de $6275 \mathrm{hm}^{3}$ y una carga de $0,6 \times 10^{6} \mathrm{t}$ de sedimento, y $2010-11$ con $4121 \mathrm{hm}^{3}$ de agua y $0,3 \times 10^{6} \mathrm{t} \mathrm{de}$ sedimento. La carga sedimentaria estimada para el río Ebro aguas arriba del embalse de Mequinenza fue de 2,0 $\times 10^{6} \mathrm{t}$ para todo el período 2008-2011, promediando una carga anual de $0,7 \times 10^{6} \mathrm{t} \mathrm{a}^{-1}(\sigma=0,4 \mathrm{t})$. Este valor representa una carga específica cercana a $13 \mathrm{t} \mathrm{km}^{-2} \mathrm{a}^{-1}$, un valor ligeramente inferior al obtenido por Sanz et al. (1999) y Vericat y Batalla (2006) durante períodos más húmedos $\left(30 \mathrm{t} \mathrm{km}^{-2} \mathrm{a}^{-1}\right)$, y superiores a los estimados por Palanques $\left(1987,7 \mathrm{t} \mathrm{km}^{-2} \mathrm{a}^{-1}\right)$ y Roura $\left(2004,10 \mathrm{t} \mathrm{km}^{-2} \mathrm{a}^{-1}\right)$ durante los años más secos. La carga estimada que entra en el embalse de Mequinenza es del orden de magnitud de otros grandes ríos europeos como el Garona y el Rin, cuya carga específica es de $21 \mathrm{t} \mathrm{km}^{-2} \mathrm{a}^{-1} \mathrm{y}$ $15 \mathrm{t} \mathrm{km}^{2} \mathrm{a}^{-1}$, respectivamente (Meybeck y Ragu, 1997). Aunque esta sección ya esté afectada por un déficit de sedimento estructural derivado de los embalses aguas arriba (ver Batalla y Vericat, 2011 para más detalles), aún mantiene un cierto comportamiento natural en cuanto a la dinámica del transporte sedimentario. Este hecho queda demostrado por la notable variabilidad interanual observada en la carga sedimentaria entre los años más húmedos y más secos (ca. 60\%). Debido a su tamaño y a la enorme capacidad de regulación, el tiempo de residencia del agua en el embalse de Mequinenza es bastante elevado (entre 1 y 2,5 meses según Prats et al. 2009), favoreciendo así la sedimentación de la mayor parte de la carga en suspensión transportada por el Ebro. Roura (2004) estima una capacidad de retención de sedimentos del 95\% para un período de estudio de 2 años (1998-2000).

\subsubsection{Entrada de sedimentos al embalse de Ribarroja}

La fracción de la carga de sedimentos en suspensión que pasa a través de la presa de Mequinenza entra directamente en el embalse de Ribarroja ya que la cola del mismo llega hasta la presa. Basado en la capacidad de retención de sedimentos del embalse de Mequinenza del 95\% obtenida por Roura (2004), nuestras estimaciones indican que llegaron al embalse de Ribarroja alrededor de $0,1 \times 10^{6}$ t durante el período 2008-2011, lo que significa una aportación anual de $0,03 \mathrm{t}(\sigma=0,02 \mathrm{t})$.

Tres kilómetros aguas abajo de la presa de Mequinenza, el Ebro recibe la aportación de los dos afluentes principales de la cuenca (Segre y Cinca, ver Fig. 1B para más detalles). La carga sedimentaria de estos dos ríos para todo el período fue de $0,49 \times 10^{6} \mathrm{t}\left(\sigma=0,02 \times 10^{6} \mathrm{t}\right)$, produciendo una carga media anual de $0,08 \mathrm{t} \mathrm{a}^{-1}$ (aproximadamente $1 / 4$ proveniente del Segre $\mathrm{y} 3 / 4$ del Cinca, a lo largo del período de estudio de 3 años). La distribución de las cargas anuales se encuentra en la tabla 2 y en la Figura 3 de manera porcentual. Específicamente, la carga en SCSE (Río Segre) para el período de estudio fue de $0,12 \times 10^{6} \mathrm{t}\left(\sigma=0,02 \times 10^{6} \mathrm{t}\right)$, produciendo una carga media anual de $0,04 \times 10^{6} \mathrm{t}$ (ver Tabla 2); a su vez, la carga obtenida en SCT (Río Cinca) fue de $0,36 \times 10^{6} \mathrm{t}\left(\sigma=0,05 \times 10^{6} \mathrm{t}\right)$, con una carga media anual de $0,12 \mathrm{t} \mathrm{a}^{-1}$. Como en el caso de la aportación hídrica, la variabilidad interanual de la carga sedimentaria es relativamente baja ( $\mathrm{CV}=39 \%$ y $50 \%$, respectivamente). La carga sedimentaria media anual fue de 3,2 $\mathrm{t} \mathrm{km}^{-2} \mathrm{a}^{-1}$ en el caso del Segre y de $12,7 \mathrm{t} \mathrm{km}^{-2} \mathrm{a}^{-1}$ en el Cinca. A pesar de que la carga es notable en estas cuencas, sobre todo en el caso del Cinca, los valores permanecen por debajo de los obtenidos en otros ríos que drenan áreas montañosas en todo el mundo (Dedkov y Moszherin, 1992) 
debido a la elevada regulación de ambas cuencas, y a otras presiones antrópicas (e.g. extracciones de áridos) que condicionan el gran déficit sedimentario al que están sujetas.

Aproximadamente $28 \mathrm{~km}$ aguas abajo de la entrada de los afluentes pirenaicos, el Matarraña (incluido su afluente principal, el Algars) desemboca en el embalse de Ribarroja. La carga sedimentaria proveniente de estos afluentes Ibéricos fue de 2260 t para todo el período de estudio, produciendo un valor medio anual de 376,15 t a ${ }^{-1}(\sigma=304 \mathrm{t})$ (ver la distribución de las cargas en la Tabla 2 y la Fig. 3). Debido a su mayor superficie, la carga obtenida en el Matarraña es mayor que la del Algars. La carga total que transitó por la sección de control Nonaspe (SCN, Río Matarraña) fue de 1426 t, produciendo una carga media anual de $475 \mathrm{t}(\sigma=385 \mathrm{t}$; ver Tabla 2). Este valor es superior a las $831 \mathrm{t}$ que pasaron por la sección de control de Batea (SCB, Río Algars) con una carga media anual de 280 ( $\sigma=233$ t; ver Tabla 2). En ambos casos, la carga sedimentaria está marcada por una fuerte variabilidad interanual, variando entre 35 t (2009-2010) y 751 t (2010-2011) en el caso de Matarraña, y entre 67 t (2009-2010) y 528 t (2010 -2011) en el Algars (ver Tabla 2). Estos valores representan CV de 123\% y 84\% para SCN y $\mathrm{SCB}$, respectivamente. La carga específica del Algars $\left(0,85 \mathrm{t} \mathrm{km}^{-2}\right.$ año-1) es más alta que la del Matarraña $\left(0,46 \mathrm{t} \mathrm{km}^{-2}\right.$ año $\left.{ }^{-1}\right)$, aunque en ambos casos los valores son muy bajos en comparación con otras cuencas mediterráneas similares (Inbar 1992; Batalla et al., 2005; Lobera et al., 2016; Piqué et al., 2017) y un orden de magnitud inferior al observado en las cuencas pirenaicas. Estas cargas no están directamente relacionadas con la aportación hídrica cuyo máximo se registró en 2008-09; en cambio, podrían estar relacionadas con la mayor crecida registrada, que tuvo lugar en el año hidrológico 201011 y que fue responsable del 61\% (322 t) y el 45\% (339t) del sedimento transportado en este año en el Algars y el Matarraña, respectivamente.

En conjunto, la carga sedimentaria que entró en el embalse de Ribarroja fue aproximadamente de $0,60 \times 10^{6} \mathrm{t}$ para todo el período de estudio, produciendo un valor medio anual de $0,2 \times 10^{6} \mathrm{ta}^{-1}(\sigma=$ $\left.0,09 \times 10^{6} \mathrm{t}\right)$. Anualmente, la carga de sedimentos en suspensión se distribuyó con $0,27 \times 10^{6} \mathrm{t}$ en $2008-$ $09,0,22 \times 10^{6} \mathrm{t}$ en $2009-10$, y casi $0,1 \times 10^{6} \mathrm{t}$ en $2010-11$. Aunque el tamaño $(35 \mathrm{~km}$ de largo y 220 $\mathrm{hm}^{3}$ de capacidad) del embalse de Ribarroja es inferior al del embalse de Mequinenza, igualmente gran parte de los sedimentos en suspensión que a él llegan quedan atrapados en él. Vericat y Batalla (2006) estimaron una tasa de retención de hasta el $90 \%$ en el embalse de Ribarroja sin tener en cuenta la aportación de los afluentes.

\subsubsection{Salidas del embalse de Ribarroja}

El efecto del complejo de embalses Mequinenza-Ribarroja sobre la carga de sedimentos del río Ebro es notable. La carga sedimentaria en SCR, inmediatamente aguas abajo de la presa, fue de $0,13 \times$ $10^{6} \mathrm{t}$ durante todo el período de estudio, con un valor medio anual de $0,04 \mathrm{t} \mathrm{a}^{-1}\left(\sigma=0,02 \times 10^{6} \mathrm{t}\right)$. Este valor representa ca. $25 \%$ de la carga total entrante en Ribarroja y ca. $5 \%$ si también tenemos en cuenta las entradas a Mequinenza. Porcentajes similares de retención (i.e. 95\%) fueron estimados por Avendaño et al. (1997) basados en datos de sedimentación en el propio embalse; Sanz et al. (1999) basados en un muestreo infrecuente; Vericat y Batalla (2005) siguiendo el método de Brune (1953) y datos de dos crecidas extraordinarias, y Vericat y Batalla (2006) basados en un muestreo frecuente.

Las cargas anuales se dieron en relación directa con la hidrología observada. De este modo, se registró una mayor carga sedimentaria en 2008-09 y menor en 2009-10 y 2010-11 (ver Tabla 2). La variabilidad del sedimento entre años fue moderada (CV 46\%). Aunque no tiene un sentido geomorfológico claro por la desconexión del punto de muestreo con la cuenca aguas arriba, se ofrece un valor de carga específica: se trata de un valor muy bajo, $0,7 \mathrm{t} \mathrm{km}^{-2} \mathrm{a}^{-1}$, como cabía esperar para un sistema fuertemente regulado. Este valor es ligeramente más bajo que el estimado previamente por los autores para un período de 10 años en dos secciones situadas aguas abajo del embalse, la primera en Pas de l'Ase $\left(1 \mathrm{t} \mathrm{km}^{-2} \mathrm{a}^{-1}\right)$ y la segunda en Xerta $\left(1,3 \mathrm{t} \mathrm{km}^{-2} \mathrm{a}^{-1}\right)$ (Tena et al., 2011, 2012). Las diferencias entre las estimaciones de este estudio y otros estudios recientes en el bajo Ebro (por ejemplo, Vericat y 
Batalla 2006; Négrel et al., 2007) pueden atribuirse al carácter seco del período de estudio 2008-2011, y a las diferencias que pueda haber entre las secciones donde se ha estimado la carga (e.g. confluencia de tributarios que aporten carga sedimentaria). Aun así, la comparación de estos valores con otros grandes ríos regulados en Europa como por el ejemplo el Rin $\left(15 \mathrm{t} \mathrm{km}^{-2} \mathrm{a}^{-1}\right.$, Meybeck y Ragu, 1997) y el Ródano (101 t km$~^{-2} \mathrm{a}^{-1}$, Serrat et al., 2001) indica la severidad del déficit sedimentario en el Ebro en su tramo final.

\section{Discusión}

El balance de sedimentos realizado corrobora que el complejo de embalses de Mequinenza y Ribarroja reduce notablemente la carga sedimentaria transportada por el río. Como diversos estudios anteriores han puesto de manifiesto, el déficit sedimentario que generan las presas del tramo bajo del río se suma al que el resto de grandes presas genera a lo largo de la cuenca, todo ello en un contexto de manifiesta reducción de la aportación sedimentaria de la cuenca desde la mitad del siglo pasado (ver los detalles del balance sedimentario completo de la cuenca del Ebro en Batalla y Vericat, 2011). Este fenómeno está asociado a varios factores, tales como la reducción de la escorrentía y, con ello, de la erosión y la carga sedimentaria debido sobre todo a la reforestación que ha tenido lugar después del abandono de tierras de cultivo durante la segunda mitad del siglo XX (e.g. Gallart y Llorens, 2004). López-Moreno et al. (2008) atribuyen esta reducción en la escorrentía a una reducción en la precipitación y acumulación de nieve, sumado a un aumento de la evapotranspiración. Además de estos factores primarios, el papel de los embalses ubicados en las regiones de cabecera es también importante, ya que interrumpen la continuidad entre las zonas de generación de sedimentos, las de transporte, y las de sedimentación. Un buen ejemplo de esto es el embalse de Barasona, que regula el sistema ÉseraIsábena. Lobera et al. (2016) estimaron una sedimentación cercana a las $300.000 \mathrm{t} \mathrm{a}^{-1}$ para el periodo 2011-2013, teniendo en cuenta tan solo el río Ésera. Sin embargo, si se incluyera el río Isábena en estos cálculos, la cifra aumentaría hasta las $550.000 \mathrm{t} \mathrm{a}^{-1}$ (según López-Tarazón y Batalla, 2014).

La carga total de sedimentos en suspensión atrapados en el complejo Mequinenza-Ribarroja durante el período de estudio se estima en $2,35 \times 10^{6} \mathrm{t}$ (lo que equivale al $95 \%$ del sedimento transportado por el río Ebro aguas arriba del embalse de Mequinenza y los principales afluentes), valor que supone una retención media anual de $0,78 \times 10^{6} \mathrm{t}$. Es importante mencionar que el periodo de estudio de este trabajo se clasifica como seco; por lo tanto, la carga de sedimentos fue probablemente más baja en comparación con los valores obtenidos a más largo plazo (Batalla y Vericat, 2011).

La retención de sedimentos en los embalses genera un déficit sedimentario en los tramos aguas abajo. Richards (1982) señaló que el efecto de la retención de sedimentos tiende a disminuir aguas abajo, y la carga sedimentaria puede llegar a recuperar unas condiciones similares a las de aguas arriba de la presa. Sin embargo, la distancia necesaria para la recuperación puede variar según la contribución de los afluentes y el tamaño de la cuenca, entre otros factores. Williams y Wolman (1984) establecieron que para grandes ríos se requería una distancia de $500 \mathrm{~km}$ para recuperar los niveles previos a la presa, aunque, en algunos casos, la carga de sedimentos pudiera no llegar a recuperarse completamente. En el caso del Ebro, el complejo de embalses analizado se encuentra a $115 \mathrm{~km}$ de la desembocadura en el mar, valor inferior al citado por dichos autores como referencia. Cabe citar que en dicho tramo los afluentes son efímeros, y el único río con capacidad suficiente para aportar cantidades apreciables de sedimentos, el Siurana, está también regulado y su lecho afectado históricamente por una importante minería de gravas. Trabajos anteriores apuntan a que, durante episodios de crecida, ya sean naturales o artificiales, por medio de la erosión de los márgenes y el lecho se puede aumentar la carga sedimentaria del río hasta algunos cientos de miles de toneladas por año (Batalla y Vericat, 2009; Vericat y Batalla, 2006; Tena et al., 2013). Otros trabajos han demostrado que las contribuciones de los pequeños afluentes efimeros durante eventos torrenciales pueden incluso superar la carga transportada durante una crecida de mantenimiento (5\% de la carga media anual para el período 1998-2008; Tena et al., 2012). 
El balance de sedimentos del embalse de Ribarroja proporciona información interesante para comprender el efecto de las presas en el comportamiento sedimentario de un gran río mediterráneo, todo ello en el contexto de un déficit estructural de sedimentos de la propia cuenca desarrollado a lo largo del siglo XX. El conocimiento adquirido en este trabajo puede y debe considerarse como una base para diseñar estrategias para la gestión de sedimentos, conducentes a la progresiva renaturalización (o rehabilitación parcial o total) de su régimen hidrológico y sedimentario. En el bajo Ebro se llevan a cabo medidas de rehabilitación hidrológica desde el año 2002 mediante desembalses regulares desde Ribarroja (crecidas de mantenimiento, Vericat y Batalla, 2009; Tena et al., 2014). Estas crecidas artificiales han sido diseñadas e implementadas, monitorizadas y modelizadas con el objetivo de controlar las poblaciones de macrófitos y analizar el transporte de sedimentos durante las mismas. Los resultados demostraron inicialmente su efectividad en la eliminación de macrófitos (hasta el 95\% en el meandro de Flix, aunque disminuyendo en dirección aguas abajo) y su potencial para erosionar y transportar sedimentos aguas abajo (Batalla y Vericat, 2009; Vericat y Batalla, 2006; Tena et al., 2013).

El potencial demostrado por estas medidas de rehabilitación, junto con la predisposición del organismo de cuenca y los gestores de las infraestructuras hidráulicas para aplicar estas medidas en otros embalses de la cuenca puede abrir nuevos horizontes y pasar de la concepción tradicional de las crecidas de mantenimiento desde una sola presa, hacia una crecida de mantenimiento en cascada en otros ríos regulados de la cuenca del Ebro. Se podrían coordinar de esta manera operaciones de mantenimiento de presas, como la suelta de sedimentos en el embalse de Barasona u otros, con conocidos problemas de sedimentación, junto con crecidas de mantenimiento generadas, por ejemplo, desde la presa de Grado en el río Cinca.

Finalmente, en un estudio dirigido por Dolz et al. (2009) sobre la dinámica sedimentaria del embalse de Ribarroja, se calculó un volumen de entre 13 y $16 \mathrm{hm}^{3}$ de sedimentos acumulados en todo el embalse (valores que corroboran los obtenidos en este estudio), de los cuales una importante proporción $\left(5 \mathrm{hm}^{3}\right)$ se encuentra en la confluencia del sistema Segre-Cinca con el Ebro. Además, estos mismos autores observaron que durante un solo evento de crecida gran parte de estos depósitos se movilizaron hasta 3 kilómetros. Esta información justificaría analizar el potencial de la coordinación de operaciones desde la cuenca del río Cinca y la presa de Ribarroja para maximizar la transferencia de sedimentos hacia el tramo bajo del río.

\section{Conclusiones}

El balance sedimentario del embalse de Ribarroja se ha elaborado a partir de datos de caudal y sedimento en suspensión en las entradas y salidas del mismo para el periodo (2008-2011). Las principales conclusiones del trabajo son:

1. La carga sedimentaria media anual entrante en el embalse de Ribarroja fue de $0,2 \times 10^{6} \mathrm{ta}^{-1}(\sigma=$ $0,09 \times 10^{6} \mathrm{t}$ ), mientras que el valor medio anual obtenido aguas abajo de la presa fue de $0,04 \times$ $10^{6} \mathrm{ta}^{-1}\left(\sigma=0,02 \times 10^{6} \mathrm{t}\right)$.

2. La tasa de retención del embalse de Ribarroja es de aproximadamente el $75 \%$ de la carga total entrante en el embalse; si se tienen en cuenta las entradas de sedimento al embalse de Mequinenza la tasa de retención aumenta hasta el 95\%.

3. Los resultados ponen de manifiesto el papel de los embalses, en este caso el de Ribarroja, en la retención de sedimentos a lo largo de la cuenca del Ebro y particularmente en su tramo bajo, hecho que exacerba el déficit sedimentario del río como consecuencia de la progresiva disminución en el suministro de sedimentos que se ha producido en la cuenca lo largo del siglo XX. 


\section{Agradecimientos}

Este trabajo se ha desarrollado con datos obtenidos en el marco de los proyectos CGL200611679-C02-01/HID y CGL2009-09770 (subprograma BTE) financiados por el Ministerio de Educación y Ciencia. El segundo autor está contratado como profesor agregado mediante el programa Serra Húnter de la Generalitat de Catalunya. El Grupo de Investigación de Dinámica Fluvial-RIUS agradece el apoyo de la Generalitat de Catalunya a través del grupo de investigación (2017 SGR 0459) y el programa CERCA. Los datos hidrológicos han sido suministrados por la Confederación Hidrográfica del Ebro.

\section{Referencias}

Avendaño, C., Cobo, R., Sanz, M.E., Gómez, J.L. 1997. Capacity situation in Spanish reservoirs. I.C.O.L.D. Ninenteenth Congress on Large Dams 74 (52), 849-862.

Balasch, J.C., Pino, D., Ruiz-Bellet, J.L., Tuset, J., Barriendos, M., Castelltort, X., Peña, J.C. 2019. The extreme floods in the Ebro River basin since 1600 CE. Science of the Total Environment 646, 645-660. https://doi.org/10.1016/j.scitotenv.2018.07.325

Batalla, R.J., Kondolf, G.M., Gomez, C.M. 2004. Reservoir-induced hydrological changes in the Ebro River basin, NE Spain. Journal of Hydrology 290, 117-136. https://doi.org/10.1016/j.jhydrol.2003.12.002

Batalla, R.J., Garcia, C., Balasch, J.C. 2005. Total sediment load in a Mediterranean mountainous catchment (the Ribera Salada River, Catalan Pre-Pyrenees, NE Spain). Zeitschrift für Geomorphology 49(4), 495-514.

Batalla, R.J., Vericat, D. 2009. Hydrological and sediment transport dynamics of flushing flows: implications for river management in large Mediterranean rivers. River Research and Applications 25(3), 297-314. https://doi.org/10.1002/rra.1160

Batalla, R.J., Vericat, D. 2011. An appraisal of the contemporary sediment yield in the Ebro Basin. Journal of Soils and Sediments 11, 1070-1081. https://doi.org/10.1007/s11368-011-0378-8

Brune, G.M. 1953. The trap efficiency of reservoirs. Transactions of the American Geophysical Union 34, 407418. https://doi.org/10.1029/TR034i003p00407

Dedkov, A.P., Mozzherin, V.I. 1992. Erosion and sediment yield on the Earth. In Erosion and Sediment Yield: Global and Regional Perspectives. IAHS Publications 236, 29-33.

Dolz, J., Armengol, J., Roura, M., De Pourcq, K., Arbat, M., López, P. 2009. Estudio de la dinámica sedimentaria y batimetría de precisión del embalse de Ribarroja. Barcelona.

Frutos, L.M., Ollero, A., Sánchez, M. 2004. Caracterización del río Ebro y su cuenca y variaciones en su comportamiento hidrológico. En Gil Olcina, A. (Coord.): Alteración de los regímenes fluviales peninsulares, Fundación Caja Murcia, Murcia, pp. 233-280.

Gallart, F., Llorens, P. 2004. Observations on land cover changes and water resources in the headwaters of the Ebro catchment, Iberian Peninsula. Physics and Chemistry of the Earth 769-773 parts A/B/C (11-12). https://doi.org/10.1016/j.pce.2004.05.004

Hu, B., Yang, Z., Wang, H., Sun, X., Bi, N. 2009. Sedimentation in the Three Gorges Dam and its impact on the sediment flux from the Changjiang (Yangtze River), China. Hydrology and Earth System Sciences Discussion 6, 5177-204. https://doi.org/10.5194/hessd-6-5177-2009

Inbar, M. 1992. Rates of fluvial erosion in basins with a Mediterranean climate type. Catena 19, 393-409. https://doi.org/10.1016/0341-8162(92)90011-Y

Lobera, G., Batalla, R.J., Vericat, D., López-Tarazón, J.A., Tena, A. 2016. Sediment transport in two Mediterranean regulated rivers. Science of the Total Environment 540, 101-113. https://doi.org/10.1016/J.SCITOTENV.2015.08.018

López-Moreno, J.I., García-Ruiz, J.M., Beniston, M. 2008. Environmental Change and water management in the Pyrenees. Facts and future perspectives for Mediterranean mountains. Global and Planetary Change 66 (3-4), 300-312. https://doi.org/10.1016/j.gloplacha.2007.10.004 
López-Tarazón, J.A., Batalla, R.J. 2014. Dominant discharges for suspended sediment transport in a highly active Pyrenean river. Journal of Soils and Sediments 14(12), 2019-2030. https://doi.org/10.1007/s11368-0140961-x

Meybeck, M., Ragu, A. 1997. River discharges to the oceans: an assessment of suspended solids, major ions and nutrients. UN Environment Programme, Nairobi, Kenya. 245 p.

Milliman, J.D., Syvitski, J.P.M. 1992. Geomorphic/tectonic control of sediment discharge to the ocean: the importance of small mountainous rivers. The Journal of Geology 100, 525-544. https://doi.org/10.1086/629606

Milliman, J.D., Farnsworth, K.L. 2011. River Discharge to the Coastal Ocean: A Global Synthesis. Cambridge University Press, Cambridge, 143-144. https://doi.org/10.1017/cbo9780511781247

Négrel, P., Roy, S., Petelet-Giraud, E., Millot, R., Brenot, A. 2007. Long-term fluxes of dissolved and suspended matter in the Ebro River Basin (Spain). Journal of Hydrology 342, 249-260. https://doi.org/10.1016/j.jhydrol.2007.05.013

Novoa, M. 1984. Precipitaciones y avenidas extraordinarias en Catalunya. Actas de las Jornadas de Trabajo sobre Inestabilidad de laderas en el Pirineo. Barcelona, 1-15.

Palanques, A. 1987. Dinámica sedimentaria, meneralogía y microcontaminantes inorgánicos de las suspensiones $y$ de los sedimentos superficiales en el margen continental del Ebro. Tesis Doctoral, Universidad de Barcelona.

Piqué, G., Batalla, R.J., López, R., Sabater, S. 2017. The fluvial sediment budget of a dammed river (upper Muga, southern Pyrenees). Geomorphology 293, 211-226. http://doi.org/10.1016/j.geomorph.2017.05.018

Poulos, S.E., Collins, M.B. 2002. A quantitative evaluation of riverine water/sediment fluxes to the Mediterranean basin: natural flows, coastal zone evolution and the role the dam construction. In: Jones SJ, Frostick LE (eds.) Sediment Flux to Basins: Causes, Controls and Consequences. Geological Society, London, Special Publications 191, pp. 227-245.

Prats, J., Dolz, J., Armengol, J. 2009. Variabilidad temporal en el comportamiento hidráulico del curso inferior del río Ebro. Ingeniería del Agua, 16(4), 259- 272. https://doi.org/10.4995/ia.2009.2960

Richards, K.S. 1982. Rivers: Form and Process in Alluvial Channels. Methuen, London, 358 p.

Roura, M. 2004. Incidència de l'embassament de Mequinensa en el transport de sòlids en suspensió i la qualitat de l'aigua del riu Ebre. Tesis doctoral. Universidad de Barcelona.

Rovira, A., Ibáñez, C. 2007. Sediment linkages between the river catchment and the sea. Journal of Soils and Sediments, 7 (5), 285-295. https://doi.org/10.1065/jss2007.08.244

Rovira, A., Ibáñez, C., Martín-Vide, J.P. 2015. Suspended sediment load at the lowermost Ebro River (Catalonia, Spain). Quaternary International 388, 188-198. https://doi.org/10.1016/j.quaint.2015.05.035

Sanz, M.E., Avendaño, C., Cobo, R. 1999. Influencia de los embalses en el transporte de sedimentos hasta el río Ebro (España). Proceedings of the Congress on Hydrological and geochemical processes in large-scale river basins. HIBAM, Shahin.

Serrat, P., Ludwig, W., Navarro, B., Blazi, J.L. 2001. Variabilité spatio-temporelle des flux de matières en suspension d'un fleuve côtier méditerranéen : la Têt (France)/ Spatial and temporal variability of sediment fluxes from a coastal Mediterranean river: the Têt (France). Comptes Rendus de l'Académie des Sciences (Series II A). Earth and Planetary Science 333, 389-397. https://doi.org/10.1016/S1251-8050(01)01652-4

Shaw, E.M. 1983. Hydrology in Practice. Van Nostrand Reinhold, London.

Syvitski, J.P.M., Kettner, A. 2011. Sediment Flux and the Anthropocene. Philosophical Transactions of the Royal Society A: Mathematical, Physical and Engineering Sciences 369, 957-975. https://doi.org/10.1098/rsta.2010.0329

Tena, A., Batalla, R.J., Vericat, D., Lopez-Tarazón, J.A. 2011. Suspended sediment dynamics in a large regulated river over a 10-year period (the lower Ebro, NE Iberian Peninsula). Geomorphology 125, 73-84. https://doi.org/10.1016/j.geomorph.2010.07.029 
Tena, A., Batalla, R.J., Vericat, D. 2012. Reach-scale suspended sediment balance downstream from dams in a large Mediterranean river. Hydrological Sciences Jounal 57, 1-19. https://doi.org/10.1080/02626667.2012.681784

Tena, A., Książek, L., Batalla, R.J., Vericat, D. 2013. Assessing the geomorphic effects of a flushing flow in a large regulated river. River Research and Applications 29 (7), 876-890. https://doi.org/10.1002/rra.2572

Tena, A., Batalla, R. J. 2013. The sediment budget of a large river regulated by dams (The lower River Ebro, NE Spain). Journal of Soils and Sediments 13 (5), 966-980. https://doi.org/10.1007/s11368-013-0681-7

Tena, A., Vericat, D., Batalla, R.J. 2014. Suspended sediment dynamics during flushing flows in a large impounded river (the lower River Ebro). Journal of Soils and Sediments 14 (12), 2057-2069. https://doi.org/10.1007/s11368-014-0987-0

Topping, D.J., Rubin, D.M. and Vierra, L.E. 2000. Colorado River sediment transport: 1. Natural sediment supply limitation and the influence of Glen Canyon Dam. Water Resources Research 36. https://doi.org/10.1029/1999WR900285.

Vericat, D., Batalla, R.J. 2005. Sediment transport in a highly regulated fluvial system during two consecutive floods (Lower Ebro River, NE Spain). Earth Surface Processes and Landforms 30, 255-272.

Vericat, D., Batalla, R.J. 2006. Sediment transport in a large impounded river: the lower Ebro River, NE Iberian Peninsula. Geomorphology 79, 72-92. https://doi.org/10.1016/j.geomorph.2005.09.017

Vericat, D., Batalla, R.J., Garcia, C. 2006. Breakup and reestablishment of the armour layer in a highly regulated large gravel-bed river: the lower Ebro. Geomorphology 76 (1-2), 122-136. https://doi.org/10.1016/j.geomorph.2005.10.005.

Vörösmarty, C. J., Meybeck, M., Fekete, B., Sharma, K., Green, P., Syvitski, J.P.M. 2003. Anthropogenic sediment retention: major global impact from registered river impoundments. Global and Planetary Change 39 (12), 169-190. https://doi.org/10.1016/S0921-8181(03)00023-7

Walling, D.E. 2006. Human impact on land-ocean sediment transfer by the world's rivers. Geomorphology 79 , 192-216.

Walling, D.E., Fang, D. 2003. Recent trends in the suspended sediment loads of the world's rivers. Global and Planearyt Change 39, 111-126. https://doi.org/10.1016/S0921-8181(03)00020-1

Williams, G.P., Wolman, M.G. 1984. Downstream effects of dams on alluvial rivers. United States Geological Survey Professional Paper 1286, 83 pp. 



\title{
HUMAN-DERIVED ENVIRONMENTAL CONSEQUENCES FOR THREE SEDIMENTARY SYSTEMS OF THE CANARY ISLANDS (SPAIN) - A STUDY OF CHANGES AND IMPACTS: A SYNTHESIS
}

\author{
AARÓN M. SANTANA-CORDERO* \\ Instituto Universitario de Análisis y Aplicaciones Textuales (IATEXT), \\ Universidad de Las Palmas de Gran Canaria, Spain.
}

\begin{abstract}
This paper explores the relationship in three sedimentary systems of the Canary Islands (Spain) between four major land change processes (resource extraction, urbanization, tourism and nature protection) and six main environmental consequences (impacts on aeolian sedimentary dynamics, changes in sand landforms, disappearance of sand landforms and entire systems, changes in vegetation, impacts on the socioeconomic system, and changes in land use patterns). The results show that all the environmental consequences have impacted all three study sites, except in one of them (La Graciosa island) in producing disappearance of sand landforms and entire systems. It should be noted that the environmental consequences described concerns different scales (from entire systems to landforms). All the impacts are described and analyzed separately, and the results are discussed in detail and in relation to the presence of these environmental consequences in other parts of the world.
\end{abstract}

\section{Consecuencias ambientales derivadas del desarrollo humano en tres sistemas sedimentarios de las islas Canarias - estudio de cambios e impactos: una síntesis}

RESUMEN. Este artículo explora la relación existente en tres sistemas sedimentarios de las islas Canarias (España) entre cuatro procesos de cambios territoriales primordiales (extracción de recursos, urbanización, turismo y protección natural) y seis principales consecuencias ambientales (impactos sobre la dinámica sedimentaria eólica, cambios en las geoformas sedimentarias, desaparición de geoformas sedimentarias y sistemas enteros, cambios en la vegetación, impactos sobre el sistema socioeconómico, y cambios en los patrones de usos del suelo). Los resultados muestran que todas las consecuencias ambientales han impactado en los tres sistemas estudiados, excepto en uno de ellos (en isla de La Graciosa), donde la desaparición de geoformas sedimentarias y sistemas enteros no se ha dado. Se debe tener en cuenta que las consecuencias ambientales descritas se refieren a diferentes escalas (desde sistemas enteros hasta geoformas). Todos los impactos han sido descritos y analizados separadamente, y los resultados son discutidos en detalle y en relación con la presencia de dichas consecuencias ambientales en otras partes del mundo.

Key words: Dunes, major land change processes, environmental consequences, coastal arid environments, Canary Islands.

Palabras clave: dunas, procesos de cambios territoriales primordiales, consecuencias ambientales, ambientes áridos costeros, islas Canarias. 
*Corresponding author: Aarón M. Santana Cordero. Instituto Universitario de Análisis y Aplicaciones Textuales (IATEXT), Universidad de Las Palmas de Gran Canaria, Spain. E-mail address: aaron.santana@ulpgc.es

\section{Introduction}

Human activity has resulted, at a global level, in the alteration of most ecosystems and landscapes (Vitousek et al., 1997), especially impacting on their structures and processes (Wenbin et al., 2018). Studies into both the natural and anthropic causes and consequences of ecosystem change are crucial to enable a better understanding of landscape dynamics. In this respect, studies on land-use/cover changes (LUCC) can contribute to establishing the main observable changes over different spatiotemporal scales (Kothari and Arnall, 2019; Müller et al., 2014). However, there are differences in such studies in terms of the relative weight of the impact of natural and anthropic-related events; while land cover changes may be due to natural and/or human processes, land use changes require human intervention. The next important step in the study of ecosystem changes is to conduct more in-depth analyses of the causes, including economic, politic and cultural questions which often remain invisible when using solely cartographic sources (e.g. Santana-Cordero et al., 2017). Lambin et al. (2001) considered this point when reviewing the underlying causes of LUCC.

The argument proposed in the present study is that a thorough LUCC investigation needs to identify the causes, analyze the processes and establish the environmental consequences, as in the following sequence: "Scenario 1 (before the changes) - Change Processes - Scenario 2 (after the changes)".

Focusing on sandy coastal areas, it has been acknowledged that natural systems have been studied in far greater detail than those altered by human activity (Jackson and Nordstrom, 2019). Nonetheless, as more than $40 \%$ of the planet's human population lives in coastal areas (Martínez et al., 2007), such systems clearly need to be studied so that their dynamics can be better understood, predictions of their future evolution made, and solutions to problems proposed. With respect specifically to sandy coastal areas, Jackson and Nordstrom (2011) highlighted the impact of the location, size and stability of dunes and the importance of sand-trapping fences, vegetation plantings and bulldozers or the replacement of dunes with shore-parallel structures. Landward zones importantly include infrastructures such as buildings, roads and parking lots. Some studies, including Goudie et al. (2000), Hoffman and Rohde (2007), Otto et al. (2007) and Pickart and Hesp (2019) have diachronically explored the changes to coastal sandy areas in different parts of the world, verifying the land changes described above.

Although the environmental consequences of human action can be observed globally, they are particularly important in islands due to their limited resources. As the present study is undertaken on sedimentary systems of the Canary Islands (Spain), some background data is first required. A good evidence of this can be seen in a study by Ferrer-Valero et al. (2017) who recorded and quantified changes along the coast of Gran Canaria which were mostly related to its occupation. Cabrera-Vega et al. (2013) reported the causes of change and the consequences for three dune and beach-dune systems of the Canary Islands (Maspalomas, Corralejo, Famara), with specific emphasis on sedimentary dynamics. Other studies have focused on Maspalomas (Gran Canaria), revealing the consequences of the establishment of a tourist resort in this system on the sedimentary dynamics (García-Romero et al., 2019a; Hernández-Calvento et al., 2014). Other (historical based) analyses have considered Maspalomas, Guanarteme (Gran Canaria) and La Graciosa island, recording the substantial changes that have occurred and the disappearance of landforms and even entire systems (Hernández-Cordero et al., 2018; Santana-Cordero et al., 2014, 2016a, 2016b, 2017).

The aim of this study is to identify and characterize human-derived environmental consequences for three sedimentary systems of the Canary Islands: Guanarteme, Maspalomas and La Graciosa island. 
It constitutes the second part of the paper $A$ century of change in coastal sedimentary landscapes in the Canary Islands (Spain) - Change, processes, and driving forces by Santana-Cordero et al. (2017), in which the causes of changes in the same three sedimentary systems were studied. In that previous study, 81 driving forces were identified, and four derived major land change processes were explained (resource extraction, urbanization, tourism, and nature protection). In the present work, the relationships between the major land change processes and the environmental consequences (impacts on aeolian sedimentary dynamics, changes in sand landforms, disappearance of sand landforms and entire systems, changes in vegetation, impacts on the socioeconomic system, and changes in land use patterns), related to different scales (from entire systems to landforms), are examined.

\subsection{Study sites}

The Canary Islands is an Atlantic archipelago situated a short distance from the coast of northwest Africa (Fig. 1). From a geological point of view, these islands are the result of a volcanic hot spot - a magmatic camera in the mantle creating a chain of islands due to the movement of the plate which is above. The location of the archipelago places it in the limit of a desert climate and a temperate one as the result of wind currents from the west. The trade winds and the cold sea current of the Canary Islands also help to reduce temperatures. The temporal distribution of temperature and precipitation follows the same pattern in different parts of the archipelago: temperatures present a normal distribution (higher temperatures in summer) with some differences between different sites (ranging from $4-25^{\circ} \mathrm{C}$ ), while precipitation rates are high during the winter but virtually non-existent in at least one month of the summer (ranging from $0-130 \mathrm{~mm}$ ). Annual average temperature is $18.5-21^{\circ} \mathrm{C}$ in coastal zones, and total average annual precipitation is $400 \mathrm{~mm}$ (Morales-Matos and Santana-Santana, 2005).

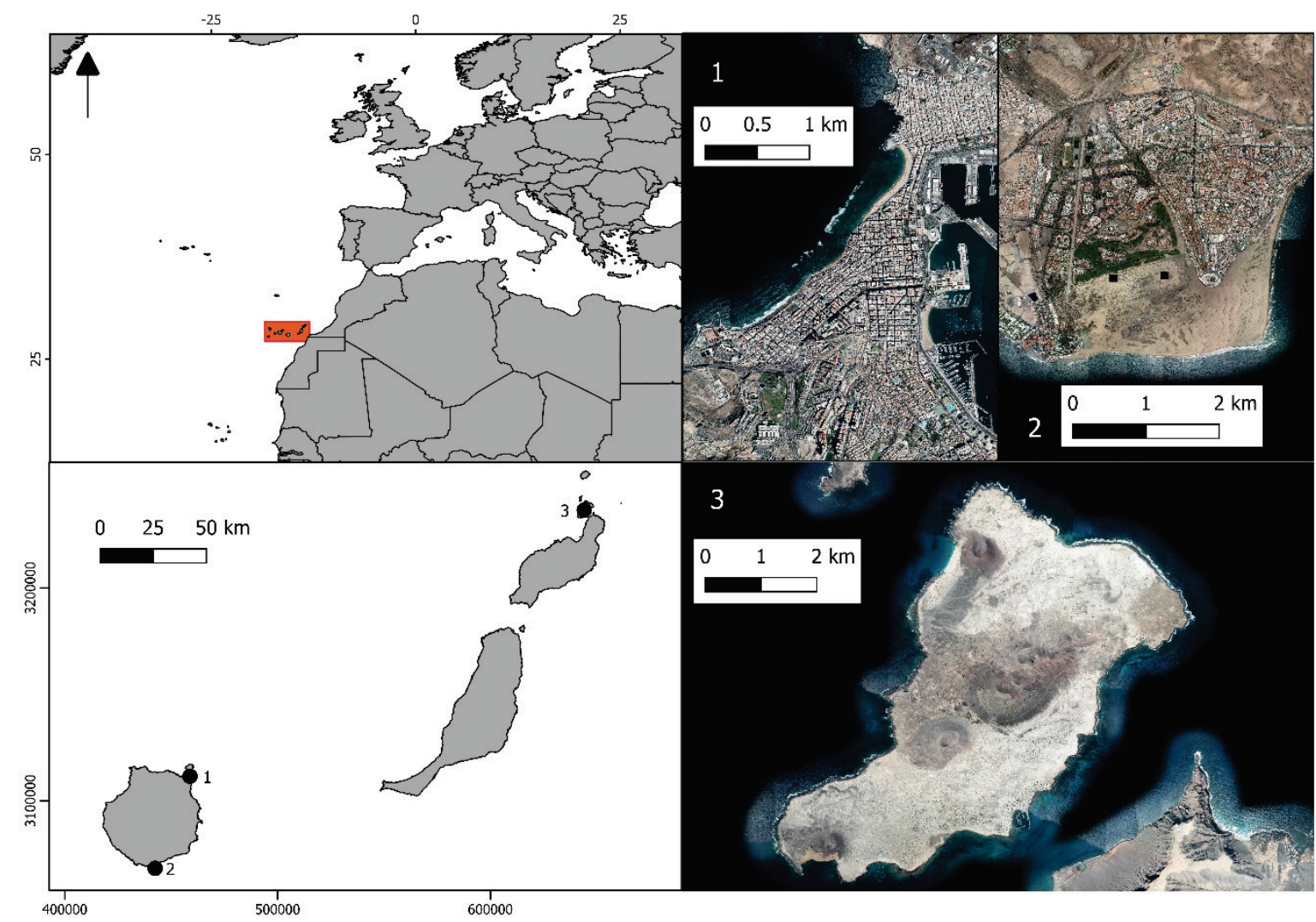

Figure 1. Study area showing the three study sites considered: Guanarteme (1) and Maspalomas (2) dune fields on Gran Canaria island; and La Graciosa island (3). In 2 (view of Maspalomas dune field); the two small black squares correspond to Masp-plot 1 (left) and Masp-plot 2 (right). 
In economic terms, the main activity of the Canary Islands is tourism, overwhelmingly of the so-called 'sun-and-beach' type. As the name itself indicates, this type of tourism tends to occupy coastal areas, with the result that several coastal systems on the islands have been subjected to alteration. In this research, three study sites are considered: the Guanarteme and Maspalomas dunefields, and La Graciosa island.

The Guanarteme dunefield is situated in the NE of Gran Canaria in the island's capital city of Las Palmas de Gran Canaria. In 1954, Guanarteme was a dune system, with an extension of $2.4 \mathrm{~km}^{2}$ (Santana-Cordero et al., 2016b). This dune system has undergone several phases in terms of the amount of sediment and the movement of its landforms throughout its history. A historical reconstruction of the dune system made by Santana-Cordero et al. (2014) reveals that there were between one and three very large, semi-stabilized dunes in the XVIII century and that in the last third of the XIX century there were barchan dunes and sand sheets which constituted a mobile dunefield. According to Santana-Cordero et al. (2014) vegetation cover was scarce in this latter period. This system disappeared towards the 1960s due to human intervention (Santana-Cordero et al., 2016b).

The Maspalomas dune system is located at the southernmost tip of Gran Canaria and had an extension of $4.8 \mathrm{~km}^{2}$ in 1961 . Since then, this system has evolved with the development of tourism tourist facilities, urbanization, golf course - both in its interior and at its borders. Typical landforms found here include a foredune, comprised of hummock dunes parallel to the eastern coastline, barchan dunes and deflation surfaces which have been shaped by tourist activity. Consequently, sectors can be found of accumulation, stabilization and erosion, parts of which interact with the vegetation cover which is more important here than it used to be in Guanarteme before its disappearance.

La Graciosa is located at the northeastern tip of the archipelago, north of the island of Lanzarote. In 1954 , the island had a sedimentary system of $13.1 \mathrm{~km}^{2}$ (that not suffered relevant variations due to its protection status). Its sedimentary system is divided into a northern and southern parts and present stabilized landforms, especially since 1987 when the prohibition of grazing and other traditional activities (as imposed by Law 12/1987, of 19 July, of the Declaration of Natural Sites in the Canary Islands) resulted in the disappearance of human pressure on the natural vegetation. This system presents sand sheets and nebkhas (small dunes to the leeward of plants, especially shrubs) stabilized by the vegetation cover (Santana-Cordero et al., 2017).

\section{Material and methods}

Different sources were used for the purposes of this study. First, a brief review was made of papers, books and reports on the morphological characteristics and the vegetation cover at the three study sites. Of these, special mention should be made about the importance and usefulness of the studies of Hernández-Cordero et al. (2018), Hernández-Calvento et al. (2014) and Hernández-Calvento (2006). Aerial photographs and orthophotos (Table 1), some of them obtained via the Web Map Service (WMS) of the IDECanarias (The Canary Islands SDI), were also used to digitize, measure and analyze some of the LUCC patterns and processes revealed in the results. Statistical data were used to support important population data and economic events related to the results of impacts on the economic system.

The results of this paper are structured to reflect the 6 main human-derived environmental consequences at the three study sites. These environmental consequences are as follows: i) impacts on aeolian sedimentary dynamics; ii) changes in sand landforms; iii) disappearance of sand landforms and entire systems; iv) changes in vegetation; v) impacts on the socioeconomic system; and vi) changes in land use patterns. 
Table 1. Aerial images used and their basic characteristics.

\begin{tabular}{|c|c|c|c|}
\hline Year & Study site & Type & Resolution (cm/pixel) \\
\hline 1949 & Guanarteme & Aerial photograph & 38.3 \\
\hline 1954 & La Graciosa & Aerial photograph & 100 \\
\hline 1961 & Maspalomas & WMS & 12.5 \\
\hline 1987 & Maspalomas & Orthophoto & 15 \\
\hline 2003 & Maspalomas & Aerial photograph & 15 \\
\hline 2006 & La Graciosa & WMS & 50 \\
\hline 2009 & La Graciosa & Aerial photograph & 10 \\
\hline 2018 & Guanarteme & WMS & 20 \\
\hline 2018 & Maspalomas & WMS & 20 \\
\hline 2018 & La Graciosa & WMS & 20 \\
\hline
\end{tabular}

The land uses have been established according to Santana-Cordero et al. (2016b) with some additions based on what it has been seen in the aerial photographs. They are as follows: abandoned land, agriculture, industrial, infrastructure, mixed, unused land, recreational and residential. Mixed land use refers to areas where different uses have been carried out. Unused lands refer to areas with forbidden uses or where natural processes appear to be the main feature, incompatible with human uses (e.g. sandy extensions). Residential land use includes the tourist resort in Maspalomas.

Continuous changes along beach shorelines due to natural processes have methodological implications in the area of the study sites. Therefore, changes in the study sites in terms of their area are registered. Furthermore, although in the case of Maspalomas the study area changed because the sandy surface has been reduced over time due to the urban expansion, the extension used here is that of 1961, namely $4.8 \mathrm{~km}^{2}$.

\subsection{Analysis}

The different data were analyzed using a number of techniques. Photo-interpretation was employed to compare geomorphologic maps, as indicated in Hernández-Calvento (2006), shedding light on the establishment of changes in sand landforms. Diachronic measurements and analyses, including a GIS-based quantification of changes, were also performed. For the study of the impacts on aeolian sedimentary dynamics, alterations were detected through photo-interpretation of the 2018 aerial photograph in Maspalomas. For the study of vegetation cover, the evolution of four aerial photographs/orthophotos was used (1961, 1987, 2003 and 2018) in Maspalomas. For this purpose, two plots (polygons) of 1 ha were established in sectors where there was no vegetation in 1961 (Masp-plot 1 and Masp-plot 2). Digitization of the vegetation elements in the other aerial photographs then gave us the corresponding vegetation cover values in each plot. After digitization of the vegetation cover, a statistical correlation was established with a good fit $\left(\mathrm{R}^{2}=0.89\right.$ and 0.83$)$. Digitization was also used to establish and measure the land uses at the three study sites. In relation to these measures, the mean, standard deviation and normalized standard deviation were calculated, with the latter employed as an indicator of the degree of homogeneity in terms of land-uses areas.

\section{Results}

The results are presented in the following subsections: causes-consequences matrix, Impacts on Aeolian sedimentary dynamics, Changes in sand landforms, Disappearance of sand landforms and entire systems, Changes in vegetation, Impacts on the socioeconomic system, and Changes in land use patterns. 


\subsection{Causes-Consequences matrix}

In Table 2, causes (understood here as major land change processes) and consequences are linked by a symbol when a relationship between a cause and a consequence exists at one of the study sites, according to the literature.

Table 2. Major land change processes and their environmental consequences. Key - circle: Guanarteme; square: Maspalomas; and triangle: La Graciosa.

\begin{tabular}{|c|c|c|c|c|c|c|}
\hline $\begin{array}{c}\text { Major land change } \\
\text { processes/ } \\
\text { environmental } \\
\text { consequences }\end{array}$ & $\begin{array}{l}\text { Impacts on } \\
\text { aeolian } \\
\text { sedimentary } \\
\text { dynamics }\end{array}$ & $\begin{array}{l}\text { Changes in } \\
\text { sand } \\
\text { landforms }\end{array}$ & $\begin{array}{l}\text { Disappearance } \\
\text { of sand } \\
\text { landforms and } \\
\text { entire systems }\end{array}$ & $\begin{array}{l}\text { Changes in } \\
\text { vegetation }\end{array}$ & $\begin{array}{l}\text { Impacts on the } \\
\text { socioeconomic } \\
\text { system }\end{array}$ & $\begin{array}{l}\text { Changes in } \\
\text { land use } \\
\text { patterns }\end{array}$ \\
\hline Resource extraction & - $\Delta$ & - $\mathbf{\Delta} \Delta$ & $\bullet$ & $\Delta$ & $\bullet$ & $\bullet \Delta$ \\
\hline Urbanization & - & $\square$ & - & - $\Delta$ & - घ & $\bullet \square$ \\
\hline Tourism & - $\Delta$ & $\square$ & - & $\square$ & $\square$ & - $\Delta$ \\
\hline Nature protection & - $\Delta$ & $\Delta$ & & $\Delta$ & $\Delta$ & $\Delta$ \\
\hline
\end{tabular}

\subsection{Impacts on aeolian sedimentary dynamics}

The sand extractions that took place in Guanarteme should have provoked alterations to the aeolian processes. The rapid changes in the topography modified the intensity of the transport of sediment through the deceleration/acceleration of the sediment flows (Fig. 2) (Santana-Cordero et al., 2016b). This effect created different landforms in terms of sediment movements, so semi-stabilized dunes were naturally revegetated while in the other side, high movement rates led to erosion.

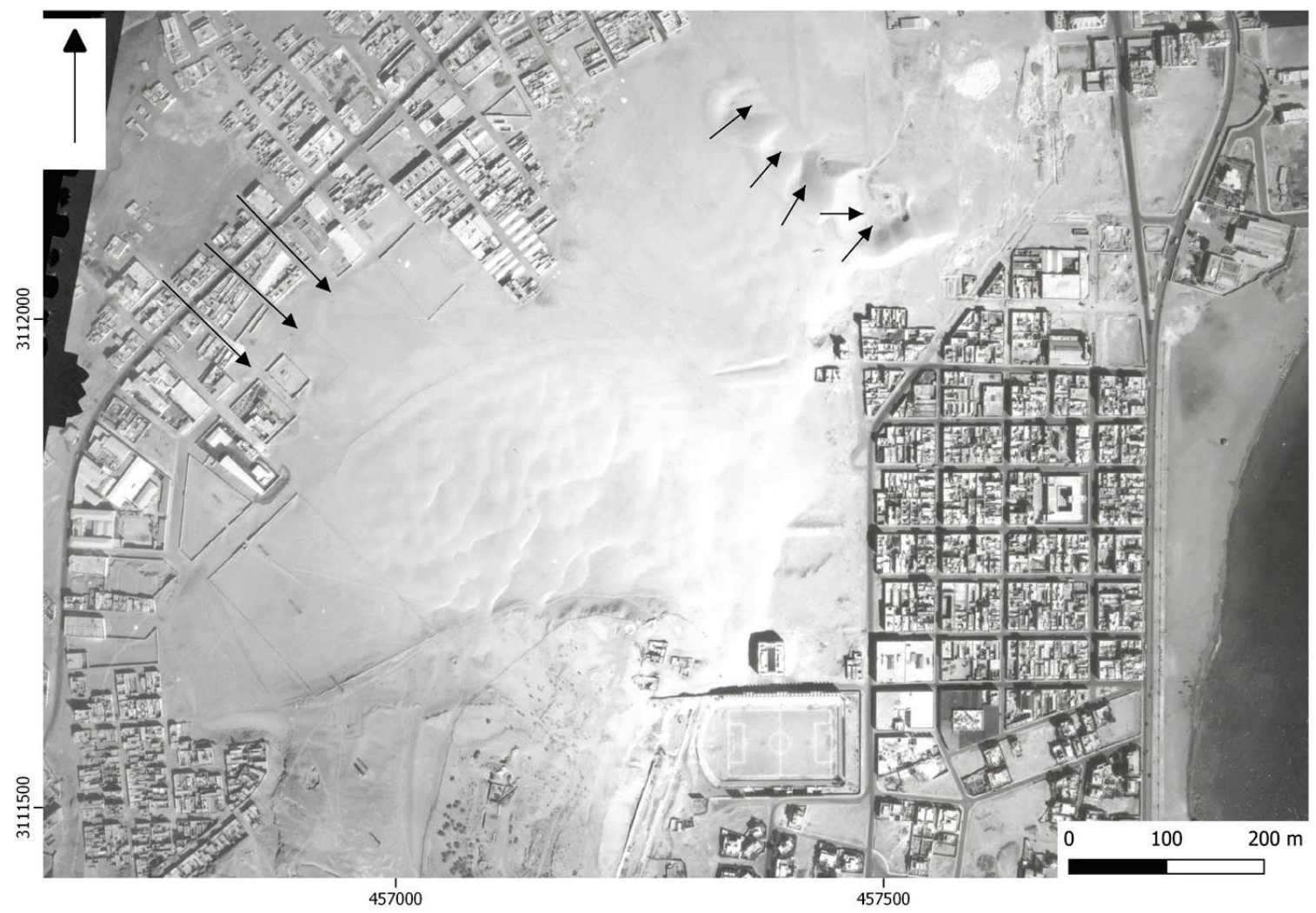

Figure 2. Guanarteme dunefield (1949). Center-top arrows represent the modification of the sediment transport; left-top arrows indicate the wind corridors. 
In La Graciosa, sand extraction has had an important impact on aeolian sedimentary dynamics, causing similar alterations to sediment flows as in Guanarteme (Pérez-Chacón et al., 2010). Sand extraction extended to 1.3 ha in 2006 and 1.0 ha in 2009, years in which this alteration had considerable dimensions (Fig. 3). The presence of a network of car tracks across the island also needs to be taken into consideration, as they cause erosion and soil compaction.

In Maspalomas, the urbanization of El Inglés over the high sedimentary terrace, which began in the early 1960s, has had two major impacts on the aeolian sedimentary dynamics due to alteration of the wind stream. Firstly, the conditions were created for the generation of a wind vortex that triggers wind acceleration and dune erosion in one sector of the dunefield (Hernández-Calvento et al., 2014). Secondly, the normally westward-flowing winds have been deviated to the southwest, which has resulted in dune stabilization in the northwestern sector of the system, leeward of the urbanization. This stabilization affected between 21.6 and 90.9 ha in this sector in the period 1961-2003 (HernándezCordero et al., 2018), also triggering changes in vegetation cover. Additionally, small dunes running above big dunes were detected in 2018, constituting new landforms caused by the wind acceleration described above (Fig. 4). These new landforms occupy 1.151 ha.
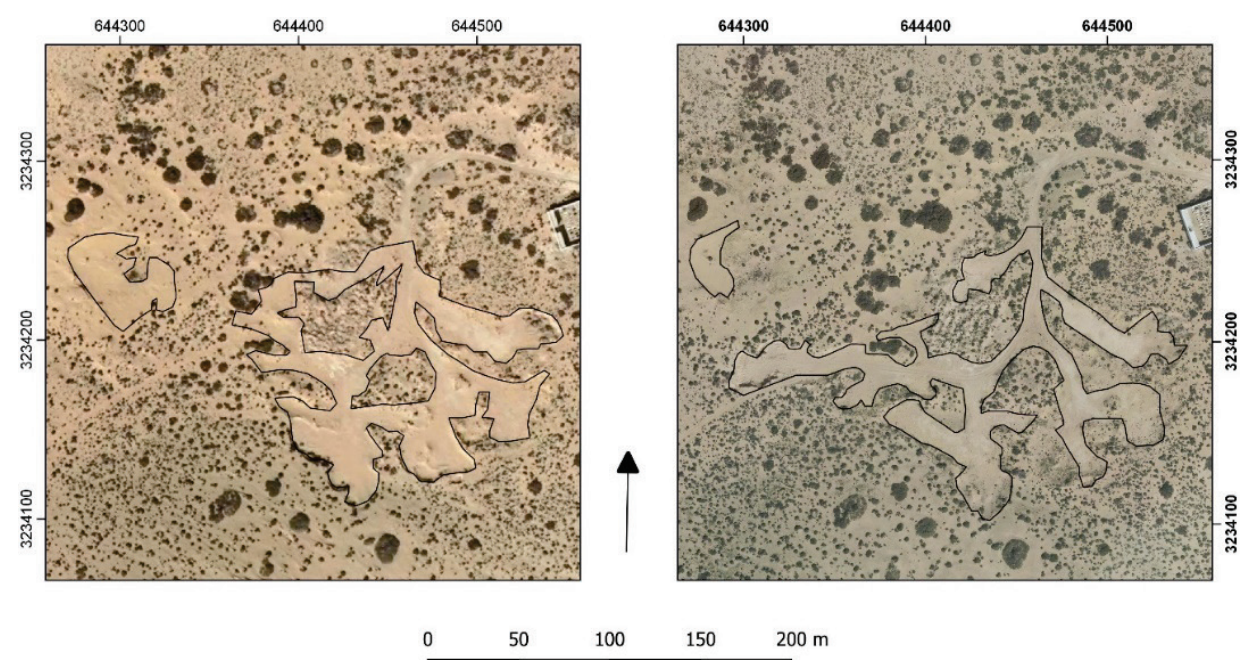

Figure 3. Extractions in La Graciosa in 2006 (left) and in 2009 (right).
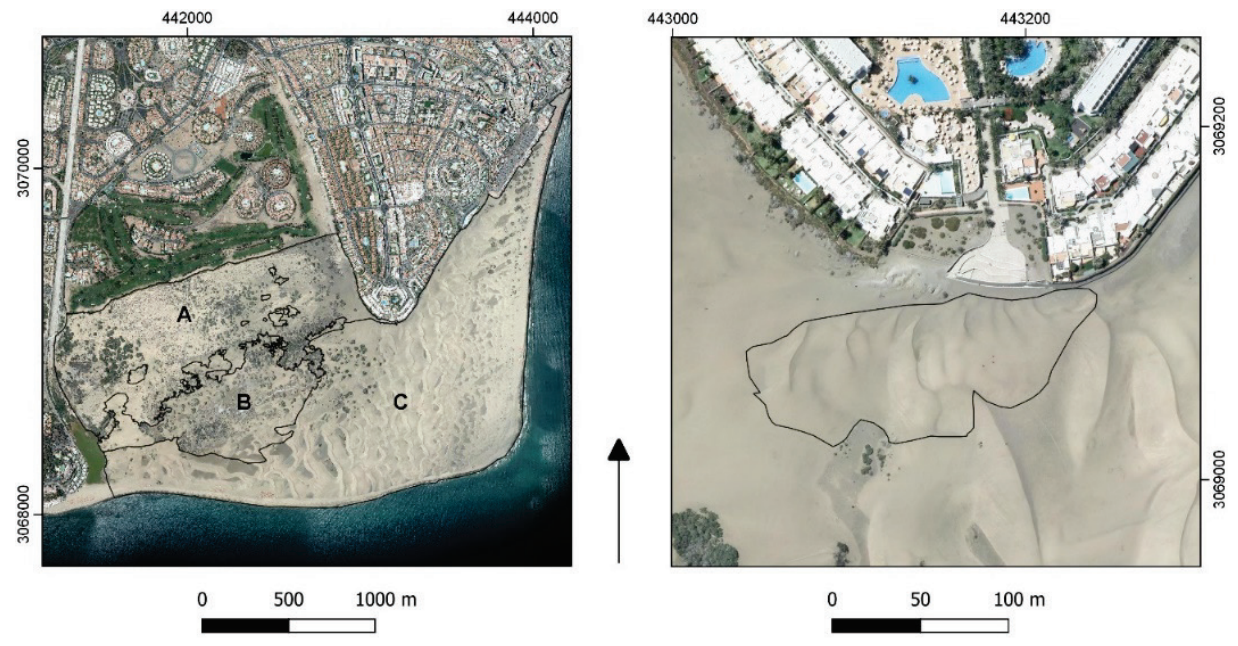

Figure. 4. Maspalomas dunefield (2018). Sedimentary dynamics sectors* (left; A: stabilized area; B: semistabilized area; and $C$ : active area) and small dunes alteration (right). *Source: Hernández-Cordero et al. (2015). 
In the case of Guanarteme, coastline urbanization had already altered the sedimentary dynamics of this dunefield, as seen in the image of 1949, with the streets acting as wind corridors (Fig. 2). This phenomenon accelerates winds, altering sediment flows inside the system and increasing the erosion process.

Tourist activities and their associated facilities have also affected the aeolian sedimentary dynamics in Maspalomas and La Graciosa. In the first case, the dynamics have been changed as the result of the presence on El Inglés beach in Maspalomas of hammocks, umbrellas, 'goros' (handmade stone shelters), Red Cross lifeguard posts and kiosks, with the latter for example generating the formation of deflation surfaces at either side of the kiosk as the result of acceleration of the wind and the accumulation of sand leeward of this obstacle ('shadow dune'), causing foredune vegetation modifications (Fig. 5). The deflation surfaces in the system, although not only provoked by kiosks, increased in the period 1961-2003 from 10.1 to 32.2 ha (Hernández-Cordero et al. 2018). In La Graciosa, the presence of tourists, especially on El Salado beach - where tents can be set up interfering in aeolian sedimentary transport - and in Caleta del Sebo, has resulted in the dumping of debris and waste and the trampling of vegetation by hikers (Fig. 6) (Pérez-Chacón et al., 2010).

Nature protection measures have also had an impact on the Maspalomas dunefield and the La Graciosa sedimentary system. Maspalomas (the dunes, not beaches) and La Graciosa island were protected in 1987 through the Declaration of Natural Sites in the Canary Islands (Canarian Law 12/1987, dated 19 July). In addition, Law 12/1994, dated 19 December, on Protected Natural Areas of the Canary Islands ensured increased protection in Maspalomas and the establishment of tracks for tourists to cross the dunefield without damaging it. Periodic displacement of the kiosks was also established in 1995-96 to minimize their impact (Hernández-Calvento et al., 2002). In La Graciosa, the prohibition of traditional land uses from 1987 onwards (grazing, agriculture, firewood extraction, lime kilns exploitation) contributed to retrieving and conserving the vegetation and sedimentary processes (Santana-Cordero et al., 2016a).

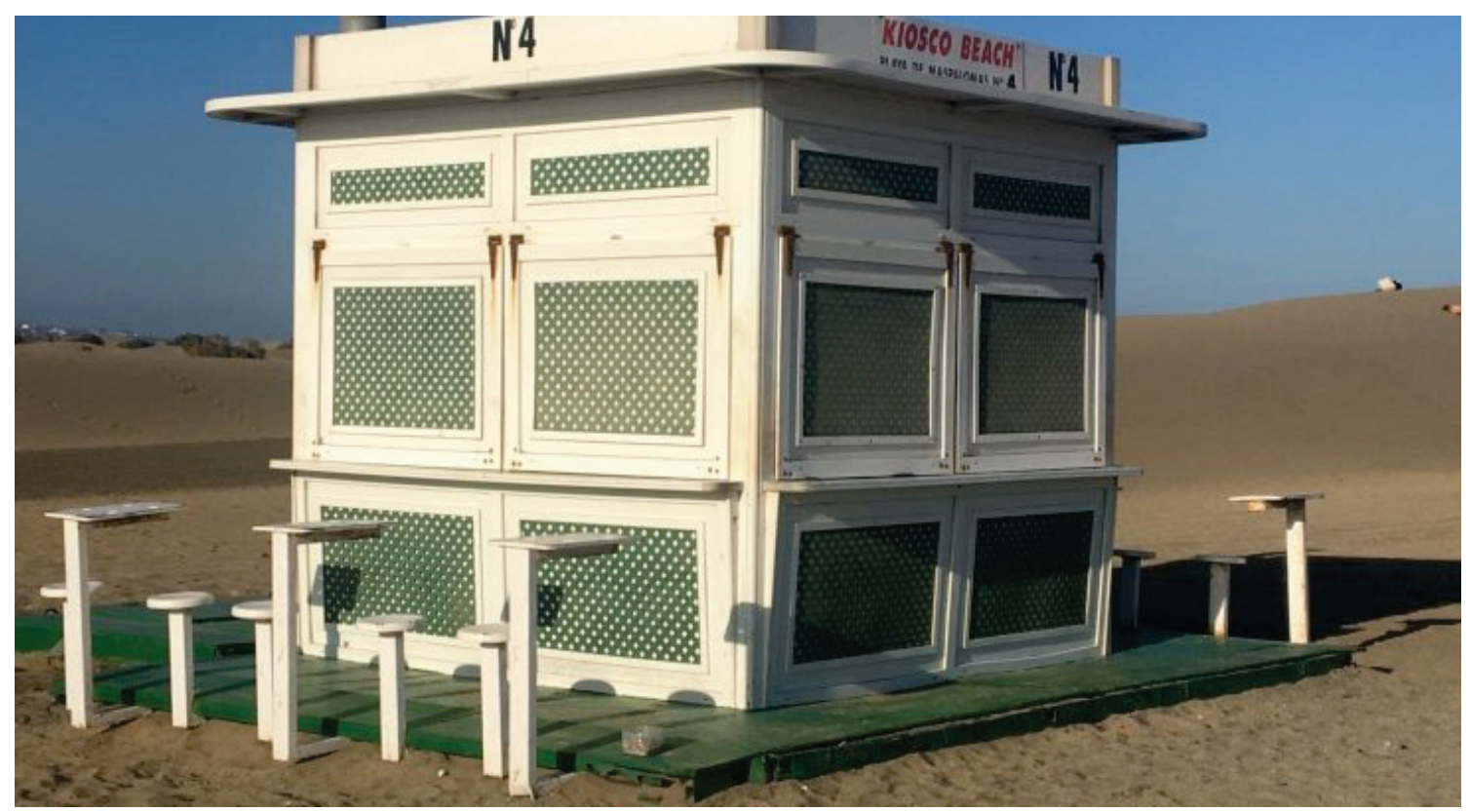

Figure 5. Kiosk on El Inglés beach; Maspalomas. 


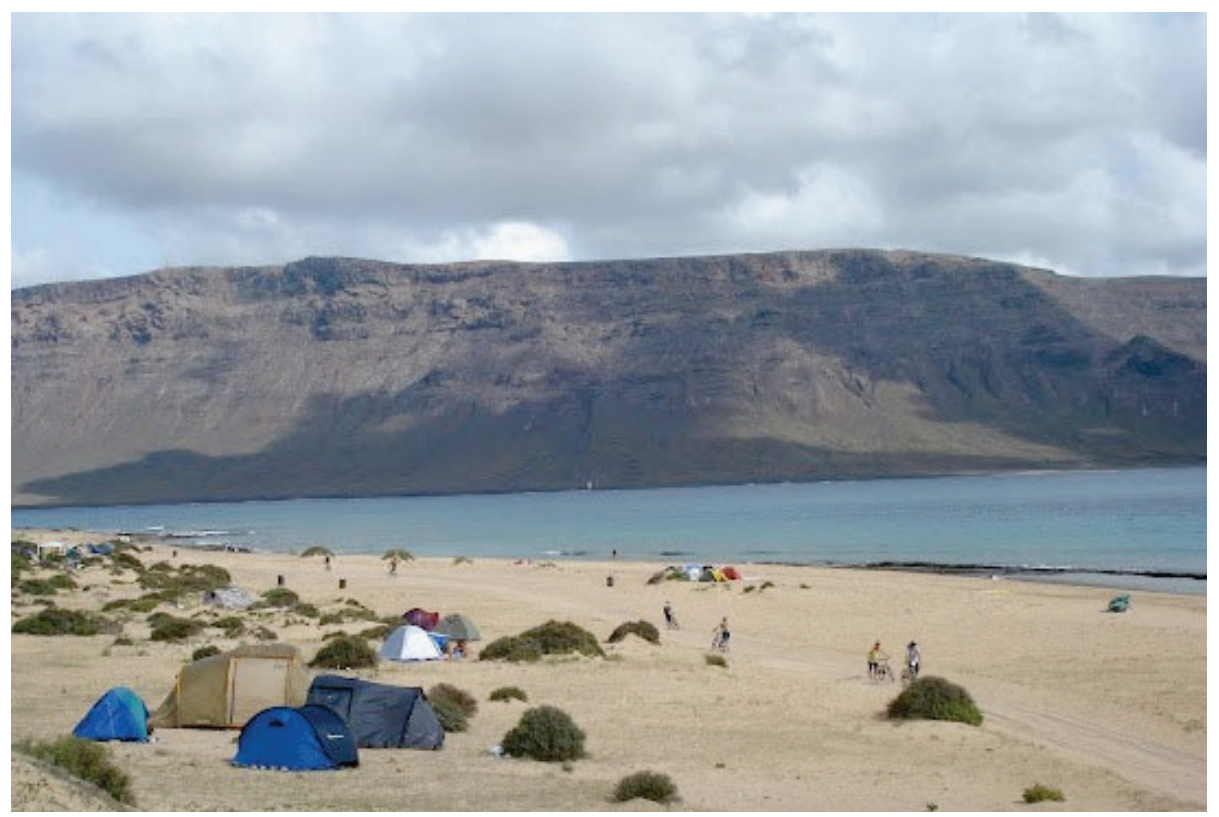

Figure 6. Tents on El Salado beach; La Graciosa. Photo: Yamilé Sarmiento, 2019.

\subsection{Changes in sand landforms}

In Guanarteme, sand extraction was detected through at least two different means: aerial photographs and oral sources. The aerial photographs allowed measurement of the extent of sand extraction in two different years (1949 and 1954) and thus calculate the mean annual extraction rate, 0.34 ha per year $\left(3412 \mathrm{~m}^{2}\right.$ per year) (Santana-Cordero et al., 2016b). This impact provoked changes in a sand sheet of the system. In Maspalomas, in 1961, sand mining was concentrated in two points in the western section of the terrace, while in 2003 this activity continued in the same location and had been extended to the western border of the dunefield (Hernández-Cordero et al., 2018). In La Graciosa, resource extraction was mainly vegetation based, as fuel for lime kilns and domestic purposes (Fig. 7). The best species for the lime kilns was Launaea arborescens, whereas Traganum moquinii and Nicotiana glauca were the best for household consumption (Santana-Cordero et al., 2016a). Extraction of this resource triggered movement of the sand, thus changing the shape or type of landforms among the sand sheets and dunes (mainly nebkhas and hummocks).

The urbanization of El Inglés terrace (Maspalomas dunefield) also triggered changes in the landforms of two parts of the system. The zone where the wind is accelerated presents landforms altered as the result of erosion processes, generating deflation surfaces. On the other hand, the wind vacuum in the northwestern system has transformed mobile dunes into stabilized ones. According to HernándezCalvento (2006), changes took places in the eastern and southwestern sections of the terrace during the 1960s and 1970s, with the eastern side transforming from a barchanoid ridge to an echo dune. In the southwestern section, erosion processes now predominate (García-Romero et al., 2019b) where falling dunes existed before.

Tourism in Maspalomas has affected, for example, the foredune, with a change in area from 13.2 ha in 1961 to 9.5 ha in 2003 (Hernández-Cordero et al., 2018). Thousands of tourists pass through it all year round, as well as numerous vehicles and other heavy machinery providing services such as cleaning. For the foredune, Traganum moquinii plays an essential role in sedimentary regulation. However, this species is under intense pressure as the result of human and vehicular traffic (Fig. 8) (Peña-Alonso et al., 2012, 2017). In La Graciosa, nature protection measure has severely reduced vegetation extraction through the prohibition of traditional land uses, releasing the system from a major anthropic pressure. In this way, the active landforms become stabilized, and it can be argued that the type of ecosystem found on the island has changed, at least in some sectors. 


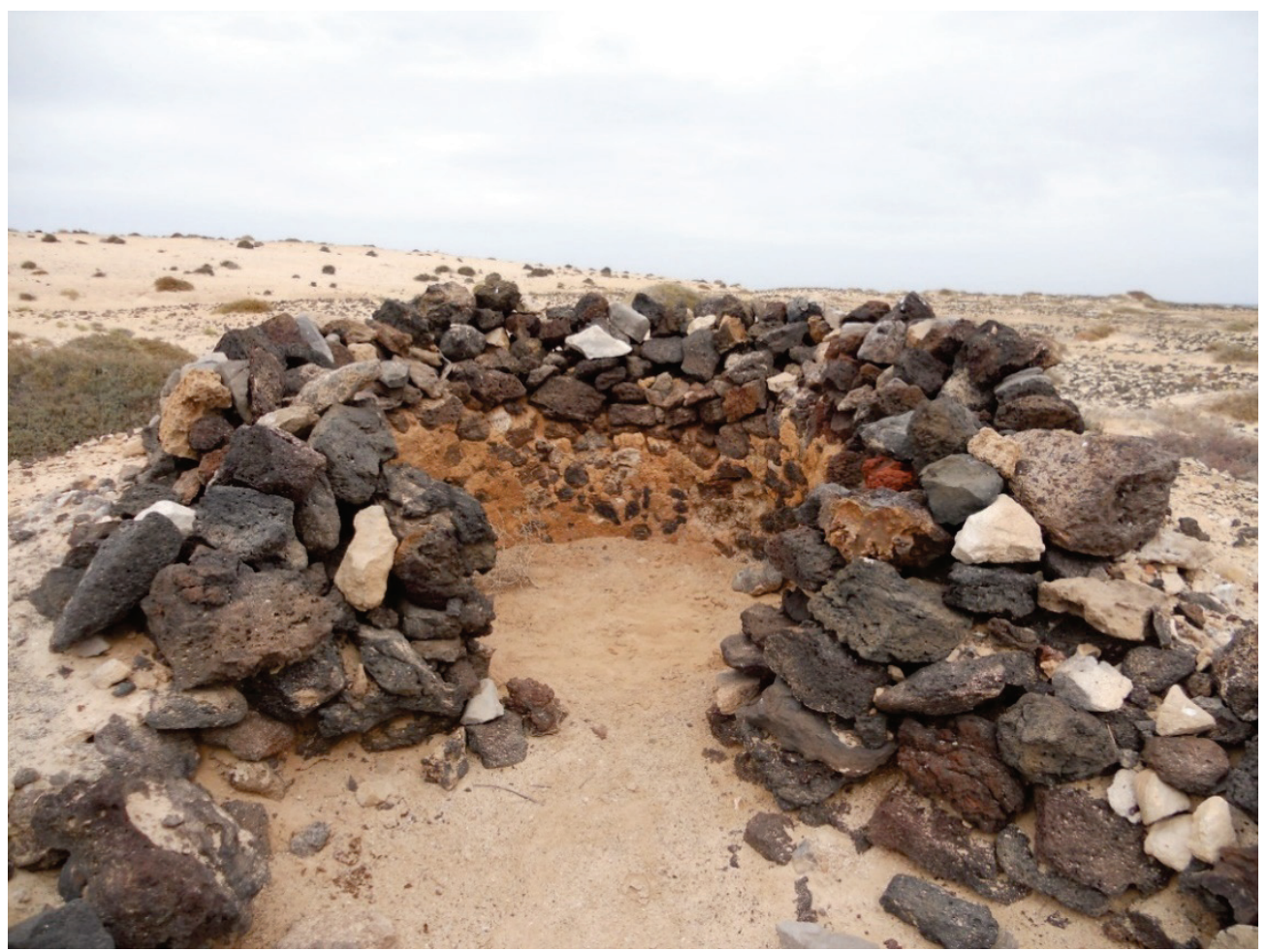

Figure 7. Remnants of a lime kiln in La Graciosa used before their prohibition in 1987. Photo: Luis Hernández Calvento, 2011.

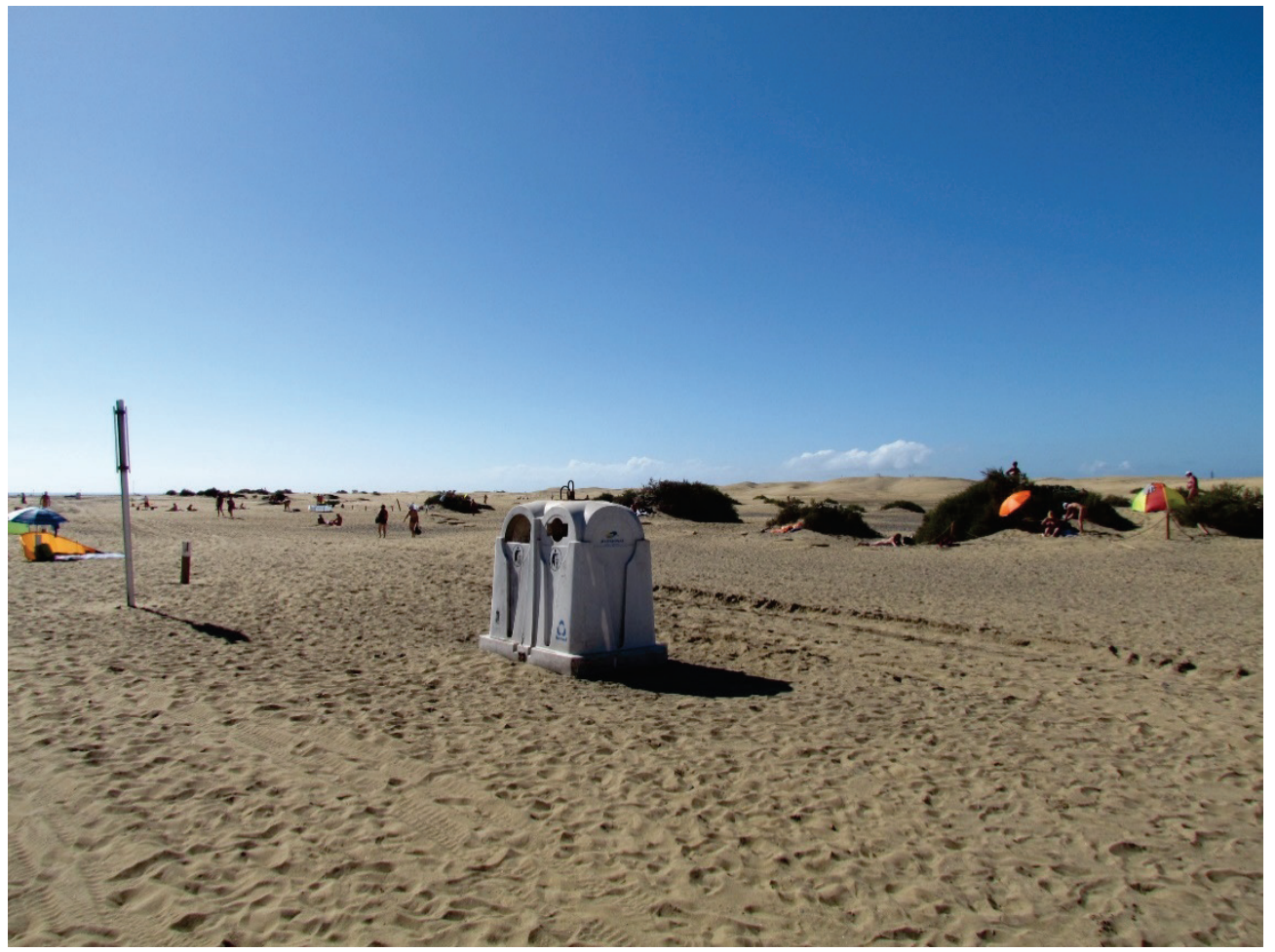

Figure 8. Impacts on the foredune of Maspalomas. Photo: Carolina Peña Alonso, 2016. 


\subsection{Disappearance of sand landforms and entire systems}

The Guanarteme dune system disappeared after sand extraction was carried out systematically from at least the last quarter of the XIX century for the purposes of brick manufacturing and freeing up land for construction (Santana-Cordero et al., 2016b). Barchan dunes and sand sheets, as well as climbing, cliff-top and falling dunes, associated with the high sedimentary terrace of this system, all disappeared. In this case, sand extraction was carried out indistinctly in sand dunes and sand sheets. The result was the loss of an ecosystem of high value to the island, partly because development of the area was planned in the mid-XIX century (Santana-Cordero et al., 2017) when ecological awareness was non-existent and the development model was based exclusively on developmental premises.

Due to the general dynamics of the extinct Guanarteme dunefield, sand continues arriving from the northwest and now accumulates on Las Canteras beach due to the obstacle or barrier to wind and sand flows caused by the urbanization of the Guanarteme isthmus (Fig. 9). This has created an environmental problem because sand on this beach has had to be extracted and deposited elsewhere to avoid its over-accumulation. Two major sand extraction operations have been carried out: $34,000 \mathrm{~m}^{3}$ of sand were extracted from the northern part of Las Canteras beach in 2003 and deposited on Las Alcaravaneras beach (also located in the northeast of Gran Canaria), and a further $56,400 \mathrm{~m}^{3}$ were extracted from the same sector of the beach in 2009 and deposited on other beaches in the south of Gran Canaria (Alonso-Bilbao et al., 2015; Copeiro del Villar Martínez and García-Campos, 2002).

In Maspalomas, cliff-top dunes, occupying 14.1 ha in 1961 (Hernández-Cordero et al. 2018), disappeared in the following twenty years with the development of the El Inglés urbanization on the terrace. These landforms had previously co-existed on the terrace with agricultural activities (Fig. 10). Part of the foredune of El Inglés beach also disappeared, losing 3.7 ha in its southern sector. This latter loss can partly be related to the presence of tourists as well as beach services (e.g. transit of vehicles), as mentioned above.

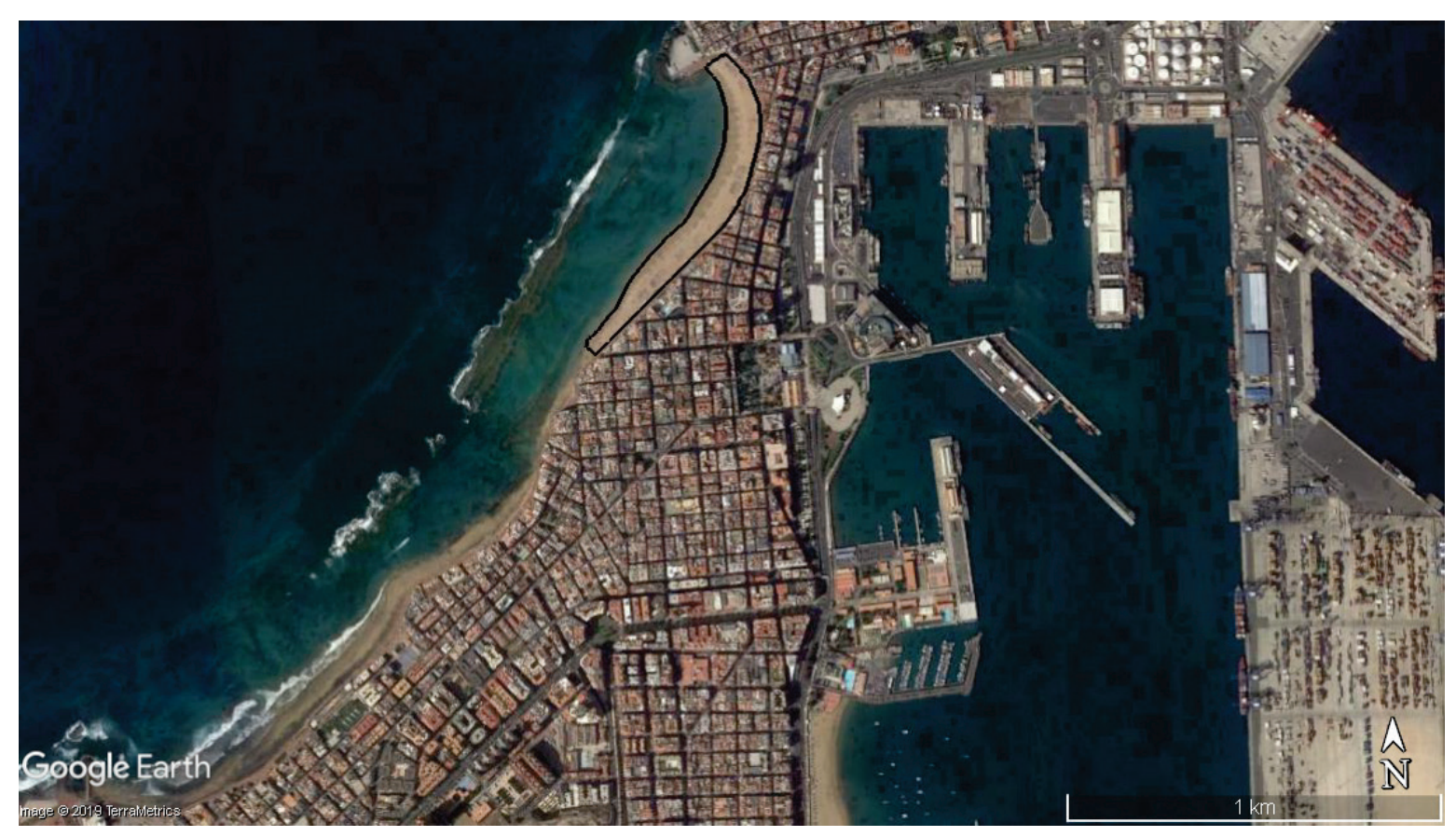

Figure 9. Las Canteras beach. Polygon: sector of the beach where the sand was extracted in 2003 and 2009. 


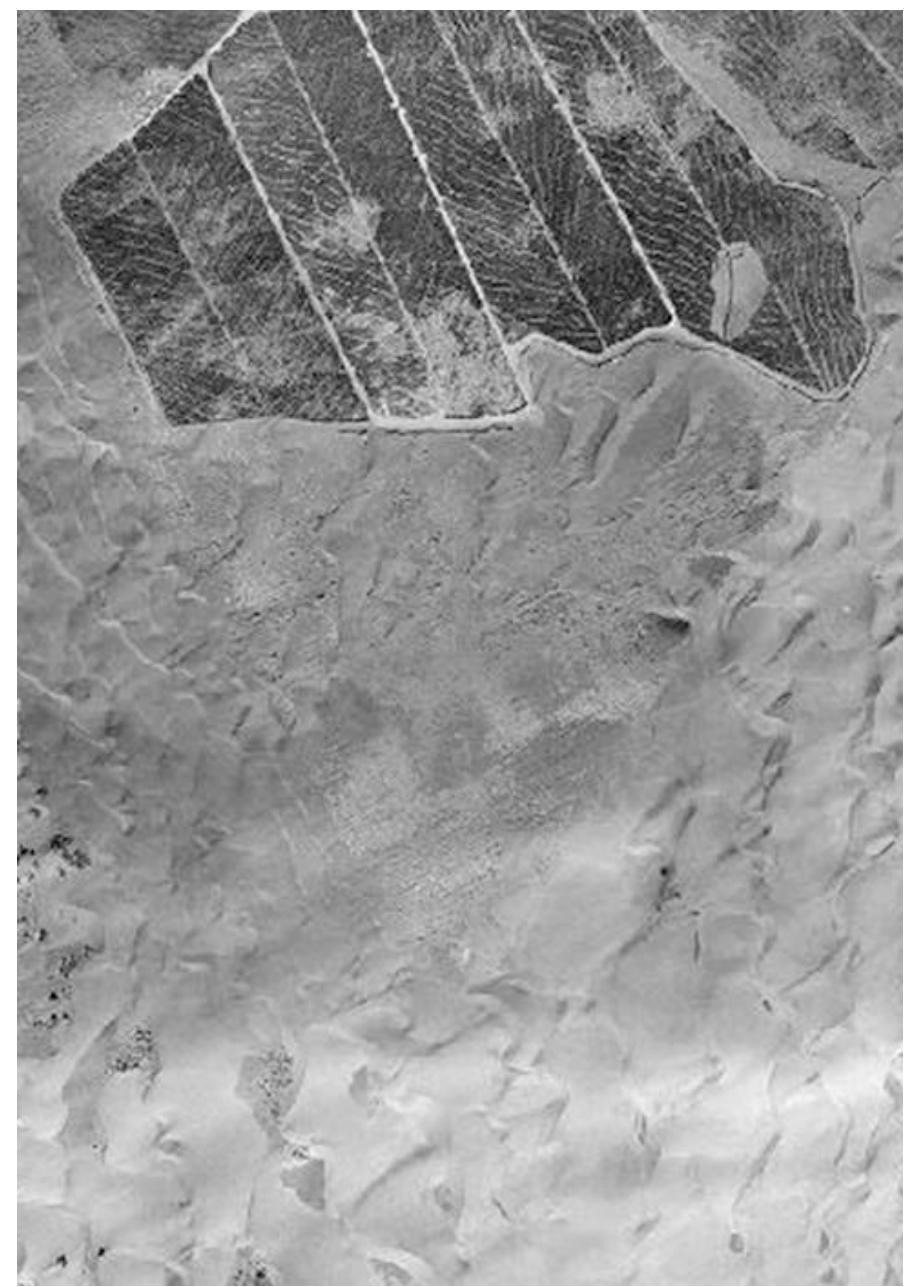

Figure 10. Cliff-top dunes and agriculture on the El Inglés terrace of Maspalomas (1961).

\subsection{Changes in vegetation}

The stabilized dune sector of Maspalomas, in the northwest sector (Fig. 4), allowed the establishment of new plant species. In this area, 92.08 ha were colonized by plant communities: 'Cyperus capitatus-Ononis tournefortii (psammophilous perennial and annual forbs), Tamarix canariensis (hygrophilous tree) and Launaea arborescens (xerophilous shrub)' (Hernández-Cordero et al., 2017, 2018). This increase has followed an exponential progression, measured through the two plots (Masp-plot 1 and Masp-plot 2) established in this study for vegetation cover monitoring, with an $\mathrm{R}^{2}$ fit of 0.89 and 0.83 , respectively (Table 3; Figs. 1 and 11).

Table 3. Evolution of main land covers (vegetation and bare sand) at the two established plots from 1961 to 2018 in the last 57 years.

\begin{tabular}{|l|l|l|l|c|}
\hline Masp-plot 1 & \multicolumn{1}{|c|}{$\left.\mathbf{1 9 6 1} \mathbf{( m}^{\mathbf{2}}\right)$} & \multicolumn{1}{|c|}{$\mathbf{1 9 8 7}$} & \multicolumn{1}{|c|}{$\mathbf{2 0 0 3}$} & $\mathbf{2 0 1 8}$ \\
\hline Vegetation & $0(0 \%)$ & $220.8(2.2 \%)$ & $1,644.0(16.4 \%)$ & $2,637.5(26.4 \%)$ \\
\hline Bare sand & $10,000(100 \%)$ & $9,779.2(97.8 \%)$ & $8,356.0(83.6 \%)$ & $7,362.5(73.6 \%)$ \\
\hline Masp-plot 2 & & & & \\
\hline Vegetation & $0(0 \%)$ & $135.6(1.4 \%)$ & $1,965.9(19.7 \%)$ & $2,586.7(25.9 \%)$ \\
\hline Bare sand & $10,000(100 \%)$ & $9,864.4(98.6 \%)$ & $8,034.1(80.3 \%)$ & $7,413.3(74.1 \%)$ \\
\hline
\end{tabular}




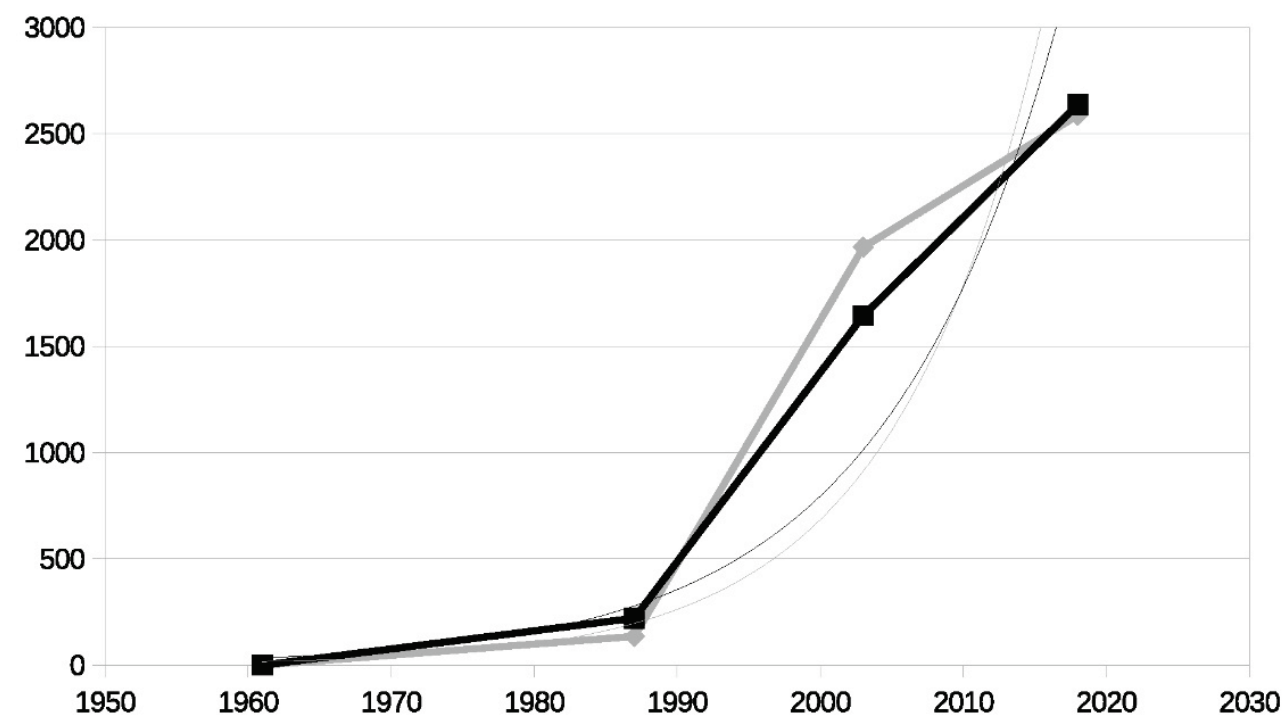

Figure 11. Evolution of vegetation cover and the exponential trend lines at the two plots. Masp-plot 1: black; Masp-plot 2: grey.

In La Graciosa, vegetation regeneration has taken place, especially since the prohibition in 1987 of resource extraction for domestic consumption and lime kilns. In 1954, the two main land covers, bare sand and vegetation, were 479.4 ha (37.4\%) and 803.5 ha (62.6\%), respectively. In 2009, the respective values were 132.7 ha $(10.5 \%)$ and 1137.1 ha $(89.5 \%)$. These data refer to two of the four sedimentary systems of this island (Lambra and El Jable Sur) (García-Romero et al., 2016). At the time of resource extraction, the various species included Mesembryanthemum nodiflorum, Mesembryanthemum crystallinum, Traganum moquinii, Nicotiana glauca and Launaea arborescens (Santana-Cordero et al., 2016a). Recently, 17 plant communities were found to be present in the island, with by far the most abundant being Salsola vermiculata, followed by L. arborescens and T. moquinii (Pérez-Chacón et al., 2010).

\subsection{Impact on the socioeconomic system}

In Guanarteme, sand extraction was a factor in the growth of the economy in the island. Urban fabric construction and the demands of the new port (mid-XIX century) and its associated services had a major impact on the socioeconomic system. These activities replaced, in part, agricultural activities in Las Palmas and resulted in the abandonment of land and the import of labor from the Gran Canaria countryside, as well as from the islands of Fuerteventura and Lanzarote. In this scenario, urbanization also occupied cultivated land, e.g. south of the Las Alcaravaneras district (Santana-Cordero et al., 2016b). Urbanization of the El Inglés terrace, in Maspalomas, contributed to changes to the socioeconomic system at regional level at a time when tourism was taking its first steps to becoming the main economic activity in the Canary Islands and coinciding with the beginning of the establishment of mass tourist destinations in other parts of the world. Tourism required the development of numerous auxiliary services, such as the development of promenades with restaurants, sea-based recreational activities (e.g. jet ski rentals) and car parking for visitors. A number of beach services were also established, as noted above: hammocks, umbrellas, kiosks, and Red Cross lifeguard posts. Unfortunately, historical data series on tourist visits to the islands are only available from 1993 onwards. Even so, they offer an overview of the growth in the volume of tourism in Gran Canaria (Fig. 12). In La Graciosa, nature protection, with the prohibition of traditional uses in 1987, resulted in a transformation to a tourism-based economy, in line with the regional economic trend. 


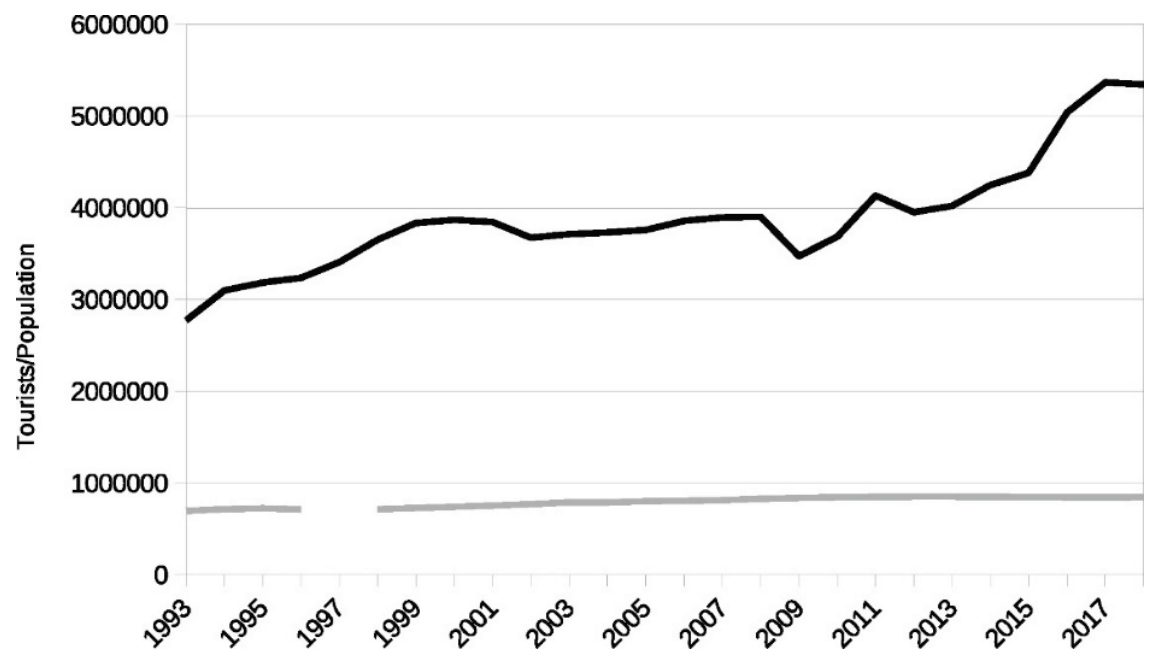

Figure 12. Evolution of tourists (black) and the population of Gran Canaria (grey) for the period 1993-2018.

\subsection{Changes in land use patterns}

Santana-Cordero et al. (2016b) identified four land uses in the XX century in Guanarteme before urbanization spread over almost all the system: agriculture, residential (urbanization), recreational, and sand extraction. Currently, residential use extends over almost all the area, except the beaches of Las Canteras and Las Alcaravaneras. Additionally, other land uses of lesser importance have been observed: industrial, mixed, infrastructure, abandoned and no use. In Maspalomas, land use in the past (before 1960) was restricted to agriculture on El Inglés terrace, and no use and recreational in the rest of the area. Currently, tourist resort use, treated here as residential, is present along with urbanization and recreational use through a golf course and the beaches. In La Graciosa, the land use pattern was defined by grazing, agriculture, firewood extraction and lime kilns exploitation, but this dramatically changed in 1987 with the prohibition of traditional land uses (Santana-Cordero et al., 2016a). Nonetheless, some farms remain today, but residential use, tourism and no use make up the current land use pattern.

In short, it can be said that land use patterns have retained the same degree of complexity. This complexity can be viewed using the normalized standard deviation, calculated for two years (1954/1961 and 2018) for each study site. The normalized standard deviation explains the degree of homogeneity by measuring the mean distance of the values to the average value, i.e. the standard deviation (Table 4). The normalized value allows the ordering (from less to more) of the degree of heterogeneity: Guanarteme 1954, 2018, Maspalomas 2018, 1961, La Graciosa 1954, and 2018.

Table 4. Land use evolution in the three study sites. Key - G: Guanarteme; M: Maspalomas; Lg: La Graciosa. The first values in each cell are expressed in ha and the second, in brackets, are \%.

\begin{tabular}{|c|c|c|c|c|c|c|}
\hline Land uses & G - 1954 & G - 2018 & M - 1961 & M - 2018 & Lg - 1954 & Lg - 2018 \\
\hline Abandoned & $\begin{array}{l}10.841 \\
(4.4) \\
\end{array}$ & $\begin{array}{l}1.551 \\
(0.6) \\
\end{array}$ & $\begin{array}{l}0 \\
(0)\end{array}$ & $\begin{array}{l}0 \\
(0)\end{array}$ & $\begin{array}{l}23.361 \\
(1.8)\end{array}$ & $\begin{array}{l}4.069 \\
(0.3) \\
\end{array}$ \\
\hline Agriculture & $\begin{array}{l}12.419 \\
(5.1)\end{array}$ & $\begin{array}{l}0 \\
(0)\end{array}$ & $\begin{array}{l}0 \\
(0)\end{array}$ & $\begin{array}{l}0 \\
(0)\end{array}$ & $\begin{array}{l}0 \\
(0)\end{array}$ & $\begin{array}{l}2.268 \\
(0.2)\end{array}$ \\
\hline Industrial & $\begin{array}{l}11.863 \\
(4.8)\end{array}$ & $\begin{array}{l}0 \\
(0)\end{array}$ & $\begin{array}{l}0 \\
(0)\end{array}$ & $\begin{array}{l}0 \\
(0)\end{array}$ & $\begin{array}{l}0 \\
(0)\end{array}$ & $\begin{array}{l}0 \\
(0)\end{array}$ \\
\hline Infrastructure & $\begin{array}{l}11.818 \\
(4.8)\end{array}$ & $\begin{array}{l}25.764 \\
(10.6) \\
\end{array}$ & $\begin{array}{l}0 \\
(0)\end{array}$ & $\begin{array}{l}5.734 \\
(1.2) \\
\end{array}$ & $\begin{array}{l}0 \\
(0)\end{array}$ & $\begin{array}{l}0.752 \\
(0.1) \\
\end{array}$ \\
\hline Mixed & $\begin{array}{l}13.996 \\
(5.7)\end{array}$ & $\begin{array}{l}0 \\
(0)\end{array}$ & $\begin{array}{l}0 \\
(0)\end{array}$ & $\begin{array}{l}0 \\
(0)\end{array}$ & $\begin{array}{l}0 \\
(0)\end{array}$ & $\begin{array}{l}0.306 \\
(0)\end{array}$ \\
\hline No use & $\begin{array}{l}46.437 \\
(18.9)\end{array}$ & $\begin{array}{l}8.305 \\
(3.4)\end{array}$ & $\begin{array}{l}441.532 \\
(92.9)\end{array}$ & $\begin{array}{l}347.09 \\
(75.1)\end{array}$ & $\begin{array}{l}1,286.837 \\
(98.2)\end{array}$ & $\begin{array}{l}1,302.822 \\
(99.4)\end{array}$ \\
\hline
\end{tabular}




\begin{tabular}{|l|l|l|l|l|l|l|}
\hline Recreational & 12.749 & 26.27 & 33.784 & 50.971 & 0 & 0 \\
& $(5.2)$ & $(10.8)$ & $(7.1)$ & $(11)$ & $(0)$ & $(0)$ \\
\hline Residential & 125.613 & 181.821 & 0 & 58.237 & 0 & 0 \\
& $(51.1)$ & $(74.6)$ & $(0)$ & $(12.6)$ & $(0)$ & $(0)$ \\
\hline Total & 245.736 & 243.711 & 475.316 & 462.032 & $1,310.198$ & $1,310.217$ \\
\hline Average & 30.717 & 30.464 & 59.415 & 57.754 & 163.775 & 163.777 \\
\hline Std deviation & 40.173 & 62.175 & 154.851 & 119.409 & 453.859 & 460.246 \\
\hline Normalization & 0.235 & 0.510 & 0.616 & 0.516 & 0.639 & 0.644 \\
\hline
\end{tabular}

\section{Discussion}

All the environmental consequences seen above can also be understood as part of the phenomenon of global change, and all are well represented in different parts of the world. Gössling (2002) listed the main factors of the environmental consequences of tourism, including two found in this study - LUCC processes and the biotic exchange and extinction of wild species - which can affect biodiversity. Defeo et al. (2009) identified the various threats to sandy shores worldwide, including recreation, cleaning, nourishment, pollution, exploitation, biological invasion, coastal development and engineering, mining, and climate change. Nordstrom (1994) reviewed anthropic alterations to coastal beaches and dunes, categorizing them as: (i) large-scale landscape conversion, (ii) creating, reshaping or eliminating landforms to suit human needs, and (iii) effects of protection structures and buildings. The paradigmatic case of large-scale landscape formation in this work can be seen in the case of the Guanarteme dunefield, which disappeared towards the 1960s. Likewise, landforms have been partially eliminated in the sedimentary terrace of the Maspalomas dunefield, where the existence of cliff-top dunes in 1961 has been corroborated. In this same dunefield, effects on the wind currents as the result of urbanization of the terrace have been verified and measured (García-Romero et al., 2019a; Hernández-Calvento et al., 2014).

In Mediterranean shores, Pintó et al. (2014) established the following, which are particularly well represented along the Costa Brava (Spain), as having the most important impact: built environments, car parking/car tracks, beach raking, erosion pathways, dune breaches, invasive species and fixed dunes. Fragmentation (or erosion pathways) of the dune front (and its vegetation cover) can result in blowouts, one of the main erosional landforms, which are frequently observed in the western Mediterranean (Mir-Gual et al., 2013). This last impact can be observed in the foredune of Maspalomas, affected by the transit of tourists walking around and through it. Garcia-Lozano et al. (2018) reported dune fragmentation along the coast of Catalonia (Spain) as the result of human activity due to trampled paths and car tracks, as also observed in the present study in Maspalomas and La Graciosa. The same authors additionally observed the disappearance of dunes due to urbanization processes, as in the dunefields of Guanarteme and Maspalomas. On the coast of Italy (Campany), the extensive erosion process of a foredune as the result of natural and human activities has been measured, with the results indicating that it has retreated 35-90 $\mathrm{m}$ landward in the last 55 years (Pennetta et al., 2011). According to the same authors, the most important anthropic impacts on the dune system have been river protection infrastructures and other urban development structures. Paradoxically, although the study area of Pennetta et al. (2011) has been declared a protected natural area, as in the case of Maspalomas and La Graciosa, this has been insufficient to impede a number of human impacts. Similarly, another study on the Northern Tyrrhenian coast (Italy), a place under touristic and residential urbanization pressure since the mid-XX century, reported a significant historical coastal retreat in a beach-dune system and an almost complete occupation of the beach, especially since the 1970s (Bertacchi and Lombardi, 2014). This last case is very similar to that of Maspalomas, where tourist activity has played the most important role in the land change.

Sedimentary areas impacted by human activities can also be found on Atlantic shores. In Valdelagrana beach (SW Spain), historical - from Roman times - and current human interventions have affected a barrier spit. This sandy platform was historically altered about 2,000 years BP by a constructed 
straight channel, and roads, port facilities, and coastal and fluvial works. Then, in 1721 an artificial cut was excavated in the inner marshlands. Additionally, the coastline has been considerably changed in the last 60 years. Whereas in the northern sector accretion rates of $6 \mathrm{~m} /$ year have been recorded, in the southern sector the rates fall to $-14 \mathrm{~m} / \mathrm{year}$ (Del Río et al., 2015). Port facilities and coastal infrastructures have similarly been determining LUCC factors in the dunefield of Guanarteme. In Belgium, Doody (2013) found that $50 \%$ of the coastal dunes had disappeared since the $1950 \mathrm{~s}$ as the result of urbanization projects. In the same study, pressure on sedimentary systems in Portugal was reported to be the result of the establishment of illegal huts in the 1970s in Leira district and Ria Formosa Natural Park. Another important impact on sand beaches and dunes is onshore sand mining. This has been reported along many Atlantic coastlines, including Northern Ireland, Scotland, the Caribbean, the Azores, Africa, and most especially northern Morocco and the USA (Doody, 2013). Hernández and Suárez (2006) characterized the sedimentary systems of Boa Vista (Cape Verde), which are very similar to those of the Canary Islands, and found that the city of Sal Rei (capital of the island) constitutes an obstacle to sedimentary dynamics. In addition, during the period 2005-2010, a tourist resort along with roads leading to and from it were developed in the Rabil coastal sector where a sand sheet and some large barchan dunes existed, as could be discerned in Google Earth (orthophoto from 2016). As in Maspalomas, in Boa Vista this urbanization is altering the sedimentary flow by stopping it and, possibly, deviating the wind current. Marcomini et al. (2017) reported that the northeastern coastal dunes of Buenos Aires (Argentina) have been altered by urbanization since 1935, causing changes in dune landforms and vegetation cover.

The Indian and Pacific shores are not exempt from human-altered sandy coasts. In South Korea, various alterations to sand dune systems found along its coastline have been caused by the development of port facilities and recreational resort facilities (Kim, 2005). Such changes are well represented in the present study in the examples of Guanarteme and Maspalomas. Land development has also been the main driver in the reduction and disappearance of coastal dunes in Japan (Iwasatu and Nagamatsu, 2018). The Tottori sand dunes, the largest coastal dune system of Japan, are being impacted by coastal erosion as the result of human activities (Yasumoto et al. 2007). As at the three study sites of the present study, beach sand excavation and sand erosion have been reported in the Maldives Islands (Kothari and Arnall, 2019). Thompson and Schlacher (2008) examined the damage that car tracks associated with beach camping areas caused to coastal dunes, especially the foredune, in Fraser Island (Australia), finding that they caused accelerated erosion, shoreline retreat and the removal of dune vegetation. Similarly, the existence of camping areas and roads on La Graciosa island has had a direct impact on the sedimentary dynamics.

Focusing on changes in vegetation cover, many dune areas have been stabilized along the Catalonian coast (Spain, Mediterranean basin) by planting pines to avoid the inland movement of the sediment. Such actions date back as far as the XIX century (Pintó et al., 2014). This contrasts with the changes in vegetation reported in this study which were unintentionally provoked by human actions (cases of Maspalomas and La Graciosa). Nevertheless, according to some historical sources, and with the purpose of stabilizing the sediments in the now-disappeared Guanarteme dunefield, measures such as the planting of Tamarix canariensis were employed to block sediment movement along the road that connected the old city with the port of La Luz in the XIX century (Santana-Cordero et al., 2014). By contrast, Moulton et al. (2019) reported an increase in vegetation in a transgressive dunefield in the Younghusband Peninsula (Australia) due to natural factors. In almost 70 years, vegetation cover grew from $7 \%$ to $40 \%$ of the area, and the authors found that the effects of human activities in this case were negligible. However, Provoost et al. (2011) highlighted the link in Europe between land use change, especially from its traditional use, and vegetation cover in such systems.

With respect to impacts on the socioeconomic system, Domínguez-Mujica et al. (2011) point out the strong correlation with the development of tourism and changes in population. All these factors are found in Maspalomas. In addition, while tourism-based economies of coastal zones may be a powerful economic resource they are also frequently the main cause of environmental consequences that 
alter and destroy such coastal environments (Hernández-Calvento et al., 2014). This is a trend which has been observed on a global scale since at least 1950 (Gormsen, 1997), and is evident in two of the sites studied here, Guanarteme and Maspalomas (Santana-Cordero et al., 2017).

Globally, the leading driving force behind LUCC is tourism (Gössling, 2002). In this respect, it is to be expected that most tourist areas in the world are undergoing or have had undergone LUCC processes. This is demonstrated in the Mediterranean coasts, the Canary Islands and the Maldives, where one of the results has been the expansion of the built environment (Kothari and Arnall, 2019). In this respect, Pintó et al. (2014) suggest that human occupation of beaches through tourist activity triggers irreversible impacts given the current legal and socioeconomic circumstances. Davenport and Davenport (2006) argue that the implementation of new land uses in tourist areas triggers ecosystem fragmentation, as also described by Mir-Gual et al. (2013), and reduces biodiversity. In the present study, ecosystem fragmentation can be observed in Maspalomas, specifically in its foredune. Finally, Goudie et al. (2000) noted that LUCC can lead to the installation of engineering structures that can modify the transport of the sediments. A similar situation in which human structures have interrupted sediment flows is found in the present study along Las Canteras beach, in Guanarteme, as a consequence of the barrier imposed by its promenade.

\section{Conclusion}

As the results of this study show, the sedimentary systems considered here have been altered by a variety of causes which have resulted in, at least, six lines of evidence (the environmental consequences), all of which are the product of human action in these coastal systems. Highlighted in this study is the importance of tourism, which has transformed the coast at an unprecedented rate. Of the six environmental consequences considered in this investigation, four are present in all three study sites: impacts on the aeolian sedimentary dynamics; changes in the sand landforms; impacts on the socioeconomic system; and changes in land use patterns. Despite the differences in the developmental models followed at the study sites (Santana-Cordero et al., 2017), the environmental consequences are shared, tending to the existence of the same impacts. The paucity of literature on human impacts on coastal sedimentary systems/dunefield systems, in line with the findings of Jackson and Nordstrom (2019), is confirmed in the present study. There is therefore a clear need for further research studies in this field to enable a proper understanding of these ecosystems and to provide a satisfactory and sufficient background for sustainable interventions. It should also be noted that the environmental consequences studied here constitute a global phenomenon, as demonstrated throughout the discussion section.

\section{Acknowledgements}

Aarón M. Santana Cordero is researcher of the Universidad de Las Palmas de Gran Canaria postdoctoral programme. The author would like to thank Dr. Antonio I. Hernández-Cordero, Dr. Carolina Peña-Alonso and Dr. Luis Hernández-Calvento for revising the results of the manuscript and for the material provided. This paper is a contribution of the Spanish National Plan for $\mathrm{R}+\mathrm{D}+\mathrm{i}$ (innovation) project CSO2016-79673-R, co-financed with ERDF funds. The author acknowledges the two anonymous reviewers for their comments aimed to improve the quality of the paper.

\section{References}

Alonso-Bilbao, I., Sánchez-García, M.J., Rodríguez-Valido, S., Casamayor-Font, M. 2015. Estudio del cálculo del balance sedimentario en la playa de Las Canteras. Informe final. Report, Las Palmas de Gran Canaria Town Council, University of Las Palmas de Gran Canaria, and Institute of Oceanography and Global Change, Spain, October. 
Bertacchi, A., Lombardi, T. 2014. Diachronic analysis (1954-2010) of transformations of the dune habitat in a stretch of the Northern Tyrrhenian Coast (Italy). Plant Biosystems 148(2), 227-236. https://doi.org/10.1080/11263504.2013.788572

Cabrera-Vega, L.L., Cruz-Avero, N., Hernández-Calvento, L., Hernández-Cordero, A.I., Fernández-Cabrera, E. 2013. Morphological changes in dunes as an indicator of anthropogenic interferences in arid dune fields. Journal of Coastal Research Special Issue No. 65, 1271-1276. https://doi.org/10.2112/SI65-215.1

Copeiro del Villar Martínez, E., García-Campos, M.A. 2002. Proyecto de extracción puntual de arena en la playa de Las Canteras. Report, Las Palmas de Gran Canaria Town Council, Spain, December.

Davenport, J., Davenport, J.L. 2006. The impact of tourism and personal leisure transport on coastal environments: A review. Estuarine, Coastal and Shelf Science 67, 280-292. https://doi.org/10.1016/j.ecss.2005.11.026

Defeo, O., McLachlan, A., Schoeman, D.S., Schachler, T.A., Dugan, J., Jones, A., Lastra, M., Scapini, F. 2009. Threats to sandy beach ecosystems: A review. Estuarine, Coastal and Shelf Science 81, 1-12. https://doi.org/10.1016/j.ecss.2008.09.022

Del Río, L., Benavente, J., Gracia, F.J., Alonso, C., Rodríguez-Polo, S. 2015. Anthropogenic Influence on Spit Dynamics at Various Timescales: Case Study in the Bay of Cadiz (Spain) In: Randazzo, G., Jackson, D., Cooper, A. (eds.), Sand and gravel spits. Switzerland: Springer, pp. 123-138. ISBN: 978-3-319-13716-2

Domínguez-Mujica, J., González-Pérez, J., Parreño-Castellano, J. 2011. Tourism and human mobility in Spanish archipelagos. Annals of Tourism Research 38(2), 586-606. https://doi.org/10.1016/j.annals.2010.11.016

Doody, J.P. 2013. Sand Dune Conservation, Management and Restoration. Dordrecht: Springer, 303p. ISBN: 97894-007-4731-9

Ferrer-Valero, N., Hernández-Calvento, L., Hernández-Cordero, A.I. 2017. Human impacts quantification on the coastal landforms of Gran Canaria Island (Canary Islands). Geomorphology 286, 58-67. http://doi.org/10.1016/j.geomorph.2017.02.028

Garcia-Lozano, C., Pintó, J., Daunis-i-Estadella, P. 2018. Changes in coastal dune systems on the Catalan shoreline (Spain, NW Mediterranean Sea). Comparing dune landscapes between 1890 and 1960 with their current status. Estuarine, Coastal and Shelf Science 208, 235-247. http://doi.org/10.1016/j.ecss.2018.05.004

García-Romero, L., Hernández-Cordero, A.I., Fernández-Cabrera, E., Peña-Alonso, C., Hernández-Calvento, L., Pérez-Chacón, E. 2016. Urban-touristic impacts on the aeolian sedimentary systems of the Canary Islands: conflict between development and conservation. Island Studies Journal 11(1), 91-112. http://hdl.handle.net/10553/52597

García-Romero, L., Delgado-Fernández, I., Hesp, P.A., Hernández-Calvento, L., Viera-Pérez, M., HernándezCordero, A.I., Cabrera-Gámez, J., Domínguez-Brito, A.C. 2019a. Airflow dynamics, vegetation and aeolian erosive processes in a shadow zone leeward of a resort in an arid transgressive dune system. Aeolian Research 38, 48-59. https://doi.org/10.1016/j.aeolia.2019.03.006

García-Romero, L., Delgado-Fernández, I., Hesp, P.A., Hernández-Calvento, L., Hernández-Cordero, A.I., VieraPérez, M. 2019b. Biogeomorphological processes in an arid transgressive dunefield as indicators of human impact by urbanization. Science of the Total Environment 650, 73-86. https://doi.org/10.1016/j.scitotenv.2018.08.429

Gormsen, E. 1997. The impact of tourism on coastal areas. GeoJournal, 42(1), 39-54. https://doi.org/10.1023/A:1006840622450

Gössling, S. 2002. Global environmental consequences of tourism. Global Environmental Change 12, 283-302. https://doi.org/10.1016/S0959-3780(02)00044-4

Goudie, A.S., Parker, A.G., Al-Farraj A. 2000. Coastal Change in Ras Al Khaimah (United Arab Emirates): a Cartographic Analysis. The Geographical Journal 166(1), 14-25. https://www.jstor.org/stable/823050

Hernández, L., Suárez, C. 2006. Characterization of the contemporary aeolian sediment dynamics of Boa Vista (Cape Verde). Journal of Coastal Research Special Issue No. 48, 64-68. ISSN 0749-0208 
Hernández-Calvento, L., Pérez-Chacón, E., Ruiz-Flaño, P., Suárez-Rodriguez, C., Alonso-Bilbao, I., AlcántaraCarrió, J. 2002. Informe sobre los impactos ocasionados por los kioscos de Playa del Inglés. Report, Ayuntamiento de San Bartolomé de Tirajana, Las Palmas de Gran Canaria, June.

Hernández-Calvento, L. 2006. Diagnóstico sobre la evolución del sistema de dunas de Maspalomas (1960-2000). Cabildo Insular de Gran Canaria: Las Palmas de Gran Canaria (Spain), 361p. ISBN: 9788481034820

Hernández-Calvento, L., Jackson, D.W.T., Medina, R., Hernández-Cordero, A.I., Cruz, N., Requejo, S. 2014. Downwind effects on an arid dunefield from an evolving urbanised area. Aeolian Research 15, 301-309. http://doi.org/10.1016/j.aeolia.2014.06.007

Hernández-Cordero, A.I., Pérez-Chacón Espino, E., Hernández-Calvento, L. 2015. Vegetation, distance to the coast, and aeolian geomorphic processes and landforms in a transgressive arid coastal dune system. Physical Geography 36(1), 60-83. http://doi.org/10.1080/02723646.2014.979097

Hernández-Cordero, A.I., Hernández-Calvento, L., Pérez-Chacón Espino, E. 2017. Vegetation changes as an indicator of impact from tourist development in an arid transgressive coastal dune field. Land Use Policy 64, 479-491. http://doi.org/10.1016/j.landusepol.2017.03.026

Hernández-Cordero, A.I., Hernández-Calvento, L., Hesp, P.A., Pérez-Chacón, E. 2018. Geomorphological changes in an arid transgressive coastal dune field due to natural processes and human impacts. Earth Surface Processes and Landforms 43(10), 2167-2180. http://doi.org/10.1002/esp.4382

Hoffman, M.T., Rohde, R.F. 2007. From pastoralism to tourism: the historical impact of changing land use practices in Namaqualand. Journal of Arid Environments 70, 641-658. http://doi.org/10.1016/j.jaridenv.2006.05.014

Iwasato, M., Nagamatsu, D. 2018. Plant species diversity and habitat conditions in a protected large coastal dune area of western Japan. Landscape and Ecological Engineering 14, 99-113. https://doi.org/10.1007/s11355-017-0334-X

Jackson, N.L., Nordstrom, K.F. 2011. Aeolian sediment transport and landforms in managed coastal systems: A review. Aeolian Research 3, 181-196. https://doi.org/10.1016/j.aeolia.2011.03.011

Jackson, N.L., Nordstrom, K.F. 2019. Trends in research on beaches and dunes on sandy shores, 1969-2019. Geomorphology 366, 106737. http://doi.org/10.1016/j.geomorph.2019.04.009

Kim, K.D. 2005. Invasive plants on disturbed Korean sand dunes. Estuarine, Coastal and Shelf Science 62, 353364. http://doi.org/10.1016/j.ecss.2004.09.023

Kothari, U., Arnall, A. 2019. Everyday life and environmental change. The Geographical Journal 185, $130-141$. http://doi.org/10.1111/geoj.12296

Lambin, E.F., Turner, B.L., Geist, H.J., Agbola, S.B., Angelsen, A., Bruce, J.W., Coomes, O.T., Dirzo, R., Fischer, G., Folke, C., George, P.S., Homewood, K., Imbernon, J., Leemans, R., Li, X., Moran, E.F., Mortimore, M., Ramakrishnan, P.S., Richards, J.F., Skånes, H., Steffen, W., Stone, G.D., Svedin, U., Veldkamp, T.A., Vogel, C., Xu, J. 2001. The causes of land-use and land-cover change: moving beyond the myths. Global Environmental Change 11, 261-269. https://doi.org/10.1016/S0959-3780(01)00007-3

Marcomini, S., López, R., Picca, P., Madanes, N., Bertolín, L. 2017. Natural Coastal Dune-Field Landforms, Plant Communities, and Human Intervention along Buenos Aires Northern Aeolian Barrier. Journal of Coastal Research 33(5), 1051-1064. http://doi.org/10.2112/JCOASTRES-D-15-00219.1

Martínez, M.L., Intralawan, A., Vázquez, G., Pérez-Maqueo, O., Sutton, P., Landgrave, R. 2007. The coasts of our world: ecological, economic and social importance. Ecological Economics 63 (2), 254-272. http://doi.org/10.1016/j.ecolecon.2006.10.022

Mir-Gual, M., Pons, G.X., Martín-Prieto, J.A., Roig-Munar, F.X., Rodríguez-Perea, A. 2013. Geomorphological and ecological features of blowouts in a western Mediterranean coastal dune complex: a case study of the Es Comú de Muro beach-dune system on the island of Mallorca, Spain. Geo-Marine Letters 33, 129-141. http://doi.org/10.1007/s00367-012-0298-7

Morales-Matos, G., Santana-Santana, A. 2005. Islas Canarias, territorio y sociedad. Las Palmas de Gran Canaria: Anroart. ISBN: 9788496577251. 
Moulton, M.A.B., Hesp, P.A., da Silva, G.M., Bouchez, C., Lavy, M., Fernandez, G.B. 2019. Changes in vegetation cover on the Younghusband Peninsula transgressive dunefields (Australia) 1949-2017. Earth Surface Processes and Landforms 44, 459-470. http://doi.org/10.1002/esp.4508

Müller, D., Sun, Z., Vongvisouk, T., Pflugmacher, D., Xu, J., Mertz, O. 2014. Regime shifts limit the predictability of land-system change. Global Environmental Change 28, 75-83. http://doi.org/10.1016/j.gloenvcha.2014.06.003

Nordstrom, K.F. 1994. Beaches and dunes of human-altered coasts. Progress in Physical Geography 18(1), $497-$ 516. https://doi.org/10.1177/030913339401800402

Otto, R., Krüsi, B.O., Kienast, F. 2007. Degradation of an arid coastal landscape in relation to land use changes in southern Tenerife (Canary Islands). Journal of Arid Environments 70, 527-539. http://doi.org/10.1016/j.jaridenv.2007.02.001

Peña-Alonso, C., Hernández-Calvento, L., Pérez-Chacón, E., Hernández-Cordero, A.I. 2012. Indicadores de vulnerabilidad de la vegetación de la duna costera de Maspalomas (Islas Canarias, España). In: Verón, E., García Sanabria, J., García Onetti, J., Chica Ruiz, J.A. (eds.) I Congreso Iberoamericano de Gestión Integrada de Áreas Litorales (Cádiz, Spain) pp. 716-724. ISBN: 978-84-695-1823-6

Peña-Alonso, C., Hernández-Calvento, L., Pérez-Chacón, E., Ariza-Solé, E. 2017. The relationship between heritage, recreational quality and geomorphological vulnerability in the coastal zone: A case study of beach systems in the Canary Islands. Ecological Indicators 82, 420-432. http://doi.org/10.1016/j.ecolind.2017.07.014

Pennetta, M., Corbelli, V., Esposito, P., Gattullo, V., Nappi, R. 2011. Environmental Impact of Coastal Dunes in the Area Located to the Left of the Garigliano River Mouth (Campany, Italy). Journal of Coastal Research Special Issue No. 61, 421-427. http://doi.org/10.2112/SI61-001.1

Pérez-Chacón, E., Hernández-Calvento, L., Fernández-Negrín, E., Romero, L., Máyer, P., Hernández-Cordero, A., Cruz, N., Fernández-Cabrera, E., Peña, C., Corbalán, Y., Mangas, J., Alonso, I., Rodríguez, S., Sánchez, I., Cabrera, L. 2010. Caracterización del sistema sedimentario eólico de La Graciosa (archipiélago canario). Informe final. Report, University of Las Palmas de Gran Canaria Centro 'Isla de La Graciosa' (OAPN-Ministerio de MAMRM), Spain, July.

Pickart, A.J., Hesp, P.A. 2019. Spatio-temporal geomorphological and ecological evolution of a transgressive dunefield system, Northern California, USA. Global and Planetary Change 172, 88-103. https://doi.org/10.1016/j.gloplacha.2018.09.012

Pintó, J., Martí, C., Fraguell, R.M. 2014. Assessing current conditions of coastal dune systems of Mediterranean developed shores. Journal of Coastal Research 30 (4), 832-842. https://doi.org/10.2112/JCOASTRESD-13-00116.1

Provoost, S., Jones, M.L.M., Edmondson, S.E. 2011. Changes in landscape and vegetation of coastal dunes in northwest Europe: a review. Journal of Coastal Conservation 15, 207-226. https://doi.org/10.1007/s11852-009-0068-5

Santana-Cordero, A.M., Monteiro-Quintana, M.L., Hernández-Calvento, L. 2014. Reconstructing the environmental conditions of extinct coastal dune systems using historical sources: the case of the Guanarteme dune field (Canary Islands, Spain). Journal of Coastal Conservation 18, 323-337. https://doi.org/10.1007/s11852-014-0320-5

Santana-Cordero, A.M., Monteiro-Quintana, M.L., Hernández-Calvento, L., Pérez-Chacón Espino, E., GarcíaRomero, L. 2016a. Long-term human impacts on the coast of La Graciosa, Canary Islands. Land Degradation and Development 27, 479-489. https://doi.org/10.1002/ldr.2369

Santana-Cordero, A.M., Monteiro-Quintana, M.L., Hernández-Calvento, L. 2016b. Reconstruction of the land uses that led to the termination of an arid coastal dune system: The case of the Guanarteme dune system (Canary Islands, Spain), 1834-2012. Land Use Policy 55, 73-85. http://doi.org/10.1016/j.landusepol.2016.02.021

Santana-Cordero, A.M., Bürgi, M., Hersperger, A.M., Hernández-Calvento, L., Monteiro-Quintana, M.L. 2017. A century of change in coastal sedimentary landscapes in the Canary Islands (Spain) — Change, processes, and driving forces. Land Use Policy 68, 107-116. http://doi.org/10.1016/j.landusepol.2017.07.028 
Thompson, L.M.C., Schlacher, T.A. 2008. Physical damage to coastal dunes and ecological impacts caused by vehicle tracks associated with beach camping on sandy shores: a case study from Fraser Island, Australia. Journal of Coastal Conservation 12, 67-82. http://doi.org/10.1007/s11852-008-0032-9

Vitousek, P.M., Mooney, H.A., Lubchenco, J., Melillo, J.M. 1997. Human domination of earth's ecosystems. Science 277, 494-499. DOI: 10.1126/science.277.5325.494

Wenbin, W.U., Verburg, P.H., Huajun, T. 2018. Understanding land system dynamics and its consequences. Journal of Geographical Sciences 28, 1563-1566. https://doi.org/10.1007/s11442-018-1562-1

Yasumoto, Y., Uda, T., Matsubara, Y., Hirano, G. 2007. Beach erosion along Tottori coast and comprehensive sediment management. Journal of Coastal Research Special Issue No. 50, 82-87. https://www.jstor.org/stable/26481561 



\title{
IMPACT OF HEATWAVE EVENTS ON LATENT AND SENSIBLE SURFACE HEAT FLUX AND FUTURE PERSPECTIVES IN SHALLOW LAKES BASED ON CLIMATE CHANGE MODELS
}

\author{
MARIA B. ALFONSO ${ }^{1 *}$, ANDREA S. BRENDEL ${ }^{1,2}$, \\ ALEJANDRO J. VITALE ${ }^{1,3}$, MARIA C. PICCOLO ${ }^{1,4}$ \\ ${ }^{1}$ Instituto Argentino de Oceanografía (IADO), Universidad Nacional del Sur (UNS)-CONICET, Florida 8000, \\ Complejo CCT CONICET Bahía Blanca, Edificio E1, B8000BFW, Bahía Blanca, Argentina. \\ ${ }^{2}$ Departamento de Agronomía, Universidad Nacional del Sur (UNS), \\ San Andrés 800, B8001FAN, Bahía Blanca, Argentina. \\ ${ }^{3}$ Departamento de Ingeniería Eléctrica y Computadoras, Universidad Nacional del Sur (UNS), \\ Av. Alem 1253, B8000CPB, Bahía Blanca, Argentina. \\ ${ }^{4}$ Departamento de Geografía y Turismo, Universidad Nacional del Sur, \\ 12 de Octubre $11984^{\circ}$ Piso, B8000CTX, Bahía Blanca, Argentina.
}

\begin{abstract}
According to model projections, increases in the frequency and intensity of heatwaves are expected all over the world. This study analyzed, for the first time, the effect of heatwaves events on long-term surface latent (LE) and sensible heat fluxes (H) from two shallow lakes: La Salada lake (LS - 39 $27^{\prime} \mathrm{S}, 62^{\circ} 42^{\prime} \mathrm{W}$ ) and Sauce Grande lake (SG - 38 $57^{\prime} \mathrm{S}, 61^{\circ} 24^{\prime} \mathrm{W}$ ). The main drivers of LE and $\mathrm{H}$ are wind speed and direction, relative humidity, and the difference between air and water temperature. We found that the daily values of both fluxes were highly fluctuating. Mean daily $\mathrm{H}$ values ranged between -309.4 to $200.5 \mathrm{~W} \mathrm{~m}^{-2}$ and -78.6 to $104.8 \mathrm{~W} \mathrm{~m}^{-2}$ in LS and SG, respectively. LE oscillated between -152 and $463.9 \mathrm{~W} \mathrm{~m}^{-2}$ and between -59.2 and $360.1 \mathrm{~W} \mathrm{~m}^{-2}$ in LS and SG, respectively. Both fluxes decreased with the passage of heatwaves events, presenting a high variation in its amplitude. Changes up to $96 \%$ in mean daily LE and $671 \%$ in mean daily H for LS and up to $25 \%$ in LE and $987 \%$ in $\mathrm{H}$ for SG were accounted in days with heatwaves respect to regular ones. We analyzed the air temperature increase for both lakes under future global warming scenarios (RCP 4.5 y RCP 8.5), expecting higher amplitudes in heat fluxes. These results contribute to the development of lake models, as well as to water resources management in the future.
\end{abstract}

\section{Impacto de las olas de calor en el flujo de calor superficial latente y sensible y perspectivas de futuro para las lagunas someras basadas en modelos de cambio climático}

RESUMEN. De acuerdo con las proyecciones de los modelos climáticos, se espera a nivel mundial un aumento en la frecuencia e intensidad de las olas de calor. Este estudio analiza, por primera vez, el efecto de los eventos de olas de calor en los flujos de calor latente (LE) y calor sensible (H) en dos lagunas someras: Laguna La Salada (LS - $39^{\circ} 27^{\prime} \mathrm{S}, 62^{\circ} 42^{\prime} \mathrm{W}$ ) y Laguna Sauce Grande ( $\mathrm{SG}-38^{\circ} 57^{\prime} \mathrm{S}, 61^{\circ} 24^{\prime} \mathrm{W}$ ). La velocidad y dirección del viento, la humedad relativa, y las diferencias de temperatura entre el aire y el agua determinaron los cambios en LE y H. Los valores diarios de ambas variables fueron altamente fluctuantes. Los valores medios diarios de $\mathrm{H}$ variaron entre 309,4 y $200,5 \mathrm{~W} \mathrm{~m}^{-2}$ y entre $-78,6$ y $104,8 \mathrm{~W} \mathrm{~m}^{-2}$ en LS y SG, respectivamente. Los valores medios diarios de LE variaron entre -152 y $463,9 \mathrm{~W} \mathrm{~m}^{-2}$ y entre $-59,2$ y $360,1 \mathrm{~W} \mathrm{~m}^{-2}$ en LS y SG, respectivamente. Ambos flujos disminuyeron con el paso de eventos de olas de calor, presentando una gran amplitud en los valores. En días con olas de calor comparados con días regulares se registraron cambios de hasta $96 \%$ en la media diaria de LE y $671 \%$ 
en la media diaria de $\mathrm{H}$ en LS y cambios de hasta 25\% en la media diaria de LE y $987 \%$ en la media diaria de $\mathrm{H}$ para SG. Finalmente, se analizó el futuro incremento de la temperatura del aire para ambas lagunas bajo dos escenarios de calentamiento global (RCP 4.5 y RCP 8.5), que permiten deducir mayores amplitudes de cambio en ambos flujos de calor. Estos resultados contribuyen al desarrollo de modelos para lagunas someras, y a las decisiones de manejo de los recursos hídricos en el futuro.

Key words: Sensible heat flux, Latent heat flux, High-frequency, Heatwave, Shallow lake, Instrumented buoy.

Palabras clave: flujo de calor sensible, flujo de calor latente, alta frecuencia, ola de calor, laguna somera, boya multi-paramétrica.

Received: 10 October 2019

Accepted: 31 March 2020

\begin{abstract}
* Corresponding author: María Belén Alfonso. Instituto Argentino de Oceanografía (IADO), Universidad Nacional del Sur (UNS)-CONICET, Florida 8000, Complejo CCT CONICET Bahía Blanca, Edificio E1, B8000BFW, Bahía Blanca, Argentina. E-mail address: mbalfonso@conicet-iado.gob.ar
\end{abstract}

\title{
1. Introduction
}

Lakes have a significant role in many environmental processes, like carbon dioxide and methane fluxes, acting as carbon sources or sinks, nutrients recycling and regional climate regulation, among others (Tranvik et al., 2009; Zhu et al., 2018). The regional climatic conditions and human activities in shallow lakes can affect the water level, limnological conditions, and, therefore, the ecological processes (Leira and Cantonati, 2008; Havens et al., 2016). The interaction between lakes and their adjacent atmospheric boundary layer is vital to understand the physical processes within them as the surface heat fluxes (Winder and Schindler, 2004; Woolway et al., 2015a). Among the drivers of the physical processes within lakes, the wind stress and turbulent mixing, together with the surface heating and cooling, are the most cited (Wüest and Lorke, 2003; Woolway et al., 2018). Lake temperature regulates many vital processes of lake ecosystems as carbon fluxes, ecosystem metabolism, nutrients dynamics, and seasonal plankton patterns, among others (Lagomarsino et al., 2011; Diovisalvi et al., 2015; Kraemer et al., 2017), and it is highly dependent of lake-atmosphere interactions. Furthermore, lakes presence regulates the regional climate, as water surface can change the microclimate of the surroundings by increasing humidity and decreasing temperature, which could change the local air circulation and enhances the airflow towards the outside of the water body (Diallo et al., 2018; Zhu et al., 2018).

Climate change produces effects on the thermal structure, heat budgets, and heat and energy fluxes at the air-water interface with consequences in the physicochemical characteristics and the biological community of lakes (Woolway et al., 2018). Water temperatures of many lakes worldwide have increased in the past decades (Fink et al., 2014). According to Arvola et al. (2010), these observed global changes in lake's heat content are attributed to increasing air temperatures. Besides, other factors as lake area, depth, residence time, optical properties, and landscape setting can influence the thermal characteristics of lakes (Arvola et al., 2010) and modify the response of the lake temperatures to changing meteorological conditions.

There are a large number of surface heat flux studies in lakes based on the eddy covariance method (Zhang and Liu, 2013; Shao et al., 2015) and also on the high-frequency measurement from instrumented buoys (Woolway et al., 2015b, 2018). Some studies have evaluated the effect of shortterm weather events as the passages of cold front (Zhang and Liu, 2013; Li et al., 2015), the intrusion 
of dry, cold air masses (Li et al., 2015), or the impact of climate-induced warming on heat fluxes (Fink et al., 2014). According to model projections, increases in the duration, intensity, and spatial extent of heatwaves in the short-term over most land regions are expected (Kirtman et al., 2013). In the context of climate change, the impact of climate extreme events, such as heatwaves, demonstrate the vulnerability of some ecosystems to climate variability (Field et al., 2013). Nevertheless, there is a lack of studies that evaluate the effect of heatwaves on latent and sensible heat fluxes on lakes.

Shallow and small lakes are the most abundant lake types globally and dominate the global area covered by continental waters (Downing et al., 2006). The Pampean Region in Argentina contains numerous shallow lakes, of which, those located in the Buenos Aires Province account for more than 13,500 shallow lakes with an area between 0.01 and $1000 \mathrm{~km}^{2}$ (Geraldi et al., 2011). Under the hypothesis that climate variability and particularly extreme events, as heatwaves, drive essential changes in the latent and sensible heat flux from shallow lakes, this study aims to describe, for the first time, the long-term surface sensible and latent heat flux and their drivers in two shallow lakes from the Pampean Region. Finally, the effect and contribution of heatwaves on the surface heat fluxes are analyzed.

\section{Materials and methods}

\subsection{Study sites}

This study comprises surface water temperature and meteorological observations from two shallow lakes situated in the Pampas Region in Argentina: La Salada lake (LS - 39 ${ }^{\circ} 27^{\prime}$ S, $62^{\circ} 42^{\prime}$ W) and Sauce Grande lake (SG - 38 $57^{\prime} \mathrm{S}, 61^{\circ} 24^{\prime} \mathrm{W}$ ) (Fig. 1). Their surface area ranges from 4 (LS) to 15.8 $\mathrm{km}^{2}$ (SG) and their maximum depths from 2.3 (SG) to $3.2 \mathrm{~m}$ (LS). The lakes are located in a temperature gradient from North (Sauce Grande) to the South (La Salada) (Alfonso et al., 2018). The climate in the region of SG is temperate; meanwhile, LS is cold temperate and mostly dry (Aliaga et al., 2017). Annual air temperature values are between 14 and $20^{\circ} \mathrm{C}$, with pronounced summers and winters. Wet and dry cycles characterize the Pampean region. The highest rainfalls occur typically in spring and summer, with an annual average of $369 \mathrm{~mm}$ for the LS area and $692 \mathrm{~mm}$ for the SG area (Aliaga et al., 2017). Prevailing winds in the region are from the NW, with a mean speed between 3.9 and $4.1 \mathrm{~m} \mathrm{~s}^{-1}$ (Aliaga et al., 2017).

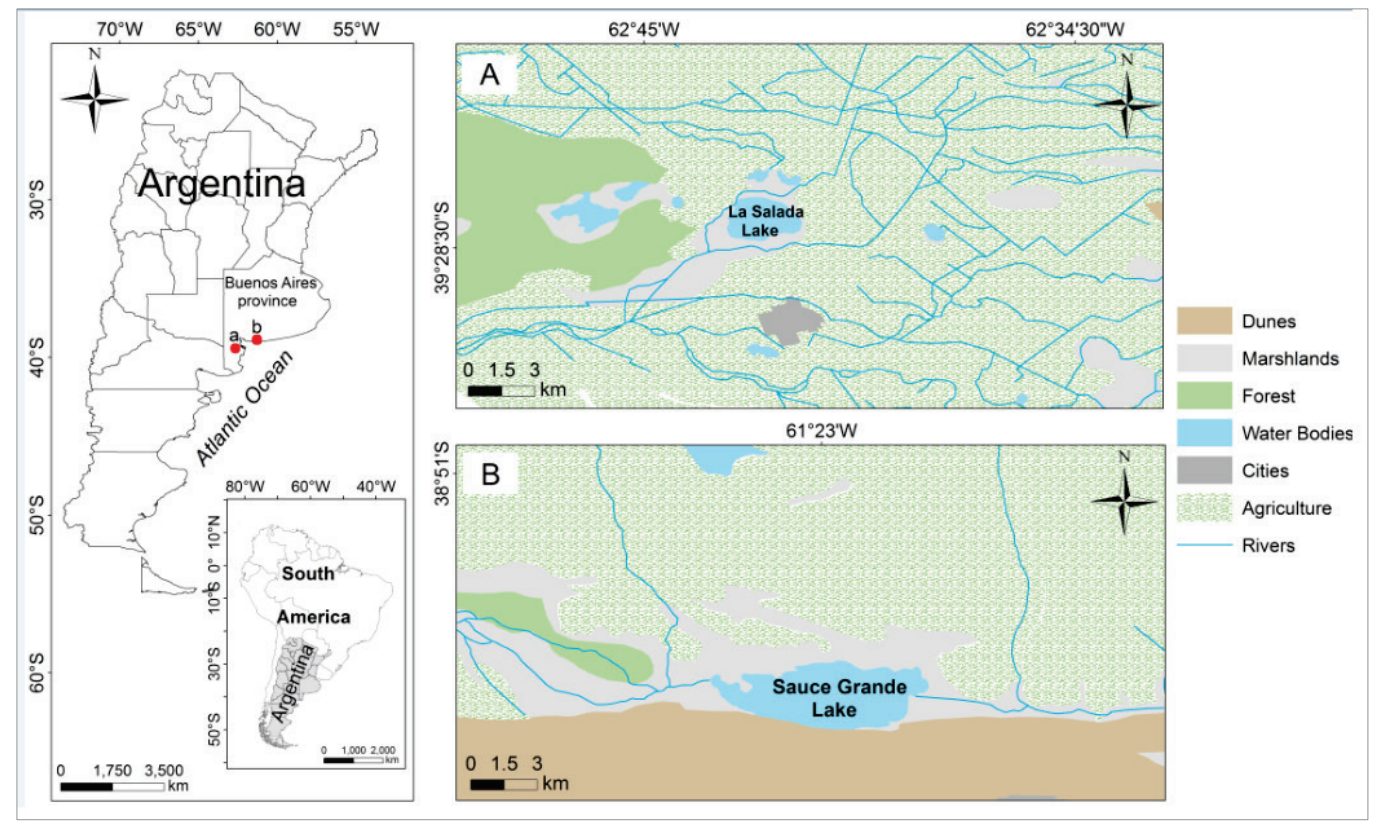

Figure 1. The geographic location of the study sites concerning their surroundings: A: La Salada lake location and land use; B: Sauce Grande lake location and land use. Modified from Alfonso et al. (2018). 


\subsection{High-frequency measurements and meteorological conditions}

We obtained high-frequency meteorological and surface water temperature data (every $10 \mathrm{~min}$ ) for four years with instrumented buoys (EMAC - Estación de Monitoreo Ambiental Costero) located in both lakes. Meteorological variables were measured at $2.10 \mathrm{~m}$ in height, including air temperature (Ta, $\left.{ }^{\circ} \mathrm{C}\right)$, relative humidity $(\mathrm{RH}, \%)$, wind speed $\left(\mathrm{U}, \mathrm{km} \mathrm{h}^{-1}\right)$, solar radiation $\left(\mathrm{Rsol}, \mathrm{W} \mathrm{m} \mathrm{m}^{-2}\right.$ ). Water temperature $\left(\mathrm{Tw},{ }^{\circ} \mathrm{C}\right.$ ) was measured at $0.5 \mathrm{~m}$ depth (Data are available at https://www.emac.iadoconicet.gob.ar/2019). For SG, we obtained Rsol data from the Coronel Dorrego meteorological station belonging to the National Institute of Agricultural Technology (INTA, for its name in Spanish). We also analyzed temporal atmospheric processes such as the passage of low and high-pressure centers during heatwave events at a regional scale by the analysis of daily synoptic maps from the National Meteorological Service (SMN, Argentina) available at https://www.smn.gob.ar/.

\subsection{Heat flux estimation}

We estimated the surface energy fluxes from lake buoy data with the Lake Heat Flux Analyzer script for MATLAB developed by Woolway et al. (2015b). The script is free to download at https:/github.com/ GLEON/HeatFluxAnalyzer. The calculated surface heat fluxes expressed in terms of the total surface heat flux (Qtot, $\mathrm{W} \mathrm{m}^{-2}$ ) are the flux of incoming short-wave radiation on the lake surface (Qsin), the reflected short-wave radiation (Qsr), the sensible (H) and the latent (LE) heat fluxes, the incoming long-wave (Qlin) and the outgoing long-wave radiation (Qlout):

$$
Q t o t=Q \sin +\text { Qlin }- \text { Qlout }-Q s r-L E-H
$$

The convention of signs applied to equation (1) was positive when the direction of heat transfer is from the lake to the atmosphere and negative when the opposite (Woolway et al., 2015b). The heat exchanges were estimated every $10 \mathrm{~min}$ and daily averaged. For more details about the equations used, see the methods of Woolway et al. (2015b). All the used abbreviations are presented in Table 1 to facilitate the lecture. One question that arose from the investigation was to determine which of the variables used in this study is the most important to determine the different heat fluxes. Therefore a Spearman (r) correlation analysis and a simple regression between LE, H, and the main meteorological variables were performed to identify those environmental factors that may have played the most significant role in heat fluxes with Infostat software (Di Renzo et al., 2018).

Table 1. Abbreviations used in the manuscript, their significance, and their units.

\begin{tabular}{|c|c|c|}
\hline Abbreviations & Significance & Unit \\
\hline HW & heatwave & \\
\hline LS & La Salada lake & \\
\hline SG & Sauce Grande lake & $\mathrm{m}$ \\
\hline Level & water level & ${ }^{\circ} \mathrm{C}$ \\
\hline Ta & air temperature & ${ }^{\circ} \mathrm{C}$ \\
\hline Tw & water temperature & $\mathrm{m} \mathrm{s}^{-1}$ \\
\hline $\mathrm{U}$ & wind speed & $\mathrm{m} \mathrm{s}^{-1}$ \\
\hline $\mathrm{U}_{10}$ & wind speed at 10 meters & ${ }^{\circ}$ \\
\hline $\mathrm{RH}$ & relative humidity & $\%$ \\
\hline $\mathrm{RH}{ }_{10}$ & relative humidity at 10 meters & $\mathrm{mm}^{-2}$ \\
\hline Rain & total rainfall & $\mathrm{W} \mathrm{m}^{-2}$ \\
\hline $\mathrm{H}$ & sensitive heat flux & $\mathrm{W} \mathrm{m}^{-2}$ \\
\hline LE & latent heat flux & \\
\hline
\end{tabular}




\begin{tabular}{|c|c|c|}
\hline Qtot & total surface heat flux & $\mathrm{W} \mathrm{m}^{-2}$ \\
\hline Qsin & incident short-wave radiation & $\mathrm{W} \mathrm{m}^{-2}$ \\
\hline Qsr & reflected short-wave radiation & $\mathrm{W} \mathrm{m}^{-2}$ \\
\hline Qlin & incoming long-wave radiation & $\mathrm{W} \mathrm{m}^{-2}$ \\
\hline Qlout & outgoing long-wave radiation & \\
\hline $\mathrm{CCSM}_{4}$ & community climate system model 4 & \\
\hline GHG & greenhouse gases & \\
\hline RCP 4.5 & representative concentration pathways at $4.5 \mathrm{~W} \mathrm{~m}^{-2}$ & \\
\hline RCP 8.5 & representative concentration pathways at $8.5 \mathrm{~W} \mathrm{~m}^{-2}$ & \\
\hline
\end{tabular}

\subsection{Future air temperature trends under future climatic scenarios}

To determine the possible future expected changes in Ta, we calculated the annual trend from a 20 years monthly mean time series (1998-2018) and for the near (2019-2039) and far future (20792099). To calculate trends, we applied the non-parametric Mann-Kendall test (Mann, 1945; Kendall, 1975) and to quantify the rates of change the Sen's slope estimator (Sen, 1968), using the "trend" package for R (R package version 1.1.1., Pohlert, 2018). We obtained the 1998-2018 time series from two weather stations, one located in the Bahía Blanca station $\left(38.44^{\circ} \mathrm{S}, 62.1^{\circ} \mathrm{W}\right)$, to describe the SG (National Weather Service (SMN, https://www.smn.gob.ar) and one in Hilario Ascasubi $\left(39.38^{\circ} \mathrm{S}\right.$, $62.62^{\circ} \mathrm{W}$ ), adjacent to La Salada (National Institute of Agricultural Technology, INTA, https://www.argentina.gob.ar/inta). We obtained the short- and long-term future series with a climate model database from the Centro de Investigaciones del Mar y la Atmosfera (CIMA) (http://3cn.cima.fcen.uba.ar). We used the annual mean temperature of the Community Climate System Model 4 (CCSM4) of the National Centre for Atmospheric Research (NCAR, USA) as this resulted in the best from 24 climate models from CMIP5 (Coupled Model Intercomparison Project 5) and CLARISLPB (A Europe-South America Network for Climate Change Issues and Impacts Assessment - La Plata Basin Project), assessed for the present time in the region after a quantile-mapping bias correction using observations from the Climate Research Unit (CRU). We used two Representative Concentration Pathways (RCP) for the emission of greenhouse gases (GHG): RCP 4.5 that represents a mitigation scenario, which stabilizes radiative forcing at $4.5 \mathrm{~W} \mathrm{~m}^{-2}$ and RCP 8.5 that represents a rising scenario with a radiative forcing of $8.5 \mathrm{~W} \mathrm{~m}^{-2}$ at 2100 .

\section{Results and discussion}

\subsection{Annual and inter-annual variations in sensible and latent heat}

Annual mean values and standard deviation of the main meteorological and limnological variables measured during the study period are presented in Table 2 and daily mean values are presented in Fig. 2 and Fig. 3. Data gaps were due to instrument failures and cleaning-calibration procedures. Both lakes have a seasonal fluctuation of the Ta, Tw and $\mathrm{RH}_{10}$ (Figs. 2 and 3). Mean wind speed at 10 meters $\left(\mathrm{U}_{10}\right)$ was higher in LS (Table 2, Fig. 2c), reaching values up to $35 \mathrm{~m} \mathrm{~s}^{-1}$ whereas in SG it presented values up to $20 \mathrm{~m} \mathrm{~s}^{-1}$ (Fig. 3c). Annual cycles of LE and $\mathrm{H}$ in both lakes are represented by the daily means derived from the 10 minutes data (Figs. 2a and 3a). In each site, LE followed a clear seasonal cycle; $\mathrm{H}$ did not present a clear temporal trend (Figs. 2a and 3a) as it was observed in previous studies in temperate lakes (Li et al., 2015; Woolway et al., 2015a, 2018; Zhang and Liu, 2013; Zhao and Liu, 2018). The warmer months (October-March) presented maximum LE values; meanwhile, the colder months (April- September) the minimum ones (Figs. 2a and 3a). A study developed in several temperate lakes also found that $\mathrm{H}$ followed a less pronounced seasonal trend, with higher $\mathrm{H}$ values during autumn as a result of the greater air-water temperature difference (Woolway et al., 2018). We did not observe it in LS and SG lakes because of the lesser water heat storage of temperate shallow lakes in comparison with deep temperate lakes. 

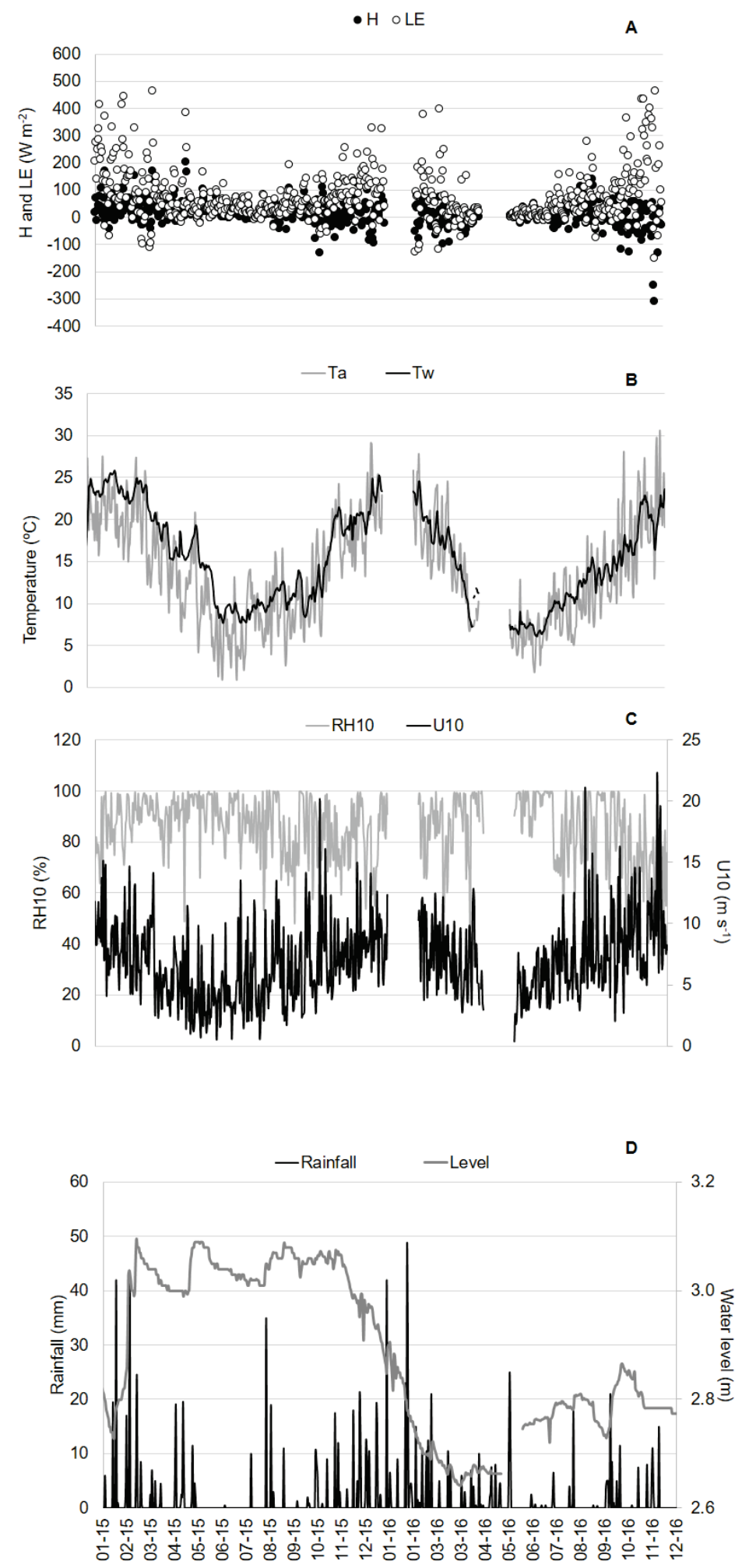

Figure 2. Mean daily values of A: latent (H) and sensible heat (E), B: Air (Ta) and water temperature (Tw), $C$ : Wind speed $\left(U_{10}\right)$ and relative humidity $\left(R H_{10}\right)$ at 10 meters and D: rainfall and water level in La Salada lake during the study period. 

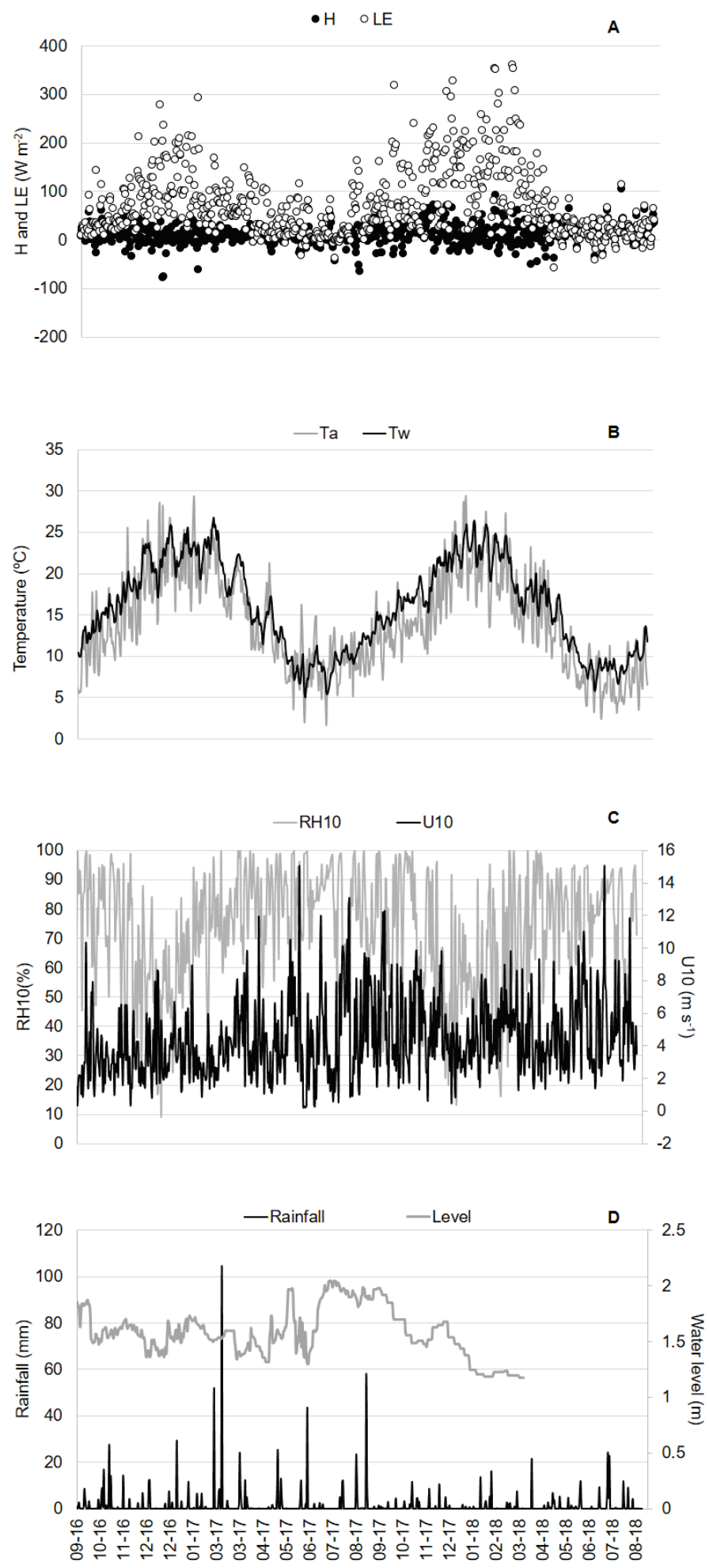

Figure 3. Mean daily values of A: latent (H) and sensible heat (E), B: Air (Ta) and water temperature (Tw), $C$ : Wind speed $\left(U_{10}\right)$ and relative humidity $\left(R H_{10}\right)$ at 10 meters and $D$ : rainfall and water level in Sauce Grande lake during the study period. 
Table 2. Mean annual values and standard deviation of lake area (Area), water level (Level), air temperature $(T a)$, water temperature $(T w)$, wind speed $\left(U_{10}\right)$, relative humidity $\left(H R_{10}\right)$, sensitive flux $(H)$ and latent flux $(L E)$, and total rainfall (Rain) in La Salada and Sauce Grande during the study period.

\begin{tabular}{|l|c|c|c|c|}
\hline Variables & $\begin{array}{c}\text { LS } \\
\mathbf{2 0 1 5}\end{array}$ & $\begin{array}{c}\text { LS } \\
\mathbf{2 0 1 6}\end{array}$ & $\begin{array}{c}\text { SG } \\
\mathbf{2 0 1 6 - 2 0 1 7}\end{array}$ & $\begin{array}{c}\text { SG } \\
\mathbf{2 0 1 7 - 2 0 1 8}\end{array}$ \\
\hline Level (m) & $3.0 \pm 0.08$ & $2.8 \pm 0.06$ & $1.6 \pm 0.2$ & $1.5 \pm 0.2$ \\
\hline Ta $\left({ }^{\circ} \mathbf{C}\right)$ & $13.9 \pm 6.2$ & $13.9 \pm 6.4$ & $14.6 \pm 5.7$ & $13.8 \pm 5.9$ \\
\hline Tw $\left({ }^{\circ} \mathbf{C}\right)$ & $16.3 \pm 5.5$ & $14.5 \pm 5.3$ & $15.9 \pm 5.6$ & $15.9 \pm 5.2$ \\
\hline $\mathbf{U}_{\mathbf{1 0}}\left(\mathbf{m ~ s}^{-\mathbf{1}}\right)$ & $6.6 \pm 3.3$ & $7.5 \pm 3.3$ & $3.9 \pm 2.4$ & $5.0 \pm 2.3$ \\
\hline RH $_{10}(\mathbf{\%})$ & $86.8 \pm 11.3$ & $83.9 \pm 15.6$ & $74.8 \pm 18.4$ & $70.9 \pm 20.1$ \\
\hline Rain $(\mathbf{m m})$ & 615 & 495 & 671 & 382 \\
\hline H $\left(\mathbf{W} \mathbf{~ m}^{-2}\right)$ & $20.5 \pm 39.95$ & $1.9 \pm 44$ & $6.83 \pm 17.2$ & $14.2 \pm 21.7$ \\
\hline $\mathbf{L E ~}\left(\mathbf{W} \mathbf{~ m}^{-2}\right)$ & $76.8 \pm 86.9$ & $66.7 \pm 99.8$ & $59.9 \pm 53.8$ & $81.5 \pm 80.3$ \\
\hline
\end{tabular}

Inter-annual variations in LE and $\mathrm{H}$ values in both lakes were detected (Table 2). For LS, a decrease of $13.2 \%$ for LE and $90.7 \%$ in $\mathrm{H}$ from 2015 to 2016 was found. Meanwhile, for SG, an increase of $107.9 \%$ for $\mathrm{LE}$ and $81.5 \%$ in $\mathrm{H}$ from the first to the second period was observed. Furthermore, $\mathrm{LE}$ and $\mathrm{H}$ daily values were highly fluctuating (Figs. $2 \mathrm{a}$ and 3a). For LS, mean daily $\mathrm{H}$ ranged between -309.4 and $200.5 \mathrm{~W} \mathrm{~m}^{-2}$ day $^{-1}$ and mean daily LE between -152 and $463.9 \mathrm{~W} \mathrm{~m}^{-2}$ day ${ }^{-1}$ (Fig. 2a). Meanwhile, for SG, mean daily $\mathrm{H}$ ranged between -78.6 and $104.8 \mathrm{~W} \mathrm{~m}^{-2}$ day $^{-1}$ and mean daily LE between -59.2 and 360.1 $\mathrm{W} \mathrm{m}^{-2}$ day $^{-1}$ (Fig. 3a). The LE and $\mathrm{H}$ amplitudes for both lakes were higher than those cited in other lakes (Li et al., 2015; Woolway et al., 2015a, 2018; Zhang and Liu, 2013; Zhao and Liu, 2018). Notably, there were several times when daily LE and $\mathrm{H}$ presented high negative values in both lakes, mainly in warmer months (Fig. 3). After a rigorous analysis of these events, most of them coincides with mean daily Ta values greater than $27^{\circ} \mathrm{C}$ for consecutive days, which are associated with heatwaves events. To analyze in detail this process, we focus the following sections on the warmer months for both lakes.

\subsection{Effect of heatwave events on heat flux diurnal variations}

We based our analysis on the definition of a heatwave (HW) as an event when maximum daily Ta was greater than $32{ }^{\circ} \mathrm{C}$ for three or more days during the warm season (October-March) (Rusticucci et al., 2016). These values correspond to the mean daily $90^{\text {th }}$ percentile calculated over the warm season of the year based on a 29 years' time series for Argentina (Rusticucci et al., 2016). LS presented two HW events during 2015 (5-7 March and 24-27 December) and four during 2016 (21-23 February, 2830 March, 22-24 November, and 15-31 December). Meanwhile, SG presented two HW events during the first period of study 2016-2017 (27-30 December 2016 and 27-29 January 2017) and four during the second period 2017-2018 (8-12 January, 20-22 January, 3-8 February, 16-18 February of 2018). There were also several times when maximum Ta was above $32^{\circ} \mathrm{C}$, but not for three consecutive days, so we do not include these events in the analysis.

The diurnal variations in $\mathrm{LE}, \mathrm{H}, \mathrm{Tw}, \mathrm{Ta}, \mathrm{RH}_{10}, \mathrm{U}_{10}$, and wind direction before, during, and after a HW event for each lake are presented in Figures 4 and 5. These two HW events were randomly selected as examples from all the HW events registered. In LS, when a high-pressure center locates over the lake area, a four days HW event on $24^{\text {th }}$ December 2015 (Fig. 4) was registered. A dry and warm air mass dominated the region, causing significant changes in the meteorological conditions and the magnitudes of LE and $\mathrm{H}$ fluxes (Fig. 6a). The previous days to the HW, Ta, and Tw were already high, accounting an increase above $18{ }^{\circ} \mathrm{C}$ in maximum Ta and above $6{ }^{\circ} \mathrm{C}$ in maximum Tw (Fig. 4b). Also, we observed an increase in $\mathrm{RH}_{10}$ and a decrease in water level (Fig. 4c, d) generated not only by the warm temperatures but also for the strong winds that mixed the lake waters, increasing evaporation. $U_{10}$ was always close to $18 \mathrm{~m} \mathrm{~s}^{-1}$ from NW direction (Fig. $4 \mathrm{c}, \mathrm{e}$ ), but being the $\mathrm{RH}_{10}$ close to the saturation point, 
originated low values of LE. The high gradient between Tw-Ta originated negatives $\mathrm{H}$ during the HW event (Fig. 4a). Previous studies also associated changes in LE with changes in $\mathrm{RH}_{10}$ and $\mathrm{U}_{10}$, and changes in $\mathrm{H}$ with changes in Tw-Ta and $\mathrm{U}_{10}$ (Li et al., 2015; Woolway et al., 2015a, 2018; Zhang and Liu, 2013; Zhao and Liu, 2018).

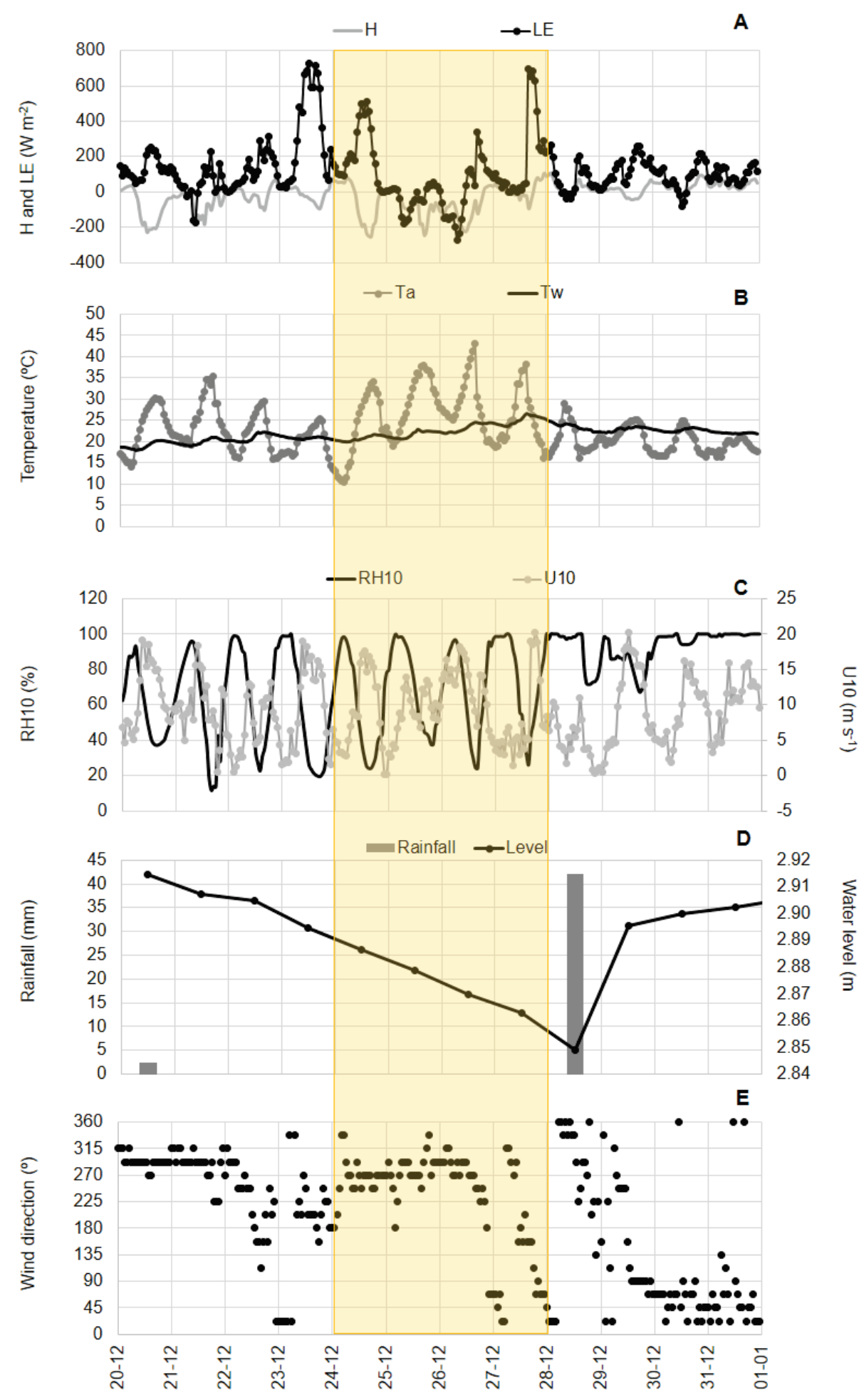

Figure 4. Variation of the measured parameters before, during, and after the passage of a heatwave in A: hourly surface latent and sensible heat, $B$ : air and water temperature, $C$ : Windspeed and relative humidity D: and E: wind direction in La Salada lake during December 2015. 


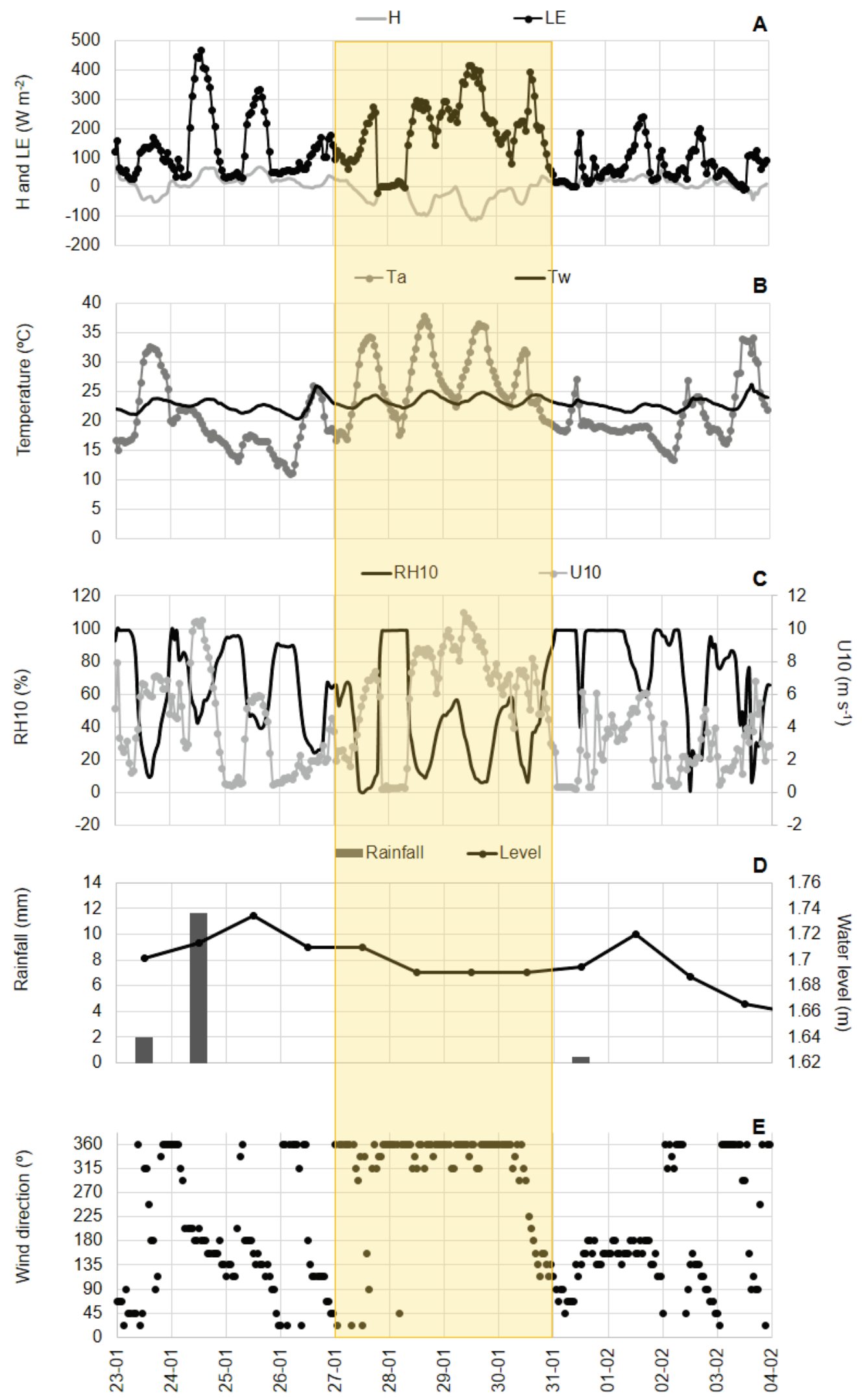

Figure 5. Variation of the measured parameters before, during and after the passage of a heatwave in A: the surface latent and sensible heat, B: air and water temperature, $C$ : Wind speed and relative humidity D: rainfall and water level and E: wind direction in Sauce Grande lake during January 2017. 
After the HW, the intrusion of a low-pressure system with a relatively cold air mass from the South (Fig. 6b) decreased the temperatures. The precipitation was $42 \mathrm{~mm}$, originating an increase of $\mathrm{RH}_{10}$ to saturation values and a rise in the water level (Fig. 4d). $\mathrm{U}_{10}$ was strong with velocities of $15 \mathrm{~m}$ $\mathrm{s}^{-1}$. This situation triggered an increase in LE and $\mathrm{H}$ returning to positive values, with a significant pulse in LE as soon as the wind direction changed from NW to SSE on $27^{\text {th }}$ December (16-17 h), reaching values over $600 \mathrm{~W} \mathrm{~m}^{-2}$. Previous studies also registered increases in $\mathrm{LE}$ and $\mathrm{H}$ after the intrusion of cold, dry air masses with strong wind speed values (Zhang and Liu 2013, Li et al., 2015).
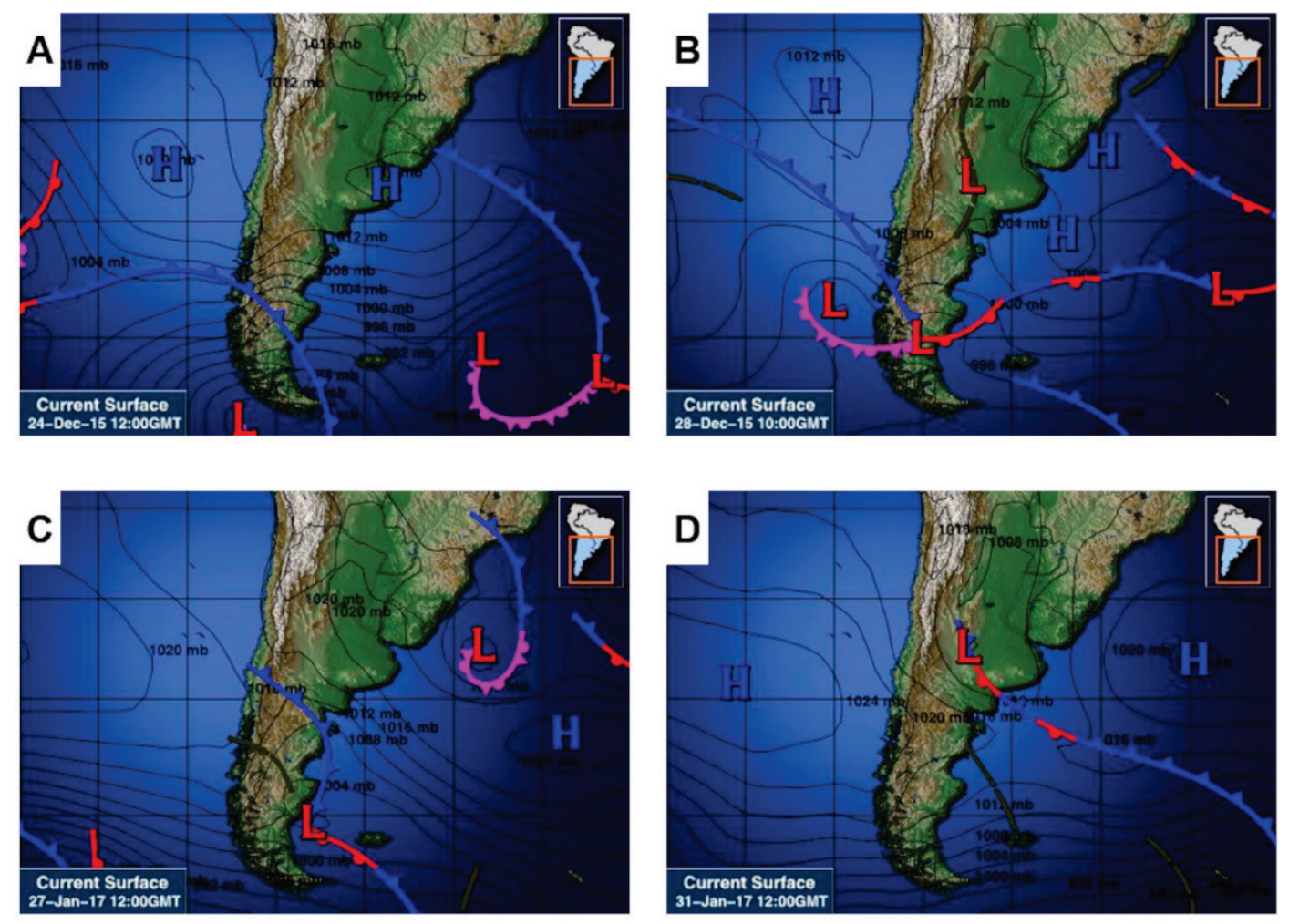

Figure 6. Synoptic maps for the study area during December 2015 (A and B) and January 2017 (C and D) (Source: https://www.wunderground.com/intellicast).

In the case of SG, from $27^{\text {th }}$ January 2017, a three days HW event was registered (Fig. 5), when a dry, hot air mass flowed over the lake area, causing significant changes in the LE and $\mathrm{H}$ fluxes and meteorological variables (Fig. 6c). In the previous days, both $\mathrm{Ta}$ and $\mathrm{Tw}$ presented values below the average for the season, and along the $\mathrm{HW}$, the Ta and $\mathrm{Tw}$ increased $11.7^{\circ} \mathrm{C}\left(37.2^{\circ} \mathrm{C}\right.$ maximum value $)$ and $3{ }^{\circ} \mathrm{C}\left(25.1{ }^{\circ} \mathrm{C}\right.$ maximum value) respectively. A significant decrease in $\mathrm{RH}_{10}$ and water level (Fig. $5 \mathrm{c}, \mathrm{d}$ ) and an increase in $\mathrm{U}_{10}$ reaching values up to $10 \mathrm{~m} \mathrm{~s}^{-1}$ from $\mathrm{N}$ direction (Fig. $5 \mathrm{c}$, e) were observed. These changes allowed a decrease in $\mathrm{H}$ values reaching values near $-113 \mathrm{~W} \mathrm{~m}^{-2}$, meanwhile LE increased reaching maximum values up to $410 \mathrm{~W} \mathrm{~m}^{-2}$ (Fig. 5a). After the event, followed an intrusion of a lowpressure center (on $30^{\text {th }}$ January) flowing from the sea on the SG area, changing LE and H rates.

According to these results, in $\mathrm{LS}$, the changes in $\mathrm{RH}_{10}$, Ta, and $\mathrm{Tw}$ ruled the $\mathrm{LE}$ and $\mathrm{H}$ variations; meanwhile, in $\mathrm{SG}$, the low $\mathrm{RH}_{10}$ and the strong $\mathrm{U}_{10}$ played a significant role in determining the fluxes. The difference among sites could be explained as LS is located in the cold temperate region, next to the Patagonian Region, where wind speeds are among the highest on a global scale (Guozden et al., 2018), therefore $\mathrm{U}_{10}$ is regularly strong (Table 2). Meanwhile, $\mathrm{SG}$ is in a temperate region with an annual mean $\mathrm{U}_{10}$ that do not exceed $5 \mathrm{~m} \mathrm{~s}^{-1}$ during the study period (Table 2). Previous studies cited that LE is ruled mainly by the changes in $\mathrm{U}_{10}$ and $\mathrm{RH}_{10}$, meanwhile, $\mathrm{H}$ is ruled by changes in $\mathrm{U}_{10}$ and $\mathrm{Tw}-\mathrm{Ta}$ (Zhang and Liu, 2013; Li et al., 2015; Woolway et al., 2015a), so, the results found in LS serve as an example of 
how $\mathrm{LE}$ and $\mathrm{H}$ behave in sites where $\mathrm{U}_{10}$ are always high, but other parameters define the sign of the fluxes. The fact that LS is twice deeper than SG explains the differences among lakes in the lag time between Ta and Tw (Table 2, Figs. $4 \mathrm{~b}$ and $5 b$ ).

\subsection{Effect and contribution of heatwaves in the diurnal and annual latent and sensible heat}

Mean diurnal cycles for $\mathrm{H}$ and LE during HW, and no heatwave events are presented in Figure 7 for the months with HW. A clear differentiation on the fluxes magnitudes along the day was observed (Fig. 7). In LS, LE was significantly correlated and responded to changes in $U_{10}$ and the $H_{10}(r=0.39$ $\mathrm{p}<0.01$ and $\mathrm{r}=-0.34 \mathrm{p}<0.01$ respectively) in accordance with studies in temperate lakes (Woolway et al., 2018). As in other studies, the diurnal cycle in $H$ was opposite to $U_{10}$, to which is related and responded to the difference in temperature between air and water. According to Woolway et al. (2018), this suggests that the Tw-Ta difference is the main driver of the diurnal variability of $\mathrm{H}$ and that the magnitude of the Tw-Ta outweighs the opposite influence of $U_{10}$ at diurnal timescales. This result is supported by the significant correlation with $\mathrm{Ta}$ and $\mathrm{Tw}(\mathrm{Ta}=-0.38 \mathrm{p}<0.01)$. In general, LE and $\mathrm{H}$ values decreased to negative values with the passage of HW events, presenting a higher variation amplitude (Fig. 7 a-f). Decreases of more than $180 \mathrm{~W} \mathrm{~m}^{-2}$ in LE (Feb-2016) and $69 \mathrm{~W} \mathrm{~m}^{-2}$ in $\mathrm{H}$ (Dec2015) were observed with the passage of HW in LS (Fig. 7 a-f).

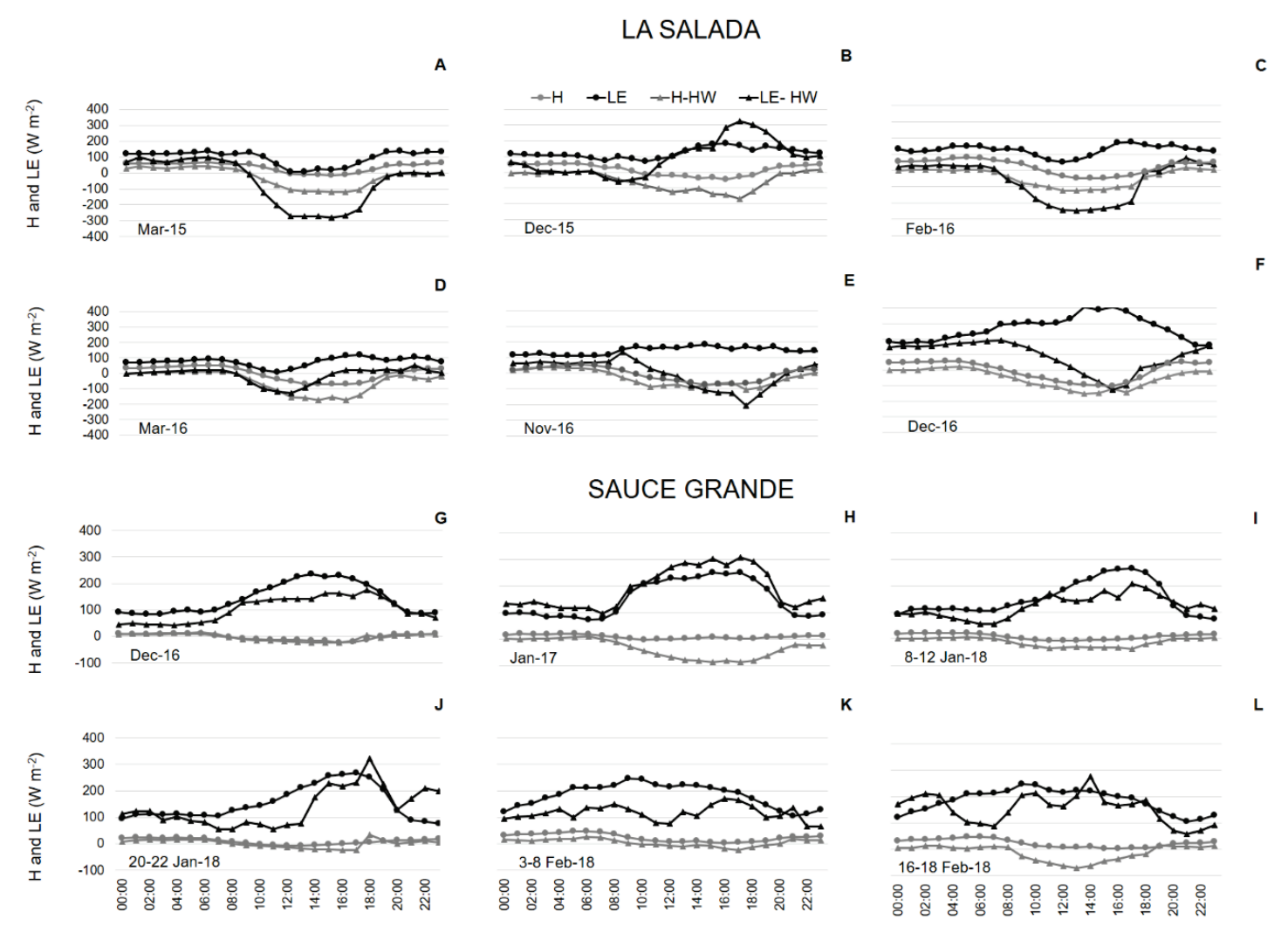

Figure 7. Monthly mean diurnal cycles of sensible heat $(H)$ and latent heat (LE) during selected heatwave events (H-HW and LE-HW) and during regular days in La Salada Lake (A-F) and Sauce Grande Lake (G-L) during the study period.

In SG lake, the increase in LE followed the increase in $\mathrm{U}_{10}(\mathrm{r}=0.39, \mathrm{p}<0.01)$; meantime $\mathrm{H}$ followed the pattern determined by the difference in temperature values $(\mathrm{r}=0.38, \mathrm{p}<0.01)$. This pattern was consistent during the warmer season, with LE values between $36.03 \mathrm{~W} \mathrm{~m}^{-2}$ (Oct-2016) and 287.31 $\mathrm{W} \mathrm{m}^{-2}$ (Dec-2017) and, $\mathrm{H}$ values between $-20.25 \mathrm{~W} \mathrm{~m}^{-2}$ (Nov-2016) and $46.69 \mathrm{~W} \mathrm{~m}^{-2}$ (Nov-2017) (Fig. $7 \mathrm{~g}-1)$. LE and $\mathrm{H}$ values also decreased with the passage of HW in SG (Fig. $7 \mathrm{~g}-1)$. Decreases of $67 \mathrm{~W} \mathrm{~m}{ }^{2}$ 
in LE (Feb-2018) and $44.8 \mathrm{~W} \mathrm{~m}^{-2}$ in $\mathrm{H}$ (Jan-2017) were observed with the passage of HW in SG (Fig. $7 \mathrm{~g}-1)$.

In order to assess the contribution of HW events among years, the daily mean values of LE and $\mathrm{H}$ during these events and regular days appear in Figure 8 for both lakes. In LS, there was a significant contribution of HW, decreasing LE and H mean daily values (Fig. 8a). In 2015, LE and H decreased $78 \%$ and $254 \%$, respectively, compared to regular days, meanwhile, in 2016 a higher decrease of $96 \%$ and $671 \%$ was calculated because twice HW events were observed respect to 2015 (Fig. 8a). In SG the difference among years was not the same (Fig. 8b), with an increase in LE of the $25 \%$ and a decrease of 987\% in $\mathrm{H}$ for the period 2016-2017, meanwhile for 2017-2018, LE showed a decrease of $13 \%$ and $\mathrm{H} \mathrm{a}$ decrease of the $11 \%$. As in LS, in the first period, the number of HW events was twice as in the second one. However, the more significant changes occurred in 2016-2017 and could be associated with the inter-annual differences in rainfall (Table 2) which influences $\mathrm{RH}_{10}$ and Ta values; meanwhile, the second period presented the higher $\mathrm{U}_{10}$ values, which also explained the increase in LE and $\mathrm{H}$ observed for the regular days (Fig. 8b).

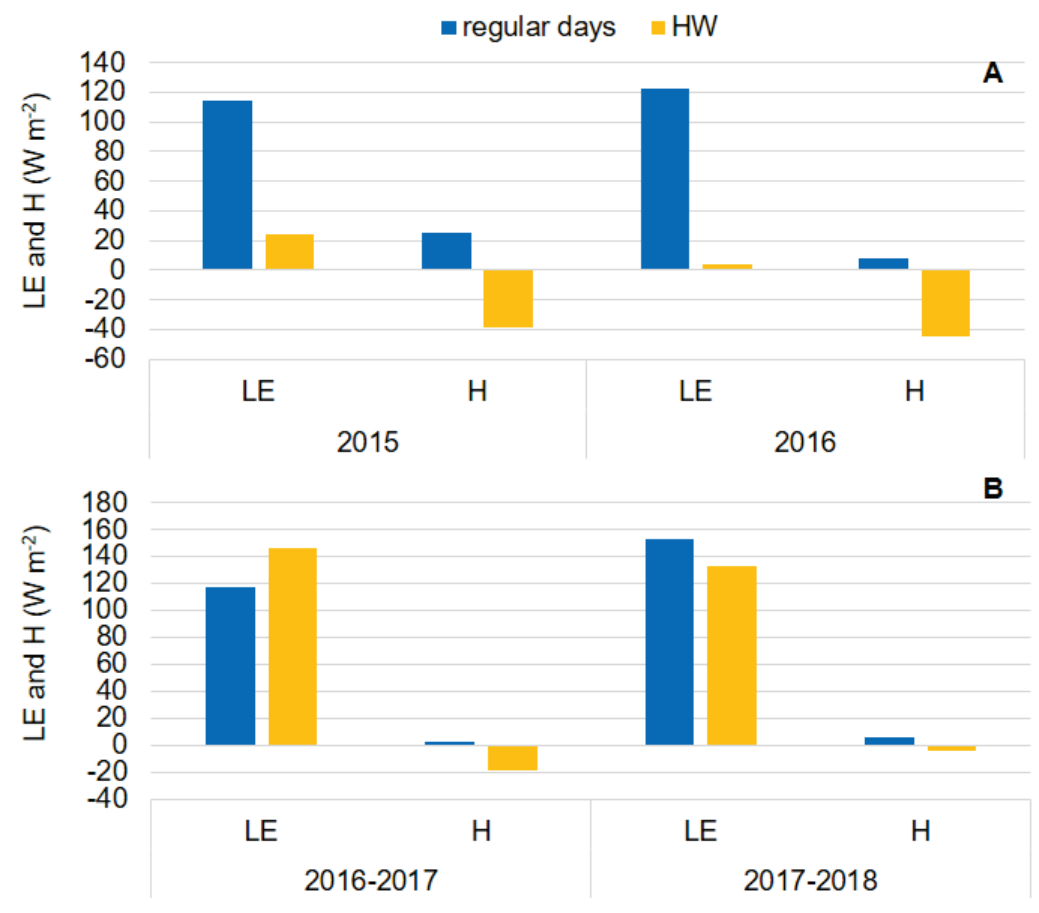

Figure 8. Mean daily $L E$ and $H$ values during regular days and heatwave events (HW) for both study periods in A: La Salada and B: Sauce Grande lakes during the study period.

\subsection{Driving forces of latent and sensible heat flux}

The results from the regression analysis are presented in Table 3. In LS, monthly LE was explained mainly by $\mathrm{U}_{10}$ changes (Table 3 ); meanwhile, monthly $\mathrm{H}$ was better explained by the product of $\mathrm{U}_{10} \times(\mathrm{Tw}-\mathrm{Ta})$ (Table 3$)$ with similar values for warmer months and the whole period. These results are following the strong $\mathrm{U}_{10}$ registered in the lake (Table 2, Fig. 2 and Fig. 4) and cited for the area (Alfonso et al., 2015, 2018; Aliaga et al., 2017).

In the case of $\mathrm{SG}$, monthly variance in $\mathrm{LE}$ was better explained by $\mathrm{RH}_{10}$, with $80 \%$ for the whole period, and $\mathrm{H}$ by the Tw-Ta difference, with $79 \%$ for warmer months. The difference between the whole period and the warmer months was higher in the case of the variance in LE and $\mathrm{H}$ explained by $\mathrm{U}_{10}$ (Table 3). The observed increase in $\mathrm{U}_{10}$ during the HW event is in accordance with these results (Fig. 
5c). A study in the Monte Hermoso beach registered the presence of sea breeze events for the beach and the area of influence, which includes the Sauce Grande lake (Huamantinco Cisneros and Piccolo, 2011). They described it as changes in wind speed and direction, with an influence on relative humidity and air temperature values. The predominance of sea breeze events was in summer, with higher mean wind speed values in December, January, and March, coinciding with the increment in $U_{10}$ found during the HW events and the relation between $U_{10}$ and $L E$ and $H$ values for the warmer months found in this study. The proximity of the sea to the SG and the presence of the sea breeze undoubtedly makes it difficult to understand the total of the processes that involve the turbulent flow of heat. Nevertheless, we need a more detailed analysis among the meteorological variables in SG and Monte Hermoso in the future for better conclusions about the relation of these events with the surface heat flux values. Other studies also attributed observed spikes in LE to substantial wind flow, which mixed the water and enhanced turbulent exchange (Blanken et al., 2003; Rouse et al., 2003; Shao et al., 2015).

Table 3. Linear regression coefficients between monthly latent heat flux $(L E)$ relative humidity $\left(R H_{10}\right)$, wind speed $\left(U_{10}\right)$ and, product of wind speed and relative humidity $\left(R H_{10} \times U_{10}\right)$; and between sensible heat flux $(H)$ and the difference in temperature between water and air $(T w-T a)$, wind speed $\left(U_{10}\right)$ and, the product of wind speed and the difference in temperature between water and air $\left(U_{10} \times(T w-T a)\right)$ in La Salada and Sauce Grande lakes For the entire study period and warmer months (October to March).

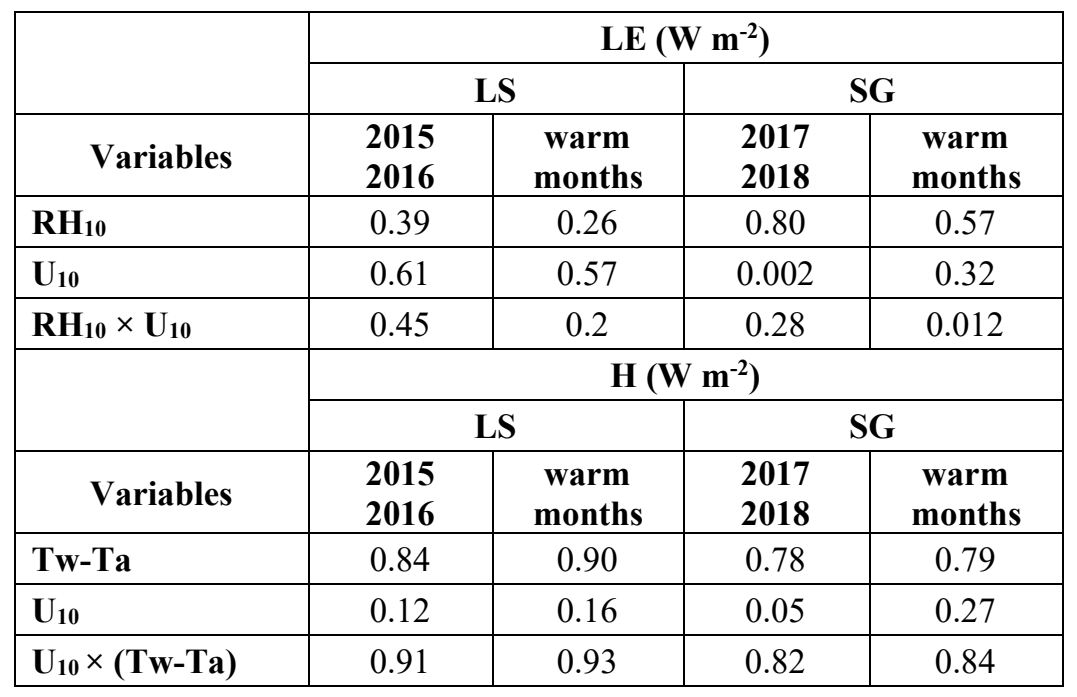

If we compared these results with other studies, similar relations are cited for $\mathrm{H}$ and the product between $\mathrm{U}_{10}$ and the Tw-Ta difference (Li et al., 2015). For LE, other investigations presented a better relationship with the product of $U_{10}$ and the difference between specific humidity in the water-air interface (Li et al., 2015) than for $\mathrm{U}_{10}$ by itself as in LS and SG lakes. The difference could be explained as other studies were developed in lakes where mean daily $U_{10}$ did not exceed the $10 \mathrm{~m} \mathrm{~s}^{-1}$ (Zhang and Liu, 2013; Li et al., 2015) whereas in this study, we found values greater than 20 and $14 \mathrm{~m} \mathrm{~s}^{-1}$ in LS and SG lakes respectively (Figs. 2 and 3), which also could explain the higher daily LE and $\mathrm{H}$ values cited here in comparison with other reports (Zhang and Liu, 2013; Li et al., 2015). Woolway et al. (2018) in a study developed in 45 lakes across different latitudinal and longitudinal gradients found that the $\mathrm{U}_{10}$ variation, which was the highest in winter, did not covary strongly with LE, H, or LE + H at seasonal timescales, meanwhile at diel timescales they found that $L E$ was related to $U_{10}$ diel changes. However, $\mathrm{H}$ was not, being the latter related to changes in the magnitude of the Tw-Ta.

Although the number of HW events was higher in the second period of study for both lakes, the mean Ta and Tw annual values remain similar between periods (Table1). However, we could register significant changes over LE and $\mathrm{H}$ fluxes on $\mathrm{HW}$ events, with the increment of Ta and Tw and significant changes in $\mathrm{U}_{10}$ and $\mathrm{HR}_{10}$ values in both lakes (Figs. 5, 6, 7, and 8). Audet et al. (2017) analyzed the 
effect of temperature on GHG emissions during HW events on shallow lakes and found a significant $\mathrm{HW}$-induced increase in $\mathrm{CO}_{2}$ emissions. Therefore, we should consider the effect of pulse events as $\mathrm{HW}$ when attempting to simulate the effect of global warming on ecosystem processes. Furthermore, previous studies reported that inland lakes have significant impacts on local and regional water and energy cycles (Long et al., 2007; Liu et al., 2012). According to Oki and Kanae (2006), evaporation from lakes returns about $60 \%$ of the annual lake precipitation to the atmosphere. Therefore, the observed decrease in LE and $\mathrm{H}$ with the occurrence of $\mathrm{HW}$ events, changes in the evaporation and precipitation cycles will be expected. As a consequence regional climate will be affected (Zhu et al., 2018), and notably, on shallow lakes, with changes in water level, physicochemical parameters, GHG emissions, nutrients concentrations and inland biological communities will be altered (Renella and Quirós, 2006; Fusé et al., 2016).

\subsection{Future perspectives in air temperature for the region based on climate change models}

For the 1998-2018 period, mean annual Ta presented a positive and significant trend in both sites. For LS, there was an annual increment of $0.8^{\circ} \mathrm{C}$ in Ta; meanwhile, for the SG, the increment was $0.2{ }^{\circ} \mathrm{C}$ (Table 4, Fig. 9). According to the CCSM4 model results, Ta will continue increasing in the near (2019-2039) and far (2079-2099) future under an RCP 8.5 scenario in both places (Table 4, Fig. 9). There will be expected a higher Ta increase for the SG region $\left(+1.1^{\circ} \mathrm{C}\right)$ than $\mathrm{LS}\left(+0.8^{\circ} \mathrm{C}\right)$. Under a mitigation scenario, with RCP 4.5, the trend results negative, but no statistically significant (Table 4, Fig. 9). These results are in accordance with previous studies in Argentina that project increases between 0.5 and $1.0^{\circ} \mathrm{C}$ for both RCP 4.5 and 8.5 scenarios (Barros et al., 2015).

According to these results and the studied effect of HW on LE and H lakes fluxes, we will expect more considerable amplitude changes in both variables with the increase in Ta. The later will increment the Tw-Ta, and consequently, the amplitude of change in H fluxes. Meanwhile, for LE, changes will also be expected as $\mathrm{Ta}$ and $\mathrm{RH}_{10}$ were negatively correlated in both lakes. According to the negative slope for the RCP 4.5 model, the impact of global warming on surface heat fluxes will be lesser if a mitigation effort in GHG emissions is applied; however, it was not statistically significant. Finally, if GHG emissions continue increasing, more considerable changes in LE and $\mathrm{H}$ fluxes will be expected, with negative consequences in shallow lakes as the evaporation process will increase. These results are in accordance with a study in the Southwest and Central Plains of Western North America, where an increase in drought severity is expected with climate change in the coming decades under moderate (RCP 4.5) and high (RCP 8.5) future emissions scenarios (Cook et al., 2015).

Table 4. Results from the Community Climate System Model 4 (CCSM4) and Mann- Kendall analysis for La Salada and Sauce Grande region present (1998-2018), near future (2019-2039) and far future air temperature time series (2079-2099).

\begin{tabular}{|l|c|c|c|c|}
\hline & \multicolumn{2}{|c|}{ La Salada } & \multicolumn{2}{c|}{ Sauce Grande } \\
\cline { 2 - 5 } & Significance & Trend & Significance & Trend \\
\hline $\mathbf{1 9 9 8 - 2 0 1 8}$ & 0.02 & 0.84 & 0.04 & 0.22 \\
\hline $\mathbf{2 0 1 9 - 2 0 3 9 ~ R C P ~ 4 . 5 ~}$ & 0.50 & -0.25 & 0.65 & -0.06 \\
\hline $\mathbf{2 0 1 9 - 2 0 3 9}$ RCP 8.5 85 & 0.03 & 0.77 & 0.01 & 1.05 \\
\hline $\mathbf{2 0 7 9 - 2 0 9 9}$ RCP 4.5 & 0.29 & -0.28 & 0.54 & -0.20 \\
\hline $\mathbf{2 0 7 9 - 2 0 9 9}$ RCP.8.5 & 0.00 & 0.91 & 0.00 & 1.13 \\
\hline
\end{tabular}




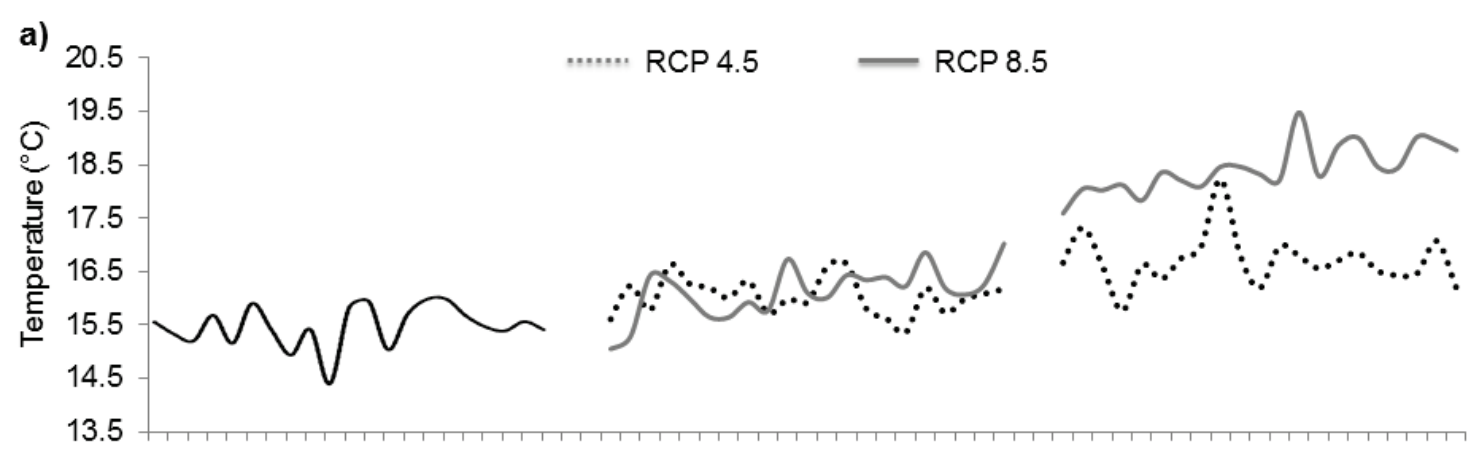

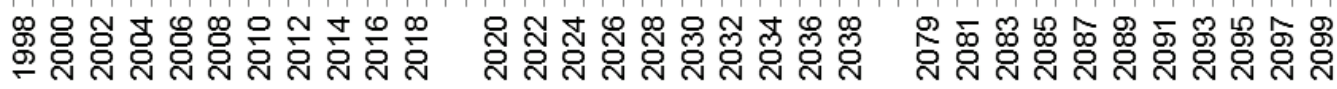

b)

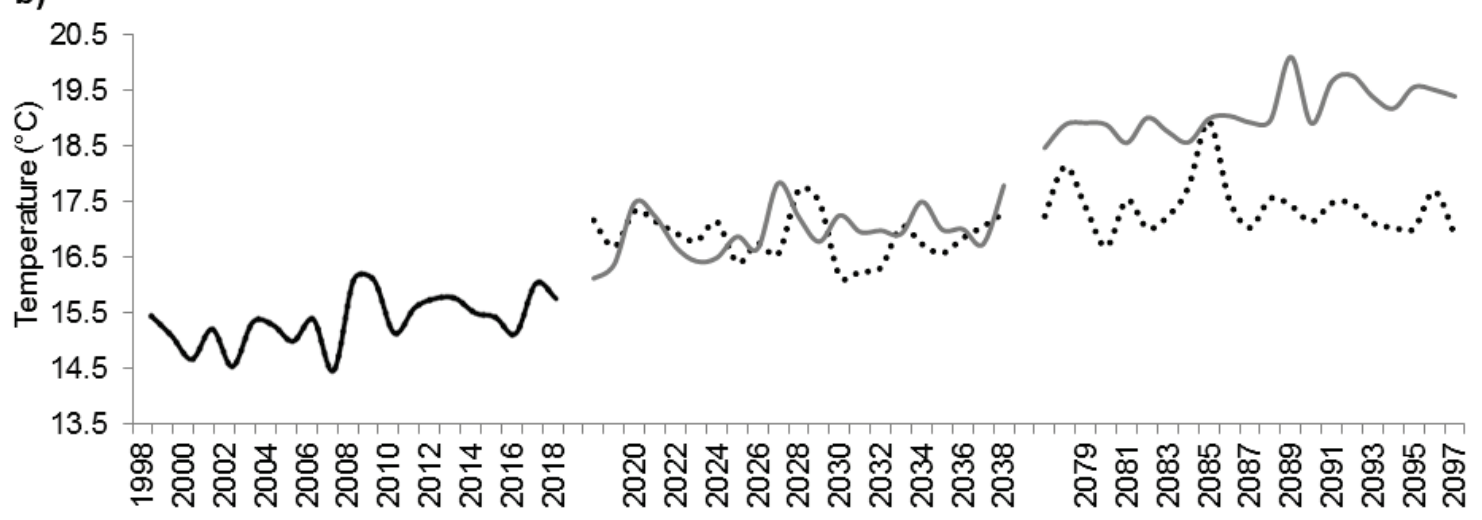

Figure 9. Trends in air temperature for La Salada and Sauce Grande region for the present (1998-2018), near future (2019-2039), and far future time series (2079-2099) under RCP 4.6 and RCP 8.5 models.

Also, a study for the United States found that extreme climate events as HW and annual extreme precipitations will increase under an RCP 8.5 scenario (Gao et al., 2012). Another study developed for the mid and high-altitude wetlands in China found that wetland distributions were predicted to decline remarkably over time under climate change scenarios with higher losses around agricultural lands (Zhao et al., 2018). For Argentina, the number and intensity of HW defined as at least three consecutive days over the $90^{\text {th }}$ percentile temperature of the 1961-1990 record has increased between 1961 and 2010 (Barros et al., 2015; Rusticucci et al., 2016), particularly those of short duration (3-5 days) as the cases studied here. Still, more detailed studies, according to projections under future climate scenarios, are needed.

\section{Conclusions}

Here we have analyzed for the first time the monthly, annual, and interannual variations of the surface latent and sensible heat on two shallow lakes from the Pampean Region. After the analysis of the effect of HW events, we found significant changes in LE and $\mathrm{H}$ heat fluxes of both lakes in response to the meteorological variables, with the increment of $\mathrm{Ta}$ and $\mathrm{Tw}$ and significant changes in $\mathrm{U}_{10}$ and $\mathrm{HR}_{10}$ values in both lakes. The main drivers of $\mathrm{LE}$ and $\mathrm{H}$ were wind speed and direction, relative humidity, and the difference between air and water temperature. Mean daily $\mathrm{H}$ values ranged between 309.4 to $200.5 \mathrm{~W} \mathrm{~m}^{-2}$ and -78.6 to $104.8 \mathrm{~W} \mathrm{~m}^{-2}$ in LS and SG, respectively. LE oscillated between -152 and $463.9 \mathrm{~W} \mathrm{~m}^{-2}$ and between -59.2 and $360.1 \mathrm{~W} \mathrm{~m}^{-2}$ in LS and SG, respectively. Both fluxes decreased with the passage of heatwaves events, presenting a high variation in its amplitude. Changes up to $96 \%$ in mean daily LE and $671 \%$ in mean daily $\mathrm{H}$ for LS and up to $25 \%$ in LE and $987 \%$ in $\mathrm{H}$ for SG were accounted in days with heatwaves respect to regular ones. Furthermore, the amplitude of changes and 
time of response to these events was different between lakes according to their geographical location, morphology, and the interannual variation in rainfall, highlighting the importance of water level and wind speed and direction on surface heat fluxes.

Also, we found increases in average air temperatures under global warming future scenarios for both lakes. According to these results and the expected change in the occurrence frequency of extreme events such as heatwaves (IPCC, 2014), we should expect substantial shifts in regional energy budget and hydrological balance in the regions with an abundance of shallow lakes. These results will contribute to the development of lake models, as well as in water resource management.

\section{Acknowledgments}

Grants from the network project PAMPA2 (CONICET), ANPCyT, Universidad Nacional del Sur (PGI 24/ G059), and the Inter American Institute for Global Change Research (IAI) CRN3038 (under US NSF Award GEO 1128040) supports the studies dealing with the article.

\section{References}

Alfonso, M.B., Brendel, A.S., Vitale, A.J., Seitz, C., Piccolo, M.C., Eduardo Perillo, G.M. 2018. Drivers of ecosystem metabolism in two managed shallow lakes with different salinity and trophic conditions: The Sauce Grande and La Salada Lakes (Argentina). Water (Switzerland) 10 (9), 1136. https://doi.org/10.3390/w10091136

Alfonso, M.B., Zunino, J., Piccolo, M.C. 2017. Impact of water input on plankton temporal dynamics from a managed shallow saline lake. Annales de Limnologie 53, 391-400. https://doi.org/10.1051/limn/2017023

Aliaga, V.S., Ferrelli, F., Piccolo, M.C. 2017. Regionalization of climate over the Argentine Pampas. International Journal of Climatology 37, 1237-1247. https://doi.org/10.1002/joc.5079

Arvola, L., George, G., Livingstone, D.M., Järvinen, M., Blenckner, T., Dokulil, M.T., Jennings E., Aonghusa, C.N., Nõges, P., Nõges, T., Weyhenmayer, G.A. 2009. The Impact of the Changing Climate on the Thermal Characteristics of Lakes. In: D.G. George (Ed.), The Impact of Climate Change on European Lakes, pp. 85-101. https://doi.org/10.1007/978-90-481-2945-4_6

Barros, V.R., Boninsegna, J.A., Camilloni, I.A., Chidiak, M., Magrín, G.O., Rusticucci, M. 2015. Climate change in Argentina: Trends, projections, impacts and adaptation. Wiley Interdisciplinary Reviews: Climate Change 6 (2), 151-169. https://doi.org/10.1002/wcc.316

Blanken, P.D., Rouse, W.R., Schertzer, W.M. 2003. Enhancement of evaporation from a large Northern Lake by the entrainment of warm, dry air. Journal of Hydrometeorology 4 (4), 680-693. https://doi.org/10.1175/1525-7541(2003)004<0680:EOEFAL>2.0.CO;2

Base de Datos Climáticos 3ra. Comunicación Nacional de la República Argentina a la Convención Marco de las Naciones Unidas sobre Cambio Climático, Centro de Investigaciones del Mar y la Atmósfera, CIMA. http://3cn.cima.fcen.uba.ar (accessed 10 October, 2019).

Cook, B.I., Ault, T.R., Smerdon, J.E. 2015. Unprecedented $21^{\text {st }}$ century drought risk in the American Southwest and Central Plains. Science Advances 1 (1), 1-8. https://doi.org/10.1126/sciadv.1400082

Davidson, T.A., Audet, J., Svenning, J.C., Lauridsen, T.L., Søndergaard, M., Landkildehus, F., larse, S.E., Jeppesen, E. 2015. Eutrophication effects on greenhouse gas fluxes from shallow-lake mesocosms override those of climate warming. Global Change Biology 21 (12), 4449-4463. https://doi.org/10.1111/gcb.13062

Di Rienzo J.A., Casanoves F., Balzarini M.G., Gonzalez L., Tablada M., Robledo C.W. 2018. Centro de Transferencia InfoStat, FCA, Universidad Nacional de Córdoba, Argentina. URL http://www.infostat.com.ar 
Diallo, I., Giorgi, F., Stordal, F. 2018. Influence of Lake Malawi on regional climate from a double-nested regional climate model experiment. Climate Dynamics 50 (9-10), 3397-3411. https://doi.org/10.1007/s00382-0173811-x

Diovisalvi, N., Salcedo Echeverry, G.E., Lagomarsino, L., Zagarese, H.E. 2015. Seasonal patterns and responses to an extreme climate event of rotifers community in a shallow eutrophic Pampean lake. Hydrobiologia 752 (1), 125-137. https://doi.org/10.1007/s10750-014-1909-2

Downing, J.A., Prairie, Y.T., Cole, J.J., Duarte, C.M., Tranvik, L.J., Striegl, R.G., et al. 2006. The global abundance and size distribution of lakes, ponds, and impoundments. Limnology and Oceanography 51 (5), 2388-2397. https://doi.org/10.4319/1o.2006.51.5.2388

Estación de Monitoreo Ambiental Costero, EMAC https://www.emac.iado-conicet.gob.ar/2019 (accessed 10 October 2019).

Fink, G., Schmid, M., Wüest, A. 2014. Large lakes as sources and sinks of anthropogenic heat: Capacities and limits. Water Resources Research 50 (9), 7285-7301. https://doi.org/10.1002/2014WR015509

Fusé, V.S., Priano, M.E., Williams, K.E., Gere, J.I., Guzmán, S.A, Gratton, R., Juliarena, M.P. 2016. Temporal variation in methane emissions in a shallow lake at a southern mid latitude during high and low rainfall periods. Environmental Monitoring and Assessment 188, 590. https://doi.org/10.1007/s10661-016-5601-z

Gao, Y., Fu, J.S., Drake, J.B., Liu, Y., Lamarque, J.F. 2012. Projected changes of extreme weather events in the eastern United States based on a high resolution climate modeling system. Environmental Research Letters 7 (4), 12 p. https://doi.org/10.1088/1748-9326/7/4/044025

Geraldi, A, Piccolo, M.C, Perillo, G.M.E. 2011. El rol de las lagunas bonaerenses en el paisaje pampeano. Ciencia Hoy 21 (123), 16-22. Retrieved from https://ri.conicet.gov.ar/handle/11336/21369

Havens, K., Paerl, H., Phlips, E., Zhu, M., Beaver, J., Srifa, A. 2016. Extreme weather events and climate variability provide a lens to how shallow lakes may respond to climate change. Water (Switzerland) 8 (6), 229. https://doi.org/10.3390/w8060229

Huamantinco Cisneros, M. A., Piccolo, M.C. 2011. Caracterización de la brisa de mar en el balneario de Monte Hermoso, Argentina. Estudios Geográficos 72 (271), 461-475. https://doi.org/10.3989/estgeogr.201118

Instituto Nacional de Tecnología Agropecuaria, INTA https://www.argentina.gob.ar/inta (accessed 10 October 2019).

IPCC, I. P. on C.C. 2014. Climate Change 2014: Synthesis Report. Contribution of Working Groups I, II and III.

Kendall, M. G. 1975. Rank Correlation Measures; Charles Griffin. London, 202 p.

Kraemer, B.M., Chandra, S., Dell, A.I., Dix, M., Kuusisto, E., Livingstone, D.M., Schladow, S.G., Silow, E., Sitoki, L.M., Tamatamah, R., McIntyre, P.B. 2017. Global patterns in lake ecosystem responses to warming based on the temperature dependence of metabolism. Global Change Biology 23 (5), 1881-1890. https://doi.org/10.1111/gcb.13459

Lagomarsino, L., Pérez, G.L., Escaray, R., Bustingorry, J., Zagarese, H.E. 2011. Weather variables as drivers of seasonal phosphorus dynamics in a shallow hypertrophic lake (Laguna Chascomús, Argentina). Fundamental and Applied Limnology 178 (3), 191-201. https://doi.org/10.1127/1863-9135/2011/0178-0191

Leira, M., Cantonati, M. 2008. Effects of water-level fluctuations on lakes: An annotated bibliography. Hydrobiologia 613, 171-184). https://doi.org/10.1007/s10750-008-9465-2

Li, Z., Lyu, S., Ao, Y., Wen, L., Zhao, L., Wang, S. 2015. Long-term energy flux and radiation balance observations over Lake Ngoring, Tibetan Plateau. Atmospheric Research 155, 13-25. https://doi.org/10.1016/j.atmosres.2014.11.019

Liu, H. P., Zhang, Q. Y., Dowler, G. 2012. Environmental controls on the surface energy budget over a large southern inland water in the United States: An analysis of one-year eddy covariance flux data. Journal of Hydrometeorology 13, 1893-1910. https://doi:10.1175/jhm-d-12-020.1

Long, Z., Perrie, W., Gyakum, J., Caya, D., Laprise, R. 2007. Northern lake impacts on local seasonal climate. Journal of Hydrometeorology 8, 881-896. https://doi:10.1175/jhm591.1 
Mann, H. B. 1945. Non-parametric Tests Against Trend. Econometrica $13 \quad(3), \quad 245$. https://doi.org/10.2307/1907187

Oki, T., Kanae, S. 2006. Global hydrological cycles and world water resources. Science 313, 1068-1072. https://doi:10.1126/science.1128845

Pohlert, T. 2018. Non-Parametric Trend Tests and Change-Point Detection, 1-18. http://docplayer.net/70194145Non-parametric-trend-tests-and-change-point-detection.html

Rennella, A., Quirós, R. 2006. The Effects of Hydrology on Plankton Biomass in Shallow Lakes of the Pampa Plain. Hydrobiologia 556, 181-191. https://doi.org/10.1007/s10750-005-0318-y

Rouse, W.R., Oswald, C.M., Binyamin, J., Blanken, P.D., Schertzer, W.M., Spence, C. 2003. Interannual and seasonal variability of the surface energy balance and temperature of central Great Slave Lake. Journal of Hydrometeorology 4 (4), 720-730. https://doi.org/10.1175/1525-7541(2003)004<0720:IASVOT>2.0.CO;2

Rusticucci, M., Kyselý, J., Almeira, G., Lhotka, O. 2016. Long-term variability of heat waves in Argentina and recurrence probability of the severe 2008 heat wave in Buenos Aires. Theoretical and Applied Climatology 124 (3-4), 679-689. https://doi.org/10.1007/s00704-015-1445-7

Sen, P.K. 1968. Estimates of the Regression Coefficient Based on Kendall's Tau. Journal of the American Statistical Association 63 (324), 1379-1389. https://doi.org/10.1080/01621459.1968.10480934

Servicio Meteorológico Nacional, SMN https://www.smn.gob.ar/ (accessed 10 October 2019).

Shao, C., Chen, J., Stepien, C.A., Chu, H., Ouyang, Z., Bridgeman, T.B., Czajkowski, K.P., Becker, R.H., John, R. 2015. Diurnal to annual changes in latent, sensible heat, and CO2 fluxes over a Laurentian Great Lake: A case study in Western Lake Erie. Journal of Geophysical Research G: Biogeosciences 120 (8), 1587 1604. https://doi.org/10.1002/2015JG003025

Tranvik, L.J., Downing, J.A., Cotner, J.B., Loiselle, S.A., Striegl, R.G., Ballatore, T.J., et al. 2009. Lakes and reservoirs as regulators of carbon cycling and climate. Limnology and Oceanography 54 (6 PART 2), 2298-2314. https://doi.org/10.4319/lo.2009.54.6_part_2.2298

Winder, M., Schindler, D.E. 2004. Climatic effects on the phenology of lake processes. Global Change Biology 10 (11), 1844-1856. https://doi.org/10.1111/j.1365-2486.2004.00849.x

Woolway, R.I., Jones, I.D., Feuchtmayr, H., Maberly, S.C. 2015a. A comparison of the diel variability in epilimnetic temperature for five lakes in the English Lake District. Inland Waters 5 (2), 139-154. https://doi.org/10.5268/IW-5.2.748

Woolway, R.I., Jones, I.D., Hamilton, D.P., Maberly, S.C., Muraoka, K., Read, J.S., Smyth, R.L., Winslow, L.A. 2015b. Automated calculation of surface energy fluxes with high-frequency lake buoy data. Environmental Modelling and Software 70, 191-198. https://doi.org/10.1016/j.envsoft.2015.04.013

Woolway, R. I., Verburg, P., Lenters, J. D., Merchant, C. J., Hamilton, D. P., Brookes, J., et al. 2018. Geographic and temporal variations in turbulent heat loss from lakes: A global analysis across 45 lakes. Limnology and Oceanography 63 (6), 2436-2449. https://doi.org/10.1002//no.10950

Wüest, A., Lorke, A. 2003. Small-scale hydrodynamics in lakes. Annual Review of Fluid Mechanics 35 (1), 373 412. https://doi.org/10.1146/annurev.fluid.35.101101.161220

Zhang, Q., Liu, H. 2013. Interannual variability in the surface energy budget and evaporation over a large southern inland water in the United States. Journal of Geophysical Research Atmospheres 118 (10), 4290-4302. https://doi.org/10.1002/jgrd.50435

Zhao, X., Liu, Y. 2018. Variability of Surface Heat Fluxes and Its Driving Forces at Different Time Scales Over a Large Ephemeral Lake in China. Journal of Geophysical Research: Atmospheres 123 (10), 4939-4957. https://doi.org/10.1029/2017JD027437

Zhu, C., Zeng, Y. 2018. Effects of urban lake wetlands on the spatial and temporal distribution of air PM10 and PM2.5 in the spring in Wuhan. Urban Forestry and Urban Greening 31, 142-156. https://doi.org/10.1016/j.ufug.2018.02.008 



\title{
UNA REVISIÓN SOBRE LAS TIERRAS ABANDONADAS EN ESPAÑA: DE LOS PAISAJES LOCALES A LAS ESTRATEGIAS GLOBALES DE GESTIÓN
}

\author{
TEODORO LASANTA ${ }^{1 *}$, ESTELA NADAL-ROMERO ${ }^{1}$, \\ MAKKI KHORCHANI ${ }^{1}$, ASUNCIÓN ROMERO-DÍAZ ${ }^{2}$
}

\author{
${ }^{1}$ Instituto Pirenaico de Ecología (CSIC), Campus de Aula Dei, \\ Avda. Montañana, 1005, 50080 Zaragoza, España. \\ ${ }^{2}$ Departamento de Geografía, Facultad de Letras, Universidad de Murcia, \\ Campus de la Merced, 30001 Murcia, España.
}

\begin{abstract}
RESUMEN. Las tierras abandonadas ocupan espacios muy extensos en algunos territorios españoles, especialmente en las áreas de montaña y ambientes semiáridos. Originan paisajes muy diversos en función de las condiciones climáticas, el lugar y la edad de abandono, la gestión previa y posterior, y las perturbaciones que sufren durante el proceso post-abandono. Estos factores condicionan la sucesión vegetal y las propiedades edáficas, dando lugar a ecosistemas y paisajes tan contrastados como bosques, matorrales, pastizales y suelos casi desnudos. En este trabajo se revisan los conocimientos actuales sobre el abandono de tierras en España a partir de la bibliografía, con especial referencia al proceso espacio-temporal del abandono, los factores que originan diferentes ecosistemas y paisajes en las tierras abandonadas y las implicaciones de éstos en el suministro de servicios ecosistémicos a la sociedad. En función del paisaje generado y de su gestión dependen la cantidad y calidad de los recursos hídricos, la protección del suelo, el secuestro y stock de carbono, los niveles de biodiversidad, la prevención y control de incendios forestales, los recursos pastorales, la estética del paisaje e incluso las posibilidades de fijar población en el medio rural a partir de aprovechamientos extensivos.
\end{abstract}

\section{A review of abandoned lands in Spain: from local landscapes to global management strategies}

\begin{abstract}
Abandoned lands occupy extensive territories in some Spanish regions, particularly in mountain areas and semiarid environments. These lands originate very diverse landscapes depending on the climatic conditions, the age of abandonment, the management before and after abandonment, and the disturbances that they suffer during the post-abandoned process. These factors condition the plant succession and soil properties, producing contrasted ecosystems and landscapes, like forests, shrublands, grasslands and almost bare soils. This study reviews the current knowledge about land abandonment in Spain, with special reference to the spatial and temporal process of abandonment, the factors that originate different ecosystems, and landscapes in abandoned lands, and the implications of these factors in the provision of ecosystem services to society. Depending on the landscape generated and its management, different services would be provided, such as the quality and quantity of water resources, soil protection, carbon sequestration, levels of biodiversity, prevention and control of forest fires, pastoral resources, landscape aesthetics, and even the possibilities of securing population in the rural environment through extensive land uses.
\end{abstract}


Palabras clave: Sucesión vegetal, degradación del suelo, paisaje cultural, servicios ecosistémicos, montaña mediterránea, zonas semiáridas.

Key words: Plant succession, soil degradation, cultural landscape, ecosystem services, Mediterranean mountains, semiarid areas.

Recibido: 26 Junio 2020

Aceptado: 26 Agosto 2020

*Correspondencia: Teodoro Lasanta, Instituto Pirenaico de Ecología (IPE-CSIC), Campus de Aula Dei, Avda. Montañana, 1005, 50080 Zaragoza, España. E-mail: fm@ipe.csic.es

\section{Introducción}

El abandono de tierras es un fenómeno global, con especial incidencia en los países desarrollados (Strijker, 2005). Ramankutty y Foley (1999) calcularon que se han abandonado aproximadamente 1,5 millones de $\mathrm{Km}^{2}$ en el mundo entre 1700 y 1992, superficie que se ha incrementado considerablemente después y que continuará incrementándose en las próximas décadas (Pointereau et al., 2008), entre otras razones porque hay alrededor de 280 Mha destinadas a agricultura nómada o itinerante (shifting agriculture) que tarde o temprano serán progresivamente abandonadas (Heinimann et al., 2017). En Europa, según Feranec (2010) entre 1990 y 2000 se abandonaron $88.000 \mathrm{~km}^{2}$ y entre los años 2000 y 2012, Kuemmerle (2016) indica que el abandono fue de $20.500 \mathrm{~km}^{2}$. En España, en el año 2019, según el Fondo Español de Garantía Agraria (FEGA), las superficies abandonadas y sin aprovechamiento agrario se estiman en más de 2,32 Mha, lo que representa el 20\% de las superficies de cultivo o el $4,5 \%$ de la superficie agraria total.

$\mathrm{El}$ abandono de tierras constituye un proceso por el que los ecosistemas y paisajes modifican su capacidad para proporcionar bienes y servicios a la sociedad (Pereira et al., 2005; Kozak et al., 2017; García-Llamas et al., 2019). Sus causas son muy diversas y sus implicaciones ambientales, paisajísticas y socioeconómicas son múltiples (Keenleyside y Tucker, 2010; Lasanta-Martínez, 2014; Alonso-Sarria et al., 2016). Es, además, un proceso en el que interactúan simultáneamente factores biofísicos, socioeconómicos, tecnológicos, demográficos, políticos y culturales (Terres et al., 2015; Lasanta et al., 2017a; Huang et al., 2020). Por otro lado, es un tema que requiere cierta perspectiva temporal, ya que la evolución de las tierras abandonadas y su diversidad de respuestas dependen en buena parte del tiempo transcurrido desde el abandono, de las características biofísicas del lugar donde ocurre el abandono de tierras y de las perturbaciones sufridas (García-Ruiz y López Bermúdez, 2009). De ahí, que sea un tema complejo de estudiar, lo que ha impulsado su interés científico. En este sentido, basta señalar que una consulta a la base de datos Scopus el 2 de febrero de 2016, introduciendo las palabras clave más utilizadas por los autores: "land abandonment" y "abandoned agricultural", y tras cruzar los resultados y eliminar los documentos repetidos quedaron 1194 referencias bibliográficas sobre tierras abandonadas en el mundo (Romero Díaz, 2016). Y tres años después, el 1 de marzo de 2019, consultada la misma base de datos con las mismas palabras clave, el número de referencias había ascendido a 1638 (Lasanta et al., 2019a), de las que 309 (18,9\%) tenían como área de estudio España, siendo el segundo país con más referencias, sólo superado ligeramente por Estados Unidos, y muy por encima de los países que le siguen: Italia, Alemania y Francia (Lasanta et al., 2019a).

En España, al igual que en los países europeos de la cuenca mediterránea, las tierras abandonadas ocupan una extensa superficie, siendo uno de los grandes cambios de uso del suelo registrados desde las primeras décadas del siglo XX (García-Ruiz y Lana-Renault, 2011; García-Ruiz et al., 2011). Las áreas de montaña y los secanos semiáridos son los territorios con mayor abandono; se 
consideran tierras marginales, espacios de escasa relevancia económica, con poca capacidad para competir en mercados muy dinámicos y competitivos. Muchos gestores del territorio y científicos consideran que deben abandonarse $\mathrm{o}$, como mucho, explotarse de manera muy extensiva para favorecer la revegetación (Lasanta y García-Ruiz, 1996; Perino et al., 2019).

Las causas que dirigen el abandono de tierras son muy diversas y varios de los autores, que han tratado de estudiarlas, consideran que deben ser tratadas de forma integrada y a diferentes escalas (Alonso Sarria et al., 2016a). Lasanta et al. (2017a) señalan que las causas globales o externas actúan como desencadenantes del proceso de abandono, mientras que las causas locales o internas condicionan la superficie abandonada y las áreas donde se localiza el abandono. Haciendo un esfuerzo de síntesis se puede resumir que el abandono de tierras en España es debido a: (i) la migración de la población rural hacia las ciudades y el envejecimiento de la que permanece (Ruiz-Sinoga et al., 2011; Kosmas et al., 2002; Meléndez-Pastor et al., 2014); (ii) las innovaciones tecnológicas y la mecanización de las tareas agrícolas, lo que ha impulsado el abandono de muchas laderas muy pendientes, los campos de pequeño tamaño y aquellas áreas poco accesibles a la maquinaria agrícola (Lasanta Martínez, 1988a; Nagendra et al., 2003; Onega-López et al., 2010); (iii) la baja productividad de algunos campos, por escasa fertilidad o suelos con horizontes poco desarrollados o muy pedregosos (Duarte et al., 2008); (iv) la elevada competitividad de los mercados, lo que hace poco rentables algunos cultivos y algunas zonas rurales (García-Ruiz et al., 2015; Van Leeuwen et al., 2019); (v) los efectos de la Política Agrícola Común (PAC), que entre 1989 y 2008 incentivó el abandono de tierras agrícolas de forma permanente (land retirement) o temporal (set-aside) (Boellstorff y Benito, 2005; Corbelle-Rico et al., 2012; Lasanta Martínez, 2014); (vi) el clima en ambientes semiáridos (Gisbert et al., 2005; Alonso Sarria et al., 2016b), que reduce la productividad y aumenta la incertidumbre frente a la irregularidad de las lluvias; (vii) la existencia de ocupaciones alternativas (Kozak et al., 2004; Rey Benayas et al., 2007); y (viii) factores vinculados a la gestión, relacionados con un inadecuado manejo de las explotaciones agrarias, como la sobreexplotación y salinización del suelo y/o de los acuíferos (Vera y Romero, 1994; Tobarra González, 2002; Chocano Vañó et al., 2007; Pérez Morales, 2008), la fatiga del suelo debida a la reiteración de determinados cultivos hortícolas intensivos (Vera y Romero, 1994; Guerrero et al., 2014), la proliferación de problemas fitopatológicos específicos (Lucas Espadas, 1996), o el abandono de determinadas prácticas y estructuras tradicionales de conservación de suelos (Vera y Romero, 1994; Franco y Calatrava, 2010). Además, Lasanta et al. (2017a) inciden en que los factores biofísicos (suelo, clima, topografía...) intervienen siempre porque determinan la productividad y, en último término, la rentabilidad y competitividad del producto ofertado. En definitiva, las causas podrían resumirse en socioeconómicas, ambientales y de gestión.

La bibliografía sobre el abandono de tierras en España es muy abundante, debido no sólo a la gran extensión directamente implicada, sino también a su destacado impacto en el territorio y sus múltiples implicaciones socio-ecológicas, hasta tal punto que es uno de los factores más destacados del Cambio Global (Herrando et al., 2014). Por otro lado, hay que tener en cuenta que las tierras abandonadas constituyen una especie de laboratorio natural para los investigadores, por su presencia en un gradiente climático muy amplio, desde ambientes subdesérticos a subalpinos, por la diversidad de sistemas de gestión pre y post-abandono, y por las perturbaciones que sufren las tierras abandonadas, como pueden ser los incendios (Azevedo et al., 2011) o los procesos de erosión (García Ruíz y Lana Renault, 2011; Romero Díaz et al., 2016). Todo ello hace que la evolución de las tierras abandonadas sea muy heterogénea, con consecuencias muy variadas a escala local y en los servicios ecosistémicos que prestan a la sociedad.

Los estudios sobre el abandono de tierras en España se iniciaron, de manera sistemática, en los años ochenta del pasado siglo, centrándose sobre todo en la distribución espacial y temporal del proceso de abandono (Díaz-Muñoz, 1984; Alguacil García, 1985; Lasanta Martínez, 1988a; Arnáez et al., 1990; Lasanta Martínez et al., 1989) y en el comportamiento hidromorfológico de las tierras abandonadas (García-Ruiz et al., 1985 y 1988; García-Ruiz, 1986). Los estudios sobre la erosión del suelo en relación con la sucesión vegetal tuvieron un gran impulso en los años ochenta y noventa, realizándose varias 
tesis doctorales a finales de los años ochenta y en la década de los noventa (Lasanta Martínez, 1988; Ruiz-Flaño, 1993, Padilla-Blanco, 1998) y más recientemente en el siglo XXI (Oserín Elorza, 2007; Cañadas Sánchez, 2008; Martínez Hernández, 2018), así como numerosos artículos y comunicaciones a congresos (ver García-Ruiz y López-Bermúdez, 2009). Posteriormente, los temas de estudio se ampliaron considerablemente, como corresponde a una cuestión con implicaciones ambientales, paisajísticas y socioeconómicas.

En Europa, se han realizado diversas revisiones bibliográficas referentes al abandono de tierras, entre las que caben citarse las relacionadas con la dinámica y restauración de las tierras abandonadas (Bowen et al., 2007; Cramer, 2007) o sobre los impactos ambientales (MacDonald et al., 2000; Haddaway et al., 2014). Y en España también se han realizado excelentes revisiones bibliográficas sobre los impactos hidromorfológicos del abandono de tierras en España (Lasanta, 2006; García-Ruiz y LópezBermúdez, 2009; García-Ruiz, 2010; García-Ruiz y Lana-Renault, 2011; Cantón et al., 2011; Arnáez et al., 2015; van Leeuwen et al., 2019; Moreno-de-las-Heras et al., 2019), pero se echa en falta un estudio que incluya información sobre el proceso espacio-temporal del abandono, sus causas, los ecosistemas y paisajes resultantes y las implicaciones de éstos en el suministro de servicios ecosistémicos a la sociedad. En este trabajo se revisan los conocimientos acumulados sobre el abandono de tierras en España. Se intenta disponer de una perspectiva holística, necesaria para gestionar, con una base científica, un espacio tan extenso como el de las tierras abandonadas en España, por sus elevadas implicaciones en el desarrollo local de muchas áreas marginales y en el suministro de bienes y servicios a otros territorios.

\section{La extensión de las tierras abandonadas}

La bibliografía sobre la extensión de tierras abandonadas en España es relativamente escasa, al igual que en el resto del mundo (Ustaoglu y Collier, 2018), quizás porque cuantificar el proceso espaciotemporal del abandono exige trabajar con documentos gráficos: mapas de usos y cubiertas del suelo, fotografías, fotogramas aéreos, ortoimágenes, imágenes de satélite. Para justificar esta escasez de datos superficiales hay que tener en cuenta cuatro limitaciones importantes: (i) que tras el abandono se inicia un proceso de sucesión vegetal que borra las huellas de la fase de cultivo en un periodo más o menos rápido, lo que exige contar con documentos próximos a la fecha de abandono $\mathrm{o}$, al menos, previos a que la cubierta vegetal haya borrado los márgenes de los campos; (ii) que una secuencia temporal de documentos gráficos es una herramienta reciente; solo a partir de 1956 existen fotogramas aéreos, a los que se añaden más recientemente imágenes de satélite que permiten cartografiar todo el proceso de abandono; (iii) que se requiere un exhaustivo trabajo de fotointerpretación, cartográfico y de campo, que exige dedicar mucho tiempo para completar todo el proceso espacio-temporal de abandono; y (iv) que en el caso del abandono de áreas de pastoreo, como ha sucedido en el piso subalpino tras la crisis de la trashumancia, el proceso de abandono es siempre progresivo y sólo puede cartografiarse en función del avance del bosque, que puede ser en algunos casos muy antiguo. De ahí, que apenas existan estudios que abarquen una superficie superior a la del municipio (Lasanta et al., 2019a), con la excepción de las tesis doctorales de Lasanta-Martínez (1998), en la que estudió el proceso de abandono de siete valles del Pirineo aragonés, Oserín Elorza (2007), que analizó los campos abandonados del sector central de la montaña riojana, y Martínez Hernández (2018), en donde se evaluó el abandono reciente de toda la Región de Murcia (11.313 km² de superficie).

En los años ochenta y noventa se realizaron algunos estudios sobre áreas de montaña, coincidiendo todos ellos en señalar una drástica contracción del espacio cultivado (Lasanta, 1996). Los abandonos se iniciaron en las primeras décadas del siglo XX y experimentaron un repunte importante entre los años cincuenta y setenta, mientras que después de 1980 fueron muy pocos los campos que se abandonaron, porque los que habían resistido hasta ese momento lograron adaptarse al nuevo sistema socioeconómico. Así, por ejemplo, en el Pirineo las parcelas que se podían trabajar con tractor, que tradicionalmente se habían cultivado con cereales, se transformaron en prados de siega para la alimentación invernal de la ganadería (García-Ruiz y Lasanta-Martínez, 1990 y 1993). Algunos datos, 
aportados por la bibliografía, contribuyen a cuantificar la extensión de tierras abandonadas en zonas de montaña. Se abandonó, al menos, el 40\% de la tierra labrada en la Cabrera leonesa (Cabero, 1980), la mitad de los valles asturianos (Rodríguez-Gutiérrez, 1989), el 89\% de la vertiente sur de la Sierra de Ayllón (Bordiú, 1985), las tres cuartas partes del Alto Tormes (Barrientos, 1978), el 36\% de la Sierra de Ronda y el 25\% en la Planicie de Ronda (Gómez Moreno, 1989), el 71\% en los Valles Centrales del Pirineo (Lasanta-Martínez, 1988a), el 99\% en Cameros Viejo (Lasanta-Martínez et al., 1989), el 42\% en el Alto Linares, Sistema Ibérico (Maiso y Lasanta, 1990), el 40\% de la Sierra de Prades (Pérez Albert, 1995) y el 80\% en Valdesamario, montaña media leonesa (Cortizo et al., 1992). El abandono también fue extenso en las montañas del sur de España, sobre todo en las Cordilleras Béticas, pese a que todavía soportaban una presión demográfica relativamente elevada, manteniéndose el cultivo, sobre todo de almendros, en campos localizados en pendientes relativamente empinadas (Faulkner, 1995). Douglas et al. (1994) señalan que los campos abandonados ocupan el 70\% de las tierras de cultivo en las Alpujarras.

El abandono de tierras es, pues, un hecho generalizado en las montañas españolas, si bien a escala temporal y espacial se observan algunas diferencias interesantes. Dado que el primer documento que permite cuantificar la extensión del abandono es la fotografía aérea de 1956 diferenciamos dos etapas: antes y después de 1956, teniendo en cuenta que después de 1980 hubo pocos abandonos en la montaña española, como ya se ha señalado.

i) La primera fase de abandono (antes de 1956) se vincula con los factores físicos locales, abandonándose las parcelas con las peores condiciones: fuertes pendientes, suelos poco fértiles y pedregosos, y mayores limitaciones climáticas. Arnáez et al. (1990) señalan que en el Pirineo aragonés se había abandonado el 63\% del espacio agrícola antes de 1956, localizándose los campos abandonados en laderas con pendientes muy acusadas, importantes limitaciones climáticas y suelos poco fértiles. Así, por ejemplo, los campos de agricultura nómada ("articas") se habían abandonado masivamente (92,7\% de su superficie) antes de 1956 (Errea Abad et al., 2015). Por el contrario, en Cameros Viejo (Sistema Ibérico riojano), sólo se había abandonado el 30\% del área agrícola tradicional al contar con condiciones menos extremas y pendientes más suaves (Ruiz-Flaño et al., 2010).

ii) La segunda fase de abandono (después de 1956) tiene que ver con la capacidad de integración de cada región en la economía nacional. Las parcelas que se pueden mecanizar, ofrecen un producto competitivo y tienen buena accesibilidad al mercado se mantienen en cultivo, mientras que se abandonan las que no admiten la maquinaria agrícola, por excesiva pendiente o mal acceso, y las alejadas de las vías de comunicación. Siguiendo con la comparación entre el Pirineo Aragonés y Cameros Viejo, la primera región mantiene el 29\% de la superficie agrícola histórica, mientras la segunda solamente el $1 \%$. La existencia de valles glaciares, rellanos de obturación glaciar y conos de deyección en el Pirineo permite disponer de campos llanos o de suave pendiente que se cultivan con prados de siega, trabajados con tractor, orientados a la alimentación de la ganadería extensiva durante la estación fría. En Cameros Viejo, la ausencia de valles glaciares limita los espacios llanos y las pocas parcelas que podrían mecanizarse encuentran grandes limitaciones climáticas para el cultivo rentable de prados (Lasanta et al., 1989; Arnáez et al., 1990).

iii) Los bancales se abandonaron lentamente en la primera fase de abandono y masivamente después. En el valle de Aísa (Pirineo aragonés), por ejemplo, el 54,4\% de los campos en pendiente se había abandonado antes de 1956, mientras que la proporción de abandono era menor en los bancales $(39,9 \%)$, en gran parte debido a que los bancales poseían suelos más fértiles y productivos que los campos en pendiente (Errea Abad et al., 2015). Después de 1956, sin embargo, los bancales se han abandonado de forma masiva, por las dificultades que plantean para la mecanización, tendencia que también se ha puesto de manifiesto en otras montañas mediterráneas (Arnáez et al., 2015; Peña-Angulo et al., 2019), mientras que se mantienen los campos de suave pendiente (Errea Abad et al., 2015). 
El abandono de tierras en áreas de montaña fue, pues, muy importante antes de los años 80 . Los espacios llanos, por el contrario, resistieron bien con apenas abandono de tierras hasta los años ochenta, aunque la presión demográfica disminuyó mucho entre 1960 y 1980. La mecanización del campo, la puesta en regadío de muchas áreas y el uso de fertilizantes químicos contribuyó a mantener activas muchas explotaciones que arrendaron las tierras de las cesantes. En otros casos, la agricultura se mantuvo como una actividad complementaria o a tiempo parcial. Sin embargo, la Unión Europea, a través de la PAC, incentivó desde 1988 la extensificación de la producción (Reglamento CEE $\mathrm{n}^{\circ}$ 4115/88, modificado por el n⿳0708/92 y el n ${ }^{\circ} 838 / 93$ ), la retirada de tierras de cultivo (Norma Comunitaria $\mathrm{n}^{\mathrm{o}} 1034 / 88$, Reales Decretos 1435/88 y 1250/90, y Normativa CEE n ${ }^{\mathrm{o}} 1765 / 92$ ), y la jubilación anticipada (Reglamento CEE n ${ }^{\circ}$ 2079/92), entre otras normas y reglamentos que fueron estableciéndose hasta 2008 y que impulsaron el cese de explotaciones y el abandono de tierras. Esta última fase es un abandono inducido por agentes externos al medio rural (García-Ruiz y Lana-Renault, 2011; Lasanta et al., 2017a), que trató de reducir en primer lugar los excedentes agrícolas y, más secundariamente, favorecer la conservación de suelos y mejorar su balance hídrico (García-Ruiz y López Bermúdez, 2009).

Al igual que en las áreas de montaña, tan sólo existe información parcial y no actualizada de la superficie abandonada en tierras llanas. En España se dejaron de cultivar 995.599 ha (el 13,3\% de la superficie cerealista) entre 1989 y 1993, afectando fundamentalmente a las comarcas de secano cerealista y con explotaciones de elevado tamaño (Lasanta, 1996). En Aragón se realizó un estudio muy detallado, con información de todas las explotaciones, sobre la influencia de la PAC en el abandono de tierras (Land retirement y set-aside) comprobándose que entre 1988 y 1994 se vieron afectadas 182.374 ha (el $12,5 \%$ de la superficie cultivada con herbáceas), concentrándose los abandonos en los secanos semiáridos (Lasanta, 1996). El abandono de tierras también ha progresado recientemente en las zonas semiáridas del sureste de España y ambientes semiáridos del centro de la Depresión del Ebro, debido a la baja productividad de las tierras de secano y a problemas ligados con la salinización y degradación de suelos (Lesschen et al., 2007; Romero-Díaz et al., 2007; Fister y Ries, 2009; Alonso-Sarría et al., 2016a, entre otros). Por su parte, Arévalo et al. (2017) señalan que más del $90 \%$ de los campos en terrazas se han abandonado en la isla de Lanzarote en los últimos 40 años. Corbelle-Rico et al. (2012) ponen de relieve que los mayores abandonos en Galicia tienen lugar tras la incorporación de España a la Unión Europea, debido a los avances tecnológicos, que fueron un importante desencadenante para el abandono de pequeñas parcelas y el cese de explotaciones minifundistas, y a la PAC.

\section{Procesos de reconstrucción: edafogénesis y sucesión vegetal}

Cuando un campo se abandona se ponen en marcha de forma simultánea dos procesos interactivos de "reconstrucción" de las condiciones naturales previas a la fase de cultivo. La Figura 1 resume las dinámicas más frecuentes en campos abandonados en función de la situación de partida. Por un lado, se inicia un proceso de edafogénesis, que trata de mejorar las propiedades del suelo. Por otro lado, ocurre un proceso de sucesión secundaria que llevará al establecimiento de un bosque, en su estadio final si se cumplen todas las fases de la sucesión. Numerosos estudios revelan la estrecha relación que se establece entre sucesión vegetal y propiedades del suelo, constituyendo un tema muy complejo: Suelo y planta interactúan condicionando la competencia y/o facilitación entre plantas, así como la heterogeneidad edáfica incluso en terrenos aparentemente homogéneos (Cañadas et al., 2010). Además, la evolución de ambos procesos está muy condicionada por diferentes factores, entre los que destacan: la situación de partida del campo (características del suelo y especies vegetales y/o semillas presentes en el banco del propio campo o en sus proximidades), las condiciones climáticas y litológicas del lugar, la gestión post-abandono y las perturbaciones que puedan surgir a lo largo del tiempo. Por otro lado, del éxito o fracaso en la reconstrucción de las condiciones naturales depende en gran medida el paisaje resultante y los servicios ecosistémicos que las tierras abandonadas proporcionan a la sociedad. 


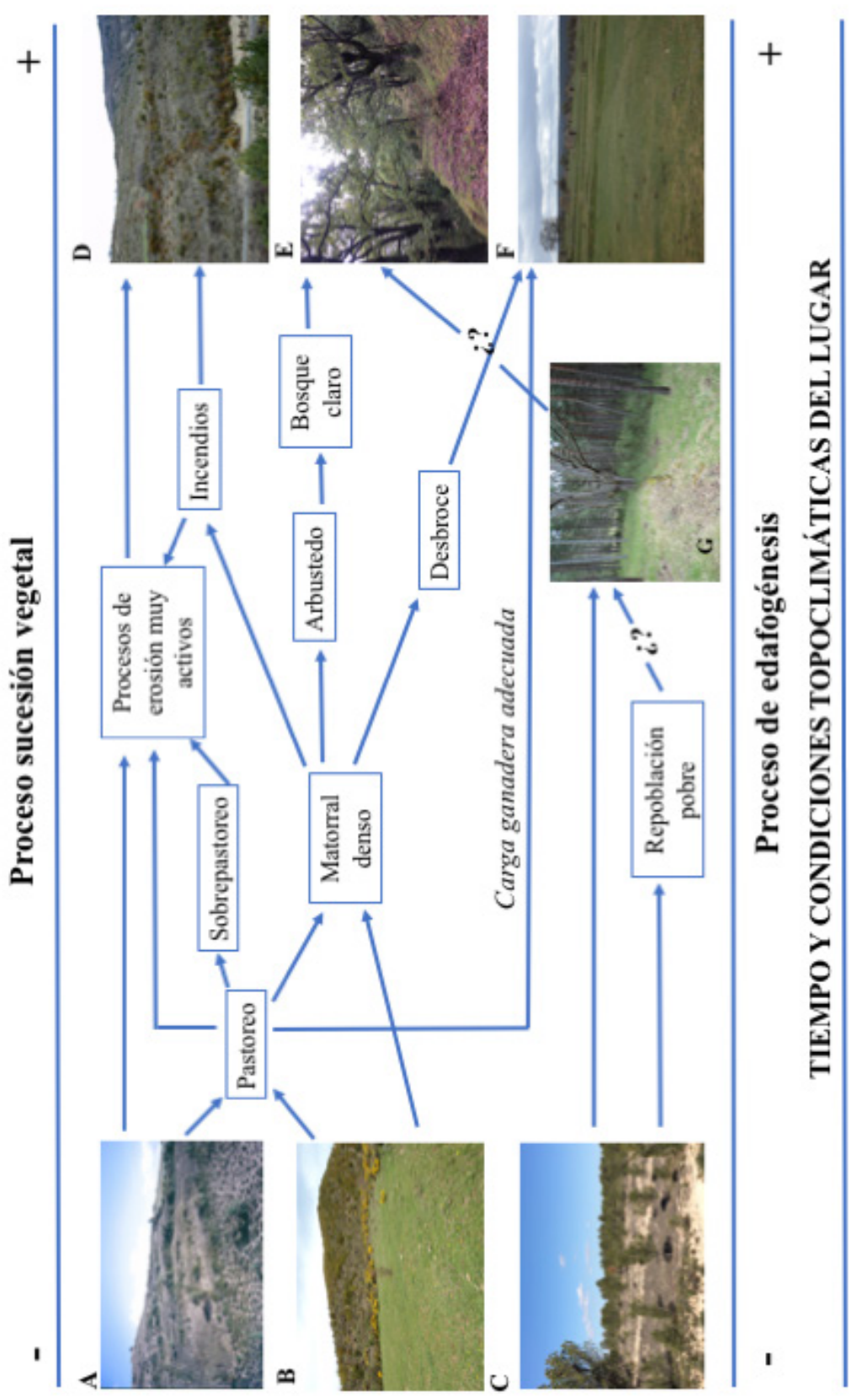

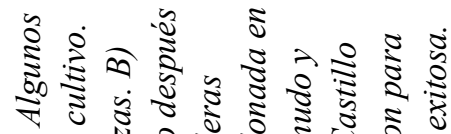

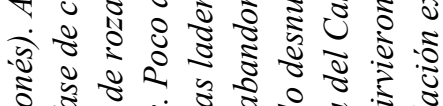

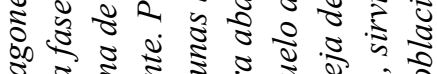

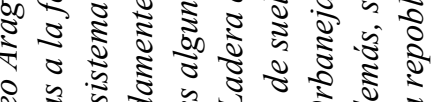

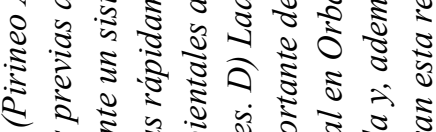

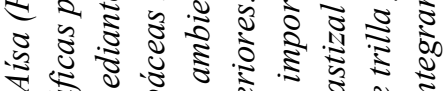

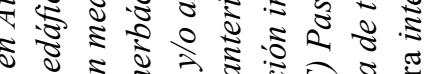

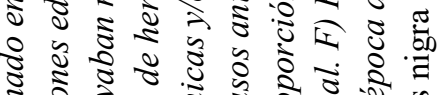

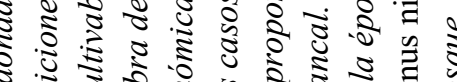

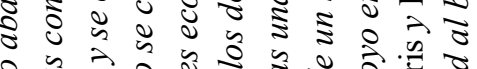

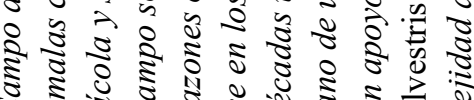

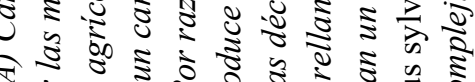

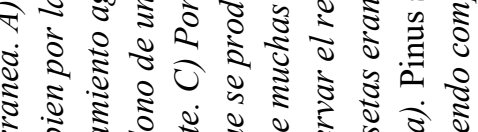

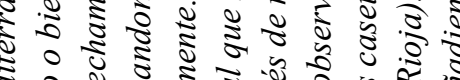

ช.

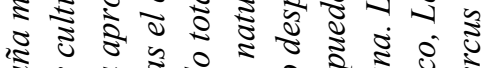

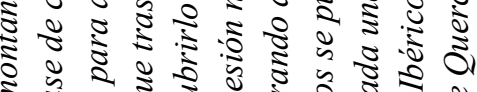

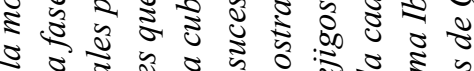

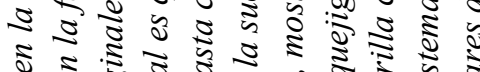

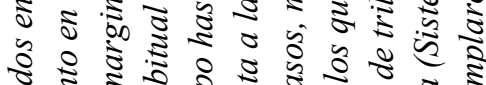

ठ ₹

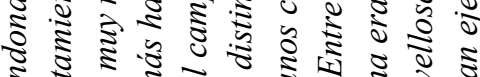

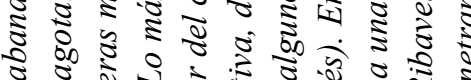

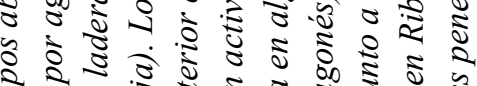

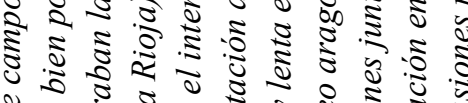

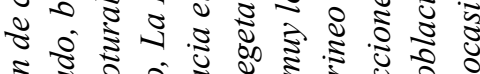

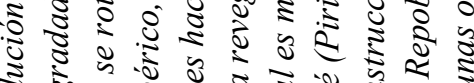

के

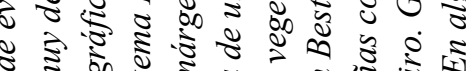

8 इ

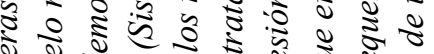

ปิ

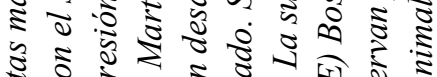

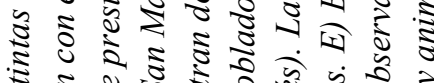

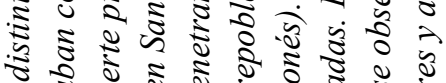

$\approx \approx 8 \%=80 \%$

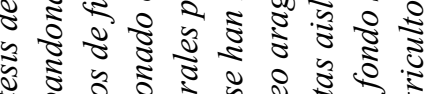

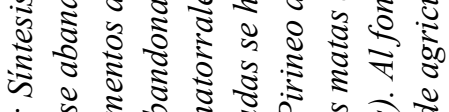

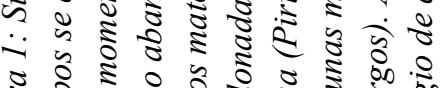

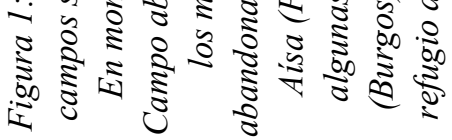


Tras el abandono de un campo agrícola se inicia un rápido proceso de sucesión secundaria o de colonización vegetal (Pugnaire et al., 2016). Inicialmente penetran las herbáceas que estaban presentes en la fase de cultivo como "malas hierbas" (especies arvenses) o bien en las proximidades del campo como ruderales. En muy pocos años (3-5 años), dependiendo de la fertilidad del suelo y de si las condiciones climáticas son favorables (humedad suficiente), el campo puede estar colonizado por una cubierta muy densa de herbáceas, formando pastizales con predominio de gramíneas y leguminosas (Lasanta et al., 2019b). Otro hecho que puede contribuir a una rápida colonización por herbáceas es la presencia de especies ruderales en el margen del campo. Pedroli et al. (2006) han comprobado la rápida colonización de bancales cuando el salto del bancal o lomo está cubierto de vegetación en la fase de cultivo. Contribuye, además, el mayor espesor y fertilidad del suelo en bancales que en campos en pendiente. Arévalo et al. (2017) señalan que los campos en terrazas de la isla de Lanzarote presentan una mejor calidad del suelo que los no aterrazados; además cuentan con suelos más profundos que les permite acumular más agua. Por otro lado, las terrazas favorecen la retención de agua contribuyendo a evitar el estrés hídrico (Bellin et al., 2009). Estudios llevados a cabo en el sureste de España (Zapata et al., 2016) han puesto de manifiesto la importancia que para la colonización vegetal de campos abandonados tiene la presencia cercana de áreas naturales y de especies endozoócoras que dispersen las semillas, y además han corroborado la idea anterior de que elementos internos que sirven de refugio (lindes, terrazas de piedra, setos...) pueden actuar como focos de colonización.

En esta fase inicial de la sucesión vegetal, el pastoreo del ganado es un factor esencial en la composición y ritmo de la sucesión vegetal. Condiciona las relaciones de colonización y extinción de especies a través del consumo, las perturbaciones mecánicas, la dispersión de semillas y la alteración de la fertilidad del suelo con la entrada de nutrientes por el estiércol (Peco et al., 2006). Si la carga ganadera es adecuada e incluso relativamente alta, los campos permanecen como pastizales durante décadas, retrasando el avance de la sucesión vegetal (Molinillo et al., 1997; Lasanta, 2010). Si se produce sobrepastoreo, la sucesión vegetal se interrumpe, el banco de semillas se empobrece, aparecen claros y el suelo se compacta. El pisoteo del ganado apelmaza la tierra y compacta el suelo, modificando algunas de sus propiedades físicas (disminución de la porosidad, ruptura de agregados), lo que implica una menor infiltración, y el incremento de la escorrentía superficial y de las tasas de erosión (Pérez y Díaz Fierros, 1988; Blanco Sepúlveda, 2008). Cuando no hay pastoreo o es muy leve, la sucesión vegetal suele ser muy rápida invadiendo los matorrales y arbustos los campos y pastizales en muy pocos años (Ferrer y Broca, 1999; Komac et al., 2013). García-Ruiz y Lana-Renault (2011) señalan que el pastoreo afecta al proceso de sucesión de distintas maneras. En unos casos retrasa la transición a la fase de matorral, al inhibir el crecimiento de los matorrales, mientras que en otros casos puede facilitar la colonización de los matorrales por el aporte de semillas con el estiércol, o por la competencia entre herbáceas. Lo expuesto aquí es una breve síntesis sobre la enorme complejidad de las interacciones entre pastoreo y sucesión vegetal (ver, por ejemplo, Chauchard et al., 2007 en un estudio en el sur de los Alpes franceses; y Lasanta, 2010 sobre la ganadería extensiva en áreas de montaña).

En un campo abandonado es muy frecuente que los matorrales penetren poco después del abandono, dando lugar a una segunda fase de la sucesión vegetal, caracterizada por el dominio de matorrales. En el proceso de sucesión se observa un patrón de colonización, en el que los matorrales van progresando desde los márgenes del campo, de forma que en la parte externa aparecen sucesivas orlas de matorrales y en el centro queda una cubierta de herbáceas. La penetración y la persistencia de una densa cubierta de matorrales depende de i) las condiciones locales (especies colonizadoras, fuentes de semillas próximas, topoclima); ii) de la actividad humana (frecuencia de incendios, desbroce de matorrales, pastoreo más o menos intenso) y iii) de la calidad del suelo (Dunjó, 2003; Arbelo et al., 2006; Lasanta et al., 2019a).

En suelos con fertilidad escasa penetran especies que tienen la capacidad de mejorar el suelo. En suelos pobres del Pirineo se ha comprobado que se introducen inicialmente especies fijadoras de nitrógeno (Genista scorpius y Echinospartum horridum, principalmente), lo que favorece la expansión de otros matorrales (Buxus sempervirens, Juniperus communis, Crataegus monogyna, Prunus spinosa, 
Rosa sp.), que continúan con el proceso de sucesión (Molinillo et al., 1997). El ritmo temporal de la sucesión puede estar muy influido por la proximidad o lejanía de semillas, así como por los vectores animales que las transportan (Martínez-López et al., 2019). La presencia de especies ruderales próximas al campo abandonado es un factor esencial para que el proceso de sucesión vegetal sea rápido (Pedroli et al., 2006). Para Pugnaire et al. (2006) la colonización vegetal en condiciones ambientales semiáridas es controlada por la dispersión de semillas y la variabilidad climática, que genera eventos de reclutamiento temporalmente separados. Escribano-Ávila et al. (2016) señalan que la dispersión de semillas por diferentes especies animales está modulada por la heterogeneidad ambiental y la estructura del ecosistema. Comprobaron que los zorzales (Turdus spp), el zorro rojo (Vulpes vulpes) y la garduña (Martes foina) dispersan una gran cantidad de semillas, mientras que los conejos (Oryctolagus cuniculus) y las ovejas, muy pocas. López-Bao et al. (2015) comprobaron la efectividad del zorro rojo en la dispersión de semillas a larga distancia, aumentando la conectividad entre las poblaciones de plantas, lo que favorece la colonización e impulsa el proceso de sucesión vegetal. Fedriani et al. (2017) estudiaron cómo la plantación de árboles frutales podría contribuir a estimular la dispersión de semillas por los animales, y con ello acelerar la restauración de tierras abandonadas. Comprobaron que plantar árboles de forma masiva (alta densidad de árboles) es menos eficiente que plantarlos de forma regular o aleatoria. Gómez-Aparicio et al. (2004) comprobaron en el sureste de España que a partir de arbustos pioneros se puede generar un bosque. Por otro lado, la presencia de árboles y arbustos en los campos abandonados puede facilitar la colonización vegetal, tanto indirectamente, actuando como "perchas" donde los pájaros reposan y aportan semillas al suelo (bien por regurgitación, bien por defecación), como directamente, mejorando las condiciones microambientales bajo planta (mayor humedad, menor estrés y mejor regulación hídrica, mayor porosidad del suelo), lo que favorece la germinación de semillas (Verdú y García-Fayos, 1996 y 1998; Puigdefábregas, 2005).

Las condiciones microtopográficas pueden resultar un factor esencial en el ritmo de la sucesión vegetal. Incluso en campos aparentemente homogéneos hay microhábitats relacionados con la topografía, el microclima, las condiciones edáficas y la redistribución de nutrientes por la fauna y flora (Cañadas et al., 2010). Además, la heterogeneidad del suelo es un elemento básico para las interacciones (competencia o facilitación) entre plantas, especialmente en ambientes con estrés hídrico, condicionando los patrones de distribución de plantas y comunidades (Cañadas et al., 2010). Rey-Benayas et al. (2005) comprobaron que el éxito de las plántulas de Quercus requiere un entorno poco competitivo de malas hierbas durante el periodo anterior a la primera estación seca. Una vez que las plántulas se establecen, un ambiente sombreado les resulta muy beneficioso.

Por otro lado, la recuperación edáfica y vegetal tras el abandono conduce a una mayor heterogeneidad a pequeña escala (especialmente en ambientes subhúmedos y semiáridos), ya que no todas las áreas siguen el mismo ritmo, generándose patrones en los que las áreas con vegetación cada vez tienen mejor suelo (Ruiz-Sinoga et al., 2011). En la media montaña mediterránea se ha observado que las laderas solanas presentan mayor estrés hídrico que las umbrías, ralentizando el proceso de colonización (Vicente-Serrano et al., 2005). Por otro lado, los procesos de erosión son más frecuentes e intensos en las laderas solanas, como se comprobó en el Pirineo Central, lo que constituye un factor muy limitante para la recuperación vegetal (Ruiz Flaño, 1993). Shakesby (2011) atribuye la mayor erosión en las laderas solanas a la mayor frecuencia de incendios, a una recuperación de la vegetación más lenta y a una mayor propensión de estas laderas a la erosión por la menor estabilidad de los agregados. Poyatos et al. (2003) comprobaron en el Pirineo catalán que las terrazas localizadas en laderas umbrías y las que tenían más pendiente se forestaban antes.

En la fase de matorrales es frecuente que la sucesión vegetal quede interrumpida, destacando como causas los incendios forestales y la eliminación de matorrales mediante quemas prescritas y desbroce. La acumulación de combustible, como consecuencia de la recolonización vegetal tras el abandono de tierras, favorece la frecuencia de incendios (Pausas, 1999; Duguy et al., 2007; Pausas \& Fernández-Muñoz, 2014; Moreno et al., 2014). Por otro lado, el fuego impulsa la homogeneización del paisaje, creando una retroalimentación positiva en la propagación de nuevos incendios (Loepfe et al., 
2010). Las zonas afectadas ven truncado el proceso de sucesión, retrasando la recuperación de la cubierta vegetal y que ésta presente menos riqueza y cobertura, debido a que las especies germinadoras se adaptan mejor que las rebrotadoras (Gallego Fernández et al., 2004). Los incendios tienen otras consecuencias, como el incremento de las tasas de erosión y la pérdida de fertilidad del suelo (Cerdá y Lasanta, 2005), lo que ralentiza la colonización vegetal y favorece la invasión de especies adaptadas al fuego. Con ello se inducen procesos de retroalimentación que conducen a cambios profundos en los ecosistemas y paisajes (Grigulis et al., 2005). El fuego puede generar distintos microambientes en un mismo campo. En este sentido, Cammeraat et al. (1999) comprobaron que en los lugares donde se produce concentración de cenizas y mantillo la vegetación arbustiva rebrota o germina posteriormente, mientras que en otras zonas se instalan procesos de erosión que degradan el suelo y limitan mucho la colonización vegetal.

En otras ocasiones, las políticas de gestión impulsan la eliminación de matorrales para evitar la acumulación de combustible y favorecer la regeneración de pastos, utilizando métodos como las quemas prescritas o controladas y el desbroce con medios mecánicos (Komac et al., 2013; Lasanta et al., 2015). La combinación de pastoreo y eliminación recurrente de matorrales truncan la sucesión vegetal, de manera que los campos no pasan de la fase inicial de dominio de las herbáceas (García-Ruiz y LanaRenault, 2011; Lasanta et al., 2019b).

La calidad del suelo es un factor esencial en el ritmo temporal de la sucesión vegetal. Esta es rápida en suelos profundos y ricos en materia orgánica. En suelos fértiles, sin estrés hídrico y con un bosque próximo al campo abandonado, se puede alcanzar un bosque joven en 2-3 décadas. Ello ha ocurrido en zonas de clima atlántico y en laderas del Pirineo (García-Ruiz y Lana-Renault, 2011; RubioBalducci, 2019).

Por lo general, la sucesión vegetal en tierras agrícolas abandonadas mejora la calidad del suelo. En campos abandonados del Sureste español, con suelos relativamente buenos de substrato calizo, se comprobó que en un corto periodo de tiempo (4-10 años) se produce un aumento significativo en el contenido de materia orgánica, en la estabilidad de agregados, la permeabilidad y la capacidad de retención de agua (López-Bermúdez et al., 1996). En un estudio comparativo de efectos del abandono en las provincias de Valencia, Murcia y Málaga (Romero Díaz et al., 2017) se puso de manifiesto que la recuperación de la vegetación contribuyó a la mejora en la calidad del suelo desde el punto de vista hidrológico, edafológico y geomorfológico. En el Maestrazgo de Castellón se observó que el abandono de tierras mejoraba el contenido de materia orgánica del suelo y la estabilidad de los agregados, con un notable incremento de su tamaño (Ruecker et al., 1988). Por su parte, Nadal et al. (2009) también observaron el incremento de la fertilidad del suelo a medida que la cubierta vegetal progresaba. Estos autores señalan que no es un proceso lineal, sino que está muy condicionado por la topografía y la fertilidad previa al abandono, así como por la gestión posterior al abandono, sobre todo por perturbaciones como el fuego. No obstante, debe tenerse también en cuenta que si los campos contaban con una adecuada estructura y contenido en nutrientes cuando se dejaron de cultivar, el abandono puede suponer inicialmente una disminución en los niveles de fertilidad, necesitándose más de 50 años para recuperar la correspondiente a la etapa agrícola. Nadal-Romero et al. (2016a) estudiaron en el Pirineo cómo evolucionan las propiedades y calidad del suelo en campos abandonados con gestión pasiva (matorrales de sucesión vegetal) y activa (reforestación con pinos). Sus resultados muestran que las propiedades del suelo evolucionan lentamente en ambos casos, a pesar de ser un clima mediterráneo subhúmedo, y que la reforestación no aceleró la recuperación de las propiedades y calidad del suelo respecto a la sucesión vegetal.

Sin embargo, una cubierta de matorrales puede permanecer durante muchas décadas en campos con suelos mal estructurados o afectados por procesos de erosión severos, aunque la precipitación sea suficiente para el desarrollo forestal y haya árboles próximos. Pueyo y Beguería (2007) comprobaron que en la media montaña mediterránea se produce un decaimiento en las tasas de sucesión vegetal, una vez que los mejores campos han sido colonizados. Lo más habitual es que los campos posean suelos de 
baja calidad cuando se abandonan, como consecuencia del agotamiento de la fertilidad en su fase de cultivo. En el Pirineo central se ha comprobado que los campos cultivados mediante un sistema de rozas (articas) tienen enormes dificultades para que progrese la vegetación, debido al agotamiento del suelo en la fase de cultivo. En estas condiciones los campos se mantienen durante décadas (más de 80 años) con muchas áreas de suelo desnudo, abundante pedregosidad y algunas matas aisladas de matorral (Lasanta et al., 2017b). Casalmiglia et al. (2017) señalan que los suelos de terrazas recientemente abandonadas poseen bajo contenido en materia orgánica y baja masa microbiana como herencia de su pasado agrícola. La calidad del suelo evoluciona en función de la relación que se establece tras el abandono entre sucesión vegetal y degradación del suelo (Lasanta et al., 2019a). Si domina el proceso de sucesión vegetal, la calidad del suelo mejora con el incremento del contenido de materia orgánica y la biomasa microbiana, la disminución de la densidad aparente y una mayor estabilidad de agregados, lo que favorece mayores tasas de infiltración y de humedad del suelo (Bautista Cruz et al., 2007; Lasanta et al., 2005; Lesschen et al., 2008a; Zornoza et al., 2009). Con estas condiciones, los procesos de erosión superficial (erosión laminar y rills) encuentran más dificultades para instalarse (Cerdà, 1996; Lasanta et al., 2001; Cantón et al., 2011), si bien las raíces pueden favorecer la acumulación de agua en el suelo e incrementar los movimientos en masa (Cammeraat et al., 2005). Si, por el contrario, la vegetación no penetra en el campo abandonado o avanza muy lentamente se instalan procesos de erosión muy activos, con la consiguiente pérdida de calidad del suelo (Pardini y Gispert, 2006; Romero Díaz et al., 2007).

La recuperación de la productividad del suelo y el progreso de la sucesión vegetal tienen mucho que ver, además, con las condiciones climáticas (Otto et al., 2006). En un clima húmedo o subhúmedo la sucesión vegetal es rápida, alcanzándose una cubierta de bosque joven en 30-40 años (Pardini et al., 1991; Lasanta et al., 2015; Peña-Angulo et al., 2019), mientras que en ambientes áridos y semiáridos es muy lenta, con un cubrimiento que con frecuencia no alcanza el $60 \%$ en campos de elevada edad de abandono (Ries et al., 2000; Lesschen et al., 2008a; Ruiz-Sinoga y Martínez-Murillo, 2009), y donde difícilmente se alcanza el estadio de bosque joven (Bonet y Pausas, 2004; Rey Benayas et al., 2015). Otra diferencia interesante entre áreas con diferentes condiciones climáticas es que en campos sin estrés hídrico la sucesión vegetal progresa de forma uniforme, mientras que en los que sufren estrés hídrico se desarrollan patrones de vegetación donde alternan manchas con cubierta vegetal y otras de suelo desnudo (Cammeraat y Imeson, 1999; Puigdefábregas, 2005). Allí donde los suelos tienen mayor capacidad de infiltración la vegetación progresa, estableciéndose una retroalimentación positiva entre agua y vegetación, ya que debajo de las plantas se incrementa la materia orgánica, la agregación y porosidad del suelo, lo que favorece mayores tasas de infiltración (Bergkamp et al., 1996; Cammeraat y Imeson, 1999). Por el contrario, en las áreas con suelo desnudo el suelo se compacta, las costras superficiales aumentan y la infiltración disminuye (Lesschen et al., 2007, 2008a; Moret et al., 2011), generándose una retroalimentación negativa entre la ausencia de vegetación, la disminución de la infiltración, el incremento de la escorrentía superficial y la degradación del suelo por erosión (Molinillo et al., 1997; Nadal et al., 2009). En el sureste español se ha comprobado que algunas plantas (Stipa tenacissima y Artemisia herba-alba) favorecen la infiltración del agua a través de sus raíces para reducir la evaporación y asegurar el mantenimiento de la cubierta vegetal, lo que contribuye a mejorar la calidad del suelo (Cammeraat et al., 2010). Otro aspecto a considerar, de gran importancia en medios semiáridos, es el papel que ejerce la litología. En la región de Murcia se ha estudiado el abandono en diferentes litologías y se ha comprobado una ralentización de los procesos de colonización en sustratos poco coherentes (como pueden ser las margas) y en condiciones climáticas más secas (piso Termomediterráneo), aunque es de mencionar que en estos ambientes las comunidades de flora y fauna existentes, por su singularidad, tienen un interés especial (Zapata et al., 2016).

La influencia de la fertilidad del suelo en la colonización vegetal se ha demostrado de manera empírica en ambientes semiáridos. En las proximidades de Zaragoza, tanto el añadido de abono orgánico (estiércol de oveja) como inorgánico (abono químico con $15 \%$ de $\mathrm{N}, 15 \%$ de $\mathrm{P}$ y $15 \%$ de $\mathrm{K}$ ) produjo una colonización vegetal más densa (cobertura entre el 75-80\%) que en las parcelas en las que no se añadió fertilizante (cubertura aproximada del 40\%) (Lasanta et al., 2000). En la Estación Experimental 
del Teuralet (Sierra de Enguera, este de España) el añadido de paja de avena contribuyó a incrementar el carbono orgánico en el suelo, la biomasa microbiana y la actividad y abundancia de hongos, mejorando la estructura del suelo (García-Orenes et al., 2012) y disminuyendo las tasas de erosión (García-Orenes et al., 2013). La importancia de la fertilidad del suelo en el proceso de colonización sugiere que la precipitación media no es el principal limitante para el desarrollo de la cubierta vegetal en medios semiáridos, sino que posiblemente el nivel de nutrientes puede ejercer mayor control en el crecimiento de la cubierta vegetal que la escasez de agua (Lasanta et al., 2000).

\section{El paisaje de campos abandonados y el suministro de servicios ecosistémicos.}

La mayoría de los estudios sobre el paisaje tradicional del medio rural español, aunque especialmente de las áreas de montaña, concluyen que era un paisaje que mostraba cierta complejidad de usos y cubiertas del suelo, con un alto grado de fragmentación y heterogeneidad, que dibujaba un paisaje en mosaico con alternancia de usos en manchas de pequeño tamaño, unidas por corredores ecológicos (Puigdefábregas y Fillat, 1986; Nogués-Bravo, 2006, entre otros). Los cambios de uso del suelo, marcados sobre todo por el abandono de tierras y la sucesión vegetal, muestran una leve simplificación del patrón tradicional, ya que el paisaje conserva un grado de complejidad bastante elevado, como herencia de su pasado con fuerte presencia de actividades humanas. No obstante, tras el abandono agrícola el paisaje pierde coherencia y heterogeneidad, caminando hacia un paisaje más simplificado, desfragmentado y muy homogéneo, donde las manchas son de tamaño mayor que en el paisaje tradicional y menor la diversidad de usos y cubiertas del suelo (Lasanta et al., 2005; Vilà Subirós et al., 2009; Arnáez et al., 2011; Heredia et al., 2013).

Estos cambios de paisaje tienen enormes implicaciones en el suministro de servicios ecosistémicos a la sociedad (Morán-Ordoñez et al., 2013; García-Llamas et al., 2019; van Leeuween et al., 2019). De ahí, que la gestión de las tierras abandonadas sea una cuestión crucial, mucho más en España, por la amplia extensión que ocupan, especialmente en áreas de montaña y ambientes semiáridos. La gestión condiciona la cantidad y calidad de los recursos hídricos, la protección del suelo, el secuestro y stock de carbono, los niveles de biodiversidad, la prevención de riesgos (incendios principalmente), los recursos pastorales y con ello el mantenimiento de la ganadería extensiva, la estética del paisaje y la actividad turística (Conti y Fagarazzi, 2004; Bernués et al., 2014; Lasanta, 2019).

El mundo científico y los gestores del territorio se debaten entre dos posturas: no intervenir en las tierras abandonadas (gestión pasiva o rewilding) o realizar una intervención activa para su restauración ecológica y reincorporación al sistema productivo (Conti y Fagarazzi, 2005; Lasanta et al., 2015). El rewilding es el proceso que permite pasivamente la invasión leñosa (sucesión secundaria) y, según sus partidarios, la reintroducción de grandes mamíferos con el fin de mejorar la biodiversidad y restaurar los paisajes originales después de siglos de actividad humana (Navarro y Pereira, 2012; Pettorelli et al., 2017). Otros científicos, por el contrario, se inclinan por una intervención moderada en el territorio, con el fin de crear un paisaje en mosaico, más parecido al paisaje tradicional o cultural, donde en el territorio se distribuían en alternancia bosques, matorrales y pastos. Se trata de restaurar ecosistemas y paisajes degradados con apoyo del desbroce de matorrales y la ganadería extensiva (Bauer et al., 2009; Regos et al., 2016; García-Ruiz et al., 2020), con el fin de impulsar el desarrollo local y mantener algunos de los muchos servicios ecosistémicos que aportaban los paisajes tradicionales (Kozak et al., 2017). La Figura 2 esquematiza los efectos de los paisajes dominantes en tierras abandonadas en los principales servicios ecosistémicos. 


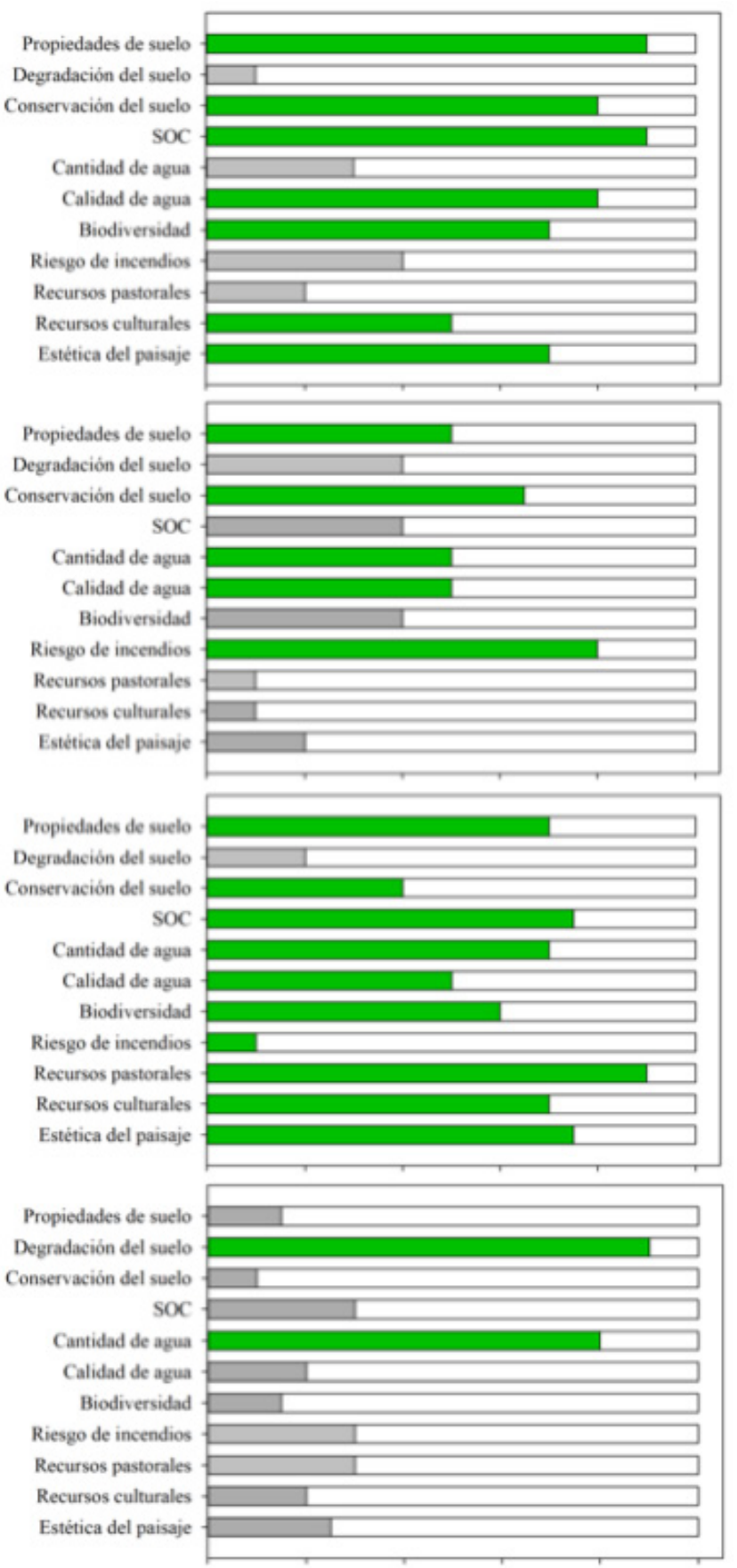

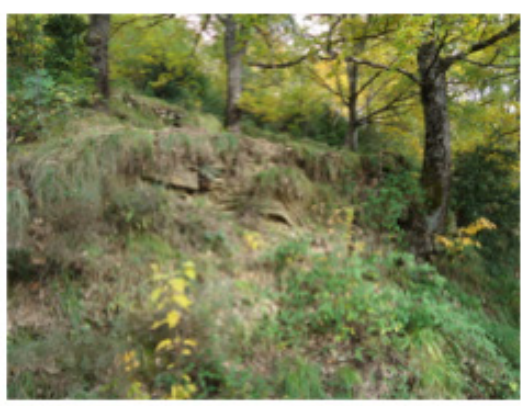

A. BOSQUE

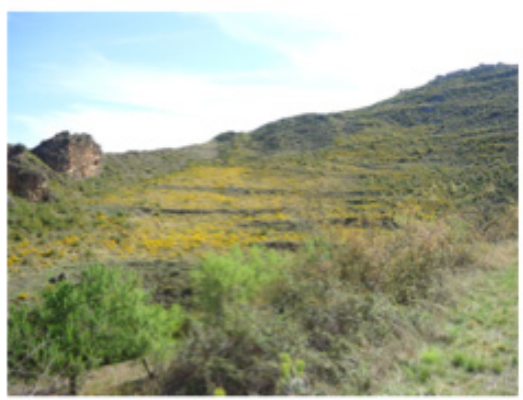

B. MATORRAL

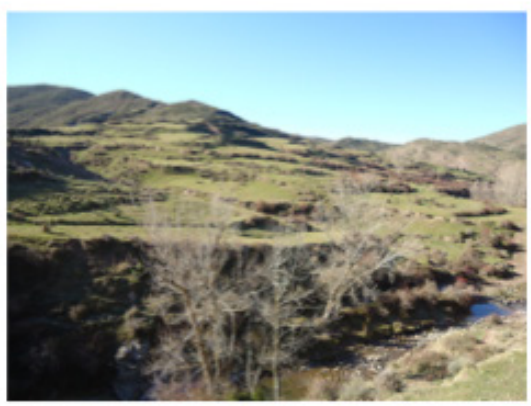

\section{PASTO MADURO}

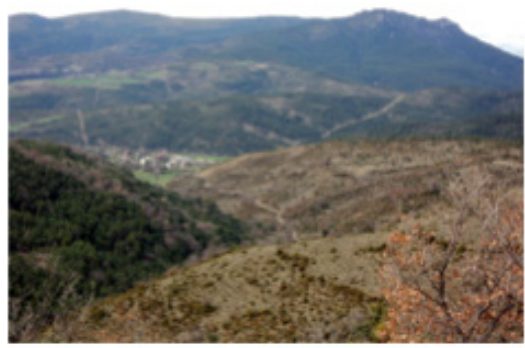

D. SUELO DESNUDO

Figura 2. Servicios ecosistémicos suministrados por los paisajes dominantes de campos abandonados. Foto A. Bosque de quejigos en bancales abandonados en Bestué (Pirineo Central). Los bancales se cultivaron con cereales hasta los años setenta del siglo XX. El proceso de revegetación ha sido muy rápido por la buena fertilidad del suelo y la presencia de ejemplares de Quercus faginea en los lomos del bancal. Foto B. Aliagas en una ladera abandonada en Jubera (Sistema Ibérico, La Rioja). Los campos se cultivaron con cereales hasta los años sesenta del siglo XX. Genista scorpius es el matorral dominante en las primeras fases de la sucesión vegetal en suelos básicos. Entre los 25 y 60 años aproximadamente presenta un cubrimiento muy denso, dejando paso después a otros matorrales y arbustos más exigentes en calidad del suelo. Foto $\boldsymbol{C}$. Ladera de pastos en Vadillos (Sistema Ibérico, La Rioja). La ladera se cultivó hasta los años setenta del siglo XX. El pastoreo continuado con distintas especies ganaderas ha generado un pasto denso, diverso y elevado valor pastoral. Foto D. Ladera de campos abandonados en Aisa (Pirineo Aragonés). Estos campos se cultivaron mediante el sistema de rozas (denominado artigueo en el Pirineo). Desde hace más de 80 años los campos se dejaron de cultivar, pero la sucesión vegetal apenas ha avanzado como consecuencia del agotamiento del suelo en la fase de cultivo y la instalación de procesos de erosión que aún continúan activos. 


\subsection{Servicios de aprovisionamiento}

Los campos abandonados tienen un elevado potencial para suministrar a la sociedad algunos servicios esenciales como agua y pastos.

\subsubsection{El suministro de agua}

La cubierta vegetal es un factor esencial en la regulación de los recursos hídricos (cantidad y calidad), mucho más en un escenario de precipitaciones irregulares e incremento de las temperaturas (García-Ruiz et al., 2011; Otero et al., 2011). En ambientes mediterráneos, donde el agua es más bien escasa, la gestión de las tierras abandonadas es fundamental para asegurar su abastecimiento. Hay que tener en cuenta que es en las montañas donde se generan la mayor parte de los recursos hídricos, actuando como islotes de humedad rodeados de tierras subhúmedas y semiáridas (Viviroli et al., 2003; García-Ruiz et al., 2011). En España existe abundante bibliografía sobre esta cuestión, coincidiendo en que la expansión superficial de la vegetación y el incremento de su densidad disminuyen los recursos hídricos (Gallart y Llorens, 2004; Cantón et al., 2011; García-Ruiz et al., 2011, entre otros). Un estudio de Gallart y Llorens (2002) sobre la evolución de la cubierta vegetal en España en las últimas décadas y sus efectos en los recursos hídricos pone de manifiesto un notable incremento de la primera y una disminución significativa de la aportación anual de los principales ríos. Beguería et al. (2003) analizaron la evolución de los caudales de varios ríos pirenaicos entre los años 1945 y 1995; observaron una disminución de los caudales en ríos no alterados por extracciones de agua, lo que explican por el aumento de la cubierta vegetal debido al abandono de tierras desde mediados del siglo XX. MoránTejeda et al. (2010) estudiaron la evolución hidrológica de las cuencas de cabecera del Duero observando una tendencia negativa generalizada en la escorrentía anual, debido a las pérdidas en el flujo de invierno y primavera. López-Moreno et al. (2011) estudiaron las tendencias climáticas e hidrológicas en 88 subcuencas de la cuenca del Ebro durante el periodo 1950-2006. Los resultados mostraron la disminución de los caudales en la mayoría de las subcuencas, que se atribuye a la combinación entre cambio climático y revegetación por el abandono de tierras, con el consiguiente incremento de la evapotranspiración.

La expansión de la cubierta vegetal, tras el abandono de la tierra, tiene una influencia directa en el ciclo hidrológico, condicionando la interceptación, infiltración, generación de escorrentía y conectividad de las laderas con los arroyos. La cubierta forestal, especialmente la arbórea y, en menor medida, la de matorrales, ejerce un papel amortiguador que, a través de los procesos de partición de la lluvia, reduce de forma considerable la cantidad de precipitación que llega al suelo (Belmonte Serrato y Romero Díaz, 1999). Además, modifica positivamente determinadas propiedades del suelo como la estructura y el contenido de materia orgánica, lo que favorece el incremento de la porosidad del suelo, el desarrollo de macroporos y la infiltración (Martínez-Fernández et al., 1995).

El primer efecto hidrológico de la cubierta vegetal es determinar la cantidad de agua de lluvia que no llega al suelo (interceptación neta). Esta puede ser muy elevada en función de la especie arbórea y de la época del año, con independencia de la intensidad y duración de las precipitaciones (GarcíaOrtiz, 2006). En bosques jóvenes de los Pirineos orientales, surgidos a partir de la colonización en campos abandonados, la interceptación representó aproximadamente el $24 \%$ de la precipitación anual (Llorens et al., 1997a). En áreas cubiertas por Pinus sylvestris, la interceptación puede variar entre el 8 y 48\% de la precipitación registrada, según se ha observado en distintas regiones españolas (Alvera, 1976 y 1977; Santa Regina, 1995; Tarazona et al., 1996; Llorens et al., 1997). En la cuenca de San Salvador (Valle de Aísa, Pirineo aragonés) los valores de interceptación fueron del 22\% para Pinus sylvestris y del $23 \%$ para Quercus faginea, tras un estudio realizado durante dos años (Serrano-Muela et al., 2012). Por otro lado, los mismos autores comprobaron que el agua que llega al suelo se reduce alrededor de un $10 \%$ más si debajo de la cubierta arbórea hay un estrato arbustivo (Serrano-Muela et al., 2012). En un estudio sobre la interceptación de la lluvia por la vegetación en España (Belmonte 
Serrato y Romero Díaz, 2013) en el que se recogen valores de interceptación de 15 áreas de estudio, se da un valor medio de 22,7 para diferentes especies de Pinus, 22,4 para Quercus y 18,3 para Fagus; respecto a especies de matorral, el valor medio de interceptación es de 29,9, lo que demuestra que el matorral, en general, supera al arbolado en pérdidas de agua por interceptación.

Una proporción importante del agua que llega al suelo se infiltra; la infiltración es mayor en suelos bien estructurados, con elevada porosidad y contenido de materia orgánica (Seeger y Beguería, 2003). Especial interés tiene la presencia de macroporos en los suelos, a lo que contribuye tanto una buena estructura como una alta densidad de raíces, especialmente si son de diversos grosores (de árboles, arbustos y plantas herbáceas), y también la actividad de la fauna dentro del suelo. Los macroporos funcionan como vías o caminos para acumular agua en las capas inferiores del suelo y en acuíferos. Bergkamp et al. (1996) comprobaron, en el sureste de España, que las raíces de los matorrales favorecían la infiltración y retención del agua por el suelo, evitando la escorrentía en distancias superiores a $1 \mathrm{~m}$.

Por otro lado, hay que tener en cuenta que parte del agua infiltrada es consumida por la vegetación. Gallart et al. (2002) y Serrano-Muela et al. (2008) demostraron la importancia del consumo de agua por los bosques, especialmente en verano, lo que reduce el contenido de agua en el suelo y la respuesta hidrológica de la cuenca de captación, que incluso puede no responder a lluvias intensas. En este sentido, Gallart et al. (2002) registraron varios casos de falta de respuesta hidrológica en verano después de lluvias de aproximadamente $50 \mathrm{~mm}$. Por su parte, García-Ruiz et al. (2008) compararon la respuesta hidrológica de tres cuencas con distintas cubiertas (bosque de frondosas y pinos; campos abandonados con matorrales y pastos; y cárcavas). La falta de respuesta hidrológica se hizo evidente en la cuenca forestal en verano, pese a la ocurrencia de fuertes tormentas; por el contrario, la cuenca de cárcavas (suelo desnudo) respondió de forma muy rápida, mientras que la de campos abandonados respondió más tardíamente y de manera moderada.

Se han realizado también estudios sobre los efectos hidrológicos de los campos abandonados cuando se encuentran en la fase de cubierta de matorrales. En la Estación Experimental "Valle de Aísa" (Pirineo central) se reprodujeron durante 20 años (1991-2011) usos del suelo que representan los sistemas tradicionales de cultivo (cereal fertilizado con abono químico, barbecho y cereal cultivado con el sistema de rozas o artigueo, es decir con añadido de cenizas como sistema de fertilización) y las diferentes etapas y alternativas del abandono (abandono a partir de cereal fertilizado con abono químico y de cereal cultivado con el sistema de rozas). Los resultados demuestran que el abandono implica una disminución clara de los coeficientes de escorrentía respecto a la fase de cultivo. Dicha disminución es paralela al proceso de sucesión vegetal, de forma que los coeficientes son más elevados cuando domina una cubierta de herbáceas (primeros años de abandono) que cuando el matorral (Genista scorpius, como especie dominante) va cubriendo la parcela, debido a que el estrato de matorral se superpone sobre el de herbáceas, lo que favorece la infiltración del agua y la disminución de la escorrentía. Por el contrario, una cubierta exclusiva de herbáceas incentiva que parte del agua de lluvia resbale sobre la hierba sin llegar al suelo (Nadal-Romero et al., 2013). Se comprobó también que el abandono de la parcela fertilizada producía menos escorrentía que la parcela abandonada tras su cultivo mediante el sistema de rozas, debido a un proceso de sucesión vegetal más rápido en la primera parcela (Lasanta et al., 2006).

En ambientes semiáridos también la gestión de la cubierta vegetal puede ser un factor esencial para los recursos hídricos. Bellot et al. (2001) simularon efectos de algunas políticas de gestión (reforestación de tierras y eliminación de vegetación mediante incendio) sobre el drenaje profundo y la escorrentía. Los resultados pusieron de relieve que la reforestación produciría una reducción tanto de la recarga del acuífero como de la escorrentía, mientras que un incendio forestal produciría los efectos contrarios. 


\subsubsection{El recurso pastoral}

Los campos abandonados forman parte de los itinerarios de pastoreo y, por lo tanto, son un recurso fundamental para alimentar a la ganadería extensiva (Molinillo et al., 1997; Reiné et al., 2014). Son escasos los trabajos que cuantifican el efecto del ganado sobre la vegetación y su dinámica en áreas de campos abandonados; pero todos ellos llegan a la conclusión de que la actual gestión ganadera (escasa diversidad de especies animales, poca carga ganadera y pastoreo muy poco gestionado; ver LasantaMartínez, 2014) es incapaz de detener el proceso de sucesión vegetal, lo que supone la pérdida de producción forrajera (Fernández-Giménez, 2015). Riedel (2007) y Riedel et al. (2013) registraron, en un estudio realizado durante 5 años en pastos arbustivos del Parque de la Sierra y Cañones de Guara (Prepirineo oscense), incrementos significativos en biomasa del estrato sub-arbóreo, tanto en áreas pastadas por ganado ovino (cargas medias de 0,15 Unidades Ganaderas Mayores, UGM/ha/año) como en zonas no pastadas, lo que condujo al embastecimiento del pasto. El pastoreo, en este caso, implicó una reducción en la dinámica de la vegetación, pero no la detuvo; el efecto difería notablemente entre especies vegetales. Casasús et al. (2007), en un estudio realizado en el Valle de la Garcipollera (Pirineo de Huesca), analizaron pastos con arbolado ralo pastoreados por vacas nodrizas y cargas de 2 UGM/ha/año. Comprobaron la eficacia del pastoreo en el control de la vegetación y su efectividad a la hora de conservar espacios forestales abiertos; vieron que el ganado es capaz de mantener estable la biomasa arbustiva, mientras que la ausencia de pastoreo produce una rápida e intensa densificación de la vegetación. Los mismos autores insisten en el papel positivo del ganado para mantener pastos productivos, lo que favorece el control de incendios y facilita el uso recreativo del territorio, una demanda cada vez más presente en la sociedad. En los dos trabajos anteriores se comprobó que el pastoreo evitó la acumulación de biomasa herbácea muerta, disminuyendo la combustibilidad y el riesgo de incendios. También se observó la mejora de la calidad de la hierba, de vital importancia tanto para animales domésticos como silvestres.

En la Cordillera Cantábrica se estudió el efecto del pastoreo con cabras domésticas como factor de control de varias especies de matorral (Cytisus scoparius, Rubus idaeus y Rosa sp.) representativas de áreas abandonadas. A partir de parcelas experimentales se analizó el efecto que ejerce el pastoreo con dos cargas ganaderas (4,5 cabras/ha/año y 9 cabras/ha/año) en el matorral y la obtención de pastos tras la quema, desbroce y poda de la comunidad de matorral. Los resultados ponen de manifiesto que el uso combinado de las cabras con apoyo de otras prácticas (quema, desbroce y poda) puede contribuir tanto a la transformación de áreas de matorral en pastos como al control del avance de los matorrales. El efecto es más positivo con carga doble ( 9 cabras/ha/año) que con carga simple (4,5 cabras/ha/año). El pastoreo de las cabras también cambia la distribución espacial de los matorrales, transformando una cobertura densa y continua en matas individualizadas, lo que permite incrementar la aportación de los matorrales a la dieta alimentaria del ganado y mejorar la accesibilidad del ganado al estrato herbáceo. Ello sugiere que mantener una estructura compleja de matorrales y pastos resulta beneficiosa para la alimentación de la ganadería extensiva y para mejorar la heterogeneidad del paisaje, incrementar la biodiversidad y controlar el riesgo de incendios (Álvarez-Martínez et al., 2013).

Bartolomé et al. (2000) comprobaron que el pastoreo de rebaños de ovino fue incapaz de detener la invasión del estrato arbustivo y mantener un paisaje abierto y diverso en la Reserva de la Biosfera del Montseny (Pirineo Catalán). A la misma conclusión llegan Komac et al. (2013) al comprobar el fuerte avance de Echinospartum horridum en los pastos subalpinos del Pirineo central, reclamando el uso combinado del pastoreo y la eliminación de matorrales por fuego o medios mecánicos. Gómez-García et al. (2011) concluyeron (a partir de parcelas experimentales) que el pastoreo con la actual presión, por sí sólo, no es suficiente para detener el avance de los matorrales en los ambientes atlánticos de Belate y Sierra de Aralar (Navarra). Estos autores aconsejan el uso simultáneo del pastoreo y el desbroce a ras del suelo en zonas de baja pendiente y alta cobertura vegetal, mientras que en áreas con pendientes medias o pronunciadas y baja densidad de cobertura vegetal concluyen que es más efectiva la combinación fuego - ganadería extensiva. Por otro lado, señalan que el equino resulta especialmente interesante para eliminar el matorral y abrir claros para la vegetación herbácea. 
Lo cierto es que los campos abandonados contribuyen muy poco a la alimentación del ganado en las últimas décadas por la presencia masiva de matorrales. García-Ruiz y Lasanta-Martínez (1993) estudiaron las fuentes de alimentación del ganado en tres valles pirenaicos (Aísa, Tena y Hecho), donde los campos abandonados ocupan amplias superficies (el 17,7\%, 4,4\% y 29,8\% del territorio, respectivamente), llegando a la conclusión de que su aportación a la dieta alimentaria del ganado era escasa: del $5 \%$ en Aísa, $1 \%$ en Tena y $2 \%$ en Hecho. Los autores atribuyen la escasa contribución a la existencia de una cubierta de matorral que impide el acceso del ganado al estrato herbáceo inferior, aunque este posee un potencial pastoral alto (Lasanta, 2005). Los campos cubiertos de matorrales dificultan, además, la conectividad entre pastos al interrumpir itinerarios de pastoreo (Valdelvira y Balcells, 1986).

Molinillo et al. (1997) estudiaron la oferta pastoral de los campos abandonados del valle de Aísa (Pirineo aragonés) seleccionando cuarenta campos con distinta edad de abandono (de 1 a 75 años). Comprobaron que el estrato herbáceo estaba compuesto por especies de mediana calidad forrajera (Achillea millefolium, Plantago lanceolata, Plantago media, Primula veris, Daucus carota, Centaurea jacea...) o buena calidad forrajera (Medicago lupulina, Trifolium pratense, Trifolium repens, Brachypodium pinnatum, Carex flacca, Dactylis glomerata, Festuca rubra...), pero que el estrato arbustivo (Genista scorpius, Rosa sp., Prunus spinosa, Crataegus monogyna...) impedía el acceso del ganado a medida que comenzaba a ser dominante.

En el valle del Leza (Sistema Ibérico Noroccidental), Rodríguez-Merino et al. (1998) estudiaron la producción pastoral en campos abandonados con suelos procedentes de litologías calcáreas y silíceas. Observaron que en las primeras dominan comunidades en la que la especie más representativa es $G$. scorpius y en las segundas Cistus laurifolius. Comprobaron también que los campos colonizados por $G$. scorpius ofrecían una productividad ligeramente superior a los de C. laurifolius, lo que vinculan con la mayor densidad del estrato herbáceo en los primeros campos, ya que la composición florística es muy parecida en ambas litologías. Por otro lado, observaron que los campos con mayor intensidad de pastoreo, sin llegar a exceder la capacidad de carga, alcanzaban mayor valor pastoral y lo mantenían durante varios años. En estas circunstancias el ganado detiene el avance de los matorrales y favorece la presencia de especies de mayor calidad forrajera. Además, el pastoreo frecuente del ganado permite aprovechar el pasto en el momento óptimo (cuando se alcanza la mayor calidad forrajera) (Montserrat, 1964). Por último, comprobaron que la productividad fluctúa a lo largo del año, alcanzando el máximo primario en primavera y el secundario en otoño. En verano desciende, especialmente en agosto, al igual que en invierno por parada del ciclo vegetativo.

Ferrer et al. (1995) estudiaron la influencia del pastoreo en bancales del Maestrazgo de la Comunidad Valenciana. Señalan que los bancales llegaron a ocupar un $21 \%$ de la superficie total, alcanzando en algunos municipios hasta el 35\%. A mediados del siglo XX se abandonaron, pastándose el $43 \%$ de ellos mientras que el $57 \%$ no se pastoreaban. Entre los resultados destacan que en los primeros años de abandono el suelo desnudo ocupa hasta el 35\% de la superficie, cubriéndose con mayor rapidez en los bancales no pastados, especialmente entre los 10 y 20 años de abandono. No obstante, comprobaron que al cabo de 40 años ya sólo quedaba un 10\% de suelo desnudo, tanto en bancales pastados como sin pastar. Los bancales pastados ven como su valor pastoral se incrementa de forma paralela a la edad de abandono, de forma que pasan de poder alimentar una carga ganadera inicial de 2,5 ovejas/ha/año a 3,5 ovejas ha/año. Por el contrario, los bancales no pastados experimentan una disminución brusca de su valor pastoral durante los 10 primeros años de abandono, un descenso más ligero entre los 10 y 30 años, para volver a disminuir bruscamente entre los 30 y 40 años, edad en la que se estabilizan admitiendo una carga de 1,2 ovejas/ha/año. Por otro lado, comprobaron que el riesgo de incendio era muy superior en los bancales no pastados. Los resultados obtenidos les llevó a concluir que sería conveniente empradizar (siembra de alfalfa, esparceta, dáctilo, Festuca alta o especies autóctonas de alto valor ganadero) los campos cuando dejan de ser cultivados para evitar problemas de erosión del suelo y favorecer el pastoreo del ganado. Además, señalan la conveniencia de complementar el pastoreo, 
dadas las bajas cargas recientes, con otras actuaciones como desbroces, resiembras con nulo o mínimo laboreo y pequeños abonados.

Errea y Lasanta (1994) estudiaron, en ambientes semiáridos del centro de la Depresión del Ebro, la contribución de campos retirados del cultivo (set-aside) como consecuencia de la PAC. Estimaron que los campos de secano eran capaces de alimentar entre 0,3 ovejas/ha/año y 1,1 ovejas/ha/año, en función de la edad de abandono y de la gestión tras la retirada. Los campos de regadío ofertaban mayor cantidad de pasto, admitiendo cargas ganaderas entre 1,1 ovejas/ha/año y 2 ovejas/ha/año. Los autores calcularon que el $10 \%$ del censo ganadero del área de estudio se alimentaba a partir de los campos abandonados debidos a la PAC. Además, comprobaron que la edad de abandono implicaba la disminución de la oferta forrajera tanto en secano como en regadío, lo que atribuyeron a la pérdida de humedad del suelo, la formación de costras, la menor densidad de la cubierta vegetal y la lignificación de algunas especies. Por otro lado, comprobaron la importancia de la gestión tras el abandono, observando que un laboreo anual con chisel (apero que realiza un laboreo ligero en la capa superior del suelo sin llegar a voltear la tierra) resultaba positivo para mantener la cubierta vegetal, al romper las microcostras superficiales y favorecer la infiltración del agua. También comprobaron que el abonado, tanto orgánico como químico, incrementaba la cubierta vegetal y con ello el potencial pastoral de los campos.

\subsection{Servicios de regulación}

Entre los servicios de regulación dos han ocupado más la atención de los estudiosos sobre campos abandonados: la erosión/conservación del suelo y el secuestro y almacenamiento de carbono por el suelo.

\subsubsection{Erosión/conservación del suelo}

El aumento progresivo de la densidad de la cubierta vegetal tras el abandono de campos de cultivo implica, generalmente, la disminución de la erosión del suelo respecto a la etapa de cultivo (García-Ruiz, 2010; García-Ruiz y López Bermúdez, 2009; García-Ruiz y Lana-Renault, 2011). Es una consecuencia lógica del efecto protector de la vegetación contra el salpicado de la lluvia (splash), el desprendimiento de partículas y la resistencia al avance de los flujos de escorrentía superficiales. Además, como ya se ha señalado, las propiedades del suelo tienden a mejorar algunos años después del abandono favoreciendo la infiltración del agua (Martínez-Fernández et al., 1995; Seeger y Beguería, 2003). En un amplio estudio sobre diferentes litologías en el sureste de España (Belmonte Serrato et al., 2016) se demostró como el abandono mejoró propiedades edáficas como la densidad aparente, estabilidad de agregados, tasa de infiltración, contenido en materia orgánica y, especialmente en margas (una litología muy erosiva) tras el abandono, se observó una tendencia a la mejora de la calidad de sus propiedades edáficas, lo que facilita la dinámica recolonizadora de la vegetación natural.

La erosión del suelo disminuye, generalmente, con la edad de abandono de los campos, a medida que la cubierta vegetal avanza y alcanza mayor densidad y una estructura más compleja. Este hecho parece sugerir que el problema está en los primeros años de abandono, cuando la vegetación no cubre el campo de forma completa (Dunjó et al., 2004). Sin embargo, el tema es mucho más complejo, ya que a veces los campos de mayor edad de abandono son los que tienen suelos más deteriorados y procesos de erosión más severos (rills, descalzamientos, procesos de piping), mientras los campos abandonados más recientemente presentan un mejor grado de conservación, dominando procesos de arroyamiento difuso débil, tanto sobre cubiertas herbáceas como de matorral (García-Ruiz y López-Bermúdez, 2009). Ruiz-Flaño et al. (1992) lo observaron en el Valle de Aísa (Pirineo aragonés), justificándolo por: i) las malas condiciones del suelo en los primeros campos abandonados, debido a que la mayoría de tales campos se cultivaron mediante el sistema de rozas, lo que dificultó el proceso de sucesión vegetal, y ii) 
porque fueron campos sometidos a fuegos recurrentes para regenerar pastos, lo que llevó a una degradación progresiva del suelo. En este sentido, Cerdà y Lasanta (2005) y Lasanta y Cerdà (2005) comprobaron, en el mismo Valle de Aísa, que tras un incendio se incrementan los flujos de escorrentía y las tasas de erosión, a la vez que se pierden cantidades importantes de nutrientes del suelo. Pasados unos años el matorral penetraba de nuevo y se frenaba la erosión, a la vez que se reproducían los problemas para el pastoreo, por lo que el campo se volvía a quemar. La repetición de este fenómeno es, muy probablemente, la causa de que algunos campos alcanzasen una alta degradación del suelo (Ruiz Flaño et al., 1992). En el valle próximo de Borau, Navas et al. (2005) utilizaron medidas de $\mathrm{Cs}^{137}$ para estudiar la redistribución del suelo en campos abandonados; comprobaron que las laderas solanas (las más utilizadas para el cultivo y las que sufrieron más quemas de matorral) tuvieron tasas de erosión más altas y un proceso de sucesión vegetal más lento.

Muchos de los estudios sobre erosión en campos abandonados se han realizado en laderas abancaladas (García-Ruiz et al., 1988; Rodríguez Aizpeolea et al., 1991; Arnáez, 2015, 2017; Gallart y Llorens, 1994; Arnáez y Ortigosa, 1997; Ruecker et al., 1998; Duiker et al., 2001; Lasanta et al., 2001; Cammeraat et al., 2005, Solé-Benet et al., 2010, entre otros). En bancales el proceso de erosión dominante son los desprendimientos en el salto o muro del bancal, formando cicatrices que deterioran la armonía del paisaje de las laderas abancaladas y favorecen la instalación de cárcavas con exportación de elevada cantidad de sedimentos. Además, las zonas internas de los bancales tienden a saturarse y a convertirse en áreas generadoras de escorrentía superficial, mientras que los sistemas de drenaje artificiales producen picos de avenida más rápidos e intensos (Gallart y Llorens, 1994). No obstante, hay que recordar que los deslizamientos también ocurrían durante la fase de cultivo tras lluvias muy intensas o cuando se registraban precipitaciones muy abundantes, pero el agricultor procedía rápidamente a reparar el muro y a rellenar el hueco de tierra desplazado (García-Ruiz et al., 1988). Sin embargo, el cese de las prácticas de conservación de los muros externos tras el abandono es una causa fundamental para el incremento de la erosión (Arnáez et al., 2015).

Lasanta et al. (2001) estudiaron la evolución geomorfológica de 86 bancales en Cameros Viejo (Sistema Ibérico). Confirmaron que el proceso de erosión más importante era el colapso de muros por pequeños deslizamientos (volumen medio: $3,3 \mathrm{~m}^{3}$ ), con un promedio total de $38,8 \mathrm{~m}^{3}$ por cada 100 metros lineales de muro de bancal. La mayor densidad de deslizamientos se observó en laderas cóncavas y parte baja de las laderas, coincidiendo con la mayor acumulación de agua, lo que favorece la saturación del suelo y, consecuentemente, la ocurrencia de movimientos en masa. A partir de la cicatriz del desprendimiento del muro externo el proceso puede avanzar por erosión remontante hasta generar pequeñas cárcavas. Los mismos autores señalan que algunas de las cicatrices de desprendimientos favorecen el paso del ganado de un bancal a otro, dibujando sendas en las que el pisoteo frecuente apelmaza el suelo y hace más efectivo el impacto de las gotas de lluvia y el arroyamiento superficial, lo que lleva a generar cicatrices muy activas en la evacuación de sedimentos. En la misma zona, GarcíaRuiz et al. (1988) mostraron que la densidad de desprendimientos en los muros de los bancales se relacionaba de forma positiva con la pendiente de la ladera y la altura del bancal (mayor en laderas más pendientes), y de forma negativa con la densidad de la cubierta vegetal, que es indicadora de la calidad de la parcela. Lesschen et al. (2008b) llegan a conclusiones parecidas en la Cuenca de Cárcavo (Murcia). Las tasas de erosión que reconstruyen para las terrazas abandonadas son de $87 \mathrm{Mg} \mathrm{ha}^{-1} \mathrm{año}^{-1}$.

En la cuenca del río Bergantes (Castellón), Pallarés Bou (1994) observó también la importancia de los deslizamientos en bancales; además comprobó el papel destacado que juegan la escorrentía subsuperficial y el desarrollo de piping en la aceleración de los movimientos en masa. Este autor señala que los muros no tenían como fin sujetar el terreno, ya que por sus características apenas proporcionaban defensa frente a los deslizamientos, sino que más bien trataban de proteger la superficie del talud frente a la erosión durante lluvias intensas.

Con precipitaciones intensas o muy duraderas, así como en áreas de fuerte acumulación de agua, pueden generarse debris flows, con longitudes de más de $100 \mathrm{~m}$, pudiendo afectar a toda una ladera 
abancalada. Costra et al. (2003) consideran que en estos casos la formación de debris flows se ve favorecida por las propiedades geotécnicas e hidráulicas del terreno, localizándose la línea de rotura en el contacto entre niveles de suelos con distintas características físico - químicas.

La influencia de la edad de abandono en la degradación de laderas abancaladas fue estudiada por Rodríguez Aizpeolea et al. (1991) en la vertiente sur de la Sierra de Mirant (Alicante). Estudiaron campos abandonados de 1, 5, 10 y 20 años mediante simulación de lluvia $\left(55 \mathrm{~mm} \mathrm{hr}^{-1}\right)$. Los resultados ponen de relieve que tanto la escorrentía como la concentración de sedimentos disminuyen con la edad de abandono, a medida que avanza el proceso de sucesión vegetal.

Una parte considerable de los estudios sobre erosión en bancales se ha dedicado a conocer el comportamiento hidromorfológico de la parte interna de bancales abandonados. Llorens et al. (1992) comprobaron en la Cuenca de Cal Parisa (Prepirineo Oriental) que la parte interna se satura con facilidad durante la estación húmeda, convirtiéndose en la principal área de generación de escorrentía. Los suelos son menos profundos y permeables en la parte interna que la externa del bancal. Eso hace que existan notables diferencias en la capacidad de infiltración dentro de cada terraza. Además, parte del agua infiltrada tiende a aflorar al pie del bancal, aumentando la probabilidad de que se sature la parte interna del bancal inferior. Por otro lado, Romero Martín et al. (1994) en el valle de Guiniguada (Gran Canaria), y Lasanta et al. (2001) en Cameros Viejo (Sistema Ibérico) comprobaron que en bancales sin rellano plano era frecuente que apareciesen procesos de arroyamiento laminar e incisiones. Hay que tener en cuenta, que los bancales se construyeron para favorecer la infiltración del agua y disminuir la escorrentía. Por ello, la génesis de un paisaje de terrazas conllevaba el trazado de una red de drenaje con pequeños canales dentro del campo que conducían las aguas sobrantes hacia otros laterales de mayor entidad (Gallart y Llorens, 1994), lo que reducía el riesgo de movimientos en masa. Sin embargo, el abandono de las terrazas implicó el cese de las tareas de mantenimiento de los drenajes, lo que incentiva la saturación del suelo y el incremento de los movimientos en masa en la parte externa del bancal (Gallart y Llorens, 1994; García-Ruiz y López Bermúdez, 2009).

En ambientes semiáridos la mayor o menor erosión del suelo está muy relacionada con las características (rapidez y composición vegetal, sobre todo) del proceso de sucesión vegetal (Cerdà et al., 1994; Ries et al., 1997 y 2000). Belmonte et al. (1999), Romero Díaz (2003) y Romero Díaz et al. (2007) comprobaron en la Región de Murcia que el abandono del cultivo sobre sustrato calizo llevaba a una rápida colonización por parte de las herbáceas y más tarde del matorral, con aumento de la materia orgánica del suelo, lo que resultaba positivo para la conservación del suelo y la diversidad del paisaje; por el contrario, sobre sustrato margoso el abandono implica la génesis de una costra superficial que hace muy lenta la colonización vegetal. Otro proceso de gran importancia que tiene lugar en campos de cultivo aterrazados y abandonados, sobre litología de margas, es el proceso de erosión por piping (López Bermúdez y Torcal, 1986; López Bermúdez y Romero Díaz, 1989). Un amplio estudio de 82 áreas, realizado en la Región de Murcia (Sánchez Soriano, 2012) ha permitido constatar como los procesos de erosión por piping aparecen ligados, predominantemente, a áreas de litología margosa, aterrazadas, de escasa pendiente y con importantes gradientes hidráulicos, generados por la altura existente entre las terrazas de cultivo. La altura entre parcelas condiciona la profundidad que alcanzan los pipes, mientras que la escasa pendiente de las parcelas favorece el estancamiento y la infiltración del agua en lugar de la escorrentía, provocando la formación de los conductos subsuperficiales (Romero Díaz et al., 2016).

Por su parte, Ries et al. (2000) comprobaron en el centro de la Depresión del Ebro que la colonización vegetal es muy lenta, de forma que a los 6-8 años de abandono no suele alcanzar el 50\%, e incluso hay campos con abandono muy antiguo que difícilmente superan el $60 \%$ de cubierta. Estos autores atribuyen las dificultades para el progreso de la vegetación al escaso contenido de agua y a la pobreza de los suelos. Por otro lado, observaron que la infiltración disminuye generalmente con el tiempo de abandono, debido a la reducción de la porosidad por falta de laboreo. A ello se une el pisoteo del ganado, que apelmaza el suelo y favorece la formación de una costra superficial. El incremento de la escorrentía, como consecuencia de la disminución de la infiltración, no acarrea, sin embargo, el 
aumento de las pérdidas de suelo, ya que la costra lo protege de la erosión, al menos hasta un umbral a partir del cual el aumento de la escorrentía implica un incremento de la erosión lineal (Ries et al., 1997). El pastoreo del ganado puede llevar a romper en ocasiones la costra y generar partículas de suelo que son fácilmente transportadas por el viento, incrementando las tasas de erosión eólica (Ries et al., 2000).

En definitiva, los estudios realizados sobre la evolución hidromorfológica de campos abandonados demuestran que se trata de un problema complejo, con resultados muy variables en función de los modelos de campos (abancalados, en pendiente, llanos), de las condiciones climáticas, que regulan el proceso de sucesión vegetal, de la gestión en su fase agrícola, que puede determinar la potencia y fertilidad del suelo en el momento del abandono, y de la gestión posterior al cese del cultivo, que influye a través de posibles perturbaciones (incendios, pastoreo,..). Además, la edad de abandono puede jugar un destacado papel en relación con la evolución de los parámetros edáficos y la colonización vegetal.

\subsubsection{El secuestro de carbono por el suelo}

El suelo constituye un almacén muy importante de carbono orgánico en la mayor parte de los ecosistemas (García-Pausas et al., 2017). En los últimos años, los estudios sobre el secuestro y almacenamiento de carbono en el suelo (SOC) se ha disparado, por sus implicaciones en la mitigación del cambio climático (Emran et al., 2012; Gabarrón-Galeote et al., 2015; García-Pausas et al., 2017). Los campos abandonados tienen un interés especial, al tratarse de áreas degradadas en recuperación, lo que ofrece un potencial significativo de secuestro de SOC (De Baets et al., 2013; Gispert et al., 2018). Así, Rodríguez-Martín et al. (2019) comprobaron en la isla de Mallorca que el SOC se duplicó entre 2006 y 2017 en laderas de bancales abandonados y en pastizales.

La capacidad de SOC guarda una estrecha relación con el ritmo temporal de sucesión secundaria y de mejora de las propiedades del suelo (Gabarrón-Galeote et al., 2015). Emran et al. (2012) comprobaron que la transformación de un espacio cultivado en pastos o en una cubierta forestal implica un incremento del SOC. Trigalet et al. (2016) señalan que la proporción de carbono lábil aumenta a lo largo del proceso de sucesión secundaria. Estruch et al. (2018) estudiaron los cambios en el balance de $\mathrm{C}$ en campos abandonados a lo largo de 100 años en un ambiente semiárido del SE de España. Señalan que fueron necesarios 65 años para que el sistema recuperase la cantidad de $\mathrm{C}$ de la zona no perturbada, lo que explican por la lentitud del proceso de sucesión vegetal. Segura et al. (2006) encontraron que en un clima semiárido los cambios de SOC en el suelo son muy lentos, por las condiciones climáticas y por los bajos aportes de materia orgánica al suelo. Por el contrario, un estudio realizado en la Sierra de los Filabres (sureste de España) concluyó que el C se acumula rápidamente durante los primeros 10-50 años tras el abandono; posteriormente los stocks de C se estabilizan (De Baets et al., 2013). Los mismos autores comprobaron que los stocks de SOC son muy variables (en suelos de $1 \mathrm{~m}$ de profundidad entre 3,16 y 76,44 t/ha), y que las áreas erosionadas que se recuperan tienen mayor capacidad para incrementar las tasas de secuestro, poniendo de relieve la importancia de gestionar campos abandonados.

Lasanta et al. (2020) estudiaron la sucesión secundaria y el SOC en campos abandonados del Sistema Ibérico. Señalan que el proceso de revegetación pasa por cinco etapas: pastos, matorrales $(C$. laurifolius, como especie dominante), arbustedos (J. communis, como especie dominante), bosque joven y bosque viejo, en ambos casos de Quercus pyrenaica). Los autores comprobaron que el contenido de SOC aumentó con la sucesión vegetal, especialmente en los $10 \mathrm{~cm}$ superiores del suelo, hasta la etapa de bosque joven, disminuyendo ligeramente el SOC en la etapa final de bosque maduro. Los autores explican el incremento de SOC por la acumulación de mantillo (hojarasca y necromasa sobre el suelo) conforme la sucesión secundaria avanza, y porque tanto los matorrales como los árboles incrementan la biomasa subterránea. El menor contenido de SOC en los bosques viejos que en los jóvenes lo justifican por el menor cubrimiento de los matorrales en el sotobosque de los primeros (cubrimiento del 14,3\%) que en los segundos (cubrimiento del 40\%). En el Pirineo, Nadal-Romero et al. (2016b) estudiaron qué implica la gestión activa (reforestación de campos abandonados con Pinus sylvestris y Pinus nigra) 
frente a la gestión pasiva (pastos y matorrales de sucesión). Comprobaron que los matorrales acumulan menos SOC que los pastos y pinares. El P. nigra es el uso del suelo con mayor contenido de SOC, seguido por los pastos, $P$. sylvestris y matorrales. Observaron también que las mayores diferencias entre los distintos usos del suelo se establecen en $\operatorname{los} 10 \mathrm{~cm}$ superiores. La evolución del contenido de SOC también se ha estudiado en laderas de pastos subalpinos abandonados en el Pirineo, comprobándose que el paso de pastizal a matorral implica una caída brusca de SOC en el suelo. En las etapas siguientes (bosque joven y maduro de $P$. sylvestris) el contenido de SOC se recupera parcialmente no llegando a alcanzar al del pasto subalpino, posiblemente por la abundancia en este último uso de raíces finas con gran capacidad para fijar $\mathrm{C}$ y por la ausencia de pastoreo en el resto de usos, lo que resta materia orgánica al suelo (Nadal-Romero et al., 2018).

\subsection{Servicios de apoyo}

La mayor parte de los estudios sobre los servicios de apoyo que pueden ofrecer los campos abandonados se han dedicado a cuantificar y valorar la biodiversidad, ya que algunos autores consideran que el abandono de tierras y la revegetación posterior son una oportunidad para la conservación de la biodiversidad (Regos et al., 2016). Por el contrario, otros autores opinan que es una amenaza, debida a la homogeneización del paisaje, la pérdida de prácticas de gestión y la desaparición de especies adaptadas a paisajes en mosaico (Otero et al., 2015). Los últimos señalan, además, que el supuesto carácter restaurador de lo que denominan transición forestal (abandono y revegetación) se basa en la subestimación de la importancia ecológica de los hábitats no forestales. Por el contrario, Regos et al. (2016) consideran que la re-naturalización o rewilding resulta positiva para la biodiversidad. Se basan en un estudio realizado en Galicia, en el que comprobaron que la revegetación de suelos desnudos tuvo como resultado que trece especies de aves de matorrales y bosques aumentaron de forma significativa, mientras que cuatro especies de ecotonos y hábitats abiertos mostraron una tendencia negativa. ReyBenayas et al. (2010) compararon los efectos en la riqueza de aves debidos a la sucesión secundaria (restauración pasiva) y la plantación de árboles (restauración activa). Llegan a la conclusión de que la gestión activa recolecta mayor número de especies (4,2 especies por cada parcela de 0,78 ha), que la restauración pasiva ( 3,5 especies por parcela). Sin embargo, los mismos autores insisten en la necesidad de combinar la restauración pasiva y activa con modelos mixtos con el fin de conciliar la producción agrícola, la restauración de la vegetación y la biodiversidad. Suárez-Seoane et al. (2002) señalan la preferencia de aves eurosiberianas por hábitats boscosos, mientras que las aves mediterráneas prefieren las áreas abiertas y los matorrales. Los mismos autores señalan que en la región mediterránea los mosaicos agrícolas de cultivo de baja intensidad mantienen los niveles más altos de diversidad de especies de aves prioritarias. Concluyen señalando que no debe darse por supuesto que el abandono de tierras agrícolas beneficia a la conservación. No obstante, Tella et al. (1988) señalan que en Monegros tanto la intensificación agrícola como el abandono de tierras son negativos para el cernícalo primilla (Falco naumanni), una especie esteparia amenazada a escala mundial.

Uno de los aspectos más abordados en el estudio de los campos abandonados es el análisis de la riqueza de especies (fauna y flora), porque de ella derivan su mayor o menor productividad, la estabilidad de los ecosistemas y la variedad genética. La diversidad biológica constituye, además, un parámetro valioso para determinar el interés ecológico de un territorio y para orientar la conservación de la naturaleza. Se la considera como un componente sintético de la organización de la estructura y función de los ecosistemas en relación con su origen y evolución (Pineda et al., 1981; Montalvo et al., 1993).

Se estima que aproximadamente el $50 \%$ de las especies de flora y fauna europeas dependen de hábitats agrícolas. De ahí, que el abandono de tierras tenga especial interés de cara a la biodiversidad (Delibes-Mateos et al., 2009a; Cohen et al., 2011). Robledano et al. (2016) en el estudio realizado en la Región de Murcia, consideran que el valor de conservación de los campos abandonados, para la flora leñosa y la avifauna, a menudo supera a superficies equivalentes de hábitat natural y bajo condiciones 
físicas rigurosas (sustratos poco coherentes y escasez de precipitaciones), los cultivos abandonados a menudo dan lugar a hábitats esteparios que albergan especies con alto valor de conservación en Europa.

Los campos abandonados reúnen algunas características que les hacen especialmente sensibles para la biodiversidad: amplia extensión, proceso de sucesión vegetal, que crea heterogeneidades en el territorio en diversidad biológica, suelos pobres y con frecuencia sometidos a dinámicas de degradación/recuperación, ser un espacio abierto rodeado habitualmente por un paisaje forestal y cese de la gestión antropogénica (Bernáldez, 1981; García-Ruiz y Lana-Renault, 2011; Lasanta, 2014).

La diversidad de especies vegetales parece estar condicionada por la edad de abandono, el mosaico vegetal circundante, las perturbaciones humanas tras el abandono, y los factores abióticos, especialmente litología, fertilidad de suelo, topoecología y clima. La edad de abandono condiciona la estructura del paisaje y su biodiversidad; se ha observado que habitualmente en las fases iniciales hay un incremento de la riqueza de especies, mientras que en fases posteriores la riqueza se reduce muchísimo (Suárez-Seoane et al., 2002; Vicente-Serrano et al., 2006). Montalvo et al. (1993) señalan el rápido incremento en la riqueza de especies como consecuencia de la entrada de herbáceas en un campo recién abandonado. Según Gutiérrez-Teira (1997) el abandono de cultivos implica, a corto plazo, un incremento en diversidad alfa (aumento de la diversidad en espacios reducidos) y una disminución en amplitud de nicho de las nuevas especies de plantas. Gómez et al. (2003) han demostrado el incremento en la riqueza de hormigas en una primera fase del abandono. Sin embargo, a medio y largo plazo la biodiversidad disminuye. Así, Suárez-Seoane et al. (2002) indican que el abandono agrícola representa una reducción en los niveles de diversidad paisajística y problemas derivados con la extinción de especies y la reducción de la biodiversidad. Por su parte, Lasanta y Vicente-Serrano (2007) comprobaron, en el Pirineo Central español, que la mayor diversidad vegetal se alcanzó coincidiendo con la penetración de forma dispersa de los primeros pies de matorral y de árboles. En esta etapa el estrato arbustivo convive con el herbáceo, compuesto por especies anuales y plurianuales, lo que se demuestra en una elevada variabilidad espacial en los valores de NDVI respecto a la situación previa. Por el contrario, en los años posteriores se asiste a una etapa de homogeneización con densificación de los matorrales y pérdida de heterogeneidad de la cubierta herbácea.

Entre las perturbaciones derivadas del cese de los cultivos destacan las producidas por la acción del ganado y los incendios forestales. Respecto al pastoreo se ha comprobado que la riqueza específica y la biodiversidad aumentan en áreas gestionadas con ganadería extensiva, que se considera como una perturbación moderada, frente a las áreas no pastoreadas (sin perturbación) o sobrepastoreadas (perturbación alta), que incentivan la degradación del suelo. No obstante, Blanco-Fontao et al. (2012) ponen en duda que haya siempre una relación directa entre ganadería extensiva y biodiversidad. Se basan en su estudio sobre la disminución del urogallo (Tetrao urogallus) en la Cordillera Cantábrica, un ave en peligro de extinción y considerada como una especie paraguas (en Biología de la Conservación, las especies paraguas son aquellas cuya protección implica la protección indirecta de otras con las que comparte hábitat) en los ecosistemas forestales de montaña. La bibliografía previa señalaba el abandono del pastoreo como la causa de la disminución del urogallo, asumiendo que la ausencia del ganado implica la disminución de la biodiversidad a través de la homogeneización de hábitats. Sin embargo, comprobaron que los censos ganaderos se habían incrementado en las últimas décadas, por lo que concluyen que los datos reales no respaldan el supuesto papel de la ganadería extensiva en la conservación de la biodiversidad.

El pastoreo actúa sobre la riqueza específica mediante la selección de especies por el ganado, lo que modifica las relaciones de competencia y cooperación entre las plantas (Ferrer y Broca, 1999). El pastoreo suele ejercer una tarea positiva, sobre todo, en los primeros estadios de sucesión vegetal, controlando por defoliación algunas especies más invasoras y favoreciendo el renuevo de las de mayor interés ganadero (Montserrat, 1964; Fillat et al., 2008); además, favorece la dispersión de semillas mediante endozoocoria (Malo y Suárez, 1996). En este sentido, Reiné et al. (2014) compararon en el Pirineo la diversidad florística entre prados de siega (laboreos de siembra, siega, pastoreo, fertilización 
y riego) y pastos en campos abandonados (tan solo pastoreo). Comprobaron que los prados reunían poca diversidad de especies, y elevada productividad, aunque la calidad del forraje era baja. Por el contrario, los pastos de campos abandonados presentaron alta diversidad de plantas y buena calidad del forraje, aunque la productividad era más baja que la de los prados. Por su parte, Tárrega et al. (2009) compararon en la provincia de León tres tipos de dehesas con diferente gestión: i) dehesas pastadas en la actualidad; ii) dehesas abandonadas (más de 20 años sin pastoreo) y iii) dehesas en las que se eliminaron arbustos tras el abandono. Los resultados señalan que las dehesas pastadas tienen mayor riqueza de especies y cobertura de especies anuales, mientras que las dehesas abandonadas presentan mayor cobertura de matorrales. Señalan, además, que la eliminación de arbustos (no seguida del pastoreo del ganado) puede causar la pérdida de riqueza de especies vegetales.

Los incendios son una perturbación importante en los paisajes de campos abandonados. Implican la eliminación de vegetación y la creación de espacios con hábitats abiertos y arbustivos (Zozaya et al., 2002). Los efectos sobre la biodiversidad parecen contradictorios. Gil-Tena et al. (2009) señalan que la expansión y maduración de los bosques como consecuencia del abandono agrícola anula los efectos negativos de los incendios sobre la distribución de aves forestales. Regos et al. (2016) consideran positivos los fuegos controlados para favorecer la creación de espacios abiertos en paisajes progresivamente cerrados derivados del rewilding, y con ello compaginar la presencia de especies de hábitats abiertos y cerrados. Vallecillo et al. (2008) precisan que los paisajes generados por fuegos controlados pueden mejorar potencialmente la resiliencia de especies amenazadas de hábitats abiertos mediante el aumento de la variedad de hábitats potenciales de ser utilizados.

En procesos de sucesión vegetal más que la cantidad de especies importa la relevancia de algunas especies concretas, por su rareza, función ecológica y significado biogeográfico y evolutivo. Fernández-Alés et al. (1992) y Laiolo et al. (2004) señalan que durante la sucesión secundaria en campos abandonados se pasa por diferentes fases o etapas. En la primera etapa aumenta la diversidad florística. A medio plazo, sin embargo, disminuye la diversidad y la biodiversidad porque las especies invasoras van arrinconando a las de mayor valor ecológico. En esta fase, muchas especies animales corren también peligro por falta de alimento o refugio; otras especies encuentran dificultades para sobrevivir por la desaparición de espacios abiertos que constituyen su hábitat natural. A largo plazo, las especies de alto valor ecológico desaparecen en favor de otras más competitivas, de menor valor. Ello causa una alta reducción de la diversidad de plantas y animales y una pérdida de riqueza. Bonet (2004) ha comprobado en el Sureste español que el pico de riqueza de especies se alcanza en campos abandonados a los 10-20 años, decreciendo posteriormente. También pone de relieve que el uso agrícola previo al abandono influye en la composición de especies y que los pájaros pueden contribuir a la dispersión de semillas incrementando la riqueza específica. Delibes-Mateos et al. (2009b) señalan que el abandono de la gestión tradicional y la revegetación posterior en las montañas de Andalucía han favorecido la expansión de especies de caza mayor y la disminución de las de caza menor. Sugieren que la conservación y regeneración de los paisajes agrícolas tradicionales debería ser una prioridad para la conservación de la biodiversidad de especies de caza menor. La misma propuesta realizan Salaverri et al. (2019) al estudiar en Galicia la disminución de la riqueza y abundancia de aves como consecuencia del abandono de paisajes humanizados. Comprobaron también que la altitud amortigua la pérdida de especies.

\subsection{Servicios culturales}

Todos los paisajes y ecosistemas humanizados guardan una riqueza histórica y cultural, al ser fruto de la simbiosis entre hombre y naturaleza durante siglos de aprovechamiento (Martínez de Pisón, 2009; García-Ruiz y Lasanta, 2018). Por otro lado, el medio rural se ha convertido en lugar de ocio para una parte importante de la población, que valora el paisaje como un servicio, y, por lo tanto, con connotaciones económicas (García-Novo, 2007). Dado que en muchas áreas de montaña la matriz del paisaje son las tierras abandonadas, y que sus cambios presentan un gran dinamismo, se entiende la 
preocupación de la sociedad y los gestores del territorio por los paisajes de espacios marginales (Lasanta et al., 2015).

Sin embargo, en España no se han realizado demasiados estudios sobre los servicios culturales del paisaje de campos abandonados, en contraste con la proliferación de estudios sobre otros servicios ecosistémicos, especialmente los relacionados con el suelo y la hidrología. La percepción y valoración del paisaje de campos abandonados es muy diversa. La sociedad los tiende a considerar en función de su calidad estética, familiaridad o experiencia con un paisaje concreto. También influyen la distancia y el recuerdo, así como la mayor consideración y aprecio que suele suponer la inminencia de la pérdida de un paisaje dado (García-Novo, 2007). El paisaje es contemplado de forma diferente por la población en función de los servicios que recibe o esperar recibir. Las personas vinculadas con el abastecimiento de productos primarios (ganaderos y agricultores) tienden a valorar menos los servicios culturales (paisaje, ocio, turismo) y de regulación (calidad del agua, control de la erosión del suelo, biodiversidad), que ejerce el medio natural, que los grupos cuya economía no depende de los recursos del territorio (Martín-López et al., 2007).

Se establece también una postura muy diferente entre los habitantes rurales y los urbanos. La población local considera que los paisajes deben ser multifuncionales para garantizar su sostenibilidad: deben producir alimentos, contribuir a la estructuración social y mantener valores ambientales; por el contrario, entre los foráneos dominan los que prefieren un paisaje muy natural y escasamente productivo. En este sentido, Rescia et al. (2008) comprobaron en el Parque Nacional de Picos de Europa (Cordillera Cantábrica) que la población local está a favor del turismo, por lo que puede suponer en la mejora de rentas; por el contrario, los representantes del Parque (gestores y trabajadores) consideran que el turismo es una amenaza para la conservación. Lasanta et al. (2016) estudiaron en Cameros Viejo (Sistema Ibérico), a partir de entrevistas a la población vinculada, la percepción sobre el abandono de tierras y el proceso de revegetación. Comprobaron que tanto los habitantes locales como foráneos perciben los cambios de paisaje derivados del abandono. Coinciden, además, en que el proceso de revegetación implica una mejor conservación del suelo frente a la erosión y un mayor riesgo de incendios por la acumulación de biomasa. Difieren, por el contrario, en los cambios hidrológicos que implica el proceso de revegetación. La población local señala que ahora hay menos producción de agua, pero que es un agua de mayor calidad, menos cargada de sedimentos. Entre la población foránea, una mayoría de los entrevistados sostiene que ahora hay más agua que en el pasado porque la mayor cubierta vegetal hace que llueva más. También manifestaron algunas matizaciones respecto a las consecuencias de la revegetación en la fauna, siendo la población local la que observa más claramente la progresión de la fauna silvestre. Respecto a la estética del paisaje, las respuestas son muy coincidentes en que el paisaje actual es más atractivo, si bien entre la población local hay quienes recuerdan el paisaje agrícola tradicional en mosaico, lo que los lleva a restar belleza al paisaje que ahora ven.

En un estudio realizado en la Alta Garrotxa (montaña mediterránea de Cataluña, caracterizada por la despoblación y el abandono durante las últimas décadas), Vila Subirós et al. (2009) concluyen que la valoración y percepción de los cambios de paisaje muestran una doble perspectiva. Una primera interpretación se basa en una visión antropocéntrica, valorando negativamente el avance de la vegetación y el incremento de la superficie forestal, lo que vinculan con un territorio más salvaje y una naturaleza más indómita, fuera del control humano. Una segunda interpretación tiene base ecocéntrica, valorando positivamente esta evolución, al considerar que responde a cánones propios de la dinámica natural.

Los bancales o terrazas agrícolas se construyeron para producir alimentos, favorecer la infiltración del agua y limitar la pérdida de suelo (Violant y Simorra, 1949; Puigdefábregas y Fillat, 1986; García-Ruiz et al., 1988; Arnáez et al., 2015, entre otros muchos). La mayoría de ellos se abandonaron en la segunda mitad del siglo XX, pero mantienen un elevado interés: se incluyen entre los paisajes culturales más valorados del mundo mediterráneo (Lasanta et al., 2013). Su interés cultural deriva de la técnica de construcción empleada, en la medida en que recopila el saber de los agricultores, plasmado en las laderas abancaladas durante siglos (milenios incluso en algunas montañas) y las 
habilidades sociales para organizar simultáneamente unos espacios con fines productivos y de conservación (Asíns, 2007). Frente a la dureza de las condiciones climáticas, la altitud y la pendiente, el agricultor dio acertadas respuestas en el uso de los recursos y materiales disponibles. Los bancales constituyen un notable recurso cultural de los sistemas de cultivo en ladera. Detrás de cada bancal, de cada murete, se esconde el saber hacer y el saber conservar de muchas generaciones de agricultores (Lasanta et al., 2013).

A todo ello se añade la labor de información que aporta un paisaje de bancales, como también ocurre en otros muchos paisajes rurales, con sus setos en los márgenes de los campos, sus caminos, acequias y construcciones (Martínez-Arnáiz, 2018). En un paisaje se encuentran siempre retazos de épocas distintas, superpuestos y entremezclados, a modo de un palimpsesto (García-Ruiz y Lasanta, 2018; García-Ruiz et al., 2017). La fecha en que se abancaló una ladera informa, por ejemplo, de las necesidades de una sociedad en una época de la Historia (Pérez-Carazo, 2008). La localización de los diferentes modelos de bancales (los más perfectos próximos siempre a los pueblos o en lugares propicios para cierta intensificación) manifiesta la discriminación espacial realizada por el hombre en función de la fertilidad del suelo y la accesibilidad. La existencia o ausencia de muretes nos anuncia las condiciones micro climáticas y litológicas de cada enclave (Lasanta et al., 2017c). En los muros y tapiales podemos encontrar piezas de calidad contrastada, llenas de equilibrio en dimensión y proporciones, adaptadas al medio, ajustadas a las necesidades, bien diseñadas y mejor ejecutadas. De gran mérito si tenemos en cuenta los pocos medios y las dificultades de todo tipo inherentes a su época (Lasanta et al., 2013).

Pueden sacarse muchas enseñanzas de un paisaje rural tradicional como el de laderas abancaladas. Con los muretes de piedra se perseguía aumentar la infiltración y disminuir la erosión. Pero los constructores, también usuarios por lo general, aprovecharon las propiedades intrínsecas de la piedra seca: retención de la humedad, aireación moderada pero continua, inercia térmica con mantenimiento de temperatura y avenamiento eficaz de los suelos. Una manera de construir que exigía un profundo conocimiento de las particularidades del terreno y de las posibilidades de la técnica de mampostería en seco o de piedra seca (Lasanta et al., 2013).

Las acequias para regar, unas veces, y para desviar el agua de escorrentía, en la mayor parte de los casos, muestran el interés del agricultor por incrementar la productividad y asegurar la conservación del suelo, pero además nos informan de la técnica y cultura del grupo que las construyó. En Cameros se diferencian las acequias de origen árabe y cristiano (Pérez-Carazo, 2008). En definitiva, en el paisaje y en las infraestructuras asociadas se puede leer a la vez el entramado de hechos y fenómenos visibles y las relaciones funcionales que han existido entre ellos a lo largo del tiempo, por lo que constituyen un patrimonio cultural de un valor irrenunciable.

Además, los paisajes de laderas abancaladas tienen un gran atractivo turístico. Biarge (2009) señala la alta valoración que reciben los paisajes abancalados del entorno del Parque Nacional de Ordesa y Monte Perdido (Pirineo Central), siendo uno de los principales atractivos para los visitantes del Parque. Jiménez Olivenza (1989-90) incluye las laderas de terrazas agrícolas de Sierra Nevada (Sistema Penibético) entre los paisajes más valorados, por su alta diversidad y belleza. Ocaña et al. (2004) indican que en la Axarquía malagueña los paisajes aterrazados con estructura compleja, formada por un mosaico de manchas con vegetación natural alternando con otras manchas de cultivos de cítricos y aguacates, cuentan con la preferencia de los usuarios; los muros de piedra seca de las terrazas están construidos con materiales extraídos del terreno, aportando una perfecta integración visual en el entorno. Sayadi et al. (2009) señalan, al estudiar los paisajes de la Alpujarra granadina, las preferencias de los turistas por las laderas con bancales cultivados frente a las áreas con campos abandonados y vegetación natural. Por otro lado, algunas laderas abancaladas constituyen áreas preferentes para la localización de segundas residencias, especialmente en las proximidades de grandes aglomeraciones urbanas y de espacios turísticos, como en los Montes de Málaga por su cercanía a la Costa del Sol (Ocaña et al., 2004). 


\section{Conclusiones y perspectivas}

Las tierras abandonadas constituyen un uso del suelo con importantes implicaciones ambientales, paisajísticas y socioeconómicas a escala global, por lo que han recibido la atención de científicos de los cinco continentes. España es el segundo país, tras Estados Unidos, con mayor producción bibliográfica, por la amplia superficie que ocupan las tierras abandonadas y por la diversidad de ambientes en que se han abandonado, que van desde las áreas de montaña a las de condiciones semiáridas. Por otro lado, el proceso de abandono se ha dilatado en el tiempo, ya que los principales abandonos se registraron a lo largo del siglo XX, especialmente entre los años cincuenta y ochenta en áreas de montaña, mientras que en los espacios llanos tienen lugar en las últimas décadas, debido tanto a las medidas e incentivos de la PAC para la retirada de tierra como a la degradación de suelos por un uso indebido (intensificación, riego, laboreos inadecuados...).

Las tierras abandonadas tienen una evolución compleja, al interactuar de forma simultánea procesos de sucesión vegetal y edafogénesis, condicionados por el tiempo de abandono, las condiciones climáticas del lugar, la gestión previa y posterior al abandono, la cubierta vegetal próxima al lugar de abandono, y posibles perturbaciones como los incendios forestales. Por ello, surgen ecosistemas y paisajes muy diversos que, además, experimentan cambios a diferente ritmo temporal. La evolución estándar crea en primer lugar ecosistemas, e incluso en ocasiones paisajes, de pastos, seguidos por los de matorrales, arbustedos, bosque claro y bosque denso en la etapa final. Sin embargo, en la realidad cualquiera de las fases anteriores puede ser la última, o al menos la más duradera, en función de los factores que interactúan en cada momento en las tierras abandonadas. Por otro lado, aún se añade más complejidad si tenemos en cuenta que el proceso de abandono ha sido muy dilatado en el tiempo, por lo que en áreas próximas pueden convivir paisajes muy diferentes de tierras abandonadas.

Los paisajes tradicionales en mosaico proporcionaban abundantes bienes y servicios ecosistémicos a la sociedad. Tras su abandono, la oferta se modifica en función del paisaje resultante. Con una perspectiva sintética cuatro son los paisajes dominantes en las tierras abandonadas y sus funcionalidades más destacadas:

i) Si se alcanza la fase de bosque, las propiedades del suelo mejoran y se incrementa el SOC, mientras que disminuyen los recursos hídricos y pastorales; se producen, además, importantes cambios en la biodiversidad (incremento de especies forestales a costa de las adaptadas a paisajes culturales) y en la estética del paisaje; este último es bien valorado por la población urbana y menos por la local.

ii) Si tras el abandono dominan procesos de degradación del suelo, el paisaje resultante se caracteriza por la presencia de laderas con suelo casi desnudo y con abundantes piedras en superficie y manchas aisladas de matorral. En estas condiciones, la biodiversidad se empobrece, las pérdidas de suelo se incrementan mientras el SOC disminuye, los recursos hídricos aumentan, pero con agua de escasa calidad y con efectos negativos en la fauna fluvial y en el aterramiento de embalses; apenas se producen recursos pastorales y el paisaje pierde todo su atractivo, restando alternativas al desarrollo local.

iii) El paisaje dominado por matorrales implica una ligera mejora de las propiedades del suelo y del SOC respecto a la fase de cultivo, pero produce menos agua y limita mucho el pastoreo del ganado; es un paisaje muy homogéneo y de escasa riqueza ecológica, además de tener un elevado riesgo de incendio.

iv) Por último, hay retazos o laderas enteras cubiertas, de forma temporal o permanente, por pastizales. Estos aportan recursos forrajeros a la ganadería extensiva, mejoran las propiedades del suelo y el SOC respecto a los cultivos y matorrales, aunque quizás, aportan valores ligeramente inferiores a los bosques; producen volúmenes de escorrentía superiores a las áreas de matorral y bosque. Si en los márgenes de los campos se mantienen matorrales, arbustos y algunos árboles, se alcanzan altas tasas de biodiversidad, ya que los márgenes 
sirven para alimento y refugio de la fauna, además de como corredores ecológicos. Reducen el combustible y actúan de cortafuegos, por lo que el número de incendios y superficie quemada se reduce.

La amplia extensión que ocupan las tierras abandonadas y la elevada diversidad de paisajes que generan, con impactos ambientales, económicos y sociales, exigen una gestión que tenga en cuenta que los impactos no sólo afectan a los habitantes locales sino también a buena parte de la sociedad. Los científicos están divididos entre los que propugnan una gestión activa (intervenir en el territorio eliminando matorrales o reforestando) y los que apoyan una gestión pasiva (dejar que la naturaleza siga su curso). También los gestores del territorio se debaten entre ambas posturas, tratando en muchos casos de conciliar la no intervención en la mayor parte del territorio, con reforestaciones en algunas laderas, quemas prescritas muy localizadas y desbroces selectivos (cortafuegos y limpieza del sotobosque próximo a vías de comunicación), si bien algunas administraciones utilizan el desbroce de matorrales como una auténtica política de gestión para favorecer la diversidad, fomentar la ganadería extensiva y fijar población, además de reducir los riesgos de grandes incendios.

De los resultados extraídos de la bibliografía se deduce que, para la conservación y el suministro de servicios ecosistémicos, dos paisajes son más positivos (los bosques y los pastos), mientras que las áreas de matorral ofrecen más impactos negativos que positivos, y las de suelo casi desnudo sólo efectos negativos, si bien la presencia de matorrales dispersos puede fomentar la formación de pequeños sectores alrededor de las matas con mayor capacidad de infiltración. Con estos resultados parece lógico compatibilizar ambas posturas, dejando algunas áreas a la evolución natural, si tienen potencial para completar el proceso de reconstrucción y alcanzar la fase de bosque, mientras que en otras es aconsejable intervenir. Así, el desbroce de matorrales en áreas seleccionadas (campos abandonados con buen suelo y accesibles al ganado) parece una buena estrategia para construir un paisaje más heterogéneo y próximo al paisaje cultural tradicional. Con ello, se incrementaría la biodiversidad, los recursos hídricos y pastorales, y se reduciría la probabilidad de grandes incendios forestales. Además, se contribuiría a mantener -e incluso incentivar- sistemas ganaderos extensivos, una actividad clave para mejorar la biodiversidad y el desarrollo socio-económico de muchos territorios rurales. En las áreas de suelo casi desnudo también parece aconsejable intervenir, bien mediante repoblaciones con diversas especies de matorral que acorten el tiempo de revegetación, a ser posible mediante aplicación de enmiendas a los suelos para mejorar sus propiedades e incentivar la sucesión vegetal, o mediante prácticas de conservación de suelos.

Las tierras abandonadas generan paisajes muy diversos, por lo que la gestión también debe ser diversa, adaptada al potencial biótico y abiótico de cada lugar. Pero teniendo siempre presente que de la gestión se derivan impactos con implicaciones ambientales y efectos en la población local y en la sociedad en su conjunto.

\section{Agradecimientos}

Este trabajo se ha realizado con el apoyo de los proyectos LIFE MIDMACC (LIFE18 CCA/ES/001099), financiado por la Comisión Europea, y MANMOUNT (PID2019-105983RB100/AEI/10.13039/501100011033), financiado por el Ministerio de Ciencia e Innovación (Gobierno de España) y FEDER. El grupo de investigación "Procesos Geoambientales y Cambio Global" financiado por el Gobierno de Aragón (E02_17R). M. Khorchani se beneficia de un contrato FPI predoctoral del Gobierno de España (BES-2016-077992) asociado al proyecto ESPAS. José María García Ruiz mejoró el manuscrito original, aportando ideas y referencias bibliográficas. 


\section{Referencias}

Alguacil García, P. 1985. Esquema metodológico para la valoración del cambio de usos del suelo (Sierra de Ayllón). Anales de la Universidad Complutense 5, 143-165.

Alonso Sarría, F., Martínez Hernández, C., Belmonte Serrato, F., Fernández Carrillo, M.A. 2016a. Principales causas del abandono de cultivos en la Región de Murcia. En: A. Romero Díaz (Coord.) Abandono de cultivos en la Región de Murcia. Consecuencias Ecogeomorfológicas. Servicio de Publicaciones de la Universidad de Murcia: 203-226, Murcia.

Alonso-Sarría, F., Martínez-Hernández, C., Romero-Díaz, A., Cánovas-Garcés, F., Gomáriz-Castillo, F. 2016 b. Main environmental features leading to recent abandonment in Murcia Region (Southeast Spain). Land Degradation \& Development 277, 654-670. https://doi.org/10.1002/ldr.2447

Álvarez-Martínez, J., Gómez-Villar, A., Lasanta, T. 2016. The use of goats grazing to restore pastures invaded by shrubs and avoid desertification: a preliminary case study in the Spanish Cantabrian mountains. Land Degradation \& Development 27, 3-13. https://doi.org/10.1002/ldr.2230

Alvera, B. 1976. Contribución al estudio de la intercepción de las precipitaciones atmosféricas en el pinar de San Juan de la Peña. Publicaciones del Centro Pirenaico de Biología Experimental 7 (1), 95-100.

Alvera, B. 1977. Interceptación de las precipitaciones atmosféricas en un pinar altoaragonés. Segundo año de observaciones. Publicaciones del Centro Pirenaico de Biología Experimental 8, 15-22.

Arbelo, C.D., Rodríguez-Rodríguez, A., Guerra, J.A., Mora, J.L., Notario, J.S., Fuentes, F. 2006. Soil degradation processes and plant colonization in abandoned terraced fields overlying pumice tuffs. Land Degradation \& Development 17, 571-588. https://doi.org/10.1002/ldr.735

Arévalo, J.R., Fernández-Lugo, S., Reyes-Betancort, A., Tejeda, M., Jiménez, C., Díaz, F.J. 2017. Relationships between soil parameters and vegetation in abandoned terrace fields vs. non-terraced fields in arid lands (Lanzarote, Spain): An opportunity for restoration. Acta Oecologica 86, 77-84. https://doi.org/10.1016/j.actao.2017.09.014

Arnáez, J., Lasanta, T., Ortigosa, L., Ruiz-Flaño, P. 1990. Le processus d'abandon de l'espace agricole dans la montagne submediterranéenne espagnole : Pyrénées centrales et Système Ibérique. Revue de Géographique des Pyrénées et du Sud-Ouest 6 (2), 237-253. https://doi.org/10.3406/rgpso.1990.3207

Arnáez, J., Lasanta, T., Errea, M.P., Ortigosa, L. 2011. Land abandonment, landscape evolution, and soil erosion in a Spanish Mediterranean mountain region. The case of Camero Viejo. Land Degradation \& Development 22, 537-550. https://doi.org/10.1002/ldr.1032

Arnáez, J., Lana-Renault, N., Lasanta, T., Ruiz-Flaño, P., Castroviejo, J. 2015. Effects of farming terraces on hydrological and geomorphological processes. A review. Catena 128, 122-134. https://doi.org/10.1016/j.catena.2015.01.021

Arnáez, J., Lana-Renault, N., Ruiz-Flaño, P., Pascual, N., Lasanta, T. 2017. Mass soil movement on terraced landscape of the Mediterranean mountain areas: a case study in the Iberian Range, Spain. Cuadernos de Investigación Geográfica 4 (1), 83-100. https://doi.org/10.18172/cig.3211

Arnáez, J., Ortigosa, L. 1997. Abandono, subutilización y erosión en un espacio de montaña: El Sistema Ibérico noroccidental (La Rioja, España). En: J.M. García-Ruiz y P. López García (Eds), Acción humana y desertificación en ambientes mediterráneos. Instituto Pirenaico de Ecología, pp. 205-220, Zaragoza.

Asíns, S. 2007. Los aterrazamientos mediterráneos. Paradigma ambiental-agro-cultural. Cuadernos de la Sostenibilidad y Patrimonio Natural 11, 81-91.

Azevedo, J.C., Moreira, C., Castro, J.P., Loureiro, C. 2011. Agriculture abandonment, land use change and fire hazard in mountain landscapes in Northerastern Portugal. In: C. Li, R. Lafortezza, J. Chen (Eds.), Landscape Ecology in Forest Management and Conservation: Challenges and Solutions for Global Change. Springer, New York, pp. 329-351.

Barrientos, G. 1978. El valle del Alto Tormes (Gredos y Aravalle: estudio geográfico). Caja Central de Ahorros de Ávila, Ávila. 
Bartolomé, J., Franch, J., Plaixats, J, Seligman, N.G. 2000. Grazing alone is not enough to maintain landscape diversity in the Montseny Reserve. Agriculture, Ecosystems \& Environment 77 (3), 267-273. https://doi.org/10.1016/S0167-8809(99)00086-9

Bauer, N., Wallner, A., Hunzinker, M. 2009. The change of European landscapes: Human-nature relationships, public attitudes towards rewilding, and the implications for landscape management in Switzerland. $\begin{array}{llllll}\text { Journal of } & \text { Environmental } & \text { Management } & 90 & \text { (9), }\end{array}$ https://doi.org/10.1016/j.jenvman.2008.01.021

Bautista Cruz A., Etchevers Barra J., del Castillo R.F., Gutiérrez C. 2004. La calidad del suelo y sus indicadores. Ecosistemas 13, 90-97.

Beguería, S., López-Moreno, J.I., Lorente, A., Seeger, M., García-Ruiz, J.M. 2003. Assessing the effect of climate oscillations and land-use changes on streamflow in the Central Spanish Pyrenees. Ambio 32 (4), 283-286. https://doi.org/10.1579/0044-7447-32.4.283

Bellin, N., van Wesemael, B., Meerkert, A., Vanacker, V., Barbera, G.G. 2009. Abandonment of soil water conservation structures in Mediterranean ecosystems. A case study from south east Spain. Catena 76, 114-121. https://doi.org/10.1016/j.catena.2008.10.002

Bellot, J., Bonet, A., Sánchez, J.R., Chirino, E. 2001. Likely effects of land use changes on the runoff and aquifer recharge in a semiarid landscape using a hydrological model. Landscape and Urban Planning 155 (1), 41-53. https://doi.org/10.1016/S0169-2046(01)00118-9

Belmonte Serrato, F., Martínez Hernández, C., Ruíz Sinoga, J.D. 2016. Análisis de las características edáficas más significativas de las áreas abandonadas. En A. Romero Díaz (Coord.) Abandono de cultivos en la Región de Murcia. Consecuencias Ecogeomorfológicas. Servicio de Publicaciones de la Universidad de Murcia, pp. 41-62, Murcia.

Belmonte Serrato, F., Romero Díaz, A. 1999. Interceptación en algunas especies del matorral mediterráneo. Colección Cuadernos de Ecología y Medio Ambiente. Universidad de Murcia, 202 pp.

Belmonte Serrato, F., Romero Díaz, A. (Coord.) 2013. Interceptación de la lluvia por la vegetación en España. Fundación Instituto Euromediterráneo del Agua. Murcia.

Belmonte Serrato, F., Romero Díaz, A., López Bermudez, F. 1999. Efectos sobre la cubierta vegetal, la escorrentía y la erosión del suelo de la alternancia cultivo-abandono en parcelas experimentales. Investigaciones Geográficas 22, 95-107. https://doi.org/10.14198/INGEO1999.22.01

Bergkamp, G., Cammeraat, L.H., Martínez-Fernández, J. 1996. Water movement and vegetation patterns on shrubland and an abandoned field in two desertification -threatened areas in Spain. Earth Surface Processes and Landforms 21, 1073-1090. https://doi.org/10.1002/(SICI)1096-9837(199612)21:12<1073::AIDESP640>3.0.CO;2-8

Bernáldez, F.G. 1991. Ecological consequences of the abandonment of traditional land use systems in Central Spain. Options Méditerranéennes 15, 23-29.

Bernués, A., Rodríguez-Ortega, T., Ripoll-Bosch, R., Alfnes, F. 2014. Socio-cultural and economic valuation of ecosystem services provided by Mediterranean ecosystems. PLoS ONE 9 (7), e102479. https://doi.org/10.1371/journal.pone.0102479

Biarge, F. 2009. Sobrarbe. Letra menuda. Diputación de Huesca, 322 pp., Huesca.

Blanco-Fontao, B., Quevedo, M., Obeso, J.R. 2011. Abandonment of traditional uses in mountain areas: typological thinking versus hard data in the Cantabrian Mountains (NW Spain). Biodiversity Conservation 20, 1133-1140. https://doi.org/10.1007/s10531-011-0016-1

Blanco Sepúlveda, R. 2008. La evaluación de la vulnerabilidad del suelo a la degradación por uso ganadero en espacios montañosos. Análisis metodológico. Estudios Geográficos 264, 51-80.

Boellstorff, D., Benito, G. 2005. Impacts of sed-aside policy on risk of soil erosion in central Spanish. Agriculture, Ecosystems \& Environment 107, 231-243. https://doi.org/10.1016/j.agee.2004.11.002 
Bonet, A. 2004. Secondary succession of semi-arid Mediterranean old-field in South-Easter Spain. Insights for contribution and restoration of degraded lands. Journal of Arid Environment 56 (2), 213-233. https://doi.org/10.1016/S0140-1963(03)00048-X

Bonet, A., Pausas J. 2004. Species richness and cover along a 60-year chronosequence in old-fields of southeastern Spain. Plant Ecology 174, 257-270. https://doi.org/10.1023/B:VEGE.0000049106.96330.9c

Bordiú, E. 1985. Valoración e infrautilización en la Sierra de Ayllón y aportación de un modelo alternativo. Anales de la Universidad Complutense 5, 167-187.

Bowen, M.E., McAlpine, C.A., House, A.P.N., Smith, G.C. 2007. Regrowth forests on abandoned agricultural land: A review of their habitat values for recovering forest fauna. Biological Conservation 140 (3-4), 273 296. https://doi.org/10.1016/j.biocon.2007.08.012

Cabero, V. 1980. Espacio agrario y economía de subsistencia en las montañas galaico-leonesas. Institución "Fray Bernardino de Sahagún" (CSIC) y Ediciones de la Universidad de Salamanca: 134 pp., León.

Calsamiglia, A., Lucas-Borja M.E., Fortesa, J., García-Comendador, J., Estrany, J. 2017. Changes in soil quality and hydrological connectivity caused by the abandonment of terraces in a Mediterranean burned catchment. Forests 8, 333. https://doi.org/10.3390/f8090333

Cammeraat, E.L.H., Cerdà, A., Imeson, A.C. 2010. Ecohydrological adaptation of soils following land abandonment in a semi-arid environment. Ecohydrology 3, 421-430. https://doi.org/10.1002/eco.161

Cammeraat, E., Van Beek, R., Kooijman, A. 2005. Vegetation succession and its consequences for slope stability in SE Spain. Plant and Soil 278 (1-2), 135-147. https://doi.org/10.1007/s11104-005-5893-1

Cammeraat, L. H., Imeson, A.C. 1999. The evolution and significance of soil-vegetation patterns following land abandonment and fire in Spain. Catena 37, 107-127. https://doi.org/10.1016/S0341-8162(98)00072-1

Cantón, Y., Solé-Benet, A., de Vente, J., Boix-Fayos, C., Calvo-Cases, A., Asensio, C., Puigdefábregas, J. 2011. A review of runoff generation arid soil erosion across scales in semiarid south-eastern Spain. Journal of Arid Environments 75, 1254-1261. https://doi.org/10.1016/j.jaridenv.2011.03.004

Cañadas Sánchez, E.M. 2008. Estudio de tierras agrícolas abandonadas en ambiente mediterráneo semiárido: vegetación, suelos y distribución espacial. Bases para la gestión. Tesis Doctoral, Universidad de Granada. Granada.

Cañadas, E.M., Jiménez, M.N., Valle, F., Fernández-Ondoño, E., Martín-Peinado, F., Navarro, F.B. 2010. Soilvegetation relationships in semi-arid Mediterranean old field (SE Spain): implications for management. Journal of Arid Environments 74 (11), 1525-1533. https://doi.org/10.1016/j.jaridenv.2010.06.007

Casasús, I., Bernués, A., Sanz, A., Villalba, D., Riedel, J.L., Revilla, R. 2007. Vegetation dynamics in Mediterranean forest pastures as affected by beef cattle grazing. Agriculture, Ecosystems \& Environment 121 (4), 365-370. https://doi.org/10.1016/j.agee.2006.11.012

Cerdà, A. 1996. Soil erosion after land abandonment in a semiarid environment of southeastern Spain. Arid Soil Research and Rehabilitation 11 (2), 163-176. https://doi.org/10.1080/15324989709381469

Cerdà, A., García-Álvarez., Cammeraat, L.H., Imeson, A.C. 1994. Agregación del suelo en una catena afectada por el abandono del cultivo en la cuenca del Guadalentín (Murcia). I. Estabilidad y distribución de los agregados del suelo. En: J.M. García-Ruiz y T. Lasanta (Eds), Efectos geomorfológicos del abandono de tierras. Sociedad Española de Geomorfología, pp. 9-19, Zaragoza.

Cerdà, A., Lasanta, T. 2005. Long-term erosional responses after fire in the Central Spanish Pyrenees. 1 Water and sediment yield. Catena 60, 59-80. https://doi.org/10.1016/j.catena.2004.09.006

Chauchard, S., Carcaillet, C., Guibal, F. 2007. Patterns of land-use abandonment control tree-recruitment and forest dynamics in Mediterranean mountains. Ecosystems 10, 936-948. https://doi.org/10.1007/s10021007-9065-4

Chocano Vañó, C., Sánchez Fuster, C., López Bermúdez, F. 2007. La agroecología como alternativa a la prevención y lucha contra la desertificación en la Región de Murcia: La Comarca del Noroeste. Agroecología $2,75-84$. 
Conti, G., Fagarazzi, L. 2005. Forest expansion in mountain ecosystems: “environmentalist's dream” or societal nightmare? Planum 11, 1-20.

Cohen, M., Varga, D., Vila, J., Barrassaud, E. 2011. A multi-scale and multi-disciplinary approach to monitor landscape dynamics: a case study in the Catalan pre-Pyrenees (Spain). The Geographical Journal 177 (1), 79-91. https://doi.org/10.1111/j.1475-4959.2010.00368.x

Corbelle-Rico, E., Crecente-Maseda, R., Santé-Riveira, I. 2012. Multi-scale assessment and spatial modelling of agricultural land abandonment in a European peripheral region: Galicia (Spain), 1956-2004. Land Use Policy 29, 493-501. https://doi.org/10.1016/j.landusepol.2011.08.008

Cortizo, J., Maya, A., Redondo, J.M. 1992. Valdesamario. Un enclave de la montaña leonesa. Imprenta Moderna, 109 pp., León.

Costra, G.B., Dal Negro, P. y Frattini, P. 2003. Soil slips and debris flows on terraced slopes. Natural Hazards and Earth System Science 3, 31-42. https://doi.org/10.5194/nhess-3-31-2003

Cramer, V. 2007. Old fields: dynamics and restoration of abandoned farmland. Island Press, 352 pp.

De Baets, S., Meerswaus, J., Vanacker, V., Quine, T. A., Van Oost, K. 2013. Spatial variability and change in soil organic carbon stocks in response to recovery following land abandonment and erosion in mountainous dry lands. Soil Use and Management 29, 65-76. https://doi.org/10.1111/sum.12017

Delibes-Mateos, M., Ferreras, P., Villafuerte, R. 2009a. European rabbit population trends and associated factors: A review of the situation in the Iberian Peninsula. Mammal Review 39 (2), 124-140. https://doi.org/10.1111/j.1365-2907.2009.00140.x

Delibes-Mateos, M., Farfán, M.A., Olivero, J., Márquez, A.L., Vargas, J.M. 2009b. Long-term changes in game species over a long period of transformation in the Iberian Mediterranean landscape. Environmental Management 43, 1256-1268. https://doi.org/10.1007/s00267-009-9297-5

Díaz Muñoz, M.A. 1984. Criterios para el análisis de evolución de usos del suelo en zona de montaña: aplicación a un sector de Somosierra. Anales de Geografia de la Universidad Complutense 4, 131-14.

Douglas, T.D., Kirkby, S.J., Critchley, R.W., Park. G.J. 1994. Agricultural terrace abandonment in the Alpujarra, Andalucía, Spain. Land Degradation and Rehabilitation 5, 281-291. https://doi.org/10.1002/ldr.3400050405

Duarte, F., Jones, N., Fleskens, L. 2008. Traditional olive orchards on sloping land: sustainability or abandonment? Journal of Environmental Management 89, 86-98. https://doi.org/10.1016/j.jenvman.2007.05.024

Duguy, B., Alloza, J.A., Röder, A., Vallejo, R., Pastor, F. 2007. Modelling the effects of landscape fuel treatments on fire growth and behaviour in a Mediterranean landscape (Eastern Spain). International Journal of Wildland Fire 16 (5), 619-632. https://doi.org/10.1071/WF06101

Duiker, S.W., Flanagan, D.C., Lal, R. 2001. Erodibility and infiltration characteristics of five major soils of Southwest Spain. Catena 45, 103-121. https://doi.org/10.1016/S0341-8162(01)00145-X

Dunjó, G., Pardini, G., Gispert, M. 2003. Land use change effects on abandoned terraced soils in a Mediterranean catchment, NE Spain. Catena 52, 23-37. https://doi.org/10.1016/S0341-8162(02)00148-0

Dunjó, G., Pardini, G., Gispert, M. 2004. The role of land use-land cover on runoff generation and sediment yield at a microplot scale, in a small Mediterranean catchment. Journal of Arid Environments 57, 99-116. https://doi.org/10.1016/S0140-1963(03)00097-1

Emran, M., Gispert, M., Pardini, G. 2012. Patterns of soil organic carbon glomalin and structural stability in abandoned Mediterranean terraced lands. European Journal of Soil Science 63, 637-649. https://doi.org/10.1111/j.1365-2389.2012.01493.X

Errea, M.P., Lasanta, T. 1994. Aportación de las tierras retiradas por la PAC a la extensificación del ovino en el “Campo de Zaragoza”. XIV Congreso Nacional de Geografía. Asociación de Geógrafos Españoles Universidad de Salamanca, pp. 217-219, Salamanca.

Errea Abad, M.P., Nadal-Romero, E., Lasanta, T. 2015. La complejidad de la cubierta vegetal en los campos abandonados del valle de Aísa (Pirineo Aragonés). Un análisis del papel de los tipos de campos. En: J. de 
la Riva, P. Ibarra, R. Montorio, M. Rodrigues, (Eds.), Análisis espacial y representación geográfica: innovación y aplicación. Universidad de Zaragoza - AGE, pp. 933-942.

Escribano-Ávila, G., Sanz-Pérez, V., Pías, B., Virgós, E., Escudero, A., Valladares, F. 2012. Colonization of abandoned land Juniperus thurifera is mediated by the interaction of a diverse dispersal assemblage and environmental heterogeneity. Plos One 7 (10), e46993. https://doi.org/10.1371/journal.pone.0046993

Estruch, C., Lozano, Y.M., Armas, C., Pugnaire, F.L. 2018. Plant community changes after land abandonment control $\mathrm{CO}_{2}$ balance in dry environment. Plant and Soil 425, 253-268. https://doi.org/10.1007/s11104018-3581-1

Faulkner, H. 1995. Gully erosion associated with the expansion of unterraced almond cultivation in the coastal Sierra de Lujar, S. Spain. Land Degradation \& Rehabilitation 6, 170-200. https://doi.org/10.1002/ldr.3400060306

Fedriani, J.M., Wiegard, T., Ayllon, D., Palomares, F., Suárez-Esteban, A., Grimm, V. 2017. Assisting seed dispersers to restore oldfields: An individual-based model of the interactions among badgers, foxes and Iberian pear trees. Journal of Applied Ecology 55, 600-611. https://doi.org/10.1111/1365-2664.13000

FEGA 2019. https://www.agronegocios.es/espana-cuenta-con-mas-de-232-mha-de-superficie-agricola-abandonaday-sin-aprovechamiento/

Feranec, J., Jaffrain, G., Soukup, T., Hazeu, G. 2010. Determining changes and flows in European landscapes 1990-2000 using Corine Land Cover data. Applied Geography 30 (1), 19-35. https://doi.org/10.1016/j.apgeog.2009.07.003

Fernández-Alés, R., Martín, A., Ortega, F., Alés, E.E. 1992. Recent changes in landscape structure and function in a Mediterranean region of SW Spain (1950-1984). Landscape Ecology 7, 3-18. https://doi.org/10.1007/BF02573953

Fernández-Giménez, M. 2015. "A shepherd has to invent" Poetic analysis of socio-ecological change in the cultural landscape of the central Spanish Pyrenees. Ecology and Society 20 (4), 29 http://doi.org./10.5751/ES-08054-200429

Ferrer, C., Ascaso, J., Maestro, M., Broca, A. 1995. Evolución de bancales no cultivados en función del grado de pastoreo en el Maestrazgo de la Comunidad Valenciana. XXXV Reunión Científica de la SEEP: pp. $197-$ 201, Tenerife.

Ferrer, C., Broca, A. 1999. El binomio agricultura-ganadería en los ecosistemas mediterráneos. Pastoreo frente a “desierto verde". Actas de la XXXIX Reunión de la S.E.E.P., pp. 309-334, Almería.

Fillat, F., García-González, R., Gómez, D., Reiné, R. 2008. Pastos del Pirineo. Diputación de Huesca, 319 pp., Huesca.

Fister, W., Ries, J.B. 2009. Wind erosion in the central Ebro Basin under changing land use management. Field experiments with a portable wind tunnel. Journal of Arid Environments 73, 996-1004. https://doi.org/10.1016/j.jaridenv.2009.05.006

Franco, J.A., Calatrava, J. 2010. Adopción y difusión de prácticas de no laboreo en el olivar de la provincia de Granada. Economía Agraria y Recursos Naturales 10 (1), 135-154. https://doi.org/10.7201/earn.2010.01.08

Gabarrón-Galeote, M.A., Trigalet, S., van Wesemael, B. 2015. Effect of land abandonment on soil organic carbon fractions along a Mediterranean precipitation gradient. Geoderma 249-250, 69-78. https://doi.org/10.1016/j.geoderma.2015.03.007

Gallart, F., Llorens, P. 1994. Papel de los cultivos de montaña y su abandono en la economía del agua. En: J.M. García-Ruiz, T. Lasanta (Eds.), Efectos geomorfológicos del abandono de tierras. Sociedad Española de Geomorfología: 43-55 pp., Zaragoza.

Gallart, F., Llorens, P. 2002. Water resources and environmental change in Spain. A key issue for sustainable catchment management. En: J.M. García-Ruiz, J.A.A. Jones, J. Arnáez (Eds.), Environmental change and water sustainability. IPE-CSIC, pp. 11-20, Zaragoza. 
Gallart, F., Llorens, P. 2004. Observations on land cover changes and water resources in the headwaters of the Ebro catchment, Iberian Peninsula. Physics and Chemistry of the Earth 29, 769-773. https://doi.org/10.1016/j.pce.2004.05.004

Gallart, F., Llorens, P., Latron, J, Regüés, D. 2002. Hydrological processes and their seasonal controls in a small Mediterranean mountain catchment in the Pyrenees. Hydrology and Earth System Science 6 (3), 527-537. https://doi.org/10.5194/hess-6-527-2002

Gallego Fernández, J.B., García-Mora, M.R., García-Novo, F. 2004. Vegetation dynamics of Mediterranean shrublands in former cultural landscape at Grazalema mountains, South Spain. Plant Ecology 172, 83-94. https://doi.org/10.1023/B:VEGE.0000026039.00969.7a

García-Llamas, P., Geijzendorffer, I.R., García-Nieto, A.P., Calvo, L., Suárez-Seoane, S., Cramer, W. 2019. Impact of land cover change on ecosystem services supply in mountain systems: a case study in the Cantabrian Mountains (NW of Spain). Regional Environmental Change 19, 529-542. https://doi.org/10.1007/s10113-018-1419-2

García-Novo, F. 2007. Los paisajes europeos en una cultura tecnológica. Cuadernos de la Sostenibilidad y Patrimonio Natural 11, 22-39.

García-Orenes, F., Morugán-Coronado, A., Zornoza, R., Scow, K. 2013. Changes in soil microbial community structure influenced by agricultural management practices in a Mediterranean agro-ecosystems. Plos One 8 (11), e80522. https://doi.org/10.1371/journal.pone.0080522

García-Orenes, F., Roldán, A., Mataix-Solera, J., Cerdà, A., Campoy, M., Arcenegui, V., Caravaca, F. 2012. Soil structural stability and erosion rates influenced by agricultural management practices in a semi-arid Mediterranean agro-ecosystem. Soil Use and Management 281, 571-579. https://doi.org/10.1111/j.14752743.2012.00451.x

García-Ortiz, E. 2006. Efecto de la estructura de la copa en la partición de lluvia de tres especies arbustivas en clima semiárido. Tesis Doctoral. Universidad de Almería: 404 pp., Almería.

García-Pausas, J., Casals, P., Camareto, I., Huguet, C., Thompson, R., Sebastià, M.T., Romanyà, J. 2007. Soil organic carbon storage grasslands of the Pyrenees: effects of climate and topography. Biochemistry 82, 279-289. https://doi.org/10.1007/s10533-007-9071-9

García-Ruiz, J.M. 1988. La evolución de la agricultura de montaña y sus efectos sobre la dinámica del paisaje. Revista de Estudios Agro-Sociales 146, 7-37.

García-Ruiz, J.M., Arnáez, J., Lasanta, T. 2017. Complejidad y diversidad en el paisaje de la montaña riojana: Una perspectiva general sobre su proceso de construcción y transformación. Berceo 173, 141-164.

García-Ruiz, J. M., Lana-Renault, N. 2011a. Una revisión de los efectos hidrológicos y erosivos del abandono de tierras en España. Geographicalia 59-60, 125-135. https://doi.org/10.26754/ojs_geoph/geoph.20115960827

García-Ruiz, J.M., Lana-Renault, N. 2011b. Hydrological and erosive consequences of farmland abandonment in Europe, with special reference to the Mediterranean region - A review. Agriculture, Ecosystems \& Environment 140, 317-338. https://doi.org/10.1016/j.agee.2011.01.003

García-Ruiz, J.M., Lana-Renault, N., Beguería, S., Lasanta, T., Regüés, D., Nadal-Romero, E., Serrano-Muela, P., López-Moreno, J.I., Alvera, B., Martí, C., Alatorre, L.C. 2010. From plot to regional scales: interactions of slope and catchment hydrological and geomorphic processes in the Spanish Pyrenees. Geomorphology 120, 248-257. https://doi.org/10.1016/j.geomorph.2010.03.038

García-Ruiz, J.M., Lasanta, T. 2018. El Pirineo aragonés como paisaje cultural. Pirineos 173, e038. https://doi.org/10.3989/pirineos.2018.173005

García-Ruiz, J.M., Lasanta, T., Nadal-Romero, E., Lana-Renault, N., Álvarez-Farizo, B. 2020. Rewilding and restoring cultural landscapes in Mediterranean mountains: Opportunities and challenges. Land Use Policy 99, 104850. https.//doi.org/10.1016/j.landusepol.2020.104850

García-Ruiz, J.M., Lasanta-Martínez, T. 1990. Land use changes in the Spanish Pyrenees. Mountain Research and Development 10 (3), 267-279. https://doi.org/10.2307/3673606 
García-Ruiz, J.M., Lasanta-Martínez, T. 1993. Land-use conflicts as a result of land-use change in the Central Spanish Pyrenees: a review. Mountain Research and Development 13 (3), 295-304. https://doi.org/10.2307/3673658

García-Ruiz, J.M., Lasanta, T., Sobrón, I. 1985. Estudio comparado de la evolución geomorfológica de campos abandonados y áreas repobladas de la Cuenca del Jubera. Comunidad Autónoma de La Rioja, 345 pp., Logroño.

García-Ruiz, J.M., Lasanta, T., Sobrón, I. 1988. Problemas de evolución geomorfológica de campos abandonados: el valle del Jubera (Sistema Ibérico). Zubía 6, 99-111.

García-Ruiz, J.M., López-Bermúdez, F. 2009. La erosión del suelo en España. Sociedad Española de Geomorfología. 441 pp., Zaragoza.

García-Ruiz, J.M., López-Moreno, J.I., Lasanta, T., Vicente-Serrano, S.M., González-Sampériz, P., ValeroGarcés, B.L., Sanjuán, Y., Beguería, S., Nadal-Romero, E., Lana-Renault, N., Gómez-Villar, A. 2015. Los efectos geoecológicos del Cambio Global en el Pirineo Central español. Una revisión a distintas escalas espaciales y temporales. Pirineos 170, e012. http://dx.doi.org/10.3989/Pirineos.2015.170005

García-Ruiz, J.M., López-Moreno, J.I., Vicente-Serrano, S.M., Lasanta, T., Beguería, S. 2011. Mediterranean water resources in a Global Change scenario. Earth Science Reviews 105 (3-4), 121-139. https://doi.org/10.1016/j.earscirev.2011.01.006

García-Ruiz, J.M., Regüés, D., Alvera, B., Lana-Renault, N., Serrano-Muela, P., Nadal-Romero, E., Latrón, J., Martí-Bono, C., Arnáez, J. 2008. Flood generation and sediment transport in experimental catchment affected by land use changes in the Central Pyrenees. Journal of Hydrology 356 (1-2), 245-260. https://doi.org/10.1016/j.jhydrol.2008.04.013

Gil-Tena, A., Brotons, L., Saura, S. 2009. Mediterranean forest dynamics and forest bird distribution changes in the late $20^{\text {th }}$ century. Global Change Biology 15, 474-485. https://doi.org/10.1111/j.13652486.2008.01730.x

Gisbert, J.M., Ibáñez, S., Pérez, M.A. 2005. Terrace Abandonment in the Ceta Valley, Alicante Province, Spain. Adv Geoecol. 36: 329-337.

Gispert, M., Pardini, G., Emran, A., Doni, S., Masciandaro, G. 2018. Seasonal evolution of soil matter, glomalin and enzymes and potential for $\mathrm{C}$ storage after land abandonment and renaturalization processes in soils of NE Spain. Catena 162, 402-413. https://doi.org/10.1016/j.catena.2017.10.019

Gómez-Aparicio, L., Zamora, R., Gómez, J.M., Hódar, J.A., Castro, J., Baraza, E. 2004. Applying plant facilitation to forest restoration: a meta-analysis of the use of shrubs as nurse plant. Ecological Applications 14, 11281138. https://doi.org/10.1890/03-5084

Gómez-García, D., Aguirre, A.J., Lizaur-Sukía, X. 2011. Recuperación del matorral tras el desbroce mecánico y quema en pastos de la Sierra de Aralar y Belate (Navarra). En: C. López-Carrasco, M.P. Rodríguez, A. San Miguel, F. Fernández, S. Roig, Eds.), Pastos, paisajes culturales entre tradición y nuevos paradigmas del siglo XXI SEEP, pp. 133-138, Toledo.

Gómez-Moreno, M.L. 1989. La montaña malagueña: estudio ambiental y evolución del paisaje. Servicio de Publicaciones de la Diputación Provincial de Málaga, 412 pp., Málaga.

Grigulis, K., Lavorel, S., Davies, I.D., Dossantos, A., Lloret, F., Vilà, M. 2005. Landscape - scale positive feedbacks between fire and expansion of the large tussock grass, Ampedelodesmos mauritanica in Catalan shrublands. Global Change Biology 11 (7), 1042-1053. https://doi.org/10.1111/j.13652486.2005.00980.x

Guerrero, M.M., Guirao, P., Martinez-Lluch, M.C., Tello, J.C., Lacasa, A. 2014. Soil fatigue and its specificity towards pepper plants in greenhouses. Spanish Journal of Agricultural Research 12 (3), 644-652. https://doi.org/10.5424/sjar/2014123-5701

Gutiérrez Teira, A. 1997. Cambios de uso del suelo y modelos de organización espacial de un paisaje de montaña mediterránea. El Valle de Lozoya (Sistema Central, Madrid). Tesis Doctoral, Universidad Autónoma de Madrid, 250 pp. + apéndices, Madrid. 
Haddaway, N.R., Styles, D., Pullin, A.S. 2014. Evidence on the environmental impacts of farmland abandonment in high altitude/mountain regions: A systematic map. Environmental Evidence 3 (1), art. no. 17. https://doi.org/10.1186/2047-2382-3-17

Heinimann, A., Mertz, O., Frolking, S., Christensen, A.E., Hurni, K., Sedano, F., Chini, L.P., Sahajpal, R., Hansen, M., Hurtt, G. 2017. A global view of shifting cultivation: Recent, current, and future extent. PLoS One 12. https://doi.org/10.1371/journal.pone.0184479

Heredia, A., Frutos, L.M., González-Hidalgo, J.C. 2013. Diferencias en la evolución del paisaje entre dos municipios prepirenaicos (Alquézar y Valle de Lierp) durante la segunda mitad del siglo XX. Pirineos 168, 77-101. https://doi.org/10.3989/Pirineos.2013.168005

Hernando, S., Anton, M., Sardà-Palomera, F., Bota, G., Gregory, R.D., Brotons, L. 2014. Indicators of the impact of land use changes using large-scale bird surveys: Land abandonment in a Mediterranean region. Ecological indicators 45, 235-344. https://doi.org/10.1016/j.ecolind.2014.04.011

Huang, J., Li, F., Xie, H. 2020. A scientometrics review on farmland abandonment research. Land 9, 263. https://doi.org/10.3390/land9080263

Jiménez-Olivenza, Y. 1989-90. Cambios ambientales que suceden al abandono de los campos de cultivo en terrazas: La acequia de Cachariche. Cuadernos Geográficos de la Universidad de Granada 18-19, 5-45.

Keenleyside, C., Tucker, G.M. 2010. Farmland abandonment in the EU: an assessment of trends and prospects. Report prepared for WWF. Institute for European Environmental Policy: 93 pp., London.

Komac, B., Kofi, S., Nuche, P., Escós, J., Alados, C.L. 2013. Modeling shrub encroachment in subalpine grasslands under different environmental and management scenarios. Journal of Environmental Management 121, 160-169. https://doi.org/10.1016/j.jenvman.2013.01.038

Kosmas, C., Danalatos, N. G., López Bermúdez, F., Romero Díaz, A. 2002. The effect of land use on soil erosion and land degradation under Mediterranean conditions. En: N.A. Geeson, C.J. Brandt, J.B. Thornes (Eds.), Mediterranean Desertification: a mosaic of processes and responses, pp. 58-70. Wiley, London.

Kozak, J., Ostapowicz, K., Szablowska-Midor, A., Widacki, W. 2004. Land abandonment in the Western Beskidy Mts and its environmental background. Ekologia (Bratislava) 23 (1), 116-26.

Kozak, J., Gimmi, U., Houet, T., Bollinger, J. 2017. Current practices and challenges for modelling past and future land use and land cover changes in mountains regions. Regional Environmental Change 17, 2187-2191. https://doi.org/10.1007/s10113-017-1217-2

Kuemmerle, T., Levers, C., Erb, K., Estel, S., Jepsen, M.R., Müller, D., Plutzar, C., Stürck, J., Verkerk, P.J, Verburg, P.H., 2016. Hotspots of land use change in Europe. Environment Research Letter 11, 064020. https://doi.org/10.1088/1748-9326/11/6/064020

Laiolo, P., Dondero, F., Ciliento, E., Rolando, A. 2004. Consequences of pastoral abandonment for the structure and diversity of the Alpine avifauna. Journal of Applied Ecology 41, 294-304. https://doi.org/10.1111/j.0021-8901.2004.00893.x

Lasanta, T. 1996. El proceso de marginación de tierras en España. En: T. Lasanta, J.M. García-Ruiz, J.M. (Eds.), Erosión y recuperación de tierras en áreas marginales. Instituto de Estudios Riojanos - Sociedad Española de Geomorfología, pp. 7-31, Logroño.

Lasanta, T. 2005. Gestion des champs abandonés pour le developpement de l'élevage extensif dans les Pyrénées centrales espagnoles. Sud-Ouest Européen 19, 109-119.

Lasanta, T. 2010. Pastoreo en áreas de montaña: Estrategias e impactos en el territorio. Estudios Geográficos 268, 203-233. https://doi.org/10.3989/estgeogr.0459

Lasanta, T. 2019. Active management against shrubland expansion: seeking a balance between conservation and exploitation in the mountains. Cuadernos de Investigación Geográfica 45 (2), 423-440. https://doi.org/10.18172/cig.3616

Lasanta, T., Arnáez, J., Nadal-Romero, E. 2019a. Soil degradation, restoration and management in abandoned and afforested lands. In: Advances in Chemical Pollution, Environmental Management and Protection. Volume 4, 71-117. https://doi.org/10.1016/bs.apmp.2019.07.002 
Lasanta, T., Arnáez, J., Pascual, N., Ruiz-Flaño, P., Errea, M.P., Lana-Renault, N. 2017a. Space-time process and drivers of land abandonment in Europe. Catena 149, 810-823. https://doi.org/10.1016/j.catena.2016.02.024

Lasanta, T., Arnáez, J., Ruiz-Flaño, P., Lana-Renault, N. 2013. Los bancales en las montañas españolas: un paisaje abandonado y un recurso potencial. Boletín de la Asociación de Geógrafos Españoles 63, 301-322.

Lasanta, T., Beguería, S., García-Ruiz, J.M. 2006. Geomorphic and hydrological effects of traditional shifting agriculture in a Mediterranean mountain, Central Spanish Pyrenees. Mountain Research and Development 26 (2), 146-152. https://doi.org/10.1659/0276-4741(2006)26[146:GAHEOT]2.0.CO;2

Lasanta, T., Cerdà, A. 2005. Long-term erosional responses after fire in the Central Spanish Pyrenees. 2. Solute release. Catena 60, 81-100. https://doi.org/10.1016/j.catena.2004.09.005

Lasanta, T., Errea, M.P., Nadal-Romero, E. 2017c. Traditional agrarian landscape in the Mediterranean mountains. A regional and local factors analysis in the Central Spanish Pyrenees. Land Degradation \& Development 28, 1626-1640. https://doi.org/10.1002/ldr.2695

Lasanta, T., García-Ruiz, J.M. 1996. Erosión y recuperación de tierras en áreas marginales. Instituto de Estudios Riojanos - Sociedad Española de Geomorfología, 211 pp., Logroño.

Lasanta, T., García-Ruiz, J.M., Pérez-Rontomé, M.C., Sancho, C. 2000. Runoff and sediment yield in a semi-arid environment: the effect of land management after farmland abandonment. Catena 38, 265-278. https://doi.org/10.1016/S0341-8162(99)00079-X

Lasanta, T., Nadal-Romero, E., Arnáez J. 2015. Managing abandoned farmland to control the impact of revegetation on the environment. The state of art in Europe. Environmental Science and Policy 52, 99-109 https://doi.org/10.1016/j.envsci.2015.05.012

Lasanta, T., Nadal-Romero, E., Errea, M.P. 2017b. The footprint of marginal agriculture in the Mediterranean mountain landscape: An analysis of the Central Spanish Pyrenees. Science of the Total Environment 599600, 1823-1836. https://doi.org/10.1016/j.scitotenv.2017.05.092

Lasanta, T., Nadal-Romero, E., García-Ruiz, J.M. 2019b. Clearing shrubland as a strategy to encourage extensive livestock farming in the Mediterranean mountains. Cuadernos de Investigación Geográfica 45 (2), 487 513. http://doi.org/10.18172/cig.3616

Lasanta, T., Nadal-Romero, E., Serrano-Muela, P. 2016. Los efectos de la revegetación en el medio natural y su percepción por la población vinculada de Cameros Viejo (Sistema Ibérico). Boletín de la Asociación de Geógrafos Españoles 70, 183-210. https://doi.org/10.21138/bage.2168

Lasanta, T., Sánchez-Navarrete, P., Medrano-Moreno, L.M., Khorchani, M., Nadal-Romero, E. 2020. Soil quality and soil organic carbon storage in agricultural abandoned fields: effects of revegetation processes in a Mediterranean mid-mountain area. Land Degradation \& Development. https://doi.org/10.1002/ldr.3655

Lasanta, T., Vicente-Serrano, S.M. 2007. Cambios en la cubierta vegetal en el Pirineo aragonés en los últimos 50 años. Pirineos 162, 125-154. https://doi.org/10.3989/pirineos.2007.v162.16.

Lasanta, T., Vicente-Serrano, S.M., Cuadrat, J.M. 2005. Mountain Mediterranean landscape evolution caused by the abandonment of traditional primary activities: a study of the Spanish Central Pyrenees. Applied Geography 25, 47-65. https://doi.org/10.1016/j.apgeog.2004.11.001

Lasanta Martínez, T. 1988a. The process of desertification of cultivated areas in the Central Spanish Pyrenees. Pirineos 132, 15-36.

Lasanta Martínez, T., 1988b. La evolución del espacio agrario en áreas de montaña: modelos en el Pirineo aragonés. Tesis Doctoral, Universidad de Zaragoza. Zaragoza.

Lasanta Martínez, T. 1989. Evolución reciente de la agricultura de montaña: el Pirineo Aragonés. Geoforma Ediciones: 220 pp., Logroño.

Lasanta-Martínez, T. 2014. El paisaje de campos abandonados en Camero Viejo (Sistema Ibérico, La Rioja). Instituto de Estudios Riojanos. Colección Ciencias de la Tierra 32, 305 pp., Logroño. 
Lasanta-Martínez, T., Arnáez, J., Ruiz-Flaño, P., Ortigosa, L. 1989. Evolución superficial del espacio cultivado en Camero Viejo (Sistema Ibérico) y su relación con algunos factores geoecológicos. Estudios Geográficos $197,553-572$.

Lesschen, J.P., Cammeraat, L.H., Kooijman, A.M., Wesamael, B. 2008a. Development of spatial heterogeneity in vegetation and soil properties after land abandonment in a semi-arid ecosystem. Journal of Arid Environments 72, 2082-2092. https://doi.org/10.1016/j.jaridenv.2008.06.006

Lesschen, J.P., Cammeraat, L.H., Nieman, T. 2008b: Erosion and terrace failure due to agricultural land abandonment in a semi-arid environment. Earth Surface Processes and Landforms 33, 1574-1584. https://doi.org/10.1002/esp.1676

Lesschen, J.P., Kok, K., Verburg, P.H., Cammeraat, L.H. 2007. Identification of vulnerable areas for gully erosion under different scenarios of land abandonment in Southeast Spain. Catena 71, 110-121. https://doi.org/10.1016/j.catena.2006.05.014

Llorens, P., Latron, J., Gallart, F. 1992. Analysis of the role of agricultural abandoned terraces on the hydrology and sediment dynamics in a small mountainous basin. Pirineos 139, 27-46. https://doi.org/10.3989/pirineos.1992.v139.180

Llorens, P., Poch, R., Latron, J., Gallart, F. 1997. Rainfall interception by a Pinus sylvestris forest patch overgrown in a Mediterranean mountainous abandoned area. I. Monitoring design and results down to the event scale. Journal of Hydrology 199, 331-345. https://doi.org/10.1016/S0022-1694(96)03334-3

Loepfe, L., Martínez-Vilalta, J., Oliveras, J., Piñol, J., Lloret, F. 2010. Feedbacks between fuel reduction and landscape homogenization determine fire regimes in three Mediterranean areas. Forest Ecology Management 259, 2366-2374. https://doi.org/10.1016/j.foreco.2010.03.009

López-Bao, J.V., González-Varo, J.P., Guitián, J. 2015. Mutialistic relationships under landscape change: carnivorous mammals and plants after 30 years of land abandonment. Basic and Applied Ecology 16, 151161. https://doi.org/10.1016/j.baae.2014.12.001

López-Bermúdez, F., Romero-Díaz, M.A. 1989. Piping erosion and badland development in South-east Spain. Catena Suppl. 14, 59-73.

López-Bermúdez, F., Romero-Díaz, M.A., Martínez-Fernández, J., Martínez-Fernández, J. 1996. The Ardal fieldsite: soil and vegetation cover. In: J. Brandt, J.B. Thornes (Eds.), Mediterranean Desertification and Land Use Wiley, 169-188 pp, Chichester.

López-Bermúdez, F., Torcal, L. 1986. Procesos de erosión en túnel (piping) en cuencas sedimentarias de Murcia (España). Estudio preliminar mediante difracción de rayos $\mathrm{X}$ y microscopio electrónico de barrido. Papeles de Geografia Física 11, 7-20.

López-Moreno, J.I., Vicente-Serrano, S.M., Morán-Tejeda, E., Zabalza, J., Lorenzo-Lacruz, J., García-Ruiz, J.M. 2011. Impact of climate evolution and land use changes on water yield in the Ebro basin. Hydrological Earth Systems Science 15, 311-322. https://doi.org/10.5194/hess-15-311-2011.

Lucas Espadas, A. 1996. Sanidad vegetal en Murcia. Agricultura 766, 398-400.

MacDonald, D., Crabtree, J.R., Wiesinger, G., Dax, T., Stamou, N., Fleury, P., Gutierrez Lazpita, J., Gibon, A. 2000. Agricultural abandonment in mountain areas of Europe: Environmental consequences and policy response. Journal of Environmental Management 59 (1), 47-69. https://doi.org/10.1006/jema.1999.0335

Maiso, E., Lasanta, T. 1990. El espacio agrario en el valle del Linares: características y utilización reciente. Berceo 118, 53-62.

Malo, J.E., Suárez, F. 1996. Las boñigas de vaca, el encespedado de Poa bulbosa y la diversidad en un pastizal de dehesa. Pastos 26 (1), 61-75.

Martín-López, B., Montes, C., Benayas, J. 2007. Influence of user characteristics on valuation of ecosystem services in Doñana Natural Protected Area (South-West, Spain). Environmental Conservation 34, $215-$ 224. https://doi.org/10.1017/S0376892907004067

Martínez Arnáiz, M. 2018. Paisaje y patrimonio en las Loras y Paramera de la Lora en Burgos y Palencia. El singular valor de un modelo de poblamiento subordinado al relieve. En: F. Molinero, J. Tort 
(coordinadores), Paisajes patrimoniales de España. Ministerio de Agricultura, Pesca y Alimentación, Ministerio para la Transición Ecológica, UAM ediciones, 1398-1421 pp., Madrid.

Martínez de Pisón, E. 2009. Los paisajes de los geógrafos. Geographicalia 55, 5-25.

Martínez-Fernández, J., López-Bermúdez, F., Martínez-Fernández, J., Romero-Díaz, M.A. 1995. Land use and soil-vegetation relationships in a Mediterranean ecosystem. Catena 25, 153-167. https://doi.org/10.1016/0341-8162(95)00007-F.

Martínez Hernández, C. 2018. El abandono de campos de cultivo en la Región de Murcia. Causas y consecuencias medioambientales y socioeconómicas. Tesis Doctoral Universidad de Murcia, Murcia.

Martínez-López, V., Zapata, V., de la Riva, P., Robledano, F. 2019. Uncovering mechanisms of bird seed dispersal in semiarid environments to helps to restore them. Ecosphere 10 (4), e02673. https://doi.org/10.1002/ecs2.2673

Meléndez-Pastor, I., Hernández, E.I., Navarro-Pedreño, J., Gómez, I. 2014. Socioeconomic factors influencing land cover changes in rural areas: The case of the Sierra de Albarracín (Spain). Applied Geography 52, 34-45. https://doi.org/10.1016/j.apgeog.2014.04.013

Molinillo, M., Lasanta, T., García-Ruiz, J.M. 1997. Managing degraded landscape after farmland abandonment in the Central Spanish Pyrenees. Environmental Management 21, 587-598. https://doi.org/10.1007/s002679900051

Montalvo, J., Casado, M.A., Levassor, C., Pineda, F.D. 1993. Species diversity patterns in Mediterranean grasslands. Journal of Vegetation Science 4, 213-222. https://doi.org/10.2307/3236107

Montserrat, P. 1964. Ecología del pasto (Ecología de los agrobiosistemas pastorales). Publicaciones del Centro Pirenaico de Biología Experimental 1 (2), 1-68.

Montserrat, P. 1976. Aspectos relacionados con la investigación en praticultura y ganadería. Anales del Instituto de Estudios Agropecuarios 2, 63-84.

Morán-Ordoñez. A., Bugter, R., Suárez-Seoane, S., de Luis, E., Calvo, L. 2013. Temporal changes in socioecological systems and their impact on ecosystems services at different governance scales: A case study of heathlands. Ecosystems 16, 765-782. https://doi.org/10.1007/s10021-013-9649-0

Morán-Tejeda, E., Ceballos-Barbancho, A., Llorente-Pinto, J.M. 2010. Hydrological response of Mediterranean headwaters to climate and land-cover changes: The mountains of Duero River basin (Central Spain). Global and Planetary Change 72, 39-49. https://doi.org/10.1016/j.gloplacha.2010.03.003

Moreno, M.V., Conedera, M., Chuvieco, E., Pezzatti, G.B. 2014. Fire regime changes and major driving forces in Spain from 1968 to 2010. Environmental Science Policy 37, 11-22. https://doi.org/10.1016/j.envsci.2013.08.005

Moreno-de-las-Heras, M., Lindenberger, F., Latron, J., Lana-Renault, N., Llorens, P., Arnáez, J., Romero-Díaz, A., Gallart, F. 2019. Hydro-geomorphological consequences of the abandonment of agricultural terraces in the Mediterranean region: Key controlling factors and landscape stability patterns. Geomorphology 333, 73-91. https://doi.org/10.1016/j.geomorph.2019.02.014

Moret, D., Pueyo, Y., Bueno, C.G., Alados, C.L. 2011. Hydro-physical responses of gypseous and non gypseous soils to livestock grazing in a semi-arid region of NE Spain. Agriculture Water Management 98, 1822 1827. https://doi.org/10.1016/j.agwat.2011.07.001

Nadal, J., Pélachs, A., Molina, D., Soriano, J.M. 2009. Soil fertility evolution and landscape dynamics in a Mediterranean area: a case study in the Sant Llorenç Natural Park (Barcelona, Ne Spain). Area 41 (2), 129-138. https://doi.org/10.1111/j.1475-4762.2008.00858.x

Nadal-Romero, E., Cammeraat, E., Pérez-Cardiel, E., Lasanta, T. 2016a. Effects of secondary succession and afforestation practices on soil properties after cropland abandonment in humid Mediterranean mountain areas. Agriculture, Ecosystems \& Environment 228, 91-100. https://doi.org/10.1016/j.agee.2016.05.003

Nadal-Romero, E., Cammeraat, E., Pérez-Cardiel, E., Lasanta, T. 2016b. How do soil organic carbon stocks change after cropland abandonment in Mediterranean humid mountain areas? Science of the Total Environment 566-567, 741-752. https://doi.org/10.1016/j.scitotenv.2016.05.031 
Nadal-Romero, E., Lasanta, T., García-Ruiz, J.M. 2013. Runoff and sediment yield from land under various uses in a Mediterranean mountain area: long term results from experimental station. Earth Surface Processes and Landforms 38 (4), 346-355. https://doi.org/10.1002/esp.3281

Nadal-Romero, E., Otal-Laín, I., Lasanta, T., Sánchez-Navarrete, P., Errea, P., Cammeraat, E. 2018. Woody encroachment and soil stocks in subalpine areas in the Central Spanish Pyrenees. Science of the Total Environment 636, 727-736. https://doi.org/10.1016/j.scitotenv.2018.04.324

Nagendra, H., Southworth, J., Tucker, C. 2003. Accessibility as a determinant of landscape transformation in western Honduras: linking pattern and process. Landscape Ecology 18 (2), 141-58. https://doi.org/10.1023/A:1024430026953

Navarro, L.M., Pereira, H.M. 2012. Rewilding abandoned landscape in Europe. Ecosystems 15 (6), 900-912. https://doi.org/10.1007/s10021-012-9558-7

Navas, A., Machín, J., Soto, J. 2005. Assessing soil erosion in a Pyrenean mountain catchment using GIS and fallout 137Cs. Agriculture, Ecosystems \& Environment 105, 493-506. https://doi.org/10.1016/j.agee.2004.07.005

Nogués-Bravo, D. 2006. Assessing the effects of environmental and anthropogenic factors in land cover diversity in a Mediterranean mountain environment. Area 38 (4), 432-444. https://doi.org/10.1111/j.14754762.2006.00709.x

Ocaña, M.C., Gómez-Moreno, M.L., Blanco-Sepúlveda, R. 2004. Las vistas como recurso territorial. Ensayo de evaluación del paisaje visual mediante un SIG. Imalgraf, 172 pp., Málaga.

Onega-López, F.J., Puppin de Oliveira, J.A., Crecente-Maseda, R. 2010. Planning innovations in land management and governance in fragmented rural areas: two examples from Galicia (Spain). European Planning Studies 18 (5). https://doi.org/10.1080/09654311003594067

Oserín Elorza, M. 2007. Cambios en la gestión del territorio en una montaña media mediterránea y sus impactos mediambientales. Hacia un nuevo paisaje (altos valles del Iregua, Leza, Jubera y Cidacos, Sistema Ibérico Noroccidental). Tesis Doctoral, Universidad de Zaragoza, Zaragoza.

Otero, I., Boada, M., Badía, A., Pla, E., Vayreda, J., Sabaté, S., Gracia, C.A., Peñuelas, J. 2011. Loss of water availability and stream biodiversity under land abandonment and climate change in a Mediterranean catchment (Olzinelles, N.E., Spain). Land Use Policy 28, 207-218. https://doi.org/10.1016/j.landusepol.2010.06.002

Otero, I., Marull, J., Tello, E., Diana, G.L., Pons, M., Coll, F., Boada, M. 2015. Land abandonment, landscape, and biodiversity: questioning the restorative character of the forest transition in the Mediterranean. Ecology and Society 20 (2). https://doi.org/10.5751/ES-07378-200207

Otto, R., Kiüsi, B.O., Burga, C.A., Fernández-Palacios, J.M. 2006. Old-field succession along a precipitation gradient in the semi-arid coastal region of Tenerife. Journal of Arid Environments 65 (1), 156-178. https://doi.org/10.1016/j.jaridenv.2005.07.005

Padilla-Blanco, M.A. 1998. Colonización vegetal en campos abandonados de la provincial de Alicante. Servicio de Publicaciones de la Universidad de Alicante, 365 pp + anexo cartográfico, Murcia.

Pallarés Bou, J. 1994. Procesos que conducen a la rotura de muros en terrazas de cultivo (Norte de Castellón). Cuaternario y Geomorfología 8 (3-4), 23-36.

Pardini, G., Aringheri, R., Plana, F., Gallart, F. 1991. Soil properties relevant to land degradation in abandoned sloping fields in Aísa Valley, Central Pyrenees (Spain). Pirineos 137, 79-93. https://doi.org/10.3989/pirineos.1991.v137.196

Pardini, G., Gispert, M. 2005. Impact of land abandonment on water erosion in soils of the Eastern Iberian Peninsula. Agrochimica 50 (1-2),13-24.

Pausas, J.G. 1999. Response of plant functional types to changes in the fire regime in Mediterranean ecosystems: A simulation approach. Journal of Vegetation Science 10, 717-772. https://doi.org/10.2307/3237086

Pausas, J.G., Fernández-Muñoz, S. 2012. Fire regime changes in the Western Mediterranean Basin: from fuellimited to drought-driven fire regime. Climatic Change 110, 215-226. https://doi.org/10.1007/s10584011-0060-6 
Peco, B., Sánchez, A.M., Azcárate, F.M. 2006. Abandonment in grazing systems. Consequences for vegetation and soil. Agriculture, Ecosystems \& Environment $113 \quad$ (1-4), 284-294. https://doi.org/10.1016/j.agee.2005.09.017

Pedroli, B., Pinto-Correia, T., Cornish, P. 2006. Landscape -what's in in? Trends in European landscape science and priority themes for concerted research. Landscape Ecology 21, 421-430. https://doi.org/10.1007/s10980-005-5204-5

Peña-Angulo, D., Khorchani, M., Errea, P., Lasanta, T., Martínez-Arnáiz, M., Nadal-Romero, E. 2019. Factors explaining the diversity of land cover in abandoned fields in a Mediterranean mountain. Catena 181, 104064. https://doi.org/10.1016/j.catena.2019.05.010

Pereira, E., Queiroz, C., Pereira, H.M., Vicente, 1. 2005. Ecosystem services and human well-being: a participatory study in a mountain community in Portugal. Ecology and Society 10 (2), 14. https://doi.org/10.5751/ES01353-100214

Pérez, T., Díaz-Fierros, F. 1988. Resistencia del suelo y susceptibilidad a la compactación en terrenos de monte sometidos a pastoreo. Anales de Edafología y Agrobiología 47 (3-4), 547-560.

Pérez-Albert, Y. 1995. Implementación de un SIG para el estudio de las tierras de cultivo abandonadas en las montañas de Prades. En: Cambios regionales a finales del siglo XX. Asociación de Geógrafos Españoles - Universidad de Salamanca, 442-445 pp., Salamanca.

Pérez-Carazo, P. 2008. Santa María de Herce y su abadengo en la Edad Media. Universidad de La Rioja-Instituto de Estudios Riojanos, Logroño.

Pérez-Morales, A. 2008. Rentabilidad socioeconómica de los cultivos con riego localizado en la Región de Murcia. En: J.M. Gómez Espín, R. Martínez Medina (Coord.), Los espacios rurales españoles en el nuevo siglo: Actas XIV Coloquio de Geografía Rural, Murcia, pp. 181-198.

Perino, A., Pereira, H.M., Navarro, L.M., Fernández, N., Bullock, J.M., Ceausu, S., Cortés-Avizanda, A., Van Klint, R., Kuemmerle, T., Lomba, A., Pe'er, G., Plieninger, T., Rey-Benayas, J.M., Sandom, C.J., Svenning, J.C., Wheeler, H.C. 2019. Rewilding complex ecosystems. Science 364, https://doi.org/10.1126/science.aav5570

Pettorelli, N., Barlow, J., Stephens, P.A., Durant, S.M., Connor, B., Schulte to Buhne, H., Sandon, C.J., Wentworth, J., du Toit, J.T. 2017. Making rewilding fit for policy. Journal Applied Ecology 55 (3), 11141125. https://doi.org/10.1111/1365-2664.13082

Pineda, F.D., Nicolás, J.P., Ruiz, M., Peco, B., Bernáldez, F.G. 1981. Succession, diversité et niche écologique dans les pâturages du centre de la Peninsule Iberique. Vegetatio 47, 267-277. https://doi.org/10.1007/97894-009-7991-8_26

Pointereau, P., Coulon, F., Girard, P., Lambotte, M., Stuczynski, T., Sánchez Ortega, V., Del Río, A. 2008. Analysis of farmland abandonment and the extent and location of agricultural areas that are actually abandoned or are in risk to be abandoned. JRC Scientific and Technical Reports (EUR 23411). http://publicationsjrceceuropaeu/repository/handle/111111111/166

Poyatos, R., Latron, J., Llorens, P. 2003. Land use and land cover change after agricultural abandonment: The case of a Mediterranean Mountain area (Catalan Pre-Pyrenees). Mountain Research and Development 23 (4), 52-58. https://doi.org/10.1659/0276-4741(2003)023[0362:LUALCC]2.0.CO;2

Pueyo, Y., Beguería, S. 2007. Modelling the rate of secondary succession after farmland abandonment in a Mediterranean mountain area. Landscape and Urban Planning 83, 245-254. https://doi.org/10.1016/j.landurbplan.2007.04.008

Pugnaire, F.I., Luque, M.T., Armas, C., Gutiérrez, L. 2006. Colonization processes in semi-arid Mediterranean old-fields. Journal of Arid Environments 65 (4), 591-603. https://doi.org/10.1016/j.jaridenv.2005.10.002

Puigdefábregas, J. 2005. The role of vegetation patterns in structuring runoff and sediment fluxes in dryland. Earth Surface Processes and Landforms 30: 133-147. https://doi.org/10.1002/esp.1181

Puigdefábregas, J., Fillat, F. 1986. Ecological adaptation of traditional land-uses in the Spanish Pyrenees. Mountain Research and Development 6 (1), 63-72. https://doi.org/10.2307/3673341 
Ramankutty, N., Foley, J.A. 1999. Estimating historical changes in global land cover: Cropland from 1700 to 1992. Global Biogeochemical Cycles 13, 997-1027. https://doi.org/10.1029/1999GB900046

Regos, A., Dominguez, J., Gil-Tena, A., Brotons, L., Ninyerola, M., Pons, X. 2016. Rural abandoned landscapes and bird assemblages: winners and losers in the rewilding of a marginal mountain area (NW Spain). Regional Environmental Change 16 (1), 199-2121. https://doi.org/10.1007/s10113-014-0740-7

Reiné, R., Barrantes, O., Chocarro, C., Juárez, A., Broca, A., Maestro, M., Ferrer, C. 2014. Pyrenean meadows in Natura 2000 network: grass production and plant biodiversity conservation. Spanish Journal of Agricultural Research 12 (1), 61-77. https://doi.org/10.5424/sjar/2014121-4617

Rescia, A.J., Pons, A., Lomba, I., Esteban, C., Dover, J.W. 2008. Reformulating the social-ecological systems in a cultural rural mountain landscape in the Picos de Europa region (Northern Spain). Landscape and Urban Planning 88, 23-33. https://doi.org/10.1016/j.landurbplan.2008.08.001

Rey-Benayas, J.M., Galván, I., Carrascal, L.M. 2010. Differential effects of vegetation restoration in Mediterranean abandoned cropland by secondary succession and pine plantations on bird assemblages. Forest Ecology and Management 260, 87-95. https://doi.org/10.1016/j.foreco.2010.04.004

Rey Benayas, J.M., Martins, A., Nicolau, J.M., Schulz, J. 2007. Abandonment of agricultural land: an overview of drivers and consequences, CAB Reviews: Perspectives in Agriculture, Veterinary Science, Nutrition and Natural Resources 2, No. 57. https://doi.org/10.1079/PAVSNNR20072057

Rey-Benayas, J.M., Martínez-Baroja, L., Pérez-Camacho, L., Villar-Salvador, P., Holl, K.D. 2015. Predation and aridity slow down the spread of 21-year-old planted Woodland islets in restored Mediterranean farmland. New Forests 46, 841-853. https://doi.org/10.1007/s11056-015-9490-8

Rey-Benayas, J.M., Navarro, J., Espigares, T., Nicolau, J.M., Zabala, M.A. 2005. Effects of artificial shading and weed mowing in reforestation of Mediterranean abandoned cropland with contrasting Quercus species. Forest Ecology and Management 212, 302-314. https://doi.org/10.1016/j.foreco.2005.03.032

Riedel, J.L., Bernués, A., Casasús, I., 2013. Livestock grazing impacts on herbage and shrub dynamics in a Mediterranean Natural Park. Rangeland Ecology Management 66, 224-233. https://doi.org/10.2111/REM-D-11-00196.1

Riedel, J.L., Casasús, I., Bernués, A. 2007. Sheep farming intensification and utilization of natural resources in a Mediterranean pastoral agro-ecosystem. Livestock Science 111, 153-163. https://doi.org/10.1016/j.livsci.2006.12.013

Ries, J.B., Langer, M., Rehberg. C. 2000. Experimental investigations on water and wind erosion on abandoned fields and arable land in the central Ebro Basin, Aragon/Spain. Zeitschrift für Geomorphology 121, 91108.

Ries, J.B., Seeger, M., Marzolff, I. 1997. El Proyecto EPRODESER. Cambios de uso del suelo y morfodinámica en el Nordeste de España. Geographicalia 35, 205-225. https://doi.org/10.26754/ojs_geoph/geoph.1997351700

Robledano Aymerich, F., Zapata Pérez, V.M., Martínez López, V., García Meseguer, A.J., García Castellanos, F.A., Zamora López, A., Pérez Navarro, M.A. 2016. Indicadores de biodiversidad en campos abandonados: flora y fauna. En: A. Romero Díaz (Coord.) Abandono de cultivos en la Región de Murcia. Consecuencias Ecogeomorfológicas. Servicio de Publicaciones de la Universidad de Murcia, 111-137pp., Murcia.

Rodríguez Aizpeolea, J., Pérez Badía, R., Cerdà, A. 1991. Colonización vegetal y producción de escorrentía en bancales abandonados: Vall de Gallinera, Alacant. Cuaternario y Geomorfología 5, 119-129.

Rodríguez-Gutiérrez, F. 1989. La organización agraria de la montaña central asturiana. Consejería de Educación, Cultura y Deportes, Principado de Asturias, 636 pp., Oviedo.

Rodríguez-Martín, J.A., Álvaro-Fuentes, J., Gabriel, J.L., Gutiérrez, C., Nanos, N., Escuer, M., Ramos-Miras, J.J., Gil, C., Martin-Lammerding, D., Boluda, R. 2019. Soil organic carbon stock on the Majorca island: temporal change in agricultural soil over the last 10 years. Catena 181, 104087 https://doi.org/10.1016/j.catena.2019.104087 
Rodríguez-Merino, E., Errea, M.P., Lasanta, T. 1998. Valoración de los recursos pastorales de campos abandonados de Cameros Viejo (Sistema Ibérico riojano). Cuadernos de Investigación Geográfica 24, 69-87. https://doi.org/10.18172/cig.1027

Romero-Díaz, A. 2003. Influencia de la litología en las consecuencias del abandono de tierras de cultivo en medios mediterráneos semiáridos. Papeles de Geografía 38, 151-165.

Romero-Díaz, A. 2016. Estado de la cuestión de los estudios de abandono. En: A. Romero-Díaz (Coord.), Abandono de cultivos en la Región de Murcia. Consecuencias ecogeomorfológicas. Servicio de Publicaciones de la Universidad de Murcia, 19-39 pp., Murcia.

Romero-Díaz, A., Marín-Sanleandro, P., Sánchez-Soriano, A., Belmonte-Serrato, F., Faulkner, H. 2007. The causes of piping in a set of abandoned agricultural terraces in Southeast Spain. Catena 69, 282-293. https://doi.org/10.1016/j.catena.2006.07.008

Romero Díaz, A., Martínez Hernández, C., Belmonte Serrato, F. 2016. Procesos de erosión en áreas abandonadas de la Región de Murcia. En: A. Romero Díaz (Coord.), Abandono de cultivos en la Región de Murcia. Consecuencias Ecogeomorfológicas. Servicio de Publicaciones de la Universidad de Murcia, 85-110 pp., Murcia.

Romero Díaz, A., Ruiz Sinoga, J.D., Robledano-Aymerich, F., Brevik, E.C., Cerdà, A. 2017. Ecosystem responses to land abandonment in Western Mediterranean Mountains. Catena 149, 824-835. https://doi.org/10.1016/j.catena.2016.08.013

Romero-Martín, L., Ruiz-Flaño, P., Hernández-Calvento, L. 2004. Diagnóstico y calidad para la conservación de los espacios agrícolas abancalados. Propuesta metodológica para la cuenca del Guiniguada (Gran Canaria, Islas Canarias). Geographicalia 45, 113-127. https://doi.org/10.26754/ojs_geoph/geoph.2004451348

Rubio Balducci, P. 2019. Natural vegetation succession after land abandonment: a soil carbon comparison in the Central Spanish Pyrenees. Tesis-Master, Universidad de Amsterdam, 53 pp., Amsterdam.

Ruecker, G., Schad, P., Alcubilla, M.M., Ferrer, C. 1998. Natural regeneration of degraded soils and site changes on abandoned agricultural terraces in Mediterranean Spain. Land Degradation \& Development 9, 179188. https://doi.org/10.1002/(SICI)1099-145X(199803/04)9:2<179::AID-LDR276>3.0.CO;2-R

Ruiz-Flaño, P. 1993. Procesos de erosión en campos abandonados del Pirineo. Geoforma Ediciones: 191 pp., Logroño.

Ruiz-Flaño, P., García-Ruiz, J.M., Ortigosa, L. 1992. Geomorphological evolution of farmland abandonment fields. A case study in the Central Pyrenees. Catena 19, 301-308. https://doi.org/10.1016/03418162(92)90004-U

Ruiz-Flaño, P., Lasanta, T., Arnáez, J., Ortigosa, L., Oserín, M. 2010. El proceso de abandono del espacio agrícola en Cameros. En: T. Lasanta, J. Arnáez (Eds.), Gestión, usos el suelo y paisaje en Cameros. Sistema Ibérico, La Rioja. Universidad de La Rioja - Instituto de Estudios Riojanos, 109-126 pp., Logroño.

Ruiz-Sinoga, J.D., Martínez-Murillo, J.F. 2009. Hydrological response of abandoned agricultural soils along a climatological gradient on metamorphic parent material in southern Spain. Earth Surface Processes and Landforms 34, 2047-2056. https://doi.org/10.1002/esp.1890

Ruiz-Sinoga, J.D., Martínez-Murillo, J.F., Gabarrón-Galeote, M.A., García-Marín, R. 2011. The effects of soil moisture variability on the vegetation pattern in Mediterranean abandoned fields (Southern Spain). Catena 85, 1-11. https://doi.org/10.1016/j.catena.2010.11.004

Salaverri, L., Guitián, J., Munilla, I., Sobral, M. 2019. Bird richness decreases with the abandonment of agriculture in a rural region of SW Europe. Regional Environmental Change 19 (1), 245-250. https://doi.org/10.1007/s10113-018-1375-X

Sayadi, S., González-Roa, M.C., Calatrava-Requena, J. 2009. Public preferences for landscape features: The case of agricultural landscape in mountainous Mediterranean areas. Land Use Policy 26, 334-344. https://doi.org/10.1016/j.landusepol.2008.04.003

Sánchez Soriano, A. 2012. Procesos de erosión subsuperficiales (piping) en la Región de Murcia. Tesis Doctoral, Universidad de Murcia, Murcia. 
Santa Regina, I. 1995. Ciclos biogeoquímicos en bosques de la sierra de Béjar (Salamanca). Retorno de bioelementos por medio de agua de lluvia. Pirineos 145-146, 65-80. https://doi.org/10.3989/pirineos.1995.v145-146.147

Seeger, M., Beguería, S. 2003. La respuesta hidrológica en dos cuencas experimentales con diferentes usos del suelo en el Pirineo aragonés. En: I. Marzolff, J. Ries, J. de la Riva, M. Seeger (Eds.), El cambio en el uso del suelo y la degradación del territorio en España. Universität Frankfurt am Main y Universidad de Zaragoza: 203-221 pp., Zaragoza.

Segura, C., Jiménez, M.N., Nieto, O., Navarro, F.B., Fernández-Ordoño, E. 2006. Changes in soil organic carbon over 20 years after afforestation in semiarid SE Spain. Forest Ecology and Management 381, 268-278. https://doi.org/10.1016/j.foreco.2016.09.035

Serrano-Muela, P., Lana-Renault, N., Nadal-Romero, E., Regüés, D., Latron, J., Martí-Bono, C., García-Ruiz, J.M. 2008. Forest and their hydrological influence in Mediterranean mountains. The case of the Central Spanish Pyrenees. Mountain Research and Development 23 (3-4), 279-285. https://doi.org/10.1659/mrd.0876

Serrano-Muela, P., Regüés, D., Nadal-Romero, E., 2012. Trascolación y escorrentía cortical en un bosque de montaña media mediterránea. Un estudio reciente en el Pirineo central español. Cuaternario y Geomorfología 26 (1), 49-72.

Shakesby, R.A. 2011. Post-wildfire soil erosion in the Mediterranean: Review and future research directions. Earth-Science Reviews 105, 71-100. https://doi.org/10.1016/j.earscirev.2011.01.001

Solé-Benet, A., Lázaro, R., Domingo, F., Canton, Y., Puigdefábregas, J. 2010. Why most agricultural terraces in steep slopes in semiarid SE Spain remain well preserved since their abandonment 50 years ago? Pirineos 165, 215-235. https://doi.org/10.3989/Pirineos.2010.165011

Strijker, D. 2005. Marginal lands in Europe -causes of decline. Basic Applied Ecology 6, 99-106. https://doi.org/10.1016/j.baae.2005.01.001

Suárez-Seoane, S., Osborne, P.E., Baudry, J. 2002. Responses of birds of different biogeographic origins and habitat requirements to agricultural land abandonment in Northern Spain. Biological Conservation 105, 333-344. https://doi.org/10.1016/S0006-3207(01)00213-0

Tarazona, T., Santa Regina, I., Calvo, R. 1996. Interception, through fall and streamflow in two forest of the "Sierra de la Demanda" in the province of Burgos. Pirineos 147-148, 27-40. https://doi.org/10.3989/pirineos.1996.v147-148.135

Tárraga, R., Calvo, L., Taboada, A., García-Tejero, S., Marcos, E. 2009. Abandonment and management in Spanish dehesa systems: Effects on soil features and plant species richness and composition. Forest Ecology and Management 257, 731-738. https://doi.org/10.1016/j.foreco.2008.10.004

Tella, J.L., Forero, M.C., Hiraldo, F., Donázar, J.A. 1998. Conflicts between Lesser Kestrel conservation and European Agricultural Policies as identified by habitat use analysis. Conservation Biology 12 (3), 593604. https://doi.org/10.1046/j.1523-1739.1998.96288.x

Terres, J.M., Scacchiafichia, L.N., Wania, A., Ambar, M., Anguiano, E., Buckwell, A., Coppola, A., Gochtf, A., Källström, H.N., Pointereau, P., Strijker, D., Visek, L., Vranken, L., Zoben, A. 2015. Farmland abandonment in Europe: identification of drivers and indicators, and development of a composite indicator risk. Land Use Policy 49, 20-34. https://doi.org/10.1016/j.landusepol.2015.06.009

Tobarra González, M.A. 2002. Agua y plan hidrológico en la Región de Murcia. Papeles de Geografia 36, $185-$ 206.

Trigalet, S., Gabarrón-Galeote, M.A., Van Oost, K., van Wesemael, B. 2016. Changes in soil organic carbon pools along a chrono sequence of land abandonment in southern Spain. Geoderma 268, 14-21. https://doi.org/10.1016/j.geoderma.2016.01.014

Ustaoglu, E., Collier, M.J. 2018. Farmland abandonment in Europe: an overview of drivers, consequences, and assessment of the sustainability implications. Environmental Reviews 26 (4), 396-416. https://doi.org/10.1139/er-2018-0001 
Valdelvira, A., Balcells, E. 1986. La pardina de Esporret como unidad empresarial de las sierras prepirenaicas meridionales. Pirineos 128, 79-118.

Vallecillo, S., Brotons, L., Herrando, S. 2008. Assessing the response of open-habitat bird species to landscape changes in Mediterranean mosaics. Biodiversity Conservation 17, 103-119. https://doi.org/10.1007/s10531-007-9233-z

Van Leeuween. C.C.E., Cammeraat, E.L.H., de Vente, J., Boix-Fayos, C. 2019. The evolution of soil conservation policies targeting land abandonment and soil erosion in Spain: A review. Land Use Policy 83, 174-186. https://doi.org/10.1016/j.landusepol.2019.01.018

Vera, F., Romero, J. 1994. Impacto ambiental de la actividad agraria. Agricultura y Sociedad 71, 153-181.

Verdú, M., García-Fayos, P. 1996. Nucleation processes in a Mediterranean bird-dispersion plant. Functional Ecology 10, 275-280. https://doi.org/10.2307/2389853

Verdú, M, García-Fayos, P. 1998. Old-field colonization by Dapnhe gnidium: distribution and spatial dependence at different scales. Journal of Vegetation Science 9, 713-718. https://doi.org/10.2307/3237289

Vicente-Serrano, S., Beguería, S., Lasanta, T. 2006. Diversidad espacial de la actividad vegetal en campos abandonados del Pirineo Central español: análisis de los procesos de sucesión secundaria mediante imágenes Landsat (1984-2001). Pirineos 161, 54-84. https://doi.org/10.3989/pirineos.2006.v161.3

Vicente-Serrano, S.M., Lasanta, T., Cuadrat, J.M. 2005. Analysis of the spatial and temporal evolution of vegetation cover in the Spanish Central Pyrenees. The role of human management. Environmental Management 34 (6), 802-818. https://doi.org/10.1007/s00267-003-0022-5

Vila-Subirós, J., Ribas-Palom, A., Varga-Linde, D., Llausàs-Pascual, A. 2009. Medio siglo de cambios paisajísticos en la montaña mediterránea. Percepción y valoración social del paisaje en la alta Garrotxa (Girona). Pirineos 164, 69-92. https://doi.org/10.3989/pirineos.2009.v164.30

Violant i Simorra, R. 1949. El Pirineo español. Editorial Plus Ultra, 675 pp., Madrid.

Viviroli, D., Weingartner, R., Messerli, B. 2003. Assessing the hydrological significance of the World's mountains. Mountains Research and Development 23, 32-40. https://doi.org/10.1659/02764741(2003)023[0032:ATHSOT]2.0.CO;2

Zapata, V.M., Robledano Aymerich, F., Martínez López, V. 2016. Dinámica recolonizadora de la vegetación en campos abandonados de la Región de Murcia. En: A. Romero Díaz (Coord.), Abandono de cultivos en la Región de Murcia. Consecuencias Ecogeomorfológicas. Servicio de Publicaciones de la Universidad de Murcia, 139-159pp., Murcia.

Zornoza, R., Guerrero, C., Mataix-Solera, J., Srew, K.M., Arcenegui, V., Mataix-Beneyto, J. 2009. Changes in soil microbial community structure following the abandonment of agricultural terraces in mountain areas of Eastern Spain. Applied Soil Ecology 42, 315-323. https://doi.org/10.1016/j.apsoil.2009.05.011

Zozaya, E., Brotons, L., Saura, S. 2002. Recent fire history and connectivity patterns determine bird species distribution dynamics in landscape dominated by land abandonment. Landscape Ecology 27, 171-184. https://doi.org/10.1007/s10980-011-9695-y 



\title{
TERRITORIAL IMPACTS OF SEA-LEVEL RISE IN MARSH ENVIRONMENTS. THE CASE OF THE BAY OF CÁDIZ, SPAIN
}

\author{
FRANCISCO J. VÁZQUEZ PINILLOS ${ }^{1 *}$, MANUEL J. MARCHENA GÓMEZ ${ }^{2}$ \\ ${ }^{1}$ Departamento de Historia, Geografía y Filosofía. Universidad de Cádiz, \\ Plaza de España, 9, 11006 Cádiz, Spain. \\ ${ }^{2}$ Departamento de Geografía Física y Análisis Geográfico Regional. Universidad de Sevilla, \\ C/ Doña María de Padilla, s/n, 41004 Sevilla, Spain.
}

\begin{abstract}
How can sea-level rise affect territory? Has territorial planning adjusted to this new situation? This paper analyses the possible ramifications of changes in tidal recurrence over the course of this century in a potentially vulnerable marsh environment, such as the Bay of Cádiz, in southern Spain, where sea-level rise is already a fact. For that purpose, the regionalisation criterion is used as a basis for adjusting the magnitude of global problems to subregional scale. Geographic information systems are applied to portray the forecasted territorial changes according to the RCP4.5 and RCP8.5 scenarios presented by the Intergovernmental Panel on Climate Change (IPCC) for the years 2050 and 2100. Projections of sea-level rise show that the tide is travelling farther inland, not just invading unoccupied areas of marshes and beaches but also reaching urban and productive areas. Estimates indicate that the floodable area could expand by more than $20 \%$ in 2050 , with a further 2,000 ha of flooded areas added in 2100 for either of the two scenarios used. The occurrence of these changes, regardless of the model used, would therefore entail an alteration of the environmental, social, cultural and economic values and elements of the Bay of Cádiz, with the Natural Park being the most affected area. In response to these consequences, the need to apply the resulting projections to other variables is insisted on, with a view to introducing territorial management tailored to this new and already present reality. Application of the regionalisation criterion to study repercussions of the climate crisis in the Bay of Cádiz could serve as a precedent for the development of adaptation strategies in other marsh environments at subregional or local level.
\end{abstract}

\section{Impactos territoriales del ascenso del nivel del mar en entornos de marisma. El caso de la Bahía de Cádiz, España}

RESUMEN. ¿Cómo el ascenso del nivel del mar puede afectar al territorio? ¿Está la planificación territorial adaptada a esta nueva coyuntura? En este trabajo se analizan las implicaciones que podrían suponer los cambios en la recurrencia mareal a lo largo del presente siglo, sobre un entorno marismeño y potencialmente vulnerable como el de la Bahía de Cádiz, al sur de España, donde el ascenso del nivel del mar ya es un hecho. Para ello, se emplea el criterio de regionalización como base para ajustar la magnitud de los problemas globales a una escala subregional y la aplicación de los Sistemas de Información Geográfica para representar las alteraciones territoriales previstas según los escenarios RCP4.5 y RCP8.5 planteados por el Panel Intergubernamental del Cambio Climático (IPCC) para los años 2050 y 2100. Las proyecciones del ascenso del nivel del mar ilustran un incremento del espacio recorrido por la marea tierra adentro, invadiendo no solo zonas desocupadas de las marismas y las playas, sino también alcanzando las áreas urbanas y productivas. De acuerdo con las estimaciones, la superficie inundable se expandiría en más de un $20 \%$ en 2050 , y se sumarían 2.000 ha de zonas inundadas en 2100 para cualquiera de los dos escenarios empleados. Por tanto, la ocurrencia de estos cambios, con independencia del modelo utilizado, implicaría una alteración de los valores y elementos ambientales, sociales, culturales y económicos de la Bahía de 
Cádiz, siendo el Parque Natural el espacio más afectado. En respuesta a estas consecuencias, se insiste en la necesidad de implementar las proyecciones resultantes a otras variables de cara a la instauración de una gestión territorial adaptada a esta nueva -y ya presente- realidad. La aplicación del criterio de regionalización para el estudio de las repercusiones de la crisis climática en la Bahía de Cádiz, podría servir de precedente para la elaboración de estrategias de adaptación en otros entornos de marisma a escala subregional o local.

Key words: Climate change, sea-level rise, RCP, IPCC, territory.

Palabras clave: Cambio climático, subida del nivel del mar, RCP, IPCC, territorio.

Received: 24 February 2020

Accepted: 16 August 2020

* Corresponding author: F. J. Vázquez Pinillos. Departamento de Historia, Geografía y Filosofía. Universidad de Cádiz. Plaza de España, 9, 11006 Cádiz (España). E-mail: fj.vazquezpinillos@outlook.com

\section{Introduction}

The current global context cannot be defined without taking into account a climate situation that affects not only the environmental dimension but also the political, social and economic dynamics that govern the world's future (Le Roy Ladurie, 2017). This reality, concurrent with other social transformations, has been identified for several decades under the label of 'climate change'. However, given the urgent need to alleviate the effects already being manifested, that term has begun to be replaced by other concepts such as 'climate crisis' or 'climate emergency' (Fundéu, 2019). In any case, in this work the second expression mentioned has been employed, as it has been the most widely used in scientific literature in recent years.

The climate crisis started at the same time that the geological period of the Anthropocene began, both originating in human activities (Trischler, 2017). This new course in climate history began with the First Industrial Revolution, with the rise of factories, expansion of transportation and use of fossil fuels. This is when an incipient temperature rise in the troposphere can be glimpsed in the recorded data - the beginning of global warming, one of the key manifestations for understanding what is happening at present (Le Roy Ladurie, 2017; Valladares et al., 2019).

The concept of climate crisis is often used interchangeably to refer to both the causes and the consequences. The increase in greenhouse gas concentrations and the rise in mean sea-level, for example, are conceived as being parts of the same idea (Vineis et al., 2011; Pongiglione, 2012). In short, it is a complex reality in which the changes occurring in recent decades have been generated at a worrisome pace. For this reason, the climate crisis has become a paramount object of study in the area of scientific research and a political concern manifested by the development of strategies meant to both mitigate (moderating the scope of the causal factors) and adapt, with a view to reducing the impacts (ONU, 2015).

Noteworthy among the environmental impacts generated by the climate crisis are the expansion of desertification, more torrential rainfall, intensification of fires and loss of forest cover, changed ecosystems and the escalation of adverse weather events, as well as the increased frequency, height and intensity of storm surges (Karl et al., 2009; Banholzer et al., 2014; Huaico et al., 2018). The climate crisis also influences increased temperature in the oceans, where an acidification process is also occurring. Since this causes a decrease in the calcium carbonate that bivalve organisms feed on to build their shells, some components of marine ecosystems are thereby altered, such as the case of coral reefs (Clarcke et al., 2007). 
The territorial consequence about which there is more consensus is the rise of mean sea level resulting from the melting of glacial and polar ice masses, as well as the surface expansion of oceans, whose waters are gradually getting warmer year after year (Masson et al., 2018). The upper 75 metres of ocean masses became between 0.09 and 0.13 degrees warmer per decade between 1971 and 2010 (Church and White, 2011; Stocker et al., 2013). In the $20^{\text {th }}$ century mean sea level rose about $20 \mathrm{~cm}$. It is currently estimated that the ongoing trend doubles the rate observed in the last century (Spencer et al., 2015; Wigand et al., 2015). In any case, it is impossible for the mean sea level to rise more than two metres by the end of the century, as that is the maximum limit that could occur under the worst circumstances (Pfeffer et al., 2008). The process does not affect all coasts the same way, as other factors such as regional changes, winds, closeness of glaciers and vertical rock movement also have influence (FitzGerald et al., 2008; Karl et al., 2009).

Regarding the European continent, its location and geographical characteristics make it especially vulnerable with respect to the anticipated climatic changes. Global warming has been more vigorous in the middle latitudes of the northern hemisphere. In Europe there has accordingly been a thermal increase of $1.4^{\circ} \mathrm{C}$ compared to pre-industrial levels. Deaths associated to heat waves have also increased. The mean sea-level rise will also have serious consequences for the European coast, particularly in the Netherlands, where more than $50 \%$ of the country's territory has an altitude of less than 5 metres. In contrast, in other states such as Portugal, Spain or Turkey this percentage is between $0.5 \%$ and $1.5 \%$ (Bozhinova et al., 2014; Echavarren et al., 2019). These figures should obviously not be neglected since most of the population lives near the coast. Mean sea level will rise more than $40 \mathrm{~cm}$ along practically the entire coastline, except in the northernmost regions. It can therefore be expected (according to the outlined scenario) that by 2100 many beaches and urban complexes will have disappeared (Vousdoukas et al. 2020; Thiéblemont et al., 2019).

In this context, coastal areas can be considered the places most vulnerable to effects of the climate crisis, especially where the relief is very flat, as in the case of marshes. Moreover, in recent decades coastal areas have become the most changing, prosperous and vulnerable areas on the planet. Although there are differences in the extent of coastal occupation, the general trend suggests that this process continues to accelerate over time. As early as 2005 the FAO forecast that the population in coastal areas would double in the next 20 to 30 years (FAO, 2005). There is no doubt that the saturation of coastal zones implies increased exposure of the population and infrastructures to the threat of increasingly recurrent extreme events (Kulp and Strauss, 2019). In sum, the climate crisis does not just mean the appearance of new territorial dysfunctions but also the exacerbation of existing problems such as floods, saline intrusions, loss of coastal ecosystems and beach erosion (FitzGerald et al., 2008).

Under these terms, the subject matter considered in this paper is fundamental for understanding the behaviour of a phenomenon of such great interest, which has garnered a great deal of media and scientific attention and become one of the pillars of major political party agendas around the world (Paglia, 2018). The Intergovernmental Panel on Climate Change (IPCC), specifically encourages this type of local studies (which are rare in Spain) (Chica, 2010) to obtain more precise and reliable information about vulnerability by comparing past and present behaviour (Stocker et al., 2013). The lack of a climate crisis discourse in coastal areas as vulnerable as the one presented in this study makes manifest the urgent need to take note of the impacts occurring in this location and the dangers it must inevitably face in the future.

The main objective pursued by this paper consists of applying the regionalisation criterion to deduce the potential implications of a rise of mean sea level at subregional or local scale. In other words, global projections are adjusted so they can be used in the particular case of the Bay of Cádiz. As a secondary objective, it is proposed on the one hand to examine how far the implications of mean sealevel rise will be reflected in the Bay of Cádiz region throughout the 21 st century. On the other hand, it is considered to depict the incidence that mean sea-level rise will have by means of extrapolation models and techniques, so that territorial management policies can be developed beforehand. 


\section{Study area}

The research has been developed in the Bay of Cádiz region, situated at the southern end of the Iberian Peninsula in the Autonomous Community of Andalusia. Located in Cádiz province, this region comprises five municipal districts: Cádiz, Chiclana de la Frontera, El Puerto de Santa María, Puerto Real and San Fernando (Fig. 1).

It is an area very likely to undergo various territorial transformations resulting from this rise of mean sea level, given its geographic characteristics: a level and marshy space where land and sea merge, with the Bay of Cádiz Natural Park inserted among the region's five large cities (Benavente et al., 2015; Gómez, 2011). Ultimately, we find ourselves before an area of transition between land and water ecosystems, a singular landscape which is becoming increasingly fragile owing to anthropic pressure and the undeniable rise of mean sea level. According to the Preliminary Analysis of the Andalusian Coast's Vulnerability to Potential Sea-Level Rise Associated to Climate Change, the Bay of Cádiz is one of the regions most likely to suffer these changes (Méndez et al., 2011).

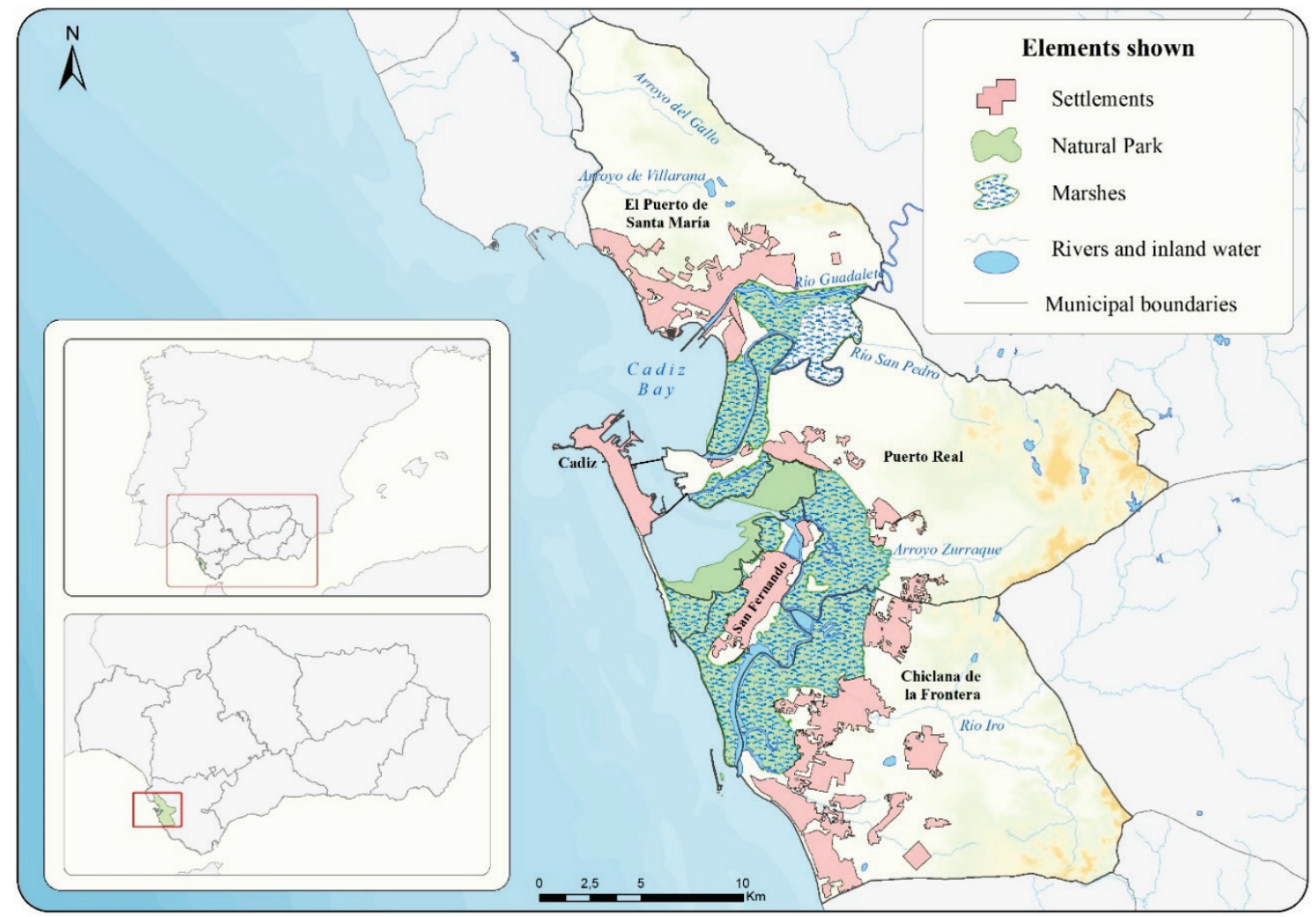

Figure 1. Location of the Bay of Cádiz region.

\section{Climate crisis and the Bay of Cádiz: two inseparable realities}

Available figures from tidal gauges close to the Bay of Cádiz prove that the rise of mean sea level is not a future estimate of a consequence of the climate crisis but rather a phenomenon that has been occurring for decades (Fig. 2). Since the early 1960s mean sea level has risen more than $20 \mathrm{~cm}$ in the Bay of Cádiz, around $3.5 \mathrm{~mm}$ annually, with reliability of $97.4 \%$. The fifth IPCC report forecast that this trend will increase over time, leading to progressive subsidence of coastal areas (Stocker et al., 2013). Mean sea-level rise in the Bay of Cádiz is the indicator that shows the most marked upward trend when compared to other variables whose behaviours are also seen to be changing with the current climate crisis, such as rising temperatures or lower precipitation (MAPAMA, 2016). 


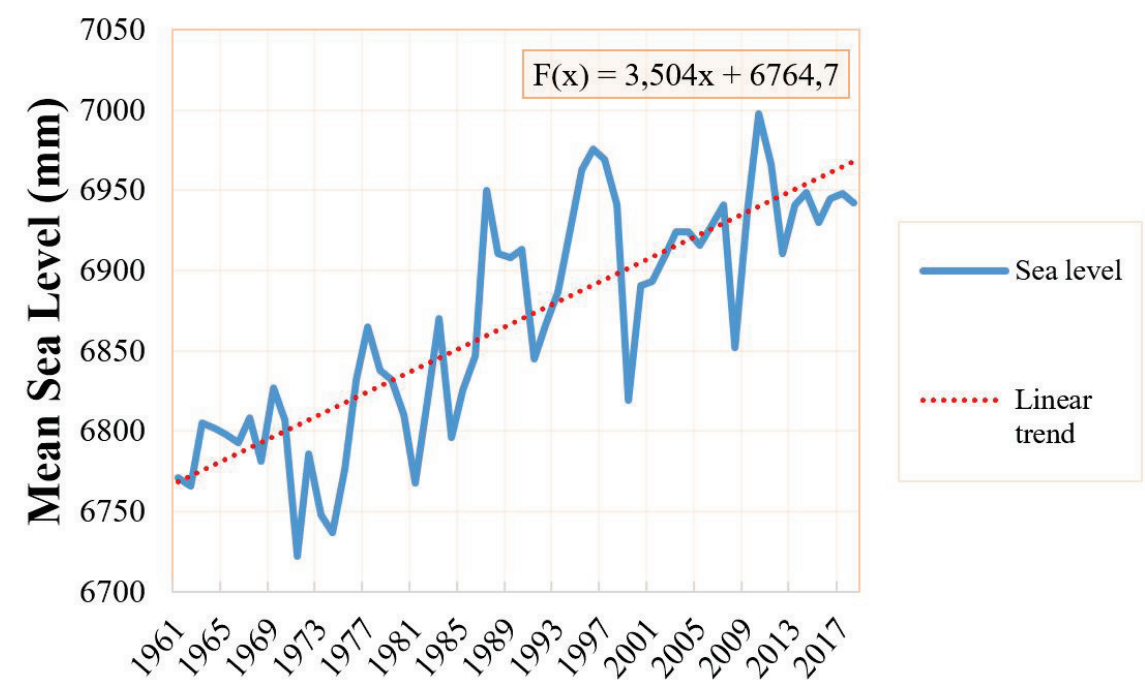

Figure 2. Evolution of mean sea level in the Bay of Cádiz (1961-2018).

The most well-known consequence is the increase in seawater flooding, an environmental problem that is already affecting various areas, such as some neighbourhoods in the municipality of Puerto Real, where every winter the streets are invaded by seawater due to storms. Moreover, these floods have worsened due to the higher number and intensity of extreme weather events also resulting from the climate crisis. On the other hand, the saline intrusions which will augmente by rising sea levels also generate worrisome impacts for the Puerto Real-Conil aquifer in the southernmost part of the region, which extends through the municipality of Chiclana and Puerto Real. This aquifer is in turn adversely affected by over-exploitation and pollution by fecal discharges due to poorly managed urbanization within city limits. The use of septic tanks has spread due to the absence of sewer systems, but they do not guarantee proper waste water treatment. The rise of mean sea level thus endangers water supply from the aquifer for the populations and agricultural sector of the Bay of Cádiz, a complex problem accentuated by the concurrently lower levels of precipitation (Chica and Barragán, 2011).

The rise of mean sea level and more storms also imply an increase in coastal erosion on Bay of Cádiz beaches, some of which are already experiencing dune regression. However, these conditions only reinforce the problems deriving from construction of breakwaters and dikes that constitute an obstacle for the proper functioning of littoral systems. The most worrisome case is the barrier spit of Valdelagrana in the municipality of El Puerto de Santa María, where the shoreline is receding around six metres per year, leading to loss affecting not just the beach but also the marshes forming part of the Natural Park (Benavente et al., 2015).

In any case, this new evolution threatens the Bay of Cádiz from various sides, beyond the direct impacts acting on natural or urban areas. The economy of the Bay of Cádiz depends on the coast and ultimately on the sea, owing to its lead role in most activities that take place, from traditional sectors such as salt collection or fishing to services. The intense exploitation of littoral resources along with uncontrolled urbanization has heightened the vulnerability and exposure of the Bay of Cádiz's populations to the threat from the current climate situation. The economic sectors most vulnerable to mean sea-level rise are aquaculture, whose facilities are situated in marshes that will undergo more tidal recurrence over time, and tourism, which is the main activity driving the local economy. A drop in sunand-sand tourism demand could occur due to water-supply problems, stronger climate conditions and, as is obvious, rising mean sea level and progressive regression of beaches (Méndez et al., 2011).

It is, therefore, clear that mean sea-level rise is the phenomenon that will cause the most damage: beaches and dunes will continue to recede as the sea invades. Extreme weather events (storms and floods) will intensify and become more frequent, even reaching areas well inland that were never 
previously threatened, and saline intrusions will augment. Also, the increased coastal vulnerability and exposure will entail serious impacts for the economy and it will become necessary to modify territorial plans.

The implications of the climate crisis on the Bay of Cádiz, which are occurring today, will predictably derive into much more alarming future alterations.

\section{Model and methodology}

This section focuses on building up projections for mean sea-level rise, given that it is the root of most of the territorial problems this area will face. For that purpose, the model put forward by the Fifth IPCC Report (2013), the latest issued to date, is used as reference. Most of the future estimates are based on studies by this body, as it is the most relevant in study of the climate crisis, although generally the model corresponding to the previous reports was used more assiduously, given that this latest one was published recently. There are different models presented by other experts, which show a considerably more pessimistic view, as in the forecasts of Pfeffer et al. (2008). Those authors indicate that over the course of the current century mean sea level will rise between $0.8 \mathrm{~m}$ and $2 \mathrm{~m}$ worldwide. Therefore, according to their studies, the most optimistic projection they put forward surpasses the most pessimistic index (RCP8.5) divulged by the IPCC, according to which a rise of $0.63 \mathrm{~m}$ is estimated. In any case, the projections of this paper are solely confined to the model from the last IPCC report.

Four scenarios are set out in the year 2013 report, known as representative concentration pathways (RCPs), indicating possible trends that will arise from greenhouse gas emissions in the $21^{\text {st }}$ century. This paper will discard two of those scenarios: RCP2.6, because it depicts an overly optimistic, even utopic, pathway; and RCP6.0, because it is the situation least considered in other studies. There is usually a tendency to use just one scenario, but in this case it was decided to approach the calculations by means of two of them (RCP4.5 and RCP8.5), the aim being to avoid presenting a biased view. Precise data is not handled, but rather complex estimates that can vary depending on the paths society takes as a whole. Therefore, by means of simultaneous use of a scenario wherein $\mathrm{CO}_{2}$ emissions would stabilize before 2100 (RCP4.5) and a pessimistic scenario of high greenhouse gas concentrations (RCP8.5), a most probable and accurate understanding of future events is obtained.

However the model bases its projections on global estimates for mean sea-level rise, i.e. it does not take into consideration spatial variability influenced by other regional factors (Karl et al., 2009). Thus, to understand the future behaviour of mean sea-level rise in a subregional context, the methodology put forward by Fraile et al. $(2013,2016,2018)$ was used. With this resource the flooding probability in each map cell is estimated, obtaining a more detailed result for cartographic depiction of the incidence of rising mean sea level. There are other methodologies for determining these changes (Titus and Richman, 2001; Gesch, 2009), though in most of them the result is presented dichotomously, i.e. identifying zones that would be affected by rising mean sea level and those that would not be harmed (Fraile et al., 2018).

The aim was accordingly to locate zones of the Bay of Cádiz that would end up below sea level and changes that would occur on the floodable surface, whose areas will also increase during this century. In this paper, the projections do not just refer to the year 2100 horizon (as put forward in the methodology used); they also apply to the year 2050. The procedures used are explained below in various phases.

The rise of mean sea level is a phenomenon marked by great spatial variability, so the figures indicated by the IPCC should not be used without considering local changes, which can differ substantially (Ministry of Agriculture, 2016). For that reason, in this first phase the local figure for sealevel rise is estimated for the selected horizons and scenarios. To analyse local sea-level rise the records of the Cádiz III tidal gauge were used, available in Permanent Service for Mean Sea Level (PSMSL, 
2019) and already used when analysing the evolution of mean sea level in the study context (Fig. 2). The database published by the Commonwealth Scientific and Industrial Research Organisation (CSIRO, 2013) was also used; it supplies information about changes in mean sea level worldwide up to the year 2013 (Fraile and Fernández, 2016). Calculation of the local value of sea-level rise is achieved using three variables, represented in the following equation:

$$
S L R=L \times T+G
$$

Being SLR: local value of mean sea-level rise for a specific year and scenario; $L$ : difference between the global and local mean sea-level rise trend; $T$ : period that runs from data collection until year of projection; $G$ : global value for mean sea-level rise per the scenario used by the IPCC.

The difference between the global and local $(L)$ trends is obtained by linear regression of the data in the period available for both registers (Table 1). The results are more accurate because one of the Spanish tidal gauges with the longest time coverage is used. Regarding the global trend, the figures were updated to the year 2013 (last year for which there is information), with respect to those used by Fraile and Fernández (2016), who took data from 2011 as the last year of reference.

Table 1. Sea-level change trend in the tidal gauge Cádiz III

\begin{tabular}{cccc} 
Period observed & Local trend & Global trend & Difference \\
\hline $1961-2013$ & $3.72 \mathrm{~mm} /$ year & $2.14 \mathrm{~mm} /$ year & $1.58 \mathrm{~mm} /$ year \\
\hline
\end{tabular}

The result of the difference is subsequently multiplied by the number of years since the last date on which the IPCC projects were based (2005) until the horizon on which the mean sea-level rise is mean to be reflected (2050 and 2100). That is, $T$ equals 46 for the year 2050 and 96 for the year 2100 , considering that the start date is included in the calculation. Finally, the product of this multiplication is added to the predicted value for mean sea-level rise according to the estimates from scenarios RCP4.5 and RCP8.5 (Table 2).

Table 2. Forecasted mean sea-level rises according to the IPCC (measured in metres)

\begin{tabular}{cccc} 
Horizon & $\boldsymbol{T}$ & RCP4.5 & RCP8.5 \\
\hline 2050 & 46 & 0.26 & 0.3 \\
2100 & 96 & 0.47 & 0.63 \\
\hline
\end{tabular}

Next, in the second phase, the level of local flooding for both scenarios and horizons is estimated, with a probability of $50 \%$. The following formula is applied for that purpose:

$$
N i=N M M L+S L R+P L
$$

Being $N i$ : local flooding level; $N M M L$ : mean sea level with respect to the topographic zero of Alicante; $S L R$ : local mean sea-level rise value; $P L$ : local high tide.

Taking only NMML and SLR, the zones that would end up below $0 \mathrm{~m}$ sea level can be found, subtracting the value of the sum from the Digital Elevations Model (DEM), with a probability of 50\%. Both the calculation and the depiction of results used the MDE generated by the Andalusian Institute of 
Statistics and Cartography (IECA) between 2008 and 2009, which has a spatial resolution of 10 meters (IECA, 2008).

For local high tide $(P L)$, the highest tide from the latest data records of the State Ports (PE, 2019) was chosen. The value used corresponds to 19 March 2011 and coincides with the measurement used by Fraile et al. (2018), in accordance with the Earth's proximity to the Sun and the position of the heavenly bodies. The Bay of Cádiz is located in a mesotidal zone, whereby the influence of tides on the territory is quite significant, as can be seen in the total surface of current floodable zones. The three identified variables are set out in Table 3.

Table 3. Values for calculating local flooding level (measured in metres)

\begin{tabular}{ccccc} 
& \multicolumn{2}{c}{ RCP4.5 } & \multicolumn{2}{c}{ RCP8.5 } \\
\hline \multirow{3}{*}{ SLR } & 2050 & 2100 & 2050 & 2100 \\
& 0.33 & 0.62 & 0.37 & 0.78 \\
\hline \multicolumn{3}{c}{ NMML } & 0.17 \\
\cline { 2 - 4 } & PL & 1.72 \\
\hline
\end{tabular}

The cartographic representation will result from subtracting the value of the local flooding level (Ni) from the DEM for each year of the selected scenarios and years, using the software ArcMap. Thus, this third phase consists of modifying the DEM so that all of the cells with a value resulting as equal to or less than 0 metres will form part of the predictable flood zone, for a probability of $50 \%$. The results are shown in Table 4.

Table 4. Values for mean sea-level rise (measured in metres)

\begin{tabular}{ccc} 
Horizon & RCP4,5 & RCP8,5 \\
\hline 2050 & 2.22 & 2.26 \\
2100 & 2.51 & 2.67 \\
\hline
\end{tabular}

Now in this paper, as in the bibliography used, the aim has been to avoid the dichotomous depiction of results, where only the flooded cells are distinguished form those that would not be affected by the rise of mean sea level. Accordingly, places where flooding probability is under $50 \%$ would not be rejected. To estimate flooding probability three typical deviations were added to and subtracted from the values for mean sea-level rise (Fraile et al., 2013).

The model used for the projections of mean sea-level rise were subjected to some changes in this paper, which can be summarised as follows:

- The calculations were projected for both years 2100 and 2050;

- Given the conditions determined, zones which would end up below $0 \mathrm{~m}$ sea level and could become flooded areas were added;

- The expression of flood probability values follows a discrete distribution. It was decided to use this representation type because it is easier to manage and to graphically analyse the results. The 'high probability' category corresponds to cells whose likelihood of flooding is above $90 \%$; 'medium probability' is associated with values with a likelihood between $50 \%$ and $90 \%$; and 'low probability' indicates a likelihood under $50 \%$. 


\section{Results}

Next, the sea-level rise projections for 2050 and 2100 are presented, according to scenarios RCP4.5 and RCP8.5.

The application of cartographic tools on the mean sea-level rise projections enables prediction of the extent of future territorial changes that will affect the urban, economic and environmental systems. This is an exercise of regionalisation, wherein global climate crisis patterns have adjusted to the specific circumstances of the Bay of Cádiz.

The first conclusion drawn from viewing the maps is the evident danger facing the study area, for regardless of the scenario selected the consequences are clearly tangible. In second place, it could be expected that minor dissimilarities between the estimates from RCP4.5 and RCP 8.5 would deliver similar results. Unsurprisingly, a slight change in the mean sea-level rise projections proves to lead to totally different scenarios.

Figure 3 considers that fluctuations in mean sea level will have important incidence for the year 2050, even though those projections correspond to scenario RCP4.5. The flooded surface, understood to be that which would end up under seawater in the absence of the tidal factor, would increase considerably in size, occupying beaches and invading a large part of the marshes northeast of San Fernando and to the south near the settlement of Sancti Petri in Chiclana de la Frontera. The extent of areas that would be situated under $0 \mathrm{~m}$ sea level would approach 2,000 ha, although it must be borne in mind that a portion of it is already currently under that threshold (Table 5). Also, this analysis does not take into account eventual topographic barriers, natural or artificial, which prevent flooding of low inland zones. However these areas are generally situated close to the shore, whereby this problem has no influence. In any case, in the cartography they are shown as zones situated under $0 \mathrm{~m}$ sea level and not as flooded zones, to avoid confusion. With respect to floodable surface, it would increase by $20.44 \%$, albeit taking into account the entire probability percentage range. The zone with a high probability of becoming floodable is particularly situated between the Guadalete and San Pedro Rivers on the region's northern fringe.

For 2100, the most notable changes would occur in the zones situated under $0 \mathrm{~m}$ sea level, as can be seen at Trocadero Island (located in the westernmost part of the municipality of Puerto Real), almost all of which would end up under water. The extent of the floodable zones would increase by just over $7 \%$ compared the year 2050 , above all in the northernmost part of the map. The zones previously considered places with medium or low flooding probability would now become high-risk zones (Fig. 4). 


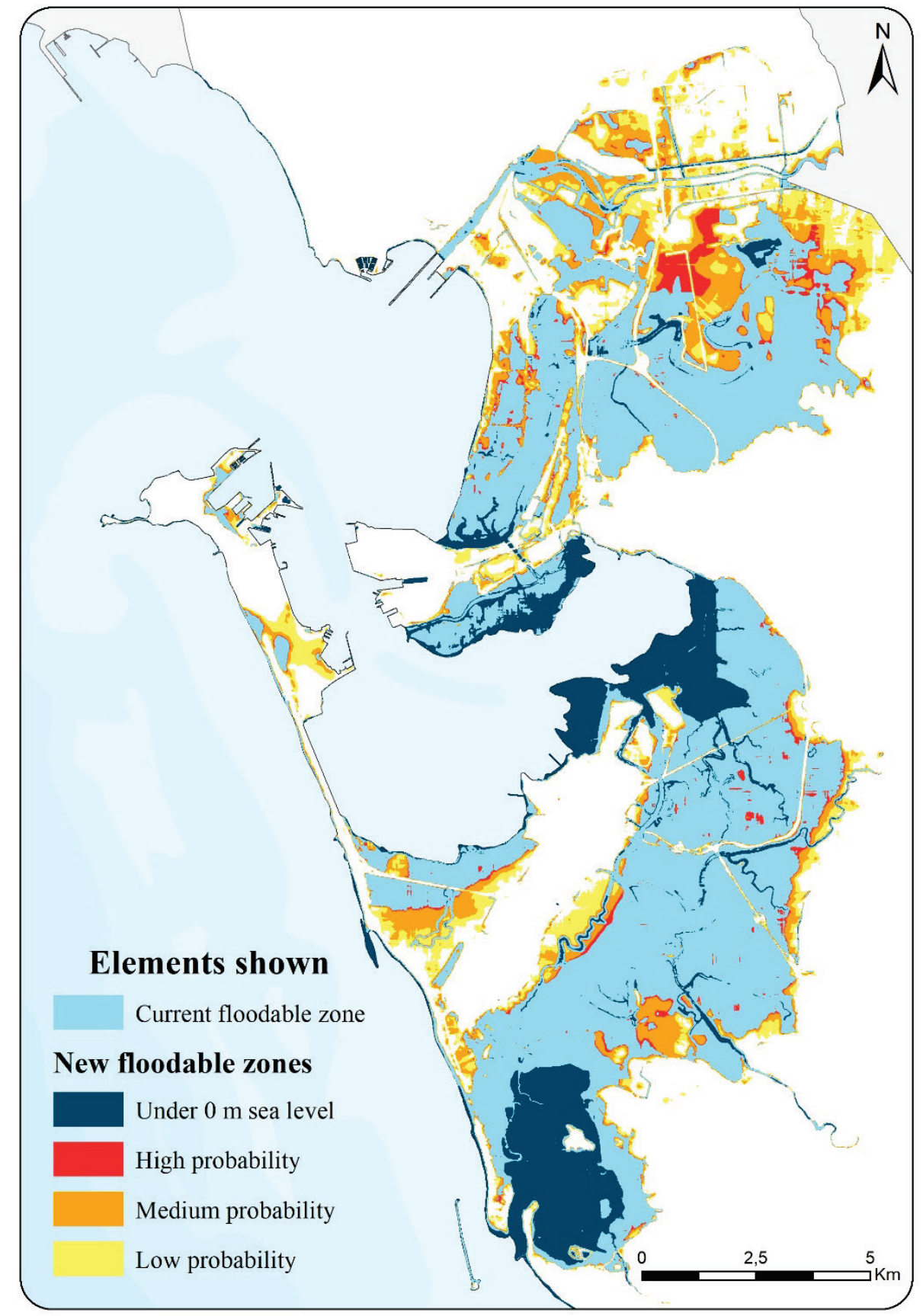

Figure 3. Mean sea-level rise projections according to IPCC scenario RCP4.5 for the year 2050.

Table 5. Changes in mean sea-level rise according to RCP4.5 (measured in hectares)

\begin{tabular}{cccccc} 
& $<\mathbf{0}$ m sea & & \multicolumn{2}{c}{ Floodable zone } & \multicolumn{2}{c}{ Total floodable } \\
\cline { 3 - 5 } RCP4.5 & level & High probability & Medium probability & Low probability & 9157.75 \\
& 358.24 & & 9157.75 & 1507.06 & 11029.95 \\
Present & 1985.21 & 7994.46 & 1528.43 & 1129.17 & 11694.78 \\
$\mathbf{2 0 5 0}$ & 2549.19 & 9084.37 & 1481.24 & \\
$\mathbf{2 1 0 0}$ & & &
\end{tabular}




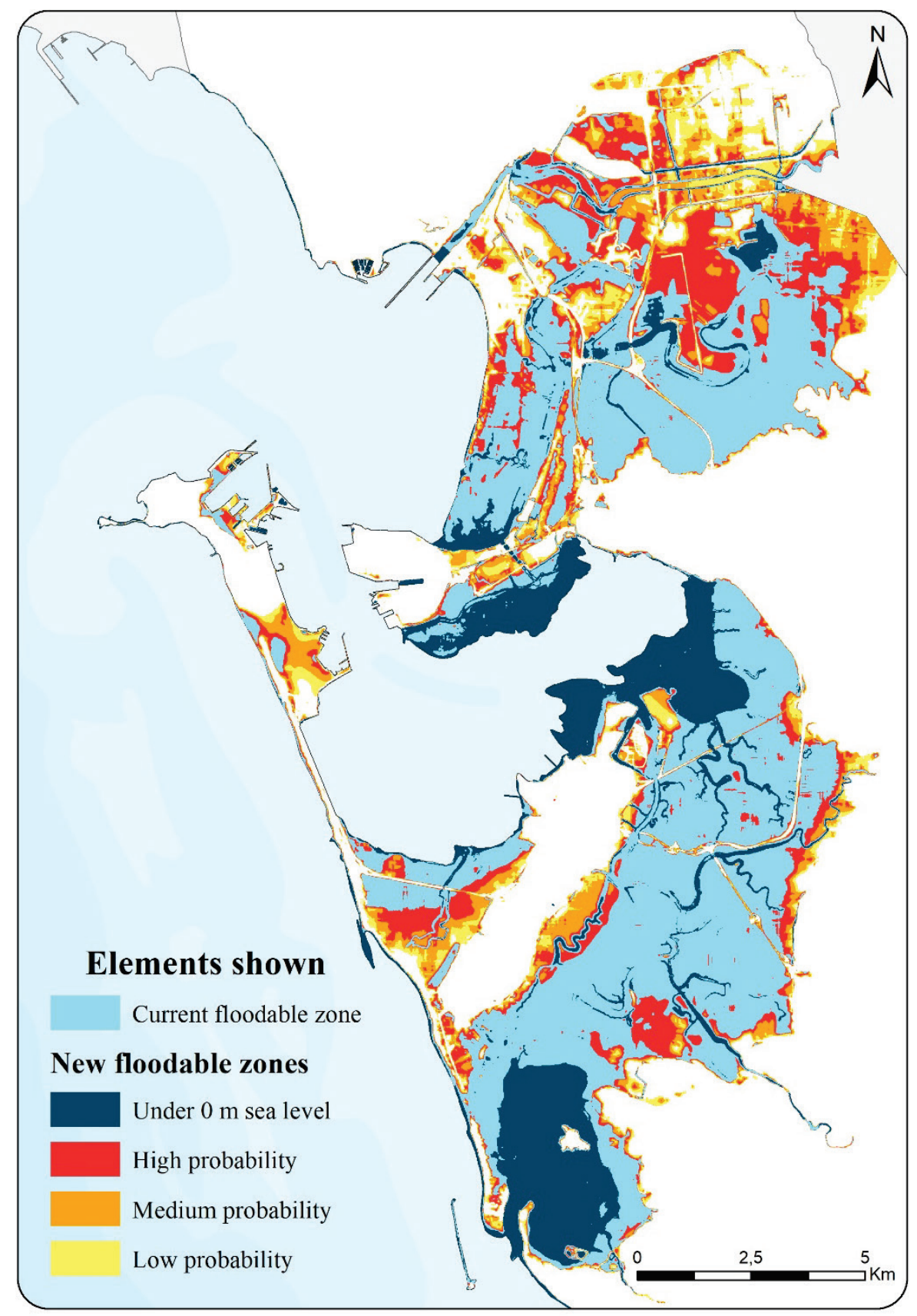

Figure 4. Mean sea-level rise projections according to IPCC scenario RCP4.5 for the year 2100.

As for scenario RCP8.5, it is striking that for 2050 a lower flooding probability with respect to RCP4.5 is indicated, though when only considering total floodable surface it can be seen that the number of hectares affected by mean sea-level rise in 2050 would be similar to what takes place in 2100 for scenario RCP4.5 (Fig. 5). This is because the typical deviation used for RCP8.5 is higher than that used in the other scenario. 


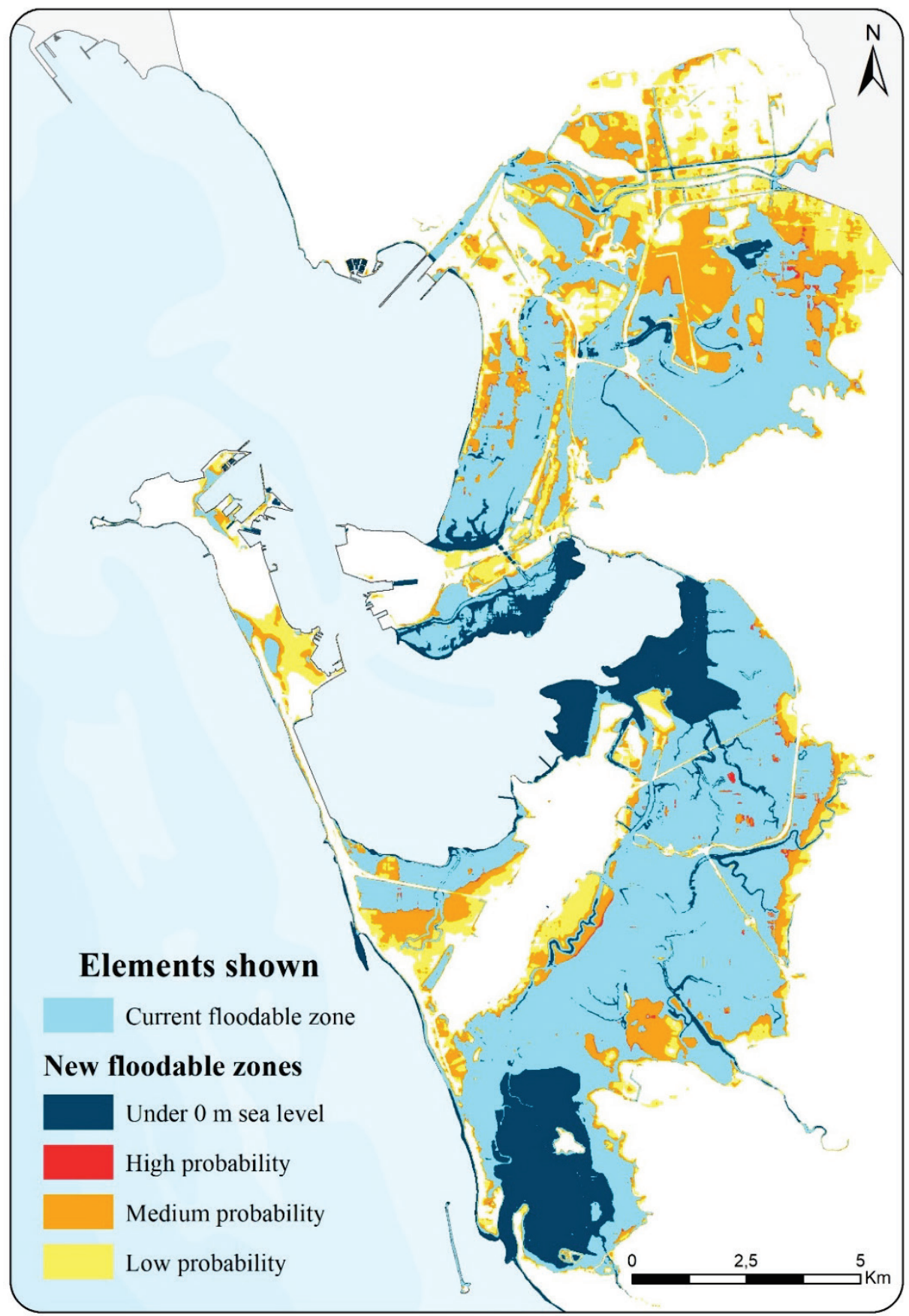

Figure 5. Mean sea-level rise projections according to IPCC scenario RCP8.5 for the year 2050.

Finally, if the most pessimistic circumstances according to the IPCC occur, at the end of the century the flooded surface would invade new areas inland from the marshes, seriously endangering some of the Bay's infrastructures. The floodable zones would grow even more and with high probability (Table 6), occupying marshland, protected spaces, agricultural zones and even some urban developments. If a high tide of this scale occurred, per the parameters of scenario RCP8.5 in the year 2100 there would be a serious problem connecting the region's five municipalities. This is even more damaging, bearing in mind that the relationships occurring in this scope are adjusted to a polycentric model. The links between Cádiz and San Fernando via the CA-33 and between Cádiz and Puerto Real via the CA-35 would be affected by the calculated mean sea-level rise and the selected high tide. The same would happen with the roads linking San Fernando and Chiclana, and between Puerto Real and El Puerto de Santa María (Fig. 6). 
Table 6. Changes in mean sea-level rise according to RCP8.5 (measured in hectares)

\begin{tabular}{cccccc}
\multirow{2}{*}{ RCP8.5 } & $<\mathbf{0}$ m above & \multicolumn{3}{c}{ Floodable zone } & \multicolumn{2}{c}{ Total floodable } \\
\cline { 3 - 5 } & sea level & High probability & Medium probability & Low probability & zone \\
\hline Present & 358.24 & & 9157.75 & 1823.44 & 1157.75 \\
$\mathbf{2 0 5 0}$ & 2062.12 & 7596.7 & 2090.97 & 1.11 \\
$\mathbf{2 1 0 0}$ & 2839.44 & 9257.84 & 1732.4 & 1053.71 & 12043.95 \\
\hline
\end{tabular}

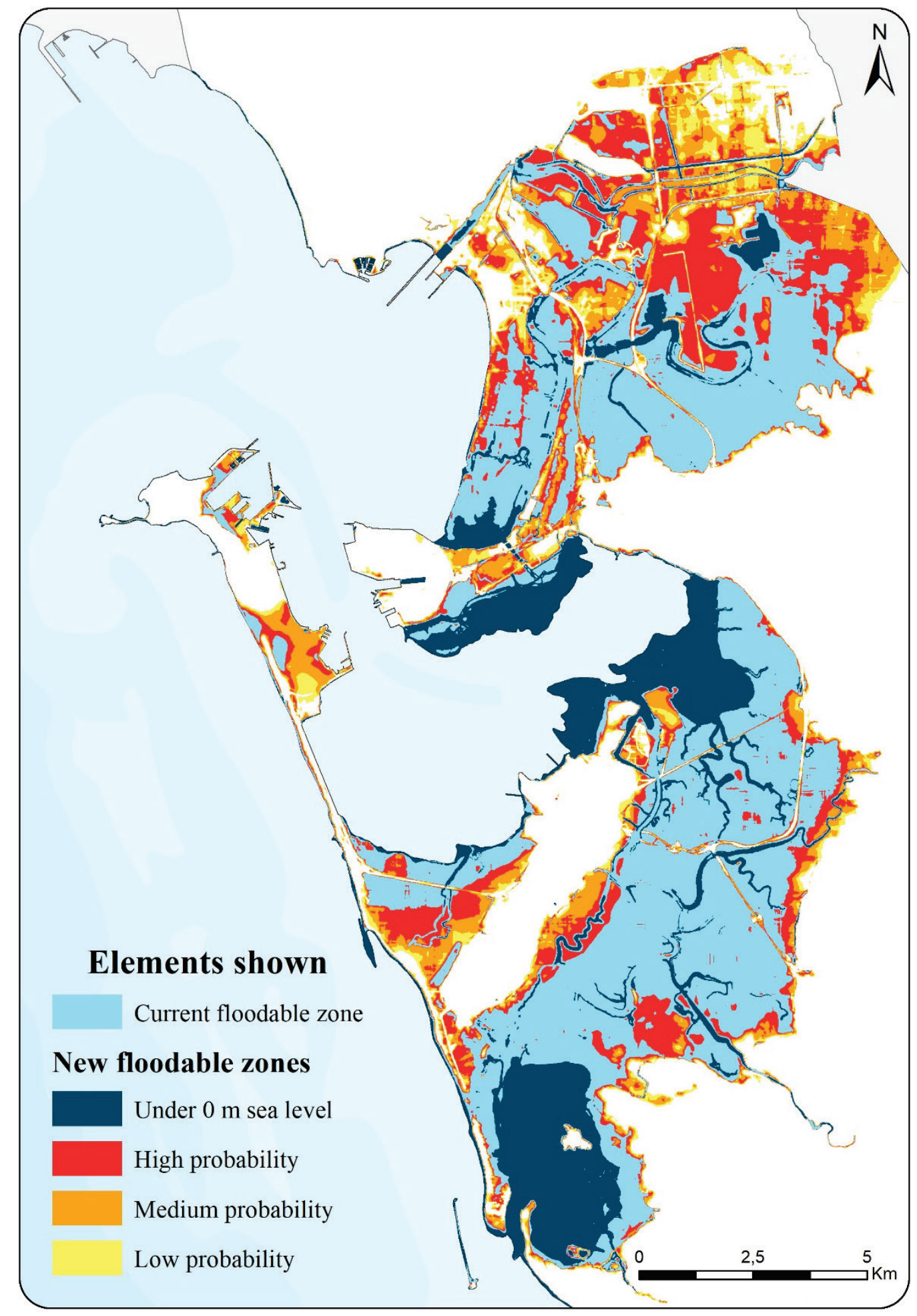

Figure 6. Mean sea-level rise projections according to IPCC scenario RCP8.5 for the year 2100. 
In short, the zones most likely to become flooded places during the $21^{\text {st }}$ century are the marshes of San Fernando and Chiclana (southern part of the region), Trocadero Island in Puerto Real and the southern end of the Valdelagrana barrier spit in El Puerto de Santa María, whose surface forms part of the Natural Park. With respect to floodable zones, the places most likely to suffer the invasion of tides expand over the region's five municipalities. In Cádiz, the most notable changes will occur on its beaches, docks and seafront promenade, as well as in urban developments and productive areas in the southernmost part of the municipality. In San Fernando, the marshes will lose part of their current surface and floodable zones will expand around the entire perimeter of the city as well as its beaches. In Chiclana it is forecast that the beach of Barrosa (the region's longest) will significantly recede and in the westernmost districts of the city the same problems already occurring in some neighbourhoods of this area may begin to be felt. In Puerto Real the most vulnerable zones are the southwest part of the municipality and the adjacent industrial poles along with the campus of the University of Cádiz. Finally, in El Puerto de Santa María the space on the left bank of the Guadalete River and the area close to the municipal limit of Jerez (northeast of the region) around Poblado de Doña Blanca will be the zones most affected by the rise of mean sea level.

Figure 7 shows the values grouped for each of the maps presented. The increase in the zones under $0 \mathrm{~m}$ sea level can clearly be seen and above all the areas with a high probability of becoming floodable.

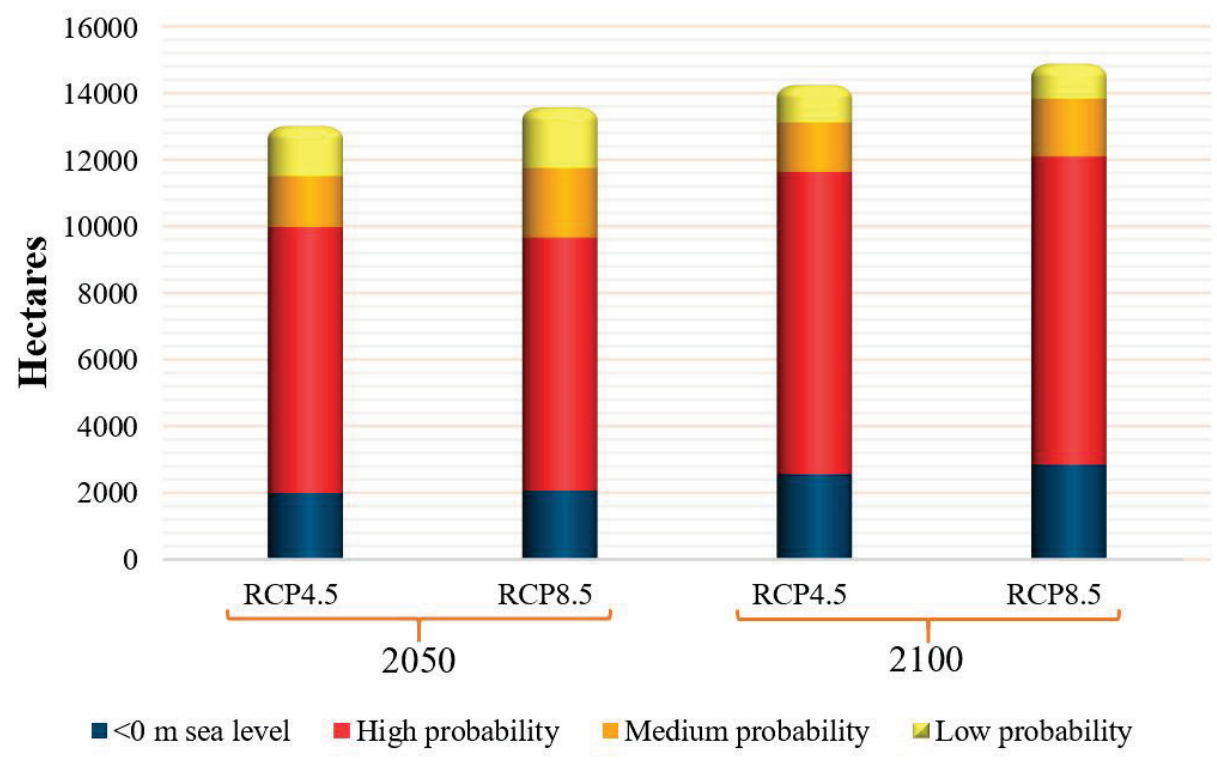

Figure 7. Changes in floodable surface of the Bay of Cádiz based on the maps.

On the other hand, the projections of mean sea-level rise may have utilities such as the study of their implications on property values (Fig. 8). Territorial planning, environmental resource management and urban planning should encourage studies about the vulnerability and exposure of assets of major environmental or cultural value in the face of upcoming changes. Scenario RCP4.5 was used because it is the one most coherent regarding current trends for rising $\mathrm{CO}_{2}$ emissions (Van Khanh, 2020). Among assets of cultural interest, several that are periodically invaded by tides because they are situated in the marshes stand out, such as salt-pond buildings. If the mean sea level continues to rise there will be more recurrent flooding and hence increased deterioration of the affected assets. It is interesting to consider how certain historical assets might not just be eroded by tidal flow but would also end up below sea level, such as the Fort of San Luis on Trocadero Island in the westernmost part of Puerto Real municipality. 

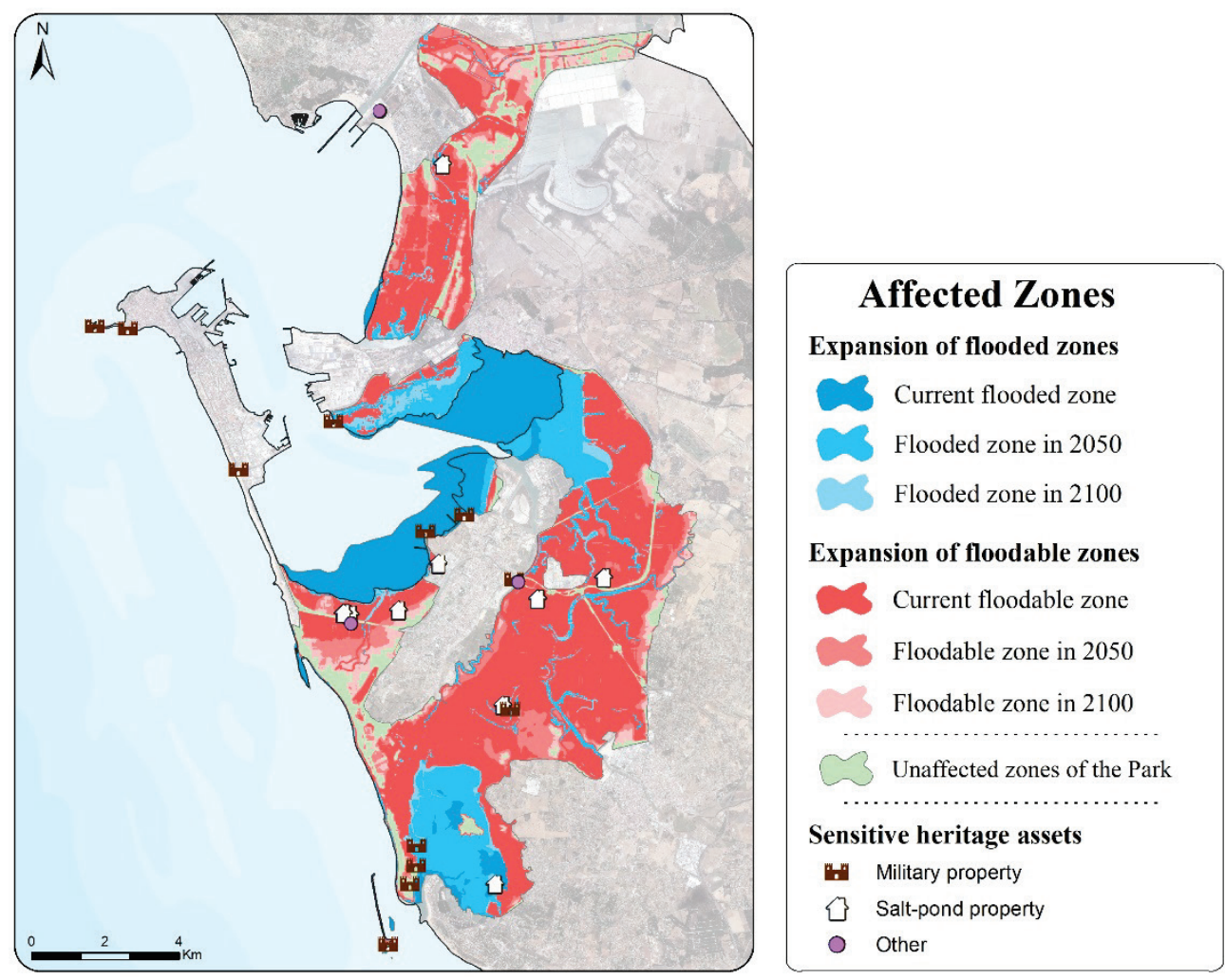

Figure 8. Implications of mean sea-level rise on the Natural Park and property assets of the Bay of Cádiz according to RCP4.5, for a probability above $90 \%$.

The Bay of Cádiz Natural Park is the element that will present a more considerable transformation due to its geographic features. The assets of cultural interest that will presumably be affected during the current century are also indicated. Only the most emblematic of the salt-pond buildings are shown. Most of these singular elements are abandoned, lack legal protection and are directly exposed to the rising mean sea level.

As for the Bay of Cádiz Natural Park, the sea depths and ecosystems it houses would deteriorate due to chemical and temperature changes of its waters, as already indicated, though also due to the rising mean sea level, which adversely affects some species used to dwelling in shallow waters. Some of the emerged surface of the park would end up under water in a not-too-distant future, with the resulting consequences for land-based life. The marshes' vegetation is distributed in bands from the shore inland, depending on tidal influence (Rendón, 2011). The accelerated expansion of floodable surface would hinder the ability of vegetation to adjust to variations in tidal periodicity and many species might consequently disappear (FOESA, 2013). The changes in flooded and floodable surface of the Natural Park are summarised in Figure 9. These graphs summarise the transformations seen in the previously shown maps, where an increase in zones situated under $0 \mathrm{~m}$ sea level is considered and a reduction of the surface unaffected by tidal recurrence. The estimates correspond to scenario RCP 4.5 for a probability above $90 \%$. 

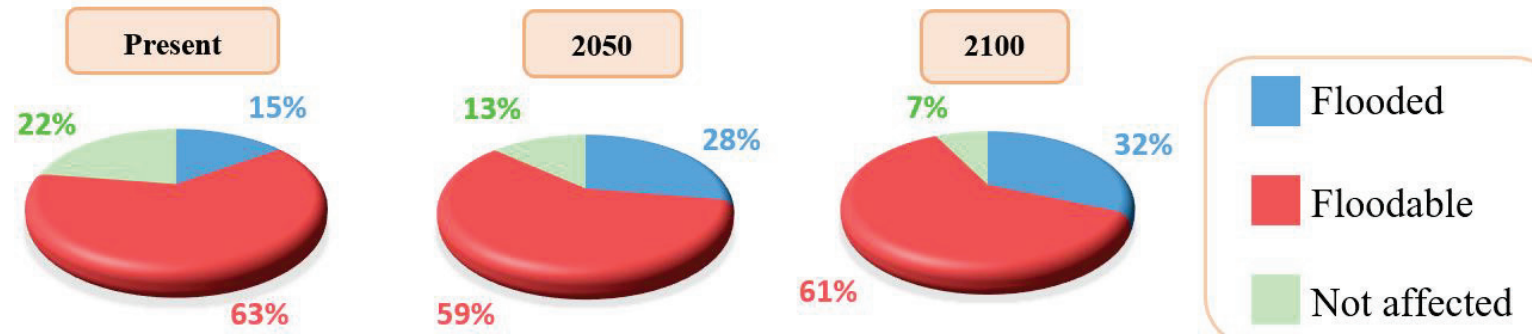

Figure 9. Changes in the surface of the Bay of Cádiz Natural Park.

\section{Discussion}

Application of the regionalisation criterion allows for construction of a subregional cartography with much higher resolution than those used to represent the behaviour of climatic phenomena on a global scale, thereby facilitating the visualisation of spatial variability among the impacts on different land uses.

The studies on mean sea-level rise considered can be used to diagnose territorial impacts from many standpoints, which could be a subject of future papers. The imminence of the expected changes (see projections for the year 2050) should spur reworked planning in matters involving adaptation to and mitigation of the climate crisis, especially at subregional and local scale (Chica, 2010). The future changes to the physical environment projected in the previous section would hence imply inordinate costs for the government. However, if passivity is chosen instead of implementing new measures, then the economic consequences will be devastating (Rodríguez, 2015).

The projections described overlook the existence of other processes unleashing the climate crisis that will intensify as sea level rises, such as the increased frequency and energy of adverse weather phenomena, barometric oscillations, wave setup, wind setup and even increased devastation from an eventual tsunami, given that as mean sea level rises large waves will have fewer obstacles to keep them out of settled areas (FitzGerald et al., 2008; Benavente et al., 2019). Likewise, to improve the rigour of the analysis, it would be worthwhile to consider changes produced in the sedimentary budget caused by reservoir construction, coastal infrastructure, dredging or aggregate extraction (Hartig et al., 2002; Pranzini and Williams, 2013). If any of these variables were added to the parameters already analysed, the cartographic result would be very different. According to the maps, for flooding to happen the involvement of weather phenomena will not be necessary; only an exceptional tide will be needed.

It should nevertheless be stressed that the projections for the rise of mean sea level are made with a high degree of uncertainty, due to both speed with which the changes are manifesting and the concurrence of multiple factors that may condition the result. However, the broad temporal spectrum of available tidal gauge data, the use of two RCP models and probabilistic calculation reduce this imprecision (Fraile et al., 2018). Although in the scientific literature it is more common to use a dichotomous representation when mapping the effects of the rise of mean sea level, to show different future situations several models and time horizons are often resorted to. For example, Hartig et al. (2002) estimated projections of mean sea-level rise in the New York City's Jamaica Bay marsh areas by using five global climate scenarios for the years 2020, 2050, 2080, and 2090. Prado et al. (2019) applied a similar criterion for the mouth of the Ebro in Spain, though based on the RCP4.5 and RCP8.5 scenarios of the last IPCC report, which coincides with this investigation.

In general, the authors agree that predictions of mean sea-level rise should be reflected in cartography using the Geographic Information Systems, with ArcMap being the most recurrent software (Hartig et al., 2002; Bornman et al., 2016; Raw et al., 2020). Also, the projections tend to conform to the models disseminated by the IPCC, both those published by the Special Report in Emissions Scenarios (SRES) and the RCP. In other studies, it is preferable to extrapolate the data collected by local tidal gauges to compare them with global prediction models (Hartig et al., 2002). 
Compared to other tidal gauges around the world, a significantly higher growth is observed in the Bay of Cádiz. The $3.5 \mathrm{~mm}$ annual rise in mean sea level in this environment contrasts with, for example, the $2.7 \mathrm{~mm}$ recorded by Hartig et al. (2002) in New York in the USA or the $2.19 \mathrm{~mm}$ observed by Raw et al. (2020) in the Knysna estuary in South Africa. However, contrary to what might be expected, the result of the calculation in this research is slightly more optimistic than in the literature used (Fraile et al., 2018). Despite all this, most researchers who have worked on the analysis and projection of mean sea-level rise in marsh areas agree that such ecosystems are diminishing as temperatures rise. The balance of coastal wetlands depends on numerous factors (wave erosion, exposure to prevailing winds, accumulation of sediments, mean sea level); but if any of those elements are altered, the ecosystem will weaken (Reed, 2002; Craft et al., 2008).

The application of cartographic projections of mean sea-level rise to other variables can be an instrument for administration. This article proposed as an example to study the consequences this phenomenon would have on heritage properties situated near the coast. If the areas vulnerable to being invaded by water are known, then the uses, activities and heritage elements most prone to suffering the consequences of floods can be identified. On that basis, adaptation policies can be implemented with a view to correcting territorial dysfunctions ahead of the expected phenomena. Various instruments of international scope meant to promote the development of plans linked to the fight against climate crisis particularly insist on the need to use geographic information systems as a methodological support (ESRI, 2010; WHO, 2012). The project Climate Change Planning for Regional and Local Authorities can serve as an example of good practices for the application of mitigation and adaptation strategies to climate crisis at subregional or local level, where a structured model is proposed that would fulfill the role of guide during development of a plan (Marletto et al., 2012).

\section{Conclusions}

This paper considers the implications the climate crisis has had for the territory of the Bay of Cádiz, a region eminently vulnerable for two reasons: its latitude close to tropical regions, making it more fragile from the thermal and hydric standpoint; and its level topography and littoral position, leaving it relatively undefended before the rising mean sea level. There is no doubt that the coast is gradually receding, especially around the marshes of the Natural Park. It is disturbing to think that these changes, already tangible, are not being dealt with or taken into account when managing the area. Most economic activities continue to operate according to the same system, ignoring the current climate situation: the aquaculture and salt-pond infrastructures are more recurrently invaded every year; some of the industrial installations could end up situated in floodable zones during this century; tourism remains dependent on the existence of beaches; some urban developments have begun to suffer periodic flooding; and all this without speaking of the lack of economic adjustment to rising temperatures and lower precipitation.

To anticipate future events and avoid unfathomable costs, the development of projections is essential, especially for the changes already taking place. As has been considered, the scenario used does not matter; the current problems will continue to follow the same upward path, although the respective pace will vary depending on the initiatives applied against the climate crisis. It must be highlighted that the forecasts for mean sea-level rise only consider the future behaviour of an extraordinary high tide. Future research could develop studies about the evolution of the intensity and frequency of storms during the course of the $21^{\text {st }}$ century, whose appearances are ever more common owing to the climate crisis.

It must be insisted that projections of mean sea-level rise should not be expressed in an isolated manner, i.e. it is not enough just to describe the distribution of new floodable zones from the scope of the study; those territorial alterations have to be applied to other also spatial variables. It is important to make clear what consequences this new scenario could have on settlements, infrastructures, protected 
spaces, assets of environmental or cultural value, economic activities, ports and beaches, etc. Indeed, a territorial problem is occurring, wherein humanity is situated as the perpetrator, though also as the possible remedy if the commitment is global. Territorial planning is a crucial departure point for regulating the development of activities that avert the effects of climate crisis by favouring restoration of natural behaviours of the environment (Pissolito, 2019). The spatial deficiencies are currently widespread, present in each of the three systems on which territorial plans are usually based.

First of all, the economic system ignores the reality. Tourism projects are sustained by exploiting increasingly scarce water resources owing to the expansion of persistent drought. In second place, the urban system of the Bay of Cádiz was traditionally characterised by its disdain regarding the ecological values of the surrounding area, resulting in high levels of erosion and increased vulnerability, given the exposure of many urban developments situated in floodable areas. Third, the environmental system is underpinned by a weakened and diminished Natural Park, due to the inundations which are and will be more recurrent, without interruption. The climate crisis generates a new urban, environmental and socioeconomic system and hence a new territorial planning.

Faced with this context of territorial fragility, regional and subregional plans should set out the guidelines to follow to prevent or in any case reduce the conditions resulting from climate crisis (Karl et al., 2009). To this must be added the insufficient cooperation between public and private bodies, the lack of interest in changes occurring in the Natural Park, the lack of citizen participation and the poor coordination between environmental planning and urban planning (Chica, 2010).

The climate crisis, as an undeniable fact, is the pillar on which any territorial planning must be grounded, especially when involving a littoral environment. Mitigation and adaptation are crucial for preventing and reducing its effects. Although the government is in charge of establishing intervention guidelines and disseminating the respective strategies, it stands to reason that the responsibility must be shouldered by each individual of society. The human being can modify the values of danger, exposure and vulnerability of its causes and impacts, as is already being done in many places. In Spain and therefore in the Bay of Cádiz, policies that are solely mitigating are being applied, as in the recent expansion of the urban bicycle network. However, the adaptation is still very rudimentary and cannot even be glimpsed in local political agendas.

The projections produced for this article were shown to various environmental officials of the Bay of Cádiz and experts in matters involving geography, territorial planning and sea sciences who have worked in the respective study context. The studies on mean sea-level rise presented in this paper open the doors to new and necessary research about the territorial conditions of the littoral environment of the Bay of Cádiz. Based on this methodology, such studies can also be conducted for other littoral environments with similar features. Study of the projections regarding the new climate reality is necessary and should be implemented in any kind of planning, whether territorial or urban and especially in littoral environments, by estimating the advance of waters caused by the rise of mean sea level.

\section{References}

Banholzer, S. Kosssin, J., Donner, S. 2014. The Impact of Climate Change on Natural Disasters. In: A. Singh, Z. Zommers (Eds.), Reducing Disaster: Early Warning Systems For Climate Change. Springer, Dordrecht pp. 21-49.

Benavente, J., Bello, E., Anfuso, G., Nachie, D., Macías, A. 2019. Sobreelevación debida a temporales y cambios producidos en las playas del litoral NE marroquí. Cuaternario y Geomorfología 21, 13-25. https://recyt.fecyt.es/index.php/CUGEO/article/view/16978

Benavente, J., del Río, L., Gracia, F.J. 2015. Riesgos de erosión costera en el litoral de Cádiz: Problemática actual y perspectivas futuras. Collectanea 204, 65-91. https://bit.ly/3jP4Caj 
Bornman, T.G., Schmidt, J., Adams, J.B., Mfikili, A.N., Farre, R.E., Smit, A.J. 2016. Relative sea-level rise and the potential for subsidence of the Swartkops Estuary intertidal salt marshes, South Africa. South African Journal of Botany 107, 91-100. https://doi.org/10.1016/j.sajb.2016.05.003

Bozhinova, D., van der Molen, M.K., van der Velde, I.R., Krol, M.C., van der Laan, S., Meijer, H.A.J., Peters, W. 2014. Simulating the integrated summertime $\Delta^{14} \mathrm{CO}_{2}$ signature from anthropogenic emissions over Western Europe. Atmospheric Chemistry and Physics 14, 7273-7290. https://doi.org/10.5194/acp-14$7273-2014$

Chica Ruiz, J.A., Barragán Muñoz, J.M. 2011. Estado y tendencia de los servicios de los ecosistemas litorales de Andalucía. Consejería de Medio Ambiente, Cádiz, 112 pp. https://bit.ly/2P31Anf

Chica Ruiz, J. A. 2010. Cambio climático y gestión costera en España. Un análisis de instrumentos. In: E. RiveraArriaga, I. Azuz-Adeath, L. Alpuche Gual, G. J. Villalobos-Zapata (Eds.), Cambio climático en México: un enfoque costero y marino. Universidad Autónoma de Campeche CTYS-Universidad, Campeche, pp. 773-787. https://bit.ly/2CWzKnM

Church, J.A., White, N.J. 2011. Sea-Level Rise from the Late 19th to the Early 21 st Century. Surveys in Geophysics 32, 585-602. https://doi.org/10.1007/s10712-011-9119-1

Clarcke, L.E., Edmonds, J., Jacoby, H., Pitcher, H., Reilly, J. 2007. Scenarios of Greenhouse Gas Emissions and Atmo-spheric Concentrations. US Department of Energy Publications, Lincoln, 166 pp.

Craft, C., Clough, J., Ehman, J., Joye, S. Park, R., Pennings, S. Guo, H., Machmuller, M. 2009. Forecasting the effects of accelerated sea-level rise on tidal marsh ecosystem services. Frontiers in Ecology and the Environment 7(2), 73-78. https://doi.org/10.1890/070219

CSIRO 2013. Reconstructed GMSL from 1880 to 2013. Available at: http://www.cmar.csiro.au/sealevel/sl_data_cmar.html (last access: 20/04/2019).

Echavarren, J.M., Balžekienè, A., Telešienė, A. 2019. Multilevel analysis of climate change risk perception in Europe: Natural hazards, political contexts and mediating individual effects. Safety Science 120, 813-823. https://doi.org/10.1016/j.ssci.2019.08.024

ESRI 2010. GIS for Climate Change. ESRI, New York, 53 pp.

FAO 2005. La ordenación integrada de zonas costeras (OIZC) y el sector forestal. Available at: http://www.fao.org/forestry/icam/es/ (last access: 13/07/2020).

FitzGerald, D.M., Fenster, M.S., Argow, B. A., Buynevich, I.V. 2008. Coastal Impacts Due to Sea-Level Rise. Annual Reviews Elanet Sciences https://doi.org/10.1146/annurev.earth.35.031306.140139

FOESA (2013). Cambio climático y acuicultura. FOESA, Madrid, España. 210 pp. https://bit.ly/39CY22c

Fraile Jurado, P., Álvarez Francoso, J., Ojeda Zújar, J. 2018. Cartografía de la probabilidad de inundación del litoral andaluz a finales del siglo XXI ante la subida del nivel del mar. Cuadernos Geográficos 57(2), 626. http://doi.org/10.30827/cuadgeo.v57i2.5899

Fraile Jurado, P., Álvarez Francoso, J., Sánchez Carnero, N., Ojeda Zújar, J. 2013. Análisis comparativo de la exposición a la subida del nivel medio del mar de la playa y marismas de Valdelagrana (Cádiz). GeoTemas 14, 167-170. https://bit.ly/3f5FkBb

Fraile Jurado, P., Fernández Díaz, M. 2016. Escenarios de subida del nivel medio del mar en los mareógrafos de las costas peninsulares de España en el año 2100. Estudios Geográficos 77(280), 57-79. https://doi.org/10.3989/estgeogr.201603

Fundéu 2019. Calentamiento global y cambio, crisis y emergencia climática. Available at: https://bit.ly/2DpDI8j (last access: 26/07/2020).

Gesch, D.B. 2009. Analysis of Lidar Elevation Data for Improved Identification and Delineation of Lands Vulnerable to Sea-Level Rise. Journal of Coastal Research 53, 49-58. https://doi.org/10.2112/SI53-006.1

Gómez Ferrer, A. 2011. Un espacio natural al ritmo de la marea. In: A. Gómez Ferrer et al., El Parque Natural Bahía de Cádiz, donde las mareas funden tierra y mar. Consejería de Medio Ambiente, Sevilla, pp. 5-9. 
Hartig, E. K., Gornitz, V., Kolker, A., Mushacke, F., Fallon, D. 2002. Anthropogenic and climate-change impacts on salt marshes of Jamaica Bay, New York City. Wetlands 22(1), 71-89. https://doi.org/10.1672/02775212(2002)022[0071:AACCIO]2.0.CO;2

Huaico Malhue, A.I., Romero Díaz, A., Espejel Carbajal, M.I. 2018. Evolución de los enfoques en desertificación: una revisión de 170 estudios de casos. Cuadernos Geográficos 57(2), 53-71. 10.30827/cuadgeo.v57i2.5530

IECA 2008. Modelo Digital de Andalucía 10 metros/pixel (2008 - 2009). Available at: https://bit.ly/2Utcwu3 (last access: 29/07/2020).

IECA 2016. Ortofotografía en Color 0.25-0.5 metros/pixel. Available at: https://bit.ly/2Utcwu3 (last access: 29/07/2020).

IECA 2019. Datos Espaciales de Referencia de Andalucía. Available at: https://bit.ly/3jIMKhr (last access: 29/07/2020).

Karl, T.R., Melillo, J.M., Peterson, T.C., Hassol, S.J. 2009. Global Climate Change Impacts in the United States. Cambridge University Press, Nueva York, 196 pp. http://aquaticcommons.org/2263/1/climate-impactsreport.pdf

Kulp, S.A., Strauss, B.H. 2019. New elevation data triple estimates of global vulnerability to sea-level rise and coastal flooding. Nature communications 10. https://doi.org/10.1038/s41467-019-12808-z

Le Roy Ladurie, E. 2017. Historia humana y comparada del clima. Fondo de la Cultura Económica, México D. F., 1087 pp.

MAPAMA 2016. Estrategia de Adaptación al Cambio Climático de la Costa Española. Dirección General de Sostenibilidad de la Costa y del Mar, Madrid, 120 pp.

Marletto, V., Johansson, H., Petersson Max, A., Prouteau, E., Brulfert, G., Chapuis, D., Chaxel, E., Girerd, I., Fritzsche, A., Röser, K., Schucht, M., Klementowski, P. 2012. Climate change planning for regional and local authorities. Regional Printing Centre of Emilia-Romagna, Bologna, 96 pp.

Masson-Delmotte, V., Zhai, P., Pörtner, H.O., Roberts, D., Skea, J., Shukla, P.R. (Co-Chairs). 2018. Global Warming of $1.5^{\circ} \mathrm{C}$. IPCC, Ginebra. https://bit.ly/39BpsVQ

Méndez Jiménez, M., Ceacero, C.J., Ordoñez, P., Guerrero Alvárez, J.J., Alvárez Peláez, S., Mesas Robles, A.I., Ojeda, J., Alvárez Francoso, J.I., Martín Cajaraville, D., Fraile-Jurado, P., Vallejo, I., Márquez, J. 2011. Análisis preliminar de la vulnerabilidad de la costa de Andalucía a la potencial subida del nivel del mar asociado al cambio climático. Consejería de Medio Ambiente, Sevilla, 154 pp. https://bit.ly/30VAfGD

ONU 2015. Acuerdo de París. Secretaria General de las Naciones Unidas, Nueva York, 29 pp. https://bit.ly/2Cn9odN

Paglia, E. 2018. The Socio-scientific Construction of Global Climate Crisis. Geopolitics 23(1), 96-123. https://doi.org/10.1080/14650045.2017.1328407

PE 2019. Datos históricos de la temperatura del agua. Available at: https://bit.ly/2X3ugOO (last access: 25/04/2019).

Pfeffer, W.T., Harper J. T., O’Neel, S. 2008. Kinematic con-straints on glacier contributions to 21st-century sealevel rise. Science 321, 1340-1343. https://doi.org/10.1126/science.1159099

Pissolito, C. A. 2019. Las consecuencias del cambio climático global en el ordenamiento territorial de Luján de Cuyo. Espacio Estratégico, Luján de Cuyo, 8 pp. https://bit.ly/30YnniW

Pongiglione, F. 2012. The key role of causal explanation in the climate change issue. Theoria 74, 175-188. https://doi.org/10.1387/theoria.3342

Prado, P., Alcaraz, C., Benito, X., Caiola, N., Ibáñez, C. 2019. Pristine vs. human-altered Ebro Delta habitats display contrasting resilience to RSLR. Science of the Total Environment 655, 1376-1386. https://doi.org/10.1016/j.scitotenv.2018.11.318

Pranzini, E., Williams, A. 2013. Coastal Erosion and Protection in Europe. Routledge, London, 488 pp.

PSMSL 2019. Mareógrafo Cádiz III. Available at: https://bit.ly/39zG6oQ (last access: 4/04/2019). 
Raw, J.L., Riddin, T., Wasserman, J., Lehman, T.W.K., Bornman, T.G., Adams, J.B. 2020. Salt marsh elevation and responses to future sea-level rise in the Knysna Estuary, South Africa. African Journal of Aquatic Science 45(1-2), 49-64. https://doi.org/10.2989/16085914.2019.1662763

Reed, D.J. 2002. Sea-level rise and coastal marsh sustaintability: geological and ecological factors in the Mississippi delta plain. Geomorphology 48, 233-243. https://doi.org/10.1016/S0169-555X(02)00183-6

Rendón Vega, J.L. 2011. La vegetación de las marismas y salinas. In: A. Gómez Ferrer et al., El Parque Natural Bahía de Cádiz, donde las mareas funden tierra y mar. Consejería de Medio Ambiente, Sevilla, pp. $45-$ 54.

Rodríguez, G. 2015. Red Natura 2000, parte de la solución para la adaptación al cambio climático. In: A. Herrero Méndez, M. A. de Zavala Gironés (Eds.), Los bosques y la biodiversidad frente al cambio climático. Ministerio de Agricultura, Alimentación y Medio Ambiente, Madrid, pp. 457-461.

Spencer, T., Brooks, S.M., Evans, B.R., Tempest, J.A., Möller, I. 2015. Southern North Sea storm surge event of 5 December 2013: Water levels, waves and coastal impacts. Earth-Science Reviews 146, 120-145. https://doi.org/10.1016/j.earscirev.2015.04.002

Stocker, T.F., Qin, D. (Co-Chairs). 2013. Cambio Climático 2013: Bases físicas. Resumen para responsables de politicas, Resumen técnico y Preguntas frecuentes. IPCC, Ginebra, 222 pp.

Thiéblemont, R., Le Cozannet, G., Toimil, A., Meyssignac, B., Losada, I.J. 2019. Likely and high-end impacts of regional sea-level rise on the shoreline change of European sandy coasts under a high greenhouse gas emissions scenario. Water 11(12), 2607. https://doi.org/10.3390/w11122607

Titus, J.G., Richman, C. 2001. Maps of lands vulnerable to sea level rise: Modeled elevations along the US Atlantic and Gulf coasts. Inter-Research Science Publisher 18, 205-228. https://doi.org/10.3354/cr018205

Trischler, H. 2017. El Antropoceno, ¿un concepto geológico o cultural, o ambos? Desacatos 54, 40-57. https://doi.org/10.29340/54.1739

Valladares, F., Magro, S., Martín-Forés, I. 2019. Antropocene, the challenge for Homo sapiens to set its own limits. Cuadernos de Investigación Geográfica 45(1), 33-59. http://doi.org/10.18172/cig.3681

Van Khanh Triet, N., Viet Dung, N., Phi Hoang, L., Le Duy, N., Duc Tran, D., Tuan Anh, T., Kummu, M., Merz, B., Apel, H. 2020. Future projections of flood dynamics in the Vietnamese Mekong Delta. Science of the Total Environment 742, 1-13. https://doi.org/10.1016/j.scitotenv.2020.140596

Vineis, P., Khan, A., D’Abramo, F. 2011. Epistemological issues raised by research on climate change. In: P. M. Illari, F. Russo, Williamson, J. (Eds.), Causality in the Sciences. Oxford University Press, Oxford, pp. 493-501. https://doi.org/10.1093/acprof:oso/9780199574131.003.0023

Vousdoukas, M.I., Mentaschi, L., Hinkel, J., Ward, P.J., Mongelli, I., Ciscar, J.C., Feyen, L. 2020. Economic motivation for raising coastal flood defenses in Europe. Nature Communications 11, 2119. https://doi.org/10.1038/s41467-020-15665-3

WHO 2012. Regional strategy for protecting health from climate change. Regional Office for South-East Asia, New Delhi, 87 pp.

Wigand, C., Ardito, T., Chaffee, C., Ferguson, W., Paton, S., Raposa, K., Vandemoer, C., Watson, E. 2015. A Climate Change Adaptation Strategy for Management of Coastal Marsh Systems. Estuaries and Coasts 40, 682-693. https://doi.org/10.1007/s12237-015-0003-y 



\title{
AN MCE-BASED INNOVATIVE APPROACH TO EVALUATING ECOTOURISM SUITABILITY USING GIS
}

\author{
PARVIZ JOKAR ${ }^{1}$, MASOUD MASOUDI ${ }^{1 *}$, FATEMEH KARIMI ${ }^{2}$ \\ ${ }^{1}$ Department of Natural Resources and Environmental Engineering, Shiraz University, Iran. \\ ${ }^{2}$ Department of Environment, Payame Nour University of Shiraz, Iran.
}

\begin{abstract}
Geographic Information System (GIS) can be used in ecotourism as a decision support tool for sustainable tourism planning, impact assessment, visitor flow management, and tourism site selection. Therefore, this system has the potential to be widely applied in tourism. The purpose of the study was to explore the potential for ecotourism development using GIS in Sepidan region, placed in the southwestern part of Iran. An innovative method was proposed for adjusting effective parameters in evaluation of ecotourism. The methods used for evaluation comprised Boolean, current multi-criteria evaluation methods (weighted linear combination), and the proposed methods of geometric mean and its calibration. Results showed that an improved method using geometric mean $(k a p p a=0.72)$ was better than Boolean (kappa $=0.56)$ and multi-criteria evaluation (kappa $=0.59$ ) models. The calibrated geometric mean was also the best among the different models used. It should be noted that the geometric mean method was simpler than multi-criteria evaluation and did not require the weighting process.
\end{abstract}

\section{Una aproximación innovadora basada en MCE y SIG para evaluar la idoneidad del ecoturismo}

RESUMEN. Para el estudio del ecoturismo los Sistema de Información Geográfica (SIG) pueden ser utilizados como una herramienta de apoyo para tomar decisiones en la planificación del turismo sostenible, la evaluación del impacto, la gestión del flujo de visitantes y la selección de áreas turísticas. En consecuencia, este método tiene potencial para ser ampliamente aplicado a la actividad turística. El objetivo del estudio es explorar el potencial para el desarrollo del ecoturismo en la región de Sepidan, localizada en el suroeste de Irán, utilizando SIG. Un método innovador se aplicó para ajustar los parámetros útiles en la evaluación del ecoturismo. La metodología utilizada para la valoración incluyó métodos Booleanos, de evaluación multi-criterio (combinación lineal ponderada), y métodos de media geométrica y su calibración. Los resultados mostraron que un método mejorado usando la media geométrica (kappa $=0,72)$ fue más apropiado que los modelos Booleanos $(k a p p a=0,56)$ y de evaluación de criterios múltiples (kappa= 0,59). La media geométrica calibrada también fue la más idónea entre los diferentes modelos utilizados. Cabe señalar que el método de la media geométrica fue más simple que la evaluación de criterios múltiples y no requirió el proceso de ponderación.

Keyword: Ecotourism, MCE, Geometric Mean, Evaluation, Boolean.

Palabras claves: Ecoturismo, MCE, Media Geométrica, Evaluación, Booleano.

Received: 13 June 2019

Accepted: 5 June 2020

*Corresponding author: M. Masoudi. Department of Natural Resources and Environment, Shiraz University, Iran.

Email: masoudi@shirazu.ac.ir 


\section{Introduction}

For a sustainable development of a region, it is important to consider the interest of its local population. Ecotourism involves visiting the nature without damaging it and disturbing habitats. In terms of ecotourism development, researchers have recently begun to examine the role of local residents in ecotourism activities. Due to the development of alternative forms of ecotourism, these activities have attracted the interest of governments, communities, and researchers. However, environmental conservation plays an important role in ecotourism development. Many scholars now agree that ecotourism requires a two-way link between tourism and environmental conservation (Ryngnga, 2008). Therefore, ecotourism has to be developed in a planned approach where Geographical Information System (GIS) and spatial decision-making tools can act as decision support systems. Spatial decisionmaking tools can be considered as a good framework for land suitability across a landscape via the actual data, criterion outcomes, and preferences and decisions of various stakeholders and. As a result, multicriteria evaluation (MCE) methods can assess decision-making alternatives for future land-use planning (Malczewski and Rinner, 2015).

MCE is a well-known method for spatial decision making in the field of geography (Voogd, 1983; Carver, 1991; Jankowski, 1995; Thill, 1999). This method is comprised of a multitude of evaluation criteria and a preference weighting scheme to evaluate decision-making alternatives. There are various MCE methods for assessing land use suitability, including simple additive scoring (SAS) (Massam, 1988; Heywood et al., 1995), multiattribute value technique (MAVT) (Pereira and Duckstein, 1993; Keeney, 1996), multiattribute utility technique (MAUT) (Janssen and Rietveld, 1990; Keeney and Raiffa, 1993), analytic hierarchy process (AHP) (Banai, 1993; Jankowski, 1995; Najafinezhad et al., 2013; Pourkhabbaz et al., 2014), ordered weighted average (OWA) (Yager, 1988; Jiang and Eastman, 2000; Malczewski et al., 2003; Malczewski, 2006), and outranking methods (Kangas et al., 2001; Joerin et al., 2001). MCE methods determine realistic results through their spatial variations in aggregation structures and weighting schemes.

Multi-criteria decision making (MCDM) methods have significantly advanced the conventional map overlay approaches to land-use suitability analysis (Zarei and Alsheikh, 2012; Oyinloye and Kufoniyi, 2013). Generally, a problem associated with land use suitability analysis methods is they do not assure a spatial pattern with contiguity or compactness in land allocations for different land use types. Furthermore, these methods are difficult to use while land suitability maps must be consistent with ground truth, and evaluation methods should be simple.

The main objective of this paper was to implement a simple innovative and quantitative model and its comparison to the current MCE approaches for ecotourism planning.

\section{Materials and Methods}

\subsection{Study area}

With an area of 286,000 hectares, Sepidan is situated northwest of Fars province, Iran. It is located between $51^{\circ} 59^{\prime}$ east longitude and $30^{\circ} 15^{\prime}$ north latitude (Fig.1). Its ten-year average rainfall is $758 \mathrm{~mm}$, with approximately $35 \%$ and $65 \%$ of evenly distributed snow and rain, respectively. It is very cold in the winter and cool and mild in the summer. "From the contemporary technicalities of climatic classification and general populace, this city is considered cool and moist to semi-dry" (Goudarzian and Yazdani, 2015). Of note, Sepidan is one of the most well-known ecotourism locations in Iran. Figure 1 represents a sketch of the area.

The present study aimed to provide a planning method for evaluating ecotourism suitability. Hence, GIS was employed to analyze the obtained data, which were of two types, namely numerical and thematic, mainly in map format. All the relevant data were obtained from the local and main offices and institutes of the Ministries of Agriculture and Energy and thoroughly processed using GIS techniques. 
The boundaries of maps were modified by Google Earth images and Global Positioning System (GPS) tools along with field work. The criteria evaluated based on expert opinion and primary model (Makhdoum, 2006), included topography, climate, soil and geology, vegetation, water, and conservation of environment.

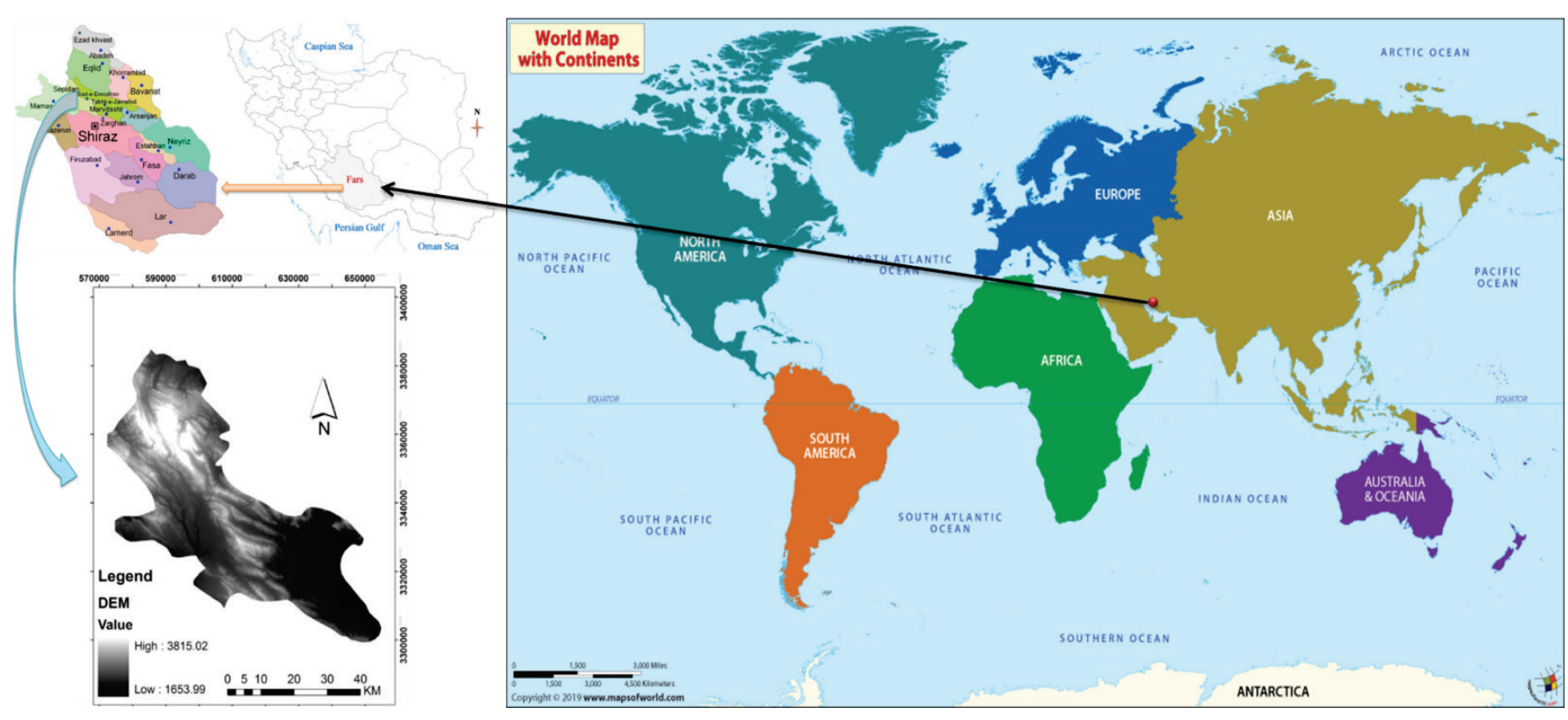

Figure 1. Location of the study area (Sepidan) in Fars Province and Iran.

\subsection{Model framework and data classification}

Model Framework involves the following steps (Fig. 2):

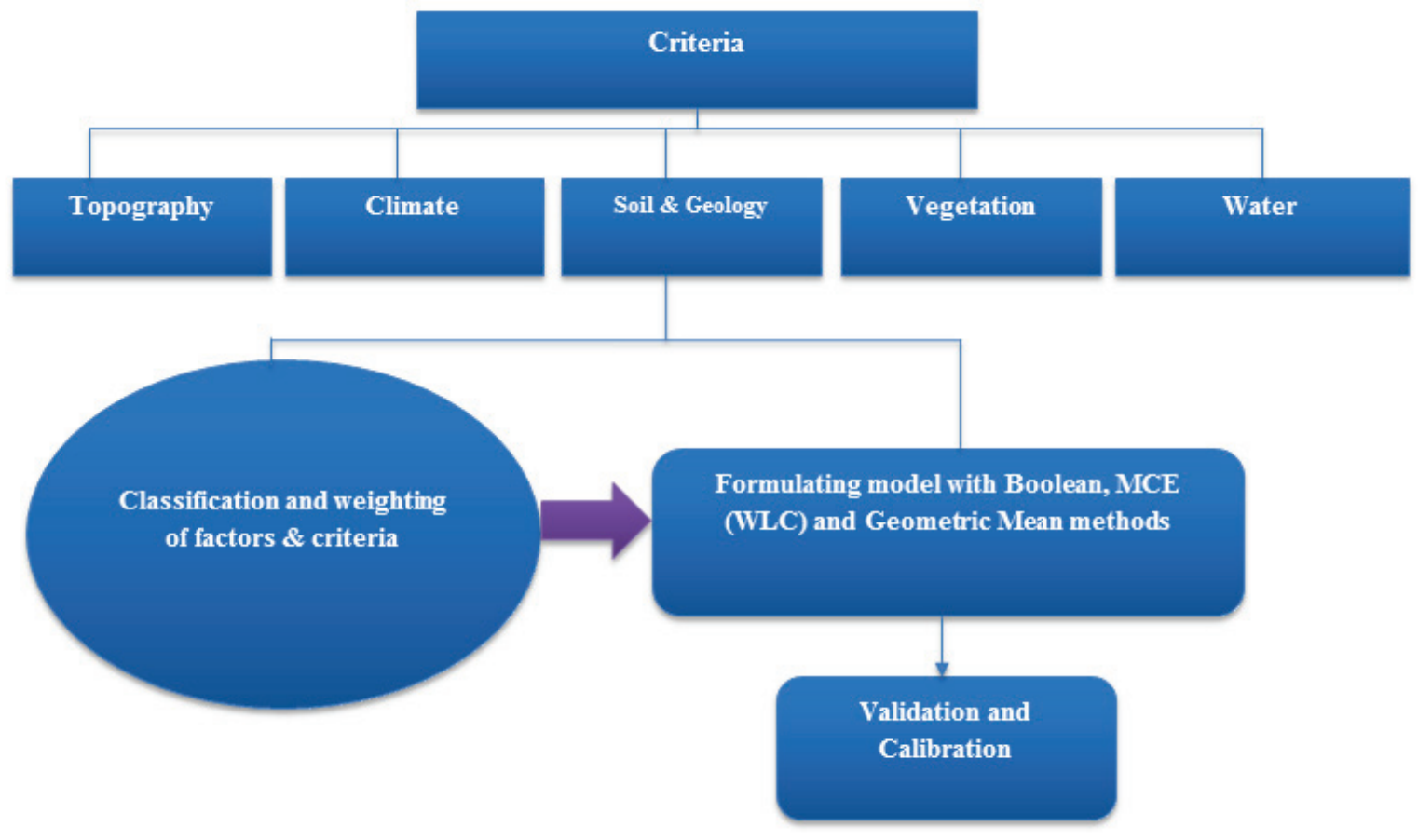

Figure 2. Platform structure of designed mode. 
i. Sampling of ecotourism regions: In this research, the data concerning the ecotourism regions of Sepidan were collected using Google Earth. These regions were modified with GPS tools during field work. In the next step, these regions were entered into ArcGIS software for analysis. In total, 187 points of ecotourism regions were systematically sampled in ArcGIS environment in order to improve and test the model.

ii. Primary model: The Iranian ecological model (Makhdoum, 2006) was run for the study area. It is composed of three classes pertaining to the ecological potentials of ecotourism and their associated capability class numbers. Class 1 is considered as suitable, class 2 is moderate, and class 3 is poor and non-suitable.

iii. Improved model: The Iranian ecological model and its indicators were improved based on the existing models (Makhdoum, 2006; Makhdoum et al., 2009; Mahdavi et al., 2015; Bunruamkaew and Murayama, 2011; Anane et al., 2012; Chakrabarty, 2011). Certain parameters such as soil depth were further ignored to increase the model efficiency (Jokar and Masoudi, 2016). As a result, effective parameters in the improved model are shown with an asterisk in Table 1. It should be noted that the next stages of evaluation were based on this model.

Table1. The indicators used in the model of land evaluation for Ecotourism (3classes)

\begin{tabular}{|c|c|c|c|c|}
\hline CRITERIA & INDICATOR & Highly Suitable (2) & Moderately Suitable (1) & $\begin{array}{c}\text { Poor \& Not Suitable } \\
(0)\end{array}$ \\
\hline TOPOGRAPHY & *Slope $(\%)$ & $0-15$ & $15-30$ & $>30$ \\
\hline \multirow{2}{*}{ CLIMATE } & $\begin{array}{c}\text { *Number of sunny days } \\
\text { (in spring \& summer months) }\end{array}$ & $>15$ & $7-15$ & $<7$ \\
\hline & $\begin{array}{l}* \text { Average of temperature }\left({ }^{\circ} \mathrm{C}\right) \\
\text { (in spring \& summer seasons) }\end{array}$ & $21-24$ & $18-21,24-30$ & $>30,<18$ \\
\hline \multirow{5}{*}{ SOIL } & *Texture & Usually moderate & Coarse, light, heavy & Very heavy \\
\hline & Depth(cm) & Deep & Semi deep & Shallow \\
\hline & $*$ Drainage $(\mathrm{cm} / \mathrm{hr})$ & Good (2-6) & \begin{tabular}{|c|} 
Moderate to poor $(0.1-2,6-$ \\
$25)$
\end{tabular} & Incomplete $(<0.1,>25)$ \\
\hline & Evolution (Structure) & Perfect (granular) & Moderate & Low \\
\hline & Fertility (organic matter \%) & Good, Moderate $(>1)$ & Low (1) & Very Low $(<1)$ \\
\hline GEOLOGY & *Lithology & $\begin{array}{c}\text { pyroclastic rocks, Granite } \\
\text { Ophiolite of melange color, } \\
\text { sand dunes, continental shelf } \\
\text { sediments }\end{array}$ & $\begin{array}{c}\text { Limestone and Dolomite, } \\
\text { sandstone, loess, schist and } \\
\text { gneiss and amphibolite, } \\
\text { quartzite, alluvial fans, } \\
\text { flood plain } \\
\end{array}$ & $\begin{array}{c}\text { marl, Shale, Clay } \\
\text { Stone, Conglomerate, } \\
\text { Salt domes, gypsum } \\
\text { dome, calcite and } \\
\text { dolomite marble } \\
\end{array}$ \\
\hline VEGETATION & *Vegetation type & $\begin{array}{l}\text { Forest lands with canopy cover } \\
\text { of } 50-80 \%\end{array}$ & $\begin{array}{l}\text { Forest lands with canopy } \\
\text { cover of } 5-50 \%\end{array}$ & $\begin{array}{l}\text { Poor Rangelands, } \\
\text { Forest lands with } \\
\text { canopy cover } \\
>80 \% \text {, Desert }\end{array}$ \\
\hline WATER & $\begin{array}{c}* \text { Quantity of water } \\
\text { For everyone (Lit/day) }\end{array}$ & $>40$ & $12-39.9$ & $<12$ \\
\hline CONSERVATION & $*$ Protected area & $\begin{array}{c}\text { Forest park of Natural and } \\
\text { planted, Nature Park, National } \\
\text { Park, Protected Area, } \\
\text { Biosphere Reserve, World } \\
\text { Heritage, Historical artefacts } \\
\text { and national and pilgrimage } \\
\end{array}$ & - & $\begin{array}{l}\text { Reserve forest, } \\
\text { Wildlife Sanctuary, } \\
\text { National natural } \\
\text { monuments }\end{array}$ \\
\hline
\end{tabular}

iv. Evaluation of the final model with mathematic methods: To evaluate the model, two basic methods (Boolean and MCE) and one proposed method (Geometric Mean) were used:

- Boolean: Boolean algebra is a mathematical system for the manipulation of variables that can have one of two values represented by: True or False, Yes or No, On or Off, 1 or 0. Boolean logic has three basic operators: Intersection (the logical term AND), Union (the logical term OR) and Inverse (the logical term NOT). These Boolean 
operators use integers or terms such as "True" and "False" as an input raster on a cellby-cell basis. Output values of True are (1) and those of False are (0) (Froja, 2013). In this paper, the primary and final models were evaluated based on the Boolean method.

- MCE (WLC) method: In the present research, MCE was used to assess the ecotourism. Accordingly, 35 questionnaires were given to experts in the field of ecotourism for weighting different criteria and factors, which was done via Expert Choice software. Table 2 shows the weight of criteria and factors with consistency ratio $(\mathrm{CR})<0.1$. Afterwards, WLC (weighted linear combination) method was used for the weighted overlay of the input data layers. With the weighted linear combination, the factors were primarily combined through applying a weight to each factor and criteria, followed by a summation of the results to yield a suitability map (Equations 1 and 2). Finally, the constraint factors $\left(\mathrm{C}_{\mathrm{i}}\right)$ were multiplied in the map (Fallahshamsi, 2004; Ghadimi et al., 2011):

Equation $1=\left[\left(\mathrm{W}_{1} \times\right.\right.$ factor 1$)+\left(\mathrm{W}_{2} \times\right.$ factor 2$) \ldots+\left(\mathrm{W}_{\mathrm{n}} \times\right.$ factor $\left.)\right] \times \mathrm{C}_{\mathrm{i}}$

$$
\text { Equation } 2=\left[\left(\mathrm{W}_{1} \times \text { Criteria1 }\right)+\left(\mathrm{W}_{2} \times \text { Criteria2 }\right) \ldots+\left(\mathrm{W}_{\mathrm{n}} \times \text { Criteria }\right)\right] \times \mathrm{C}_{\mathrm{i}}
$$

Table 2. Weight of criteria and indicators by AHP.

\begin{tabular}{|c|c|c|c|}
\hline Criteria & Criteria Weight & factor & factor Weight \\
\hline Topography & 0.16 & Slope (\%) & 1 \\
\hline \multirow{2}{*}{ Climate } & \multirow{2}{*}{0.13} & $\begin{array}{c}\text { Number of sunny days (in spring \& } \\
\text { summer months) }\end{array}$ & 0.5 \\
\hline & & $\begin{array}{c}\text { Average of temperature }\left({ }^{\circ} \mathrm{c}\right) \text { (in spring } \\
\& \text { summer seasons) }\end{array}$ & 0.5 \\
\hline \multirow{3}{*}{ Soil and Geology } & \multirow{3}{*}{0.16} & Texture & 0.33 \\
\hline & & Drainage $(\mathrm{cm} / \mathrm{hr})$ & 0.37 \\
\hline & & Lithology & 0.3 \\
\hline Vegetation & 0.18 & Vegetation type & 1 \\
\hline Water & 0.19 & $\begin{array}{c}\text { Quantity of water } \\
\text { For everyone (Lit/day) }\end{array}$ & 1 \\
\hline Conservation & 0.18 & Protected area & 1 \\
\hline \multicolumn{4}{|c|}{ CR (Consistency Ratio) $<0.1$} \\
\hline
\end{tabular}

- Geometric mean method (proposed): In the geometric mean method (such as MEDALUS model (Kosmas et al., 1999)), every index (Table 1) was given a weight from 0 (non-suitability of ecological conditions for ecotourism use such as deserts) to 2 (the most suitable ecological condition for ecotourism). Next, every criterion was calculated based on the geometric mean of indicators according to Equation 3:

$$
\text { Equation 3: Criterion_X }=\left[\left(\text { Layer-1) } \times(\text { layer-2) } \times \ldots \times(\text { Layer-n })]^{1 / n}\right.\right.
$$

where Criterion_ $\mathrm{X}$ is a defined criterion, Layer is an indicator map of the criterion, and $\mathrm{n}$ is the number of used indicators. The criteria were then multiplied through geometric mean (Equation 4):

$$
\text { Equation 4: Final Criterion }=\left[\left(\text { Layer-1) } \times(\text { layer-2) } \times \ldots \times(\text { Layer-n })]^{1 / n}\right.\right.
$$


where Final Criterion is the final layer of ecological capability, and $\mathrm{n}$ is the number of used criteria. For ecotourism planning, qualitative and suitability classes of ecological capability were identified by use of GIS (Jokar et al., 2015) (Table 3).

Table 3. Suitability classes in the Capability Maps and models for 3 classes uses regarding the scores of polygons.

\begin{tabular}{|c|c|c|c|}
\hline \multirow{2}{*}{ Their score } & \multicolumn{3}{|c|}{ Suitability classes } \\
\cline { 2 - 4 } & Good (1) & Moderate (2) & Poor \& Not suitable (3) \\
\cline { 2 - 4 } & $1.5-2$ & $0.5-1.5$ & $<0.5$ \\
\hline
\end{tabular}

v. Validation and Calibration: The accuracy of the identified classes was assessed by detailed ground reality (Makhdoum et al., 2009). For this purpose, ecotourism regions (as suitable classes of ground reality) were primarily created by use of Google Earth, GPS, and field work. After that, the samples of these areas were systematically gathered by "Create Fishnet" algorithm in ArcGIS 9.3 environment (Fallahshamsi, 1997). Of note, sampling of suitable classes (ecological and socioeconomical) of ground reality were more than others (such as moderate and non-suitable classes), as shown in Figure 3 (Fallahshamsi, 1997). This means the current ecotourism areas were sampled more than those with less suitability for ecotourism. In fact, these regions were selected as ground reality and had to be compared with potential conditions. Afterwards, these points (ground reality) were overlaid to the land capability maps. The obtained results are shown in Table 4 (error matrix or agreement matrix), and quantitative indices such as overall accuracy, kappa coefficient, and in class coefficient were calculated.

vi.
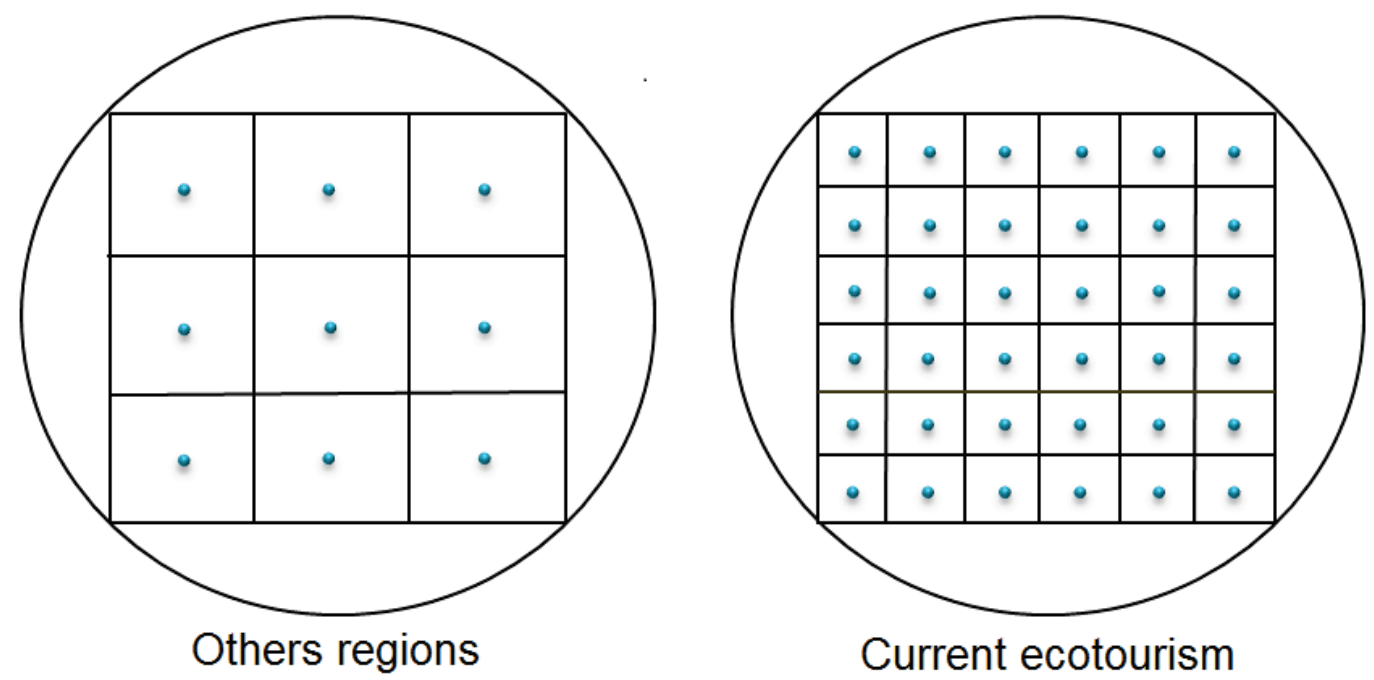

Figure 3. Sampling of suitable classes of ground reality comparing to other. 
Table 4. Error Matrix for ecotourism use in study area.

\begin{tabular}{|c|c|c|c|c|}
\hline Model & \multicolumn{4}{|c|}{ Ground Reality } \\
\hline \multirow{4}{*}{ Classify } & Class & $\begin{array}{c}\text { Current } \\
\text { ecotourism }\end{array}$ & $\begin{array}{c}\text { Forest lands with canopy cover 5-50\% } \\
\text { (exception current ecotourism and Non- } \\
\text { suitable) }\end{array}$ & $\begin{array}{c}\text { Poor } \\
\text { Rangelands, } \\
\text { Desert }\end{array}$ \\
\cline { 2 - 5 } & Suitable & $*$ & & \\
\cline { 2 - 6 } & Moderate & & $*$ & $*$ \\
\cline { 2 - 6 } & Non-suitable & & 440 & 469 \\
\hline
\end{tabular}

A particular aspect of the proposed accuracy assessment is that capability classes indicate the relative rather than the absolute values of capability for a particular area (Sepehr et al., 2007). Omission and commission errors were used to increase the accuracy and calibrate the model. Hence, according to these errors in the geometric mean method, the quantitative ranges of suitability classes (Table 3 ) were slightly changed. This kind of calibration has been performed in other classifications such as MEDALUS method (Kosmas et al., 1999). In the present research, class 1 ranged from 1-1.47 to 2.

\section{Results and Discussion}

Using ArcGIS software, the geospatial data were processed and the Boolean, MCE, and the proposed methods were implemented. The output maps obtained for ecotourism land capability are presented in Figure 4. The maps include primary and improved models by Boolean algebra, MCE, geometric mean, and calibration of geometric mean. Figure 5 also presents land percentage under different capability classes in different maps and the flexibility of each model. For instance, in Boolean methods, most regions tended towards the weaker classes while in MCE methods, the opposite was the case. However, the proposed methods (geometric mean and calibration of geometric mean) showed a balanced state between the foregoing models. They showed all three classes in the maps and had more agreement with the ground reality of the region. According to Figures 4 and 5, the primary model (Iranian ecological model) with Boolean has the least suitable class $(0.018 \%)$. In this method, the most widespread area was in poor and none-suitable class (79.63\%), showing the highest percentage for this class among all methods. Its improved method (with Boolean view) showed a slight increase in good class $(0.08 \%$ of region). The moderate class increased $(56.05 \%)$ and the none-suitable class $(43.87 \%)$ decreased compared to the primary model. Results showed that among the evaluation methods, the averaging methods (MCE, geometric mean, and calibration) had a better classification compared with Boolean methods. It should be noted that geometric mean and its calibration were the most optimal methods, hence useful and flexible for ecotourism purposes. The suitability of each class has to be accurately determined. Table 5 shows the results related to the number of accuracy indices for all methods used. As observed, the method improved using geometric mean was better than Boolean and MCE models, calibrated geometric mean was the best, and primary model (Iranian ecological model) had the least accuracy. 


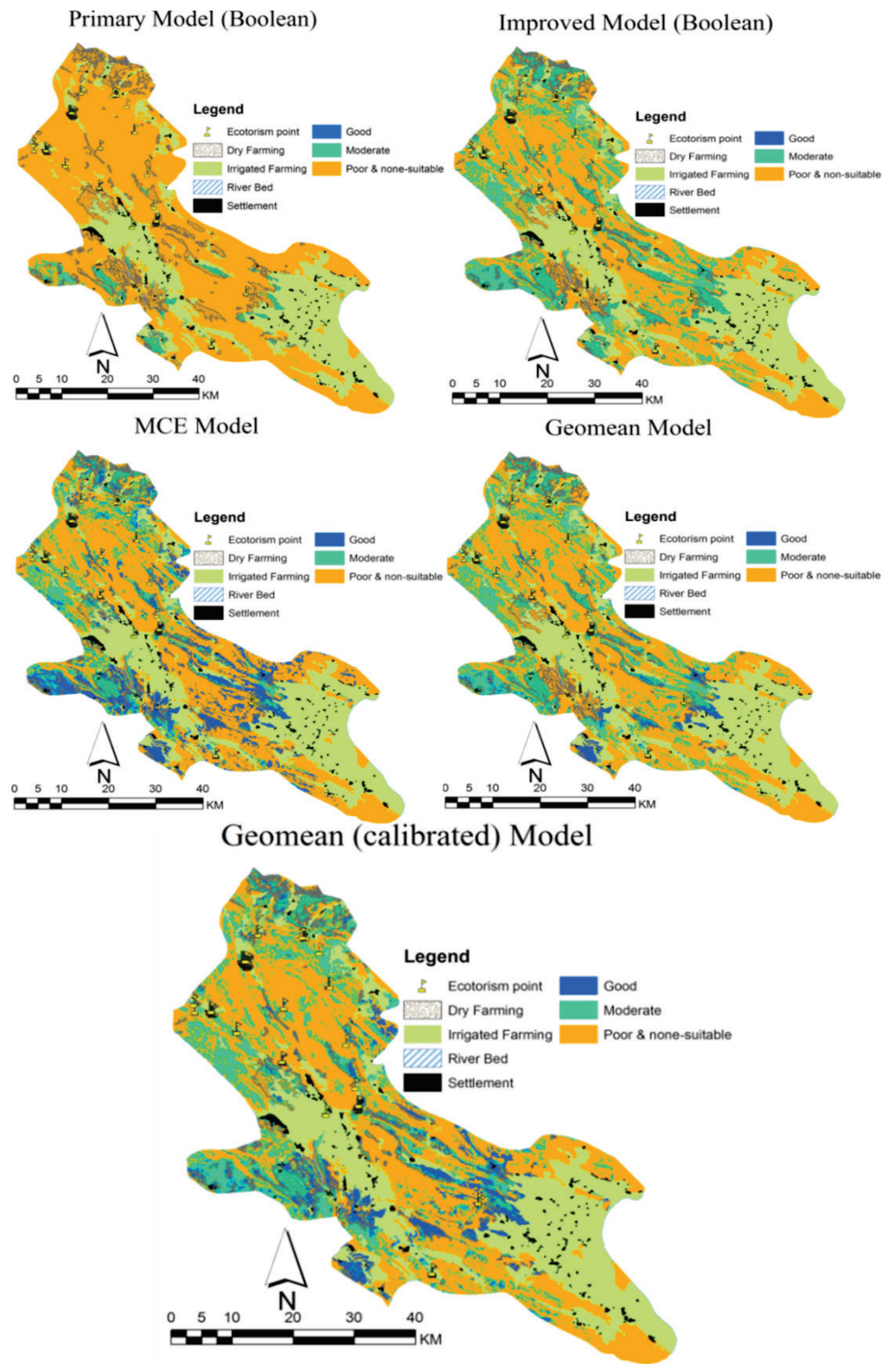

Figure 4. Ecological capability maps prepared for used models. 


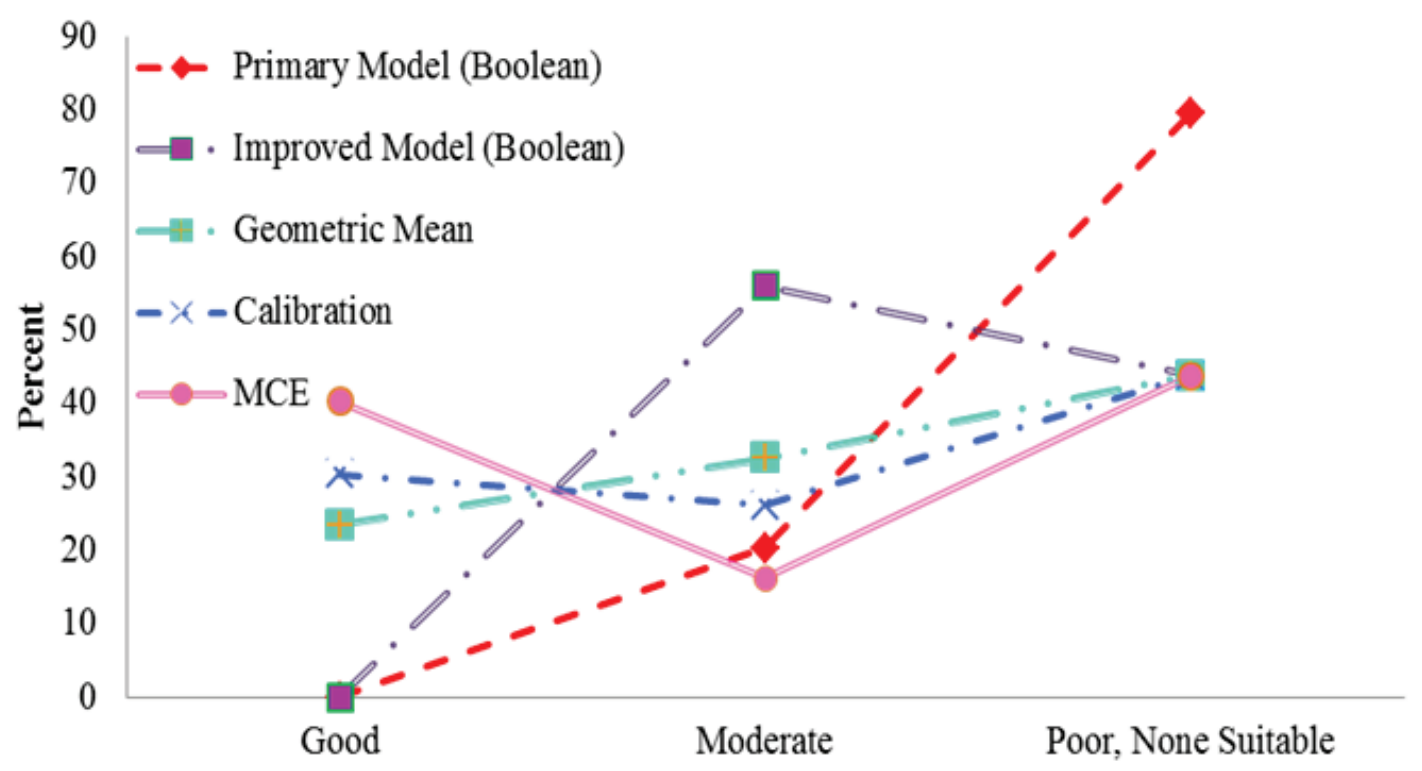

Figure 5. Percent of land under different capability classes for different methods of ecotourism use.

Table 5. Overall Accuracy, Inclass and Kappa Coefficients in the used models.

\begin{tabular}{|c|l|c|c|c|c|c|}
\hline \multirow{2}{*}{ Land Use } & \multirow{2}{*}{ Index } & Podel & Primary & \multicolumn{4}{|c|}{ Improved } \\
\cline { 3 - 7 } & & Boolean & Boolean & MCE & Geometric & Calibration \\
\hline \multirow{3}{*}{ Ecotourism } & Overall Accuracy & 54 & 74 & 73 & 81 & 82 \\
\cline { 2 - 7 } & Kappa Coefficient & 0.2 & 0.56 & 0.59 & 0.7 & 0.72 \\
\cline { 2 - 7 } & Inclass Coefficient & 0 & 0 & 0.76 & 1.14 & 1.35 \\
\hline
\end{tabular}

\section{Conclusion}

This study successfully implemented a GIS-based method as an advanced and more precise MCE approach to evaluating the capability and suitability of ecotourism area. The proposed model can be applied to all types of study areas. The geometric mean method is simpler than MCE and does not require weighting process whereas most studies (Kangas et al., 2001; Joerin et al., 2001; Najafinezhad et al., 2013; Pourkhabbaz et al.,2014) have assessed land capability based on subjective opinions. Also, among the models generated by Boolean, MCE (WLC), and geometric mean methods, the results showed that the primary model (Iranian ecological model) by Boolean had the lowest accuracy, which is line with Najafinezhad et al. (2013) and Fallahshamsi (2004). However, after modifying the model (by the same Boolean method), the results and accuracy were further improved (Table 5). Next, the evaluation methods of the improved model were changed by averaging methods (MCE, geomean and calibration, Table 5). Results (Table 5) showed that these methods had a higher accuracy compared with Boolean methods (in accordance with Fallahshamsi, 2004; Ghadimi et al., 2011; Najafinezhad et al., 2013; Elaalem et al., 2010). Geometric mean method had a higher accuracy than MCE method, and its calibration was the most optimal method for ecotourism planning as man-made use. Additionally, compared to Boolean model, geometric mean reduced the dependency on indicator numbers (like soil with higher indicators compared to those criteria that include fewer indicators such as topography). Hence, this point can be considered by geometric mean and its type of evaluation. Therefore, for example, climate and topography with only two indicators had the same weight as soil factor with more indicators, which is in good agreement with other studies (Jokar et al., 2015; Jokar and Masoudi, 2016). 
Due to the importance of natural hazards, parameters such as drought and climate change should be considered in future research. It is recommended that the results of this study be considered by managers and other stakeholders in management planning processes.

\section{Acknowledgement}

Especial thanks to the official organizations of Iran (especially Shiraz University) for providing the data, maps and reports for this project.

\section{References}

Anane, M., Bouziri, L., Limam, A., Jellali, S. 2012. Ranking suitable sites for irrigation with reclaimed water in the Nabeul-Hammamet region (Tunisia) using GIS and AHP-multicriteria decision analysis. Resources, Conservation and Recycling 65, 36-46. https://doi.org/10.1016/j.resconrec.2012.05.006

Banai, R. 1993. Fuzziness in geographic information systems: contributions from the analytic hierarchy process. International Journal of Geographical Information Systems 7(4), 315-329. https://doi.org/10.1080/02693799308901964

Bunruamkaew, K., Murayama, Y. 2011. Site suitability evaluation for ecotourism using GIS \& AHP: A case study of Surat Thani Province, Thailand. Procedia Social and Behavioral Sciences 21: 269 - 278. https://doi.org/10.1016/j.sbspro.2011.07.024

Carver, S.J. 1991. Integrating multi-criteria evaluation with geographical information systems. International Journal of Geographical Information Systems 5(3), 321-339. https://doi.org/10.1080/02693799108927858

Chakrabarty, A. 2011. Ecotourism Development and Security Restructuring: A GI Based Planning for Peaceful Dissuasion of Anarchism in Forest Provinces of India. Procedia Social and Behavioral Sciences 21, 108-115. https://doi.org/10.1016/j.sbspro.2011.07.006

Elaalem, M., Comber, A., Fisher, P. 2010. Land Suitability Analysis comparing Boolean logic with fuzzy analytic hierarchy process. Accuracy 2010 Symposium, July 20-23, Leicester, UK. pp. 245-247.

Fallah Shamsi, S.R. 1997. Accuracy assessment of satellite-based maps using sampling. M.Sc Dissertation, Faculty of Natural Resources, University of Tehran, Iran, pp. 86. (In Persian).

Fallahshamsi, H.R. 2004. Economic Evaluation of Different Land uses in Kalibar - chai Forest -covered Watershed, Using Linear Programming and Geographical Information Systems, Ph.D. Thesis Report, Natural Resource Faculty, Tehran University, Karaj. [In Persian]

Froja, N.M. 2013. Fuzzy-GIS Development of Land Evaluation System for Agricultural Production in North West Libya. PhD thesis, School of the Built Environment, Heriot-Watt University.

Ghadimi, M., Hosseini, M., Pourghasemi, H.R., Moradi, H.R. 2011. Protection Modeling of Manesht and Ghalarang Protected Areas Using Fuzzy logic. Environmental Sciences, 8: 85-106. [In Persian]

Goudarzian, P., Yazdani, M.R. 2015. Climate diversity in line with agroforestry systems: studying technicalities of agroforestry systems and allied components in two diverse climatic regions (Warm climate vs. cold climate) (Case study: Kazeroun \& Sepidan in Fars Province, I. R. Iran). Desert 20(2): 157-166.

Heywood, I., Oliver, J., Tomlinson, S. 1995. Building an exploratory multi-criteria modelling environment for spatial decision support. In P. Fisher (Ed.) Innovations in GIS, Taylor and Francis, London, UK, pp. 127-136.

Jankowski, P. 1995. Integrating geographical information systems and multiple criteria decision-making methods. International Journal of Geographical Information Systems 9(3), 251-273. https://doi.org/10.1080/02693799508902036

Janssen, R., Rietveld, P. 1990. Multicriteria analysis and geographical information systems: an application to agricultural land use in the Netherlands. In H. J. Scholten and J. C. H. Stillwell (Eds.) Geographical information systems for urban and regional planning, Kluwer Academic Publishers, Dordrecht, The Netherlands, pp. 129-139. 
Jiang, H., Eastman, J. R. 2000. Application of fuzzy measures in multi-criteria evaluation in GIS. International Journal of Geographical Information Science 14(2), 173-184. https://doi.org/10.1080/136588100240903

Joerin, F., Theriault, M., Musy, A. 2001. Using GIS and outranking multicriteria analysis for land-use suitability assessment. International Journal of Geographical Information Science 15(2), 153-174. https://doi.org/10.1080/13658810051030487

Jokar, P., Masoudi, M. 2016. Land Suitability for Urban and Industrial Development by a Proposal Model, Case Study: Jahrom Township, Iran. Journal of Environmental Studies 42, 135-149.

Jokar, P., Masoudi, M., Fallah Shamsi, S.R., Afzali, S.F. 2015. Developing a Model for Ecological Capability Evaluation of Ecotourism (A Case Study: Jahrom Township, Iran). International Journal of Scientific Research in Environmental Sciences 3(1): 1-8. https://doi.org/10.12983/ijsres-2015-p0001-0008

Kangas, A., Kangas, J., Pykäkäinen, J. 2001. Outranking methods as tools in strategic natural resources planning. Silva Fennica 35(2), 215-227. https://doi.org/10.14214/sf.597

Keeney, R.L. 1996. Value-focused thinking: identifying decision opportunities and creating alternatives. European Journal of Operational Research 92, 537-549. https://doi.org/10.1016/0377-2217(96)00004-5

Keeney, R.L., Raiffa, H. 1993. Decisions with Multiple Objectives: Preferences and Value Tradeoffs (2nd Edition). Cambridge: Cambridge Press.

Kosmas, C., Poesen, J., Briassouli, H. 1999. Key indicators of desertification at the Environmentally Sensitive Areas (ESA) scale, The Medalus Project: Mediterranean Desertification and Land Use. Manual on Key Indicators of Desertification and Mapping Environmentally Sensitive Areas to Desertification. Project report. European Commission.

Mahdavi, A., Niknejad, M., Karami, O. 2015. A fuzzy multi-criteria decision method for locating ecotourism development. Caspian J. Env. Sci. 13 (3), 221-236.

Makhdom, M. 2006. Principles of Land use planning. 7th ed, University of Tehran.

Makhdom, M., Darvishsefat, A., Jafarzadeh, H., Makhdom, A. 2009. Environmental Evaluation and Planning by Geographic Information System. 4th ed, University of Tehran.

Malczewski, J., Rinner, C., 2015. Multicriteria Decision Analysis in Geographic Information Science. Springer, New York, p. 1-331.

Malczewski, J. 2006. Ordered weighted averaging with fuzzy quantifiers: GIS-based multicriteria evaluation for land-use suitability analysis. International Journal of Applied Earth Observation and Geoinformation 8(4), 270-277. https://doi.org/10.1016/j.jag.2006.01.003

Malczewski, J., Chapman, T., Flegel, C., Walters, D., Shrubsole, D., Healy, M.A. 2003. GIS-multicriteria evaluation with ordered weighted averaging (OWA): case study of developing watershed management strategies. Environment and Planning A 35(10), 1769-1784. https://doi.org/10.1068/a35156

Massam, B.H. 1988. Multi-criteria decision making (MCDM) techniques in planning. Progress in Planning 30(1), 1-84. https://doi.org/10.1016/0305-9006(88)90012-8

Najafinezhad, A., Pishdad Soleimanabad, L., Salmanmahini, A. 2013. Comparison of the efficiency of systematic and multi objective land allocation methods for land use planning using Geographic Information System. Journal of Applied RS \& GIS Techniques in Natural Resource Science 4, 1-11. [In Persian]

Oyinloye, M., Kufoniyi, O. 2013. Application of IKONOS Satellite Images in Monitoring of Urban Landuse Change in Ikeja, GRA, Lagos, Nigeria. International Journal of Engineering Science Invention 2, 1-10.

Pereira, J.M.C., Duckstein, L. 1993. A multiple criteria decision-making approach to GIS-based land suitability evaluation. International Journal of Geographical Information Systems 7(5), 407-424. https://doi.org/10.1080/02693799308901971

Pourkhabbaz, H.R., Javanmardi, S., Faraji Sabokbar, H.A. 2014. Suitability Analysis for Determining Potential Agricultural Land Use by the Multi-Criteria Decision-Making Models SAW and VIKOR-AHP (Case study: Takestan-Qazvin Plain). J. Agr. Sci. Tech. 16, 1005-1016. 
Jokar et al.

Ryngnga, P.K. 2008. Ecotourism Prioritization: A Geographical Information System Approach. South Asian Journal of Tourism and Heritage 1, 49-56.

Sepehr, A., Hassanli, A.M., Ekhtesasi, M.R., Jamali, J.B. 2007. Quantitative assessment of desertification in south of Iran using MEDALUS method. Environ. Moni.t Assess. 134, 243-254. https://doi.org/10.1007/s10661-0079613-6

Thill, J.C. 1999. Multicriteria decision-making and analysis: a geographic information sciences approach. Ashgate, New York, USA.

Voogd, H. 1983. Integrating multi-criteria evaluation with geographical information systems. Taylor \& Francis, London, UK.

Yager, R. 1988. On Ordered weighted averaging aggregation operators in multicriteria decision making. IEEE Transactions on Systems, Man, and Cybernetics 18(1), 183-190. https://doi.org/10.1109/21.87068

Zarei, R., Alsheikh, A. 2012. Modeling of Urban Development Using CA and Genetic Algoritm (A Case Study: Shiraz City). Urban Planning 3, 1-16. [In Persian] 


\section{CUADERNOS DE INVESTIGACIÓN GEOGRÁFICA GEOGRAPHICAL RESEARCH LETTERS Normas de publicación}

Cuadernos de Investigación Geográfica (Geographical Research Letters) publica trabajos científicos inéditos sometidos a una revisión por pares. Los artículos no deberán superar las 7500 palabras, aunque pueden publicarse trabajos de mayor dimensión si su interés así lo aconseja. También publica notas y crónicas que no deberán superar las 2500 palabras.

El envío de trabajos se realiza online a través de la página web de la revista http://publicaciones.unirioja.es/revistas/cig.

\section{Preparación de manuscritos}

Los manuscritos estarán escritos preferentemente en inglés. Aquellos autores que tengan dificultades con este idioma deberán enviar el texto a un nativo (o a una persona con excelente dominio de este idioma). Aquellos manuscritos que sean difíciles de entender debido a una redacción en inglés deficiente serán devueltos a los autores antes de ser evaluados científicamente.

Los manuscritos deben incorporar necesariamente la siguiente información:

Carta de presentación. Los autores deberán dirigir al Editor una carta de presentación del manuscrito resaltando los aspectos más sobresalientes del trabajo. En esta carta se sugerirán cinco expertos en la materia (nombre, institución y correo electrónico) -no vinculados directamente con los autoresque podrán actuar de evaluadores.

Título. Debe ser conciso e informativo del contenido del manuscrito.

Nombres de los autores y afiliaciones. Indique los nombres y apellidos de los autores, añadiendo la afiliación profesional de cada autor: institución, dirección postal, país y correo electrónico.

Autor de correspondencia. Indique el autor de contacto, su centro de trabajo, su dirección y el correo electrónico. Con este autor el equipo editorial mantendrá la correspondencia en todo el proceso de edición.

Abstract/Resumen. Es necesario incluir un resumen (entre 300 y 400 palabras) del contenido del manuscrito que incorpore información sobre los objetivos de la investigación, los resultados y las conclusiones principales. Si el artículo es publicado en español, obligatoriamente debe incorporarse un resumen en inglés.

Palabras clave. Inmediatamente después del resumen, se debe proporcionar un máximo de 5 palabras clave. Estas palabras clave son utilizadas con fines de indexación.

Agradecimientos. Incluya los agradecimientos en una sección independiente, al final del manuscrito, antes del listado de referencias bibliográficas. Si en los agradecimientos se desea incorporar a los responsables de la financiación de la investigación, debe añadir el título del proyecto (número), organización financiadora y país.

\section{Estructura del manuscrito}

La estructura del manuscrito será lo más sencilla posible. Se aconseja dividir el texto en secciones numeradas $(1,2,3 \ldots)$. Las subsecciones también deben numerarse 1.1 (luego 1.1.1, 1.1.2, ...), 1.2, etc. En cualquier caso, en el manuscrito es necesario incluir: Introducción, Área de Estudio, Métodos y Materiales, Resultados, Discusión y Conclusiones como secciones. Las subsecciones pueden tener un breve encabezado.

Se deberán numerar consecutivamente las ecuaciones que vayan en el texto principal.

En los textos escritos en español, los decimales estarán separados por una coma (por ej., 27,3). Se utilizará un punto para el separador de miles en números de cuatro o más cifras (por ej., 24.237). En los textos escritos en inglés, los decimales estarán separados por un punto (por ej., 27.3). Se utilizará una coma para el separador de miles en números de cuatro o más cifras (por ej., 24,237).

\section{Tablas}

Las tablas deben presentarse como texto editable y no como imágenes. Las tablas se pueden colocar en el interior del texto o en páginas separadas al final. Es necesario numerar las tablas e incorporar un encabezamiento.

\section{Figuras}

Las figuras, mapas y fotos se entregarán en archivos separados y numerados consecutivamente en relación con su posición en el texto. Dichos archivos se enviarán juntos en uno o varios archivos comprimidos (.zip, rar) que tengan un tamaño máximo de $4 \mathrm{Mb}$. Se admiten archivos EPS (o PDF) o TIFF (o JPEG) con una resolución mínima de 300 dpi. Figuras, mapas y fotos se publicarán en color, sin coste adicional. Los pies de figura se incluirán al final del texto. Se admitirá hasta un máximo de 10 figuras por artículo.

\section{Referencias bibliográficas}

Asegúrese de que todas las referencias citadas en el texto estén presentes en la lista de referencias (y viceversa). Los trabajos de investigación regulares (artículos) tienen un límite de 50 referencias y las comunicaciones breves no deben exceder las 20 citas.

En el texto se citará el nombre del autor y el año entre paréntesis. Caso de que el trabajo citado corresponda a más de dos autores se especificará el primero, añadiendo posteriormente et al. Al final del texto se incluirá la lista de referencias por orden alfabético, siguiendo el siguiente modelo:

Badding, M.E., Briner, J.P., Kaufman, D.S. 2013. 10 Be ages of late Pleistocene deglaciation and Neoglaciation in the north-central Brooks Range, Arctic Alaska. Journal of Quaternary Science 28, 95-102. http://doi.org/10.1002/jqs.2596

French, H.M. 2007. The Periglacial Environment. Third Edition. Wiley, Chichester, 458 pp.

Camuffo, D., Enzi, S. 1995. Reconstructing the climate of northern Italy from archive sources. In: R. Bradley, P.D. Jones P.D. (Eds.), Climate since A.D. 1500. Routledge, London, pp. 143-154.

IDERM 2011. Ortofoto regional del vuelo de 1981. Disponible en: http://cartomur.imida.es/visorcartoteca/ (Fecha de acceso: 20/06/2011).

Los autores añadirán, cuando sea posible, el número de DOI al final de cada artículo citado.

Cuando el artículo sea aceptado, en la página web de la revista estará disponible una versión pre-print con su correspondiente DOI.

Las pruebas de imprenta se enviarán como archivo PDF y por correo electrónico al autor de contacto.

La evaluación y publicación de artículos en Cuadernos de Investigación Geográfica es gratuita y responde a la política de la revista de poner los avances en Geografía Física al alcance del mundo científico. 


\section{CUADERNOS DE INVESTIGACIÓN GEOGRÁFICA GEOGRAPHICAL RESEARCH LETTERS Guide for authors}

Cuadernos de Investigación Geográfica (Geographical Research Letters) publishes scientific, unedited, peer-reviewed works. These should normally not exceed 7500 words, although articles of major dimension can be published if the Editor approves it. Notes and chronicles should not exceed 2500 words. Submission to this journal proceeds totally online via http://publicaciones.unirioja.es/revistas/cig.

\section{Preparation}

Manuscripts should preferably be written in English. Authors who are not fluent in English should have their manuscript checked by someone proficient in the language. Manuscripts in which the English is difficult to understand may be returned to the author for revision before scientific review.

Manuscripts must necessarily incorporate the following information:

Cover letter. The authors should send the Editor a presentation letter of the manuscript highlighting the most outstanding aspects of the work. Five experts in the subject (name, institution and email), who could act as referees, will be suggested in this letter. These experts will have no direct link with the authors.

Title. This should be concise and informative.

Author names and affiliations. Please clearly indicate the given name(s) and family name(s) of each author and check that all names are accurately spelled. Present the authors' affiliation address: institution, mailing address, country and email.

Corresponding author. Indicate who will handle correspondence at all stages of refereeing and publication, also postpublication. Ensure that the email address is given.

Abstract. It is necessary to include an abstract (300-400 words). The abstract should state briefly the purpose of the research, the principal results and major conclusions. If the article is published in Spanish, an English abstract must be included.

Keywords. Immediately after the abstract, provide a maximum of 5 keywords. These keywords will be used for indexing purposes.

Acknowledgements. Include acknowledgements in a separate section, at the end of the article, before the references. If you want to add research funding, you must add the project title (number), funding organization and country.

\section{Article structure}

Divide your article into clearly defined and numbered sections. Sections and Subsections should be numbered 1.1 (then 1.1.1, 1.1.2, ...), 1.2, etc. In any case, the manuscript must include introduction, study area, methods and materials, results, discussion and conclusions. Subsections may have a short heading.

Number consecutively the equations that are displayed in the text.

In the papers written in English, decimals will be separated by a point (e.g., 27.3). A comma will be used for the thousands separator (e.g., 24,237). In the papers written in Spanish, decimals will be separated by comma (e.g., 27,3). A point will be used for the thousands separator (e.g., 24.237).

\section{Tables}

Please submit tables as editable text and not as images. Tables can be placed inside the text, or on separate page(s) at the end. Number tables consecutively in accordance with their appearance in the text and place the table heading.

\section{Figures}

Illustrations (figures, maps and photographs) should be submitted as separate files and numbered according to the sequence in the text. The files should be sent together in one or several compressed files (.zip, rar) not exceeding 4Mb. Please convert the illustrations to one of the following formats: EPS (or PDF), or TIFF (or JPEG) with a minimum of 300 dpi. Illustrations will be published in colour at no additional charge. Figure captions should be supplied at the end of the text. Each article should include no more than 10 figures.

\section{References}

All the references cited in the text should be given in the reference list, and vice versa. Regular research papers have a reference limit of 50 cites and shor communications should not exceed 20 cites.

References should be quoted in the text as name and year within brackets. When more than two authors are referred, the name of the first author should be used, with the addendum et al. At the end of the text references should be listed alphabetically by author's name, in the following style:

Badding, M.E., Briner, J.P., Kaufman, D.S. 2013. 10 Be ages of late Pleistocene deglaciation and Neoglaciation in the north-central Brooks Range, Arctic Alaska. Journal of Quaternary Science 28, 95-102. http://doi.org/10.1002/jqs.2596.

French, H.M. 2007. The Periglacial Environment. Third Edition. Wiley, Chichester, 458 pp.

Camuffo, D., Enzi, S. 1995. Reconstructing the climate of northern Italy from archive sources. In: R. Bradley, P.D. Jones P.D. (Eds.), Climate since A.D. 1500. Routledge, London, pp. 143-154.

IDERM 2011. Ortofoto regional del vuelo de 1981. Available at: http://cartomur.imida.es/visorcartoteca/ (last access: 20/06/2011).

The authors will provide, if possible, the DOI number at the end of each cited article.

Once the paper is accepted, a pre-print version will be available at the website of the journal with its corresponding DOI number.

One set of page proofs will be sent by e-mail to the corresponding author as a PDF file.

Each author will receive, at no cost, a PDF file of the article and one printed copy of the corresponding issue of the journal.

The publishing policy in Cuadernos de Investigación Geográfica makes the evaluation and publication processes free, in agreement with the policy of making accessible the advances in Physical Geography to the scientific community. 
Universidad de La Rioja

Servicio de Publicaciones

Piscinas, 1, 26006 LOGROÑO (España)

Tel.: (+34) 941299187 Fax: (+34) 941299193

Correo-e: publicaciones@unirioja.es

\section{Edición electrónica / E-Journal}

\section{EISSN: 1697-9540}

http://publicaciones.unirioja.es/revistas/cig

cig@unirioja.es

\section{Indización y Calidad / Indexation and Quality Analysis}

Bases de Datos y Recursos para el análisis de la calidad de las revistas científicas /

Databases and Resources for the analysis of the Scientific Journal's Quality:

CARHUS Plus +2018

CIRC (EC3 Metrics)

Dialnet Métricas (Fundación Dialnet)

DOAJ (Directory of Open Access Journals)

ERIH Plus (European Science Fundation)

ESCI, Emerging Sources Citation Index (Web

of Science ${ }^{\mathrm{TM}}$, Clarivate Analytics)

Fuente Académica Plus (EBSCO)

\section{Latindex (Catálogo)}

MIAR (Matriz de Información para el Análisis

de Revistas)

Periodicals Index Online (ProQuest)

SCIMAGO Journal \& Country Rank (SJR)

Scopus (Elsevier)

Sello de Calidad FECYT

\section{Política del editor sobre copyright y autoarchivo}

- Sólo se puede archivar la versión final del editor/PDF.

- En el sitio web del autor o repositorio institucional o cualquier repositorio designado por los organismos financiadores a petición de dichos organismos o como resultado de una obligación legal desde el momento de su publicación.

- El uso debe ser no comercial, no permitiéndose obras derivadas.

- La fuente editorial debe reconocerse. Debe incluirse la declaración establecida por el editor: "Publicado originalmente en Cuadernos de Investigación Geográfica / Geographical Research Letters en [volumen y número, o año], por la Universidad de La Rioja (España)".

- Debe enlazar a la versión del editor con la inclusión del número DOI a partir de la siguiente frase: "La publicación original está disponible en Cuadernos de Investigación Geográfica / Geographical Research Letters a partir de http://doi.org/[DOI]".

\section{Publisher copyright \& self-archiving policies}

- Authors may self-archive publisher's version of the article (final PDF version) on their own websites.

- Authors may also deposit this version of the article in institutional or funder repositories, at the request of the fundind organizations or as a result of legal obligation after publication.

- The final published version can only be used for non-commercial purposes. Derivative work is not allowed.

- Acknowledgement to the original source of publication must be given as follows: "First published in Cuadernos de Investigación Geográfica / Geographical Research Letters in [volume and number, or year] by Universidad de La Rioja (Spain)".

- A link must be inserted to the published article on the journal's website by including the DOI number of the article in the following sentence: "The final publication is available at Cuadernos de Investigación Geográfica / Geographical Research Letters via http://doi.org/[DOI]".

\section{Cuadernos de Investigación Geográfica / Geographical Research Letters} A scientific journal of Physical Geography
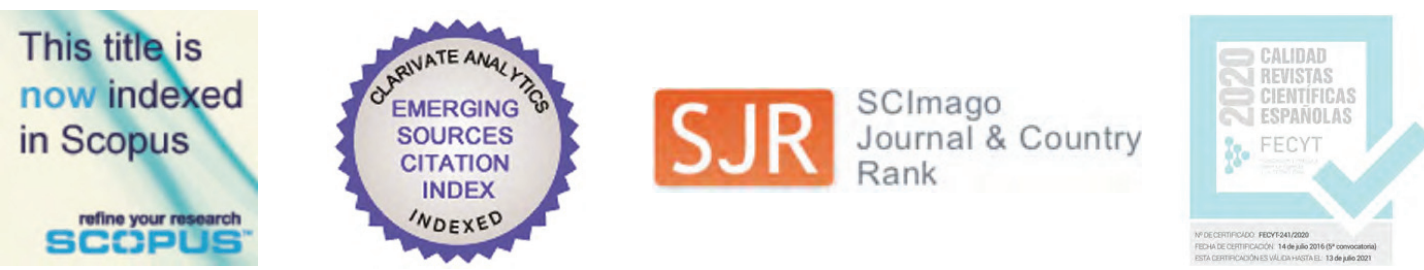


\section{CUADERNOS DE INVESTIGACIÓN GEOGRÁFICA GEOGRAPHICAL RESEARCH LETTERS}

TOMO 47 (2), Año 2021

Josep Bonsoms, Ferran Salvador-Franch, Marc Oliva. Snowfall and snow cover evolution in the Eastern Pre-Pyrenees (NE Iberian Peninsula)

Ana Paula Forte, Cristian Daniel Villarroel, María Yanina Esper Angillieri. Rock glacier and protalus rampart inventory in Las Salinas river basin, Central Andes of Argentina

Felix I. Contreras, Gian M. Mavo Mastretta, María C. Piccolo, Gerardo M. E. Perillo. Spatio-temporal variability monitoring of the floods in the center-west of the Buenos Aires Province (Argentina) using remote sensing techniques

Carolina Lauro, Alberto Vich, Stella M. Moreiras, Luis Bastidas, Sebastián Otta, Emilce Vaccarino. Regionalización del caudal máximo anual en cuencas del sistema hidrográfico del río Colorado, Argentina

Annick Delapierre, Juan A. Ballesteros-Canovas, Jorge Buzzi Marcos, Vera I. Slaveykova, Markus Stoffel. Trees as sentinels of metallic pollution induced by mining along the Odiel River (Southern Iberian Peninsula)

Michał Fojutowski, Piotr Gierszewski, Dariusz Brykała, Alicja Bonk, Mirosław Błaszkiewicz, Mateusz Kramkowski. Spatio-temporal differences of sediment accumulation rate in the Lake Gościąż (Central Poland) as a response of meteorological conditions and lake basin morphometry

Álvaro Tena, Damià Vericat, Ramón J. Batalla. Balance sedimentario del embalse de Ribarroja

Aarón Santana Cordero. Human-derived environmental consequences for three sedimentary systems of the Canary Islands (Spain) - a study of changes and impacts: a synthesis

María B. Alfonso, Andrea S. Brendel, Alejandro J. Vitale, María C. Piccolo. Impact of heatwave events on latent and sensible surface heat flux and future perspectives in shallow lakes based on climate change models

Teodoro Lasanta, Estela Nadal-Romero, Makki Khorchani, Asunción RomeroDíaz. Una revisión sobre las tierras abandonadas en España: de los paisajes locales a las estrategias globales de gestión

Francisco J. Vázquez Pinillos, Manuel J. Marchena Gómez. Territorial impacts of sea-level rise in marsh environments. The case of the Bay of Cádiz, Spain

Parviz Jokar, Masoud Masoudi, Fatemeh Karimi. An MCE-based innovative approach to evaluating ecotourism suitability using GIS 